UC-20

Reporting Date: July 1975

Issued: January 1976

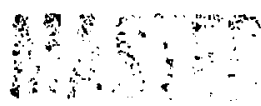

\title{
Conceptual Design Study of a Scyllac Fusion Test Reactor
}

\author{
Compiler
}

Keith 1. Thomassen

Contributors

James L. Anderson

Sumner Barr

Robert Barthclomew

David Blevirs

William Bradiey

Emmett Brazier

Richard Browning

James Dickinson

Donald Dudziak

Siegfried Gerstl

Roy Haarman

Kenneth Hanks

Lash Hansborough

Donald Houck
Franz Jahoda

Roland Jalbert

Donald Keigher

Edwin Kernp

Charles R. King

David Kirby

Robert Krakowski

John Lillberg

Ruion Linford

Thomas McDonald

Clair Nielson

William Nunnally

Thomas Oliphant

H. Don Orr
William Purtymun

John Rand

John Rogers

Donaild Sandstrom

Richard Siemon

Walter Stone

Clarke Swannack

Keith Thomassen

Robert W. Turner

Dwayne Vier

Herbert Vogel

John L. Warren

David Weldon

K. E. Williamson, Jr. 
Work supported by the Division of Controlled Thermonuclear Reseatch, U.S. Energy Revearch and Development Administration.

Printed in the United States of America. Avallable from

U.S. Department of Commerce

6285 Port Royal RoRd

Price: Printed Copy $\$ 8.76$ Microfiche $\$ 2.25$

Thin report whe prepared as en tecount of work sponoored by the Uniled Stater Government. Neither the United State

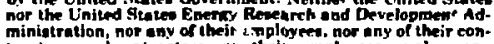
ministration, nor eny of (hej 2 Tployeren, nor any of their conwhrranty, exprean or imglied, ur nomumet any tecal liability of

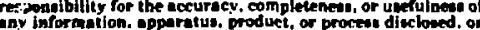

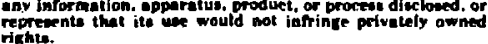




\section{SCYLLAC FUSION TEST REACTOR}

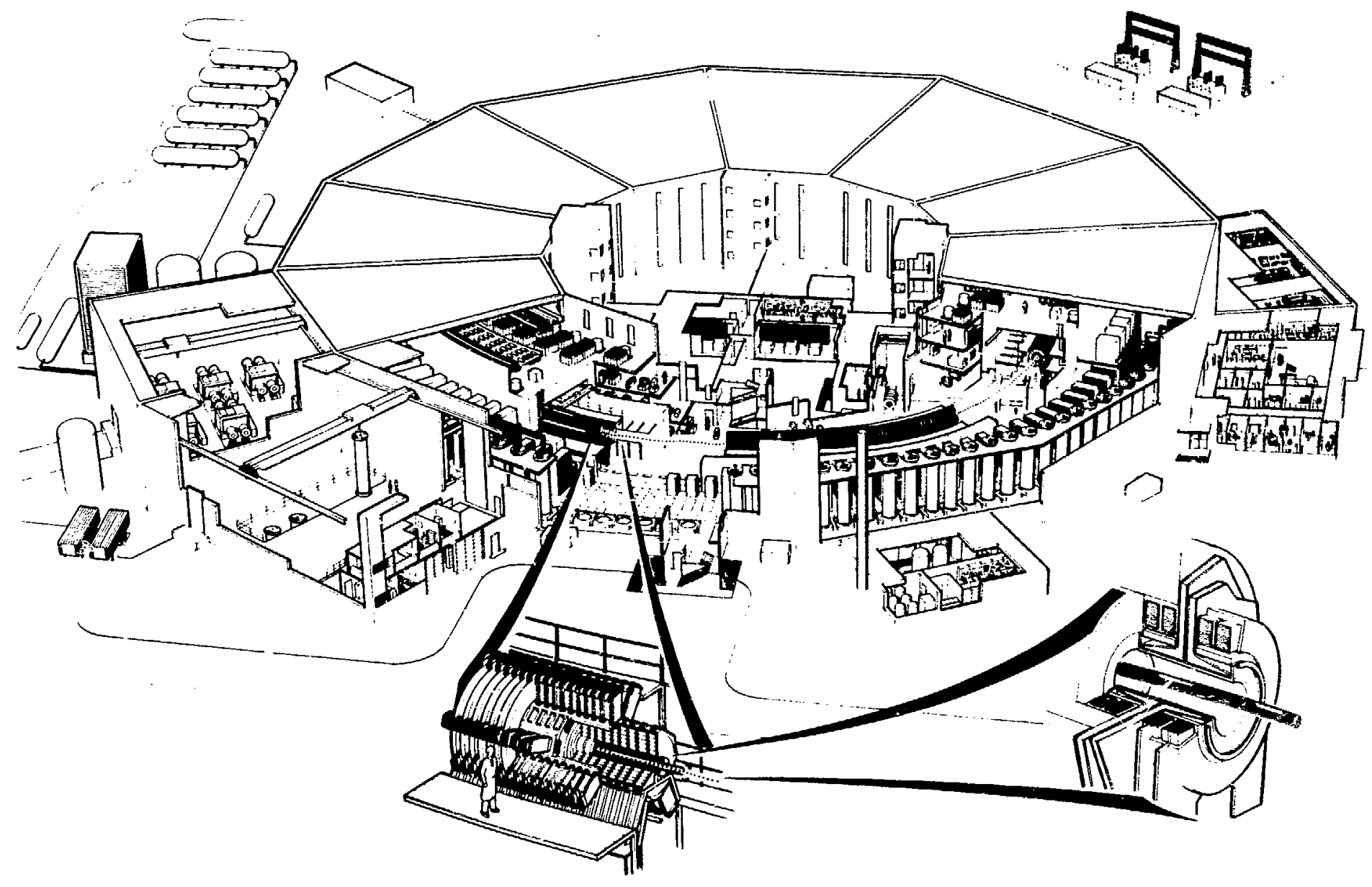




\section{CONTENTS}

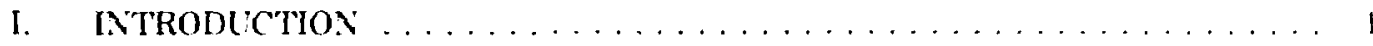

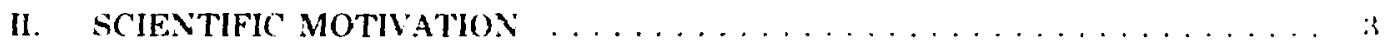

2.1 Background . . . . . . . .

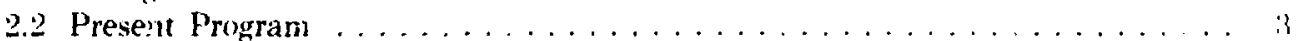

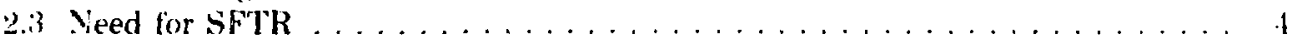

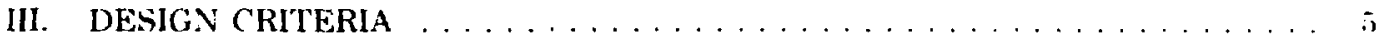

IV. THEORETICAL CONCEPTS $\ldots \ldots \ldots \ldots \ldots \ldots \ldots \ldots \ldots \ldots \ldots$

4.1 Objectives .......................

4.1 .1 General Description .................. T

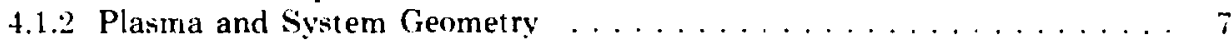

4.1 .3 System Requirements ................. 7

4.1 .3 .1 Definition and Signiticance of $Q \ldots \ldots \ldots \ldots$

4.1.3.2 Plasma Equilibrium and Stability . . . . . . . . . . .

4.1 .4 Proposed Approach . . . . . . . . . . . . . . . . . . . . . 9

4.1.4.1 Staging Concept of Plasma Heating . . . . . . . . . .

4.1.4.2 Wall Stabilization and Slow Feedhack Control . . . . . . . . . 12

4.1.4.3 Operating Point Determination . . . . . . . . . . . . . 14

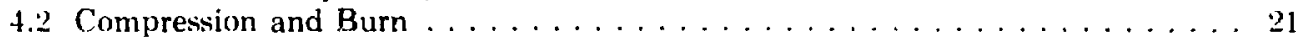

4.2 .1 General Description . . . . . . . . . . . . . . . . . 21

4.3 .2 Analytic Model . . . . . . . . . . . . . . . . . . . 21

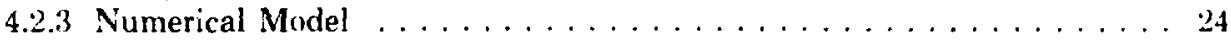

4.3 Implosion Heating . . . . . . . . . . . . . . . . 24

4.3.1 General Description ... . . . . . . . . . . . . . . . . 24

4.3 .2 Resonant Heating Concept . . . . . . . . . . . . . . . 24

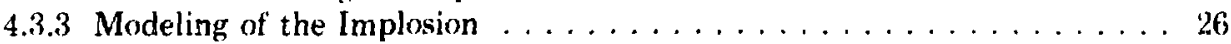

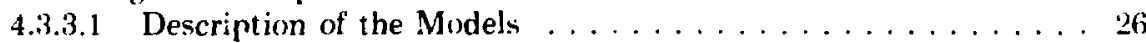

4.3 .3 .2 Initial Implosion . . . . . . . . . . . . . .

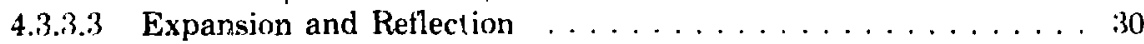

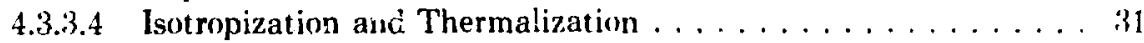

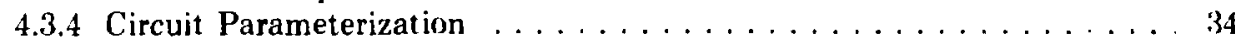

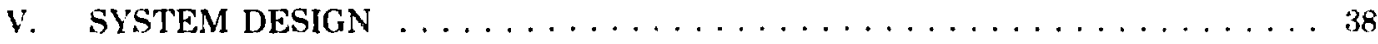

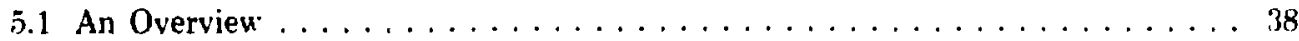

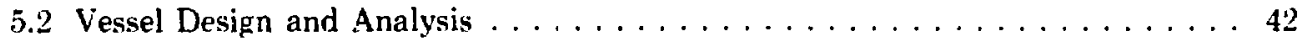

5.2 .1 Summary . . . . . . . . . . . . . . . . . . . 42

5.2 .2 Engineering Design of Containment System $\ldots \ldots \ldots \ldots \ldots \ldots \ldots 42$

5.2.2.1 Flectrical Aspects of Vessel Design . . . . . . . . . . . 42

5.2 .2 .2 Thermal Analysis of Containment System . . . . . . . . . . 42

5.2.2.3 Stress Analyses of Containment System . . . . . . . . . . . . 50

5.2 .3 Fabrication of Containment Vessel . . . . . . . . . . . . . . . 59

5.2.3.1 Implosion-Heating Coil/Vacuum Vessel . . . . . . . . . 59

5.2.3.2 Magnetic Flux Concentrator . . . . . . . . . . . . . . 59

5.2.3.3 Mechanical Design and Fabrication of the Compression Coil . . 60

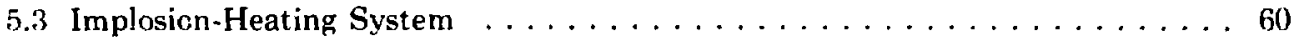

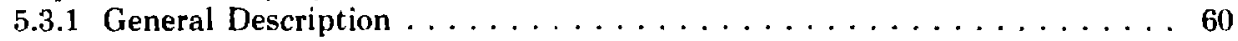

5.3.2 Capacitor and Feedplate Design . . . . . . . . . . . . 61 


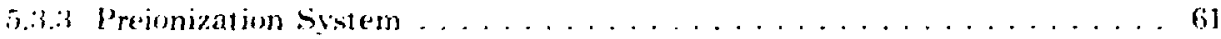

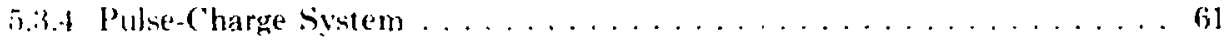

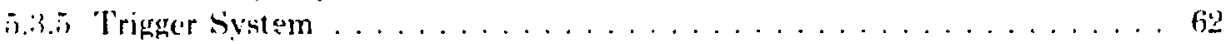

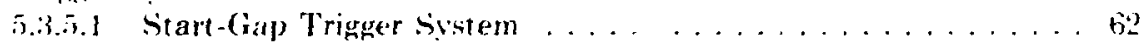

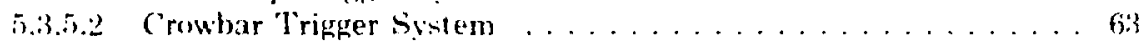

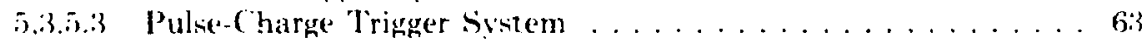

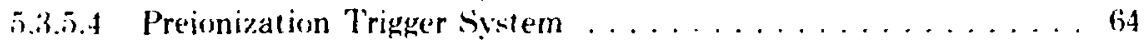

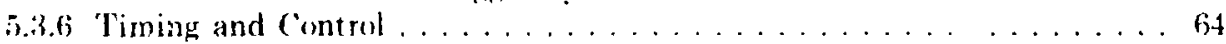

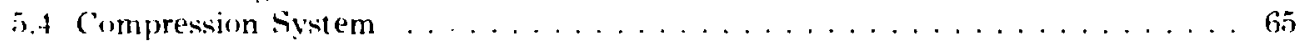

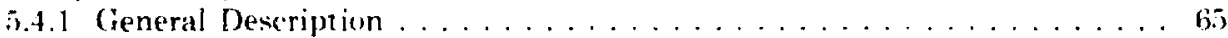

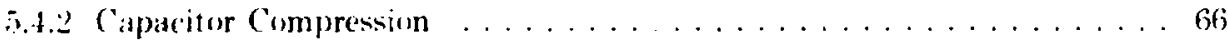

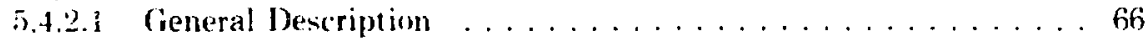

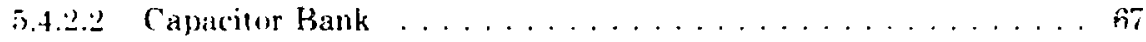

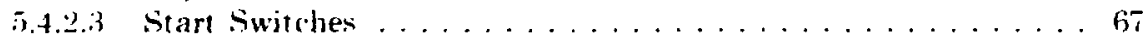

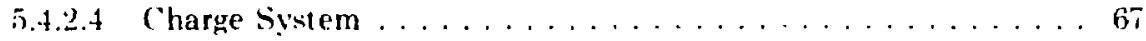

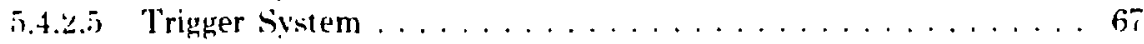

¿.4.3 Magnetic Fnergy storage ..................... 68

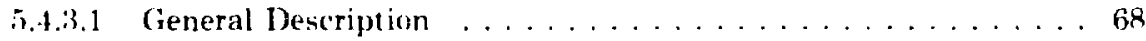

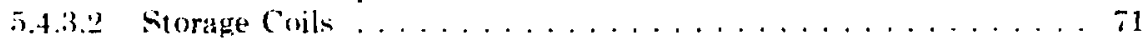

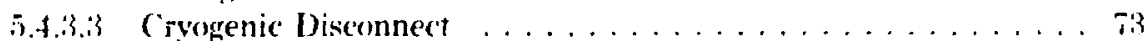

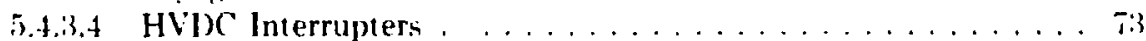

$5.4 .3 .5 \quad$ Interrupter Counterpulse system $\ldots \ldots \ldots \ldots$

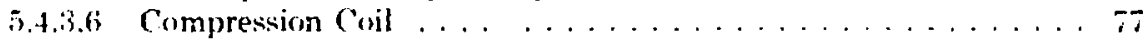

5.4 .3 .7 Fast and Slow Compression Cril Crowhar Switches . . . . . . is

5.4 .3 .8 Charging Power Supply for Storage Coils . . . . . . . . . . . 88

5.4.3.9 Counterpulse Storage Power Supply . . . . . . . . . . . . 79

i.4.3.10 Capacitor Transfer Bank .................... 79

5.4 .3 .11 Electrical Leads . . . . . . . . . . . . . . . 79

5.4.3.12 Component Connections and Cabling ............. 80

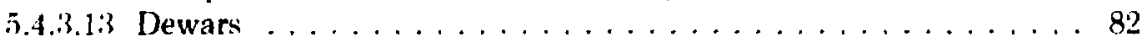

5.4 .3 .14 Liquid and Gas Distribution Systems . . . . . . . . . 84

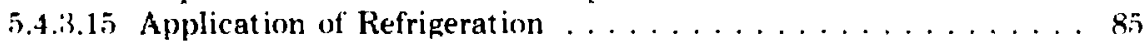

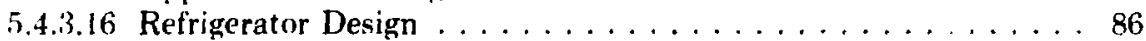

$\$ .4 .3 .17$ Utilities, Cryogen. and Gas Suppiies . . . . . . . . . . . 87

5.4 .3 .18 Cryogenic System Operation ................. 89

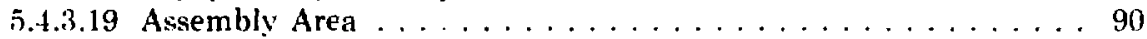

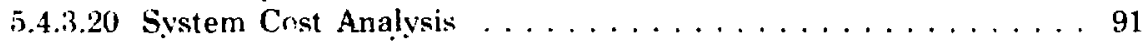

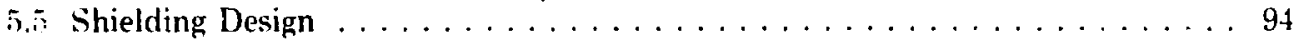

5.5 .1 Design Ohjectives ......................... 94

5.5 .1 .1 Biological Shield Design Criteria . . . . . . . . . . . . 94

5.5.1.2 Activation and Radiation Damage Criteria . . . . . . . . . . 94

5.5 .2 Shielding Concept and Layout $\ldots \ldots \ldots \ldots \ldots \ldots$

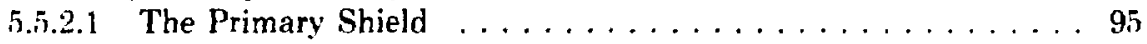

5.5 .2 .2 The Secondary, Biological Shield ............... 97

5.5 .3 Radiation Transport Analysis and Results . . . . . . . . . . . . . . 98

5.5.3.1 Method of Analysis and Nuclear Data . . . . . . . . . . . . . . . . . . . .

5.5.3.2 Computational Results ...................... 99

5.6 Tritium Handling System . . . . . . . . . . . . . . . . . . . 104

5.6.1 Overview of Tritium Handling System Function, Design

Requirements, and zayout ................... 104

5.6.2 Physical, Chemical, and Radiological Nature of Tritium . . . . . . . 105

5.6.3 Description of Subsystems . . . . . . . . . . . . . . . . . 107

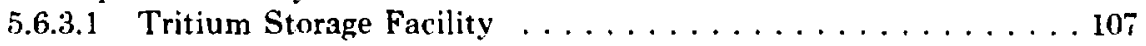

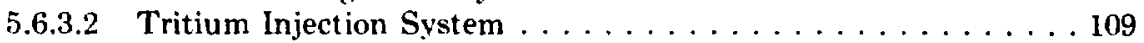

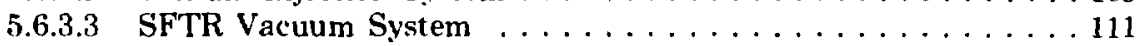


5.6.3.t Tritiam Recovery System . . . . . . . . . . . 118

5.6.3.5 SFTR Cleanup Systems. . . . . . . . . . . . . . . 119

5.6.4 Instrumentation, Control. and Gperational Procedures . . . . . . . . 1:4

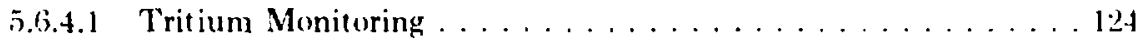

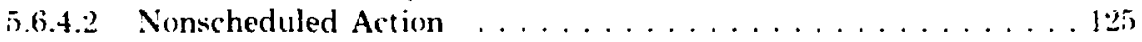

5.6.4.3 Postshot Diagnosis . . . . . . . . . . . . . . . . 12:

5.6.4.4 Maintenance of Tritium Handling Equipment . . . . . . . . 127

5.7 Diagnostics . . . . . . . . . . . . . . . . . . . . . . . . . . . 1:30

5.7 .1 Introduction . . . . . . . . . . . . . . . . . . . .

5.7.1.1 Experience at Los Alamos . . . . . . . . . . . . . . .:30

5.7.1.2 Importance of Diagnosties . . . . . . . . . . . . . . 131

5.7.1.3 Increased Role of Automatic Data

Processing and Its Significance . . . . . . . . . . . . .

5.7.2 Basic Measurements and Equipment Required . . . . . . . . . 1:31

5.7.2.1 Plasma Luminosity for Profile and Location

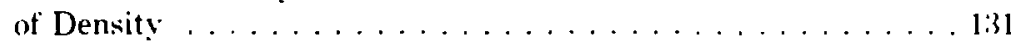

5.7.2.2 Neutron Detectors for Ion Tenıperature . . . . . . . . . 1333

5.7 .2 .3 Interferometry for Plasma Density . . . . . . . . . . 1:333

5.7.2.4 Light Scattering for Temperature and

Density Profile . . . . . . . . . . . . . . . . 13:4

5.7.2.5 Excluded Flux Loops $\ldots \ldots \ldots \ldots \ldots \ldots \ldots \ldots \ldots \ldots$

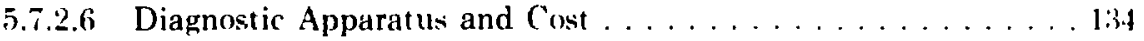

5.7.3 Advanced Techniques for Deveinoment . . . . . . . . . . . . 1:35

5.7.3.1 Light Scattering at 10.2 Microns . . . . . . . . . . 135

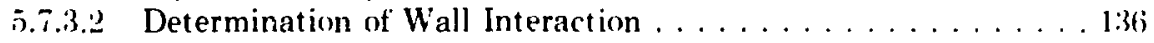

5.7.3.3 Veutron Measurements . . . . . . . . . . . . 1.36

5.7.4 Predicted Bremsstrahlung Level of Luminous

Radiation in SFTR . . . . . . . . . . . . . . . . . . . . .

5.7 .5 Predicted Intensity for Thomson Scattering $\ldots \ldots \ldots \ldots \ldots \ldots$. . . . . . .

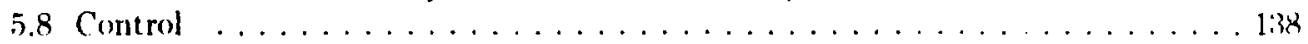

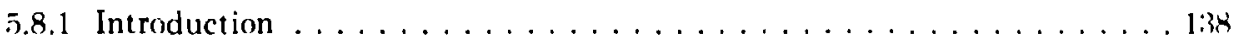

5.8 .2 System Design . . . . . . . . . . . . . . . . . . . 1:39

5.8 .2 .1 Tritium Handling and Vacuum system . . . . . . . . 140

5.8 .2 .2 METS Refrigeration System . . . . . . . . . . . 140

5.8 .2 .3 METS Energy Transfer System . . . . . . . . . . . 141

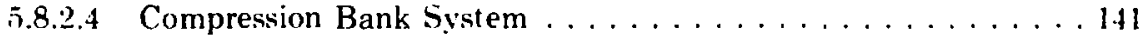

i.8.2.5 Implosion-Heating System . . . . . . . . . . . . . . 141

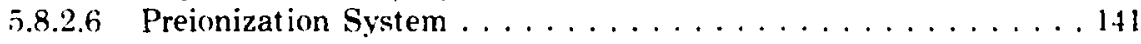

5.8 .2 .7 Diagnostics System . . . . . . . . . . . . . 141

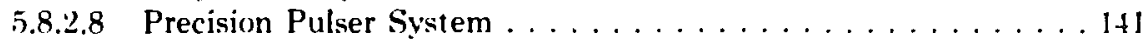

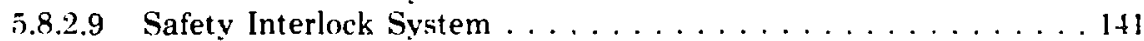

5.8 .3 Basic Computer Design . . . . . . . . . . . . . . . 142

5.8.3.1 Computer Protection . . . . . . . . . . . . . 142

5.8.3.2 Standard Nomenclature . . . . . . . . . . . . . . . . . 142

5.8.3.3 Main Control Computer and Operator Consoles . . . . . . . . 144

5.8.3.4 Cathode Ray Tube Display System Computer . . . . . . . . 144

5.8.3.5 Precision Pulser (Timing) Syst ¿m . . . . . . . . . . . . 144

5.8 .4 Software Development Machine $\ldots \ldots \ldots \ldots \ldots \ldots \ldots \ldots \ldots \ldots \ldots$

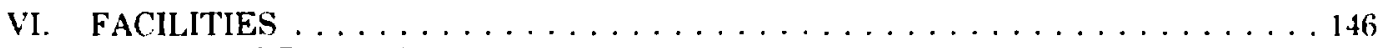

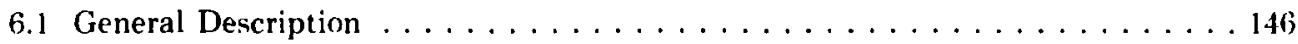

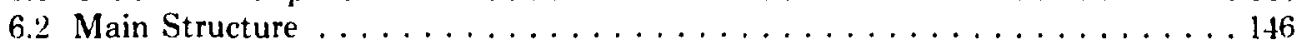

6.3 METS Refrigeration and Maintenance Area $\ldots \ldots \ldots \ldots \ldots \ldots 1$

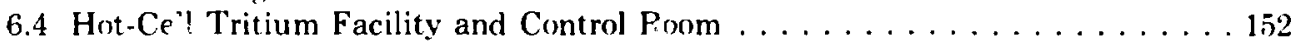

6.5 General Maintenance and Administrative Areas . . . . . . . . . 152 


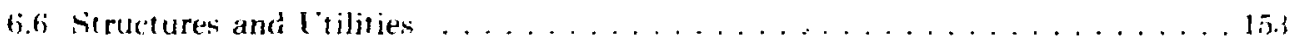

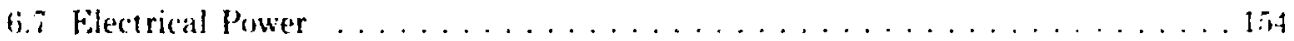

6.T.1 Power Requirements . . . . . . . . . . . . . . . . . .154

6.7.2 Impact on Flectric Power System . . . . . . . . . . . . . . 15ist

6.7.3 Power Distribution Sistem . . . . . . . . . . . . . . . . 15:

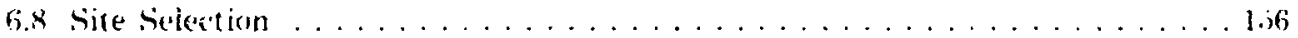

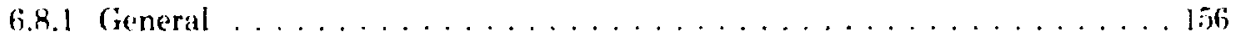

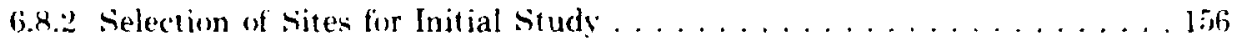

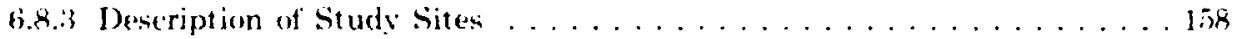

6.8.4 Site Selection . . . . . . . . . . . . . . . . . . . 159

VII SAFETY AND ENVIRONMENTAL CONSHDERATIONS $\ldots \ldots \ldots \ldots \ldots$. .

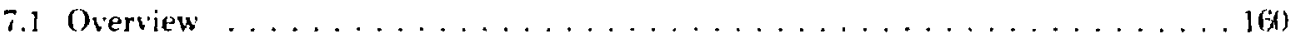

7.2 Description of SFTR Site . . . . . . . . . . . . . . . 160

T.2.1 Demographic Description . . . . . . . . . . . . . . . 160

7.2 .2 Meteorological Deseription . . . . . . . . . . . . . . 16:3

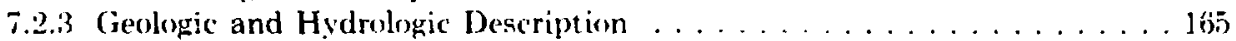

7.2 .4 Access and Security Aspects . . . . . . . . . . . . . 166

7.3 Safety and Environmental Considerations During Normal Operation . . . . 166

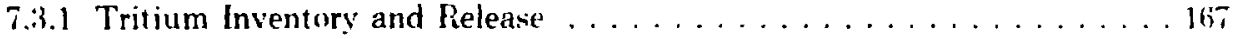

7.3.1.1 Tritium Control and Inventory Procedures . . . . . . . . . 168

7.3.1.2 Tritium Monitoring Procedures . . . . . . . . . . . . . 169

T.3.1.3 Radiation Dose Rate Resulting from

Routine Tritium Releases. . . . . . . . . . . . . . . . 170

7.3 .2 Direct Neutron Irradiation Exterior to the SFTR Coll $\ldots \ldots \ldots \ldots \ldots 171$

7.3.3 Veutron Activation of Equipment. Cell Atmosphere.

and Shielding . . . . . . . . . . . . . . . . . . . . .

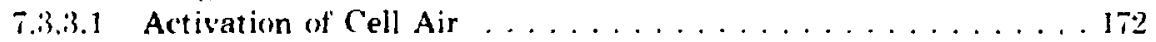

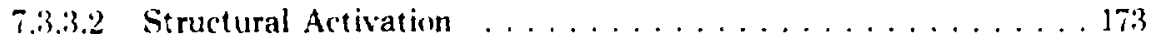

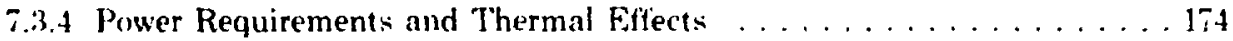

7.3 .5 Radioactive Waste . . . . . . . . . . . . . . . . . . . 175

7.3 .6 Chemical, Sanitary and Other Wastes . . . . . . . . . . 175

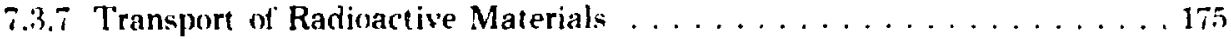

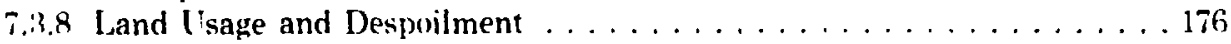

7.4 Safety and Environmental Considerations During Otf-Normal

or Accident Conditions . . . . . . . . . . . . . . . . . 176

7.4.1 Consequences of a Sudden and Total Release of Tritiun . . . . . . . 176

7.4.2 Catastrophic Failure of Discharge Tube During a D.T

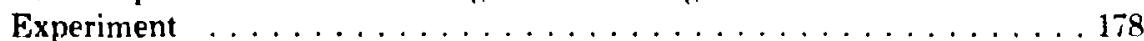

7.4 .3 Fire Hazards within the Cell $\ldots \ldots \ldots \ldots \ldots \ldots \ldots$

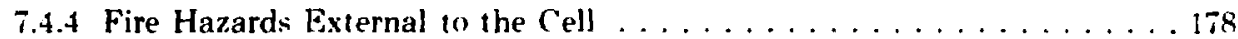

7.4.4.1 Fire within Tritium Storage and Treatment

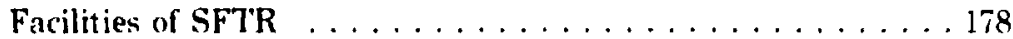

7.4.4.2 Fires Associated with the Transfer Capacitor System . . . . . 179

7.4.4.3 Hydrogen Explosions . . . . . . . . . . . . . . . . . 179

7.4.5 Magnet Malfunctions . . . . . . . . . . . . . . . . 181

7.4.6 Failure of Cryogenic Energy-Storage and Transfer

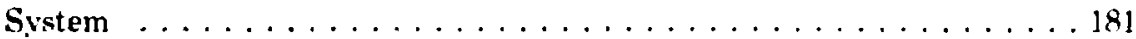

7.4.7 Fuel Injection Accidents . . . . . . . . . . . . . . . . 182

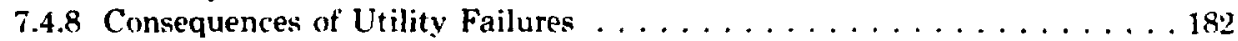

7.4 .9 Unusual Occurrences . . . . . . . . . . . . . . . . . 182

7.5 Summary and Conclusions $\ldots \ldots \ldots \ldots \ldots \ldots \ldots$

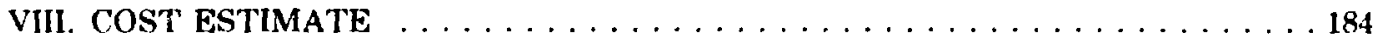

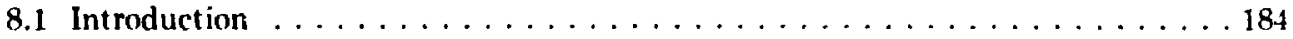




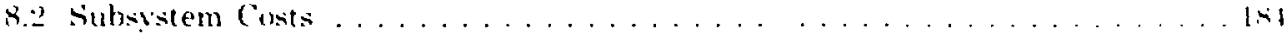

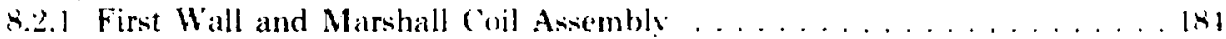

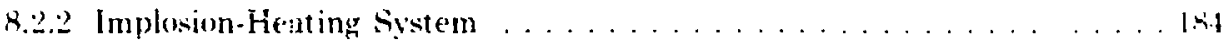

8.2 .3 Prelonization System . . . . . . . . . . . . . . . . . .

8.2 .4 Primary stield . . . . . . . . . . . . . . . . . L Lis

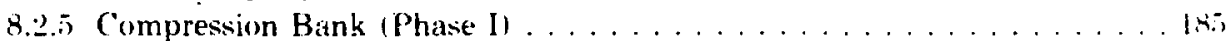

8.2 .6 METS (Phase Il) . . . . . . . . . . . . . . . . . . . Isti

8.2.7 Tritium Handling and Vacuum system . . . . . . . . . . L Rit

8.2 .8 Diagnostics Sustem . . . . . . . . . . . . . . . . . . KRi

8.2 .9 Control system . . . . . . . . . . . . . . . . . Liti

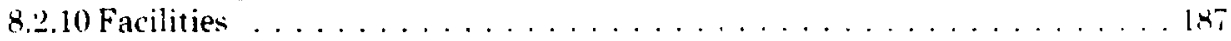

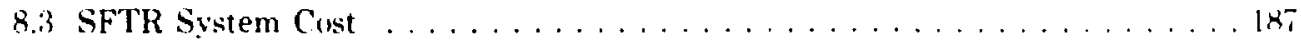

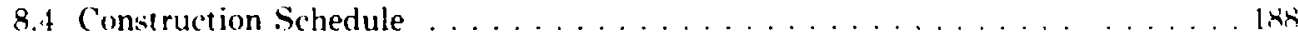

APPENDIX I. ROOM TEMPERATT RE MAGNETIC ENERGY STORAGF, . . . . 1SM

A.1 Summary . . . . . . . . . . . . . . . . . . . . . . . . . 190

A.2 Circuit Description . . . . . . . . . . . . . . . . . . 1901

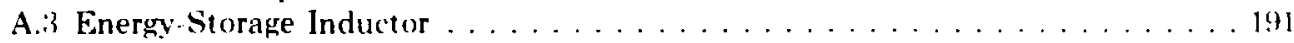

A.3.1 General Description . . . . . . . . . . . . . . . . . . 191

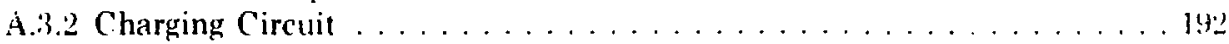

A.3.3 Flux Extraction . . . . . . . . . . . . . . . . . . 19.4

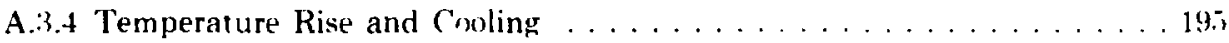

A.3.5 Structural Considerations . . . . . . . . . . . . . . 1966

A.5.5.1 Torus Inner Support Structure . . . . . . . . . . . . . 1965

A.3.5.2 Hoop Stress in Coil . . . . . . . . . . . . . . . . 197

A.3.5.3 Cradle Support Design for Foundation of Toroid . . . . . . . 197

A.3.6 Optimization . . . . . . . . . . . . . . . . . .

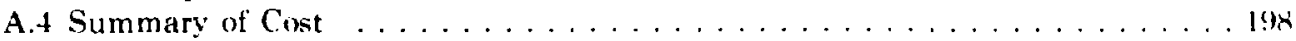

A.i Operational Considerations $\ldots \ldots \ldots \ldots \ldots \ldots \ldots \ldots$

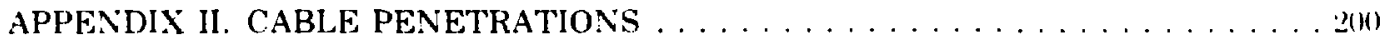




\title{
CONCEPTLAL DESIGN STUDY OF A SCYLLAC FUSION THST REACTOK
}

\author{
Compiled br
}

\author{
Keith I. Thomassen
}

\section{INTRODUCTION}

The report describes a conceptual design study of a fusion test reactor based on ine Scyllac toroidal theta-pinch approach to fusion. It is not the first attempt to describe the physiss and technology reyuired for demonst rating scientili, frasibility of the approach. but it is the most complete design in the sense that the physics necessary to achieve the device goals is extrapolated from experimentally tested MHD theories of toroidal systems, and it uses technological systems whose engineering performance has been carefully calculated to ensure that they meet the machine requirements.

Developmental effort is required in some of the systems. and the extrapolations from presently understood MHD theory and experiment are such that an intermediate device of large major radius is clearly indicated before construction of the Scyllac Fusion Test Reactor (SFTR. The major reason for this early conceptual design is, in fact, to bring into focus the issuts in both the theory and technology of the device. In this way, developmental guidance will be available for decisions on technological programs. and the course to be pursued by the experimental physics program will be more sharply defined.

An earlier study ${ }^{l}$ proposed a number of the central ideas, such as the separated shock and superconducting energy storage. Here we build on these ideas. give them substance using current knowledge of plasma physics, and propose a complete engineering design.

The machine is designed to achieve one central objective. to demonstrate "breakeven" or feasibility of the concept. The objective can be and has been defined many ways. The first study used the Lawson criterion" to define feasibility. As the SFTR is a pulsed high- $\beta$ machine, one could also define breakeven during some arbitrary time interval during the burn and require the average power output from neutrons to balance the average plasma power loss by radiation and other energy transport mechanisms. For example, the peak neutron power output is $\sim 110 \mathrm{MW}$, while the bremsst rahlung is $\sim 7$ MW. Internal plasma Josses are $\sim 20) \mathbf{M W}$ ifor classical diffusion losses), hence we mort than break even at peak compression. The most restrictive definition, which we adopt here, is that the total energy output in neutron:- during the pulse (2 M.J) equals the stored energy in the plasma lat its ma:imum. In so doing we exceed the Lawson criterion.

To meet the design objective. subject to restrictions on equilibrium. stability. and high voltage technology, a large aspect ratio (major to minor radius) machine is needed. After some (rial designs. a $40-\mathrm{m}$ major radius, $10-\mathrm{cm}$ minor radius device was chosen. The minor radius is chosen so as to have a reasonable volume of piasma between the 2- to 3 cm-thick sheaths which form on implosion. The major radius was also chosen somewhat arbittarily after a preliminary assessment to determine an approximate minimum value.

On completion of this design it was heartening to note that only a few untested ideas or technologies stand between the present program and SFTR. These include wall stabilization of the $\mathrm{m}=1$ mode. fabrication of the end-fed Marshall coil for implosion heating, and the large superconducting Magnetic Energy Transfer and Storage (METS) system. The last of these can be replaced by very conventional systems. such as capacitors, or by simplified inductive energy storage (room temperature, with motorgenerator or line charging). Of course, plasma transport on the millisecond time scale. if it is much faster than classical theory dictates, may a!so be a problem. However. these studies are to be part of the experimental program for SFTR. 


\section{REFERENCES (Chap. I)}

1. F. L. Ribe, "Parameter Study of a Lung. Separated Shock $\Theta$-Pinch with Superconducting Inductive-Energy Storage," Los Alamos Scientific Laboratory Report LA-4828-MS (December 1971).
2. J. D. Lawson. "Some Criteria for a Power Producing Thermonuclear Reactor," Proc. Phys. Soc. (Lomdon) B70, 6 (1957). 


\section{SCIENTIFIC MOTIVATION}

\subsection{BACKGROUND}

Theta-pinch research. since its inception in the inid-1950)'s, has been centered at los Alamos. The earliest device. Sovlla, was a $10-\mathrm{cm}$ linear device driven by a $30 . k$.j capacitor bank. The linear work culminated with scylla-IV, a $1-m$ linear machine in which $\sim 4-k e l$ ions at $\beta \sim 0.8-1.0$ were produced.

Find losses dominated the linear pinches, so the closed Scylla, known as Scrllac. was first proposed in 1966. To provide an equilibrium in toroidal geometry the MdS bumpy torus was first proposed, hut stability analyses led to the chroice of the more favorable stellarator conf:guration with $/=0.1$ tields providing equilibrium.

The first experiments, in April 1971, were with a 5 . $m$ arc of the 2.4-m-radius machine. These experiments demonst rated the existence of the toroidal equilibrium. but plasma was lost to the ends and by gross motion to the wall. The wall motion was either by loss of equilibrium as a changed (with plasma end-coolingl. or by an $m=1$ mode as predicted therretically. There was insufficient evidence to distinguish between the two.

The next experiments, in 1973), on an $8 . \mathrm{m}$ sector of a 4-m-radius torus, verified the MHD scaling of the plasma equilibrium with major radius. Again. wall motion was observed but its cause could not be unambiruously identified. Conversion to the rull Scyllac arus and operation of the device began in April 1974.

ro control the unstable $m=1$ gross motion of the plasmd, the first scheme envisioned was to oscillate the magnetic field for dynamic stabilization. The required amplifier power was quite large, so the idea was soon rejected. Since 1970, a program to develop feedback control amplifiers for Scyllac has been in progress. These amplifier: drive auxiliary $/=0$ coils in the $i=0.1$ system, or $t=2$ coils in an $t=0.1 .2$ system.

The main results of experiments on the full torus without feedback were to confirm the existence of the $m=1$ roode and verify theoretically predicted MHD growth rates of the toroidal mode numbers $n$ (around the torus). The growth rates decrease with increasing $n$, with $\mathrm{n}=6$ being the last unstable mode.

One further result was that the maximum growth time equaled the delay time of the feedback amplifiers, which is the limiting condition for a sucressful feedhack esptriment. At this limit. stability can be achieved only if the initial position and velocity of the plasma are zero wher the amplifiers are switched on. To accommodate a realistic range of initial conditions. the growth rate times delay time should be $-1 / 3$ rather than unity. Consequently, the $t=0,1$ wavelength. $\delta$. and toroidal field were changed to lower the growth rate by a factor of :3. A sector of the derated machine has been operated. contirming the expected lower growth rate. and feedback amplifiers are being installed.

\subsection{PRESENT PROGRAM}

The coroidal theta-pinch program presently has several components. The major experiment of course is Scyllac. and feedback experiments on the derated sector will soun be under way. Another major direction centers on implosion and staging experiments. designed to create hot, wall-stabilized plasmas. Wall stabilization requires a large ration of plasma-to-wall radii la/bl. In scyllac. implosion and compession are inseparahle, and the plasmas are too far from the wall for stability.

Implosion heating with a ver: fast rising magnetic field leaves the plasma radius relatively large. but to bring the tield up in a time which is short compared to the time for the plasma boundary to move to its minimum radial position requires very high voltage circuits. The technology of producing the required magnetic field waveforms is being pursued in the Staged Theta Pinch (STP) experiment.

Experiments aimed at understanding the physics of the implosion process are being pursued both in STP and in the Impiosion-Heating Experiment (IHX). In the latter. one produces. as nearly as possible. a step function magnetic field waveform in order to tompare the dynamics of the process with predictions from plasma simulation codes and other plasma models.

The Scyllac program is expected to culminate in a Large Staged Scyllac (LSS) experiment. In LSS. the major radius will be large $(\sim 30 \mathrm{~m})$ to reduce the MHD growth rates and to allow for wall stabilization of the imploded. staged plasma. The ratio a/b which provides for wall stabilization can be smailer for larger major radii. hence more adiabatic compression heating is permitted. 


\subsection{NEED FOR SFTR}

The present Scyllac program is expected to terminate with LSS in which gross MHD stability (to $m \geq 1$ mode) is to be demonstrated. The Scyllac program as such $h$ wil have tested the salient features of the tornidal higl: $-\beta$ stellarator configuration. It remains to show long-term stability and confinement, to reach ignition temperatures, and meet Lawson or breakeven conditions.

The achievement of the Lawson criterion has long been recognized as a milestone toward pure fusion systems. Its achievement is a significant feat, not only for the physics it represents, but for the technology it implies. In the Tokamak program this step lies beyond the Tokamak Fusion Test Reactor (TFTR), but short of planned Engineering Power Reactors (EPR's).

To meet the Lawson criterion one must burn a $\mathrm{D}$ $T$ fuel, a step of major signiticance for CTR. The intended use of tritium in an experiment has major consequences in the design of the machine, particuiarly in handling and containment of the tritium. Interestingly, the amounts to be handled are not large, and the techniques for doing so are rather straightforward extensions of present practice at Los Alamos.
A more significant impact of D-T burning is the neutron production and associated activation of structures in the machine. The shielding, remote handling, and safety aspects of the device require very special care and zidd to the cost and complexity of the rnachine. 'Thus, D-T burning is an important step for the program, to be faced for the first time in SFTR, and links the present physics program with the Reference Theta Pinch Reactor (R'TPR) and intermediate experimental reactors.

In the theta-pinch program, the experiment is needed to investigate long-term confinement. beyond the 1000 - to $300-\mu \mathrm{s}$ time scale of LSS, to the tens of milliseconds, where localized MHD modes or enhanced transwort may be important. Also, several new technological systems associated with the longer time scales of the experiment must be tested on the scale of SFTR. The intplosion-heating circuitry, including the Marshall coil and ferrite isolation, involves departures from present systems and requires a system test. The METS superconducting system will receive its first large-scale test in SFTR.

The merging of physics and technology in SFTR to achieve breakeven is the primary purpose for the device. The results of the work there form the basis for further steps toward power-producing fusion reactors based on the Scyllac principle. 


\section{DESIGN CRITERIA}

There is one primary onjective of the design-the achievement of' breakeven. However, a number of secondary criteria which have influenced tha design are described in this section, as is the primary goal.

We first define $Q$, the ratio of thermonuclear output energy to the maximum internal plasma energy $w$ w

$$
Q(t)=\frac{\int_{0}^{t} P_{n}(x) d x}{W_{p n}}
$$

where $P_{n}$ is the power out put from neutrons, taken at $20 \mathrm{MeV}$ per neutror, to account for released binding energy in the structure around the plasma. As the burn proceeds, $Q$ increases, and the objective of breakeven is defined as reaching $Q=1$, which occurs near the end of the pulse.

Another criterion is to minimize the machine cost. and this is done intuitively by limiting the major radius. However, a larger aspect rat in gives a greater stability margin. hence a delicate balance must be struck. The major radius is alsu dependent on the technique chosen for MHD stability, with wali stabilization permitting the least compression. hence requiring a larger radius to maintain the required aspect ratio. If feedback control of the $m=1$ mode were feasible, and we believe it may be, a snaller machine could be constructed to reach $Q=$ 1. However. this appears to be the less conservative of the two approaches to stability.

The implosion-heating system involves a number of tradeoffs, centering on the high voltage requirements to achieve significant initial temperatures. One cannot rely almost entirely on slow compression heating since the plasma would be overcompressed for stability. Also. Jow initial temperatures permit the field 10 diffuse into the plasma. Finally, too much adiabatic compression implies a large field-to-plasma volume, hence a large magnetic energy to plasma energy ratio. Such a scheme does not extrapolate well to a reactor, which must minimize the amount of puised magnetic energy.

The efficiency of energy transfe: by implosion is significarstly higher than the efficiency of transfer of adiabatic compression energy. Thus, it would appear that one should do as much implosion heating as possible, limited only by high volt age constraints. In fact, one should ninimize the implosion- heating energy consistent with maintaining a large plasma radius for stability, and consistent with avoiding too large a compression supply. In so doing. one arrives at a system with voltages near the technological limit.

One technique for getting higher azimuthal electric field st rengths in the implosion coil is to use parial turn coils. In this way a given voltage produces more field. hence more implosion heating. The limit to this method is the internal and source induct ance of the structure, which should tot exceed the load inustance. $A$ partial turn coil to produce a toroidal weld, which could be fed from one end, was devised by Whn Marshall at Los Alamos, hence is called a Marshall coil.

Since the implosion coils are fed by radial feed plates at the ends, the structures resemble nested top hats, with current entering the brim. flowing along the outer hat, back on the inner hat, and out the other brim. The current in the Marshall coil is forced $t o$ follow helices on the hats by slotting them in a helical manner, thus producing only toroidal fields inside the structure. The fabricability of this structure, which serves as a first-wall vacuum (and tritium) barrier is one of the more difficult technical developments required in SFTR. There also are limirations on the voltages it can withstand. hence the amount of implosion heating is limited.

The compressional energy source for SFTl can he one of a variety of systems, and we present here a discussion of these choices. The choices are the con. ventional capacitor bank and many forms of inductive energy-storage systems. At first glance, the 500 M.J requirement would appear to weigh heavily in favor of inductive systems, since the multiplic to of switches and timing circuits in the capacitor bank gives that scheme a reliability problem. However. the inductive energy sustem must also be modularized because of limitations set by current interrupting switches. The limitations come from the fact that the current tis be interrupted, multiplied by the voltage on the switch after interruption. is approximately equal to the eneigy to be transferred. divided by the transfer tine. Since the voltagecurrent product is a function of the switch characteristics. and the transfer time is fixed, a maximum energy is set by the switch rating. For SFTR. by using two parallel and two series switches, the composite rating of $25 \mathrm{kA}$ times $60 \mathrm{kV}$ limits the energy to $381 \mathrm{~kJ}$, therefore requiring 1280 modules. 
Although the multiplicity of components in the in ductive sistem diminishes its inhesent advantage of high-energy density storage, we believe the system still has an edge over pure capacitor systems. Further, the energy requirements of fut ure systems almost surely dictate inductive systems. so early development for SFTR should be fruitful.

Varrous forms of inductive storage and transfer sistems were considered. These include superconducting storage, with either superconducting switches or vacuum interrupters, and room temperature or nitrogen-cooled copper or aluminum storage coils, charged from the line, from motorgenerator sets, or from a homopolar machine. The option of resonant transfer through a capacitor bank or straight transier from the storage coil was also considered. The former allows nearly $100^{\circ} ;$ efficiency of transter. while the latter necessarily dissipates $50^{\circ} ;$ in the switch and leaves $25^{\circ} ;$ in the store. Thus, it requires four times the initial storage. Resonant transfer requires a capjacitor bank with half the storage capacity of the inductive store. but cost comparison favors the resonant system. Since there are no start switches and trigger systems for the ban'i. the reliability is not in question.

The system we chose uses superconducting energy stcrage, vacuum interrupters, and the resonant transfer scheme. This system makes the least demand on charging supplies and has the most favorable impact on future technology for thetapinch devices. It is neither the least expensive nor the most straightforward system to develop. Should we run into technical diffieulties in superconducting develc pment, we have designed a conventional room tempercture inductive storage system with line charging. That design is described in Appendix I.

The tritium handling system is designed to minimize the amount of tritium that could be released in an accident, and is simplified by sending the spent tritium off-site for reclamation. Only l week's supply $(300 \mathrm{mg}$ for 20 discharges) will be in the vacuum system at any one time, being stored on uranium beds for injection or after-recovery. An onsite vault will store $2 g$ in gaseous form.

The vacuum wall of the Marshall roil is the primary cont ainment system, and a surrounding cell with concrete walls is the secondary slstem. There are ventilation hoods over the areas where transfer into the vacuum system occurs, and these hoods are vented to a waste treatment facility. Leakage into the main experimental cell will be handled by replacing all the air in the cell using a large blower (in $1.5 \mathrm{~h}$ ) and processing the old air in the waste treatment facility. Even after leakage of the entire $15 \mathrm{mg}$ DT in one discharge, une can have routine access to the cell after $1.5 \mathrm{~h}$.

To shield personnel from the $14-\mathrm{Me} V$ neutrons from SFTR requires $\sim 2 \mathrm{~m}$ of shielding material. This is accomplished by a $1 / 2-\mathrm{m}$ primary shield around the toroidal structure between the compres. sion coils and the implosion-heating capacitors. Surrourding this vessel is a cell with $1-1 / 2-m \cdot t h i c k$ concrete walls, allowing experimenters unlimited access just outside the cel:.

The primary shield allows access to the cell shortly after a discharge, and also protects organic material in the implosion capacitors from radiation damage. Without such a shield, all maintenance inside the cell would have to be done by remote control. As is, only the modules inside the primary shield need be removed by remote handling equipment and transferred to hot cells for repair or transfer.

In designing subsystems for SFTR, many design choices must be made. The major criteria, those with significant impact on the shape and size of the structure or which determine its major characteristics, have been presented here. 


\section{THEORETICAL CONCEPTS}

\subsection{OBJECTIVES}

\subsubsection{General Description}

The basic objectives of SFTK are outlined in Chaps. II and III. Chapter IV presents the theoretical basis of one approach to achieving the two specific goals: (1) $Q=1$ and (2) plasna stability. In Sec. 4.1 the plasma geometry and the requirements for the mathematical model are detined. and the general engineering pian to achieve the goals is presented.

\subsubsection{Plasma and System Geometry}

The parameyers of the basic toroidal system are defined in Fiy. 4-1. The mathematical model assumes that the plasma has a sharp boundary at an average radius a(t). The system, including the plasma, has a toroidal variation to provide plasma equilibrium. ${ }^{1-4}$ This variation, shown in Fig. 4-2, has a wavelength $\lambda$ and consists of three perturbations: the $t=0$ (sausage-like) parameterized hy $i_{11}$. the $t=i$ (helical) parameterized by $\delta_{1}$, and the $I$ $=2$ (elliptical) parameterized by $\delta_{2}$. Using the coordinates defined in Fig. 4-2, the plasma surface can be described (for small is's by

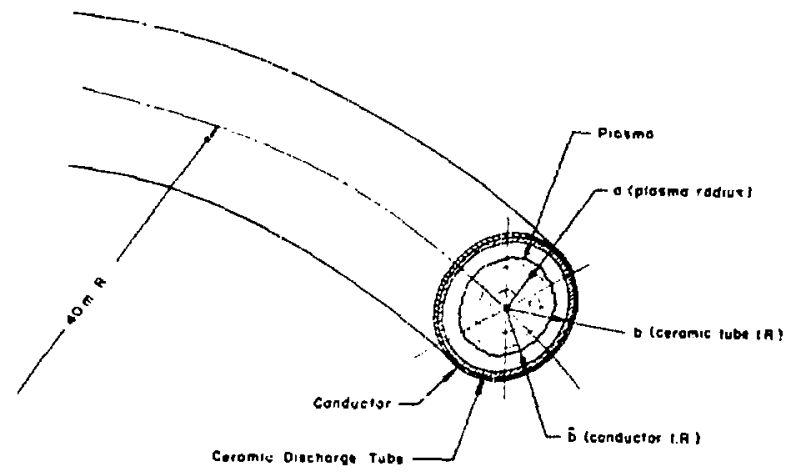

Fig. 4-1.

Geometry of the implosion coil conductor, the vacuum wall, and the plasma.

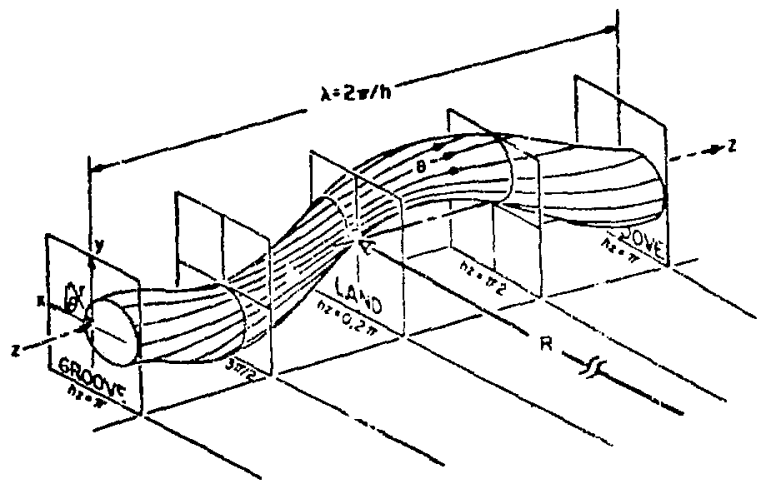

Fig. 4-2.

Plasma surface geometry showing the $1=0,1$, and 2 perturbations.

$$
\begin{aligned}
& r=\mathrm{a}\left[1+\delta_{2} \cos (2 \theta-\mathrm{hz})\right. \\
& \left.\quad+\delta_{1} \cos (\theta-\mathrm{hz})-\delta_{0} \cos h z\right]
\end{aligned}
$$

The addition of the $/=2$ perturbation 5 to the usual Scyllac configuration ${ }^{6}$ eases the technological requirements of the SFTR.

\subsubsection{System Requirements}

The mathematical model is used to determine the possibility of achieving $Q=1$ with projected technology while maintaining plasma stability. In this section, $Q$ is mathematically defined and the stability requirements are established.

\subsubsection{Definition and Significance of $Q$}

For the SFTR system $Q$ is defined as the ratio of the total neutron energy output to the maximum plasma energy. During the burn cycle the plasnia density $\mathbf{n}$ is assumed to be constant inside the average radius $a(t)$ and the velocity distributions are assumed to be Maxwellian. Thus $Q$ can be defined as 


$$
Q \equiv \frac{Y \int_{0}^{\infty}\left(\frac{n}{2}\right)^{2}\langle\sigma v\rangle d t}{\frac{3}{2} n_{c} k_{B}\left(T_{e c}+T_{i c}\right)} .
$$

The average esergy yield per neutron $\mathrm{Y}$ is assumed to be $20 \mathrm{MeV} / \mathrm{n}$. The subscripts $c$ on the density and temperature in the denominator refer to the peak values which occur at maximum compression.

The goal of $Q=1$ is assumed to be the "scientific breateven" point and it should be noted that for the system described in this report $Q=1$ is more stringent than $n \tau=10^{14}$.

\subsubsection{Plasma Equilibrium and Stability}

The criterion presented here for equilibrium and stability is based on the sharp boundary. MHD model developed for Scyllac, ${ }^{6}$ but it has been exiended to include an $i=2$ perturbation." The anaivtic expressions are based on the so-called "old ordering" $\left(\delta_{1} \ll \text { ha } \leq 1, h \equiv 2 \pi / \lambda\right)^{1,2}$ which is violated by the SFTR parameters. As is explained in the Scyllac theory review in Ref. 6, the old ordering vields surprisingly accurate results (for $\beta \leq 0.95$ and $\left.3 \delta_{1}<\pi^{2} / 16\right)$ when compared with the numerical results of a more accurate theory. ${ }^{T}$ This new theory is based on an appropriate ordering where ha $<1$ and $\delta_{1}$ is arbitrary. Recently, the accuracy of the old ordering has been experimentally confirmed at Garching 8 for $\delta_{1}=3$. The usefulness of analytic expressions and the apparent accuracy of the old ordering motivate its use in this report.

Plasma equilibrium requires that the outward grad $B$ force be balanced by the inward force arising from the plasma surface perturbation. For an $l=0$, 1,2 perturbation the equilibrium condition is

$$
f_{0} \delta_{0}+f_{2} \delta_{2}=\frac{1}{h^{2} a R \delta_{1}}
$$

where $f_{0}$ and $f_{2}$ are functions of the plasma $\beta$ as follows:

$$
\begin{aligned}
& f_{0} \equiv \frac{3-2 B}{2} \\
& f_{2} \equiv \frac{2-B}{2} .
\end{aligned}
$$

The condition for stability against growth of the $\mathrm{m}$ $=1$ mode is

$$
\left(\frac{a}{b}\right)^{4} \geq g_{0}\left(\frac{\delta_{0}}{\delta_{1}}\right)^{2}+g_{2}\left(\frac{\hat{o}_{2}}{\delta_{1}}\right)^{2}+g_{1}(h a)^{2}
$$

where the g's are the following functions of $\beta$ :

$$
\begin{aligned}
& g_{0} \equiv \frac{(3-2 \beta)(1-\beta)}{\beta(2-\beta)} \\
& g_{1} \equiv \frac{(4-3 \beta)(2-\beta)}{8 \beta(1-\beta)} \\
& g_{2} \equiv \frac{(2-\beta)}{2 \beta} .
\end{aligned}
$$

This st ability criterion cont ains the st abilizing effect of the wall on the left side of the inequality. and the destabilizing effect due to bad curvature in the $t=0$. 1 , and 2 perturbations on the right side. The SFTR design requires that $(3)$ and $(6)$ be satisfied.

The $m=2$ mode might also be unstable in the SFTR. The stability requirement for an $t=0,1$ perturbation is given by

$$
\left(\frac{x_{I}}{a}\right)^{2} \geq g_{4} h^{2} E^{2} \delta_{1}^{2}
$$

where $\eta$ is the ion Larmor radius and

$$
g_{4} \equiv \frac{2 \beta^{2}(1-\beta)}{2-\beta}
$$

The $m=2$ mode has not been experimentally observed in the collisionless regime, so that neither the time asymptotic behavior of the mode nor the accuracy of (10) is krown. Because of this lack of information, (10) has not been made a requirement of the SFTR design. However, $(10)$ is never violated for the proposed operating conditions. Proposed experiments on Scylla IV-P (now under construction) will provide the needed information on the $m=2$ instability for future iterations of the SFTR design.

The effects of these equilibrium and stability re. quirements on the design of SFTR are described in Secs. 4.1.4.2 and 4.1.4.3. 


\subsubsection{Proposed Approach}

The proposed method of achieving $Q=1$ and satisfying the stability requirements of Sec. 4.1.3 consists of heating the plasma by implosion and adiabatic compression, using the staging concept. ${ }^{9}$ Plasma stability is achieved by a combination of wall stabilization and "slow" feedback control. This approach is explained and the reasons for choosing it are presented in the remainder of this section.

\subsubsection{Staging Concept of Plasma Heating}

Standard theta-pinch systems, including Scvllac. use a single capacitor bank to form one magnetic field pulse. The plasma is implosion or "shock" heated at the beginning of this pulse until the average speed of the plasma ions exceeds the magnetic piston speed. At this point the heating process begins the transition from implosion heating 10) adiabatic compression as the magnetic field con . tinues to increase to its maximum value. When the peak field is reached the capacitor bank is crowbarred, allowing the field to decay exponentially.

The inductance in these standard systems must be sufficiently low to provide the tast-rising fjelds required by implosion heating. However, this low inductance limits the $L / R$ time of the crowbarred field to the order of $100 \mu \mathrm{s}$. In present theta pinches, the plasma confinement time has always been less than the L/R time. In future systems such as SFTR the confinement times must exceed this $100 \mu \mathrm{s}$ by 2 or 3 orders of magnitude and yet the induciance must be even lower to allow for more implosion heating.

The staging concept was proposed several years ago ${ }^{9}$ to meet these disparate requirements of low inductance and long confinement times. Instead of a single bank, two separate systems are used. The low inductance implosion system provides the required fast-rising field $(\sim 10) \mathrm{ns})$ which is crowbarred or "staged" into the slowly rising ( $\sim 1 \mathrm{~ms}$ ) compression field. The compression system is sufficiently inductive to provide the long $L / R$ times required by SFTR.

The staging approach is also economically sound. The major portion of the magnetic energy is supplied by the low cost $([-5 d / J)$ compression system as compared with the expensive $(\sim 75 d / d)$ implosion svstem. This difference in cost exemplifies the fact that the faster the energy must be transferred, the more technologically difficult and expensive the system. Thus, from an economic and technological view. the amount of implosion leating should be minimized.

\subsection{Importance of Implosicin Heating}

Some implosion heating is required by plasma physics and reactor efficiency constraints. If an adiabatic compression field were applied to the typical 1- to 10-eV preionized plasma, the field would diffuse into the low conductivity plasma. producing virtually no compression. Some method such as electron-cyclot ron resonance might be used in place of the implosion heat ing to increase the electron temperature and hence the conductivity; however. the efficiency requirements of future reactors will not allow the use of pure adiahatic compression to heat the ions.

The inefficiency of pure compression is emphasized by the following example. The adiabatic law re. quires a volumetric compression of nearly 32000 to heat a $6-\mathrm{eV}$ plasma to the $6 \mathrm{keV}$ required for burning. If $Q=25$ is assumed, as for RTPR. " the energy in the compression field would be more than 800 times greater than the thermal energy extracted from the reactor. This would limit the efficiency of the compression system intolerably.

These requirements of reactor efficiency and of high electrical conductivity in the plasma imply that some implosion heating will always be necessary in future theta-pinch reactors unless other techniques are used to heat both ions and electrons. The optimum ratio of implosion and compressional heating must be determined by assessing the plasma physics. technological, and economic goals and constraints of the specific device under consideration. The relevant discussion of SFTR constraints and design is presented in Sec. 4.1.4.3.

\subsection{Efficiency of Implosion and Com- pressional Heating Systems}

A few fundamental concepts of plasma heating efficiency presented here are helpful in the design of SFTR and perhaps other theta-pinch systems. To simplify the development, several assumptions are made. A sharp boundary model is assumed and the heating process is defined by an initial state (1) and a final state (2). The surface of the plasma volume in state (1) is chosen as the fixed boundary between the driving circuit and the "load" as shown in Fig. 4-3. The plasma heating efficiency in the load is discuss ed separately from the circuit transfer efficiency to the load.

The total efficiency of the system $e_{T}$ is defined by

$$
e_{T} \equiv e_{c} e_{p}
$$




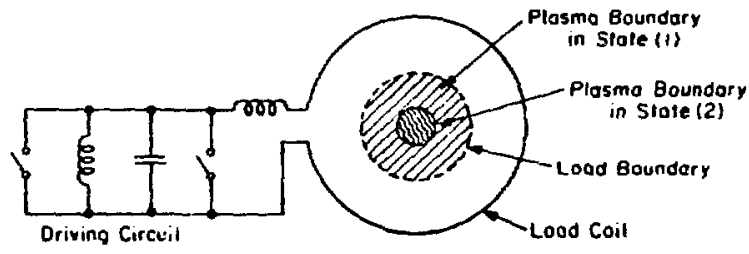

Fig. 4-3.

Schematic diagram of a driving circuit showing the spatial relation of the load coil and the plasma in states (1) and (2). The load boundary' separates the "circuit" from the "load" for the purpose of calculating circuit and plasma heating efficiencies. The circuit shown here is similar to a compression circuit describ. ed in Sec. 5.4.2, but in this figure it represents a general circuit and no special emphasis is intended.

where $e_{c}$ is the circuit efficiency and $t_{p}$ is the plasma heating efficiency within the load boundary as defined by

$$
\begin{gathered}
e_{c}=\frac{\Delta E_{L}}{E_{s}} \\
e_{p}=\frac{\Delta E_{p}}{\Delta E_{L}}
\end{gathered}
$$

The change in energy in the load $\Delta \mathrm{E}_{1}$, includes the sum of the changes in both plasma energy $\lrcorner E_{p}$ and the field energy $\Delta E_{B}$ inside the load boundary. but does not include the field stored between the load boundary and the load coil. The symbol $E_{\text {s }}$ represents the total stored energy in the circuit in state (1). This distinction between circuit efficiency and plasma heating efficiency allows a more lucid comparison of the implosion and compression systems.

The plasma efficiency can be determined by recognizing that the energy imparted to the plasma by the magnetic field is always given by

$$
\Delta E_{p}=-\int_{V_{p 1}}^{V} \frac{B^{2}}{2 \mu_{0}} d V_{p}
$$

independent of the detailed state of the plasma. The numerical subscripts refer to the plasma volume $V_{p}$, in states (1) and (2).

The adiabatic plasma efficiency $e_{p a}$ can be calculated by assuming that the plasma is isotropic over three dimensions and adiabatic so that

$$
\mathrm{PV}_{\mathrm{P}}^{5 / 3}=\text { constant }
$$

and that pressure balance exists

$$
\frac{B^{2}}{2 \mu_{0}}=n k_{B}\left(T_{e}+T_{i}\right)=p
$$

Equations (16) and (17) yield the required relation between $B$ and $V_{p}$ needed in (15). The resulting plasma heating efficiency for adiabatic compression is

$$
e_{p a}=\frac{3}{2} v \frac{1-v^{2 / 3}}{1+\frac{1}{2} v-\frac{3}{2} v^{5 / 3}}
$$

which is plotted in Fig. 4-4. The value $v$ is the volumetric compression ratio

$$
v \equiv \frac{v_{p 2}}{v_{p 1}}
$$

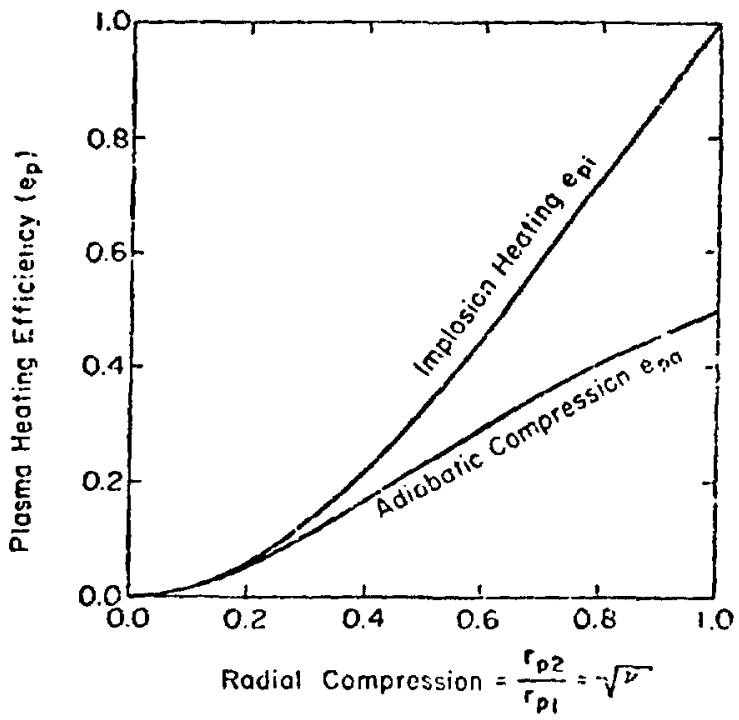

Fig. 4-4.

Comparison of the plasma heating efficiencies for implosion heating and adiabatic compression. 
The least restrictive assumptions required to calculate the plasma heating efficiency for an implosion $e_{p i}$ are that the change in plasma energy is large compared with the initial plasma energy.

$$
\Delta E_{p} \gg E_{p_{l}}
$$

and that in state (2) the plasma thermalizes, isotropizes into three dimensions, and comes into pressure balance with the field

$$
\frac{\Delta E_{D}}{E_{B_{2}}} \simeq \frac{E_{P_{2}}}{E_{B_{2}}}=\frac{\frac{3}{2} V_{p_{2}}}{V_{L}-V_{P_{2}}}
$$

The plasma heating efficiency for implosion heating $e_{1}$, is obtained directly from (21)

$$
e_{p i}=\frac{3}{2} v\left[\frac{1}{1+\frac{1}{2} v}\right] \text {. }
$$

This efficiency is compared with $e_{p a}$ in Fig. 4-4.

Several important observations can be made by examining Fig. 4-4 and the assumptions leading to Eqs. (18) and (22). First, nonadiabatic heating is always more "efficient" than adiabatic heating. Note that the asymptotic behavior of (18) and (22) for small $\nu$ is a result of the fact that for small $\nu$ the adiabatic assuniptions are a special case of the implosion assumptions. Also, if a plasma is heated by a process that greatly changes the plasma energy withiut significantly changing the plasma volume, the process must be nonadiabatic. Finally, in contrast with economic and technological considerations, implosion heating appears most attractive when plasma heating efficiency is considered.

The general statements that can be made about circuit efficiency $e_{c}$ are much less quantitative than those just made about $e_{p}$. One reason for the lack of simplicity in $e_{c}$ is the virtually limitless variety of circuits that could be used in the implosion and compression systems. However, some qualitative statements can be made with approximate numerical examples for the systems proposed for the SFTR.

Con pression circuits like the one in Fig. 4.3 can be built (with rise times on the order of $1 \mathrm{~ms}$ ) to transfer virtually all of the stored energy $E_{\text {s }}$ into the load coil. In this case, the efficiency $e_{c}$ depends mainly on the relative volume of the load boundary $V_{m}$ and the load coil $V_{L}$.

$$
e_{c a}=\frac{1+\frac{1}{2} v-\frac{3}{2} v^{5 / 3}}{\frac{v_{L}}{v_{p_{1}}}+\frac{1}{2} v-\frac{3}{2} v^{5 / 3}}
$$

The total efficiency becomes, using (12), (18), and (23),

$$
e_{T a}=\frac{3 v\left(1-v^{2 / 3}\right)}{2 \frac{v_{L}}{v_{P_{1}}}+v-3 v^{5 / 3}} .
$$

Using $V_{1} N_{p l}=8.2$ for the SFTR compression system, Eq. (24) is plotted in Fig. 4-5. The value of $\sqrt{v}$ for the compression system in SFTR is about 0.5 , thus

$$
\mathrm{e}_{\mathrm{Ta}} \simeq 0.03 \text {. }
$$

In general, implosion circuits are much more dissipative than compression circuits because of the greater speed of energy transfer. In addition to resistive losses, the circuit described in Sec. 4.3 is optimized with the source inductance comparable

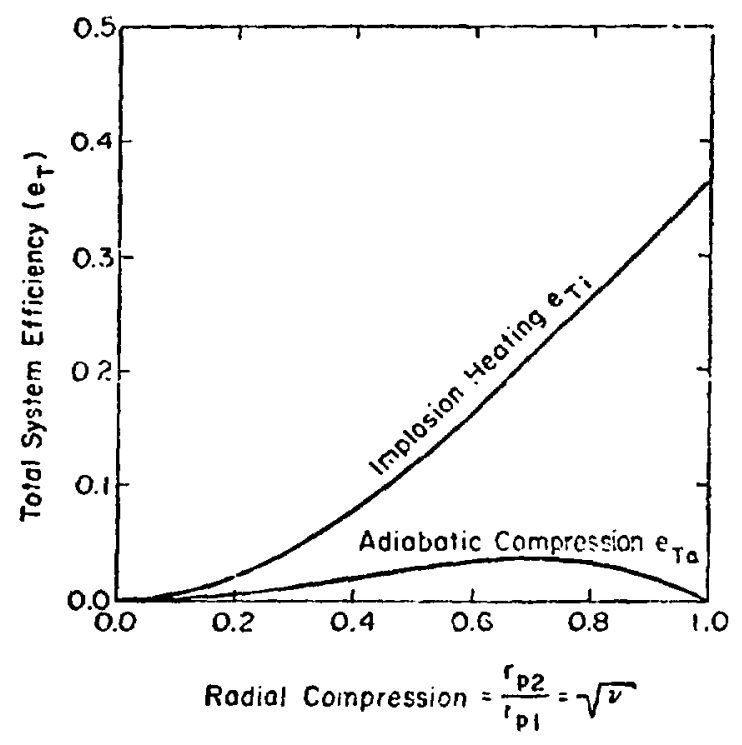

Fig. 4-5.

Comparison of total system ffficiency for implosion heating and adiabatic compression in the SFTR sustem. 
with the load coil inductance. These resistive and inductive losses limit the efficiency of energy transfer into the load coil to about $49 \%$. Hence.

$$
e_{c i} \approx 0.4 \frac{1+\frac{1}{2} v}{\frac{v_{L}}{v_{p_{1}}}+\frac{1}{2} v} .
$$

The total implosion efficiency becomes. using (12). $(22)$, and (26).

$$
\mathrm{e}_{\mathrm{Ti}} \simeq 0.6 \frac{v}{\frac{\mathrm{V}_{L}}{\mathrm{v}_{\mathrm{P}_{1}}}+\frac{1}{2} v} .
$$

Using $V_{\mathrm{L}} / N_{p 1}=1.14$ for the SFTR implosion system, Eq. $(16)$ is compared with $\mathrm{T}_{a}$ in Fig. 4-5. The value of $\sqrt{1}$ for the implosion system in SFTR is about 0.7 . so

$$
\mathrm{e}_{\mathrm{T} i} \simeq 0.3
$$

The comparison in Fig. $4-5$ indicates that, in spite of the dissipative nature of the implosion circuit, the implosion system transfers a far laryer fraction of its stored energy to the plasma than does the compression system. This fact might mislead one into doing all of the heating with the implosion system. If this mistake were made, the compression system would still have to supply essentially the same amount of energy simply to contain the plasma (no compression, i.e, $\sqrt{v}^{v}=1.0$ ) during the long burn cycle. Hence the same compression system could have supplied a major portion of the plasma energy with essentially the same amount of stored energy. Obviously, the more heating done by the compression system. the more the cost and the technological difficulty of the implosion system are reduced. Looking beyond SFTR, in future reactor systems the compression field energy will be retrieved by the circuit and recycled with a high degree of efficiency in contrast to retrieving only a small portion of the wasted im. plosion field energy in the form of heat.

All these considerations indicate that as much of the plasma heating as possible should be done by adiabatic compression. The limitation on the fraction of heating done by compression will arise from some plasma physics consideration such as electrical conductivity or plasma stability, or it may be set by an efficiency requirement of the reactor. The remainder of the heating will have to be provided by the implosion system which should be made as efficient as possible by minimizing the complexity of the circuit and by maximizing the value of $\sqrt{v}=\mathbf{x}$, for the implosion. A quantitative analysis of the dominant factors limiting the fraction of heating done by compression in SFIKR is presented in siec. 4.1.4.3.

\subsubsection{Wall Stabilization and Slow Feedback Control}

There are two conceptual techniques for achieving plasma stability. The design of the SFTR presented in this report is based on the concept of wall stabilization in conjunction with slow feedback control. Straight feedback (like that being developed for Scyllac) appears to have merit, but its impact on an SFTR design has not been fully investigated. The properties of both concepts are discussed and briefly compared in the remainder of this section.

\subsection{Necessity for Slow Feedback Control in a Wall-Stabilized SFTR}

The $m=1$ mode cannot be wall-stabilized by the finite (noninfinite) conductivity implosion coil ${ }^{11}$ even though the growth rate of the mode might be greatly reduced. The mode can be examined as the conductivity of the wall is theoretically increased rom $\sigma=0 .{ }^{12}$ In general, $\gamma$ decreases as $\sigma$ increases. When the inertial effects are no longer important compared with the dissipative effects of the conducting wall, the growth rate is given by the field diffusion rate throv $h$ the wall. Thus, for a wall of finite conductivity, $\gamma$ can be reduced but rot to zero. Since the confinement time required in SFTR exceeds the field diffusion time, some feedback control is necessary.

The field diffusion time depends on the specific geometry of the system shown schematically in Fig. 4-6. The separation of the implosion and compression systems discussed in Sec. 4.1.4.1 requires the use of two distinct coils. The fractional turn implosion coil (see Sec. 5.2.2.1.1) provides the low inductance load for the implosion system. The multiturn, high-inductance compression coil, described in Sec. 5.2 .2 .1 .2 , is required to produce the $250-\mathrm{ms}$ decay time for SFTR. The field diffuses through the metal of the implosion coil with the characteristic time

$$
\tau_{0}=\frac{\mu_{0} \sigma \delta^{2}}{2} \text {. }
$$




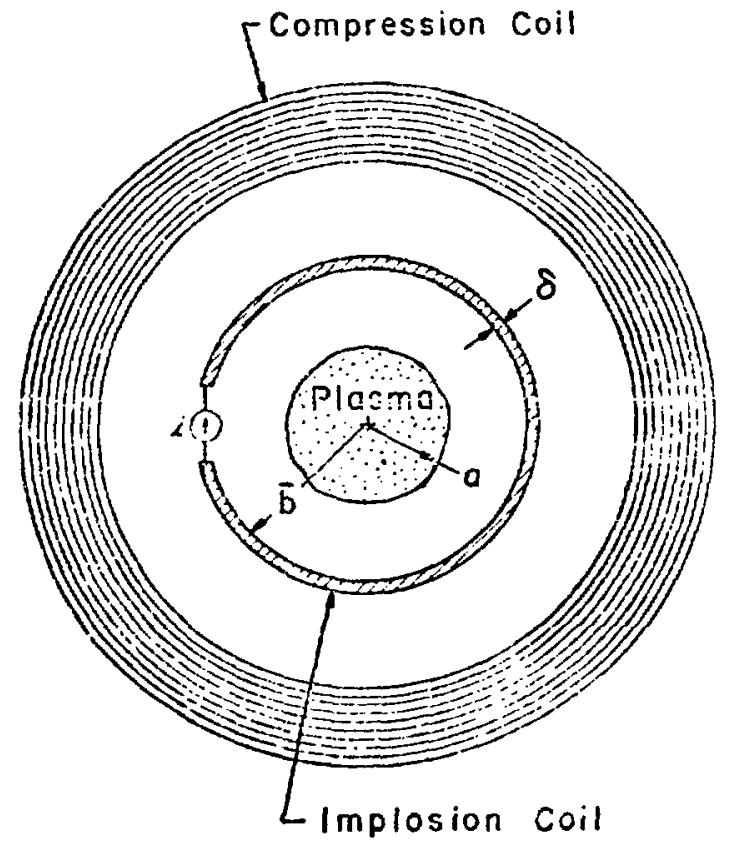

Fig. 4-6,

Sichematic representation of the fractional turn implesion coil and multiturn compression coil.

However, the field enclosed by the coil decays at a slower rate given by the $\mathrm{L} / \mathrm{R}$ time of the coil. For the simple coil shown in Fig. 4-6 with no plasma present,

$$
\tau_{L / R}=\frac{\mu_{0} \sigma \bar{b} \delta}{2} .
$$

Two complexities modify this simple model of field diffusion. The implosion coil is crowbarred by a switch. If the resistance of the switch is sufficiently high compared with that of the coil, the switch will dominate the $L / R$ decay, invalidating Eq. (30). Even if the crowbar were perfect, the presence and motion of the plasma in conjunction with the $l=1$ perturbation generate field perturbations that are responsible for wall stabilization. In the perfect crowbar condition, these field perturbations will decay at a rate given by $(30)$ with $\bar{b}$ r'placed with some characteristic length of the perturbation which could range from $\bar{b}$ to $\delta$.

It is advantageous to increase the field deca:" time as much as possible because as $\tau$ inc:eases, $\gamma$ decreases. In addition, the required rise time of the compression field increases. The thickness of the conductor $\delta$ can be increased until the crowbar switch resistance dominates the $\mathrm{L} / \mathrm{K}$ time. If $\delta$ were increased further, the $\mathrm{L} / \mathrm{R}$ time would not increase appreciably. but the dissipation and distortion of the rising compression field would begin to increase sharply.

To maximize the $\mathrm{L} / \mathrm{R}$ time and minimize dissipa. tion of the compression field, the following limits are placed on $\delta$

$$
\frac{\mu_{0} \sigma \delta^{2}}{2}<\tau_{s} \ll \frac{\mu_{0} \sigma \bar{b} \delta}{2},
$$

where $\tau_{\mathrm{s}}$ is the crowbar-dominated $\mathrm{L} / \mathrm{R}$ time of the implosion circuit. The subscript $\mathrm{s}$ is used to refer to the implosion or "shock" process to be consistent with the literature and to avoid confusion with subscript $i$ for ions. As was mentioned in Sec. 4.1.4.1 the maximum crowbar times achieved on present systems have been on the order of $100 \mu \mathrm{s}$. The value projected for SFTR is $\tau_{s}=230 \mu \mathrm{s}$. Using this $\tau_{s}$ in (31) yields

$$
0.059 \ll \delta \ll 2.5 \mathrm{~mm}
$$

The value chosen for SFTR is $\delta=1.5 \mathrm{~mm}$.

With $\tau_{\mathrm{s}}$ and $\delta$ determined, the foll $r$ wing limits can be placed on the wall-stabilized growth rate

$$
\frac{1}{\tau_{s}} \approx \gamma_{w s}<\frac{1}{\tau_{0}} \text {. }
$$

Simply stated, the growth rate is bounded on the lower side by the field decay rate due to the crowbar gap, and on the upper side by the diffusion rate of the field into the metal of the implosion coil. Evaluating (33) yields

$$
4.3 \times 10^{3} ₹ \gamma_{w s}<1.2 \times 10^{4} s^{-1} .
$$

Since $\gamma_{w b} T_{r}<0.4$ is required for stabilization in a practical feedback system ${ }^{13}$ with rise time $\tau_{r}$.

$$
\tau_{r} \approx 33 \mu \mathrm{s}
$$

in the wall-stabilized SFTR.

4.1.4.2.2 Comparison of Straight Feedback with the W'all-Stabilization Feedback Combination

The major advantage of the wall-stabilized approach over straight feedback is the slower rise time required of the feedback system. In the case of straight feedback it is assumed that the plasma is compressed significantly beyond the limit imposed by (17), thus eliminating the effect of wall stabilization. Equation (17) was obtained from the growth 
rate of the $\mathrm{m}=1$ mode for infinilely conducting walls $(\sigma=\infty)$.

$$
\begin{aligned}
Y= & \operatorname{lv}_{a} 8\left[-\left(\frac{a}{b}\right)^{4} \delta_{1}^{2}+g_{0} \delta_{0}^{2}\right. \\
& \left.+g_{2} \delta_{2}^{2}+g_{1}(\text { na })^{2} \delta_{a^{2}}^{2}\right]^{y / 2}
\end{aligned}
$$

where $v_{11}$, the Aiven speed, is

$$
v_{\alpha} \equiv \frac{B}{(j, n m)^{\sqrt{2}}} .
$$

The straight feedback sistem must be capable of responding to a growth rate given by (3til when the wall-stabilizing term thirst tern in the bracketsl is innored. Csing typical sfork parameders, this growth rate is

$$
Y_{f} \simeq 3.5 \times 10^{5} \mathrm{~s}^{-1}
$$

Since is is $<0.4$, the required rise time is

$$
\tau_{\mathbf{r}} \approx 1.1 .1 \mathrm{~s}
$$

A comparison of $(39$ ) with $(3 i \overline{0})$ indicates that the st raight teedback system would have (1) be :30 times taster than the slow teedback system used in the wall-stabilized appruach.

This taster rise time makes it more difficult to electrically isolate the straight leedback slstem from the imploxion system. Buth types of feedbatis sylem would have to use the implosion covil as the feedback drive coil. Feedback coils cannot le placed inside the implosion coil as in Scyllac necause of the stringent. low source inductance requirements of the FFTR implosion system. They cannot he placed out side the implosion coil because the field penteration time through the implosion coil would be ton slow to satisfy $r_{r}<0.4$. Since the feedback system must be attached to the $270-k V$ implosion system, very effective electrical isolation is required to protect the feedback system. The greater the difference in the rise time between the feedback system and the implosion system $(100 \mathrm{~ns})$ the more effective the isolation can be made.

The major disadvantage of the wall-stabilized approach is that it requires a larger ratio of $a / b$ and hence more implosion heating than the straight feedback approach. However, the reduct ion in implosion heating allowed by straight feedback may be small because of a constraint on the minimum DT siiiing pressiture described in siece 1.1.4.3. Fhis perensure rest rietion may prodece enomentic limitations on the reduction of implesion heange that woshd dominate. wer the plasma endurtivity limenteion discussed in sese 4.1.4.1.1. Since the present siflele design

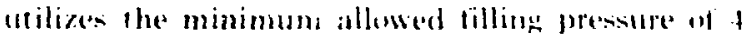

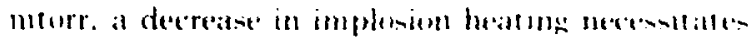
high eompressional heating. The resule is a plasma with similar temperature hut higher demsits and this higher phasma pressure must he contined by a

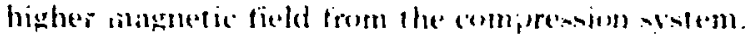
A preliminary insestigation indicales that limitations in the cost of the complessiun sistem may present the lowering of the implosion bank voltage by more than 2li'. Althomgh smatl, this reduetion combl be techmologically important

At this time the wall-siabilized appresth appears the most promising. but the stratertit feedback sytem is not without merit. 'lhe expermenental ex perience with wall sabiligation. implesion hesting. and feedhack stabilization is much tow -e'ant 1u pere mit a final decision between wall stabilisation and

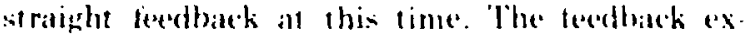
periments on sevflac and the remulto from the sile'. IHX. and resomallt heating experimeme will aid erreatly in making that decision.

\subsubsection{Operating Point Determination}

The staging toncept and wald-stabilization ap. proath torm the hasis of this sif"lh design. This atp. proach was chosen as a result of the comerepts presented in Seses. 4.1.4.1 and 4.1.4.2. In the remainder of this soction, relationships deris:d elsewhere in ('hay). IV are used to determine the numerical value or limits on a variety of machine and plasma parameters.

To aid in the following presentation. she temperal behavior of the magnatic field is plotted in fin. 4.7 on a logarithmic time base. IA more complete discussion of the field hehavior and the implusion and rompression circuits is found in secs, 4.2 and 4.3 .1 The implosion field rises in about $10(1 \mathrm{~ns}$. oscillatc: briefly. and settles down to a value $B$, at a lime $t$.. The inplosion field decass at an exponential rate $t$. associated with the $1, \mathrm{R}$ time of the (rowharred implosion or "shock" circuit. During this decay. the compression field rises to a peak value of $\mathrm{H}$, in a time t. The compression circuit is crowbarred at $t=t_{1}$. allowing the ficld to decay at the exponemtial rate $\tau$, .

Several reasons for minimizing the amount of implosion heating were presented in sec. 4.1.4.1.1. Another reason is apparent from a study of fig. $4-7$. As the ratio $B_{,} / B_{1}$ is reduced, $r, / t$ c can be reduced, 


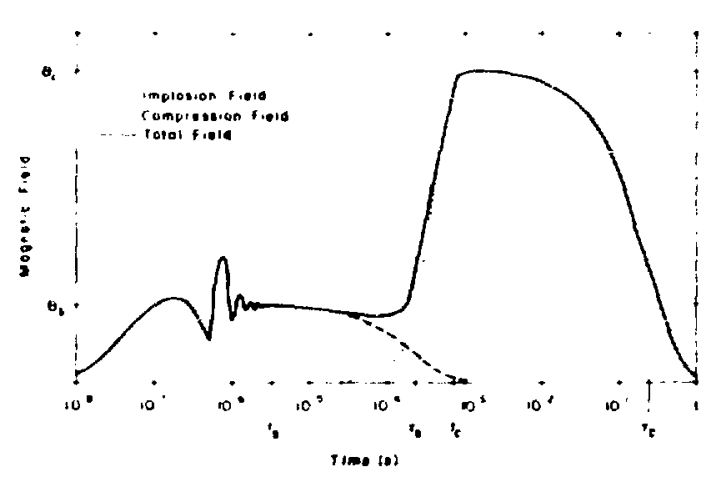

Fig. 1-7.

Timpural hehas ior of the magnetic field produced he the implesion and compression $\rightarrow \cdot n+m \mid$

thus decreasing the reguirements of the implesion

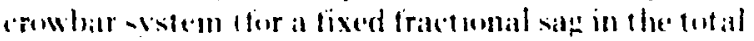
field during the staning process.

f1.131 Minimeatlom of the Flectric Fields and

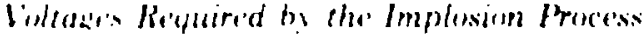

An is dincussed in Sire 4.1.4.1. one of the molin reisons for minimizing the amonent on implosion heationg is that the implosion sistem is terhmolughalls mash mere diticult than the compresion sistem. A major problem is the high fields

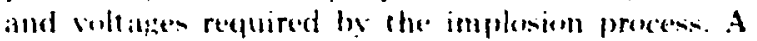
number of the stotem and plasma parameters are

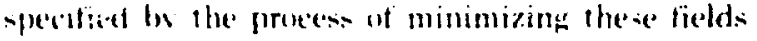
and coltagres.

Plasma physies considerations determine the required value of the electric field F... induced around the plasmat by the implosion sistem. In turn. Foand the eirenit efticiency determine the voltage $l$. needed on the implusion bank. Thus Fo is minimized tir:t, then the cireuit is opmimized to reduce $V$. Io the smalle'st allenwed value.

Six impurtant parameters determine the value of lis (sere Sere 4.3):

$$
\begin{aligned}
& x_{c}=\frac{a\left(t_{c}\right)}{\tilde{b}}, \\
& x_{s}=\frac{a\left(t_{s}\right)}{\vec{b}},
\end{aligned}
$$

the maxi.num average plasma temperature

$$
T_{c}=\frac{T_{i c}+T_{E c}}{2} \text { at } c=t_{c} \text {, }
$$

the enmpresson tield decay time $x_{\text {. }}$ the lot filling iressure $p$. and $Q$. The value of $Q=1 \mathrm{mus}$ be reached in a time $t_{y}<t$ since the neutron production drops sharply as platma expands and conds. Thus i, should be made as large as possible. Results trom sece ist indicate that the maximum reasomable $1 . / 6$ lime is

$$
\tau_{c}=250 \mathrm{~ms} \text {. }
$$

To minimize the chance of losing the plasm a before $\mathrm{Q}=1$ is reached, the value of t, is set at

$$
t_{Q}=150 \mathrm{~ms} \text {. }
$$

From the code described in See 4.2 .3 , the diulat value af i) required to satisti 1.41 is

$$
Q_{f} \simeq 1.1 \text {. }
$$

In ingerment with sed 4.1.4.3.2. $x$, should be maximized to optimize the implosion-hotating of. ficiency. and $x_{1}$ should be minimized as minimize the atmunn of required implusion heating. Implesien codes indiente tsee sece 4.3 .31 that

$$
x_{s}=0.7
$$

is obtainable. rielding an implosion eafficieney of

$$
c_{I i} \simeq 0.21
$$

from Fig. 4-i. The minimum value of $x_{\text {, }}$ consistent with stability requirements is obtained in sec. 4.1.4.3.2.

The quantities $p_{1}$ and $T$, are related $t o x_{\text {, }}$ through the definition of $Q$. If mo additional const raints are placed on p.. $T_{1}=8$ kel yelds the lowest value of F. The filling pressure $p_{0}$ is then determined hy the valus of $Q$ cee sec. 4.3.33). Infirtunately: the resulting value of $p_{1}$ is less than the minimum value of $f$ mtorr at which standard theia pinches have

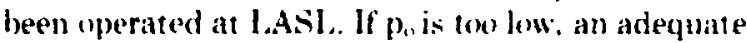
sheath for implosion fails to form. Although this hwer limit has not been thoroughly explored. past experience indicates that it is hetween 1 and $f$ mitorr. If the pressure is set at

$$
p_{0}=4 \text { mtorr }
$$


then $T_{1}$ can be reduced as far as Eq. (45) will allow. Even though the ralue of $T_{c}$ depends on $x_{c}$, the strong dependence of the $\langle\sigma v\rangle$ curve (see Sec. $4.2 .2)$ on $T_{1}$ limits the range of $T_{1}$ to

$$
4.6 \approx \mathrm{T}_{\mathrm{c}} \approx 6 \mathrm{keV}
$$

for $0.23<x .<0.4$

Esing the values of the four parameters $Q, x_{n}, T_{C}$ and $p_{1}$ just established in this section, the value of $E_{H}$ is approximately given by [see Eq. (199)]

$$
\mathrm{E}_{\theta}[\mathrm{kV} / \mathrm{cm}] \simeq 65 \mathrm{x}_{\mathrm{c}}{ }^{4 / 3} / \ln \left(5.3 / \mathrm{x}_{\mathrm{c}}{ }^{2} \mathrm{Q}\right) .
$$

To obtain the minimum value of $\mathrm{E}_{\mu} . \mathrm{x}_{\mathrm{c}}$ must be minimized within the constraints imposed by $(2)$ and $(6)$.

\subsection{Minimization of $x_{\text {. for a }}$ Wall-Stabilized} Sistem

As discussed in the previous section, a reduction in $F$, reduces the technological problems associated with the implosion system. From (50) it is apparent that $F_{*}$ is reduced by minimizing $x_{*}$ within the plasma equilibrium and stability constraints given by $r i 3$ and $(6)$.

This minimization is easily accomplished by eliminating one of the variables between $(: 3)$ and $(6)$ and minimizing a witti respect to the rest of the variables. Since $h$ has essentially no effect on the "rist or complexity of the sistem, it is the ideal variable tw eliminate. giving

$$
\begin{aligned}
a^{4}- & \frac{B_{1} \bar{b}^{4}}{k \delta_{1}\left(f_{0} \delta_{0}+E_{2} \hat{v}_{2}\right)} a \\
& -\frac{\bar{b}^{4}}{\delta_{2}^{2}}\left(g_{0} \delta_{0}^{2}+g_{2} \delta_{2}^{2}\right)=0 .
\end{aligned}
$$

From $(51)$ it can be shown that the following inequalities hold. independent of the other variables

$$
\begin{aligned}
& \frac{\partial a}{\partial R}<0, \\
& \frac{\partial a}{\partial \bar{b}}>0, \\
& \frac{\partial a}{\partial \delta_{1}}<0 .
\end{aligned}
$$

These inequalities indicate that $R$ and $\delta_{1}$ should be chosen as large as possiole and b should be chosen as small as possible.

The upper bound on $\mathrm{R}$ is set by economics and the operational and reliability problems associated with a large system. A compromise between those limitations and the relatively weak dependence of a on $R$ [see $(63)$ ] resulted in the choice of

$$
R=40 \mathrm{~m} \text {. }
$$

The ineguality $(5 ; 3)$ and the desire to minimize the cost of SFTR imply that $\bar{b}$ shoukd be made as small as possible. However, the plasma sheath thickness must be small compared with $b$ or $\bar{b}$ for an adequate implosion. In present theta-pinch sistems the sheat thiclness is 2 or $3 \mathrm{~cm}$. The sheat h thickness is often assumed to be $c / w_{p}$. although the sheath formation is probably much more complex than is indicated by this simple relation. For a filling pressure of 4 mtorr lsee $(18)$ this retation yields

$$
\frac{c}{\omega_{p i}}=2.1 \mathrm{~cm},
$$

which agrees with the experimental observations. Thus, to insure an adequate implosion. the vacuum wall radius is set at

$$
\mathrm{b}=10 \mathrm{~cm} \text {. }
$$

The radius of the implosion coil conductors is chosen to be

$$
\bar{b}=10.7 \mathrm{~cm}
$$

to allow for the $7 \mathrm{~mm}$ of insulation required by the fabrication process described in Sec. 5.2.2.3.1.

The maximum allowed value of $\delta_{1}$ is really not known. As described in Soc. 4.1.3.2, the analytic model used here theoretically breaks down for $\delta_{1}>1$. and yet the model has been experimentally verified ${ }^{*}$ for $\dot{b}_{1}=3$. There is a strong motivation to maximize $\hat{n}_{1}$ because a is more strongly dependent on $\delta_{\text {t than on }}$ either $B$ or $\bar{b} 7$ see (63) |. One is also reluctant to set $\delta_{1}$ $=3$ or larger because of the lack of extensive experimental experience at these large values.

Before the final design of SFTR is made. future experiments will provide additional informatiom abrut the limitations on $p_{1 .}$. F $F_{1 \prime}$, and $\delta_{1}$. This information will alıw a more rational choice of design values for these parameters than can be made at the present time. In this report, the pressure is set 
at $p_{12}=4$ ratorr. and $\hat{o}_{1}$ remains a free parameter within the range

$$
\text { 1. } ₹ \delta_{1} ₹ 4 \text {. }
$$

This variation in $\delta_{1}$ will produce a corresponding range of values for $x_{i}, E_{t}, V_{0}$, and other related parameters. 'This range of operating conditions will allow the impact of future experiments on the SFTR design to be more readily assessed.

Now, $R, \bar{b}$, and $\delta_{1}$ can be considered fixed quantities in $\mathrm{Eq}$. (51). When $\delta_{0}$ and $\delta_{2}$ are varied to minimize a.

$$
\begin{aligned}
& \delta_{0}=\frac{f_{0}}{g_{0}}\left(\frac{g_{1} \delta_{1} a}{2 R} \Lambda^{2}\right)^{2 / 3}, \\
& \delta_{2}=\frac{f_{2}}{g_{2}}\left(\frac{g_{1} \delta_{1} a}{2 R} \Lambda^{2}\right)^{2 / 3}
\end{aligned}
$$

where

$$
\Lambda \equiv-\frac{g_{0} g_{2}}{f_{0}{ }^{2} g_{2}+f_{2}{ }^{2} g_{0}} .
$$

Substituting $(60)$ and $(61)$ into (51) yieids

$$
\frac{a}{\bar{b}}=\frac{\left(3 A^{1 / 3}\right)^{3 / 10}}{\delta_{2}^{2 / 5}}\left(\frac{g_{1} \bar{b}}{2 \bar{k}}\right)^{1 / 5} \text {. }
$$

The corresponding wave number becomes

$$
h=\left(\frac{2 \Lambda}{g_{1}}\right)^{1 / 5}\left(\frac{1}{a^{2} k \delta_{1}^{2}}\right)^{1 / 3} .
$$

Fquations (60) through (64) can be numerically evaluated (except for $\delta_{1}$ ) for an appropriate value of 13. This analytic old-ordering model is normally evaluated with $\alpha=0.8$ for high- $\beta$ plasmas because results agree well with the more accurate model ${ }^{7}$ for $0.8<\beta<1.0$. For $\beta \rightarrow 1.0$ the old ordering possesses unphysical singular behavior as is evident in $\mathrm{Eq}$. (19). I!sing

$$
\begin{aligned}
& \beta=0.8, \\
& \delta_{0}=0.13 \delta_{1}^{1 / 5},
\end{aligned}
$$

$$
\begin{aligned}
& \delta_{2}=0.044 \delta_{1}^{3 / 5}, \\
& \lambda\left[\mathrm{m}_{i}\right]=2.7 \delta_{1}^{2 / 5}, \\
& z[\mathrm{~cm}]=\frac{4.0}{\delta_{1}^{2 / 5}},
\end{aligned}
$$

and from (40) and (58)

$$
x_{c}=\frac{0.37}{\delta_{1} 2 / 5} \text {. }
$$

Equation $(70)$ yields the desired minimum value of $x$. dependert only on the choice of $\delta_{1}$.

From an engineering point of view. the length of individua! implosion and compression coils should be an integral fraction of $\lambda / 4$. To facilitate the design of these coils and their related circuits. a fixed length

$$
\ell=20 \mathrm{~cm}
$$

was chosen. This choice is a compromise between minimizing the multiplicity of coils and minimizing the voltage on the implosion circuit. As a result. $\lambda$ must be an integral multiple of $4:=80 \mathrm{~cm}$ instead of the continuous function (68).

If $x$ is minimized for a fixed value of $\lambda, h$ cannot be eliminated between (14) and (17). and the following sixth order equation replaces $(51)$

$$
\begin{gathered}
a^{6}-g_{1} h^{2} \vec{b}^{4} a^{4}-\frac{g_{0} f_{2}^{2}+g_{2} f_{0}^{2}}{f_{0}^{2}} \\
\left(\frac{\delta_{2}}{\delta_{1}}\right)^{2} \bar{b}_{a^{4}}^{2}+\frac{g_{0} f_{2}}{f_{0}^{2}}\left(\frac{2 \delta_{2} \vec{b}^{4}}{h^{2} R_{\delta_{1}}^{3}}\right) a \\
-\frac{g_{0}}{f_{0}^{2}}\left(\frac{\bar{b}}{h \mathrm{hK}^{1 / 2} \delta_{1}}\right)^{4}=0 .
\end{gathered}
$$

Equations (60) and (61) become

$$
\delta_{0}=\frac{E}{\varepsilon_{0}} \frac{i}{h^{2} \mathrm{aRS} S_{1}} ;
$$

$$
\delta_{2}=\frac{f_{2}}{g_{2}}-\frac{\Lambda}{h^{2} \text { aRS }}
$$


but ne simple solution similar to (63) can be ob. tained from the combination of $(72),(73)$, and $(74)$

$$
a^{6}-g_{2} h^{2} \bar{b}^{4} a^{4}-\frac{\Lambda b^{-4}}{h^{4} R^{2} \delta_{1}}=0 .
$$

Figure 4.8 compares $\mathrm{Eq} .(70)$ with the numerical solution of $(75)$ for $\lambda=3.2 \mathrm{~m}$ and $\lambda=4.0 \mathrm{~m}$. These iwo values of $A$ are integral multiples of $4 /$. This figure indicates that $(70)$ is an acceptable approximation of $(75)$ if

$$
\begin{array}{ll}
\lambda=3.2 \mathrm{~m} & 1 \approx \delta_{1} \approx 2 \\
\lambda=4.0 \mathrm{~m} & 2 \approx \delta_{1} \approx 4 .
\end{array}
$$

Thus, to retain the analytic simplicity of $(70)$, the constraint on $\lambda$ given by (76) will be assumed. Now that $x$. has been minimized, the plasma and field parameters can be calculated.

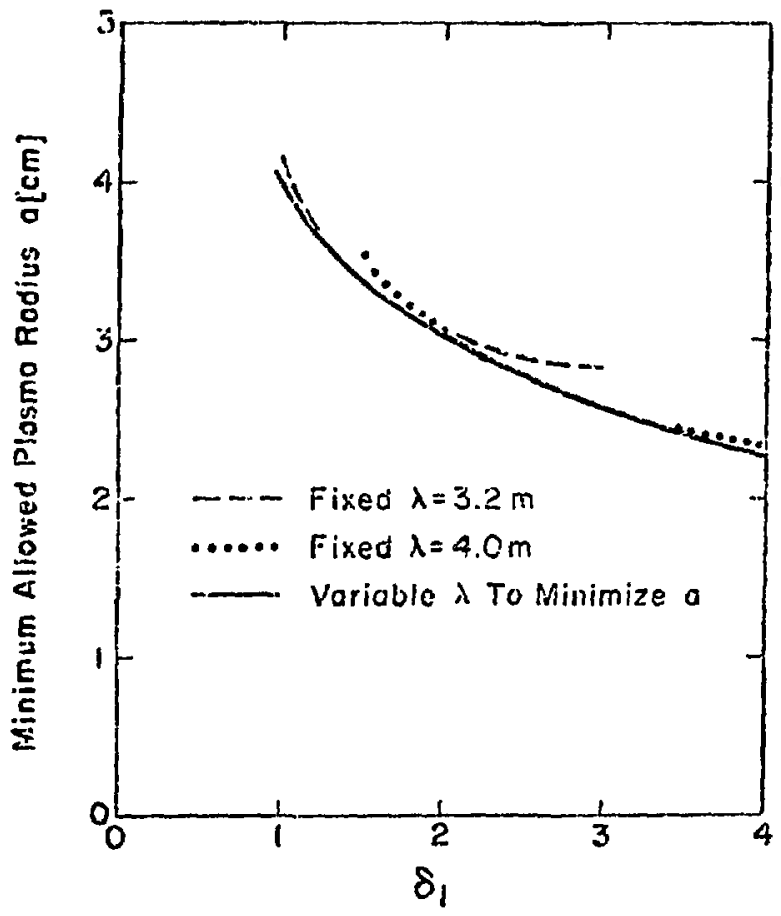

Fig. 4-8.

('imparison of the effect of fixed and variable $\lambda$ on the minimization of the stable $(m=1)$ plasma radius.
4.1.4.3.3 Determination of the Plasma and Field Parameters

Using the minimum allowed $x_{1}$ given by (70). the plasma and field parameters can be expressed in terms of $\delta_{1}$. From $(50)$ the maximum induced electric field $\mathbf{F}_{\| \text {becomes }}$

$$
\mathrm{E}_{\theta}[\mathrm{kV} / \mathrm{cm}] \simeq \frac{24}{\delta_{1}{ }^{8 / 15} \ln \left(70 \delta_{1}\right)} .
$$

The plasma parameters at maximum compresision are

$$
n_{c}\left[\mathrm{~cm}^{-3}\right]=1.8 \times 10^{35} \delta_{1}^{4 / 5}
$$

and

$$
T_{c}[k \in V] \simeq \frac{26}{\ln \left(70 \delta_{1}\right)}
$$

These formulas are derived in Sec, 1.2 .2 . The values of $r_{c}, Q, x_{i}$, and $p_{1}$, are assumed to be those given by (43), (45), (46), and (48). The value of the compres. sion lield can be obtained from (78) and (79) by assuming $\alpha=1$ :

$$
B_{c}[k G] \simeq 61 \delta_{1}^{2 / 5} /\left[\ln \left(70 \delta_{1}\right)\right]^{1 / 2} .
$$

Note that $T_{r}$ is the average ptasma temperature given by

$$
T_{c}=\frac{T_{e c}+T_{i c}}{2} .
$$

The corresponding parameters $n_{s} . T_{*}$, and $B_{*}$ evaluared at $t=t_{s}$ can be obtained from the adiabatic law for 3 degrees of freedom

$$
\frac{B_{S}}{B_{c}}=\left(\frac{x_{c}}{x_{S}}\right)^{5 / 3}=\left(\frac{T_{S}}{T_{c}}\right)^{5 / 4}=\left(\frac{n_{S}}{n_{c}}\right)^{5 / 6}
$$

Evaluating (82) yields

$$
\begin{aligned}
& \mathrm{n}_{\mathrm{s}}\left[\mathrm{cm}^{-3}\right] \simeq 5.8 \times 10^{14}, \\
& \mathrm{~T}_{\mathrm{s}}[\mathrm{keV}] \simeq \frac{12}{\delta_{2}^{8 / 15} \ln \left(70 \delta_{1}\right)},
\end{aligned}
$$




$$
B_{S}\{k G]=\frac{24}{\delta_{1}{ }^{4 / 15}\left[\ell_{i}\left(70 \delta_{1}\right)\right]^{1 / 2}} ;
$$

again, $T_{s}$ is an average temperature given by

$$
w_{s}=\frac{T_{e s}+T_{i . s}}{2} \text {. }
$$

These parameters are plotted as a function of $\delta_{1}$ in the next section, which is a summary of all the parameter values derived in Sec. 4.1. The parameter, such as capacitance and voltage. associated with implosion and compression circuits are presented in Secs. 4.2 and 4.3 .

\subsubsection{3.+ Operating Point Summary}

Tabie 4-1 and Figs. 4-9, 4-10. and 4-11 summarize the SFTR parameters discussed in Sec. 4.1. Where applicable, the relevant equation numbers are listed to aid in locating the discussion of a given variahle in the text. When the parameter is dependent on $\delta_{1}$, a figure number is listed instead of the parameter value. The denoted figure contains a geaph of the parameter's dependence on $\delta_{1}$ for $1<\delta_{1}<4$.

The values of the circuit parameters and voit ages are discussed in other sections. The compression cirruit is clescribed in Secs. 5.2.2.1.2 and 5.4, while the implosion circuit is discussed in Sec4. 4.3, 5.2.2.1.1. and 5.3 .

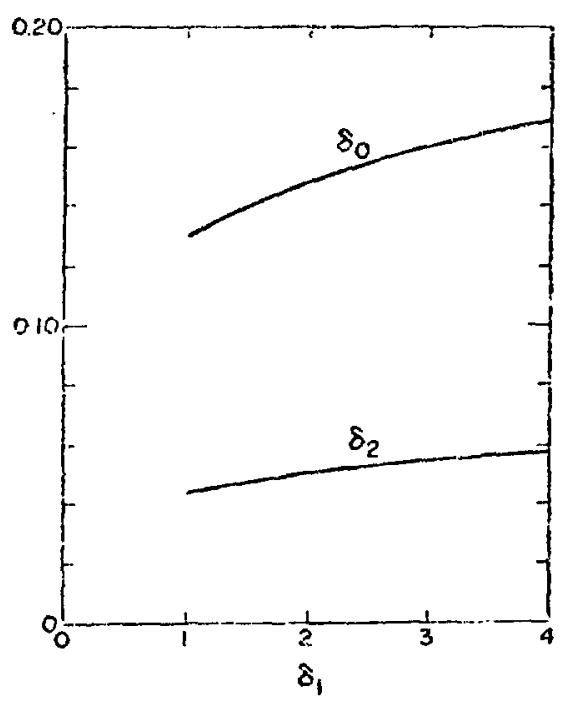

Fig. $4-9$

Dependence of $\delta_{l}$ and $\delta_{2}$ on $\delta_{l}$, as given by Egs. (66) and (67).

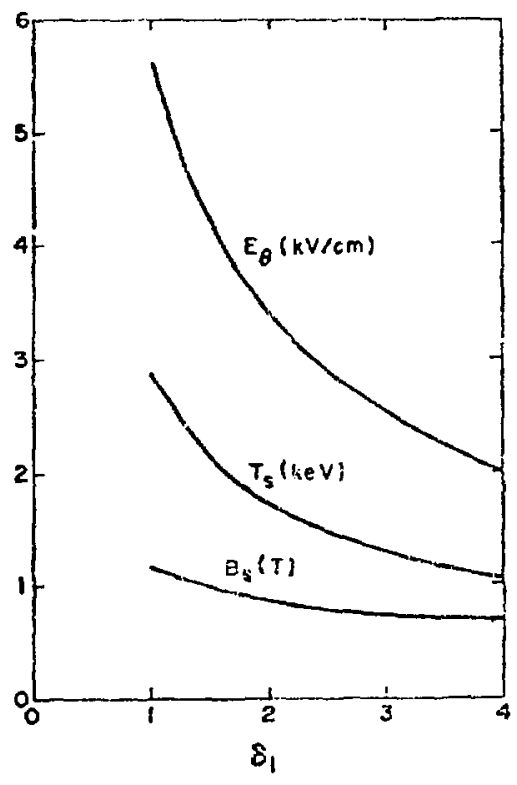

Fig. 4-10.

Dependence of $E_{\theta}, T_{s}$, and $B_{s}$ on $\delta_{l}$, as given by Eqs. (77), (84), and (85).

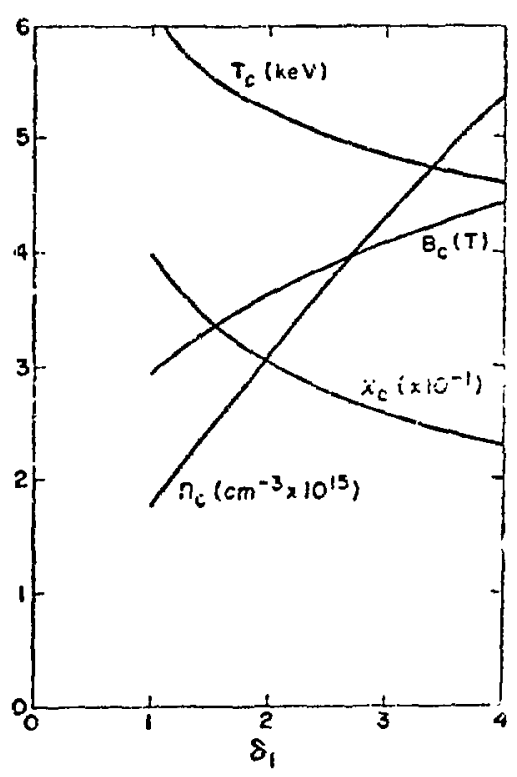

Fig. 4-11.

Dependence of $T_{c}, B_{c} . x_{i}$, and $n_{c}$ on $\delta_{l}$, as given by Eqs. (79), (80), (70), and (78). 
TABIE 4-

OPEHATING POINT SI MMARY

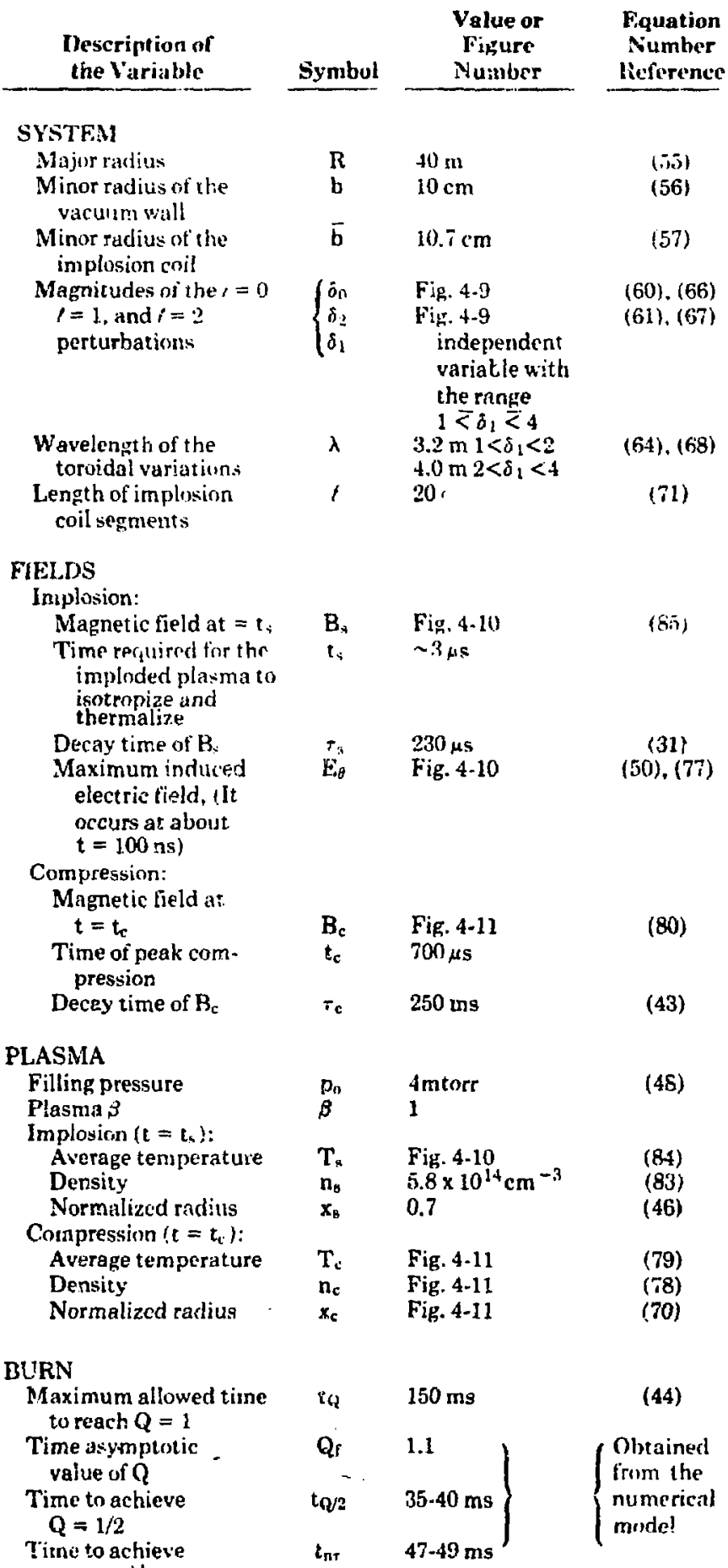




\subsection{COMPRESSION AND BURN}

\subsubsection{General Description}

The plasma is brought o ignition by adiabatic compression, and the magnetic field is then heid while thermonuclear burning occurs. Theoretical models are needed to describe the processes of heating, equilibration of species. neutron and $\alpha$ particle production, and bremsistrahlung as the external magnet ic field is changed. An analytic roodel has been derived which gives approximate answers for several of the above precesses. This model is described in the next section.

The best model available of us is a thermonuclear burn code developed by Oliphant. ${ }^{1.4}$ It is a numerical solut ion of the Fokker-Planck equation and has been used in determining the thermonuclear burnup and wher parameters. It uses the external masnetic field waveform for the SFTR circuit. That waveform is a $\sin ^{2}-\pi t / 2 t$. variation between the initial implasion field and final compression fietd, followed by an exponential tield decay with a 250 -ms time constant. A description of the numerical model and results is riven below.

\subsubsection{Analytic Model}

The approximate model developed in this srction relates the gual: of $Q=1$ and $i n=1$ plasmat stability to the plasma parameters at the beginning of the compression cycle. In order to generate a tractable. analytic model, several effects such as electrou-ion equilibration. a-particle beating and bremsitrahlung are omitted. These effects are included in the numerical model described in the next section.

The following assimptions form the basis for the model:

1. The adiabatic law holds for $\mathrm{t}>\mathrm{t}$, with $\gamma=5 / 3$. The time 1, , definedi in Fig. 4-7, marks the end of the implosion phase and the beginning of the compression phase. The adiabatic law for $\gamma=5 / 3$ is given in (R2).

2. The magnetic field decavs with a simple exponential from its peak value at $t=t_{c}$.

$$
B=-B_{C} s^{-t / x_{C}}
$$

where $\tau_{i^{\circ}}=250 \mathrm{~ms}$ as in (43).

3. For this model $t$ is set to zero at $t_{\text {( }}$ since neutron production for $t<t$, can be ignored in the equation for Q (12).

4. The energy yield per neutron is assurned to he

$y=20 \mathrm{MeV} /$ neutrón.

5. The $\langle\sigma u\rangle$ curve is approximated by

$$
\langle\sigma u\rangle=\mathrm{Ge}^{-\theta / T}
$$

where

$$
G=10^{-21} u^{2} / s
$$

and

$$
\theta=20.8 \mathrm{~kat}
$$

Fquation (39) is compared with the known D.T reaction rates ${ }^{15}$ in Fig. 4-12.

6. A shanp boundary model is assumed. with ru radial variations inside the boundary. Since the classical energy loss time is long compared with $r$. the radial particle and energy transports are innored.

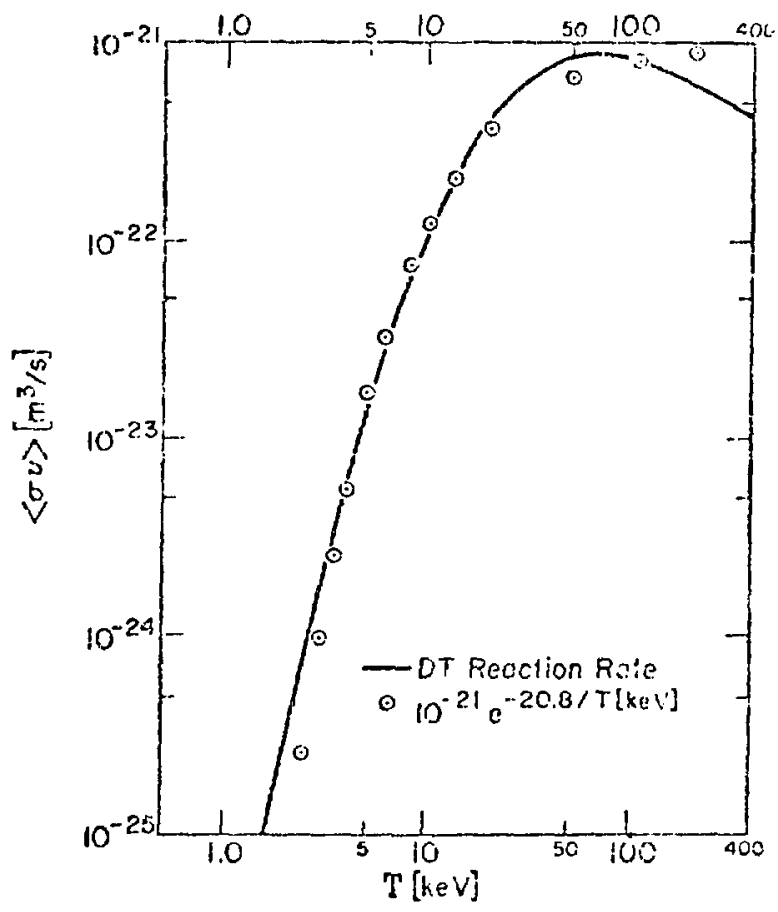

Fig. 4-12.

Comparison of the D-T thermonuclear reaction rate ${ }^{5}$ with the approximation given by (78). 
i. The electrons and ions are assumed to be equilibrated for $\mathrm{t}>\mathrm{t}_{\mathrm{c}}$ (or from assumption $3, t>0$ )

$$
\mathrm{T}=\mathrm{T}_{\mathrm{e}}=\mathrm{T}_{\mathrm{i}} \text {. }
$$

8. The effects of bremsstrahlung, $\alpha$-particle heating, and electron-ion equilibration are ignored except for a single parameter $\alpha$ which is used to correlate the numerical model with this analytic model.

9. To evaluate the integral in (13), it is assumed that

$$
\mathbf{T}<\theta \text {. }
$$

Equation (13) can now be evaluated using (82). (87), (89), and (92)

$$
\begin{aligned}
Q & =\frac{Y_{{ }_{11}}}{12 k_{B} T_{c}} x \\
& \int_{0}^{\infty} \exp \left\{-\frac{12}{5} \frac{t}{\tau_{c}}-\frac{\theta}{T_{c}} e^{4 t / 5 T_{c}}\right\} d t .
\end{aligned}
$$

When $(93)$ is satisfied, the major contribution to the integral in (94) occurs for $t \ll \tau_{r}$. This restriction allows the second term in the exponential to be expanded. thus rendering a tractable integral. The result is

$$
Q=\frac{r_{n}}{H}
$$

where

$$
H \equiv \frac{3+\frac{\theta}{T_{c}}}{\theta / T_{c}}\left(\theta / T_{c}\right)
$$

and

$$
\eta \equiv \frac{5}{48} \frac{G Y \tau_{c}}{k_{B} \theta} .
$$

Equation (95) uniquely determines the relation between the plasma density and temperature at maximum compression for a given value of $Q$ in the SFTR systems. The value of $\eta$ is a constant for the SFTR determined by (88), (90), (91), and (4:3)

$$
n=2.5 \times 10^{-20} \mathrm{~m}^{3} / \text { neutron } .
$$

The variable $\mathrm{H}$ depends only on $\mathrm{T}$, , as is evident in (96). The time asymptotic value of $Q$ is chosen $t o$ be

$$
Q_{\mathrm{f}}=1.1
$$

so that $Q=1$ will be reacied before $150 \mathrm{~ms}$ into the compression.

The numerical model described in the next section indicates that the $\mathrm{o}$-particle heating dominates bremsstrablung losses and ion cooling due to electron-ion equilibration. The result is a slightly larger $Q$ than predicted by (95). A comparison of (95) with the numerical results shows that by using a constant

$$
\alpha \simeq 0.72
$$

excellent agreement can be obtained between the numerical model and the empirical relation

$$
Q_{f}=\frac{n n c}{\alpha H}
$$

Equation (101) can be easily solved for $\mathbf{n}_{\mathbf{t}}$ in terms of $T$.

$$
r_{c}=\frac{a Q_{\mathrm{f}} \mathrm{H}\left(\mathrm{T}_{\mathrm{C}}\right)}{\eta}
$$

and by using (98), (99), and (100)

$$
\mathrm{n}_{\mathrm{C}}\left[\mathrm{cm}^{-3}\right]=3.2 \times 10^{13} \mathrm{H}\left(\mathrm{T}_{\mathrm{C}}\right) .
$$

In the SFTR design it would be most useful to solve (103) for $T_{t}$, since $n_{c}$ is already determined by p. and the maximum compression allowed by stability. The form of (96) does not allow a simple solution, but the following approximate solution is compared to (96) in Fig. 4-1:3

$$
T_{c} \simeq \frac{\theta}{\ln (0.537 \mathrm{H})}
$$

Substituting $(103)$ into $(104)$ vields

$$
T_{\varepsilon}=\frac{\theta}{\ln \left[\frac{n_{c}\left[m^{-5}\right]}{6.0 \times 10^{13}}\right]} .
$$




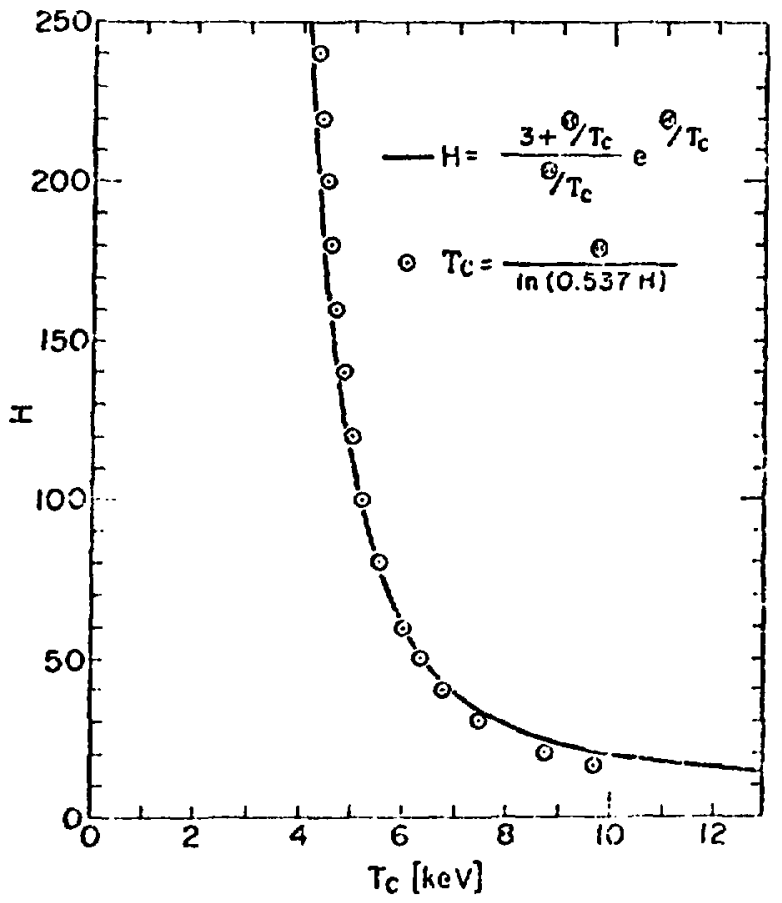

Fig. 4-13.

Comparison of the approximate relation for $T_{\text {. }}$ (10-4) with the definition of $\mathrm{H}$ given by (96).

Conservation of particles requires that

$$
\mathrm{n}_{\mathrm{c}}=\frac{\mathrm{n}_{\mathrm{o}}}{\mathrm{x}_{\mathrm{c}}^{2}}
$$

and by using $(48)$ and $(50), 1106)$ becomes

$$
n_{c}\left[\mathrm{~cm}^{-3}\right]=1.8 \times: .0^{15} \delta_{1}^{4 / s} \text {. }
$$

Combining (107), (105) and (91) yields

$$
T_{c}[k e V]=\frac{26}{\ln \left(70 \delta_{1}\right)}
$$

Equations: $(107)$ and $(108)$ appear as $(78)$ and 179$)$ in the previous section. The values of $n_{\text {, am ' }} T_{\text {' given by }}$ $(107)$ and $(108)$ describe the plasma that can provide the desired $Q$ and st ahility for the minimum induced field Fi.
The plasma parameters at $t=t_{s}$ are related to $n_{c}$

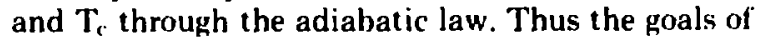
$Q=1$ and $m=1$ stability have been related to $n_{5}$ and T. under the constraint that Et should be minimized.

Minimizing $\mathrm{F}_{*}$ minimizes the technological strain on the implosion system. but it also increases the energy required from the compressym systems. Lsing pressure balance, the peak magne: ir energy density required during compression can be expressed in terms of $T$. by using (96) and 1033 )

$$
W_{B}[M J / m]=0.11 \frac{\left(3+\theta / T_{C}\right)}{\left(\theta / T_{c}\right)^{2}} e^{\theta^{\prime} T_{C}} .
$$

This function is plotted in Fig. 4-14. The minimum required energy density of $0.98 \mathrm{M} . \mathrm{J} / \mathrm{m}^{3}$ is required at $T_{c}=12.6 \mathrm{keV}$. To minimize $\mathrm{F}_{\mathrm{n}}$ for $\dot{b}_{1}=4$ 1 see Fig. 4 $111 . T_{c}=4.6 \mathrm{keV}$. and the required energy density is nearly four times larger. However. since the required fi) $\mathrm{kC}$ is still within reasunable techmological Jimits. Fu should still be minimized.

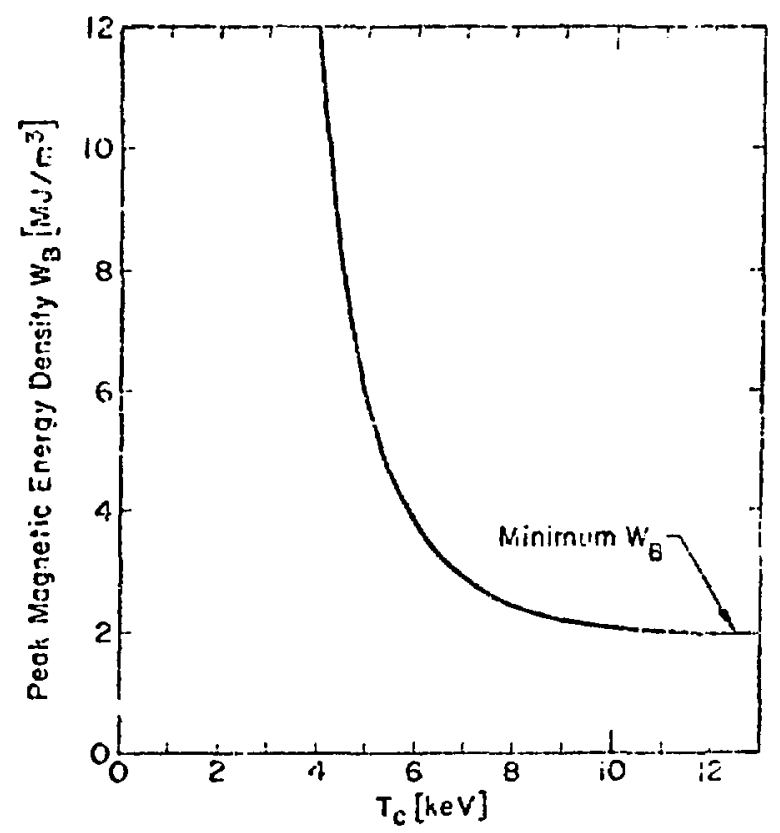

Fig. 4-14.

Magnetic energy density at peak compression $W_{H}$ as a function of the acerage temperature $T_{i}$. 


\subsubsection{Numerical Model}

The thermonuclear burn inodel assumes that there is a sharp boundary between a unitorm ciensits crlindrical plasmat and the magnetic tield. The field is excluded from the plasma. so $s=1$ and the plikma and field pressures are eayual at all times.

The ion and electron distribution functions are Maxwellian, with different temperatures. The aparticles are born at 3.5 .Mel. with a Dopplerbroadened half-width determined by the ion lemperature. in at'ordance with the D).T cross stec. tion. The a-particle production is assumed uniform. but sines the "i-particle orbits take some of the a. particles ont of the hot core we assume at certinin trace. tion 1 interact with the plasma only om part of their urbirs.

These three distribution functions are determined trum the Fokker-Planck equation. and the distributions exolve in time according to the coulumb collision rate and adiahatic changes in the external field. One can therefore moni:or electron and ion cemperature. "r-particle production and heat input (1) the plasmat. neutron and bremsitrahlung emission. direst conversion work by expansion of the plasma anainst the external field, and plasma densi15. and size.

The analytic model in used to explore pussible operating points and to de parameter variation studies. The more promising parameter sets are used in the numerical model to compute the repuired information.

\subsection{IMPLOSION HEATING}

\section{i.3.1 General Description}

The implowion-heating ststem must heat the plasma to the conditions specified in Table 4.1 so that the compression and burn cycle can achiese o $=1$ while maimaining $m=1$ stabilits. The theoretic.1! model presented in this section is needed to connect the field and plasma parameters in Table 4. I 16 the implosion circuit parameters.

The resenant heatio:s appronch involves tuning the implession bank and crowbar switching time to the natural implosion time of the plasma. For this

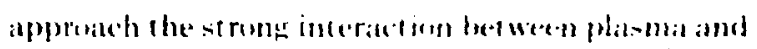

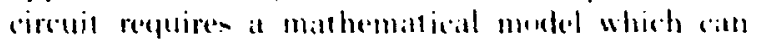
handle the phasma and rescuit denamion

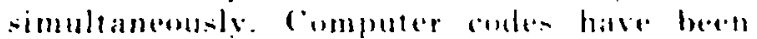
developed that satiste thi- regutement." hat the present sophistication of the platentat merteling stones mut vield resulte that are significantl ditteremt from

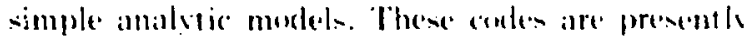

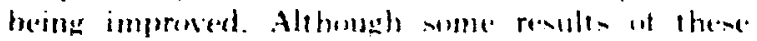

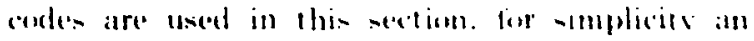

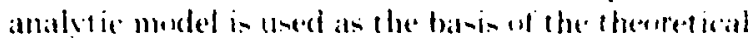
description.

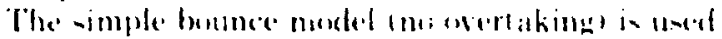

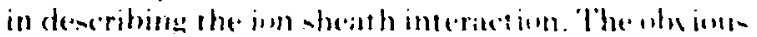
crors insolved in usin: stath a simple midel have.

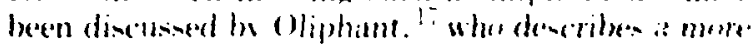
accurate but more complex medel. The simple. model is uned to illu-tratle the bataic plasmat circui. interatetion. leavine the mumerical atcourace to the

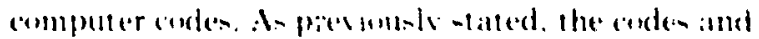
the simple medel de mot deviate signitianthe exeren where indieated in sie. 4.3 .3$.

\subsubsection{Resonant Heating ('oncept}

The optimization of the implesion-heating sy-tem

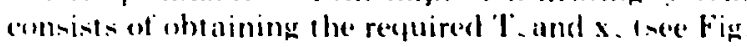
1.10 and Table t-11 for the smallest movible buand $V$. using the simplewt pessible cirenit $1 V^{\text {. }}$ is the capacitor hank voltagel. Freidbers. Morse. and Ribe" determined that the programmed tield shown in Fig. 4.15 vielded larger $x$. and $T$. thr a griver: $l^{\circ}$ than a simple implission field. The lirst tield mulse impludes the plasma, but the field is drupped to zero during the expansion of the plasma so that the plasmat does not give its enerery baek te the field. As the platsma approache's the wall. the lield is raised to the minimum value reeguired to prevent the plasmat from hitting the wall.

An error was made in Ref. s in calculating the relative sizes of the tirst and second field pulse. Figure 4.15 shows the correct lacetor of 2 instead of $\sqrt{2}$. As a result of this error the value of $x$. is mistakenly given as 6.66 instead of the correct wakle (1)

$$
x_{s}=\left(\frac{22}{45}\right)^{1 / 2}=0.70 \text {. }
$$



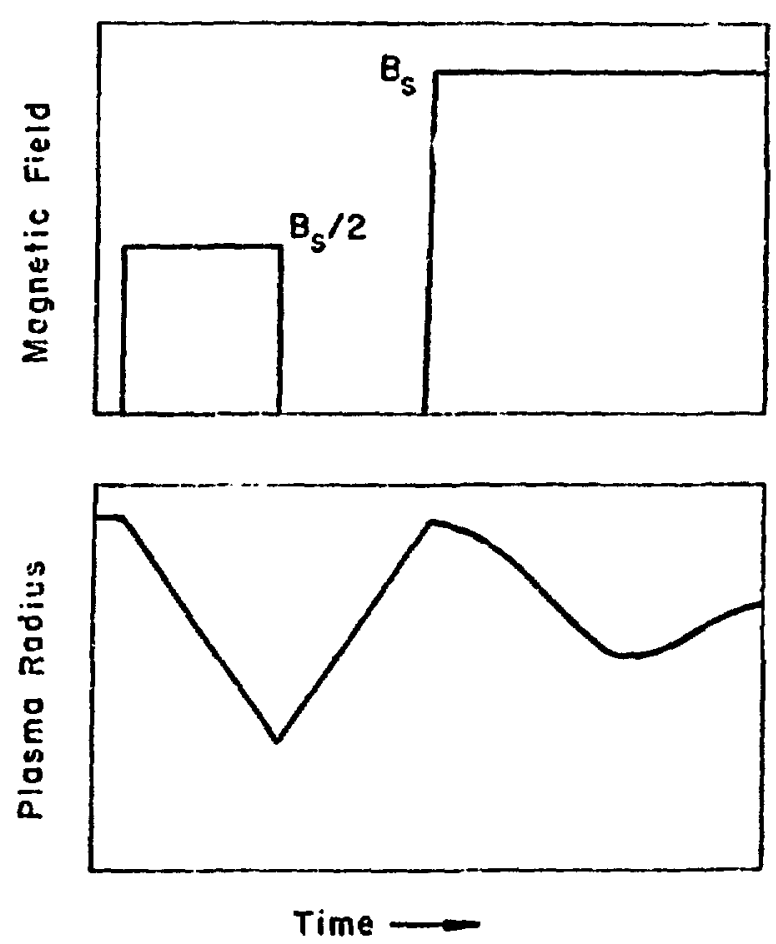

Fig. 4-15.

Programmed implosion field allowing free expansion of the plasma.

Alt hough the field waveform of Fig. $4-15$ cannot be generated by a realistic circuit, the basic features can be produced by the simple circuit shown in Fig. 4-16. The temporal behaviors of the magnetic field and plasma radius shown in this figure were ob. tained from a hybrid simulation code developed by Nielson. ${ }^{16}$ The field is not zero during the plasma expansion. but it is less than the initial implosion fjeld. As a result. only a small fraction of the plasma energy is given back to the field during the expansion.

The circuit is topologically the same as the classical theta-pinch circuit used in Scyllac. However, the source and crowbar inductances $L_{s}$ and $1_{4}$. are made smaller than the empty load coil inductance $L_{1}$. In addition the bank capacitance $\mathrm{C}$ is chosen so that the circuit is tuned to the implosion time of the plasma, hence the name resonant heating.

More precisely, the circuit is tuned so that by the time of the maximum implosion $t_{\mathrm{cr}}$ the field has peaked and decayed to a specific value $B_{c r}$. At this time $t_{c r}$ the crowbar switch is closed and the magnetic flux corresponding to $B_{\mathrm{cr}}$ is compressed by the expanding plasma. If $L_{r}$ and $L_{c}$ are sufficiently small and if $B_{t r}$ is chosen properly, the pulse of field at 4 will be just sufficient to prevent the plasma

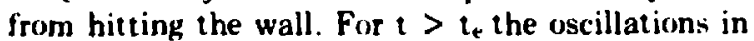
the field and the plasma radius dampen rapidly as the plasma thermalizes and isotropizes. Of course the field exponentially decays on a much longer time scale as is shown in Fig. 4.7.

The second field pulse at $t_{t}$ (Fig. 4-16) is not twice as large as the initial pulse mainly because of the plasma energy lost during the expansion against a nonzero field.

Any analysis of this resonant heating system must handle the circuit and plasma dynamics simultaneously. The interaction of the circuit anc plasma cannot be ignored hecause of the dominant effect of $L_{p}(t)$ in the circuit. The analytic model described in the next section includes this plasmacircuit interaction. The model is used to determine
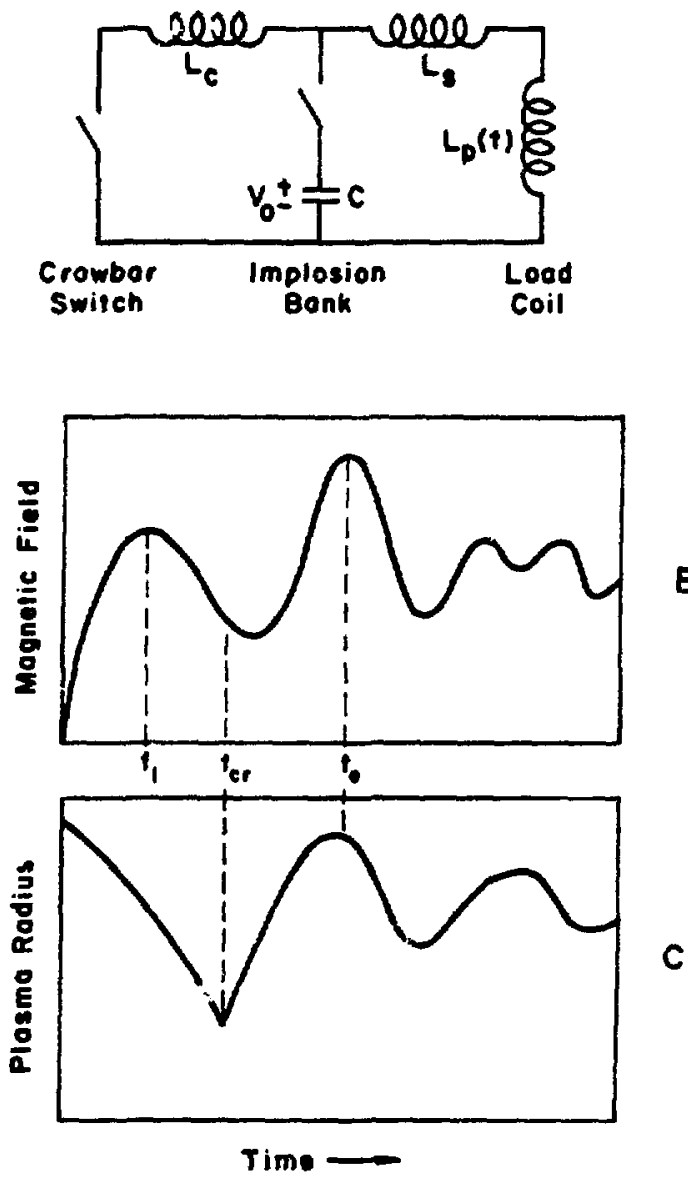

Fig. 4-16.

Resonant heating circuit showing the temporal behaviar of the B-field and the plasma radius. 
the values of the circuit components and the relation between $T_{s}, x_{i}, E_{t}$, and $V_{0}$. In addition, the minimum $V_{0}$ is obtained by optimizing the number of turns on the implosion coil. The relation between the turns and the inductance of the implosion coil is derived in Sec. 5.2.2.1.1.

\subsubsection{Modeling of the Implosion}

The analytic model for the implosion process described in this section actually consists of three separate models used in sequence. Each of the models describes a separate portion of the time history of the plasma. The segments of time are delineated by $t_{\mathrm{cr}}$ and $t_{\mathrm{p}}$ in Fig. 4-16, and are venoted by the following descriptive names:

1. Initial Implosion $0<t<t_{c r}$

2. Expansion and Reflection $t_{\mathrm{cr}}<t<t_{t}$

3. Isotropization and Thermalization $t_{\mathrm{e}}<\mathrm{t}<\mathrm{t}_{\mathrm{s}}$. The isotropization and thermalization are assumed to be complete by $t=t_{*}$, where $t_{s}$ is defined by Fig. 4-7. A brief description of the three models is given hefore any calculations are presented.

\subsubsection{Description of the Models}

\section{Initial Implosion}

The plasma is described by a sharp boundary. simple bounce model with no overtaking. That is. each ion is assumed to bounce elastically off the magnetic piston only once. Physically, the ions struck by the piston at very early times will be overtaken one or more times by the accelerating piston, but this fact is ignored by the model. This problem of overtaking as well as inelastic collisions between piston and ions (snowplo'v model) has been treated analy+ically by Oliphant. ${ }^{17}$ Finite sheath thickness and cvertaking have been included in simulation codes. ${ }^{9.16}$ Although the effects of finite sheath thickness, overtaking, and snowplowing are observable, they do not appear to dominate the gross plasma-circuit interaction. Thus for the sake of simplicity they are omitted from the models prosented here. The end of the initial implosion at $t$ $=\mathrm{C}_{\mathrm{r} r}$ is defined by the time when the first accelerated ions strike the sheath on the opposite side of the plasma after passing through the center of the system.

The circuit used in this portion of the model is one loop of the circuit shown in Fig. 4-16. The loop containing the crowbar switch is not needed since the switch is not closed until $t=t_{\mathrm{cr}}$. The plasma effects are introduced to the one loop equation through
L, (t). A single time varving inductor to model the plasma effects can be used only if a sharp boundary plasma model is used.

The equation arising from this simple model has a closed form solution in only a few special cases. Analytic scaling laws obtained from the equation are helpful in understanding the system, but the equation must be solved numerically for some of the final results. The results include the required relations between the induced electric field $E_{t}$, the bank voltage $V_{0}$, the peak circuit current $i_{1}$, and the peak plasma energy $E_{\mathrm{rr}}$ (the subscripts 1 and $\mathrm{cr}$ refer to the times $t_{1}$ and $t_{c r}$ shown in Fig. 4-16).

\section{Expansion and Reflection}

The simple bounce concept used in the first model breaks down at $t=t_{\mathrm{cr}}$ when fast ions begin striking the piston. At this time the crowbar switch is closed and this also complicates the circuit model. Thus a new model is needed for $t_{1 \cdot r}<t<t_{\text {t }}$ that takes advantage of the new boundary conditions of the system.

Two basic assumptions are made. First, the crowbar is assumed to be "perfect" thus causing the tot al magnetic flux $\phi_{\mathrm{T}}$ and the total energy (magnetic plus plasma) to be constants of the motion. This assumption is justified if the transfer time of the current from the capacitor branch to the crowbar is short compared with the implosion time. The second assumption is that pressure balance exists during the reflection at $t=t_{\mathrm{e}}$. An accurate description of the dynamic plasma pressure at $t=t_{\mathfrak{c}}$ would require a knowledge of the phase space distribution of the plasma. Since this information is not obtainable in analytic form, the plasma is assumed to be thermalized with a finite degree of isotropization parameterized by $\epsilon$.

The results of this model are combined with the initial implosion results to determine requirements for tuning the circuit to the plasma implosion time. The actual calculation of the sizes of the circuit parameters is presented in Sec. 4.3.4

\section{Isotropization and Thermalization}

The purpose of this model is to determine the $x$ : and $T_{s}$ of the plasma as a function or initial plasma and circuit parameters. The conservation of flux and total energy is assumed as in the Expansion and Reflection model. The plasma is assumed to be thermalized, isotropized in three dimensions, and in pressure balance with the field at $t=t_{k}$. As a pessimistic assumption

$$
T_{\text {es }} \simeq \frac{1}{2} T_{\text {is }}
$$

which is less than that observed in Scyllac. ${ }^{14}$ Equation (111) is in approximate agreement with a 
The least restrictive assumptions required to calculate the plasma heating efficiency for an implosion $e_{p 1}$ are that the change in plasma energy is large compared with the initial plasma energy.

$$
\Delta E_{p} \gg E_{p_{1}} \text {, }
$$

and that in state (2) the plasma thermalizes, isotropizes into three dimensions, and comes into pressure balance with the field

$$
\frac{\Delta E_{D_{1}}}{E_{B_{2}}} \simeq \frac{E_{p_{2}}}{E_{B_{2}}}=\frac{\frac{3}{2} V_{D_{2}}}{V_{L}-V_{P_{2}}} .
$$

The plasma heating efficiency for implosion heating $e_{p 1}$ is obtained directly from (21)

$$
e_{p 1}=\frac{3}{2} v\left[\frac{1}{1+\frac{1}{2} v}\right] \text {. }
$$

This efficiency is compared with $e_{\mathrm{pa}}$ in Fig. 4-4.

Several important observations can be made by examining Fig. 4-4 and the assumptions leading to Eqs. (18) and (22). First, nonadjabatic heating is always more "efficient" than adiabatic heating. Note that the asymptotic behavior of (18) and (22) for small $u$ is a result of the fact that for small $\nu$ the adiabatic assumptions are a special case of the im. plosion assumptions. Also, if a plasma is heated by a process that greatly changes the plasma energy without significantly changing the plasma volume, the process must be nonadiabatic. Finally, in contrast with economic and technological considerations. implosion heating appears most attractive when plasma heating efficiency is considered.

The general statements that can be made about circuit efficiency $e_{c}$ are much less quantitative than those just made about $e_{p}$. One reason for the lack of simplicity in $e_{c}$ is the virtually limitless variety of circuits that could be used in the implosion and compression systems. However, some qualitative statements can be made with approximate numerical examples for the systems proposed for the SFTR.

Compression circuits like the one in Fig. 4.3 can be built (with rise times on the order of $1 \mathrm{~ms}$ ) to transfer virtually all of the stored energy $E_{s}$ into the load coil. In this case, the efficiency $e_{c}$ depends mainly on the relative volume of the load boundary $V_{p 1}$ and the load coil $V_{1}$.

$$
e_{c a}=\frac{1+\frac{1}{2} v-\frac{3}{2} v s / 3}{\frac{V_{L}}{V_{p_{1}}}+\frac{1}{2} v-\frac{3}{2} v^{5 / 3}}
$$

The total efficiency becomes, using (12), (18), and (23),

$$
e_{T a}=\frac{3 v\left(1-v^{2 / 3}\right)}{2 \frac{V_{L}}{V_{P:}}+v-3 v^{5 / 3}} \text {. }
$$

Using $V_{\mathrm{L}} N_{\mathrm{pl}}=8.2$ for the SFTR compression system, Eq. (24) is plotted in Fig. 4-5. The value of $\sqrt{v}$ for the compression system in SFTR is about 0.5 , thus

$$
\mathrm{e}_{\mathrm{Ta}}=0.03 \text {. }
$$

In general, implosion circuits are much more dissipative than compression circuits because of the greater speed of energy transfer. In addition to resistive losses, the circuit described in Sec. 4.3 is optimized with the source inductance comparable

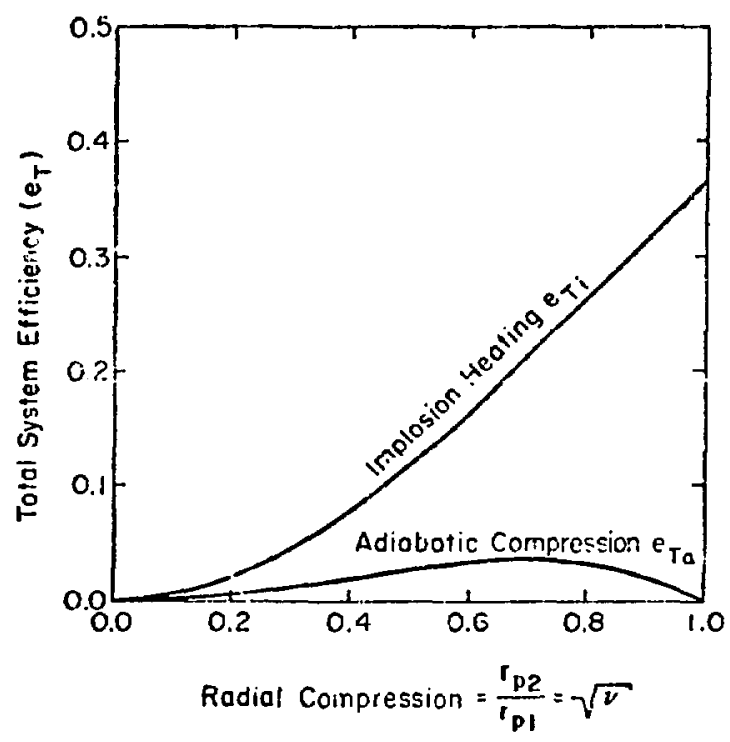

Fig. 4-5.

Comparison of total sustem efficiency for implosion heating and adiabatic compression in the SFTR system. 
with the load coil inductance. These resistive and inductive losses limit the efficiency of energy transfer into the load coil to about $40{ }^{\circ}$. Hence.

$$
e_{c i} \approx 0.4 \frac{1+\frac{1}{2} v}{\frac{v_{L}}{v_{p_{1}}}+\frac{1}{2} v} .
$$

The total implosion efficiency becomes, using (12). $(2 \cdot 2)$, and (26).

$$
\mathrm{e}_{\mathrm{Ti}} \simeq 0.6 \frac{v}{\frac{\mathrm{V}_{\mathrm{L}}}{\mathrm{v}_{\mathrm{p}_{i}}}+\frac{2}{2} v} .
$$

I sing $V_{1} N_{p 1}=1.14$ for the SFTR implosion system, Eq. $(16)$ is compared with $\mathrm{e}_{\mathrm{Ta}}$ in Fig. 4-5. The value of $\sqrt{1}$ for the implosion system in SFTR is about 0.7 . so

$$
e_{\mathrm{Ti}} \simeq 0.3 \text {. }
$$

The comparisor in Fig. 4-5 indicates that, in spite of the dissipative nature of the implosion circuit, the implosion system transfers a far larger fraction of its stored energy to the plasma than does the compression system. This fact might mislead one into doing all of the heating with the implosion system. If this mistake were made, the compression system would still have to supply essentially the same amount of energy simply to contain the plasma (no compression, i.e. $\sqrt{y}=1.0)$ during the long burn cycle. Hence the same compression system could have supplied a major portion of the plasma energy with essentially the same anount of stored energy. Ohviously, ne more heating done by the compression system, the more the cosi and the technological difficulty of the implosion system are reduced. Looking beyond SFTR, in future reactor systems the compression field energy will be retrieved by the circuit and recycled with a high degree of efficiency in contrast to retrieving only a small portion of the wasted implosion field energy in the form of heat.

All these considerations indicate that as much of the plasma heating as possible should be done by adiabatic compression. The limitation on the fraction of heating done by compression will arise from some plasma physics consideration such as electrical conductivity or plasma stability, or it may be set by an efficiency requirement of the reactor. The remainder of the heating will have to be provided by the implosion system which should be made as efficient as possible by minimizing the complexity of the circuit and by maximizing the value of $\sqrt{v}=\mathrm{x}$, for the implosion. A quantitative analysis of the dominant factors limiting the fraction of heating done by compression in SFTR is presented in sece. 4.1.4.3.

\subsubsection{Wall Stabilization a:1d Slow Feediback Control}

There are two concept ual techniques for achieving plasma stability. The design of the SFTR presented in this report is based on the concept of wall stabilization in conjunction with slow feedhack contro!. Straight feedback (like that being developed for S(yllac) appears to have merit, but its impact on an SFTR design has not been fully investigated. The projerties of both concepts are discussed and brietly compared in the remainaer of this section.

\subsection{R cessity for Slow Feedback Control in a Wall-Stabilized SFTR}

The $m=1$ mode cannot be wall-stahilized by the finite (noninfinite) conductivity implosion coil ${ }^{11}$ even though the growth rate of the mode might be greatly reduced. The mode can be examined as the conductivity of the wall is theoretically increased from $\sigma=0,{ }^{12}$ In general, $\gamma$ decreases as $\sigma$ increases. When the inertial effects are no longer important compared with the dissipative effects of the conducting $w . ~ U$, the growth rate is given by the field diffusion rate through the wall. Thus, for a wall of finite conductivity, $\gamma$ can be reduced but not to zero. Since the confinement time required in SFTR exceeds the field diffusion time, some feedback control is necessary.

The field diffusion time depends on the specific geometry of the system shown schematically in Fig. 4-6. The separation of the implosion and compression systems discussed in Sec. 4.1.4.1 requires the use of two distinct coils. The fractional turn implosion coil (see Sec. 5.2.2.1.1) provides the low inductance load for the implosion system. The multiturn, high-inductance compression coil, described in Sec. 5.2 .2 .1 .2 , is required to produce the $250-\mathrm{ms}$ decay time for SFTR. The field diffuses through the metal of the implosion coil with the characteristic time

$$
\tau_{0}=\frac{\mu_{0} \sigma \delta^{2}}{2} .
$$




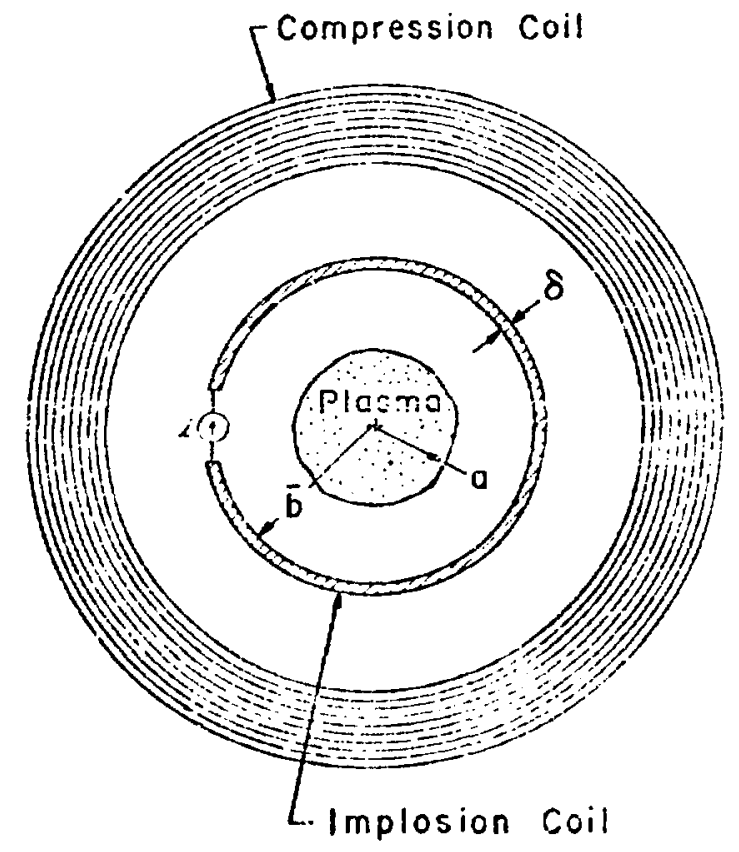

Fig. $1-f$

Sir hemafic repressinfation of the fractional surn implesion coil and multiturn compression coil.

However. the field enclosed by the coil decays at a slower rate given by the $L / R$ time of the coil. For the simple coil shown in Fig. 4-6 with no plssma present.

$$
\tau_{L / R}=\frac{1 ! 0 \cap \bar{b} \dot{0}}{2} \text {. }
$$

Two complexities modify this simple model of field diffusion. The in piosion coil is crowbarred by a switch. If the resistance of the switch is sufficiently high compared with that of the coil. the switch will dominate the L/R decay, invalidating Eq. (30). Even if the crowbar were perfect. the presence and motion of the plasma in conjunction with the $t=1$ perturbatiun generate fieid perturbations that are responsible for wall stabilization. In the perfect crowbar condition. these field perturbations will decay at a rate given by $(30)$ with $\bar{b}$ replaced with some characteristic length of the perturbation which could range from $\bar{b}$ to $\dot{b}$.

It is advant andenss to increase the fiedd decay time as moch as possible becaluse an : increases. deereat:ies. In addition, the required rise lime of the compression lield increases. The thicklless of that conductur a ran be increised umil the crewhar swith renist ance dombinates the 1 , $R$ time. Il o were

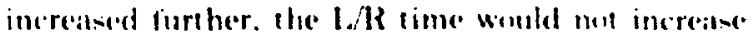

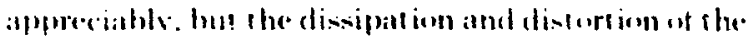

rising compression field wouid begin 10 increast sharply.

To maximize the $L / R$ time and minimize dissipa. tion of the compression field, the following !imits are placed on $\delta$

$$
\frac{\mu_{0} \sigma \delta^{2}}{2}<\tau_{s} \ll \frac{\mu_{0} \sigma \bar{b} \delta}{2},
$$

where $\tau_{n}$ is the crowbar-dominated $L / R$ time of the implosion circuit. The subscript $\mathrm{s}$ is used to refer to the implosion or "shock" process to be consistent with the literature and 10 avoid confusion with subscript $i$ for ions. As was mentioned in Sec. 4.1.4.1 the maximum crowbar times achieved on present systems have been on the order of $100 \mu \mathrm{s}$. The value projected for SFTR is $\tau_{*}=230 \mu \mathrm{s}$. Lising this $\tau_{*}$ in (31) yields

$$
0.059 \ll \delta<2.5 \mathrm{rm} .
$$

The value chosen for SFTR is $\delta=1.5 \mathrm{~mm}$.

$W$ ith $\tau$, and $\delta$ determined, the following limits can be placed on the wall-stabilized growth rate

$$
\frac{1}{i_{s}} \approx \gamma_{h s} \approx \frac{1}{\tau_{0}} \text {. }
$$

Simply stated. the growth rate is bounded on the lower side by the field decay rate due to the crowbar gap. and on the upper side by the diffusion rate of the field into the metal of the implosion coil. Fsaluating (3:3) yields

$$
4.3 \times 10^{3} \div r_{w s}<1.2 \times 10^{4} \mathrm{~s}^{-1} \cdot 134
$$

Since $\gamma_{u_{x}} \tau_{t}<0.4$ is required for stabilization in a practical feedback system ${ }^{1:}$, ith rise time $\tau_{r}$.

$$
\tau_{r} ₹ 33 \mathrm{Hs}
$$

in the wall-stabilized SFTR.

4.1.4.2.2 Comparison of Straight Feedback with the Wall-Stabilization Feedback Combination

The major advantage of the wall-stabilized approach over st raight feedback is the slower rise time requited of the feedback system. In the case of straight feedback it is assumed that the plasma is compressed significantly beyond the limit imposed by (17). thus eliminating the effect of wall stabilization. Equation (1i) was obtained from the growt to 
rate of the $m=1$ mode for infinitely conducting walls $(\sigma=\infty)$.

$$
\begin{aligned}
y= & h v_{\alpha} \beta\left[-\left(\frac{a}{b}\right)^{4} \delta_{i}^{2}+g_{d} \varepsilon_{a}^{2}\right. \\
& \left.+g_{2} \delta_{2}^{2}+g_{1}(h a) \varepsilon_{i} \varepsilon^{2}\right]^{1 / 2}
\end{aligned}
$$

where $v_{,}$, the Alven speed, is

$$
v_{\alpha} \equiv \frac{b}{(\mu, n m)^{1 / 2}} .
$$

The straight teedback sistem mus be capable of respending to a growth rate given by $1: 36$ when the wall-stabilizing term lfirst term in the hrackets) is ignored. l'sing typical sFl'k parameters. rhis growth rate is

$$
\gamma_{f}=3.5 \times 10^{5} \mathrm{~s}^{-1}
$$

Since it ir 211.4 . the required rine time is

$$
\tau_{I} ₹ 1.3 .115 \text {. }
$$

A comparison of 6351 with $1: 3 i$ indicate that the straight feedhack sistem wouid have (1) he :3) times faster than the slow feedhack sistem used in the wall-wabilized approach.

This faster rise time makes it more difficult (1) electrically isolate the straight feedhack sistem from the implusion system. Buth ! ypes of feedhack system would have tes tise the implosion a . il as the feedhack drive croil. Feedhack coils canmen be plateed inside the implosion coil as in Scyilac because of the stringent. low source inductance requirements of the SFTR implosion system. They cannot be placed out. side the implosion coil because the lield penct ration time through the implosion cosl would be cors slow to" sat isty $; i_{,}<0.4$. Since the feedhack sistem must be attached to the 2-70.kl implosion stitem. very effectise electrical isolation is required to protect the teedback switem. The greater the ditierence in the rise time between the feedback sistem and the im. plosion sistem (10) nsl the more effective the isolation can he made.

The major disadwntage of the wall-stabilized appronch is that it requires a larger ratio of $a / b$ and hence more implosion heat ing than the st raight feedback approach. However. the reduct ion in implosion heating allowed by straight feedback may be small because of a constraint on the minimum DT tilling

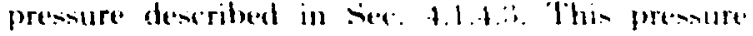

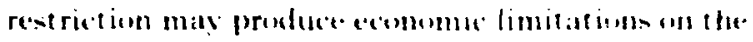
reduction of implesent heating that weruld dommate

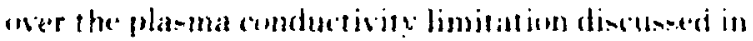

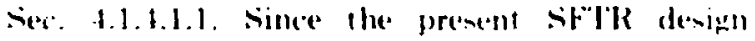
utilizes the minimum allowed filling presisure of 1

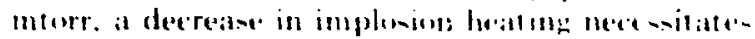
high orinpressional heat ing. The result in a plasmat with similar temperature but higher density. and this higher plasmat presisure must be contined bs a

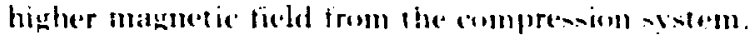
A preliminary insestigation andicates that

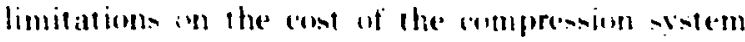
maty presemel the fowering of the implesisen bank soltage by more thats ger Althetash smatle thireduction could be terhmolugsolls important.

At this time the wall-atabilized approith appearthe mos promising. but the straight teedback syetem is not without merit. The experimental experience with wall stabilisation. implesion heating.

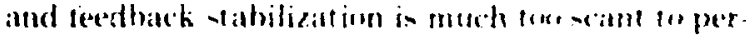
mit a tinal decision beriseen wall stabilization and,

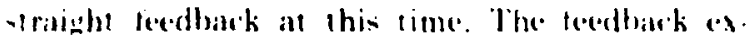

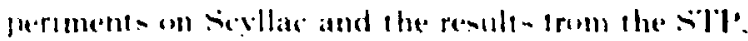
IHX. atnd resolnam heating experiment- will asd wreatly in making that decivion.

\subsubsection{Operating Point Determination}

The taging coneept and wall stabilization ap. proach form the basio of this Slelle resign. This ap. proach was ehosen as a result of the concepts presented in sees. 4.1.4.1 and 4.1.t.2. In the remainder if this sectim, relationships derived elsewhere in ('hisp. It are used of determine the numerical value or limits on a variety of machine and plasma parameters.

To aid in the following presentation, the temperal behasior of the magnetir field is ploted in Fig. 4-7 in a logarithmir lime base. A more eomplete diseussion of the lield behavior and the implesiten and compression circuits is found in sers. 1.2 and 3.3 .1 The implosirm field rises in about l(n) ns, oscillatpe briotly, and settle: down to a value $B$, at a time $i$.. The implosion field decays at an sxponemial rate $t$. assesiated with the $\mathrm{J} / \mathrm{K}$ time of the crowblared im. plosion or "shock" circuit. During this decay. The irmpression field rises to a peak value of $B$, in a time $t_{1}$. The compression circuit is crowbarred at $1=1$, allowing the field tudecay at the exponential rate $r_{2}$.

several reasins for minimizing the amount of im. plession heating were presented in sece 4.1.4.1.1. Another reason is apparent from a study of $\mathrm{Fig} .4-7$.

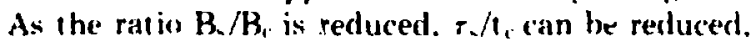




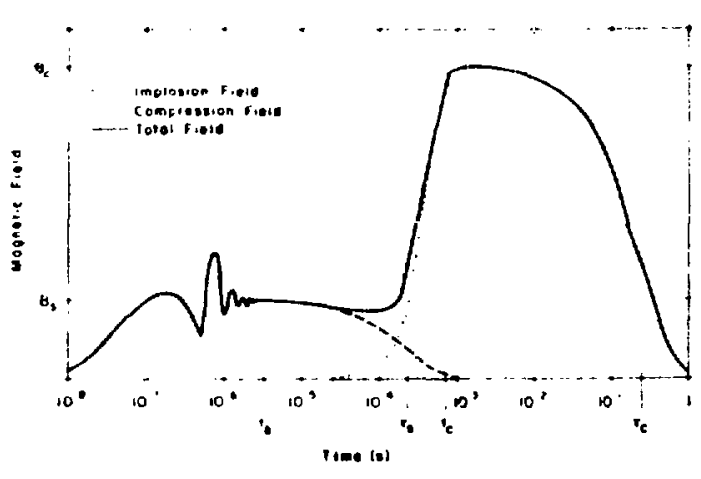

Fing. $1-7$

T'mporal hehatiur of the masnetic field produced the the implession and compression $\therefore$ stim

thus decreasing the requirements of the implosions crewbar -ristem ther a fixed fractional sag in the total field during the staging processil.

1.1.1.3.1 Mirimization of the Electric Fields and listrage's Reculuired bs the Impiosion Process.

As is discussed in Sece 4.1.4.1, one of the main reasons for minimizing the amount of implosion healing is that the implosion sistem is technolongicalls much more difficult than the com. presison system. A major prohlem is the high fields and voltages required by the implosion process. A number of the sistem and plasma parameters are speified by the process of minimizing these field:i and voltages.

Plasma physies considerations determine the re. quired value of the electric field $E_{\text {w }}$ induced around the plasma by the impksion stistem. In turn. Ewand the circuit efficiency determine the voltage $\mathrm{V}^{\prime}$. needed on the implosion bank. Thus Fo is minimized first. then the circuit is optimized to reduce V'to the smallest allowed value.

Six important parameters deternine the value of H. (sere tieg. 4.3):

$$
\begin{aligned}
& x_{c}=\frac{a\left(t_{c}\right)}{\vec{b}}, \\
& x_{s}=\frac{a\left(t_{s}\right)}{\vec{b}},
\end{aligned}
$$

the maximum average plasma temperature

$$
T_{c}=\frac{T_{j c}+T_{e c}}{2} \text { at } t=t_{c}
$$

the compresion field decay time ${ }_{1}$. the oT filling pressire $p$. and $Q$. The value of $Q=1$ unust be reacked in a time to $<T_{\text {, since the neutron }}$ production drops sharply as plasma expands and cooks. Thus $\tau_{1}$ should br made as large as possible. Results from sece. 5.4 indicate that the a:axirnum reasmable $\mathrm{L} / \mathrm{R}$ time is

$$
\tau_{c}=250 \mathrm{~ms} .
$$

To minimize the chance of losing the plasma bogores $\mathbf{Q}=1$ is reached, the value of to is set at

$$
t_{Q}=150 \mathrm{~ms} \text {. }
$$

From the code described in seo. 4.2.3. 1 he tinal valur: of $Q$ required to satisfy $14+1$ is

$$
Q_{f} \simeq 1.1 \text {. }
$$

In agreement with Sec. 4.1.4.i.2. x. should he maximized to optimize the implosion-heating etficiency. and $x_{c}$ should be minimized to minimze the amount of reciluired implosion heating. implosion codes indicate (see Sec. $4.3,3$ ) that

$$
x_{s} \simeq 0.7
$$

is obtainable, vielding an implosion efficiency of

$$
\varepsilon_{\mathrm{T} i} \simeq 0.21
$$

from Fig. 4-i). The minimum value of $x$ consistent with stability requirements is obrained in sec. 4.1.4.3.2.

'The quantities $p_{1}$ and $T_{1}$ are related $10 x_{1}$ through the definition of $Q$. If no additionai constraints are placed on $p_{1}, T_{c}=8 \mathrm{keV}$ yields the lowest value of $E_{*}$. The filling pressure $p_{n}$ is then determined by the value of $Q$ tsee Sec. 4.3.3). Infortunately. the resulting value of $p_{\text {. }}$ is less than the minimum value. of 4 morr at which standard thet a pios hess bave been operated at LASL. If $p_{1}$ is too low. an adequate sheath for implosion fails to form. Aithough this lower limit has not been thoroughly explored, past experience indicates that it is berween 1 arid 4 mtorr. If the pressure is set at

$$
p_{0}=4 \text { mtorr }
$$


then $T_{r}$ can be reduced as íar as Eq. (45) will allow. Even though the value of $T_{c}$ depends on $x_{e}$, the strong dependence of the $\langle\sigma v\rangle$ curve (see Sec. $4.2 .2)$ on $T_{c}$ limits the range of $T_{c}$ to

$$
4.6 \approx \mathrm{T}_{\mathrm{c}} \approx 6 \mathrm{keV}
$$

for $0.23<\mathrm{x}^{\prime}<0.4$

Using the values of the four parameters $Q, x_{s}, \tau_{c}$, and $p_{1}$ just established in this section, the value of $E_{t}$ is approximately given by [see Eq. (199)]

$$
E_{\theta}[\mathrm{kV} / \mathrm{cm}] \simeq 65 \mathrm{x}_{\mathrm{c}}^{4 / 3} / \ln \left(5.3 / \mathrm{x}_{\mathrm{c}}^{2} \mathrm{Q}\right) .
$$

To obtain the minimum value of $E_{t}, x_{c}$ must be mininized within the constraints imposed by (:i) and (6).

\subsection{Minimization of $x_{\text {. for a Wall-Stabilized }}$} Sirstem

As discussed in the previous section, a reduction in $F_{4}$ reduces the technological problems associated with the implosion system. From (50) it is apparent that ${ }_{d}$, is reduced by minimizing $x_{c}$ within the plasma equilibrium and stability constraints given hy $! 31$ and (6).

This minimization is easily accomplished by climinating one of the variables between $(3)$ and $(6)$ and minimizing a with respect to the rest of the variables. Since $h$ has essentially no effect on the cust or complexity of the system, it is the ideal variable to eliminate. giving

$$
\begin{aligned}
a^{4}- & \frac{{E_{2}}_{1} \bar{b}^{4}}{R \delta_{1}\left(f_{0} \delta_{0}+E_{2} \dot{U}_{2}\right)} a \\
& -\frac{\bar{b}^{4}}{\delta_{1}^{2}}\left(g_{0} \delta_{0}{ }^{2}+\delta_{2} \hat{\delta}_{2}{ }^{2}\right)=0 .
\end{aligned}
$$

Fron 1511 it can be shown that the following inequalities hold. independent of the other variables

$$
\begin{aligned}
& \frac{\partial a}{\partial R}<0, \\
& \frac{\partial a}{\partial \bar{b}}>0, \\
& \frac{\partial a}{\partial \varepsilon_{1}}<0 .
\end{aligned}
$$

These inequalities indicate that $R$ and $\delta_{1}$ should be chosen as large as possible and $\bar{b}$ should be chosen as small as possible.

The upper bound on $\mathrm{R}$ is set by economics and the operational and reliability problems associated with a large system. A compromise between those limitations and the relatively weak dependence of a on $R$ [see (63)] resulted in the choice of

$$
R=40 \mathrm{~m} \text {. }
$$

The inequality (53) and the desire to minimize the cost of SFTR imply that $\bar{b}$ should be made as small as possible. However, the plasma sheath thickness must be small compared with $b$ or $\bar{b}$ for an adequate implosion. In present theta-pinch sysiems the sheath thickness is 2 or $3 \mathrm{~cm}$. The sheath thickness is often assumed to be $c / \omega_{p}$, although the sheath formation is probably much more complex than is indicated by this simple relation. For a filling pressure of 4 mtorr |see (48)| this relation yields

$$
\frac{c}{\omega_{p i}}=2.1 \mathrm{~cm}
$$

which agrees with the experimental observations. Thus, to insure an adequate implosion, the vacuum wall radius is set at

$$
\mathbf{b}=10 \mathrm{~cm} \text {. }
$$

The radius of the implosion coil conductors is chosen to be

$$
\bar{b}=10.7 \mathrm{~cm}
$$

to allow for the $7 \mathrm{~mm}$ of insulation required bv the fabrication process described in Sec. 5.2.3.1.

The maximum allowed value of $\delta_{1}$ is really not known. As described in Sec. 4.1.3.2, the analytic model used here theoretically breaks down for $\delta_{1} \overline{>}_{1}$, and yet the model has been experimentally verified ${ }^{8}$ for $\delta_{1}=3$. There is a strong motivation to maximize $\delta_{1}$ because a is more strongly dependent on $\delta_{1}$ than on either $R$ or $\bar{b}$ [see (63)]. One is also reluctant to set $\delta_{1}$ $=3$ or larger because of the lack of extensive experimental experience at these large values.

Before the final design of SFTR is made, future expriments will provide additional information about the limitations on $\mathrm{p}_{n}, \mathrm{E}_{n}$, and $\delta_{1}$. This information will allow a more rational choice of design values for these parameters than can be made at the present time. In this report, the pressure is set 
at $\mathrm{p}_{t}=4$ mtorr. and $\delta_{1}$ remains a free parameter within the range

$$
\text { 1. } ₹ \delta_{1} \approx 4_{4} \text {. }
$$

This variation in $\delta_{1}$ will produce a corresponding range of values for $x_{C}, E_{\theta}, V_{0}$ and other related parameters. This range of operating conditions will allow the impact of future experiments on the SFTR design to be more readily assessed.

Now, $R, \bar{b}$, and $\delta_{1}$ can be considered fixed quantities in Eq. (51). When $\delta_{0}$ and $\delta_{2}$ are varied to minimize a.

$$
\begin{aligned}
& \delta_{0}=\frac{f_{0}}{g_{0}}\left(\frac{g_{1} \delta_{1} a}{2 R} \lambda^{2}\right)^{1 / 3}, \\
& \delta_{2}=\frac{f_{2}}{g_{2}}\left(\frac{g_{1} \delta_{1} a}{2 R} \Lambda^{2}\right)^{1 / 3}
\end{aligned}
$$

where

$$
\Lambda \equiv \frac{g_{0} g_{2}}{f_{0}{ }^{2} g_{2}+f_{2}^{2} g_{0}} .
$$

Substituting (60) and (61) into (51) yields

$$
\frac{a}{\bar{b}}=\frac{\left(3 \Lambda^{1 / 3}\right)^{3 / 10}}{\delta_{1}^{2 / 5}}\left(\frac{g_{1} \bar{b}}{2 \bar{k}}\right)^{1 / 5} .
$$

The corresponding wave number becomes

$$
\mathrm{h}=\left(\frac{2 \lambda}{g_{1}}\right)^{1 / 5}\left(\frac{1}{a^{2} k \delta_{1}^{2}}\right)^{1 / 3} .
$$

Equations $(60)$ through $(64)$ can be numerically evaluated (except for $\delta_{1}$ ) for an appropriate value of p. This analytic old-ordering model is normally evaluated with $\beta=0.8$ for high $-\beta$ plasmas because results agree well with the more accurate model ${ }^{\top}$ for $0.8<\beta<1.0$. For $\beta-1.0$ the old ordering possesses unphysical singular behavior as is evident in Eq. (19). Using

$$
\begin{aligned}
& \beta=0.8, \\
& \delta_{0}=0.13 \hat{o}_{1}^{1 / 5},
\end{aligned}
$$

$$
\begin{aligned}
& \delta_{2}=0.044 \delta_{1}^{1 / 5}, \\
& \lambda[\mathrm{m}]=2.7 \delta_{1}{ }^{2 / 5}, \\
& z[\mathrm{~cm}]=\frac{4.6}{\delta_{2} 2 / 5},
\end{aligned}
$$

and from $(40)$ and $(58)$

$$
x_{c}=\frac{0.37}{\delta_{1}^{2 / 5}} \text {. }
$$

Equation (70) yields the desired minimum value of $x_{4}$, dependent only on the choice of $\dot{b}_{1}$.

From an engineering point of view, the length of individual implosion and compression coils should be an integral fraction of $\lambda / 4$. To facilitate the design of these coils and their related circuits, a fixed length

$$
\ell=20 \mathrm{~cm}
$$

was chosen. This choice is a compromise between minimizing the multiplicity of coils and minimizing the voltage on the implosion circuit. As a result. $\lambda$ must be an integral multiple of $4 /=80 \mathrm{~cm}$ instead of the continuous function (68).

If $x$ is minimized for a fixed value of $\lambda . h$ cannot be eliminated between (14) and (17), and the following sixth order equation replaces (51)

$$
\begin{aligned}
& a^{6}-g_{1} h^{2} \bar{b}^{4} a^{4}-\frac{g_{0} f_{2}^{2}+g_{2} f_{0}^{2}}{f_{0}^{2}} \\
& \left(\frac{\delta_{2}}{\delta_{1}}\right)^{2} \bar{b}^{4} a^{2}+\frac{g_{0} f_{2}}{f_{0}^{2}}\left(\frac{2 \delta_{2} \bar{b}^{4}}{h^{2} R_{\delta_{1}}^{3}}\right) a \\
& -\frac{g_{0}}{f_{0}^{2}}\left(\frac{\bar{b}}{h^{1 / 2} \delta_{1}}\right)^{4}=0 .
\end{aligned}
$$

Equations (60) and (61) become

$$
\begin{aligned}
& \delta_{0}=\frac{\varepsilon_{0}}{g_{0}} \frac{\Lambda}{h^{2} a R \delta_{1}}, \\
& \delta_{2}=\frac{f_{2}}{g_{2}} \frac{\Lambda}{!_{1}{ }^{2} a k \delta_{2}}
\end{aligned}
$$


but no simple solution similar to (63) can be ob)tained from the combination of $(72),(73)$, and (7t)

$$
a^{6}-g_{1} h^{2} \bar{b}^{4} a^{4}-\frac{\Lambda \bar{b}^{4}}{h^{4} R^{2} \delta_{1}}=0 .
$$

Figure 4-8 compares $E c_{\mathrm{i}}$. $\{70\}$ with the numerical solution of (75) for $\lambda=3.2 \mathrm{~m}$ and $\lambda=4.0 \mathrm{~m}$. These two values of $\lambda$ are integral multiples of 4/. This figure indicates that $(70)$ is an acceptable approximation of $(75)$ if

$$
\begin{array}{ll}
\lambda=3.2 \mathrm{~m} & 1 \approx \delta_{1} \approx 2 \\
\lambda=4.0 \mathrm{~m} & 2 \approx \delta_{1} \approx 4 .
\end{array}
$$

Thus, to retain the analytic simplicity of $(70)$, the constraint on $\lambda$ given by (76) will be assumed. Now that $x_{i}$ has been minimized, the plasma and field parameters can be calculated.

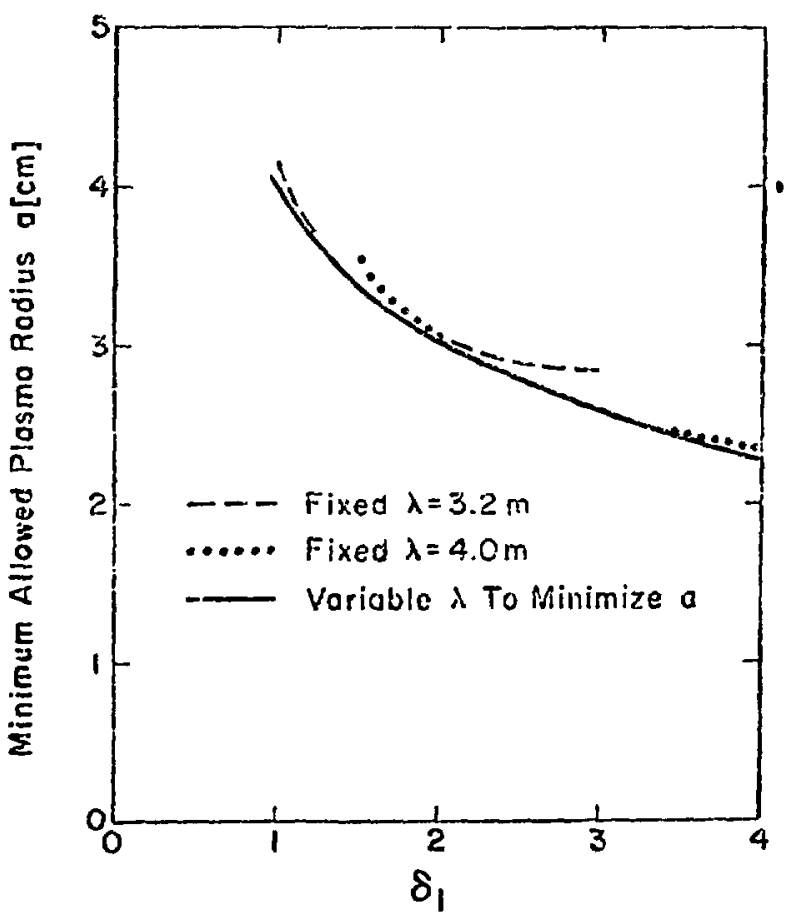

Fig. 4-8.

('omparison of the effect of fixed and variable $\lambda$ on the minimization of the stable $(m=1)$ plasma radius.

\subsection{Ditermination of the Masma and Field} Paramieters

Using the minimum allowed $x_{x}$ given $b y$ (70), the plasma and field parameters can be expressed in terms of $\delta_{1}$. From (50) the maximum induced electric field $\mathrm{F}_{4 i}$ becomes

$$
E_{\theta}[\mathrm{kV} / \mathrm{cm}] \simeq \frac{24_{4}}{\delta_{1}^{8 / 15} \ln \left(70 \delta_{1}\right)} .
$$

The plasma parameters at maximum compression are

$$
\mathrm{n}_{\mathrm{c}}\left[\mathrm{cm}^{-3}\right] \approx 1.8 \times 10^{35} \delta_{2}^{4 / 5}
$$

and

$$
\mathrm{T}_{a}[\mathrm{keV}] \simeq \frac{26}{\ln \left(70 \delta_{1}\right)}
$$

These formulas are derived in Sec. 4.2.2. The values of $\tau_{r}, Q, x_{n}$, and $p_{0}$ are assumed to be those given by (43). (45). (46), and (48). The value of the compression field can be obtained from $(78)$ and $(79)$ by assuming $\beta=1$ :

$$
B_{c}[k G] \simeq 61 \delta_{1}^{2 / 5} /\left[\ln \left(70 \delta_{2}\right)\right]^{1 / 2} .
$$

Note that $T_{c}$ is the average plasma temperature given by

$$
T_{c}=\frac{T_{e c}+T_{i c}}{2}
$$

The corresponding parameters $n_{s} . T_{s}$, and $B_{s}$ evaluated at $t=t_{s}$ can be obtained from the adiabatic law for 3 degrees of freedom

$$
\frac{\mathrm{B}_{\mathrm{S}}}{\mathrm{B}_{\mathrm{c}}}=\left(\frac{\mathrm{x}_{\mathrm{c}}}{\mathrm{x}_{\mathrm{S}}}\right)^{5 / 3}=\left(\frac{\mathrm{T}_{\mathrm{S}}}{\mathrm{T}_{\mathrm{c}}}\right)^{5 / 4}=\left(\frac{\mathrm{n}_{\mathrm{S}}}{\mathrm{n}_{\mathrm{c}}}\right)^{5 / 6} .
$$

Evaluating (82) yields

$$
\mathrm{n}_{\mathrm{s}}\left[\mathrm{cm}^{-3}\right] \simeq 5.8 \times 10^{14}
$$

$T_{\mathrm{S}}[\mathrm{keV}] \simeq \frac{12}{\delta_{1} 0 / 15 \ln \left(70 \delta_{1}\right)}$, 


$$
8_{S}[k G]: \therefore \frac{24}{\delta_{1}^{4 / 15}\left[\ln \left(70 \delta_{1}\right)\right]^{1 / 2}}
$$

again, $T_{s}$ is an average temperature given by

$$
T_{S}=\frac{T_{e s}+T_{i s}}{2} .
$$

These parameters are plotted as a function of $\delta_{1}$ in the next section, which is a summary of all the parameter values derived in Sec. 4.1. The parameters, such as capacitance and voltage, associated with implosion and compression circuits re presented in Secs. 4.2 and 4.3 .

\subsection{Operating Point Summary}

Table 4-1 and Fins. 4-9, 4-10, and 4-11 summarize the SFI'R parameters discussed in Sec. 4.1. Where applicable, the relevant equation numbers are listed to aid in locating the discussion of a given variable in the text. When the parameter is dependent on $\delta_{1}$, a figure number is listed instead of the parameter value. The denoted figure contains a graph of the parameter's dependence on $\delta_{1}$ for $1<\delta_{1}<4$.

The values of the circuit parameters and voltage. are discussed in other sections. The compression circuit is described in Secs. 5.2.2.1.2 and 5.4, while the implosion circuit is discussed in Secs. 4.3, 5.2.2.1.1, and 5.3 .

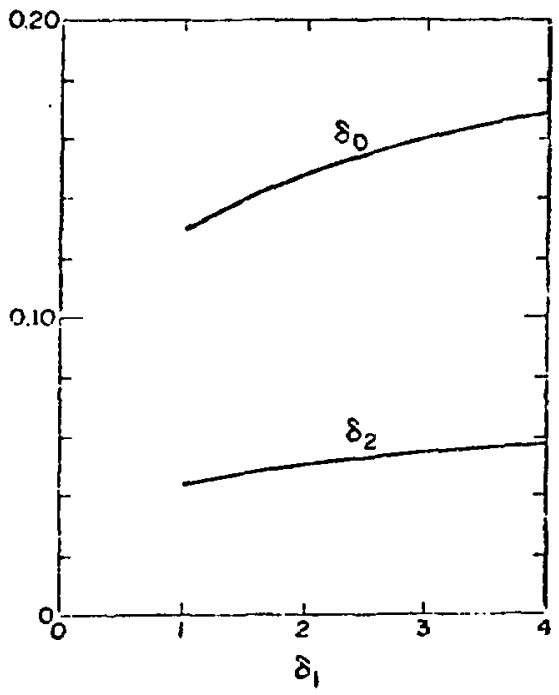

Fig. 4-9

I) (pendence of $\delta_{i}$ and $\delta_{2}$ on $\delta_{l}$, as given by Eqs. (66) and (67).

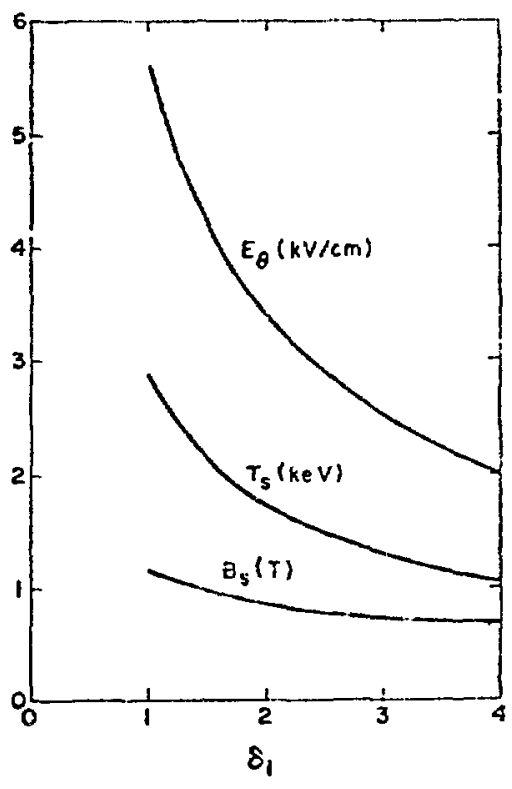

Fig. 4-10.

Dependence of $E_{\theta}, T_{s}$, and $B_{s}$ on $\delta_{3}$, as given by Eqs. (77), (84), and (85).

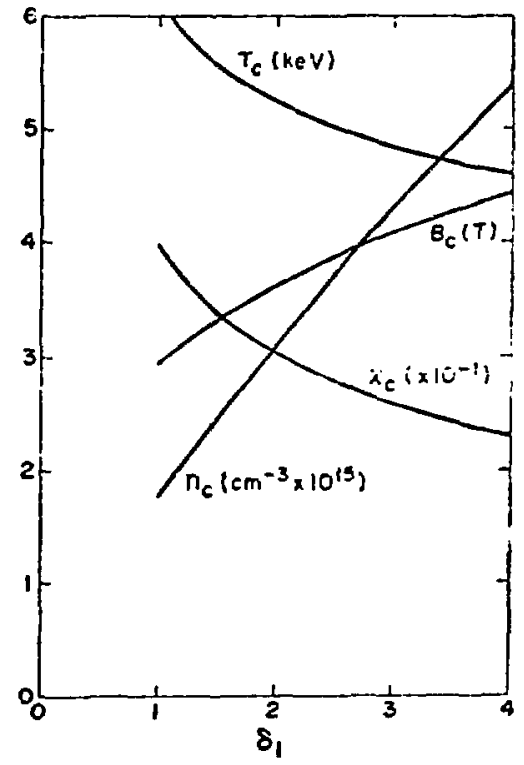

Fig. 4-11.

Dependence of $T_{c}, B_{c}, x_{c}$, and $n_{c}$ on $\delta_{7}$, as given by Eqs. (79), (80), (70), and (78). 
TABL.E +-1

OPFRATING POINI SLMMARY

\begin{tabular}{|c|c|c|c|}
\hline $\begin{array}{l}\text { Iescription of } \\
\text { the Variable }\end{array}$ & Synubul & $\begin{array}{l}\text { Value or } \\
\text { Fisure } \\
\text { Nuitiocs }\end{array}$ & $\begin{array}{l}\text { Equation } \\
\text { Number } \\
\text { lieference }\end{array}$ \\
\hline \multicolumn{4}{|l|}{ SYSTFMI } \\
\hline Majur radius & $\mathbf{R}$ & $+10 \mathrm{~m}$ & {$[i, i]$} \\
\hline $\begin{array}{l}\text { Minor radicis ot the } \\
\text { vacunn wall }\end{array}$ & b & $10 \mathrm{~cm}$ & (j56) \\
\hline $\begin{array}{l}\text { Minor radius of the } \\
\text { implosion coil }\end{array}$ & $\bar{b}$ & $10.7 \mathrm{~cm}$ & $(57)$ \\
\hline Magnitudes of the $t=0$ & $\left(\hat{\boldsymbol{o}}_{3}\right.$ & Fig. 4.3 & $(60), 666)$ \\
\hline $\begin{array}{l}l=1, \text { and } t=2 \\
\text { perturbations }\end{array}$ & $\left\{\begin{array}{l}\delta_{2} \\
\delta_{1}\end{array}\right.$ & $\begin{array}{l}\text { Fig. } 4-9 \\
\text { independent } \\
\text { variable with } \\
\text { the range } \\
1<\delta_{1}<4\end{array}$ & $(611 .(67)$ \\
\hline $\begin{array}{l}\text { Wavelength of the } \\
\text { toroidal variations }\end{array}$ & $\lambda$ & $\begin{array}{l}3.2 \mathrm{~m} l<\delta_{1}<2 \\
4.0 \mathrm{~m} 2<\delta_{1}<4\end{array}$ & $(64) .(68)$ \\
\hline $\begin{array}{l}\text { Length of implosion } \\
\text { coil segments }\end{array}$ & $f$ & $20 \mathrm{~cm}$ & $(71)$ \\
\hline \multicolumn{4}{|l|}{ FIELDS } \\
\hline $\begin{array}{l}\text { Magnetic field at = } \mathrm{t} \text {, } \\
\text { Time reigliecd for the } \\
\text { implnded plasma to } \\
\text { isotropize and } \\
\text { thermalize }\end{array}$ & $\begin{array}{l}\text { B. } \\
t_{4}\end{array}$ & $\begin{array}{l}\text { Fig. } 4-111 \\
-3 \mu \mathrm{s}\end{array}$ & (85) \\
\hline $\begin{array}{l}\text { Deray time of } \mathrm{H} \text {. } \\
\text { Maximum induced } \\
\text { elect ric tield. I lt } \\
\text { occurs at about } \\
t=100 \mathrm{~ns})\end{array}$ & $\stackrel{T}{E_{v}}$ & $\begin{array}{l}230 \mu \mathrm{s} \\
\text { Fig. } 4-10\end{array}$ & $\begin{array}{c}(31) \\
(50),(76)\end{array}$ \\
\hline $\begin{array}{l}\text { Compression: } \\
\text { Mngnetic field at } \\
t=t_{c} \\
\text { Time of peak com- } \\
\text { pression }\end{array}$ & $\begin{array}{l}B_{c} \\
t_{c}\end{array}$ & $\begin{array}{l}\text { Fig. } 4 \cdot 11 \\
\operatorname{TON} \mu \mathrm{s}\end{array}$ & $(80)$ \\
\hline Decay time of $B_{c}$ & $T_{c}$ & $250 \mathrm{~ms}$ & (43) \\
\hline \multicolumn{4}{|l|}{ PLASMA } \\
\hline $\begin{array}{l}\text { Filling pressure } \\
\text { Plasma } 3^{3} \\
\text { Inplosion }\left(t=t_{4}\right) \text { : }\end{array}$ & $\mathbf{p}_{n}$ & $l_{1}^{\text {tmorr }}$ & (46) \\
\hline $\begin{array}{l}\text { Average temperature } \\
\text { Density } \\
\text { Normalized radius } \\
\text { Compression }\left(t=t_{\mathrm{t}}\right) \text { : }\end{array}$ & $\begin{array}{l}T_{s} \\
n_{s} \\
x_{n}\end{array}$ & $\begin{array}{l}\text { Fig. } 4-10 \\
5.8 \times 10^{14} \mathrm{~cm}^{-3} \\
0.7\end{array}$ & $\begin{array}{l}(84) \\
(83) \\
(46)\end{array}$ \\
\hline $\begin{array}{l}\text { Compression }\left(t=t_{c}\right) \text { : } \\
\text { Average temperature } \\
\text { Density } \\
\text { Normalized radius }\end{array}$ & $\begin{array}{l}T_{c} \\
n_{c} \\
x_{c}\end{array}$ & $\begin{array}{l}\text { Fig. 4-11 } \\
\text { Fig. 4-11 } \\
\text { Fig. 4-11 }\end{array}$ & $\begin{array}{l}(79) \\
(78) \\
(70)\end{array}$ \\
\hline \multicolumn{4}{|l|}{ BURN } \\
\hline $\begin{array}{l}\text { Maximum allowed time } \\
\text { to reach } Q=1\end{array}$ & ta & $150 \mathrm{~ms}$ & (44) \\
\hline $\begin{array}{l}\text { Time asymplotic } \\
\text { value of } Q\end{array}$ & $Q_{r}$ & 1.1 & $\begin{array}{l}\text { Obrained } \\
\text { frem the }\end{array}$ \\
\hline $\begin{array}{l}\text { Time to achieve } \\
Q=1 / 2 \\
\text { Tisnt to achieve }\end{array}$ & $t_{n / 2}$ & $\begin{array}{l}35.40 \mathrm{~ms} \\
47.49 \mathrm{~ms}\end{array}$ & $\begin{array}{l}\text { mumerical } \\
\text { minde! }\end{array}$ \\
\hline
\end{tabular}




\section{2 (OMIPRISSION AND BLTN}

\subsubsection{Ceneral Deseription}

The plasma is brought to ignition by adiabatic compression. and the magnetic field is then held while thermonuclear burning occurs. Theoretical models are needed to describe the processes of heating. equilibration of speriges. neutron and arparticle production. and bremsstrahlung as the ex. termal mannetic field is changerl. An analytic model has been derived which gives approximate answers for several of the above processests. This model is described in the next section.

The best model available (1) us is a thermomuclear burn conde developed by Oliphant. "It is a numerical solution of the Fokker-Planck equation and has been uned in determining the thermunuclear burnup and wher parameters. It uses the external maynetic field wavetiorm for the SFTR circuit. That waveform is a

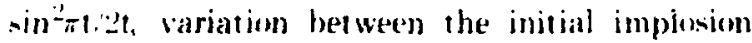
field and final compression ficeld. fullowed by an exponential field decay with a 2 int-ms time comstant. A description of the numerical model and results is given below.

\subsubsection{Analytic Model}

The approximate model deseloped in this section relat es the goals of $(Q=1$ and $m=1$ plasma stability to the plasma parameters at the beginning of the compression evele. In urder to generate a tractable. analytic model. several effects such as electron-ion equilibration. a-particle heating and bremsitrahlum are omilted. These effects are in. cluded in the numerical model described in the next sections.

The following assumptions form the basis for the model:

1. The adiabatic law holds for $t>1$, with $\}=5 / 3$. The time 1. . defined in Fig. $4-7$. marks the end of the implowion phase and the beginning of the compres. sion phase. The adiabatic law for $;=5 / 3$ is griven in (N2).

2. The mannetic field decars with a simple exponential tronn its peak value at $t=1$,

$$
B:=B_{c} e^{-t / 7 c}
$$

where $\tau_{6}=250 \mathrm{~ms}$ as in $(4.3)$

3. For this model $t$ is set 10 zero at $t_{1}$ since neutron production for $t<t$. can be ignored in the equation for $(9) 112)$

1. The energy vield per neutron is assumed to be

$\because-20 \mathrm{MeV} /$ nautron.

5. The $\langle\pi \cdot\rangle$ curve is approximated by

$$
<\sigma u\rangle=\mathrm{Ge}^{-\theta / \mathrm{I}}
$$

where

$$
G=10^{-21} \mathrm{i}^{2} / \mathrm{s}
$$

ind

$$
\theta=20.8 k .6
$$

Equation 189 ) is compared with the known D.T reactiom rates ${ }^{1.5}$ in Fig. $4-j 2$.

i. A sharp boundary model is assumed, with nu rad al variations inside the boundary. Since the clas.ical eneroy losis time is long compared with $T_{\text {. }}$, the radial particle and energy t ransports are ignored.

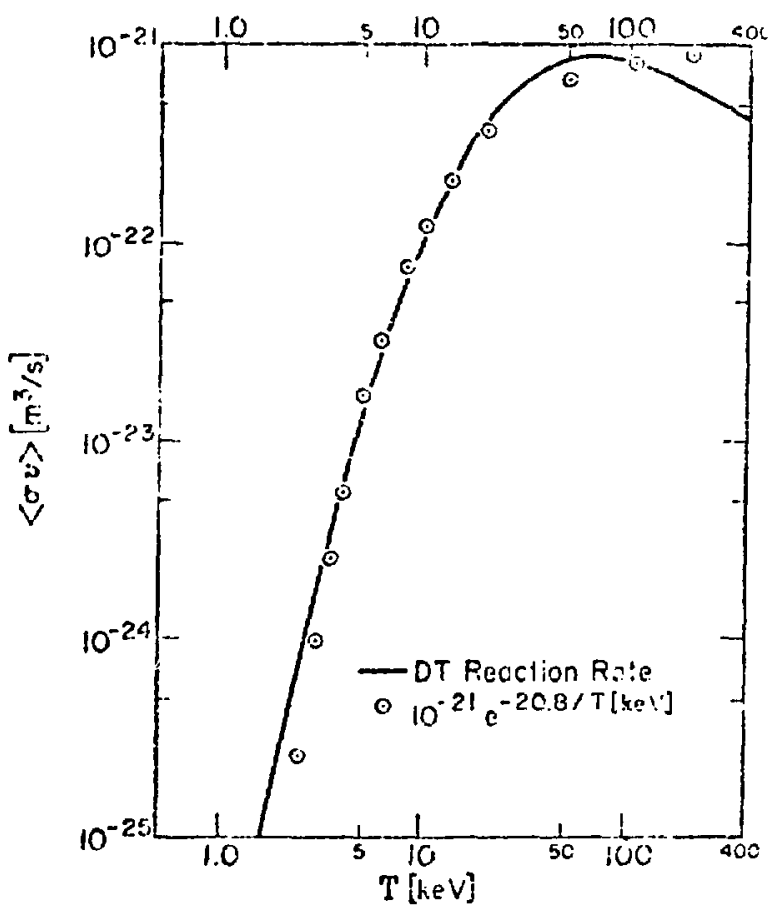

Fig. 4-12.

Comparison of the D-T thermonuclear reaction rate ${ }^{5}$ with the approximation given by (78). 
7. The electrons and ions are assumed to be equilibrated for $t>t_{t}$ (or from assumption $3, t>0$ )

$$
\mathrm{T}=\mathrm{T}_{\mathrm{e}}=\mathrm{T}_{\mathrm{i}} \text {. }
$$

8 . The effects of bremsstrahlung, $\alpha$-particle heating, and electron-ion equilibration are ignored except for a single parameter $\alpha$ which is used to correlate the numerical model with this analytic model.

9 . To evaluate the integral in (13), it is assuned that

$$
\mathbf{T}<\Theta .
$$

Equation (13) can now be evaluated using (8:2). (8i). (89), and (92)

$$
\begin{aligned}
Q & =\frac{Y_{n_{C}}}{12 k_{B} T_{C}} x \\
& \int_{0}^{s} \exp \left(-\frac{12}{5} \frac{t}{T_{C}}-\frac{\theta}{T_{C}} e^{4 t / 5 \tau_{c}}\right) d t .
\end{aligned}
$$

When (9:3) is satisfied. the najor contribution to the integral in $(94)$ occurs for $t \ll \tau_{1}$. This restriction allows the second term in the exponential to be expanded. thus rendering a tractable integral. The result is

$$
Q=\frac{n_{c}}{H}
$$

where

$$
H \equiv \frac{3+\frac{\theta}{T_{c}}}{\theta / T_{c}}\left(\theta / T_{c}\right)
$$

and

$$
\eta \equiv \frac{5}{48} \frac{G Y c}{k_{B}} .
$$

Fquation 1951 uniquely determines the relation between the plasma density and temperature at maximum compression for a given value of $Q$ in the SFTR sistems. The value of $\eta$ is a constant for the SFTR determined by (88). (90): (91), and 1431$)$

$$
n=2.5 \times 10^{-20} \mathrm{~m}^{3} / \text { neutron } .
$$

The variable $\mathrm{H}$ depends only on $\mathrm{T}_{r}$. as is evident in (96). The time asymptotic value of $Q$ is chosen to be

$$
Q_{f} \approx 1.1
$$

so that $Q=1$ will be reached before $150 \mathrm{~ms}$ into the compression.

The numerical model described in the next section indicates that the a-particle heating dominates bremsstrahlung losses and ion cooling due to electron-ion equilibration. The result is a slightly larger $Q$ than predicted by (95). A comparison of (95) with the numerical results shows that by using a constant

$$
\alpha=0.72
$$

excellent agreement can be obtained between the numerical model and the empirical relation

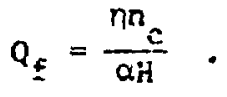

Equation (loll can be easily solved for $n$, in terms of $T$.

$$
n_{c}=\frac{\alpha Q_{f} H\left(T_{c}\right)}{n}
$$

and by using $(98),(99)$, and 1100$)$

$$
\mathrm{ac}_{\mathrm{c}}\left[\mathrm{cm}^{-3}\right]=3.2 \times 10^{13} \mathrm{H}\left(\mathrm{T}_{\mathrm{c}}\right)
$$

$110: 31$

(n) the SFTR design it would be most useful to solve (10:3) for $T_{r}$. since $n$, is already determined by $p_{1}$, and the maximum compression allowed by stability. The form of 1961 does not allow a simple solution. but the following approximate solution is compared $10(96)$ in Fig. $4-1: 3$

$$
T_{c} \simeq \frac{\theta}{\ln (0.537 i)}
$$

Substituting (103) into 1104$)$ yields

$$
I_{s}=\frac{\theta}{\ln \left[\frac{n_{c}\left[\mathrm{~cm}^{-3}\right]}{6.0 \times 10^{r 3}}\right]} \text {. }
$$




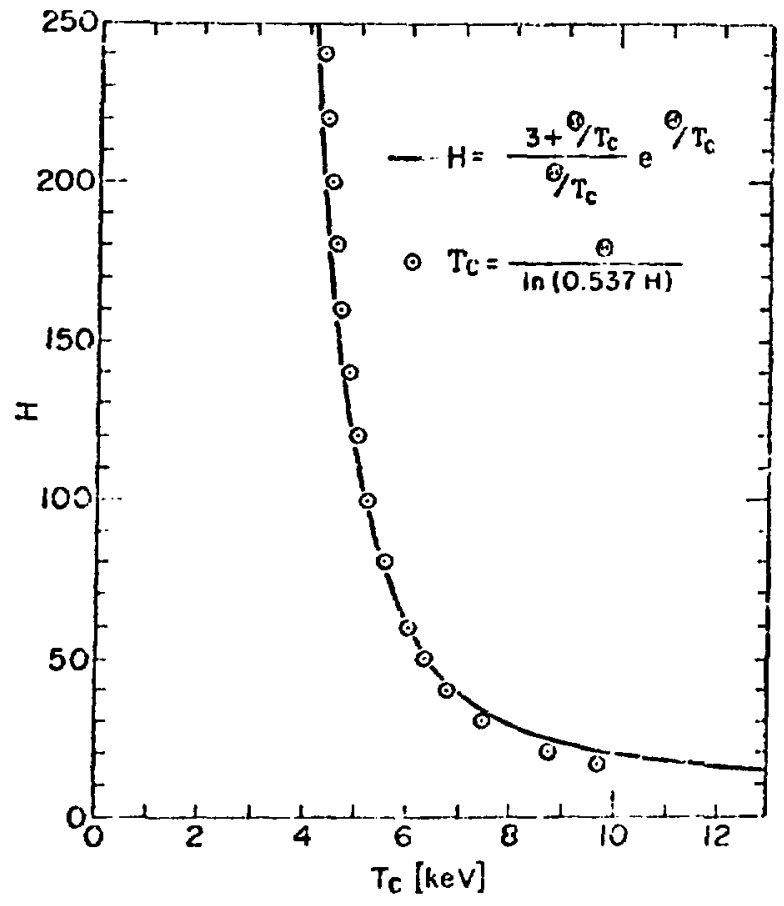

Fig. 4-13.

Comparison of the approximate relation for $T_{r}$ (l(1)4, with the definition of $H$ given by (96).

Consersation of particles requires that

$$
\mathrm{n}_{\mathrm{c}}=\frac{\mathrm{n}_{\mathrm{o}}}{\mathrm{x}_{\mathrm{c}}^{2}}
$$

and by using $(48)$ and $(70)$. (1106) becomes

$$
n_{c}\left[\mathrm{~cm}^{-3}\right]=1.8 \times 10^{15} \delta_{1}^{4 / 5} \text {. }
$$

Combining (107), (105) and (91) yields

$$
T_{c}[k e V]=\frac{26}{\ell_{1}\left(70 \delta_{1}\right)}
$$

E(puations $(107)$ and $(108)$ appear as $(78)$ and $(79)$ in the previous section. The values of $n_{1}$ and $T$, given by. $(107)$ and $13(0)$ describe the plasma that can provide the desired $Q$ and st ability for the minimum induced field Fin.
The plasma parameters at $t=t$, are related to $n$, and $T_{\text {, through the adiabatic law. Thus the goals of }}$ $Q=1$ and $m=1$ stability have been related to $n_{s}$ and ' $T$, under the constraint that $\mathrm{E}_{\text {" }}$ should be minimized.

Minimizing Eu minimizes the technological strain on the implosion system, but it also increases the energy required from the compression sustems. lising pressure balance, the peak magnetic energy density required during compression can be expressed in terms of $T_{i}$ by using (96) and (103)

$$
W_{B}[M J / m]=0.11 \frac{\left(3+\theta / T_{c}\right)}{\left(\theta / T_{C}\right)^{2}} e^{\theta^{\prime} T_{C}} .
$$

This function is plotted in Fig. 4-14. The min:mum required energy density of $0.98 \mathrm{M} \cdot / \mathrm{m}^{3}$ is required at $T_{c}=12.6 \mathrm{keV}$. To minimize $E_{t i t}$ for $\delta_{1}=4$ ( see Fig. 4 11). $\mathrm{T}_{c}=4.6 \mathrm{keV}$. and the required energy density is nearly four times larger. However, since the required $45 \mathrm{kC}$ is still within reasonable technological limits. Fe should still be minimized.

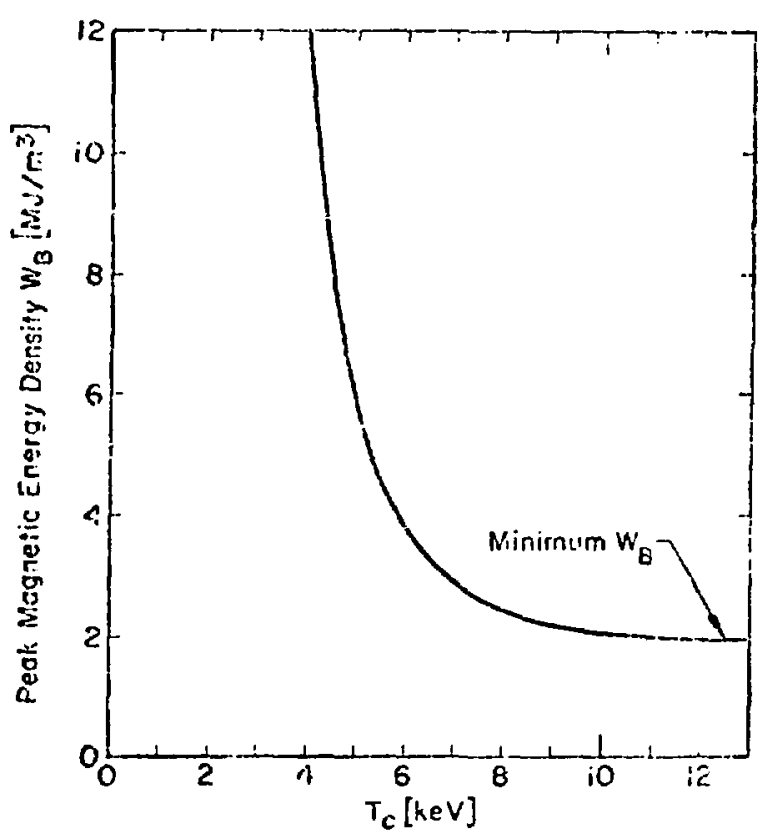

Fig. 4-14.

Magnetic energy density at peak compression $W_{H}$ as a function of the average temperature $T$. 


\subsubsection{Numerical Model}

The thermonuclear burn model assumes that there is a sharp boundary between a uniform density crlindrical plasma and the magnetic field. The field is excluded from the plasma. so $s=1$ and the phlisma and tield pressures are equal at all limes.

The ion and electron distribution functions are Maxwellian, with different temperatures. The aparticles are horn at 3.5 Mel. with a Dopplerbroidened half-width determined by the inn remperature, in accordance with the D-T cross sec'tion. The a-particle production is assumed uniform. but since the a-particle orbits take some of the aparticles rut of the hest core we assume a certitin trate tion en interate with the plasma only on part of the.ir intrits.

These three dist ribution tunctions are determined trum the Fokker-Planck equation, and the distributions evolve in time according to the coulomb (ollision rate and acliabatic changes in the external field. One tan therefore monitur electron and ion temperature, w-particle production and heat input (1) the plasma, neutron and bremsitrahlung enissimn. direct conversion work by expansinn of the plasma against the external tield, and piamma densiIV and size.

The analytic model is used 10 explure pussible operating puints and $t o$ do parameter variation studies. The more promising parameter sets are uned in the numerical model to compute the required in. fiormation.

\subsection{IMPLOSION HEATING;}

\subsubsection{General Description}

The inplonion-heating sistem nusis heat the plasma to the conditions specified in Table $4-1$ so that the compression and burn evele can achieve? $=1$ while maintaining $\mathrm{m}=\mathrm{l}$ stability. The theoretical model presented in this section is needed to connect the field and plasma parameters in Table f-I to the implosion circuit parameters.

The resonamt heating approach involves tuning the implosion bank and crowbar switching sime or the natural implosion time of the plasma. For this

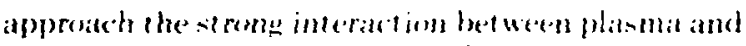
circuit recutures a mathematlical medel which catn handle the plasma and eireuit donamic simulaneously. Computer codes have been developed that satisf this requirement."16 hut the present sepphisteation of the plasmit modeding does nen vield resules that are significants ditferent from simple analytie modeje. These codes are presemly being improsed. Although sonse results ut these codes are used in this soction. tor simplicity an analyere moded is used as the banin al the theoret ical description.

The simple bounce model tho wrertiakingt in uned in dexeribing the ion sheat h interatetion. The obrious errors invelved in usine such a simple molel haw been disensised be oliphant. " whe describer a more accurate but more complex model. The simple model is used to illustrate the batsic plasmat circuit interatetion. leaving the numerical accuratey to the computer codes. As presinusty stated. the condersand the simple molel do mot deviate signifieantly except where indieated in sies $1.3,3$.

\subsubsection{Resonant Heating Concept}

The optimization of the implosion-heating sistem

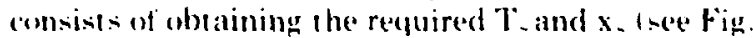
f-10 and Tatble $4-11$ for the smallest possible Fen alld $\therefore$ using the simplest possible eirevit $\left(b_{2}\right.$, is the capacitur bank voltage). Freidherg. Mlorse. and Ribe" determiated that the programmed field shomn in Fig. 1 15 vielded larger $\mathrm{x}$, and ' $\mathrm{T}$ '. for a given $\mathrm{V}$. than a simple implusion field. The first field pulse. implodes the plasma, but the field is dropped to zero during the expansion of the plasma so that the ptasma does not give its eneray back to the field. As the plasma approaches the wall. the field is raised to the minimum value regused le prevent the plasma from hitting the wall.

An error was made in Ref. 9 in calculating the relative sizes of the first and secound field pulse. Figure $4-15$ shows the correct farcor of 2 instead of $\sqrt{2}$. As a result of this error the value of $x$. is mistaken!y given as 6.76 instead of the correct villue of

$$
x_{s}=\left(\frac{22}{45}\right)^{1 / 2}=0.70 \text {. }
$$



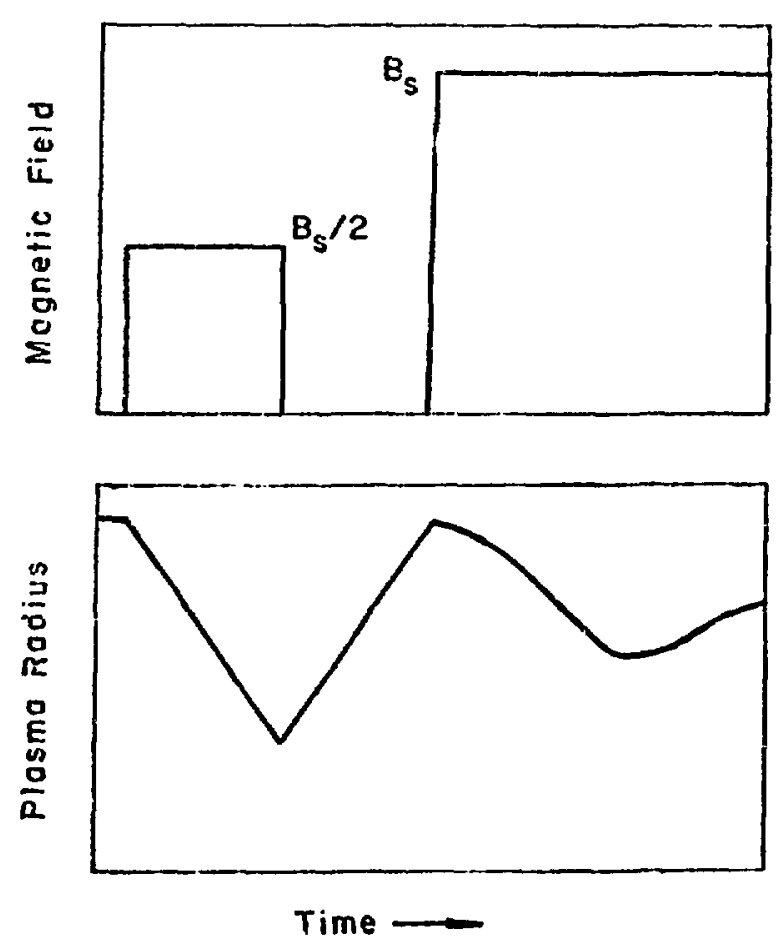

Fig. 4-15.

Programmed implosion field allowing free expansion of the plasma.

Although the field waveform of Fig. 4-15 cannot be generated by a realistic circuit, the basic features can be produced by the simple circuit shown in Fig. 4-16. The temporal behaviors of the magnetic field and plasma radius shown in this figure were obtained from a hybrid simulation code developed by Nielson. ${ }^{16}$ The field is not zero during the plasma expansion, but it is less than the initial implosion field. As a result. only a small fraction of the plasma energy is given back to the field during the expansion.

The circuit is topologically the same as the classica! theta-pinch circuit used in Scyllac. However, the source and crowbar inductances $L_{s}$ and 1. are imade smaller than the empty load coil inductance $L_{*}$. In addition the bank capacitance $C$ is chosen so that the circuit is tuned to the implosion time of the plasma, hence the name resonant heating.

More precisely, the circuir is tuned so that by the time of the maximum implosion $t_{c r}$ the field has peaked and decayed to a specific value $B_{c r}$. At this time $t_{\mathrm{cr}}$ the crowbar switch is closed and the magnetic fux corresponding to $\mathrm{B}_{\mathrm{cr}}$ is compressed by the expanding plasma. If $L_{s}$ and $L_{r}$ are sufficiently small and if $B_{c r}$ is chrsen properly, the pulse of field at 4 will be just sufficient to prevent the plasma from hitting the wall. For $t>t_{e}$ the oscillations in the field and the plasma radius dampen rapidly as the plasma thermalizes and isotropizes. Of course the field exponentially decays on a much longer time scale as is shown in Fig. 4-7.

The second field pulse at $t_{k}$ (Fig. 4-16) is not twice as large as the initial pulse mainly because of the plasma energy losi during the expansion against a nonzero field.

Any analysis of this resonant heating system must handle the circuit and plasma dynamics simuitaneously. The interaction of the circuit and plasma cannot be ignored because of the dominant effect of $L_{p}(t)$ in the circuit. The analytic model described in the next section includes this plasmacircuit interaction. The model is used to determine
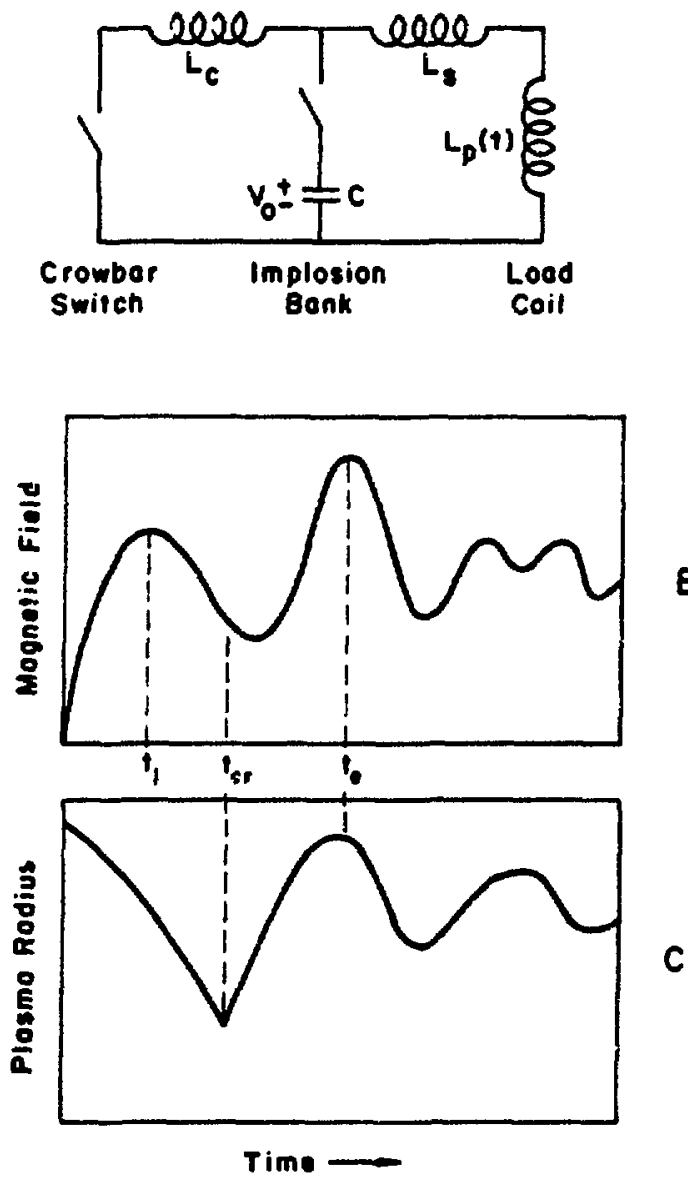

Fig. 4-16.

Resonant heating circuit showing the temporal behavior of the B-field and the plasma radius. 
the values of the circuit components and the relation. between $T_{s}, x_{s}, E_{4}$, and $V_{0}$. In addition, the minimum $V_{0}$ is obtained by optimizing the number of turns on the implosion coil. The relation between the turns and the inductance of the implosion coil is derived in Sec. 5.2.2.1.1.

\subsubsection{Modeling of the Implosion}

The analytic model for the implosion process described in this section actually consists of three separate models used in sequence. Each of the models describes a separate portion of the time history of the plasma. The segments of time are delineated by $t_{c r}$ and $t_{e}$ in Fig. 4-16, and are denoted by the following descriptive narnes:

1. Initial Implosion $0<t<t_{r r}$

2. Expansion and Reflection $\mathrm{t}_{\mathrm{cr}}<\mathrm{t}<\mathrm{te}_{\mathrm{e}}$

3. Isot ropization and Thermalizat ion $t_{\mathrm{e}}<t<t_{\text {, }}$.

The isotropization and thermalization are assumed $t$ t) be complete by $t=t$, where $t$, is detined by Fig. 4-- A briet description of the three models is piven before any calculations are presented.

\subsubsection{Jescription of the Models}

\section{Initial Implosion}

The plasma is described by a sisarp houndary. simple bounce model with no orertaking. That is. each ion is assumed (n bounce elastically off the magnetic piston only once. Physically. the ions struck by the piston at very early times will be over. taken one ar more tinues by the accelerating p:ston. but this fact is ig:oored by the model. This problem of overtaking as well as inelastic collisions between piston and ions (snowplow model) has heen treated analytically by Oliphant. "Finite sheath thickness and overtaking have been included in simulation corles." 91 Although the effects of finite sheath thicktress. overtaking. and snowplowing are obser. vable. they do not appear to dominate the grosis plasma-circuit interaction. Thus for the sake of simplicity they are omitted from tila models presented here. The end of the iritial implosion at : $=t_{r r}$ is defined by the time when the first acrelerated ions strike the sheath on the opposite sic s of the plasma after passing through the center of the sistem.

The circuit used in this portion of the model is one loop of the circuit shown in Fig. 4-16. The loop containing the crowbar switch is not needed since the switch is not closed until $t=t_{r r}$. The plasma effects are introduced to the one loop equation through
$L_{p}(t)$. A single time varying inductor $t o m$. plasma effects can be used only if a shary isoundary plasma model is used.

The equation arising from this :umple model has a closed form solution in only a few special cases. Analytic scaling law's obtained from the equation are helpful in understanding the system. but the equa. tion rausi be solved numerically for some of the final results. The results include the required relations between the induced electric field $E_{H}$. the bark voltage $V_{c}$, the peak circuit current $i_{1}$, and the peak plasma energy $F_{a}$, (the subscripts $I$ and cr refer to the times $t_{1}$ and $t_{\text {cr }}$ shuwn in Fig. 4-16).

\section{Expansion and Reflection}

The simple bounce concept used in the first nuedel breaks down at $t=t_{\text {.r }}$ when fast ions begin striking the piston. At this time the crowhar switch is clused and this also complicates the citcuit model. Thus a new model is needed for $t_{10}<t<t$, that takes: advantege of the new boundary conditions of the ivstem.

Two basic assumptions are made. First, the crow. bar is assumed to be "perfect" thus causing the total magnetic flux or and the total enera (magnetic plus plasma) to be constants of the motion. This assump. tion is justified if the transter time of the current from the capacitor branch to the crowbar is short compared with the implosion time. The second assumption is that pressure balance exists during the reflection at $t=t_{r}$. An accurate desrription of the dynamic plasma pressure at $:=1$, wouid require a knowledge of the phase space distribution of the plasma. Since this information is not obtainable in analytic form, the plasma is assumed to be thermalized with a finite degree of isotropization parameterized by $\mathrm{c}$.

The results of this model are combined with the initial implosion results to determine requirements for tursing the circuit to the plasma implosion time. The actival calculation of the sizes of the circuit parameters is presented in Sec. 4.3.4

\section{Isotropization and Thermaivation}

The purpose of this model is th determine the $x$. and $T$, of the plasma as a functiste if initial plasma and circuit parameters. The conser ot in on thux and total enersy is assumed as in the Expansion and Roflection model. The piasma is assirned lo be thermutized. isotropized in three dimeretions. and in pressure halance with the field at $t=t$. As a pessimistic assumption

$$
T_{e s} \sim \frac{1}{2} T_{i s}
$$

which is less than that observed in fryllac. It Fquation $(1111)$ is in approximate agreeny zet with a 
simple model for ohmic heat ung due to field diffusion for sheath widths comparable with those experimentally observed (on the arder of $2 \mathrm{~cm}$ ).

The results of this model agrec regsonably well with simulation codes for the adiabatic invariant $T$. $x^{+3}$. However, the value of $x_{*}$ is typicaliy higher thall that generated by the codes because the eleciron lemperature is inserted in the model in an ad hoc inanner: increasing the plasma pressure and radius. Since the major electron heating probably occurs during the initial implosion before the crow. bar limits the flux, a smaller $x$. is expected than this model predicts. Fur this reason

$$
x_{s}=0.7
$$

in assumed becantse it agrees with the simulation reder as well as the simple model" previously described in Sect 4.3.2 |sece 111011).

"The three moulels described above are nutlined mathematically in the remainder of this section

\subsubsection{Initial Implosion}

A new unrmalized radisal cariable simplities the algebraic description of the plasma inestel

$$
2 \equiv 1-x \text {. }
$$

I simg this variable, the momentuat balance equation for $t<I_{1}$, is

$$
\frac{B^{2}}{2 \mu_{0}}-2 \operatorname{nom}(2 b)^{2}
$$

where 12 . is the filling density and on the average ion matsis. Solving for the piston velocity yields

$$
b i=\frac{v_{\alpha}}{2}
$$

where $\mathrm{s}$ is the Alven velecity

$$
v_{\alpha} \equiv \frac{B}{\sqrt{\mu_{0} n_{\text {gnl }}}} \text {. }
$$

The relation between the magnetic ficld $B$ and the

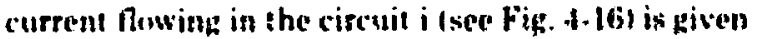
by the Ixundary conditions:

$$
B=\frac{M_{2} N_{i}}{k}
$$

where $I$ is the length of the implosion coil and $X$ is the effective number of turns in the coil [see (16) in Sec 5.2.2.1.11.

The form of the loop equation for the circuit in Fip. 4.16 can be simplified by defining several new variables. Let $Q_{r}$ be the initial churge on the capacitor $\mathrm{C}$.

$$
Q_{c} \equiv v_{0} c
$$

while $Q_{1}$ is an effective "rharge" associated with the plasma

$$
Q_{p} \equiv \frac{\partial L}{N}\left(\frac{n_{0}{ }^{m}}{u_{0}}\right)^{1 / 2} .
$$

The ratio of these charges is defined as

$$
q=\frac{Q_{p}}{Q_{c}}
$$

The inductance assincisted with the empty vacuum vesisel is

$$
1_{0} \equiv \frac{\mu_{0} 3 m b^{2}}{\ell}
$$

and the normalized source incuctance

$$
\Lambda_{s} \equiv \frac{L_{s}}{L_{0}}
$$

is evaluated in 1261 of soc. 5.2.2.1.1. ( sing 1113 and 11211. the time-dependent load inductance can be expressied as

$$
L_{p}(t)=L_{0} 2(2-z) .
$$

Finally, the cursent and time are nurmalized to

$$
\begin{aligned}
& I \equiv \frac{1}{i_{r}} \\
& I=\frac{t}{t_{0}}
\end{aligned}
$$

whore

$$
\begin{aligned}
& i_{0} \equiv v_{0}\left(q \frac{c}{l_{0}}\right)^{2 / 2} \\
& t_{0} \equiv\left(q i_{s} L_{s} c\right)^{i / 2} .
\end{aligned}
$$


lsing these variables, the loop equation can be writcen in the following form

$$
\begin{aligned}
& \frac{d I}{d T}\left[1+\left\langle\int I d T\right)\left(1-\frac{1}{4} \Lambda_{S} \int I d T\right)\right] \\
& \quad+I^{2}\left(I-\frac{1}{2} \Lambda_{S} \int \operatorname{IdT}\right)=1-q \Lambda_{S} \int d d T .
\end{aligned}
$$

In addition. $11151, \ldots$ be integrated sielding

$$
z=\frac{1}{2} \Lambda_{S} \int \operatorname{IdT}
$$

Thus ine displacement of the piston from the wall is linearly related to the charge remosed from the capacitor.

Some uriderst anding of the solution of $(128)$ can be ohtained by examining the equation at early times. i.e..

$$
z<\frac{1}{2} \Lambda_{s}<1
$$

If

$$
q>2
$$

then (1:28) can be approximated by

$$
\frac{d I}{d T}+I^{2}=1 \text {. }
$$

The solution of (132) is

$$
I=\operatorname{tann} T
$$

which is plotted in Fig. 4-17. Condition (130) breaks down for $T>1 / 2$ as is evident in Fig. 4.17 where $1133)$ is compared with a numerical solution of $(128)$. The agreement with (133) for $T<1 / 2$ is good for a wide range of .1 , and $q$.

The effects of $q$ and.. , on the solution of (128) can he compared with the solution in Fig. 4-17. As is stated in (118). (119), and (120), q essentially compares plasma mass with the charge on the capacitor bank. If $q-1$ then the current is similar to the plonted example. As q is decreased (bank capacity or voltage increased), the normalized peak current increases and the drop in current following the peak is slower. Thus it approaches $(1: 33)$ for $y \ll 1$. This condition corresponds to classical theta-pinch systems such as Scyllac. The model then is only valid for $T \ll$ 1 since ions traverse the plasma in a time short compared with the current rise time. As $q$ is increased beyond 1 , the peak normalized current decreases and the current falls to zero more quickly. In fact. for

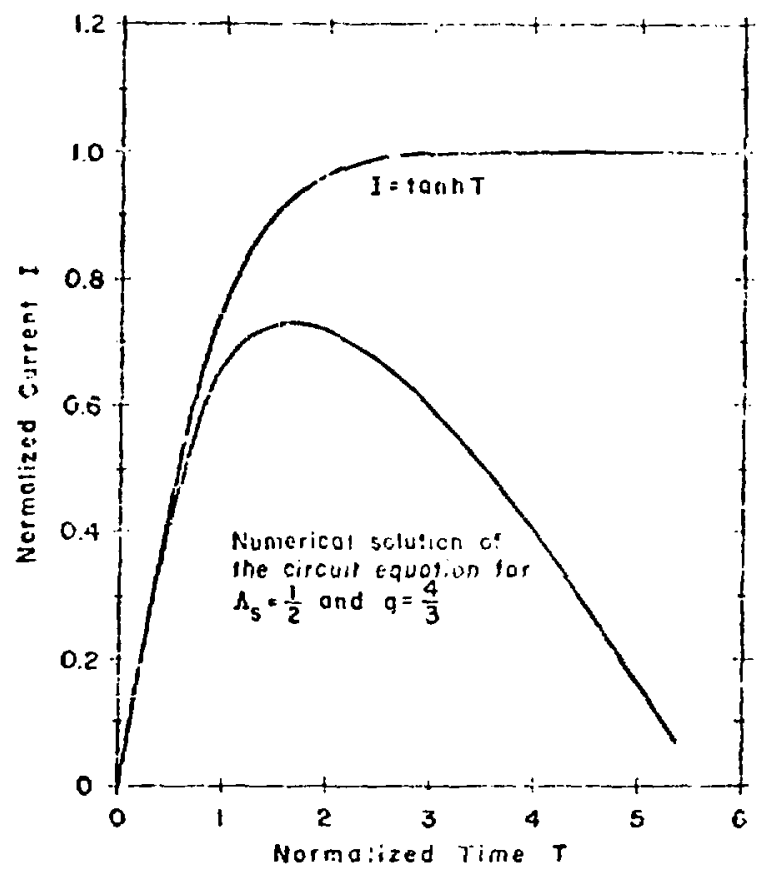

Fig. 4-17.

Comparison of the numerical solution of (128) and the solution of (132) given by (133).

$q>1.5$ the current goes negative before the ions traverse the plasma. Equation $(128)$ can be made valid for $1<1$ if the l's in the integrands on the left side of $1128 ;$ are bracketed by absolute magnitude signs / the same with the I in (129)]. This is required to correctly model the field pressure as being independent of the direction of $B$ (or I).

This range of $q>1.5$ resulting in negative current is an inefficient and hence uninteresting regime. As has been described in the Expansion and Reflection model, a specified amouni of flux must be in the system at $t=t_{\mathrm{cr}}$ when the first ions traverse the plasma. If the current has passed through zero one or more times hefore $t=t_{\mathrm{cr}}$. the average field pressure and the resulting plasma energy are smaller than if the current remains positive during the initial inplosion.

The effect of 1 , on the normalized current is quite similar to the effect of $y$ on the current. For $\Lambda, \gg 1 / 2$. most of the energy is stored in the source induct ance. resulting in small values of peak normalized current. If $I, \ll 1 / 2$, the current is still high when the plasma hegins to expand $\left(t=t_{\mathrm{cr}}\right)$ and thus the plasma gives up an excessive amount of energy to the field during the expansion. 
As a result of numerical studies

$$
\Lambda_{\mathrm{s}}=\frac{1}{2}
$$

and

$$
q=\frac{4}{3}
$$

appear io be close to the optimum values.

Two additional variables are defined here for future reference. The first is the peak current $I_{1}$ which can be evaluated hey sel ing $\mathrm{dl} / \mathrm{d} \mathrm{T}=0$ in $(128)$

$$
I_{l}^{2}=\frac{1-2 q z_{1}}{1-2_{1}} \text {. }
$$

I'nfort unately $z_{1}$ is dependent on the time history of the currens up to its peak value as is evident from (12.91. Hence. I Inust be determined numericall!.

The second variable $z_{1}$, is the position of the piston at $t=t_{1}, I_{n}$ ine simple model illustrated in Fig. 4-15 the value of $z_{4}$ is $2 / 3.3$. In the nore complicated model under ronsideration. $z_{c}$, is still alout $2 / 3$ but the small variations are important to the timing of the crowhar switch. Thus $z_{1}$, should also be determined numericaily:

Nin that the current has been defined over the time period $0<t<t_{i r}$. the peak energy of the plasnia, which occurs at $t=t_{\text {cr }}$, can be related to the current 1 . Define $W_{\text {ir }}$ as the average ion energy at $t=$ $t_{4}$.

$$
W_{c r^{*}}=\frac{1}{2} m \int v^{2} f_{i}\left(v, t=t_{c r^{\prime}}\right) d v
$$

where $f_{1}(v)$ is the ion velocity distribution. Since each ion is given a velocity characterized by the Alven speed at the instant the piston strikes the ion and since the ions are collisionless. $(137)$ can be I ransformed io

$$
W_{c r}=\frac{1}{2} m v_{\alpha_{1}}^{2} \int_{x_{c r}}^{1}\left(\frac{v_{\alpha}(x)}{v_{\alpha_{1}}}\right)^{2} 2 x d x
$$

where $v_{i, l}$ is the maximum Alven speed which occurs $a t=t_{1}$. By defining

$$
\gamma \equiv 2 \int_{c}^{2} c r\left(\frac{I}{I_{1}}\right)^{2}(1-2) d z
$$

(i:38) becumes

$$
W_{c r}=\gamma \frac{1}{2} m v_{\alpha}^{2} \text {. }
$$

It is apparent from (139) that $\gamma \leq 1$ where $\gamma=1$ corresponds to the constant implosion field in Fig. 4-15. The more realistic field or current behavior of Fig. 4 Ii is obviously less effective at heating the ions. As an example the ; associated with 1 , and $y=4 / 3$ in Fig. $4-17$ is

$$
\gamma=0.628
$$

from numericai calculations of (139) and 11281.

The peak Alven speed $v_{\text {crl }}$ can be related to the current using 11161 and 1117 . Substituting the result into $(140)$ sields

$$
w_{c r}=\gamma \sqrt{\frac{m}{n_{0} \mu_{0}}} \frac{v_{0}}{2 \pi b N} I_{2}^{2} .
$$

This is the desired relation between the peak ion energy and tise peak current

It is also import ant to relate the induced electric field Fon at the coil $(r=\hbar)$ to $W_{\text {cr }}$ and $I_{1}$. By defining the area $A_{1}$ inside the coil

$$
A_{1} \equiv \pi\left[\bar{b}^{2}-a(t)\right]^{2} \text {, }
$$

Eit can be written as

$$
E_{\theta t}=\frac{\dot{j}}{2 \pi \dot{b}}\left[A_{1} \frac{\partial B}{\partial t}+B \frac{\partial A_{2}}{\partial t}\right] .
$$

Evaluating (1+1) appropriately vields

$$
\begin{aligned}
& \mathbb{E}_{\theta t}=\frac{b}{\vec{b}} \frac{\mathbb{E}_{\theta}}{I_{I}} \\
& \left\{1-2 q z-\frac{d I}{d T}\left[1-\frac{\left.\left(\frac{\bar{b}}{b}\right)^{2}-1\right]}{\Lambda_{s}}\right]\right\}
\end{aligned}
$$

where

$$
E_{\theta} \equiv \frac{v_{0}}{2 \pi s N} I_{1}{ }^{2}
$$

[rompare (146) with (142)]. When the $E_{*}$ in $(145)$ is maximized with respect to I. the result $E_{t \mid \max } c a n$ be skown to be

$$
\left.E_{\theta}\right|_{\text {max }}=E_{\theta} \text {. }
$$

Thus $E_{\text {a }}$ is a good measure of the peak induced electric field.

The Initial Implcsion model has provided th: average ion energy $W_{c r}$ at $t=t_{c r}$ and the peak electric field $E_{*}$ as $\&$ function of circuit parameters. and the 
numerically obtainable parameters $\gamma, I_{1}$, and $z_{c r}$. The equations needed to cbtain these parameters are (128), (129), (136), and (139). Obviously the numerical code must track the ions to determine the time $t_{r r}$ and position $z_{c r}$ of the piston at which the first ions traverse the plasma. The system parameters $i_{\mathbb{A}}$ and $q$ are given by (134) and (135). The value chosen for $q$ is confirmed in the calculations of the Expansion and Reflection model. The optimum value of $\Lambda_{\mathrm{s}}$ is discussed in Sec. 4.3.4 where the optimum values of $V_{0}, N$, and $C$ are calculsted.

\subsubsection{Expansion and Reflection}

During the expansion and reflection of the plasma. the total flux $\phi_{T}$ is given by

$$
\Phi_{T}=\int_{A_{C}} \bar{B} \cdot d \bar{A}
$$

where $A_{r}$ is the sum of the effective areas of flux storage in the circuit. The indicated calculation parallels the derivation of the source inductance in Sec. 5.2.2.1.1 so only the result is presented here:

$$
\Phi_{T}=\pi b^{2} B[\Phi+z(2-z)] \text {. }
$$

The normalized flux $\phi$ can be compared with $\Lambda_{\text {s }}$ given by (26) in Sec. 5.2.2.1.1

$$
\begin{aligned}
\Phi \equiv & \frac{\Delta l}{2 \pi^{2} b^{2} N} \cdot\left\{\ln \left(\frac{r_{c}}{b}\right)\right. \\
& \left.+\frac{\bar{\Delta}}{\Delta} \ln \left(\frac{r_{D}}{r_{c}}\right)+\frac{\ell}{2 b}\left(1-\frac{\Delta}{b}\right)\right\}+\frac{2 s}{b} .
\end{aligned}
$$

Evaluating (150) generates a result similar to (27) in Sec. 5.2.2.1.1

$$
\Phi=\frac{\Delta}{100 \mathrm{~N}}\left[2.5-\frac{\Delta}{10}\right]+0.14 .
$$

In fact. $\Phi$ differs from $\Lambda_{\mathrm{s}}$ in only two respects. The contribution from the capacitor is omitted because the crowbar shorts it out, and the first term differs by a factur of $\mathbf{N}$ which arises from the basic difference between flux and magnetic energy density.

The plasma energy $E_{p}$ and field energy $E_{B}$ are defined as the energies contained in the lensth / of an implosion coil. An appropriate expression of the con- servation of total energy is that the change in plasma and field energy between $t=t_{c r}$ and $t_{e}$ is equal in magnitude but opposite in sign

$$
\begin{aligned}
& \Delta E_{B} \equiv E_{B e}-E_{B c r} \\
& \Delta E_{B}=-\left(E_{p e}-z_{p c r}\right) .
\end{aligned}
$$

The basic purpose of this model is to determine the value of $\mathrm{q}$ which results in the reflection of the plasma at $r=b$. The requirement for reflection is that dynamic pressure balance must exist at the time of reflection. Since the plasma has not thermalized at $t=t_{e}$, the contribution of the electrons to the plasma pressure is ignored. The phase space distribution of the ions is not known, so the ion pressure is modeled by the thermalized pressure and a parameter $\epsilon$ which represents the degree of isotropization. The pressure balance is given by

$$
\varepsilon E_{\text {pe }} \Lambda_{s}=E_{B e}
$$

This equation simply states that the plasma and field energy densities are equal if $\epsilon=1$. Thus $\epsilon=2$ for one-dimensional motion and $t=2 / 3$ for complete istritopization.

The magnitude of the energy change $\lrcorner E_{B}$ is given by the amount of work done by the plasma on the field as it expands from $z=z_{c r}$ to $z=0$

$$
\Delta E_{B}=-\int_{V_{c r}}^{V_{C}} \frac{b^{2}}{2 \mu_{0}} d V_{B} .
$$

Because $\phi_{T}$ is a constant, (155) yields

$$
\Delta E_{B}=E_{B C r}{ }^{\Psi} \mathrm{Cr}
$$

where

$$
\Psi_{c r} \equiv \frac{1+A_{c r} / \Phi}{1+\Lambda_{s} / A_{c r}}
$$

where

$$
A_{c r} \equiv z_{c r}\left(2-z_{c r}\right) \text {. }
$$

One more relation is needed to solve for the five variables in (152) and (153). To establish the value of $q$, these energies must be related to the initial stored energy $\mathrm{E}_{\mathrm{r}}$ 


$$
\mathrm{E}_{\mathrm{T}} \equiv \frac{1}{2} \mathrm{CV}_{0}^{2}
$$

Conservation of energy requires

$$
\frac{E_{C c r}}{E_{T}}+\frac{E_{P C r}}{E_{T}}+\frac{E_{P C r}}{E_{T}}=1
$$

where

$$
\mathrm{E}_{\mathrm{ccr}} \equiv \frac{1}{2} \mathrm{CV}_{\mathrm{cr}}{ }^{2} \text {. }
$$

The first ratio in (160) can be determined by use of (129), the relation between $z$ and charge. By the time $t=t_{c r}$, the capacitor has been completely discharged and is being charged in the opposite polarity, hence

$$
c\left(v_{0}+v_{c r}\right)=\int_{0}^{t} c r i d t .
$$

Using (129) yields

$$
C V_{c r}=2 Q_{p} z_{2}-Q_{c}
$$

Since the ratio of the energies is equal to the ratio of the charges squared,

$$
\frac{E_{c c r}}{E_{T}}=\left(2 q z_{c r}-1\right)^{2} \text {. }
$$

The second ratio in (160) is essentially the energy transfer efficiency to the plasma during the initial implosion. The plasma energy $E_{p c r}$ is related to the average ion energy $W_{c r}$ by

$$
E_{p c r}=W_{c r} V_{l} n_{0}
$$

where $l^{\prime}$, is the volume of the vacuum chamber of length $\%$. Recasting (140) and substituting it into (165) yields

$$
\frac{E_{p c r}}{E_{T}}={\gamma q I_{1}}^{2} \text {. }
$$

The third ratio in (160) can be determined by using (152), (153), (154), and (156), and by modifying (159) in the following way:

$$
\frac{E_{\text {ccr }}}{E_{T}} \cdot \frac{E_{\text {pcr }}}{E_{T}}\left[1+\frac{E_{B c r}}{E_{\text {per }}}\right]=1 .
$$

When the term $E_{\mathrm{Bcr}} / \mathrm{E}_{\mathrm{pcr}}$ is evaluated and substituted into the equation along with (164) and (165), the following constraint on $q$ is obtained:

$$
q=\frac{1}{z_{c r}}\left\{1-\frac{\gamma I_{1}^{2}}{4 Z_{c r}}\left[I+\frac{1}{\Omega}\right]\right\} \text {. }
$$

The value of $\Omega$

$$
\Omega \equiv \frac{1+\left(1+\varepsilon \Lambda_{s}\right) \Psi_{c \Omega}}{\varepsilon \Lambda_{s}}
$$

is always much greater than unity for interesting parameter values. Since the second term in the bracket of (168) is always much less than unity, it is justifiable to simplify (168) to

$$
q=\frac{1}{z_{c r}}\left[1-\frac{Y I_{1}^{2}}{4 z_{c r}}\right] \text {. }
$$

Equation (170) gives the desired constraint on q. It is apparent that $q$ is only weakly dependent on $\gamma$ and $I_{1}$, and from numerical results, $z_{2}$ varies only $\pm 5 \mathrm{c}^{\mathrm{r}}$ over a wide range of parameters. As a result, $q$ is also rather constant. To verify $(135),(170)$ is evaluated with $\gamma$ given by (141), $I_{1}$ obtained from Fig. 4-17, and $z{ }^{\prime} r$ is given by its value from the constant field model (Fig. 4-15)

$$
z_{c r}=\frac{2}{3} \text {. }
$$

The result is

$$
q=1.31
$$

compared with $4 / 3$ in (135).

\subsubsection{Isotropization and Thermalization}

The purpose of this part of the model is to relate the plasma energy $E_{p s}$ at $t=t_{s}$ to the peak ion energy $E_{p c r}$ at $t=t_{c r}$. The value of $x_{s}$ is also determined. Finally the relation between $\mathrm{E}_{\theta}, \mathrm{x}_{\mathrm{c}}$, and $q$ [see $(50)$ ] is derived by relating $T_{s}$ and $x_{s}$ with $T_{c}$ and $x_{c}$ through the adiabatic law. 
From experimental observation and from simple theories, it appears that the electron temperature is at least half of the ion temperature at the end of the implosion phase [see (111)]. For the purpose of this model

$$
T_{\text {es }}=\frac{1}{2} T_{\text {is }}
$$

is assumed. Even though a large portion of this energy is probably given to the electrons during the initial implosion, this energy was not included in the eneray balance. The inclusion of electron pressure has two minor effects. One effect is to decrease slightly the required value of $\mathrm{q}$. The second term in the brackets in $(170)$ would be increased by $50 ;$; 10 aciount for electron heating. This lowers $q$ by 5 to $10 \%$ and increases the required capacitance of the bank by the same percentage for a fixed filling pressure $p_{n}$.

The second effect is a result of "adding" the electron energy in the Isotropization and Thermalization model. The model assumes that the energy comes from the magnetic field after the crowbar, thus unrealistically reducing the magnetic pressure at $t=$ $t$. This results in an abnormally high $x_{s}$ and low $T_{s}$ where

$$
T_{s} \equiv \frac{T_{\text {es }}+T_{\text {is }}}{2} .
$$

The adiabatic invariant $T_{\mathrm{s}} \mathbf{x}_{\mathrm{s}}{ }^{4 / 3}$ is in good agreement with the numerical codes so that the inaccuracies in $x_{*}$ and $T_{s}$ present no significant drawback to the model.

The plasma energy at $t=t_{s}$ is related to the temperatures by

$$
E_{p s}=\frac{3}{2} n_{0} V_{L} k_{B}\left(T_{e s}+T_{i s}\right) .
$$

Using (174), $E_{p s}$ becomes

$$
E_{p s}=3 n_{0} V_{L} k_{B} T_{s} \text {. }
$$

Pressure balance requires that

$$
n_{s} k_{B}\left(T_{e s}+r_{i s}\right)=\frac{B_{s}^{2}}{2 \mu_{0}} .
$$

Combining (176) and (177) yie'ds

$$
E_{p s}=E_{B s} \frac{3}{2}\left[\frac{1-A_{s}}{\Lambda_{s}+A_{s}}\right]
$$

where

$$
A_{s} \equiv z_{s}\left(2-z_{s}\right) \text {. }
$$

Now that the system is described at $t=t_{s}$ and $t=$ t. the change in field energy between the two times can be calculated [see (155), (156), and (15i)]

$$
\Delta E_{B s}=-\int_{V_{e}}^{V_{S}} \frac{B^{2}}{2 \mu_{0}} d V_{B} .
$$

By assuming that the total flux $\phi_{T}$ is conserved. (180) becomes

$$
\Delta \mathrm{E}_{\mathrm{BS}}=-\mathrm{E}_{\mathrm{Be}}{ }_{\mathrm{S}}
$$

where $\Psi_{i}$ is defined by

$$
\Psi_{s} \equiv \frac{A_{s} / A_{s}}{1+A_{s} / \phi} .
$$

Using the pressure balance at $t=t,(i 54)$. (181) becomes

$$
\Delta E_{B s}=-E_{p e} \varepsilon \Lambda_{s} \Psi_{s} .
$$

The conservation of energy requires

$$
E_{p s}=E_{p e}-\Delta E_{B:}
$$

or hv combining (183) and (184)

$$
E_{p s}=E_{p e}\left[1+\varepsilon \Lambda_{s} \psi_{s}\right] \text {. }
$$

Equation (185) relates the plasma energies at $t=t$ and $t=t_{s}$. By a similar technique using (152), (i53), (154), and (156), $E_{p e}$ can be related to the energy at $t$ $=\mathrm{t}_{\mathrm{cr}}$

$$
E_{p e}=E_{p c r}\left[\frac{1+\Psi_{c r}}{1+\left(1+\varepsilon \Lambda_{s}\right) \psi(r r}\right]
$$

Combining (185) and (186) yields the desired result

$$
E_{p s}=E_{p c r}\left[\frac{\left(1+\psi_{c r}\right)\left(1+\varepsilon \Lambda_{s} \psi_{s}\right)}{1+\left(1+\varepsilon \Lambda_{s}\right) \psi_{c r}}\right]
$$


Equation (187) will be used later in this section to evaluate $\mathrm{E}_{t \prime}$.

Even though the value of $x_{s}$ | needed to evaluate (187) can be assumed to be 0.7 . it is of interest to determine the value of $x_{n}$ from the code in order $t$ ) compare it with numerical models. The value is readily determined by using the energy conservation law

$$
E_{p s}-E_{p e}=E_{B e}-E_{B s}
$$

in conjunction with the two pressure balance equations $(154)$ and $(178)$ and the energy relation (185). The result is a quadratic equation in $A$.

$$
A_{S}{ }^{2}+C_{1} A_{S}+C_{2}=0
$$

where

$$
c_{1}=\frac{\left(\frac{2}{3}+\varepsilon\right) \phi+\left(\frac{2}{3}-\varepsilon\right) \Lambda_{s}+\frac{5}{3} \varepsilon \Lambda_{s} \phi}{\frac{2}{3}+\varepsilon\left(\Lambda_{s}-\frac{1}{3} \Phi\right)}
$$

and

$$
C_{2}=\frac{\left(\frac{2}{3}-\varepsilon\right) \Phi \Lambda_{s}}{\frac{2}{3}+\varepsilon\left(\Lambda_{s}-\frac{1}{3} \phi\right)} .
$$

If $(189)$ and $(179)$ are evaluated for $t=1 . \perp=2.7$ $\mathrm{cm}$. and $L_{\mathrm{s}}=1 / 2$, then $\mathrm{N}=0.47, \Phi=0.27$ and

$$
x_{s}=0.96
$$

which is unrealistically high as previous!y mentioned. If $(187)$ is evaluated with compatible parameter values.

$$
\frac{E_{p s}}{E_{p c r}}=0.74 .
$$

Numerically it is found that

$$
\gamma x_{s}^{4 / 3} \frac{E_{p s}}{E_{p c r}}=0.45
$$

is virtually independent of $q$ and $\lambda_{s}$. As a check on the model, use of the values in (141), (192), and (193) yields 0.44 .
In addition. if the plasma were adiabatically com. pressed from $\mathrm{x}=0.96$ to 0.70 , then $\mathrm{T}$. would increase to make (19:3) become

$$
\frac{E_{\text {ps }}}{\text { Epr }_{\text {pcr }}}=1.1 \text {. }
$$

This corresponds to about a $30 \mathrm{r}$; decrease in total plasma energy from $t_{1}, t o t$, if the electron energy is added to the ion energy described by $E_{p}$. This 30 ', decrease agrees well with siniulation code results. and the correlation adds further support to the assumption that $x_{x}=0.70$.

The tinal objective of this se'tion is to relate the maximum induced field $E_{n}(1)$ the plasma parameters at $\mathrm{t}=\mathrm{t}$ and also $\mathrm{t}=\mathrm{t}$. Combining (142) and $(146)$ vields

$$
E_{0}=\sqrt{\frac{u_{0} \mu_{0}}{m}} \frac{W_{c r}}{r} .
$$

Substituting in (16i) and (176) provides one of the desired results

$$
E_{g}=\sqrt{\frac{n_{0} \mu_{0}}{m}}\left(\frac{E_{p c r}}{E_{p s}}\right) \frac{3}{\gamma} k_{B} T_{s} .
$$

The electric field can be expressed in terms of compression parameters by using the adiabatic relations and (102) and (104)

$$
\begin{aligned}
& F_{\theta}=\sqrt{\frac{n_{0} H_{0}}{m}}\left(\frac{E_{p c r}}{E_{p s}}\right) \\
& \cdot \frac{3}{\gamma}\left(\frac{x_{c}}{x_{s}}\right)^{4 / 3} \frac{k_{B} \theta}{\ln (0.537 \theta)} .
\end{aligned}
$$

Equation (198) can be evaluated by using the follow. ing parameter values:

$$
\begin{aligned}
& m=2.5\left(1.67 \times 10^{-27} \mathrm{~kg}\right) \\
& P_{0}=4 \mathrm{mtorr} \\
& \gamma \times_{s}{ }^{4 / 3} \frac{E_{p s}}{E_{p c r}}=0.45 \\
& \frac{k_{B} \theta}{e}=2.08 \times 10^{4} \mathrm{ev}
\end{aligned}
$$

|see $(91) \mid$ 


$$
\begin{aligned}
& H=\frac{\eta^{n_{0}}}{x_{c}^{2} \alpha Q_{f}} \\
& n=2.5 \times 10^{-2} m^{3} \text { izeutron } \\
& \alpha=0.72 \\
& Q_{E}=1.1
\end{aligned}
$$

The result is

$$
E_{\theta}=65 x_{c}^{4 / 3} / \ln \left(5.3 / x_{c}^{2} Q_{E}\right) .
$$

This is the equation used in Sec. 4.1.4.3.1 |see (50)| to motivate the minimization of $x_{c}$ in Sec. 4.1.4.3.2.

This section has been devoted to the derivation of field and plasmg parameters associated with the im:plosion system. In the next section. some of these results will be used to optimize and evaluate the im. plosion circuit parameters.

\subsubsection{Circuit Parameterization}

The parameters of the implosion circuit shown in fig. $4-16$ are determined in this section by using some of the results obtained in the previous section. The values of the source inductance $L_{s}$ and the load inductance $L_{p}(t)$ are described in Sec. 5.2.2.1.1, including their functional dependence on the bank voltage $V_{1}$ and the number of turns $N$ on the Marshall-type implosion coil. The crowbar inductance $l_{\text {a }}$ should be made as small as possible, which is about 2 to $3 \mathrm{nH}$. The sizes of $\mathrm{N}, \mathrm{V}_{0}$, and the bank capacitance $\mathrm{C}$ are dependent on the implosionheating requirements described in Sec. 4.3.3.

The first step in determining the circuit parameters is to find the optimum value of $N$. In general. the optimum value of $\mathrm{N}$ matches the circuit impedance to the plasma so that the desired plasma heating is achieved with the minimum $V_{0}$. The best indicator for determining the required amount of $\mathrm{im}$ plosion heating is the peak electric field $E_{\theta}$. This fact can be determined from (198) with $\mathrm{H}$ given by (101). Equation (198) essentially states that the amount of implosion heating (parameterized by $E_{\theta}$ ) required to achieve the goal of a given $Q$ is uniquely related to $Q$. The other parameters in $(198)$ and (101) are $x_{c}$ (which is set by the $m=1$ stability criteria), $p_{1}$ (which is set at the minimum allowed pressure of 4 mtorrl. other parameters of the compression system, constants, and a group of iriplosion-heating parameters

$$
\gamma x_{s}^{4 / 3} \frac{c_{p s}}{E_{p c r}} \simeq 0.45
$$

[see (194)] which is found to be essentially const ant within the relevant range of implosion-heating parameters. Since no parameter of the implosion circuit affects this relation between $E_{\|}$and $Q$. E E can be used as the "goal" of the implosion system.

By using $E_{4}$ as a measure of the required implosion heating. $N$ can be optimized by minimizing $V_{1}$ in the following equation

$$
v_{0}=2 \pi b N \frac{E_{\theta}}{I_{1}{ }^{2}} .
$$

This equation comes from (146) where $E_{t t}$ is defined. The variable $I_{1}$ is dependent on $N$ through the normalized source induct ance Isee (122) in Sec. 4.3.3 and (26) in Sec. 5.2.2.1.11

$$
\begin{aligned}
\Lambda_{s} & \equiv \frac{L_{s}}{L_{0}}=\left(\frac{1}{10 N}\right\}^{2} \\
& \left\{2+\Delta\left[2.5-\frac{\Delta}{10}\right]\right\}+0.14
\end{aligned}
$$

where $J$ is the coil insulator thickness in centimeters.

The dependence of $I_{1}$ on $I_{s}$ is briefly deseribed in Sec. 4.3.3 in conjunction with Fig. 4-17. For $\Lambda_{s} \ll 1, I_{1}$ approaches unity, $I_{1}$ is only weakly dependent on $\Lambda_{\text {s. }}$. and $V_{0}$ is linearly proportional to $\mathrm{N}$. This agrees with a simple circuit analog where the load inductance $L_{\text {, }}$ is the dominant impedance in the circuit so that the voltage available to the plasma $2 \pi \mathrm{bE}_{\|}$is just

$$
2 \pi b E_{\theta} \simeq \frac{v_{o}}{N}
$$

where $\mathrm{N}$ is the turns ratio of the transformer-like Marshall coil. As $\Lambda_{\mathrm{*}} \rightarrow 1$ more of the voltage is dropped across $\mathrm{L}_{4}$, hence reducing $2 \pi \mathrm{bE} \mathrm{E}_{\theta}$. This decrease in $2 \pi \mathrm{bE}$ occurs in (201) because of the decrease in $I_{1}$ with increasing $\Lambda_{s}$. As $\Lambda_{s}$ is increased toward infinity. $I_{1}^{2}$ goes to zero faster than $N$, thus forcing $E_{a t}$ to zero for a finite $V_{0}$. Physically, $N=0$ corresponds to a Marshall coil (see Fig. 5-11) with straight conductors (no helical twist). Such a coil cannot produce $B_{z}$ or $E_{t i}$ fields, which agrees with the above analysis.

Obviously, the minimum $V_{b}$ for a fixed $E_{t}$ has to occur for $\Lambda_{s} \sim 1$. A circuit theorem states that the 
maximum power to the load occurs when the source impedance equals the load impedance. This does not imply $\Lambda_{s}=1$ for this system, since $L_{o}$ is the empty (no plasma) load inductance, and the imploding plasma is causing $L_{p}(t)$ to change with timse. If the load inductance in the circuit theorem were ap. proximated by the time average of $L_{p}(l)$ during the implusion, then

$$
\Lambda_{\mathrm{S}}=\frac{1}{2}
$$

is very close to the optimum value. Fortunately this choice of $A_{s}$ [see (134)] agrees with numerical results. A direct analytic optimization is, unfortunately, impossible due to the complexity of (128) and (136).

Once the value of 1 , is fixed by (204), the optimum $\mathrm{N}$ can be determined from (202)

$$
N=\frac{\left[2+\Delta\left(2.5-\frac{\Delta}{10}\right)\right]^{3 / 2}}{6}
$$

However, $J$ is dependent on $V_{0}$, so that (28) (from Sec. 5.2.2.1.1), (205), and (201) must be solved simultaneously. The result is

$$
V_{o}[M]=\frac{8}{25} \psi\left[\psi+\left(\psi^{2}+\frac{1}{2}\right)^{1 / 2}\right]
$$

where

$$
\psi=\frac{2 \pi b E_{\theta}}{\left(16 \times 10^{5}\right) I_{1}{ }^{2}\left(\Lambda_{s}-0.14\right)^{1 / 2}} .
$$

Using $I_{\mathrm{s}}=1 / 2, \mathrm{~b}=10 \mathrm{~cm}, I_{1}=0.73$ from Fig. 4-16, and (77) for $E_{1}$, to evaluate (207) yields

$$
\psi=\frac{3.0}{\delta_{1}^{8 / 15} \ln \left(70 \delta_{1}\right)} .
$$

The dependences of $V_{0}$ and $N$ on $\delta_{1}$ are plotted in Fig. 4-18 by using (208), (206), and (205) where

$$
\Delta[\mathrm{cm}]=10 \mathrm{~V}_{\mathrm{O}}[\mathrm{MV}]
$$

The result clearlv indicates the advantage of using large $\delta_{1}$.

The capacitance $\mathrm{C}$ is easily determined by using (118), (119), (120), and the values of $N$ and $V_{n}$ in Fig. 4-17. The value of $q$ is decreased to

$$
q=\frac{5}{4}
$$

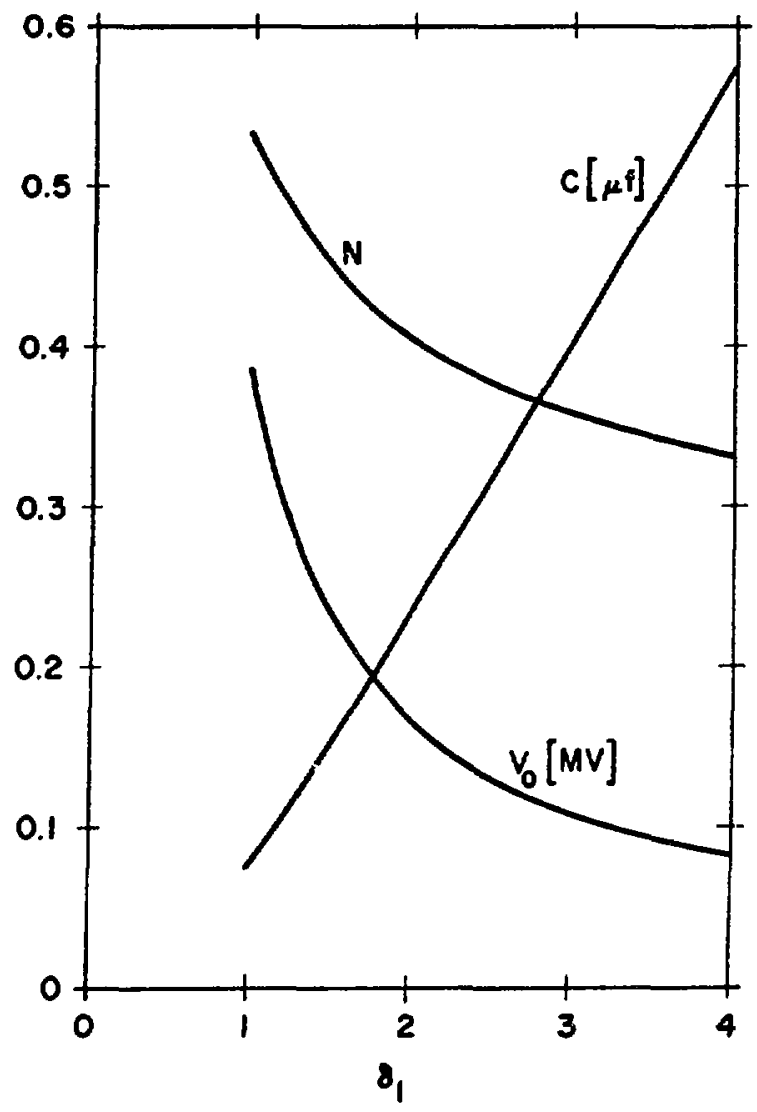

Fig. 4-18.

Effect of $\delta_{I}$ on the implosion bank voltage $V_{0}$, number of turrs on the implosion coil $N$, and the implosion bank capacitance $C$ for a singie implosion coil $20 \mathrm{~cm}$ long.

from the $4 / 3$ value in (135) to allow for the electron heating as discussed in the Isotropization and Thermalization model in Sec. 4.3.3. The result is

$$
\mathrm{C}[\mu f]=\frac{1.6 \times 10^{-2}}{\mathrm{~N} V_{\mathrm{O}}[\mathrm{MV}]}
$$

which is plotted in Fig. 4-18.

The total energy required in the implosion bank is obtained from

$$
E_{\mathrm{TB}}=(1280) \frac{1}{2} C \mathrm{~V}_{\mathrm{o}}^{2}
$$

where 1280 is the number of implosion coils in the system. The dependence of $\mathrm{E}_{\mathrm{TH}}$ on $\delta_{1}$ is plotted in Fig. 4-19. 


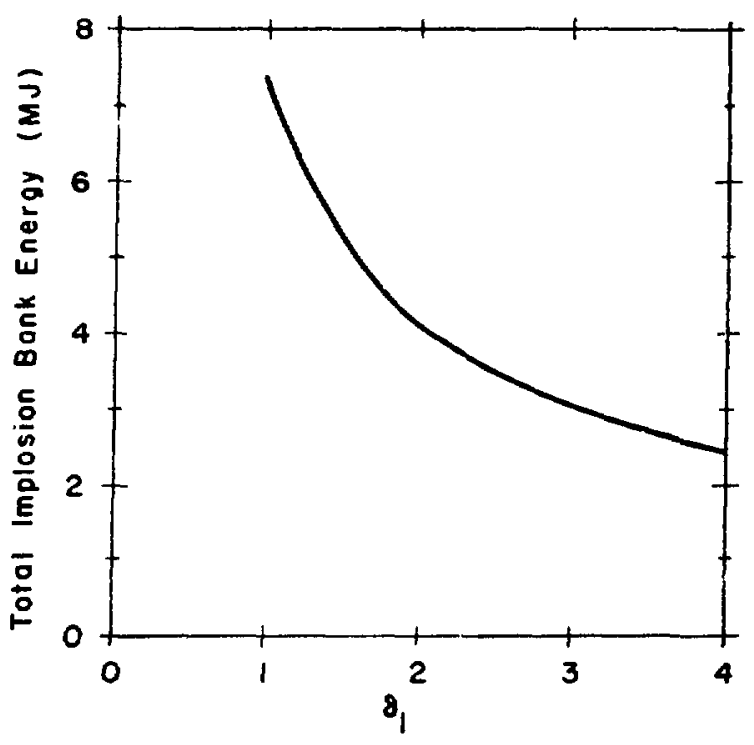

Fig. 4-19.

Dependence of the total implosion bank energy on $\delta_{l}$.

The efficiency of the implosion system is estimated in Sec. 4.1.4.1.2 by using simple pressure balance and impedance matching arguments. An in. dependent method is possible using (166), (187), and he relation

$$
\mathrm{e}_{\mathrm{T} i}=\frac{\mathrm{E}_{\mathrm{pcr}}}{\mathrm{E}_{\mathrm{T}}} \frac{\mathrm{E}_{\mathrm{ps}}}{\mathrm{E}_{\mathrm{pcr}}} .
$$

The result can be evaluated using Fig. 4-17, (141), (210), and (193) (hence pessimistically ignoring electron heating)

$$
\mathbf{e}_{\mathrm{Ti}} \simeq 0.31 \text {. }
$$

This result compares favorably with the previous result in (28) or Fig. 4-5 with $\mathrm{x}_{\mathrm{s}}=0.7$.

'Two general conclusions can be made about implosion heating if experiments confirm the results of the resonant heating model. First, the method is an efficient way of preheating the plasma for the compression phase and, second, the larger $\delta_{1}$ is made, the more attractive the system becomes technologically. The engineering design presented in this report is based on the pessimistic choice of $\delta_{1}=1.5$, but we hope future experiments will add to the Garching results ${ }^{8}$ which indicate that $\delta_{1}>3$ is an acceptable and attractive operating range.

\section{REFERENCES (Chap. IV)}

1. M. N. Rosenbluth. J. L. Johnson. J. M. Greene. and K. E. Weimer. "Stability Limitations for Stellerators with Sharp Surfaces." Phys. Fluids 12. 726 (1969).

2. H. Grad and H. Weitzner. "Critical $\beta$ from Stellerator and Scyllac Expansions," Phys. Fluids 12. 1725 (1969).

3. F. L. Ribe, "Free-Boundary Solutions for HighBeta Stelleiators of Large Aspect Ratio," Los Alamos Scientific 1 ,aboratory report LA-4098-MS (May 1969).

4. F. L. Ribe and M. N. Rosenbluth, "Feedback Stabilization of a High- $\beta$, Sharp-Boundaried Plasma Column with Helical Fields," Phys. Fluids 13. 2572 (1970).

5. J. P. Freidberg and W. Grossmann, private communication (1974).

6. W. R. Ellis, F. C. Jahoda, R. Kristal, W. E. Quinn, F. L. Ribe, G. A. Sawyer, and R. E. Siemon, "Plasma Equilibrium and Stability in the Scyllac Toroidal Sector Experiments," Nucl. Fusion 14, 841 (1974).

7. J. P. Freidberg, "Stability of the Straight $f=1$ Scyllac Configuration," Phys. Fluids 14, 2454 (1971).

8. M. Kaufmann, private communication (June 1975).

9. J. P. Freidberg, R. L. Morse, and F. L. Ribe, "Staged Theta Pinches with Implosion Heating," Symp. Technology of Controlled Thermonuclear Fusion Experiments and Engineering Aspects of Fusion Reactors, Austin, TX, Nov. 20-22, 1972.

10. R. A. Krakowski, F. L. Ribe, T. A. Coultas, and A. J. Hatch, "An Engineering Design Study of a Reference Theta-Pinch Reactor (RTPR)," Los Alamos Scientific Laboratory report LA-5336/ANL8019 (March 1974). 
11. I). P'irsch and H. Tasso. "A. Theorem on MHI)Instability of Plasmas with Rissistive Walls," Nucl. Fusion 11, 259 (1971).

12. J. P. Goedbloed, D. Plirsch, and H. Tasso, "Instability of a Pinch Surrounded by a Resistive Wall," Nucl. Fusion 12, 649 (1972).

13. E. I. Cantrell, W. R. Ellis, H. W. Harris, F. C. Jahoda, R. Kristal, M. D. Machalek, J. R. McConnell, W. E. Quinn, F. L. Ribe, G. A. Sawyer, F. T. Seibel, and R. E. Siemon, "Plasma Experiments in the Scyllac Toroidal Theta Pinch," Proc. Fifth IAEA Conf. on Plasma Physics and Con. trolled Nuclear Fusion Research, Tokyo, Nov. 11-15, 1974.
14. T. A. Oliphant, "Fuel Burn-Up aind Direct Conversion of Energy in a D-T Plasma," Proc. BNES Conf. on Nuclear Fusion Reactors, UKAEA Culham Laboratory, Sept. 17-19, 1969, p. 309.

15. J. L. Tuck. "Thermonuclear Reaction Rates," Nucl. Fusion 1, 201 (1961).

16. A. G. Sgro and C. W. Nielson, to be published. 17. T. A. Oliphant, "A Mixed Snowplow-Bounce Model for Shock Heating in a Staged Theta Pinch," Nucl. Fusion 14, 377 (1974). 


\section{SYSTEM DESIGN}

\subsection{AN OVERVIEW}

The SFTR facility can be subdivided into a number of somewhat isolated svstems. This chapter describes each in detail, and this section gives an overview of their integration into the SFTR.

The electrical systems and the implosion and compression circuits provide two-stage heating of the plasma. The implosion circuit heats the ions in the preionized plasma from $-1 \mathrm{eV}$ to $\sim 3 \mathrm{keV}$. The electron heating is determined by anomalous processes in the sheath during the shock. hence we cannot calculate it. Experience from Scyllac and particle simulation codes suggests $T_{e} \sim 1 / 2 T_{i}$ after implosion, so we assume that value.

The implosion circuit must produce a fast-rising magnetic tield to accomplish the initial heating, and two different approaches are possible. The first is an adaptation of the Staged Theta Pinch circuit, and uses a complex circuit to program the magnetic field waveform. The field is essentially removed when the sheath is at its minimum radius, allowing it to expand freely toward the wall. Before contact, the field is reapplied to hold the plasma at a large radius. In this circuit, the plasma impedance is small compared to the circuit impedance so that the latter controls the current waveform. Figure 5.1-1 shows the circuit and field waveform of a pulse-forming network.

An alternative approach, termed resonant heating.' uses a simple capacitor circuit with minimum source impedance, such that the plasma impedance is comparable or larger. The plasma initially appears inductive, and the current rises as in an $L C$ circuit. As the sheath moves off the wall, the changing inductance (which appears resistive) is the dominant plasma impedance, and the resulting $\mathrm{RC}$ behavior (see Fig. 4-16) resembles a crowbarred capacitor circuit. If the quarter period $\pi \sqrt{L C / 2}$ is set approximately equal to the time for the sheath to reach its min mum radius (hence the term resonant heating), and the capacitor is then crowbarred, the plasma will deliver energy back to the field, increasing the circuit current (see Fig. 4-16).

The major advantages of the resonant circuit are its simplicity and its efficiency. It is efficient because the low source inductance permits more energy to be delivered to the plasma. It is simple because the capacitor switching is simultaneous as opposed to the programmed switching of thf; other approach. Because of these advantages we use the resonant circuit in this design.
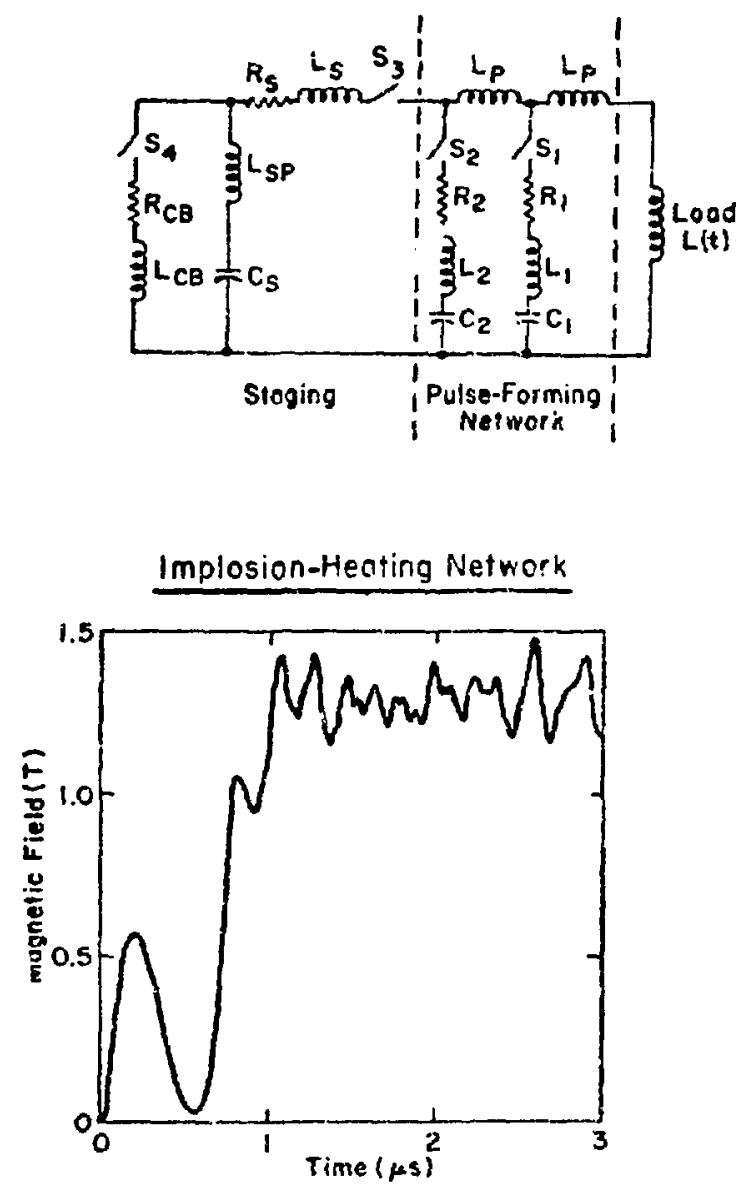

Fig. 5.1-1.

A pulse-forming netuork and the magnetic field waveform it produces.

The resonant approach is made possible by the Marshall coil which can be designed to have an even lower inductance than the single turn coils in present theta pinches. A separate end-fed Marshall-type implosion coil is a necessity in SFTR since a high inductance is required for the slow compression circuit. The end feed is forced by the geometry of the situation.

The implosion coils are nested inside the compression coils, and to avoid inducing high voltage on the compression coils a shurting capacitor is placed on the compression coil to force flux from the implosion coil to return in the annular region between the two 
coils. 'This region is filled with magnetic material to reduce the energy stored in that region. The shorting capacitor allows a slow-rising compression field to rise, but shields the compression coil from the fast implosion field. The geometry is shown in Fig. $5.1-2$.

Outside the compression coil there is $1 / 2 \mathrm{~m}$ of primary shield, penetrated by radial feed plates $t$ the Marshall coil. Figure 5.1-3 shows the feed plates and supporting structure. Beyond the shield these radial plates are fed with pairs of $135 . \mathrm{kV}$ implosion capacitors placed on the periphery. Azimuthally splaced between the capacitors are the crowbar gaps. A cross section perpendicular to the toroidal axis is shown in Fig. 5.1-4.

A toroidal vacuum manifold runs under the main torus, with 188 connecting paths periodically placed around the torus. The $\sim 8000-t$ volume is pumped at 4 stations with $2000-1 / \mathrm{s}$ pumps at each station. The vacuum and tritium handling systems are intimate-
Iy connected. The tritium injection uses standarc volumes and vacuum transfer methods, and the recovery is at the cryogenic vacuum pump station.

The entire vessel, from the plasma radially out ward to the implosion capacitors and vacuum system, is set in a cell with 1.5 -m-thick concrete walls for biological shielding. Electrical leads for energizing capacitors and coils, and for diagnostics. penetrate the wall at feedthroughs located around the cell as shown in Fig. 5.1-5, which stows a cross section of the cell. By maintaining a pressure differential of a few pounds per square inch between the cell and the outside, with the cell pressure lower. contamination of outside air is minimized in the event of a leak.

The magnetic energy transfer and storage (METS) system stores and transfers 488 M.J of energy to the pancake-type compression coils. The

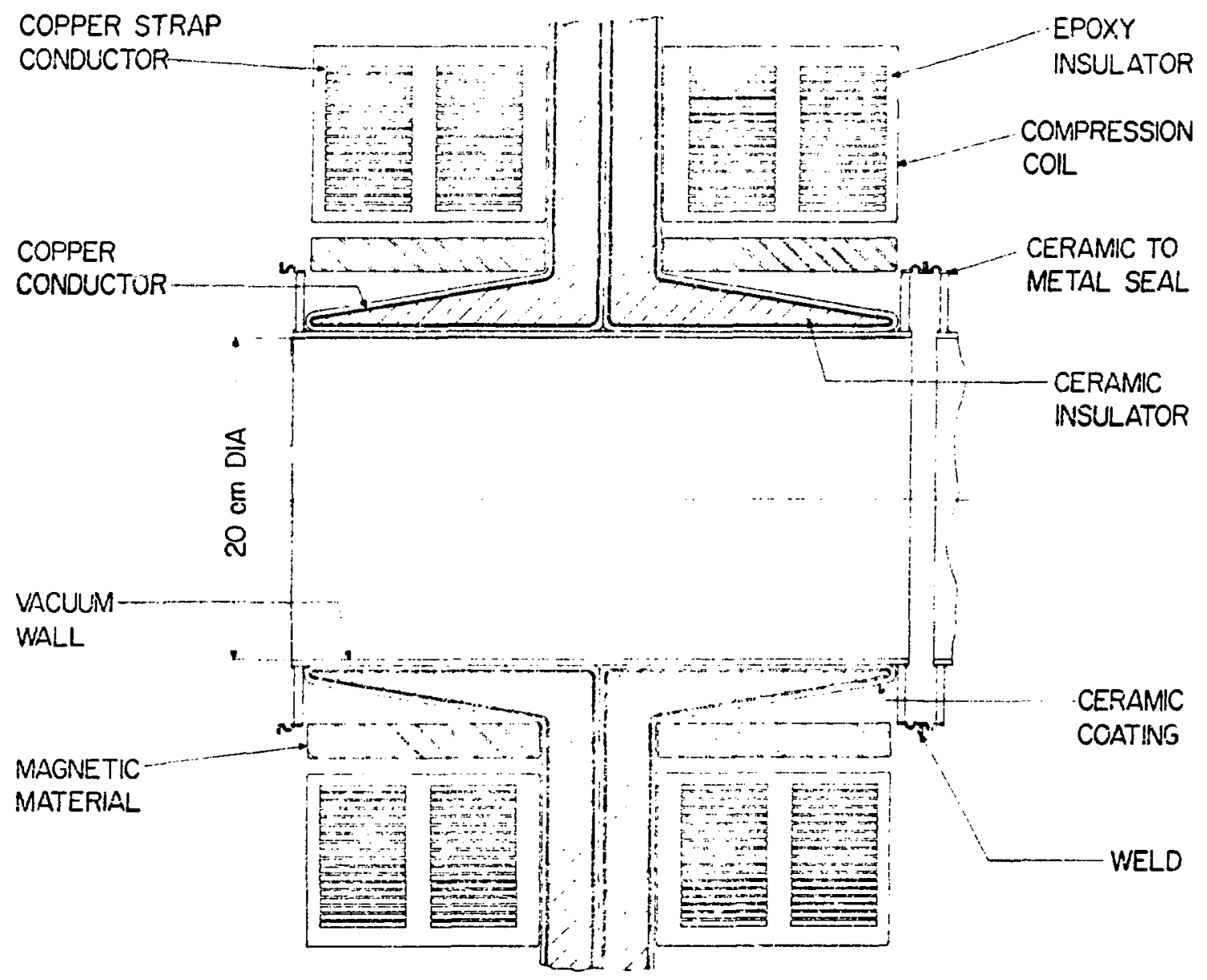

Fig. 5.1-2.

Coil assembly. 


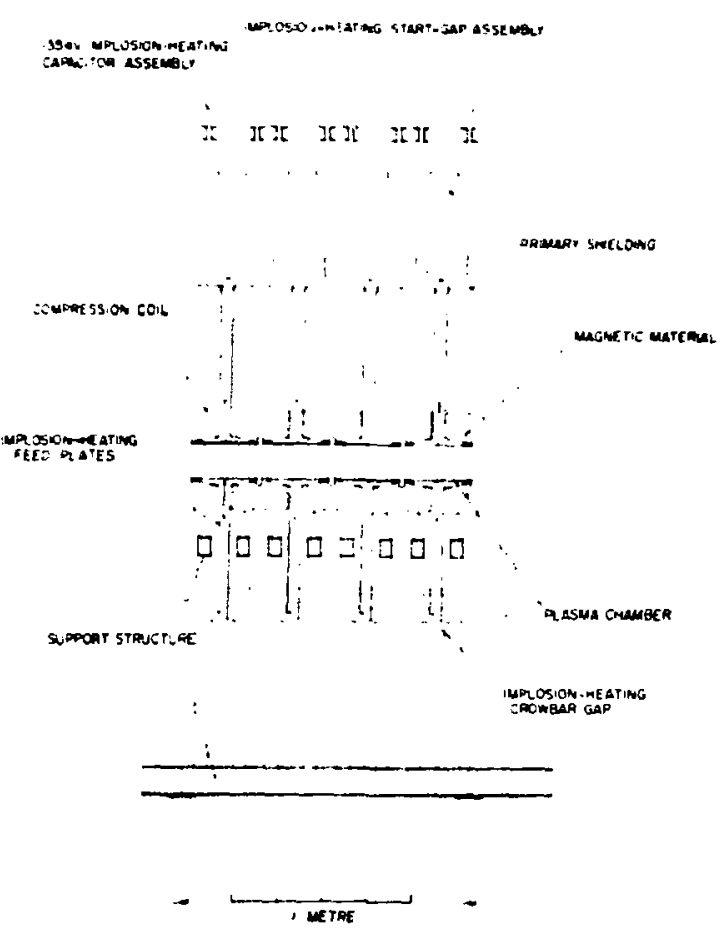

Fig. 5.1-3.

Section of the torus, shouing the plasma chamber, implosion and compression coils, and physical layout of the implosion circuit com. punents.

system is modular. with 1280 units of storage coil. interrupter. transfer capacitor bank, and compression coil. Each module has $381 \mathrm{~kJ}$ and energizes $20 \mathrm{~cm}$ of the torus. The storage coils are stacked in 128 dewars, 10 to a dewar, around the machine. The 1280 vacuum interrupter modules lise 4 vacuum bottles each, 2 in series and 2 in parallel, to break $26 \mathrm{kA}$ and hold $60 \mathrm{kV}$. Each transfer capacitor module has 18 series-parallel, connected capacitors storing $185 \mathrm{k} . J$. The compcinents are located outside the cell, as in Fig. 5.1-6.

The storage coils are series charged with a $125-\mathrm{V}$. $26-k A$ de supply. Each storage coil is separated by a low resistance $(-10-100 \mathrm{n} \Omega)$ switch, which is opened to isolate the modules for parallel discharging. Energy transfer to the compression coil is initiated be opening and then counterpulsing the vacutem in terrupters. forcing a momentary current zero in ex. tinguish the are. Entrgy for the coumserpulse cirtuit (10) M.J eomes from the transfer calpacitors which were charged io a lew kilowoll. for that purposes.

The refrigerator for the helium load liqueties :3014: kg/h. about 2.2 simes more than any plant in operation loday. 'The power reguirement for the refrigerator and compressors is 12 $\mathrm{MW}$. The heal losses include foth steady-state and ptised loads. The steady-state dewar losses are $7.16 \mathrm{~kW}$. including 5. $4 \mathrm{kll}$ for the current leads to the dewar. The pulsed dewar losies are R.t $\mathrm{kW}$. of which $5.5 \mathrm{~kW}$ are associated with the discommed switches betwern storage coils. The transfer lines bet ween compunousts of the refrigeration system consume another $1.1 \mathrm{kH}$. for a total helium refrigeration requirement of 17.3 kW'.

'The crvogenic plant and tritium waste treatment facility are located on the outside of the building housing the cell. An artist seonception of the SFTK. showing the layout of the various facilities is shown in the frontispiece of this report.

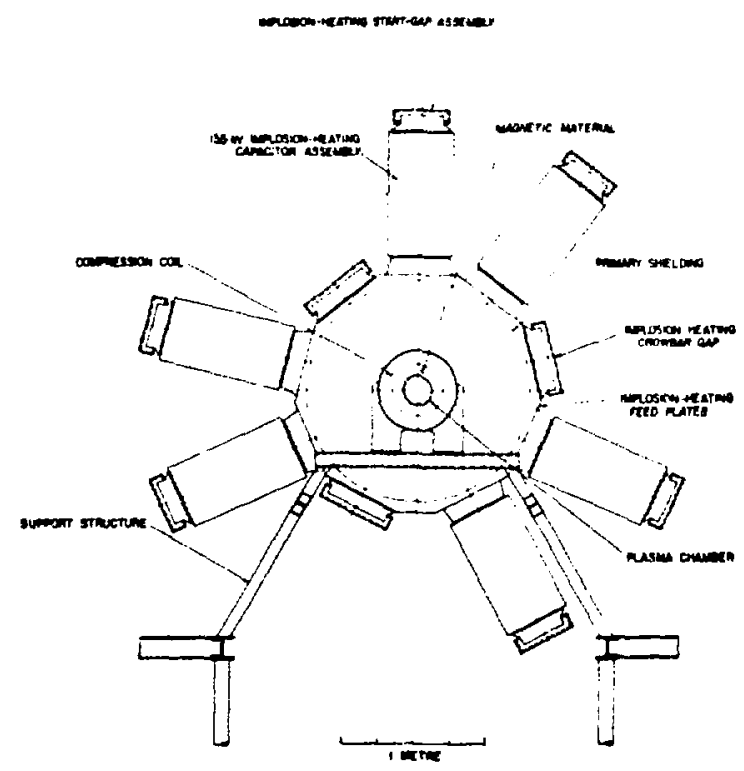

Fig. 5.1-4.

Axial view of the implosion system and coil assembly. 


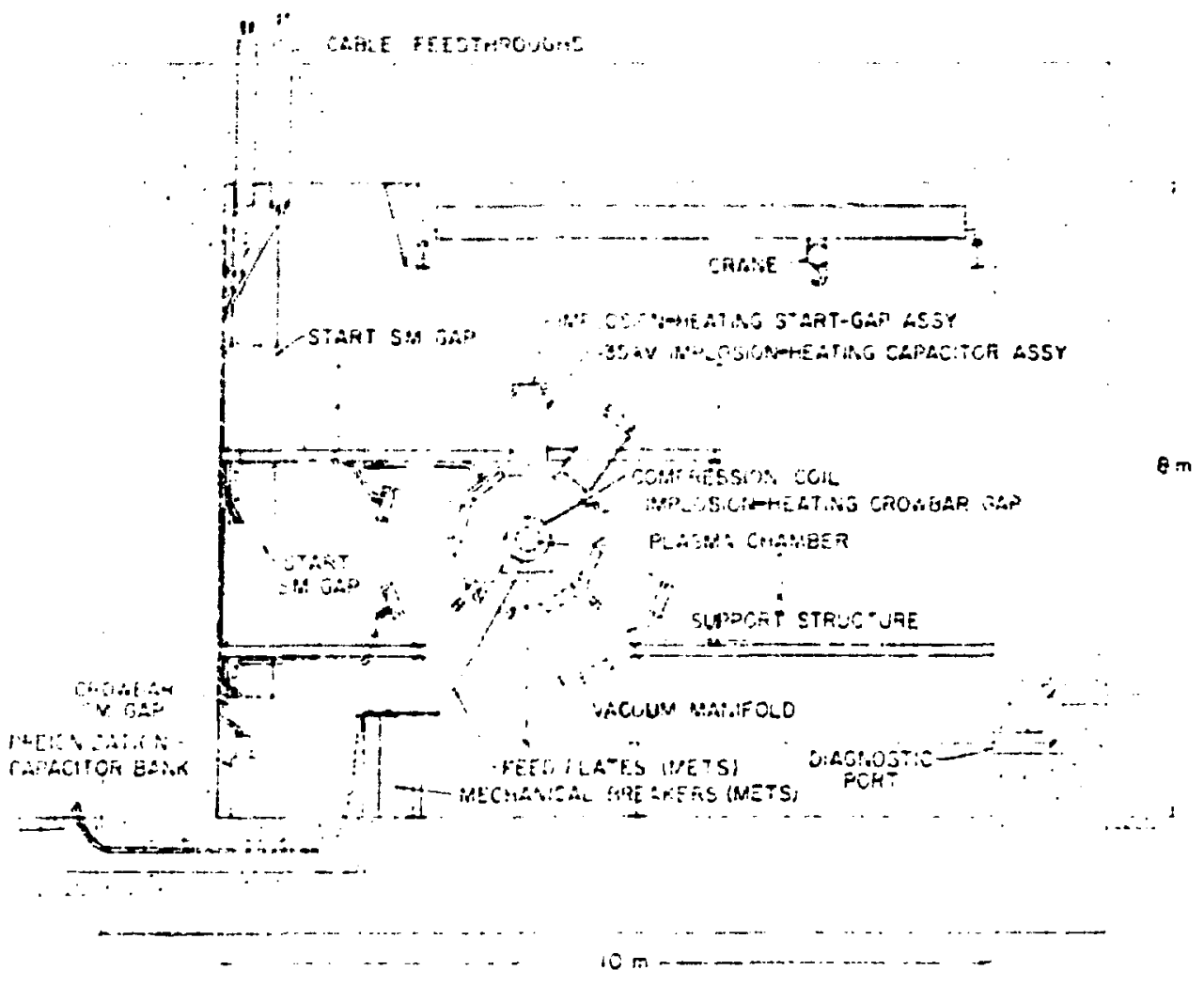

Fig. 5. $1-\overline{5}$.

('ross section of cell with the experimental rhamber, cabling, and submaster (SM) trigger gaps.

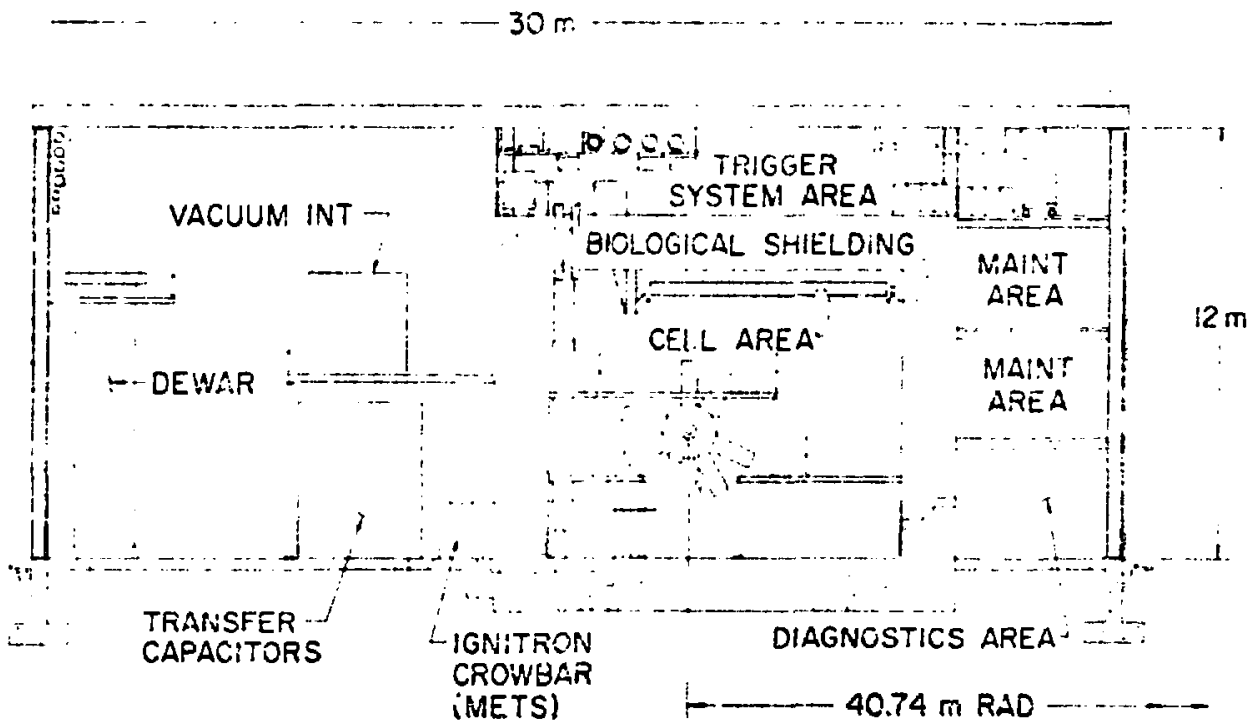

Fig. 5.1-6.

Building cruss section shouing location of METS components. 


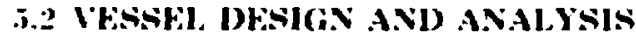

\section{..2.1 Summary}

In this section the enginesering design and fabrica. tion of the containment sistem is described. The imponents include the tirst wall. implusion aroil. masnet ic material. compression cont. start and crowbatr wilps, feedplates, and st ructural support. These components are shown in Fig. 5.1-4. in sarticular. We consider the electrical design. thermal and stress andivies. and the constraints impused by the elecerical and mechanical properties of materials.

The ceramic lirst wall and Marshall coil are both difficult components to fabricate. hence have a sub. tamtial iniluence on the design. The dielectric strength of the ceramic insulator in the implosion coil determines the internal inductance of the strueture. hence limits the soltage for efficient energy transter. The inductances of the coil and feed structure are computed.

The implosiom coil is also subject to magnetic stresises. and both static and dynamic stresses have been calculated using a tinite element code. The quasimagnetustatic tields produce a dynamic stress which is about twice the static stress but well within material linats.

The first wall is subject to neutron. particle. and pinoton tluxes which induce a significant thermal stress on the ceranic material. As determined here. these stresst's are within limits but the margin is not large and is dependent on details of the rate of entergy deposition from the plasma which are not precisely known. This rate determines the thickness of the temperature gradient region. hence the stress level.

\subsubsection{Engineering Design of Containment System}

\subsubsection{Electrical Aspects of Vessel Design}

The electrical requirements of the implosion and compression systems strongly affect the design of the coil assembly shown in Fig. 5.1-2. Some of these aspects are discussed briefly here. while those that reed more ext ensive explanation are discussed in the three subsections which follow.

The implosion coil must have a low inductance and be fed with a low inductance line to produce resonant heating. The implosion coil shown in Fig. $5.1 \cdot 2$ has the necessary low induct ance, as does the parallel plate line (Fig. 5.2-1 and 5.1-4) which feeds it. The line alco allows the required coaxial relation between the implosion and compression coile.

The electrical interaction between the irnplosion and compression coils must also be considered. A

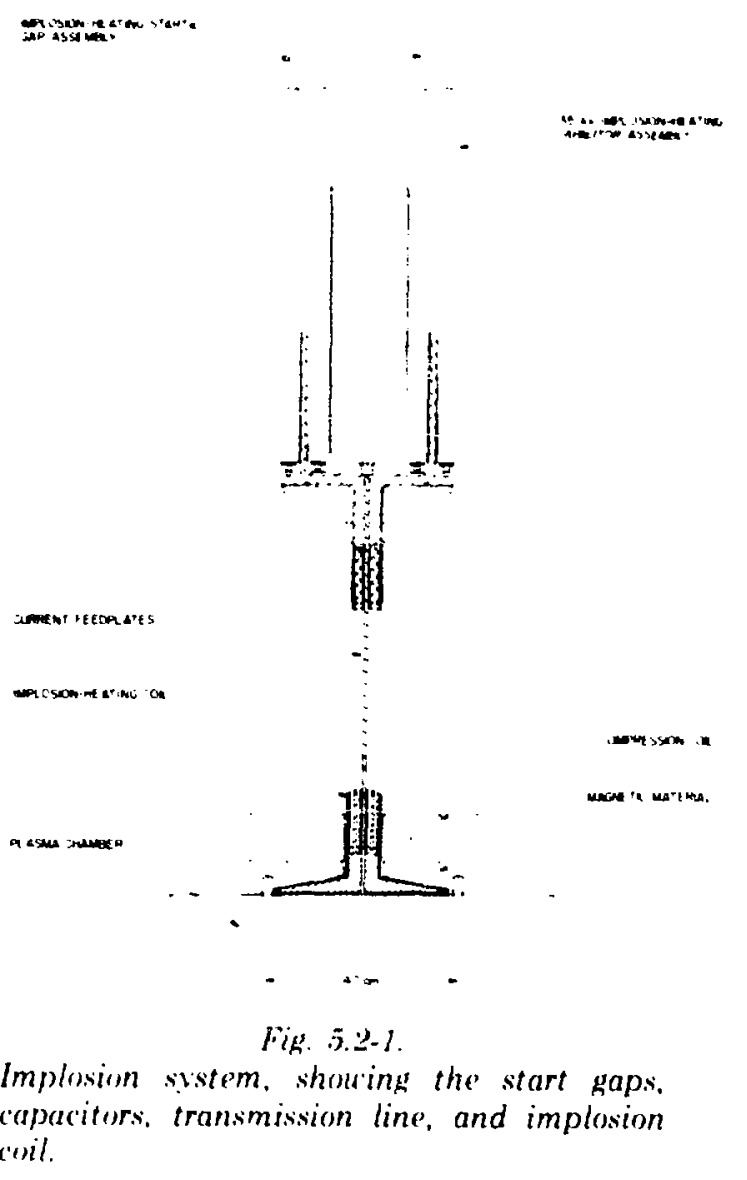

shorting capacitor can be placed across the terminals of the compression coil to prevent voltage multiplication in the multiturn compression coil when the implosion coil is energized. This shorting of the compression coil, although necessary, causes the undesirable storage of implosion field energy in the area between the coils $\mathrm{A}_{2}$ shown in Fig. 5.2-2. If $\mathrm{A}_{1}=$ $A_{2}$, and if both regions bave the permeability of free space, then it is obvious from Fig. 5.2-2 that the energies stored in $A_{1}$ and $A_{2}$ will be equal.

To reduce the stored energy in $A_{2}$ without increasing the required implosicn energy, magnetic material is inserted in area $A_{2}$ (see Fig. 5.1-2). If $\mu_{2}$ 》) $\mu_{1}$, and if $A_{1} \simeq A_{2}$, then the implosion energy stored in $A_{2}$ will be made smaller than in $A_{1}$. In addition, the compression system will not be adversely affected.

The magnetic material not only minimizes the extra energy storage, but it also reduces the possibility of voltage breakdown inside the compression ccil. Even though the compression coil terminals are shorted on the outside, flux from the implosion field 


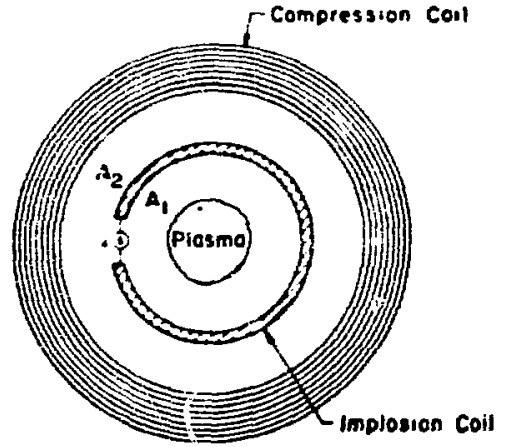

(a) Schemotic Cross Section of the SFTR Module

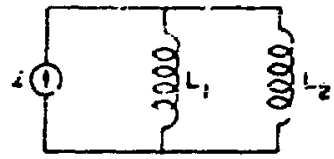

$$
\begin{aligned}
& L_{1} \cdot \frac{\mu_{1} A_{1}}{l} \\
& L_{2} \cdot \frac{\mu_{2} A_{2}}{l}
\end{aligned}
$$

(b) Equivolant Circuit for the Implosion Coil With the Compression Coil Terminals Shorted

Fig. 5.2-2.

Schematic diagram and circuit which model the electrical interaction between the implosion and compression coils.

will still penetrate into the body of the coil. This changing flux induces an electric field which is largest between the inter two windings of the coil. The presence of the magnetic material reduces this fieid from the free-space condition by the ratio of $\mu_{1.2} / \mu_{10}$.

The magnetic material must satisfy four requirements:

1. The saturation field must be as large as possible to minimize $A_{2}$.

2. We require an effective value of $\mu_{2} S 10 \mu_{0}$ (including air gaps imposed by the feedplates). Thus, the $\mu$ of the bulk material for pulsed application must exceed $30 \mu_{0}$.

3 . The field must be able to penetrate the material during the rise time of $100 \mathrm{~ns}$.

4. The indiuced electric field generated by the penetrating $B$-itield must not break the material down.

The proposed fabrication of this material is discussed in Sec. 5.2.3.2.
The first wall, or vacuura wall, must be as close to the implosion coil conductors as pessible to maximize wall stabilization (see Sec. 4.1.4.2), and to minimize the source inductance of the implosion system (see Sec. 4.3.2). This implies that the vacuum wall should be made is thin as possible and stili maintain mechanical integrity.

Electrical requirements of the system also impose strong constraints on the vacuum seals between adjacent vacuum wall segments. Organic materials cannot be used because of tritium contamination and radiation damage. Bolted metal flanges cannot be used because the magnetic fields cannot penetrate the massive flanges and unacceptable field distortion results. Thin welded metal joints, shown in Fig. 5.1-2, can be used only if they are at a sufficiently large radius that the implosion field (including the flux passirg between segments of magnetic material) is not seriously distorted by the metal. The slowly rising compression field car diffuse through this welded seal without serious distortion. The position of the joint in Fig. 5.1-2 is the only acceptable one since the joint's radius is barely large enough to prevent serious implosion field distortion and barely small enough to allow the compression coil to slide over it.

\subsection{1.1 Impiasion-Heating Coil}

A more detailed description of the coil is presented here than in the previous section. The constraints on the conductor shape and size are discussed along with insulator requirements. Finally, the fields produced by the coil are described and the coil inductance is calculated.

The current path in the coil is shown by the arrows in Fig. 5.2-3. It follows the conductive strips radialty in on the transmission line, axially down the outside of the coil, helically back up the inside of the coil. and back out the transmission line. On the inside of the coil the conductor is made of strips to force the current to take the desired helical path. Elsewhere, the conductor is made of strips to allow fields to penetrate.

The $500^{\circ}$; transparency achieved by equal conductor and gap widths insures that the implosion field. passing through the transmission line between magnetic material segments, will not be increased by more than a factor of 2 . The slowly rising com. pression field will diffuse through the copper conductors and hence will not be greatly affected by the transmission line. The equal widths of conductor and gap do not increase the coil inductance appreciably over the solid conductor case as long as the conductor width is small compared with the insulator thickness of the transmission line. 


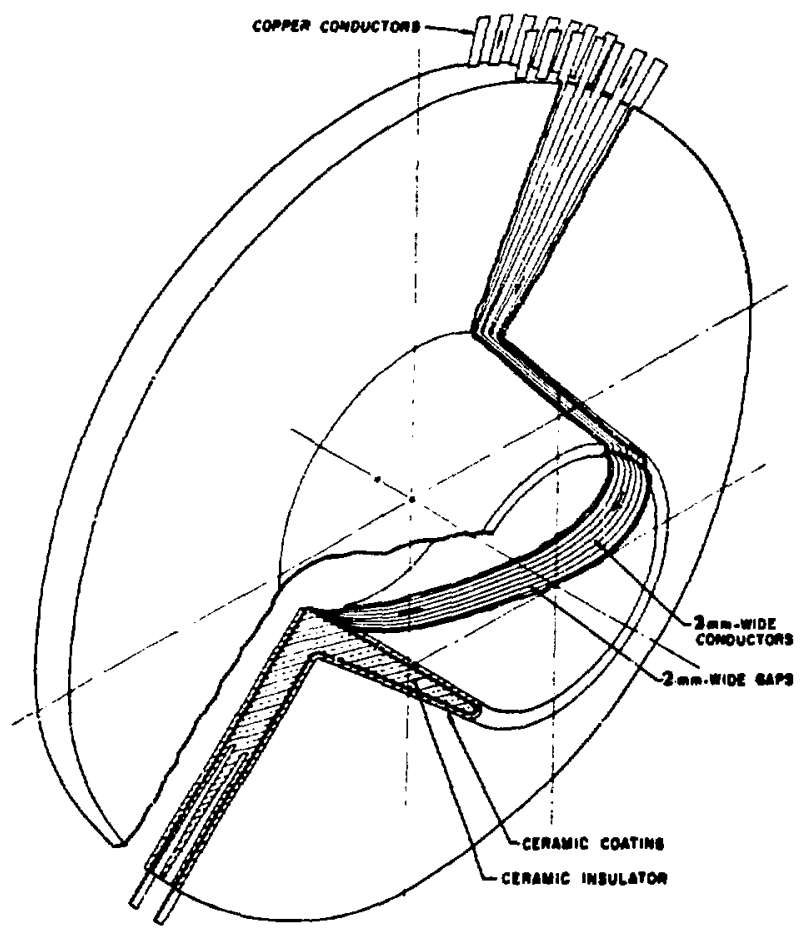

Fig. 5.2-3.

Marshall-type implosion coil.

The thickness of the conductors has been chosen to be $1.5 \mathrm{~mm}$ in Sec. 4.1.4.2.1 [see (21)). The conductor is thus thick enough to allow the crowbar gap to dominate the $L / R$ decay time of the implosion field. but thin enough so that the compression field can diffuse through the coil.

The insulator should be kept as thin as the dielectric strength will allow so that the coil inductance can be minimized. The coil thickness is tapered because the voltage across the sissulator decreases linearly along the coil. For adequate reliability in a system as large as SFTR, the dielectric strength of the $\mathrm{Al}_{2} \mathrm{O}_{3}$ ceramic has been estimated at

$$
\mathrm{E}_{\mathrm{D}}=10^{5} \mathrm{~V} / \mathrm{cr} \text {. }
$$

Additional testing is needed before a firm value can be assigned to this dielectric strength under the appropriate pulsed conditions. The ceramic coating added to the outside of the conducting layer is needed to prevent arcing between adjacent conductors. Using a maximum $\mathrm{E}_{i}$ of $5 \mathrm{kV} / \mathrm{cm}$ (see Fig. 4-10), the maximum field between the coriductors is $10 \mathrm{kV} / \mathrm{cm}$. or $4 \mathrm{kV}$ between conductors.
For the purpose of calculating the inductance of the coil, the magnetic fields can be approximated by a simple model. The field inside the ceramic insulator is in the $\theta$ (azimuthal) direction arising from the radial and $z$ (axial) components of the current. The $\theta$ component of the current inside the coil gives rise to the axiul magnetic field $\mathrm{H}_{z}$ surrounded by the coil. The $\mathrm{H}_{2}$ field outside the coil is assumed to be negligible because of the magnetic material.

The dimensions used in the indur:tance calculation are shown on the schematic coil cross section in Fig. 5.2-4 (compare with Fig. 5.2-1). Starting with the capacitors and working toward the coil, the best estimate of the inductance $L_{c s}$ for the six parallel capacitor-gap combinations shown in Fig. 5.1-4 is

$$
\mathrm{L}_{\mathrm{cs}}=4_{\mathrm{nh}} \text {. }
$$

The inductance of the transmission line $\mathrm{L}_{f}$ with two thicknesses $\bar{J}$ and $\triangle$ can be calculated using

$$
H_{e}=\frac{i}{2 \pi r}
$$

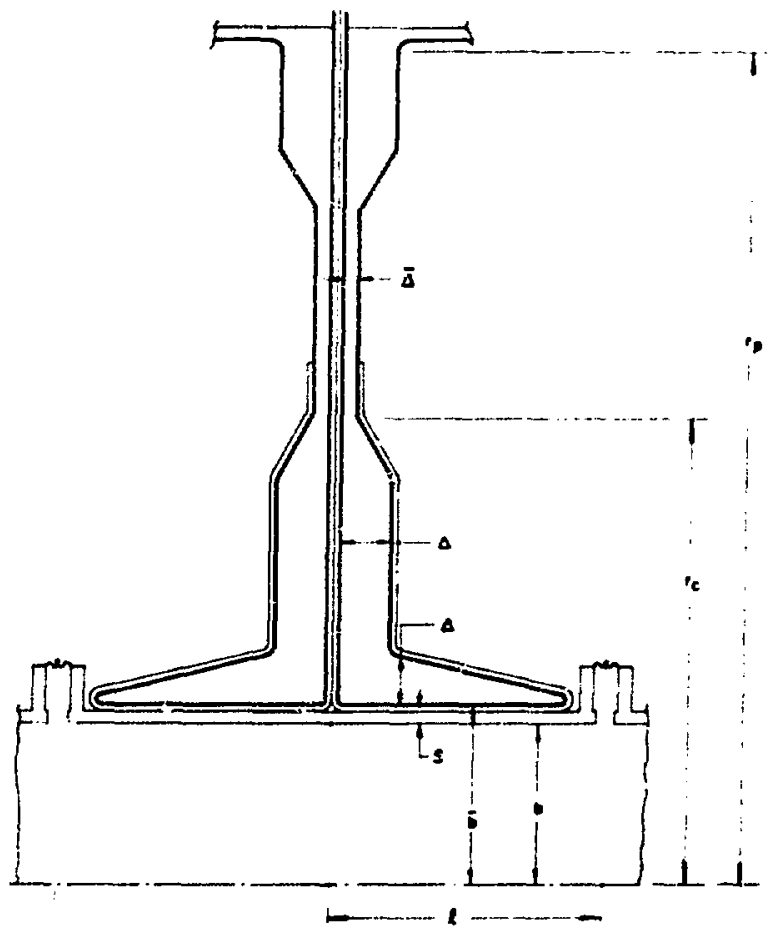

Fig. 5.2-4.

Schematic cross section of the im, olosion coil shouing dimensions used in deriving the induc. tance. 
and

( $A_{1}$ was defined in Fig. 5.2-2). In addition,

$$
L_{\ell}=\mu_{0} \int\left(\frac{H_{\theta}}{i}\right)^{2} d V .
$$

The result is

$$
\mathrm{L}_{\ell}=\frac{\mu_{0} \Delta}{2 \pi}\left\{\ln \left(\frac{\mathrm{r}_{\mathrm{c}}}{\bar{b}}\right)+\frac{\bar{\Delta}}{\Delta} \ln \left(\frac{r_{\mathrm{p}}}{\mathrm{r}_{\mathrm{c}}}\right)\right\}
$$

The inductance $L_{1+1}$ associated with the $H_{n}$ field in the coil insulator is obtained from (3) and an equation similar to (4) yielding

$$
\begin{aligned}
& \mathrm{L}_{c \theta}=\frac{u_{0}}{2 \pi} \int_{0}^{\ell} \\
& \cdot d z \int_{b}^{\Delta\left(1-\frac{z}{l}\right)+b} \frac{d r}{r}
\end{aligned}
$$

and finally

$$
\begin{aligned}
& L_{c \theta} \frac{u_{0} l}{2 \pi}\{-1 \\
& \left.+\left(1+\frac{b}{\Delta}\right) \ln \left(1+\frac{\Delta}{b}\right)\right\} .
\end{aligned}
$$

For $\lrcorner / h \ll I,(7)$ can be approximated by

$$
\omega_{c \theta}=\frac{\mu_{c} \Delta}{2 \pi} \frac{\ell}{2 b}\left(1-\frac{\Delta}{b}\right) \text {. }
$$

The last component of inductance that is needed is the inductance $L_{c z}$ of the coil associated with the $\mathrm{H}$, field. The derivation is simplitied by defining the following areas

$$
\begin{aligned}
& A_{v} \equiv \pi\left(b^{2}-a^{2}\right) \\
& A_{W} \equiv \pi\left(\bar{b}^{2}-b^{2}\right)
\end{aligned}
$$

so that

$$
A_{2}=A_{v}+A_{w}
$$

$$
A_{2} \equiv A_{2}+\left(\frac{\mu_{2}}{\mu_{0}}-1\right) A_{m}
$$

where $A$ is the cross-sectional area between the compression coil and the implosion coil surface of $r$ $=\overline{\mathrm{b}}$ (see Fig. 5.2-2(a)), and $\mathrm{A}_{\mathrm{m}}$ is the cross-sectional area of the magnetic material.

If $\mu_{2 .}>10 \mu_{11}$ then $A_{2} \gg A_{1}$. and therefore the $H$, lield for $r>\bar{b}$ is negligible. Inder this assumption the surface current $K$, in the azimuthal direction at $r$ $=\bar{b}$ is related on the axial component $k, b y$

$$
K_{g}=\frac{\theta \bar{b}}{\grave{l}} K_{2}
$$

where $"$ is the angle through which the conductors rotate from the front edge to the back edge of the coil. Since

$$
k_{z}=\frac{i}{2 \pi \bar{b}}
$$

then

$$
\mathrm{k}_{\theta}=\frac{\mathrm{Ni}}{\ell}
$$

where

$$
N \equiv \frac{\theta}{2 \pi} \text {. }
$$

From the boundary conditiun on $\mathrm{H}_{2}$ and $(15)$

$$
\mathrm{H}_{2}=\frac{N}{l}
$$

and the inductance becomes

$$
I_{z_{z}}=\frac{\mu_{0} N^{2} A_{1}}{\ell} \text {. }
$$

A similar but more involved derivation can be made which includes the fields in area $A_{2}$. The result is identical with (18) if $A_{1}$ is replaced by

$$
A_{1} \rightarrow A_{1}\left\{\frac{1}{1+\frac{A_{1}}{A_{2}^{*}}}\right\}
$$

For the SFTR design. (18) is sufficiently accurate. 
For the implosion model described in Sec. 4.3, the sistem inductance is divided into the source inductance $L_{\alpha}$ and the load inductance $L_{p}$. The load inductance is associated only with the field inside the vacuum wall. This is given by (Is) with $A_{1}$ replaced by $A_{1}$. Using (9) yields

$$
L_{p}(t)=\frac{\mu_{0} N^{2} \pi\left(b^{2}-a(t)^{2}\right)}{l} .
$$

The appropriate inductance to use as a base for normalizing is the vacuum inductance

$$
L_{0} \equiv \frac{\mu_{0} N^{2} \pi b^{2}}{l} .
$$

The source inductance $L$. includes all of the inductances not included in $(20)$

$$
I_{s}=L_{c s}+L_{\ell}+L_{c \theta}+L_{w z}
$$

where

$$
L_{w z} \equiv \frac{\mu_{0} N^{2} A_{W V}}{l}
$$

Since $s \ll \underline{b}$.

$$
L_{w Z} \simeq \frac{\mu_{0} N^{2} 2 \pi b s}{\ell}
$$

Substituting (5). (8). and (24) into (22) yields

$$
\begin{aligned}
L_{s} & =L_{c s}+\frac{u_{0} \Delta}{2 \pi}\left\{\ln \left(\frac{r_{c}}{\bar{b}}\right)\right. \\
& \left.+\frac{\Delta}{\Delta} \ln \left(\frac{r_{p}}{r_{c}}\right)+\frac{\ell}{2 b}\left(1-\frac{\Delta}{b}\right)\right\} \\
& +\frac{\mu_{Q} N^{2}}{\ell} \frac{2 \pi b s}{\ell} .
\end{aligned}
$$

Define $\Lambda_{\text {, }}$ as L, normalized to $L_{0,1}$ [see (21)]

$$
\begin{aligned}
\Lambda_{s} & =\frac{L_{c s}}{L_{o}}+\frac{\Delta \ell}{2 \pi^{2} N^{2} b^{2}}\left\{\ln \left(\frac{r_{C}}{\bar{b}}\right)\right. \\
& \left.+\frac{\pi}{\Delta} \ln \left(\frac{r_{P}}{r_{c}}\right)+\frac{\ell}{2 b}\left(1-\frac{\Delta}{b}\right)\right\}+\frac{2 s}{b} .
\end{aligned}
$$

l'sing $(2)$ and SFTK dimentsions yields

$$
\Lambda_{s}=\left(\frac{1}{10 . i}\right)^{2}\left[2+\Delta\left[2.5-\frac{\Lambda}{10}\right] !+0.14\right.
$$

where $A$ is in centimeters. The insulation thickness $\triangle$ is controlled by the dielectric strength En of the ceramic | see (1)| and the voltage V'., used to drive the coil

$$
\Delta=\frac{V_{o}}{E_{D}} .
$$

The relation hetween $V_{1}$ and the required $F_{\text {" }}$ is derived in sec. 4.3. For a given F... $V_{.,}$can be minimized by chousing the value of $X$ which properly matches the circuit to the plasma.

\subsubsection{Electrical Iescription of the Compres- sion crill}

The electrical design of the compression coil is dictated by the required magnetic field st rength as a function of time, the present limitations in the superconducting technology of magnetic energy slorage. and finally by various const raints on the coil dimellsions. The peak magnetic lield is $5.5 \%$ and the rise time for the field is $0.7 \mathrm{~ms}$. At peak field the compression coils are crowbarred and the time constant of the field decay is $250 \mathrm{~ms}$. Both the peak field and decay time are specified by the plasma heating and burn time requirements. The rise lime is specified by the decay time of the magnetic field produced by the shock heating coils and the need for the magnetic field of the compression coils to pick up the plasma confinement before the field of the shock heating coils decayss too much.

The limitations on conductor current and voltage imposed by present-day superconducting technology are uncertain, but for design purposes a peak current of $25 \mathrm{kA}$ and a peak voltage of $60 \mathrm{kV}$ have been selected. Beyond this, the technological problems associated with the magnetic energy storage system powering each compression coil become more difficult. The inside radius of the compression coil must be at least $17 \mathrm{~cm}$ to enclose the discharge tube. shock heating coils, etc. Parallel plate transmission lines feeding the shock heating coils are brought in every $40 \mathrm{~cm}$ along the theta-pinch's major circumference. Midway between entry points for the shock heating feeds, a 2-cm-long access space to the discharge tube must be left open for either vacuum pumping ports or diagnostic ports. Consequently, individual compression coils must fit within the $14.75-\mathrm{cm}$ length between the shock heating feeds and the vacuum 
pumping ports. The compression coils may extend radially to the distance required to contain the windings of the coil and its insulation. Enough copper must be included to give the compression coils a decay time of $270 \mathrm{~ms}$ since the bus bar and crowbar resistance will bring this down to $250 \mathrm{~ms}$.

All of the above electrical requirements can be met by compression coil modules with an inside radius of $17 \mathrm{~cm}$, an outside radius of $27.9 \mathrm{~cm}$, and a length of $14.75 \mathrm{~cm}$. These dimensions include insula$t i c n$ and are also average dimensions since the $t=1$ and $t=0$ modulations will cause small nodifications of the radial dimensions. Figures 5.1.2 and 5.2.1 show these coils and their locations around the implosion coil. Each module provides field for $20 \mathrm{~cm}$ along the theta-pinch's major circumference. Each coil is wound with 36 turns of flat copper strap 0.38 $\mathrm{cm}$ thick. No further subdivision and transposition of the conductor are needed. although a single sub. division and transposition could be done with very little additional cost.

\subsection{Current Feedplates and Structural Sup-} ports

Around the 81.48-m-diam torus, 640 machine modules are positioned. Each module is $40 \mathrm{~cm}$ wide and is: composed of two $20-\mathrm{cm}$-wide implosionheating coils positioned back-to-back with a common collector plate feed in the center of each module. The compression coil and magnetic material surround each implosion-heating coil. The collector plate has an inside diameter of $66 \mathrm{~cm}$ where the implosion-heating coil is attached and an outside diameter of $166 \mathrm{~cm}$ where the implosion-heating capacitor and rail-gap assemblies and crowbar railgap assemblies are at tached. Two implesion-heating capacitor assemblies are installed side by side as shown in Fig. 5.2-1. Four additional assemblies are installed in pairs. each $\pm 120^{\circ}$ from the first set of implosion-heat ing capacitor assemblies, as shown in Fig. 5.1-4. Three implosion-heating crowbar gaps are installed, each between a pair of implosion-heating capacitor assemblies. Space at the bottom of the collector plate is reserved for diagnostic access. the vacuum system. and the METS parallel plate compression coil leads.

Each of the implosion-heating capacitor assemblies and crowbar gaps is designed to plug into the outside diameter of the collector plate with an air connection to hold off the $270-\mathrm{kV}$ system voltage. The electrical connections between the collector plates and the implosion-herting coil are also made in a similar geometry. The dielectric medium bet ween aluminum conductors in the collector-plate region will be polyethylene.
The structural supports for the implosion-heating coil and compression coil systems are an integral part of the structural platforms in the main cell area. Each of the coil systems is supported independently from a central frame geometry (as in Figs. 5.1-3 and 5.1-4) located between and below adjacent machine modules. Structural cross members tie supports together het ween machine modules. Each support system can be adjusted independently for positioning and alignment. Rectangular 304 stainless-steel tubing will be used for the main structural material. Four modules will be supported with one span of structure and will weigh approximately $10000 \mathrm{lb}$. Part of this structure will remain permanently attached to the four modules for installation and removal.

Even though the implosion-heating capacitor assemblies and crowbar-gap assemblies are supported from the outside diameter of the collector plates, secondary support members to each of these assemblies from the structural plat forms ahove and below the machine will be required.

\subsubsection{Thermal Analysis of Containment System}

As can be seen ir. Fig. 5.1.2. the first wall of the SFTK is formed from a $0.1-\mathrm{m}$-radius. 4.0 - $1 \mathrm{~nm}$-thick alumina $\left(\mathrm{Al}_{2} \mathrm{O}_{3}\right)$ tube. During a D.T burn cycle a flux of bremsstrahlung, energetic D-T ions, alpha particles, fusion neutrons, and gamma rays will be incident on the first wall. Since the neutron and gamma energy densities within the first wall material are less than $1 \%$ that of the plasma. bremsstrahlung, and alpha particles, the heattransfer analysis reduces simply to one described by a time-dependent wall flux. The penet rability of the bremsstrahlung radiation into the first wall is on the order of $0.1 \mathrm{~mm}$ for $\mathrm{Al}_{2} \mathrm{O}_{3}$. and is less than the thermal diffusion distance for time scales equal to the hurn time $11.0 \mathrm{~mm}$ at $50 \mathrm{~ms}$ for $\mathrm{Al}, \mathrm{O} ; 1$. The bremsstrahlung source term, therefose. is also treated analytically as a heat flux rather than a volumetric heat-source term.

In view of the small ratio of wall thickness $\Delta b$ to inner radius $b$ of the first wall, the heat-transfer analysis uses a one-dimensional slab approximation. During the early stages of the burn a semi-infinite slab approximation is adequate (for times less than $0.010 \mathrm{~s}$, the diffusion dist ance is $\sim 0.4 \mathrm{~mm}$. compared to $\lrcorner b=4 \mathrm{~mm}$ ). For this case the temperature rise $T(x, t)$ at a distance $x$ from the inner radius $b$ at time $t$ is given for a constant wall flux $F_{0}$ by ${ }^{1}$ 


$$
\begin{gathered}
\mathrm{T}_{(x, t)}=\frac{2 E}{\mathrm{k}}\left[\sqrt{\frac{k t}{\pi} e^{-x^{2} / 4 k t}}\right. \\
\left.+\frac{x}{2} \operatorname{sic}(x / \sqrt{4 k t})\right] .
\end{gathered}
$$

The increase in the surface temperature is given by

$$
T_{(o, t)}=\frac{2 F_{o}}{k} \sqrt{\frac{K E}{\pi}}
$$

where $\mathrm{k}$ is the thermal conductivity and $\mathrm{k}$ is the thermal diffusivity. Tabir $5.2-1$ summarizes relevant thermal/mechanical properties of dense $\mathrm{Al}_{2} \mathrm{O}_{3}$.

The average heat flux $F_{0}$ is assumed to occur for a time $\tau$ and is estimated as follows. If $Q$ is the ratio of fusion neutron energy ( $20 \mathrm{MeV} / \mathrm{n}$ ) to plasma internal energy $3 / 2 \mathrm{nkT}$. and $f_{\mathrm{Bk}}$ is the fraction of the plasma internal energy emitted as bremsst rahlung radiation during the effective burn time $\tau$, then

$$
\begin{aligned}
& F_{0}\left(W / m^{2}\right)=3 n_{0} \\
& \cdot k T\left[1+0.175 Q+e_{B R}\right] \frac{b}{2 \tau} .
\end{aligned}
$$

The terms in the bracket correspond, respectively. to the plasma internal energy, :he alpha-particle energy (3.5 MeV/alpha particle), and the bremsstrahlung energy contributions to $F_{n}$. The density $n_{1}$ corresponds to the initial filling density and is related to the average plasma density $n$ by $n_{1} x^{2}$. where $x$ is the average plasma-to-wall radius ratio. With temperature expressed in $\mathrm{keV}$ and $p_{0}$ (mtorr) equal to the initial filling pressure. Eq. (3i) becomes

$$
\begin{aligned}
& F_{0}\left(H / m^{2}\right)=1.70 \times 10^{4} T_{0}^{T} \\
& \left(1+0.175 Q+f_{B R}\right) \frac{b}{2 T} \\
& f_{B R}=0.0708 P_{0} \tau / x^{2} \sqrt{T} .
\end{aligned}
$$

For the typical SFTR conditions indicated in Table 5.2-2. $\mathrm{f}_{\mathrm{sk}}=0.15, \mathrm{~F}_{11}=0 ., 2 \mathrm{MW} / \mathrm{m}^{2}$. and $\mathrm{T}(0, \tau)=$ $16.4 \mathrm{~K}$. I sing the conditions given in 'Table 5.2 .2 .2 . the melting point of $\mathrm{Al}_{2} \mathrm{O}_{3}$ will be exceeded at the surface if $;$ is less than $1 \mu$ s when the only source of energy is that of the (5-keV) plasma. Under "normal $^{*}$ conditions, therefore. the first-wall temperature rise is modest from the viewpoint of phase change and chemical reaction. The question of thermal stress is addressed in the following sections.

Although simplistic. the above-described analytical estimate is useful for an order-ofmagnitude value of the first -wall temperature excursion: the major uncertainty in this estimate is the approximation of a constant first-wall heat tlux. In order to assess the thermal behavior of the SFTR first wall for a given dynamic burn case, the finiteelement code $A Y E R^{2}$ was programmed to follow the thermal excursion as driven by first-wall energy fluxes given by the DTBIRN thermonuclear burn code. ${ }^{3}$

Figure 5.2-5 gives the bremsstrahlung, alphaparticle, and plasma (ion/electron) first-wall energy fluxes as a function of time for a "typical" SFTR burn: the bremsstrahlung was treated as a wall flux rather than a volumetric heat source, and the plasma flux at the first wall was assumed to follow the alpha-particle flux, a classical diffusion model being used to describe the latter. ${ }^{4}$ Neutron and gamma heating were treated as a volumetric heat source, but contribute insignilicantly to the induced temperature rise. These approximations will change

TABLE 5.2-1

PHYSICAL PROPERTIES FOR (DENSE) $\mathrm{Al}_{2} \mathrm{O}, \mathrm{AT} 300 \mathrm{~K}$

$c_{p}\left(\mathrm{~J} / \mathrm{k}_{\mathrm{p}} \mathrm{K}\right)$
$\mathrm{k}\left(\mathrm{J} / \mathrm{msK}_{\mathrm{K}}\right)$
$\rho\left(\mathrm{kg} / \mathrm{m}^{13}\right)$
$\kappa\left(\mathrm{m}^{2} / \mathrm{s}\right)$
$\alpha\left(\mathrm{K}^{-1}\right)$
$\mathrm{E}(\mathrm{Pa})$
$\hat{\sigma}(\mathrm{Pa})$
$\hat{\sigma}_{\mathrm{T}}(\mathrm{Pa})$
$\mathrm{T}_{\mathrm{MP}}(\mathrm{K})$
$\nu$

Specific heat

Thermal conductivity

Density

Thermal diffusivity

Coefficient of thermal expansion

Young's modulus

Ultimate compressive strength

Ultimate tensile strength

Melting point

Poisson's ratio
8.30

50.0

3800

$1.59 \times 10^{-5}$

$9.36 \times 10^{-6}$

$3.1 \times 10^{11}$

$7.0 \times 10^{8}$

$1.0 \times 10^{8}$

2323

0.3 
TABLE 5.2-2

\section{SFTR BURN PARAMETERS USED FOR THERMAL/MECHANICAL ANALYSES}

$\begin{array}{llc}\text { P., (mtorr }) & \text { Initial filling pressure } & 5 \\ b(\mathrm{~m}) & \text { First-wall radius } & 0.1 \\ Q & (20 \mathrm{MeV}) \text { neutron-to-plasma energy ratio } & 1.0 \\ r(\mathrm{~s}) & \text { Average burn time } & 0.050 \\ T(\mathrm{keV}) & \text { Mean plasma temperature } & 5.0 \\ \mathrm{X} & \text { Mean plasma-to-first wall radius ratio } & 0.4\end{array}$

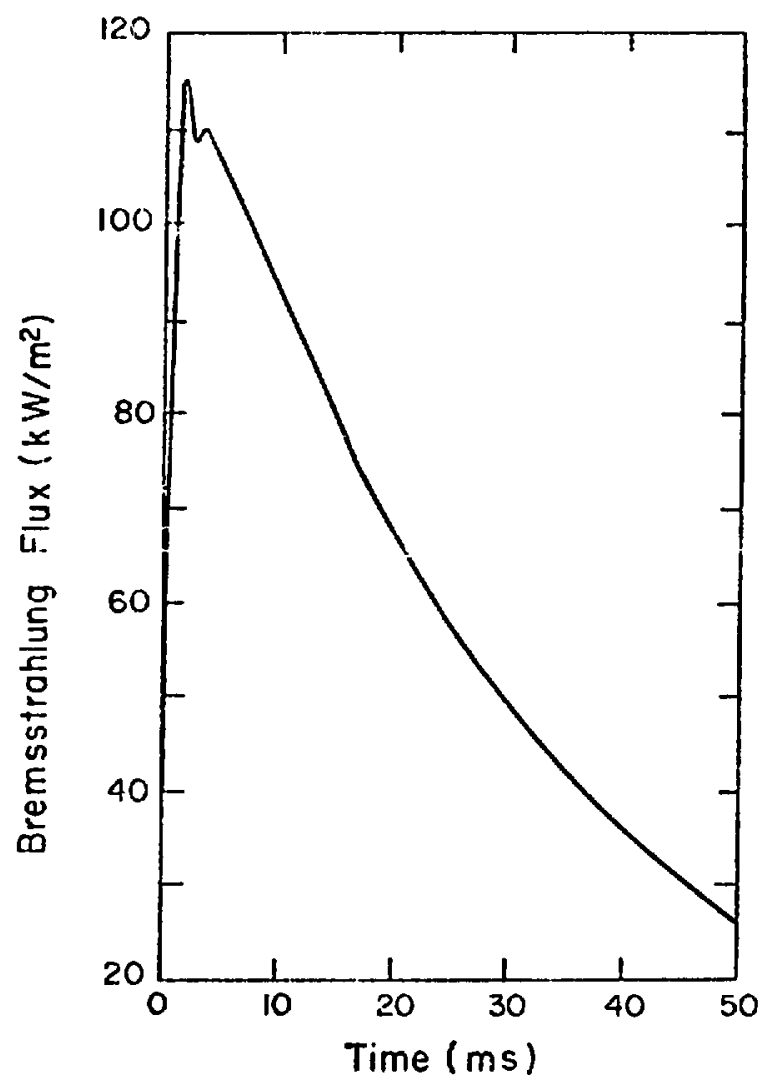

Fig. 5.2-5(a).

Bremsstrahlung energy flux at the first wall vs burn time.

by a factor of $\sim 2$ the time history and form of the computed first-wall temperature profiles, but should not significantly alter the overall temperature rise computed.

The time history of the first-wall surface temperature for these dynamic conditions is depicted in Fig. 5.2-6, whereas Fig. 5.2-7 gives the spatial dependence of the temperature vs time. The

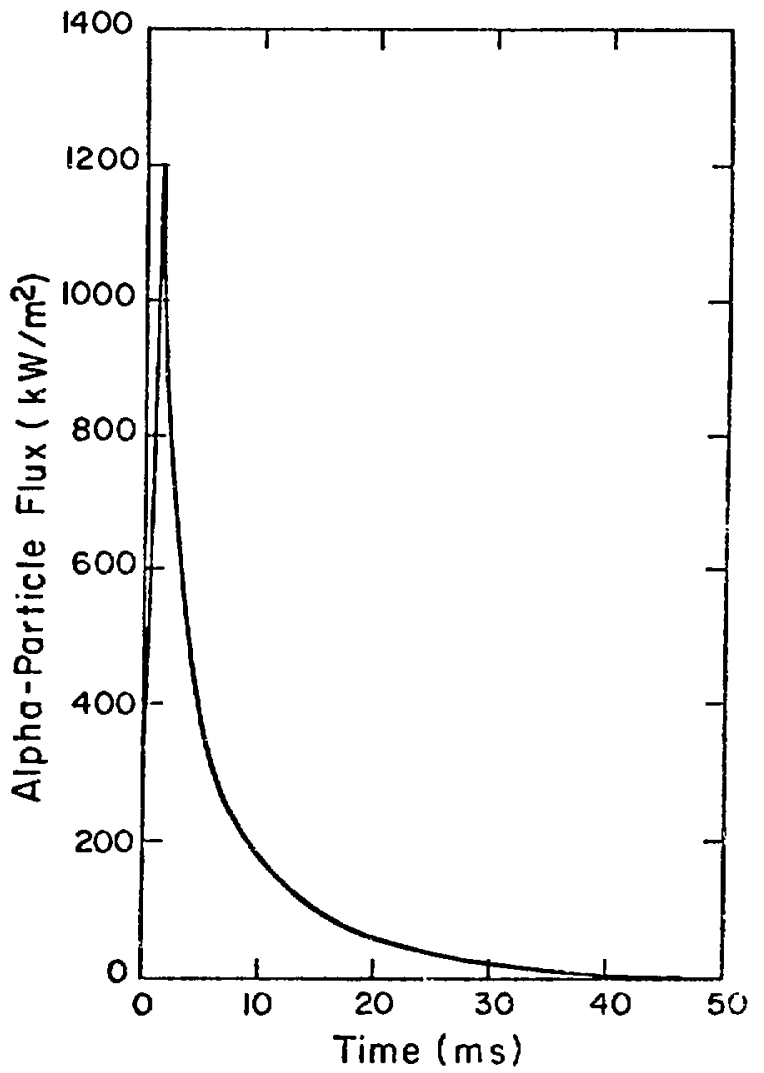

Fig. 5.2-5 (b).

Alpha-particle energy flux (3.5 MeV) at the first wall vs burn time.

maximum temperature rise is $28 \mathrm{~K}$ and occurs at 5 $\mathrm{ms}$ into the burn cycle. The temperature rise computed by AYER is a factor of $\sim 2$ above that predicted by the analytical model, and is a result of the higher total energy deposition predicted for the plasma conditions used in the AYER computation. Hence, with the range of uncertainties in both the heat-transfer and the D-T burn models and 


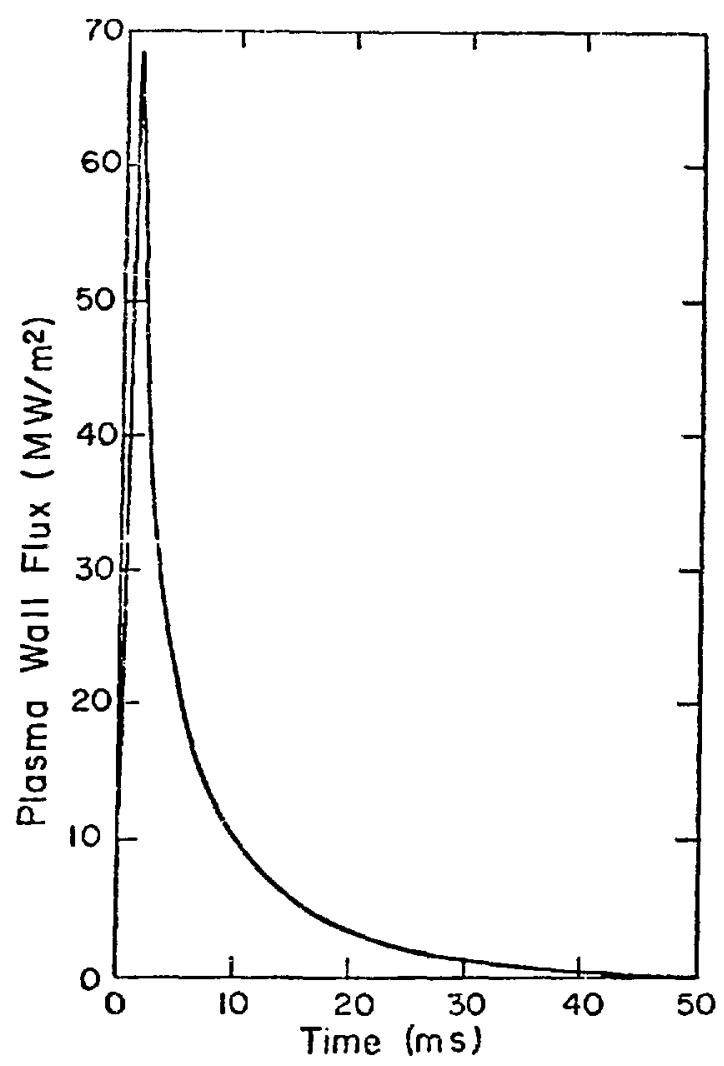

Fig. 5.2-5(c)

Plasma energy flux at the first wall ws burn lime for a $Q=1$ plasma.

parameters, a first-wall temperature rise of $\sim 10-30$ $\mathrm{K}$ is anticipated for the SFTR. This temperature increase is caused primarily by the plasma dump and will occur, therefore, for D-D as well as D-T experiments. On the basis of both the simple analytic model and computer computation, the thermal stresses in the $\mathrm{AlO}_{3}$ first wall will be estimated.

\subsubsection{Stress Analyses of Containment System}

The major mechanical stresses anticipated for the SFTR confinement system will be thermally driven stresses in the $\mathrm{Al}_{2} \mathrm{O}_{3}$ first wall and electromagnetically driven stresses occurring within the implosion coil and the adiabatic compression coil. Both analytical and computer estimates of these stresses are given below.

\subsection{Thermal Stresses within the First Wall}

An analytical estimate of the thermal stresses induced within the $\mathrm{Al}_{2} \mathrm{O}_{3}$ first wall can be obtained in

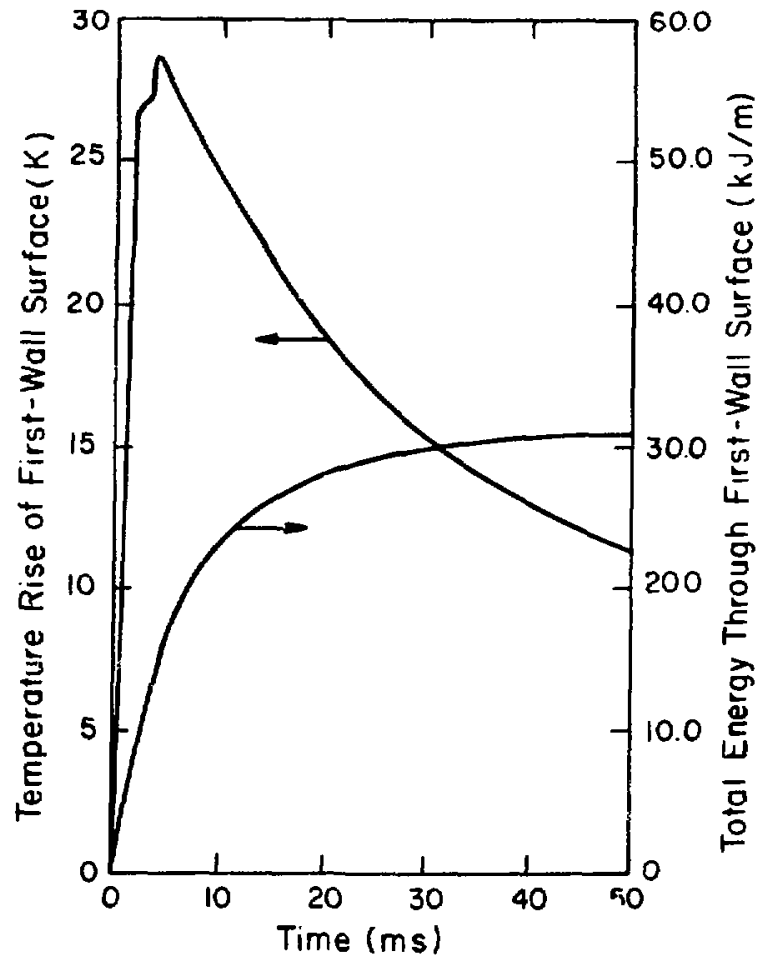

Fig. 5.2-6.

Time dependence of first-uall surface temperature and total energy deposited at the first-uall inner surface.

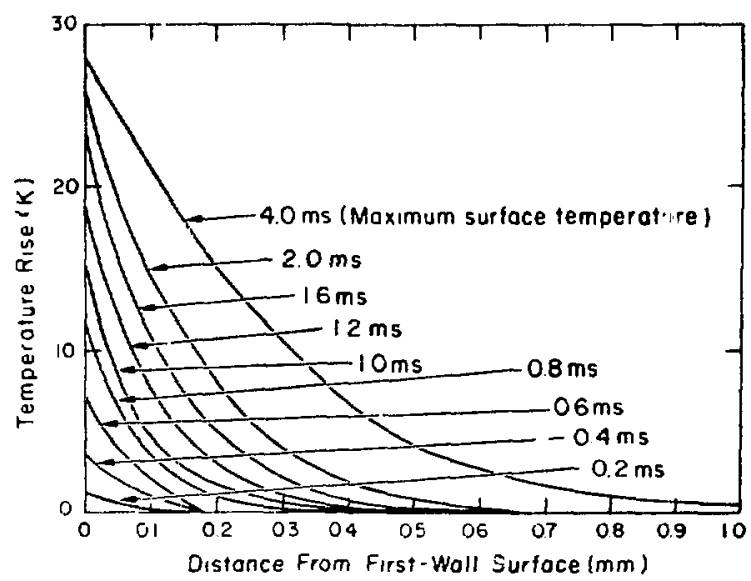

Fig. 5.2-7.

Time dependence of temperature profiles in the SFTR first wall for the conditions described in Table 5.2-1 and Fig. 5.2-5. 
cylindrical geometry by solving the following equations under the plane strain approximation ${ }^{5}$

$$
\begin{aligned}
& \frac{1}{r^{3}} \frac{d}{d r}\left(r^{3} \frac{d \sigma r r}{d r}\right)=-\frac{2 \alpha E}{1-v} \frac{1}{r}\left(\frac{d T}{d r}\right) \\
& \frac{d \sigma r}{d r}+\frac{1}{r}\left(\sigma_{r r}-\sigma_{\theta \theta}\right)=0 .
\end{aligned}
$$

The thermal expansivity is given by $\alpha$, whereas Young's modulus and Poisson's ratio are given by $\mathbf{E}$ and $\nu$, respectively (Table 5.2-1). The following approximation to the first-wall temperature distribution at any given time is lised

$$
T_{(r)}=T_{0} e^{-\lambda(r-b)}
$$

$T_{1}$ is the temperature of the inner surface and $1 / \lambda$ is a thermal attenuation distance, assumed here to be much shorter than the inner radius $b$. More realistic computer calculations verify this general behavior for small times (Fig. 5.2-7).

Using these approximations, the radial and azimuthal (hoop) stresses are given by

$$
\begin{aligned}
& { }_{r r} / \sigma_{0}=\left[\frac{e^{-\lambda(r-b)}}{\lambda r}-\frac{1}{\lambda b}\right] \\
& +\left[\frac{e^{-\lambda(r-b)}}{(\lambda r)^{2}}-\frac{1}{(\lambda b)^{2}}\right] \\
& +\frac{c_{1} \lambda^{2}}{2}\left[\frac{1}{(\lambda r)^{2}}-\frac{1}{(\lambda b)^{2}}\right] \\
& \sigma_{\theta \theta} / \sigma_{0}=-e^{-\lambda(r-b)}\left[1+\frac{1}{\lambda r}+\frac{1}{(\lambda r)^{2}}\right] \\
& -\left[\frac{1}{\left.(\lambda b)^{2}+\frac{1}{(\lambda b)}\right]}\right. \\
& -\frac{c_{1} \lambda^{2}}{2}\left[\frac{1}{(\lambda r)^{2}}+\frac{1}{(\lambda b)^{2}}\right]
\end{aligned}
$$

where the constants $C_{1}$ and $\sigma_{0}$ are given by

$$
\begin{aligned}
& \frac{c_{1} \lambda^{2}}{2}= \\
& \frac{e^{-\lambda \Delta b}[\lambda b(1+o)+1]-(1+\lambda b)(1+p)^{2}}{\rho(2+\rho)} \\
& \sigma_{0}=2 \alpha E T_{0} /(1-v)
\end{aligned}
$$

and $\rho$ has been defined as the ratio of wall thickness Ib to the inner radius $b$. The radial stresses $\sigma_{\mathrm{rr}}$ have been tssumed to be zero at the inner and outer radii, $h$ and $b+j b$, respectively. For the case where $\rho \ll 1$ (thin si:ell) and $\lambda \gg b, \Delta b$ (true for most of the burn time f. the hoop stress is given by

$$
\sigma_{\theta \theta}(r) \simeq-\sigma_{0} e^{-\lambda(r-b)}
$$

and is maximum in compression at the inner radius of the first wall. For the thermal conditions given by the analytic model in Sec. 5.2.2.2 and Tables 5.2-1 and $5.2-2$, the maximum stress predicted by this approximate mode! amounts to $86 \mathrm{MPa}(1 \cdot 2.6 \mathrm{kpsi})$ The ultimate compression strength of $\mathrm{Al}_{2} \mathrm{O}_{3}$ is 700 $\mathrm{MPa}$ and is achieved for the same thermal loading. $F_{11} T\left(1 / 1 / \mathrm{m}^{2}\right)$, given in Sec. 5.2.2.2 when $\mathrm{t}$ falls below $0.8 \mathrm{~ms}$.

The simple analytical treatment given above is probably accurate to within a factor of 2 . The major thermal problem appears to be related to mechanical stress rather than phase change or chemical reactions. The stress scales directly with temperature rise $T_{n}$, which in turn scales more or less directly with the burn time $\tau$. These problems can be ameliorated for $\mathrm{Q} Q=1$ plasma only by extending the time during which the plasma dump occurs. To within 10-20 $r$, these conclusions are independent of whether or not thermonuclear burn occurs for the $\mathbf{Q}=1$ plasma described in Table 5.2-2.

To model more accurately the two-dimensional nature of the thermal-stress problem associated with the $\mathrm{Al}_{2} \mathrm{O}_{3}$ SFTR first wall, a finite element, thinshell code, RASH, ${ }^{6}$ was used ${ }^{7}$ in conjunction with the temperature profiles depicted in Fig. 5.2-7. The structural properties given in Table 5.2-1 were used. and Figure 5.2-8 gives the geometric model used in the 2-D stress calculation. It should be noted that the neutral axis $\xi$ varies in direction within regions (1) and (2). 


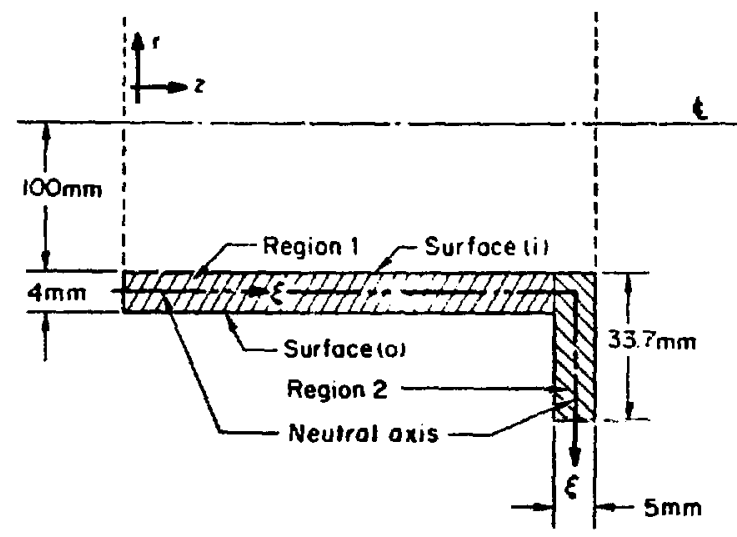

Fig. 5.2-8.

SFTR $\left(\mathrm{Al}_{2} \mathrm{O}_{3}\right)$ first-wall model used for thermal stress analysis.

Figure 5.2-9 gives the time dependence of the maximum tensile and compression (hoop) $\sigma_{\theta \theta}$ stress, and Fig. 5.2-10 gives the spatial distribution of the (longitudinal) $\sigma_{z z}$ and (hoop) $\sigma_{\theta y}$ stresses for the time

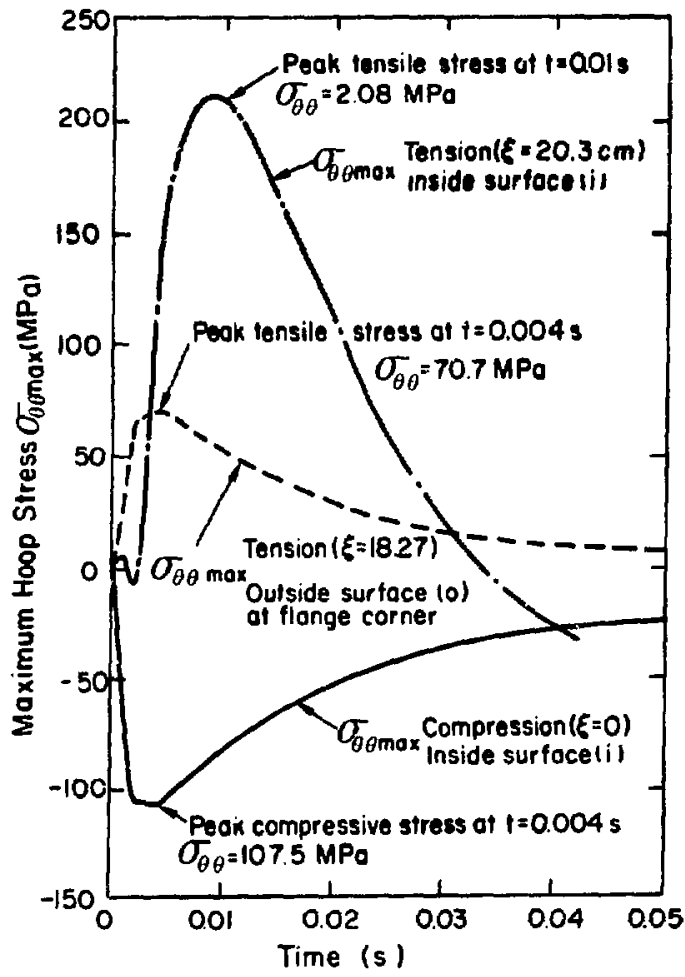

Fig. 5.2-9.

Maximum hoop stress quasi-steady thermal transient.

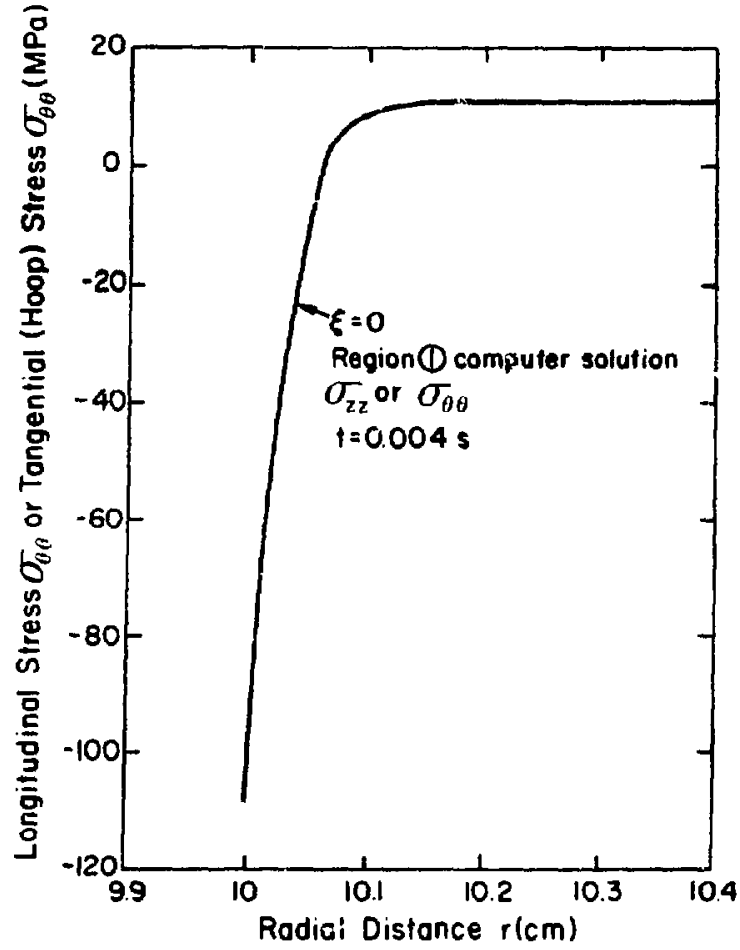

Fig. 5.2-10(a).

Computer solution radial distribution of longitudinal stress or hoop stresses at the transierse plane farthest from the end flange at time of maximum compressive stress.

(4-5 ms) when these stresses are maximum. The (radial) $\sigma_{\mathrm{rr}}$ stresses are not computed by RASH because this code is restricted by thin-shell theory. Because of the finite-difference nature of RASH, stress discontinuities exist at the common plane between regions (1) and (2); although stress gradients are steep in this region, the stress trajectories should be continuous in the actual situation. A large tensile hoop stress is predicted in the region of the flange on surface $(0)$, and this stress amounts to $200 \mathrm{MPa}$. This stress exceeds the tensile strength (Table 5.2-1) and can be reduced by rounding both inside and outside corners of the joint between regions (1) and (2). The degree of rounding and the effectiveness of rounded edges on stress reduction in the vicinity of the joint can be determined only by more detailed, finite-element computations. The maximum compressive stress is $107 \mathrm{MPa}$ and is within a factor of 7 of the ultimate compressive strength of $\mathrm{Al}_{2} \mathrm{O}_{3}$. 


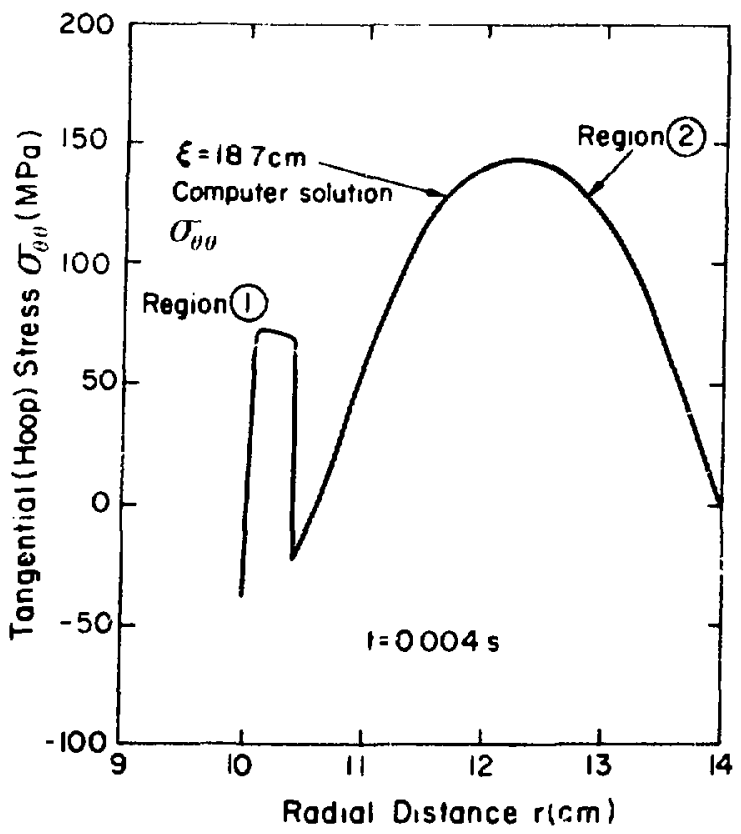

Fig. 5.2-lo(b).

('omputer solution radial distribution of hoop stress at the flanged end of the cylinder at time of maximum compressice stress.

The crucial problem with the $\mathrm{Al}_{2} \mathrm{O}_{3}$ first-wall structure, therefore, appears to be one of tensile failure driven by steep thermal gradients. The severity of this problem depends not only on the mode of SFTR operation and the details of the firstwall design, but also on the quality of the ceramic used to fabricate the first wall. ${ }^{8}$ Given a highquality, fine-grained ceramic, the primary concern is the presence of "stress-rising" flaws, which can have a stress-concentration factor of $\sim 30$. The following steps are suggested to reduce the failure via stressrising flaws: ${ }^{8}$

- good surface finish (i.e., 325-grit diamond paste for final grinding).

- avoid surface damage to ceramic during fabrication and installation.

- avoid introduction of parasitic stresses during assembly.

- reduce moisture in the atmosphere around installed ceramic components to avoid chemical effects at int rinsic crack tips.

- institute a thorough system of nondestructive inspection (x-radiography) and proof testing (gas pressurization).

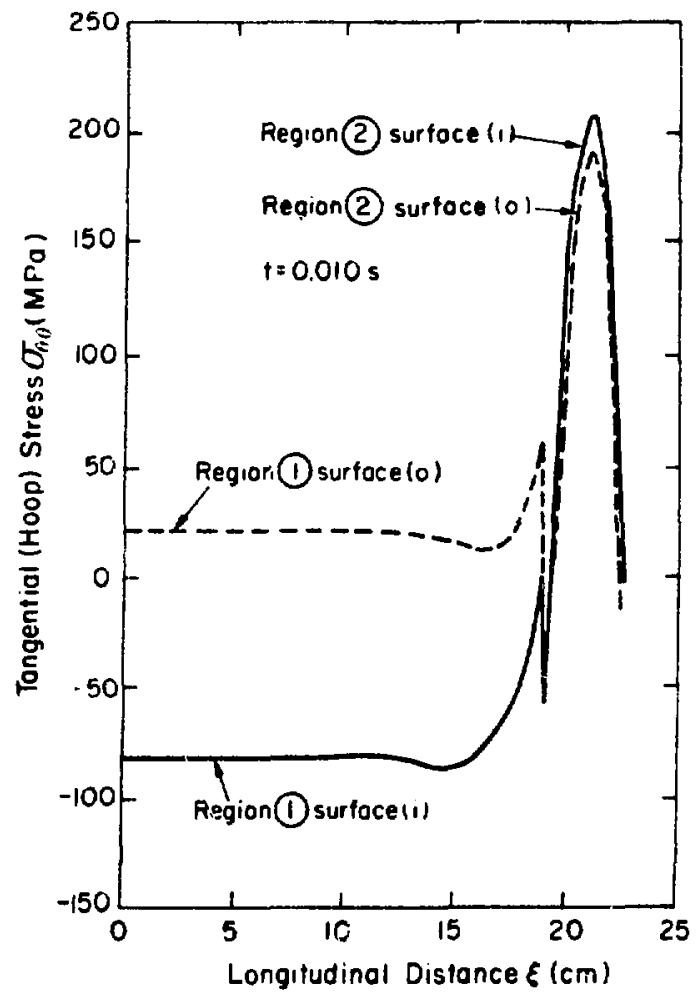

Fig. 5.2-10(c).

C'umputer solution longitudinal distribution of hoop stress at time of maximum stress.

\subsection{Mechanical Stresses in the Implosion} and Compression Coils

Both the implosion-heating coil (IHC) and the adiabatic compression coil (ACC) will be subjected to elertromagnetic force fields which are complex in both space and time. This complexity, coupled with the composite construction (insulators, metallic conductor. support structurel and the complicated geometry (short and stubby cylinder, $l=1,0$ asymmetries, feedplate restraints, etc.) makes a purely analytic solution to the mechanical stress problem virtually impossible. The added complexity associated with the dynamic loading of both the coils and the support structure necessitates multidimensional. time-dependent computer calculations.

The approach used here evokes static, elastic, plane-strain theory for a preliminary estimate of mechanical loading. Separate, multidimensional computer calculations are used to model specific aspects of the problem that may appear crucial. 
Representing either the IHC or the Al' as a thick-walled reflinder, and using the plane-strain theory approximately. the radial and azimulal stress, or and rim. respectively, are given beluwi for an internal pressure loading equal to $\mathrm{B}^{2} \cdot \vartheta_{\mu} .$.

$$
\begin{aligned}
& \sigma_{r r}=-\frac{E^{2}}{2 \mu_{0}} \frac{(1+\rho)^{2}}{\rho(2+\rho)}(b / r)^{2}-1 /(1+\rho)^{2} \\
& \sigma_{\theta \theta}=\frac{B^{2}}{2 \mu_{0}} \frac{(1+\rho)^{2}}{\rho(2+\rho)}(b / r)^{2}+1 /(1+\rho)^{2}
\end{aligned}
$$

where $y=3 b / b$. The maximum stresinecours in tension tior

$$
\sigma_{\theta \theta}=\frac{B^{2}}{2 \mu_{0}} \frac{(1+\rho)^{2}+1}{\rho(2+\rho)} .
$$

For the IHC and $A C C$. respectively, $b=0.1 .0 .18 \mathrm{~m}$ : $\Delta b=0.00 .0 .08 \mathrm{~m}: B=1.0 .6 .0 \mathrm{~T}$. and $\mathrm{B}^{2}: 2_{\mu_{1},}=0.80$. 28.7 MPa. For these desinn conditions, $\sigma_{m}$ IH $\left.\mathrm{IH}^{\prime}\right)=$

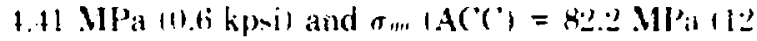
kpsil. These preliminary estimates. therefore. in. dicate no severe mechanicai problems with either the IHC or ACC. The unique grometry dynamic loading. and close design tolerances associated with the IHC however, warrant a more detailed examination of this crucial component, particularly since the ceramic support structure for the IHC will undoubtedly be placed in tension and may be required to support the mechanical load. The results of a twodimensional, tinite-element stress calculation of the IHC are summarized below.

The computer code TSAAS $^{9}$ was used ${ }^{10}$ to estimate the static stresses in the implosion coil shown in Fig. 5.2.4. The finite-element mesh. material boundaries, and static loadings are shown in Fig. 5.2-11; the overall dimensions of this mesh are roughly scaled from Fig. 5.2-4, and most dimensions and blend radii should be considered approximate. The inaterial properties given in Table 5.2-1 were used, and $100000 \mathrm{MPa}$ was used for the Young's modulus of copper.

To simulate the (static) electromagnetic loading. the pressure conditions were applied at the copper/insulator $\left(\mathrm{Al}_{2} \mathrm{O}_{3}\right)$ interface over the straight boundary regions plus one element into the various radii. The pressures were computed as a function of average radius $\mathrm{r}(\mathrm{mm})$ at the $\mathrm{Cu} / \mathrm{Al}_{2} \mathrm{O}_{33}$ interface by the following expressions

$$
\begin{aligned}
& H_{3}=P, 1: 1-110111, \\
& r_{1}=l \cdot 11-1+f(n) r^{2}-1 \\
& \left.P_{1}=P_{1},(1)(x) / \mathrm{r}^{\prime \prime}\right)
\end{aligned}
$$

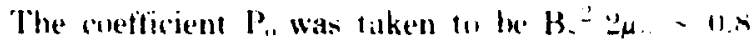

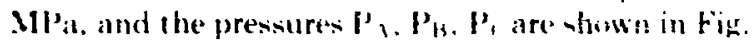
5.2.11. The results presented nere are lor a "champed" ar gero displanement benundary al the muter edine of the feedplate region tFig. 5.2.111. A calculation which restraned onty axial motion at the muter bundarien resulted in substantially the same defurmations and stressis.

Figure 5.2-1:" gives the ratial and axial displace. ment contosurs, and the circumferential (how)) $\sigma_{\text {tet }}$ stress ontours are given in Fig. 5.2-1:3. The maxima of the principal $\left|\sigma_{r r} . \sigma_{i \cdots}, \sigma_{r^{\prime \prime}}\right|$ stresies are shown in contour on Fig. \$.2-lt. The maximum tensile stresses occur at the tip of the IHC llarthest point axially from teedplate and are howp stresses of b.8 MPa in the insulating coil torn and $7.2 \mathrm{MPa}$ in the insilation which surrounds the copper conductor. The st resses fall to very low levels around and within the feedplate region. Although placing a brittle ceramic in tension should be avoided. the low levels of the tensile loading relative whe ultimate tensile

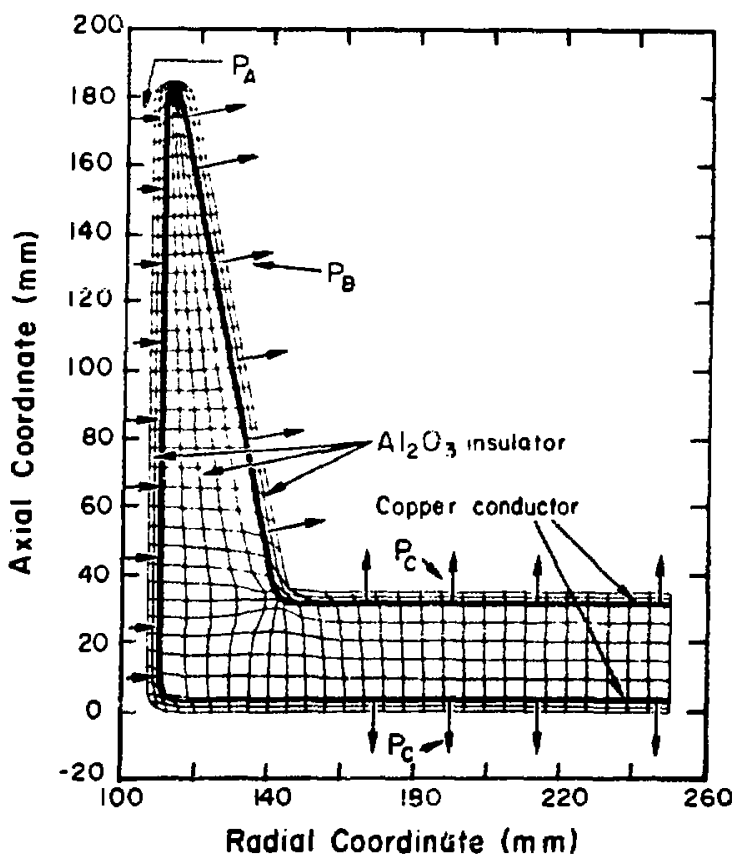

Fig. 5.2-11.

Finite-element mesh, material bourdaries, and static loadings used to model static stresses in the $I H C$. 


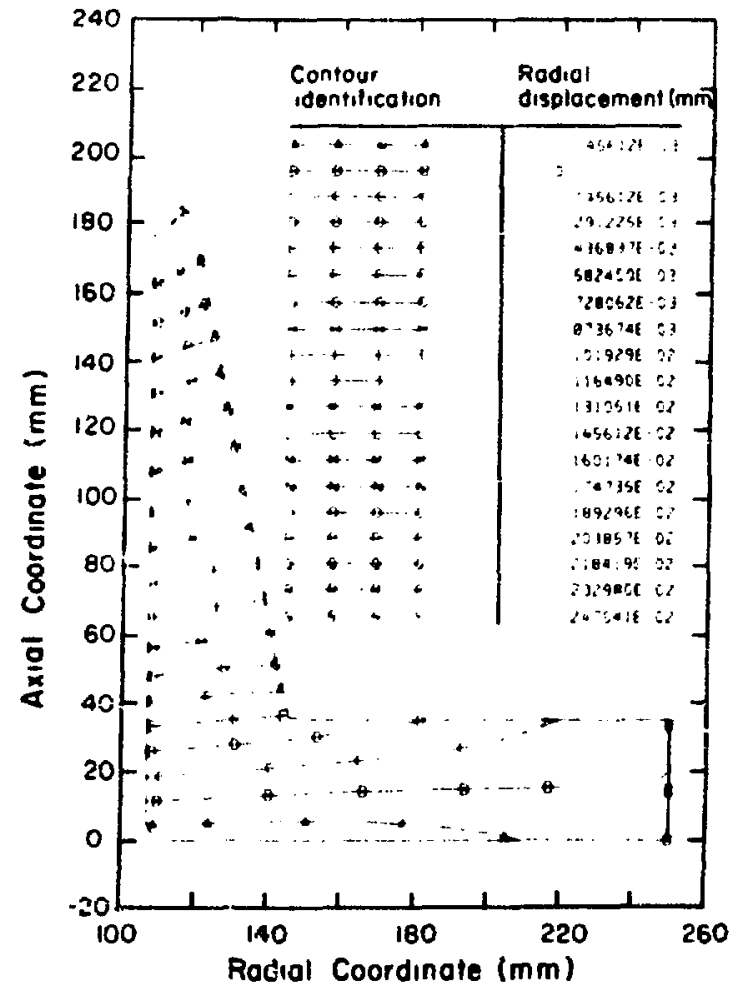

Fig. 5.2-l2 $(a)$.

Radial displacement contrurs for the stutic lesadings gicen by Eq. (45).

stress $(\sim 100 \mathrm{MPa})$ should present no serious problems: even with "stress-riser" amplification by local defects of $\sim 30$, the ultimate tensile stress should not be exceeded.

The finite-element mesh depicted in Fig. 5.2-11 and the material properties given in Table $5.2-1$ were used in conjunction with an approximation to the field waveform shown in Fig. 4-16 for a dynamic analysis of the implosion-heating roil. The computer program NONSAP ${ }^{11}$ was used and the magnetic field waveform was approximated by: (i) a linear rise from zero to the maximum value in $0.2 \mu \mathrm{s}$. (ii) a maximum field for $0.1 \mathrm{us}$. and (iii) a linear decrease to half the maximum in $0.05 \mu \mathrm{s}$ followed by a constant (one-half maximum) field. A static analysis with NONSAP verified to within $3^{\mathrm{C}}{ }^{*}$ the previous calculations made with TSAAS.

The eigenvalue option in NONSAP was used to obtain the first five modal shapes and resonant frequencies for the IHC system. Table 5.2-3 sum-

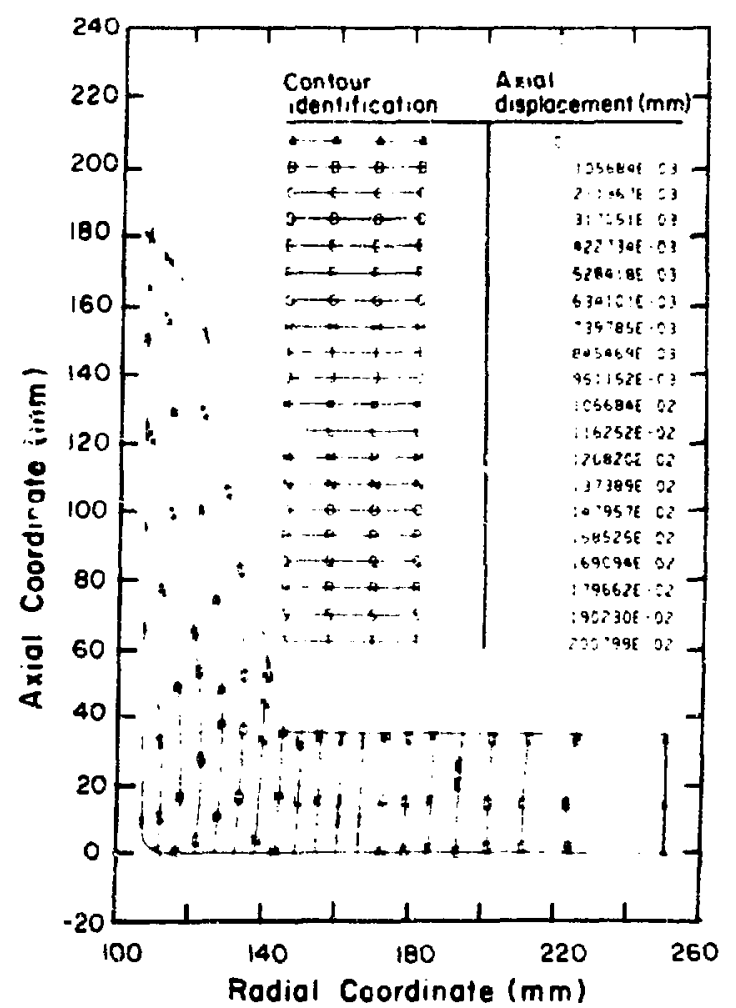

Fig. 5.2-12(b).

Axial displacement contours for the static loadings given by Eq. (45).

marizes the resonant frequencies. and indicates that a structurally significant response would probably take many tens of microseconds. The calculational

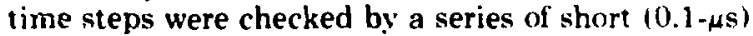
time steps to test for a tendency of acoustic waves to interact and to build up large stresses: longer time sleps $(1.2 \mu \mathrm{s})$ were used to examine the long-term structural response.

The largest tensile stresses were computed at the small tip of the IHC and are summarized in Table 5.2-4. The response for the (tip) element of maximum hoop stress is nearly sinusoicial with a period of $\sim 90 \mu \mathrm{s}$ and a peak stress of $\sim 7.8 \mathrm{MPa}$ (i.e., slight ly twice the static stress with the same magnitude of pressure). Most of the deformation occurs in one mode, so the system response is nearly sinusoidal. The peak stresses from the dynamic calculation, therefore, are essentially the same as for the static calculation, and the conclusions are the same. 


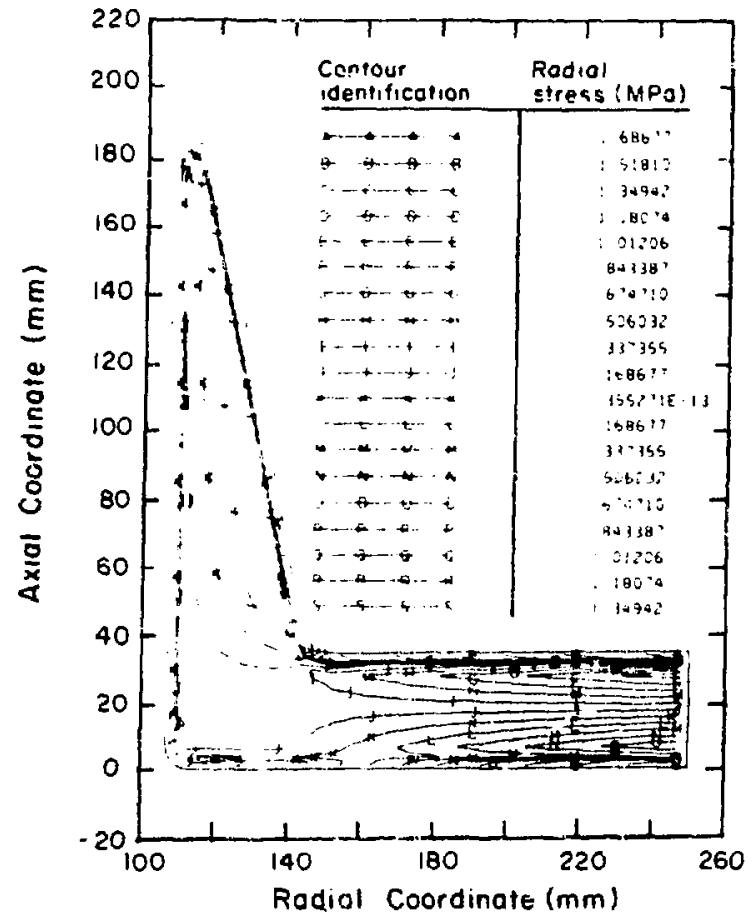

Fig. 5.2-13(a).

Radial stress contours for the static loading given by $E q$. (45).

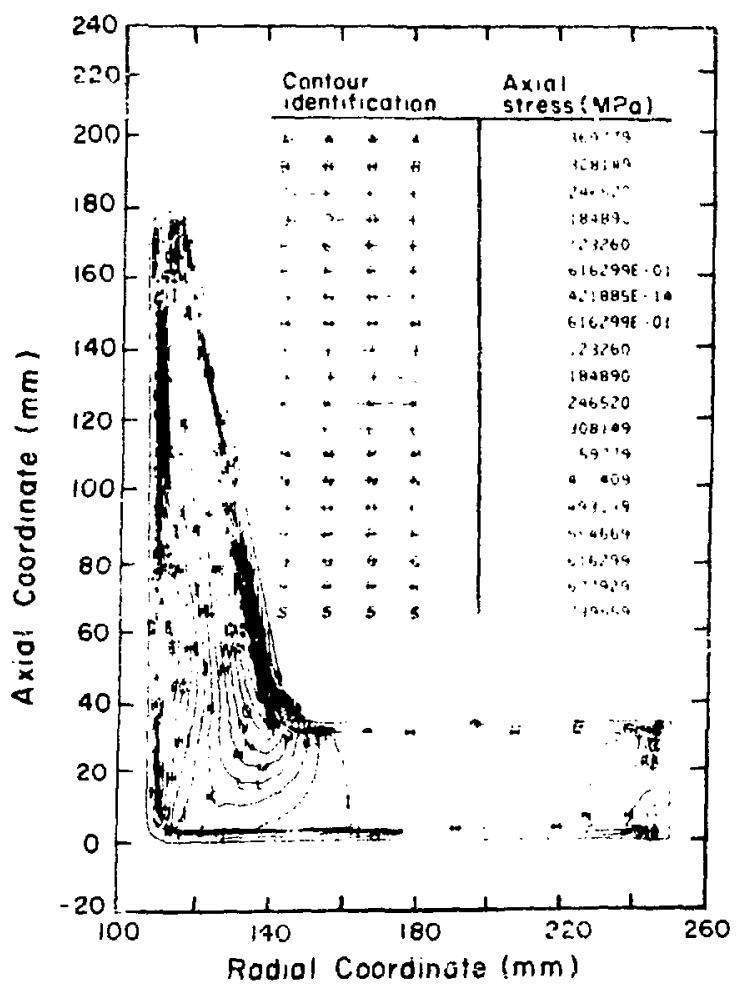

Fig. 5.2-1:3(b).

Axial stress contours for the static loading given by Eq. (45). 


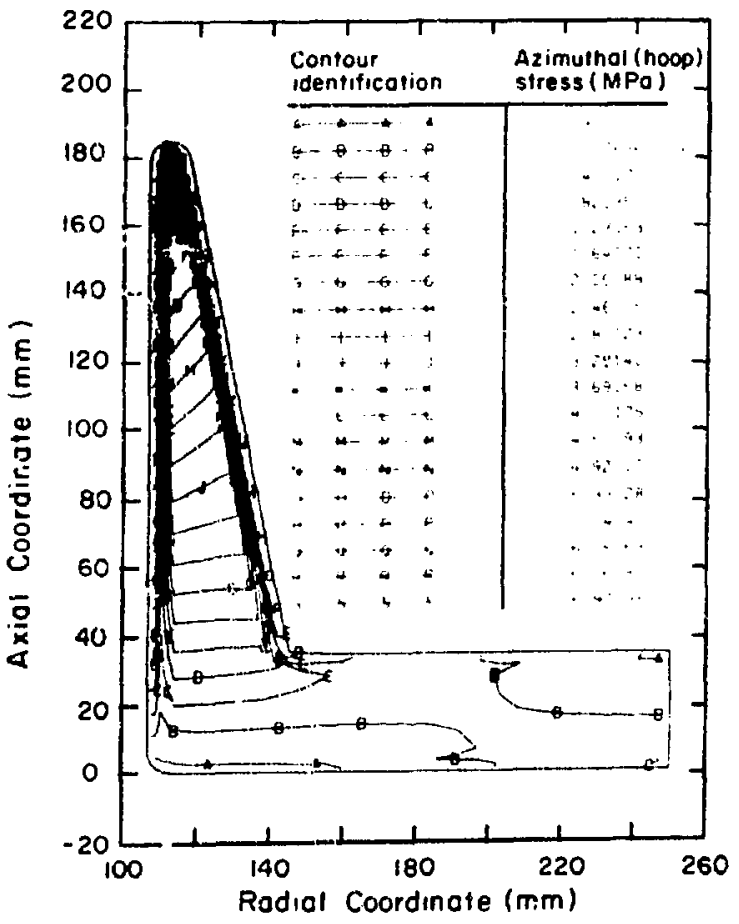

Fig. 5.2-1:3(c).

Azimuthal (hoop) stress contours for the static loading given iv $F_{q}$. (45).

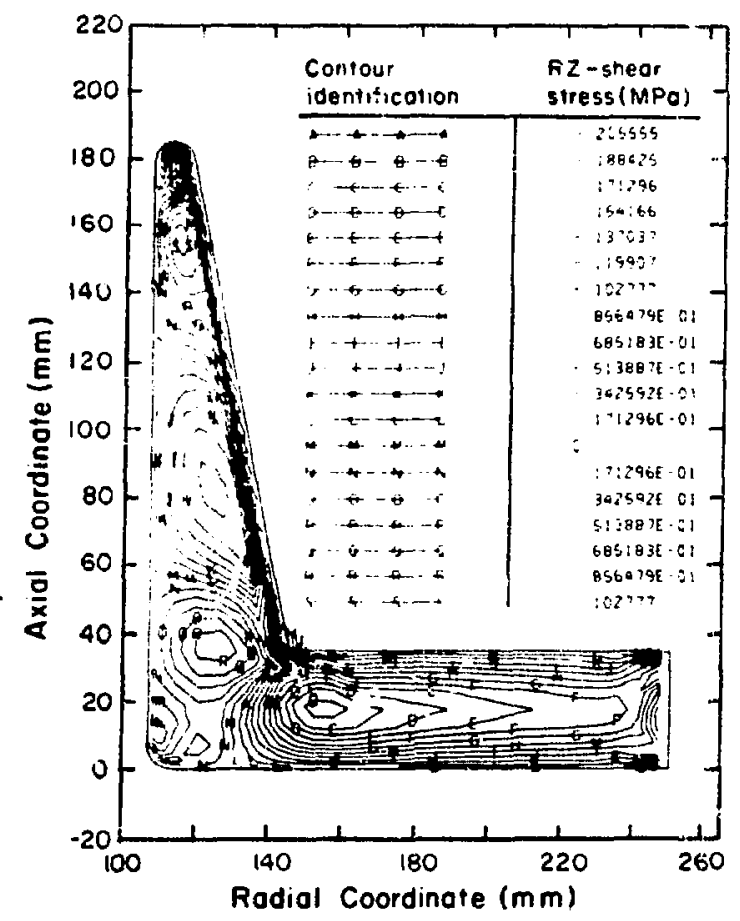

Fig. 5.2-13(d).

Radial/axial shear stress contours for the static loading given by Eq. (45). 


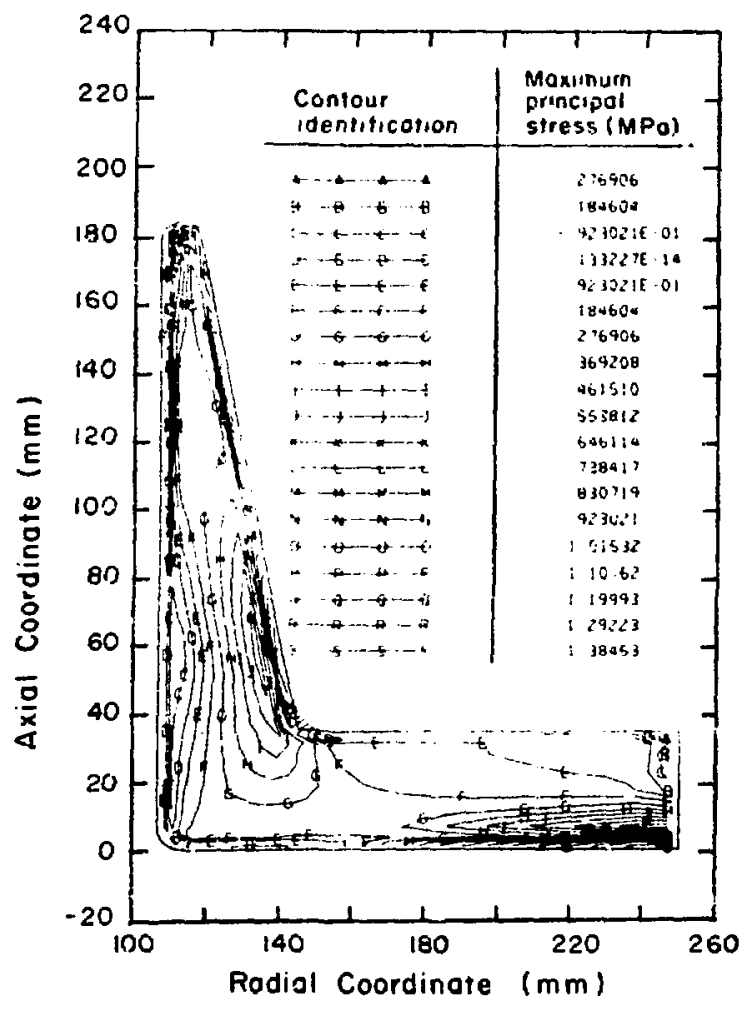

Fig. 5.2-14.

Maximum principal stress contours for the loading given by Eq. (45).

TABLE 5.2-3

MODAL FREQUENCIES OF IHC

$\begin{array}{cccc}\text { Mode Number } & \text { Frequency (kHz) } & \text { Period }(\mu \mathrm{s}) \\ 1 & 7.78 & & 359 \\ 2 & 10.98 & 91 \\ 3 & 12.24 & \\ 4 & 14.94 & 82 \\ 5 & 17.31 & & 67 \\ & & \end{array}$

TABLE 5.2-4

PEAK TENSILE STRESSES OVER FIRST $600 \mu \mathrm{s}$

\begin{tabular}{|c|c|c|c|c|}
\hline \multirow[b]{2}{*}{ Material Region } & \multicolumn{2}{|c|}{$\begin{array}{l}\text { Maximum } \\
\text { Hoop Stress }\end{array}$} & \multicolumn{2}{|c|}{$\begin{array}{l}\text { Maximum } \\
\text { Stress in } \\
r-z \text { Plane }\end{array}$} \\
\hline & (MPa) & $(\mu \mathrm{s})$ & $(\overline{\mathbf{M P a}})$ & (p \\
\hline Core & 7.90 & 320 & 2.33 & 1 \\
\hline Cop & 2.65 & 320 & 1.02 & \\
\hline Outer Ceramic & 8.43 & 320 & 3.67 & 18 \\
\hline
\end{tabular}




\subsubsection{Fabrication of Containment Vessel}

\subsubsection{Implosion-Heating Coil/Vacuum Vessel}

The fabrication of the first wall and implosion (shock heat ingl coil for the SFl'K requires a number of operations which are beyond the current state of the art. It is felt that with a concerted effort these operations can be accomplished. gicen sufficient lead time and resources for R\&D.

'Two concepts of the SFTR first wall-implosion coil assembly have been examined: (1) a unit structure with all components bonded yogether, and $12 i$ a separate first-wall liner coupled with an implosion coil assembly. Becrause of the inherent prolsem of the nistnatch of the thermal expansion coefficients of mosi ceranics and copper. the first concept presents very difficult fabrication problems. A coil based on the second concept appears to have the best chance of being successfully fabricated.

The implusion coil assembly is shaped like a wide. brimmed "top hat." as shown in Figs. 5.1.2 and 5.2. 3. 'The current e:iters at the outer edge of one side of tive brim and is routed over the exterior of the hat. down parallel conductors in a slow spiral pattern on the inside of the hat hack to the brim. and exits on ihe outer edge of the brim opposite the entering point. The hat is a ceramic insulator and support structure of $-2.5-\mathrm{cm}$ thickness for the $\sim 1.5 \cdot \mathrm{mm}$. thick copper condictors, and it is coated with a ceramic insulator. The following materials have heen considered for the hat: alcmina bodies. electrical procelains, mica, glass-bonded mica, and glass-ceramics. At present, the most suitable material appears to be an impervious alumina body. It would have good mechanical st rength and no open porosity. Much is known about its fabricability. techniques exist for dewositing copper conductor patterns on its surface, and its dielectric strength is comparable to other ceramic bodies ${ }^{12}$; however, a fundarnental problem is the assignment of a realistic and safe dielectric strength (which determines the insulation thickness) in the 200 . to $300-\mathrm{kV}$ region. No data exist for alumina in this voltage region for pulse-type operation and the type of geometry found in the SFTR implosion coil.

Fabrication of the insulator will be difficult. Initial forming would probably be done by slip casting followed by a considerable amount of slow diamond-yrinding. This type of fabrication is technic ally feasib!e but expensive: however, if $\sim 85$ '\% dense. high purity $\mathrm{Al}_{2} \mathrm{O}_{3}$ boty can be used instead of fully dense, high purity $\mathrm{Al}_{2} \mathrm{O}_{3}$, the cost of the insulator couid be reduced by a factor of $\sim 5$.

Althnugh severa! technigues exist for depositing thin copper conductor patterns on alumina. ${ }^{13.14}$ some fabrication problems remain because of the thermal expansicin mismatch between alumina and copper. ('urrent electroplating techniques tend to produce only a mechanical bond between the alumina and thin copper. and a development program is needed to perfect an electrochemical method of forming thick layers of stress-free, tightly. adherent copper on alumina substrates which can withst and a moderately high $\left(\sim 400^{\circ} \mathrm{C}\right)$ temperature.

Once the insulator hat is formed and the copper conductor with the required pattern is deposited on the hat. the hat must be coated with electrical insulation to prevent arcing to nearbs components or between adjacent current feeds. This insulating laver would be a ceramic coating. Firing techniques must be developed which will not cause the insulator to crack and yet will not exceed the $-400^{\circ} \mathrm{C}$ temperature limit imposed by the thermal expansion of the electroplated copper coil. An alternate method may be a flame- or arc-plasma-spraved oxide-plass conating if sufficient coating tightness and dielectric strength can be achieved.

The first wall for the SFTR must be vacuum-tight, impermeable to tritium, fabricable. and able to withstand the plasma environment without causing erosion or contamination of the plasma. Such a material may not exist, but investigations have begun on several promising materials.

The material for a separate first-wall liner must be capable of being joined to other parts by ceramic. metal and cerarnic-ceramic sealing techniques, have reasonable resistanco to thermal shock, and have good mechanical strength. Both alumina and silica glass are candidates for the first-wall liner, with alumina being the first choice. Fabrication of the first -wall liner would be similar to that of the implosion coil insulator hat. Conventional ceramic-tometal sealing techniques appear suitable: however. ceramic-to-ceramic joining techniques (low melting glass with infrared heating. a normal glazed joint utilizing a laser "bıazing" operation. or a fusion joint using a laser "welding" operation) are untested and are now considered to be beyond the present state of the art. However, recent developments in laser welding technology indicate that the techniques for making such joints will be developed within the required time.

\subsubsection{Magnetic Flux Concentrator}

No commercially available material will satisfy the four requirements of the magnetic material (Sec. 5.2.2.11. However. the development of a suitable material does not appear difficult.

Oriented silicon steel seems to be the best maieriai. it meets the requirement of $\mu / \mu_{0}>30$. and the saturation field in a powdered or fiake core is 14 $15 \mathrm{kG}$. Tape-wound cores are ruled out because it is 
tois difficu!t to roll the material to a thickness less than a skin depth. The only problem with present powdered or flake oriented silicon steel cores is that the dielectric st rength is marginal. Modifications are being made that may correct the problem.

The material will be manufactured into trapezoidal rross-section "logs" that will be stacked around the implosion coil to form the cylinder shown in Fig. 5.1.2.

\subsubsection{Mechanical Design and Fabrication of the Compression Coil}

Each compression coil is of split pancake design. that is. it is made of two pancake soils wound with upposite helicity and connected ioget her electrically at the inside turns. In this way all other electrical connections can be convenient ly brought to the outer radius. Insulation is made of vacuum impregnated fiber glass, and a dielectric strength of $10(6) \mathrm{V} / \mathrm{mil}$ is assumed. (vils can be fabricated by rolling up ropper strap with a spacer of tiber glass cloth and then vacuum impregnating the composite. The paired pancake coils can be welded together at the center turn before impregnation, or the entire split pancake couid be made of a continuous piece ol copper with a bend between paired pancakes. The , $=1$ and $t=0$ modulations of the coils can be of difterent radii. The maximum hoop stress in the coil is well below the endurance limit of the copper strip since enough copper was used in the winding to keep the $\mathrm{I} / \mathrm{R}$ time of the coil large. Dynamic hoop stress is also negligible for the same reason.

\section{REFEKENCES (Chap. V, Sec. 5.2)}

1. H. S. Carslaw, J. C. Jaeger, Conduction of Heat in Solids (Oxford at the Clarendun Press, and ed. NY. 1959).

2. R. G. Lawton, "The AYFR Heat Conduction Computer Program." Los Alamos Scientific Laboratory report LA-5613-MS (197: ).

3. T. A. Oliphant, personal communication (1975).

4. K. I. Thomassen and T. A. Oliphant, "First-Wall Fluxes in a Theta Pinch Feasibility/DT Experiment." J. Nucl. Mater. 53. 48 (1974).

5. C. F. Bonilla, Ed., Nuclear Engineering. (McGraw-Hill Publ. Co., NY, 1975), p. 538.
6. RASH. a LASI thin-shell st ress code.

7. R. J. Bartholomew, personal comnunication (1975).

8. F. W. Clinard, personal communication 11975).

9. R. V. Browning. D. G. Miller and C. A. Anderson. "TsAAs: Finite Element Thermal and stress Analysis of Axisymmetric Solids with Orthotropic Temperature-Dependent Material Properties," IAs Alamos Scientific Laboratory report LA-ijig9-MS (May 197t).

10. R. V. Browning, personal communication (1975).

I1. K. I. Bathe, F. L. Wilson, and R. H. Iding. "NONSAP-A Structural Analysis Program for Static and Dynamic Response of Nonlinear Sistems." SFSM Report 74-3. Dept of Civil Engineering. University of California. Berkeley (February 1974 .

12. W. S. Kingery, Introduction to Ceramics (John Wiley and Sons, NY, 1960), p. 726.

13. R. E. Cowan, U.S. Patent No. 3180756.

14. G. L. Babcock et al., U.S. Patent No. 3766634 .

\subsection{IMPLOSION-HEATING SYSTEM}

\subsubsection{General Description}

The inplosion-heating sustem provides the fast rising magnetic field which initially heats the plasma. The basic circuit for the system is shown in Fins. 4-16 and 5.3.1. The inductance of the capacitors and start gap is $L_{r}$. $L_{f}$ is the inductance of the feedplate, and $L_{i}$ is the inductance of the load coil. The storage capacitance is $C$, and $S_{k}$ and $S_{c}$ are. respectively, the start gap and crowbar switches. The basic operation is: (1) charge the capacitor with $S_{\text {. }}$ and $S_{k}$ open, (2) close $S_{k}$ so that the capacitor discharges through the load coil, (3) at peak current close $S_{c}$ which maintains the current in the load cril until the compression field can be raised. The implosion field must rise to $1.1 \mathrm{~T}$ in approximately $100 \mathrm{~ns}$ and be sustained for approximately $200 \mu \mathrm{s}$. 


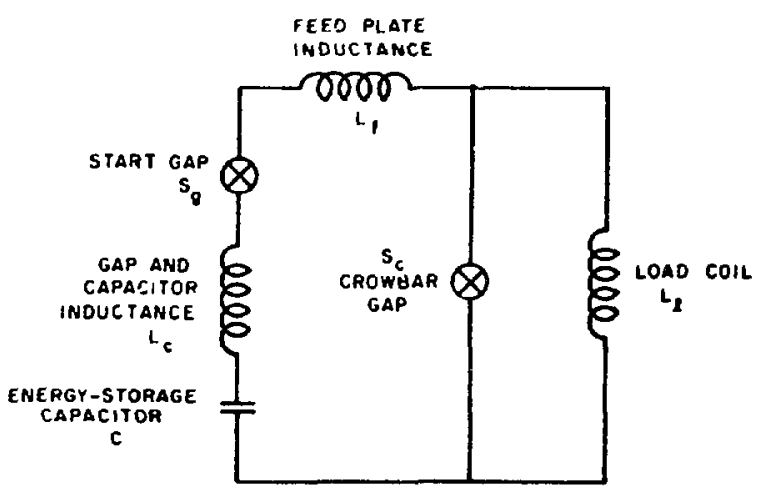

Fig. 5.3.1.

Basic circuit of the implosion-heating system.

\subsubsection{Capacitor and Feedplate Design}

To utilize the energy stored in the capacitor most efficiently, the source inductances $L_{c}$ and $L_{r}$ must be as small as possible consistent with dielectric breakdown st re:ngths and physical dimensions. This implies that the feedplates should be as short as possible and the capacitors and st art gaps should be designed for low inductance. Several capacitors and start gaps will be placed in parallel to achieve a low effective inductance of the capacitor and gap.

The approach used in the design is to configurate the feedplates as circular discs which extend from the load coil (Marshall coil) to just outside the primary radiation shield with six capacitors feeding around the periphery of the feedplates as was shown earlier in the layout of Fig, 5.1-4. Three crowbar gaps are placed between every two capacitors, with a spare being provided for the compression coil leads and the vacuum downcomers. A cross section of the feedplate and capacitor is shown in Fig. 5.2-1. The offiset feedplates displace the capacitors from the neutron streaming path along the feedplates and allow space to place shielding in this streaming paih.

An important feature of the design is that it allows ready acress to the capacitors and gap for maintenance purposes. The radia! feedplate shown in Fig. 5.2-1 is common to two adjacent Marshall coils with the outer feedplates at a common voltage of $270 \mathrm{kV}$. The center feedplate is connected to the machine ground grid. The $270 \mathrm{kV}$ is achieved by connecting two $0.04-\mu \mathrm{F}, 135-\mathrm{kV}$ capacitors in series through a single $270-\mathrm{kV}$ spark gap. The energy stored in each capacitor is $365 \mathrm{~J}$. The feedplate assembly feeds two load coils which are assembled into a unit referred to as a module. The length of a module is $40 \mathrm{~cm}$. and there are eight modules in a wavelength $\lambda$.
The estimated inductance of the capacitor and gap for this design is approximately $27 \mathrm{nH}$ and the calculated inductance of the feedplate corresponding to one capacitor unit is $23 \mathrm{nH}$. Thus, the total source inductance for one capacitor assembly is $\mathbf{5 0}$ $\mathrm{nH}$ and with six capacitors in parallel the equivalent source inductance to the load coil is $8.3 \mathrm{nH}$. The overall electric circuit for a module is shown in Fig. $5.3-2$. Each $40-\mathrm{cm}$ module is made up of two load coils, six gajs, twenty-four $0.04-\mu \mathrm{F}$ capacitors at 135 $\mathrm{kV}$, and $270-\mathrm{kV}$ start gaps. The preionization and pulse-charging systems also shown on the schematic are discussed in the following sections.

\subsubsection{Preionization System}

The preionization system consists of a capacitor and start gap connected across the iozd coil through a relatively large inductance. One capacitor per module will be required. The capacitor to be used will be a standard $1.85-\mu \mathrm{F}, 60-\mathrm{kV}$ capacitor with a standard Scyllac-type start gap.

The capacitor will be discharged through the load coil approximately $10 \mu \mathrm{s}$ before the implosionheating capacitors are discharged.

\subsubsection{Pulse-Charge System}

The pulse-charge system is used to charge the 135$\mathrm{kV}$ implosion-heating caparitors. It is a relatively high-inductance system with the charge being accrmplished in approximately $5 \mu \mathrm{s}$. The implosionheating capacitors are pulse-charged to avoid corona problems in the implosion-heating system and to avoid the necessity of designitig a start gap having the capability of withstanding $270 \mathrm{kV}$ for a long period.

The basic system consists of a two-stage Marx generator which has the capability of charging half the capacitors in eight modules cone wavelength of

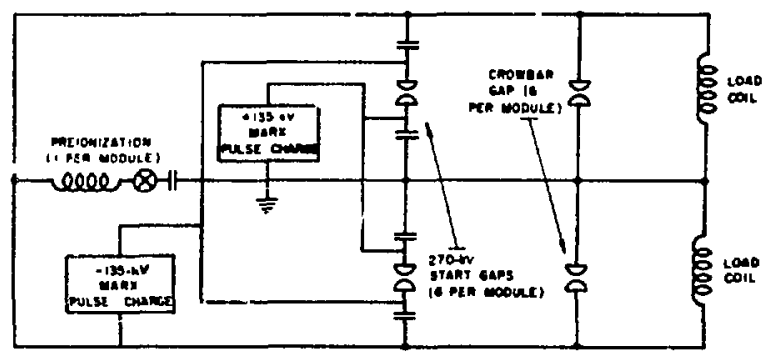

Fig. 5.3-2.

Electrical circuit of implosion-heating system module. 
the plasma chamber). There are 192 capacitors in 8 modules: 96 must be charged positively and 96 negatively. Two Marx generators are required per eight modules: one for positive charging and one for negative charging.

Fach Marx generator consists of two $7.7-\mathrm{kV}, 7.7$ ${ }_{\mu} \mathrm{F}$ capacitors connected as shown in the circuit of Fig. 5.3.3.

\subsubsection{Trigger System}

Independent trigger systems are required for the start gaps. the crowbar gaps, and the preionization system to allow adequate flexibility during operation. The trigger-system design follows the standard approach. An 8-kV pulser is triggered from central control; the pulser triggers a master gap which in turn triggers several submaster gaps, and so on until the actual start gaps are triggered. The synchronization of the trigger signals is controlled by maintaining proper cable lengths through the various levels of the system. The implosion-heating trigger system has been segmented into four identical systems, each of which triggers all gaps in one-fourth of the machine. For example, to trigger the start gaps, a trigger pulse is sent from the control system which triggers the 8-kV pulser at each quadrature of the niachine and this in turn starts the chain of trigger pulses resulting in the triggering of the start gaps in that quadrature.

The schematic of a typical trigger-system chain is shown in Fig. 5.3-4. An important subsystem of the trigger system is the trigger-system Marx generator

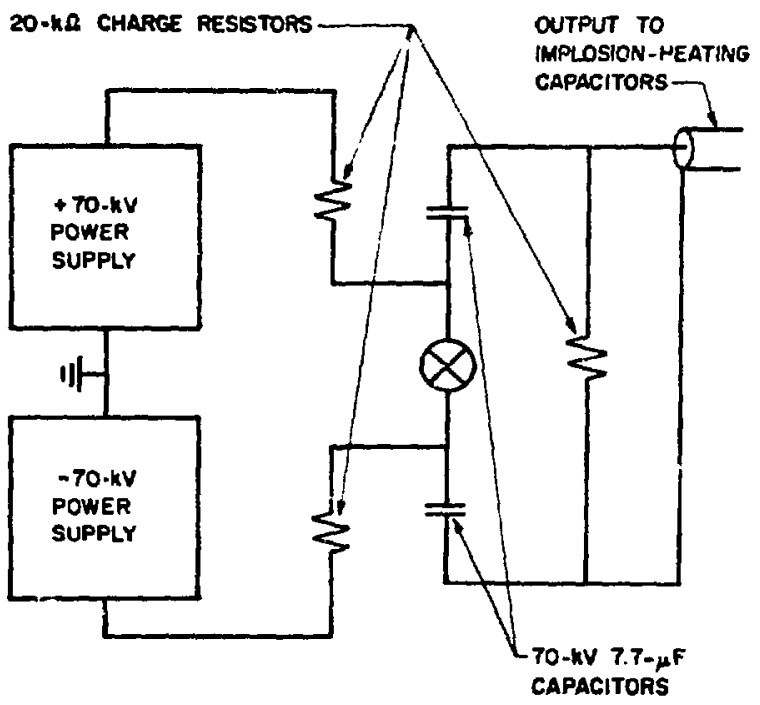

Fig. 5.3-3.

Circuit of pulse-charge Marx generator.

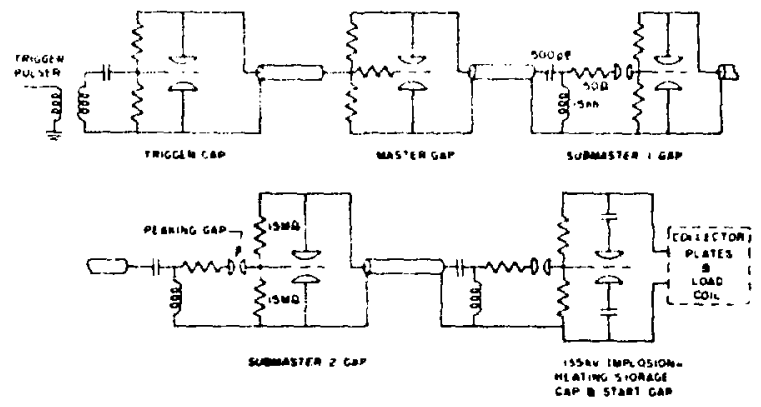

Fig. 5.3-4.

Tipical trigger swstem schematic.

which must also be triggered from central control just before the start gaps are triggered.

\subsubsection{Start-Gap Trigger System}

The start-gap trigger system consists of four identical subsystems, each of which triggers the start gaps in a quadrature of the machine. The iotal number of start gaps triggered by a subsystem is 1920. Each subsystem has been designed with a master gap and two levels of submasters. The master gap triggers a submaster-one gap; each submasterone gap triggers 16 submaster-two gaps: and each submaster-two gap triggers 12 start gaps.

Four trigger-system Marx generators are required for each of the four subsystems of the trigger system. A trigger-system Marx generator consists of four 75$\mathrm{kV}, 7.7-\mu \mathrm{F}$ capacitors connected as shown in the circuit of Fig. 5.3-5. Each Marx generator charges the trigger-system circuits for 40 modules, or for 480 start gaps.

The puise-charge trigger system consists of two master gaps, each of which triggers 20 pulse-charge

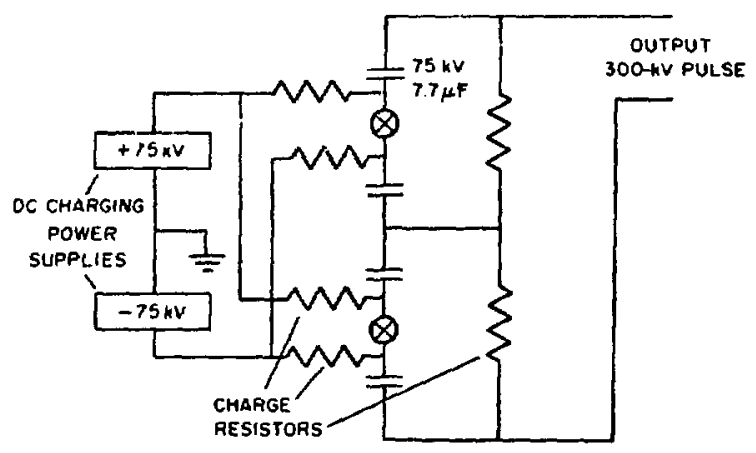

Fig. 5.3-5.

Circuit of start gap trigger system, Marx generator. 
Marx generators. Each pulse-charge generator charges half the implosion-heating capacitors in a module.

A diagram of the major components in the start gap trigger system is given in Fig. 5.3-6.

\subsubsection{Crowbar Trigger Systern}

The crowhar trigger system is almost identical to the start-gap trigger system. The only difference is that there are half as many crowbar gaps as start gaps. Like the start-gap trigger system, the crowbar trigger system consists of four identical subsystems. each of which triggers all the crowbar gaps in a quadrature of the machine. There are 962 crowhar gaps in a quadrature. Fach subsystem consists of a master gap and two levels of submasters. The master gap triggers 5 submaster-one gaps; each submasterone gap triggers 16 submaster-two gaps: and each submaster-two gap triggers 12 crowbar gaps.

Two trigger-system Marx generators are required for each of the four subsystems. The trigger-system Marx generators are identical to the generators discussed in Sec 5.3.5.1 and shown in the schematic of Fig. i. 3-5.

A diagram of the major components in the crow. bar trigger system is given in Fig. 5.3-7.

\subsubsection{Pulse-Charge Trigger System}

As discussed in Sec. 5.3.4, two pulse-charge Marx generators are required for each eight modules or one wavelength of the discharge chamber. There are, therefore, 40 Marx generators in a quarter of the

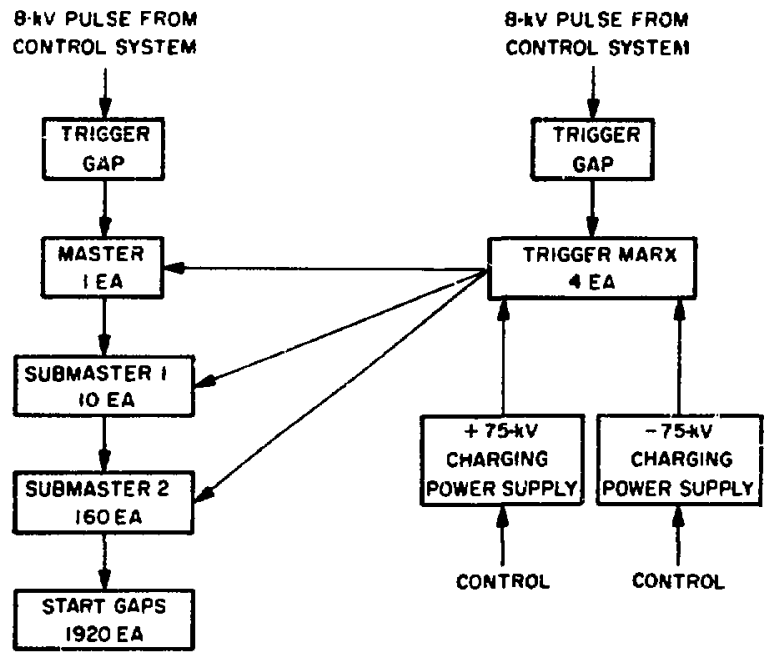

Fig. 5.3-6.

Diagram of start-gap trigger system.

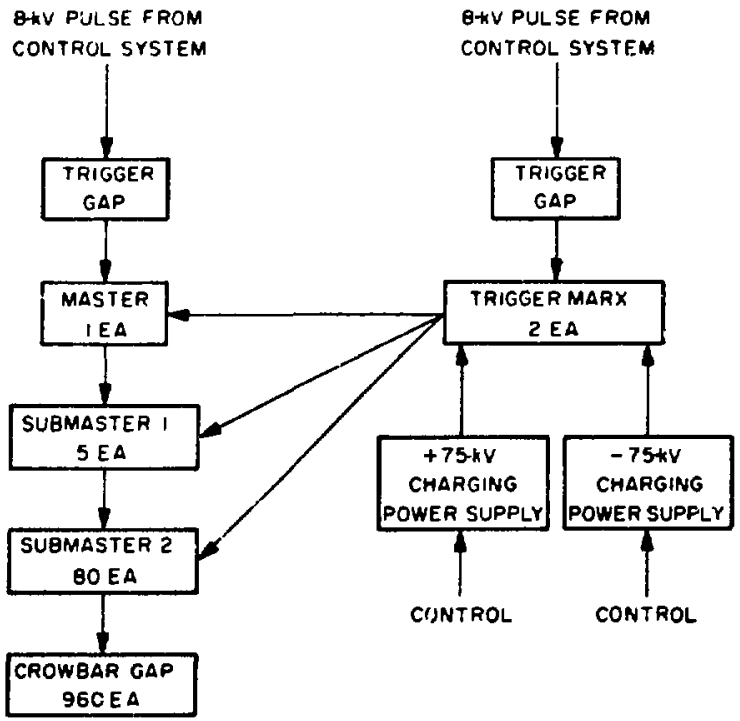

Fig. 5.3-7.

Diagram of croubar trigger system.

machine. 20 positive and 20 negative. The trigger system consists of two master gaps, one triggering 20 positive Marx generators and one triggering 20 negative Marx generators. A diagram of the system is shown in Fig, 5.3-8.

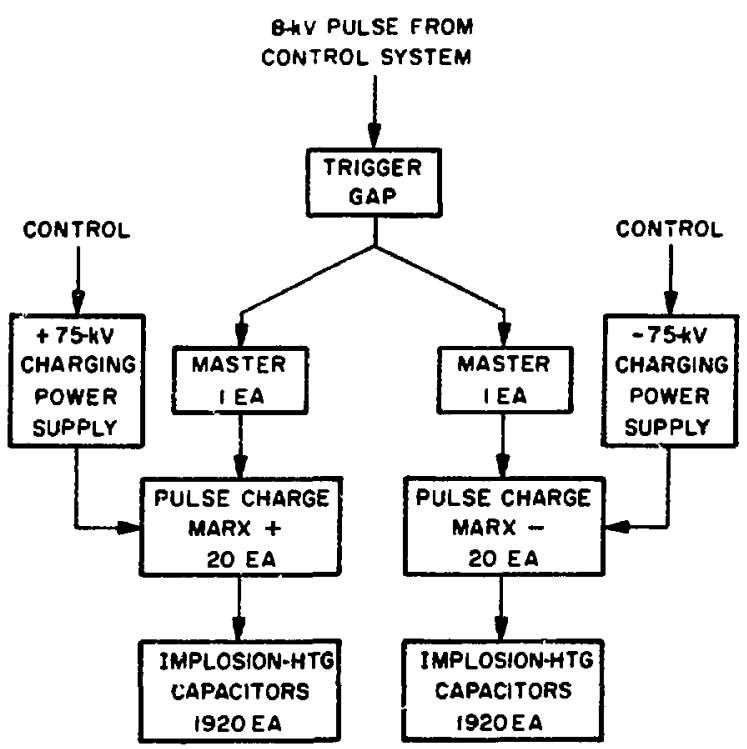

Fig. 5.3-8.

Diagram of pulse-charge trigger system. 


\subsubsection{Preionization Trigger System}

The preionization system consists of one capacitor and start gap per module. Thus, there are 160 capacitors and gaps in one quadrature. The trigger system consists of a master gap which triggers 20 submasters; each submaster triggers 8 gaps. A diagram of the system is shown in Fig. 5.3-9.

\section{8-kV PULSE FROM CONTROL SYSTEM}

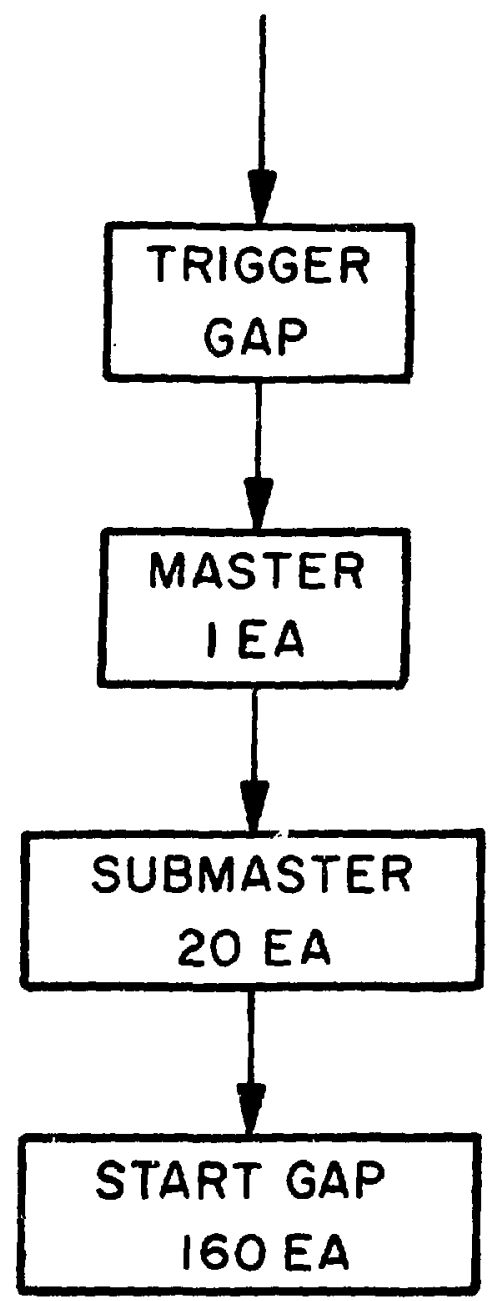

Fig. 5.3-9.

Diagram of preionization trigger system.

\subsubsection{Timing and Control}

The triggering of the various systems and sub. systems in the implosion-heating sivstem must be accomplished within microseconds and nanoseconds. The speed and precision of the various trigger pulses are such that a computer-type control is impractical. The control system is, therefore, designed around pulse-delay generators for generating precisely timed pulses to trigger the master gaps in the various systems. The central control computer will ensure that all systems parameters, such as pulse delays. are properly set and all slow functions accomplished. such as de charging of the Marx generators. When the sustem is ready for triggering, the cent ral control will trigger the pulse-delay generator which in turn will emit properly timed triggering pulses to the various subsystems. Timing through the system wili be controlled by maintaining proper cable lengths: between the various pulsos and gaps.

The timing of the functions of the implosionheating system is described below. The firing time of the implosion-heating start gaps is designated $t_{1, .}$

1. $t=t_{11}-30 \mathrm{~ms}$

a. Initiate charge of start-gap trigger-system Marx.

b. Initiate charge of pulse-charge sy'stem Marx.

c. Initiate charge of crowbar-trigger-system Marx.

d. Initiate charge of preionization sistem.

This $\mathrm{de}$ charging will require approximately $30 \mathrm{~ms}$.

2. $t=t_{1 .}-10 \mu \mathrm{s}$

a. Trigger the start-gap trigger-system Marx.

b. Trigger the crowbar trigger-sustem Marx.

c. Trigger the pulse-charge system.

The $10 \mu \mathrm{s}$ is the time required to complete the pulse-charging of the implosion-heating capacitors and the trigger systems. This is a design value of the system components and will not be changed after the machine has been constructed.

3. $\left.t=t_{11}-(5) t 010 \mu s\right)$

a. Trigger the preionization system.

The range of 5 to $10 \mu \mathrm{s}$ will be the typical operating range of the prejonization system: however, the system is designed to be able to vary this time over a range as wide as necessary.

4. $\mathrm{t}=\mathrm{t}$,

a. The start-gap system is triggered.

5. $\mathrm{t}=\mathrm{t}_{1}+(\sim 100 \mathrm{~ns})$

a. The crowbar system is triggered. The timing of the triggering of this system must be flexible and nust also be precise within a few nanoseconds. 


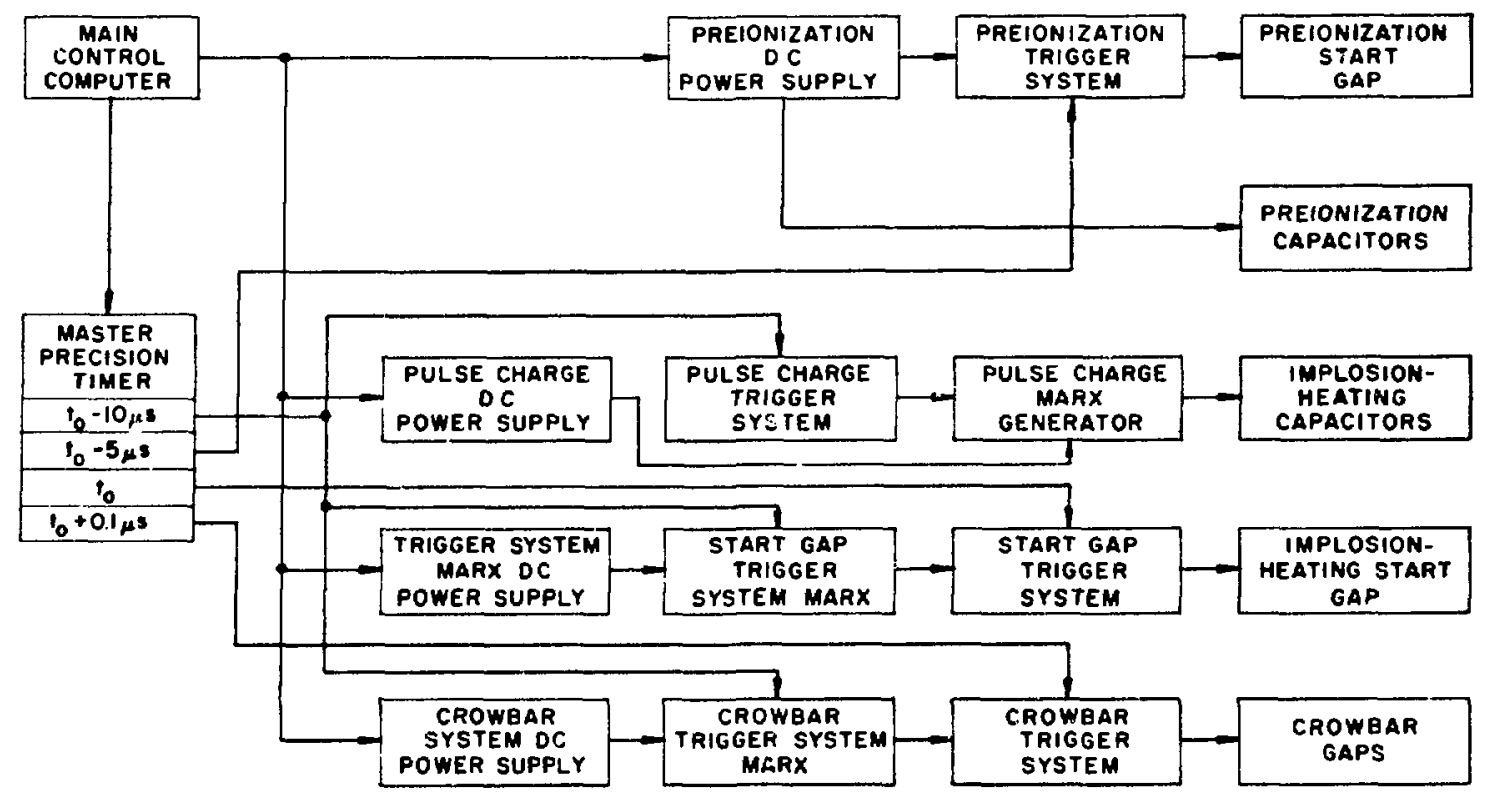

Fig. 5.3.10.

Trigger system for one quarter of the machine.

Figure 5.3-10 shows a diagram of the overall trigger system for one-fourth of the machine. Each quadrature of the machine has a system identical to that shown in Fig. 5.3-10. The central contro! computer and the central pulse generator are located in the machine control rooru, while the $8-\mathrm{kV}$ pulsers and the master trigger gaps are located in the trigger-system area of the machine.

\subsection{COMPRESSION SYSTEM}

\subsubsection{General Description}

The purpose of the compression field system is to provide the necessary magnetic field to compress adiabatically the plasma for the burn cycle. A circuit diagram of a basic module of the energy-storage and transfer system is shown in Fig. 5.4-1. The energy required for the compression field is stored initially in the superconducting storage coil. The current is transferred by opening the current interrupter and allowing the current to resonate through the transfer capacitor into the load coil. At peak current in the load coil the crowbar switch is closed and the current decays with an $L / R$ time constant.

The compression field reaches $55 \mathrm{kG}$ in $700 \mu \mathrm{s}$ and decays with a $250-\mathrm{ms}$ time constant. There are 1280 compression load coils in the machine, one for each implosion load coil, with each compression coil hav- ing a crowbar switch, transfer capacitor, vacuum interrupter, and storage coil. The energy required to produce $55 \mathrm{kG}$ in each compression coil is approximately $345 \mathrm{~kJ}$ and the energy-storage capacity of the transfei capacitor is somewhat less than half of that $(185 \mathrm{~kJ})$ when losses are taken into account. The $55 \mathrm{kG}$ is in excess of the field required according to Fig. 4-11, but we chose to have a margin for exploration.

Although the energy for the compression field could be supplied from a capacitor bank, an inductive energy-storage system has been selected in order to advance the necessary technology required for future reactors. Although capacitor technology is presently well developed and the energy-storage requirements of the SFTR could be handled by large capacitor banks, we believe that capacitors will not

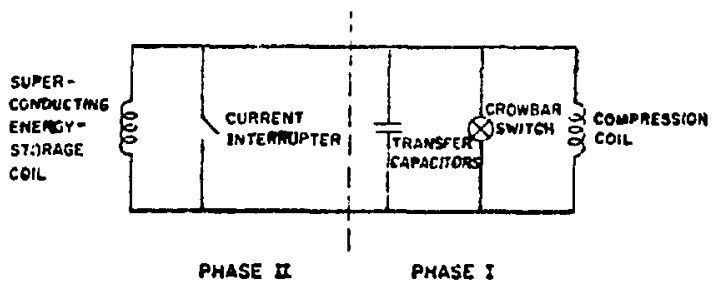

Fig. 5.4-1.

A basic module of the compressional energy supply. 
be the economical choice in a reactor where approximately 1 G.J of energy storage will be required for staging between the capacitive implosion system and the homopolar compression svitem. The primary reasons that superconduct ing and inductive storage are expected (o) be more atcractive than capacitive storage for that purpose are:

- Inductive storage has a much higher energy density trom capacitors. thus, less space is required.

- Because of the higher energy density. lewer elements are required in an inductive energy-storage sistem, and maintenance requirements are reduced.

- An inductive energy-storage syitem is projected to have a lower cost per joule than a capacitive system.

For smaller experiment al machines, the reduced volume requirements of the inductive sistem are not a major consideration: however, in a reactor the energy-storage requirements for staging syitems. which must be discharged in the $1 / 2-101-\mathrm{m}$ s time frame. are of the order of a gigajoule. and higher enesgr density beromes an important advantage for the inductive storage stiten. In a commercial reactor, the reliability and maintenance of the system are major considerations and the system having the fewest components consistent with acceptable reliability, such as the inductive storage sistem. also has the advantage. The cost of an inductive energystorage system that would be used in a reactor can only be roughly approximated. The present cost estimate for installation of an inductive system is substantially higher than for a capacitive system. However, the technology and manulacturing capability for capacitors are well developed. and increased production of inductive storage systems will significantly reduce their cost. The cost of a capacitive storage system would be approximately $4 \mathrm{c} / \mathrm{J}$. whereas the cost of a superconducting induc. tive energy-storage system would be approximately $2 d / .3$ in a mature superconducting industry.

The compression field system will be installed in two phases. Phase I consists of the installation of the compression load coils, the crowbar switches, and the transfer capacitor bank. The experiment will be operated initially with just the transfer capacitor bank supplying the compression field. This will permit operation of the machine with a relatively reliable capacitor system similar to systems used previously in theta-pinch experiments at Los Alamos. Phase l operation will also allow more time for the development of the superconducting system. After all systems hàve been checked out, initial plasma experiments aimed at producing long confinment times (tens of milliseconds) will be carried out. Installation of the inductive system, or Phase III. consists of adding the superconducting energy-storage coils and current interrupters and doing innition experiments. Under Phase I the peak magnetic field wil! lye $39 \mathrm{~kg}$; with a rise time of $53014 \mathrm{ss}$ : under l'hase Il the peak magnetic tield will be $55 \mathrm{k}$ ( $;$ with at rise time of $7(10) \mu \mathrm{s}$.

\subsubsection{Capacitor Compression}

\subsubsection{General Description}

The energy-storage capacity of the transter capaciurs associated with a single compression field load coil is $185 \mathrm{~kJ}$. The system is designed for a peak voltage of to $\mathrm{kJ}$ and would transfer energy from the inductive supply in $7(10) \mu$. Without the industive store the transter time is 53010 s. At $60 \mathrm{kl}^{\mathrm{k}}$ the capacitance required per module is $100 \mathrm{fH}$. Bute to the large inductance of the load coil, at relatively high inductance can be toleroted in the caljacitur bank. This allow's the less expensive construction of connectiag low voltage capacitors in series to achieve the required $60 \mathrm{kl}$. The 100$)-\mu \mathrm{F}$ capasitance will be

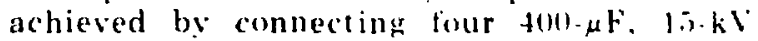
"apacitors" in series in a Marx generator arrangement as illustrated in the schematic shown in Figr. 5.4-2. Each $400-\mu F$ "capacitor" comsists of four 1011)$\mu F$ capacitors in parallel. As well as allowing the use of relatively inexpensive low voltane capacitor and ignitron start switches. the arrangement aiso allows the capacitor to be charged by a simple dc charging supply.

The reliability of the system has been determined by calculating the mean tume between component failures, and using this to assess reliubility. It was found that despite the large number of components. approximately 30 energy transters can be expected between component failures. This analysis was based on the following assumptions:

- The prefire rate of the start switches (ignitronsl is 1 to 2000 .

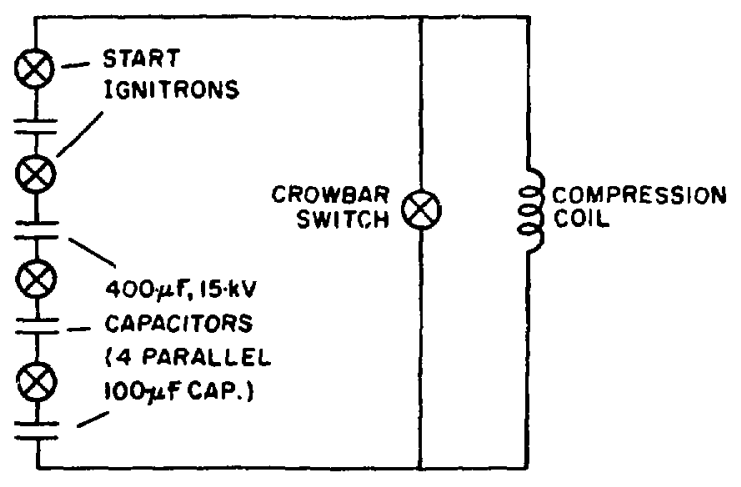

Fig. 5.4-2.

Schematic of Phase I energy-storage capacitor for one compression load coil. 
- The prefire rate of the igsitrons used in the crowbar switch is 1 in 10000 . The crowbar swit.h consist. of four size D ignitrons in series.

- Prefires do not cause component failures.

- Failure of any component terminates the shot.

- Prefire of a single ignitron does not cause a total switch prefire but a simultaneous prefire of two ignit rons in series does.

- Failure of any component does not cause failure of any other component.

This level of reliability has proved satisfactory in Scyllac, where approximately 10 shots are expected between component failures, and it is considered satisfactory in the SFTR.

\subsubsection{Capacitor Bank}

The basic capacitor used in the cumpression system is a $15-\mathrm{kV}, 1(\mathrm{~K})-\mu \mathrm{F}$ unit. The sy'stem is designed for minimum voltage reversal on the capacitor so that. following current practices. a relatively high energy density can be stored per unit. The total energy stored in a single capacitor is $11.2 \mathrm{~kJ}$. With $3.25 .3 /$ in $^{3}$, which is the approximate energy density in a standard high-density capacitor. the size of the capacitor is the sam as the slandard Scyllac-type unit. 24 in. $x 14$ in. $x 11$ in. For a single compression coil, 4 capacitors will be connected in parallel (to) give the $4(0)-\mu \mathrm{F}, 15-\mathrm{kV}$ "(apacitor" in Fig. 5.4-2). with 4 groups of these parallel capacitors connected in series for a total of 16 capacitors per compression coil. The capacitors will be placed in racks just outside the concrete cell as shown in Fig. 5.1-6. Fach rack contains 160 capacitors-sufficient for 10 load coils. A total of 128 racks is required for the machine.

\subsubsection{Start Switches}

The locations of the start switches are shown in the schematic in Fig. 5.4-2. Each start switch must have a peak current rating of $18 \mathrm{kA}$. be able to withstand at least $15 \mathrm{kV}$. and carry at least $6 \mathrm{C}$. Suit able commercial ignitrons have been selected for the start switch application. ignitrons having a volt age rating of $20 \mathrm{kV}$ will be emploved to provide a maryin of safety against prefires. The ignitrons and header: are located at one end of the capacitor racks.

\subsubsection{Charge System}

In the design of the capacitor bank charge sistem one must take into account not only the charging of the capacitors but also persomnel safety and fault modes. The charging circuit is shown in Fig. $5.4-3$. Four $100-\mu \mathrm{F}$ capacitors are cumected in parallel to a single start switch with 5 -k $\Omega$ charge resistors. Failsafe shorting balls are connected across the

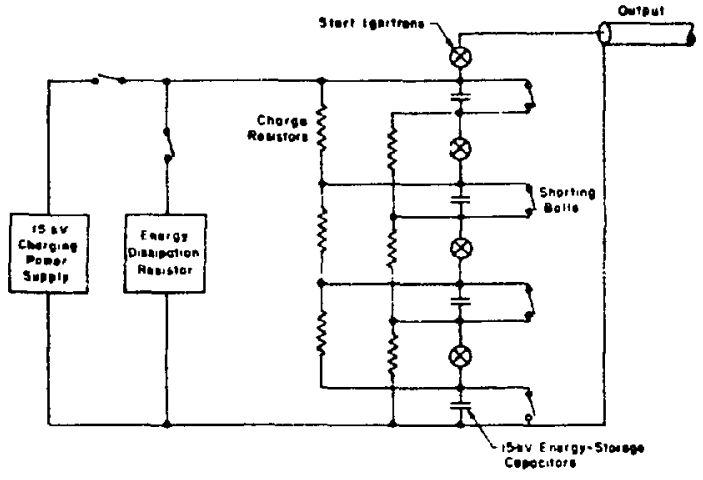

Fig. 5.4-3.

Electrical design of capacitor-bank charge sistem.

capacitors to ensure complete discharge of the capacitors during maintenance. A shorting switch across the bank can be closed to dump the energy into a resistor should the charge cycle ever need to be aborted.

Sixteen racks of capacitors are charged from a single controlled power supply. Eight power supplies will be required for the facility and will be located just outside the main building. Following standard Scyllac practice, the capacitor banks will be charged in $30 \mathrm{~s}$; each power supply must therefore supply 128 $A$ at a nominal $15 \mathrm{kV}$ for a peak power rating of approximately $2 \mathrm{MW}$.

\subsubsection{Trigger System}

The trigger system for the 128 racks will be divided into \& equal subsystems similar to the trigger system for the implosion-heating system. Each subsistem consist a of a master pulser which triggers : 320 Krytron drivers, with each driver triggering the 4 sturt ignitrons in a 16-capacitor unit which supplies the energy for 1 compression coil. The four master pulsers are triggered from the control computer.

An important consideration of the trigger circuit is the isoiation of the start ignitrons. Since ignitrons are prone to prefire, the ratings on the four series ignitrons are chosen such that when one prefires the remaining three will hold off the voltage: two ignitrons must, therefore, prefire simultaneously for the system to malfunction, which is a much less likely event than a single prefire. However. to gain this increased protection from prefires, the trigger system must provide sufficient isolation of the trigger pulses so that a prefire in itself does not trigger the remaining ignitrons. Such a circuit is shown in Fig. 5.4-4. The pulse transformers supply the trigger pulses to the ignitrons. but when a prefire 


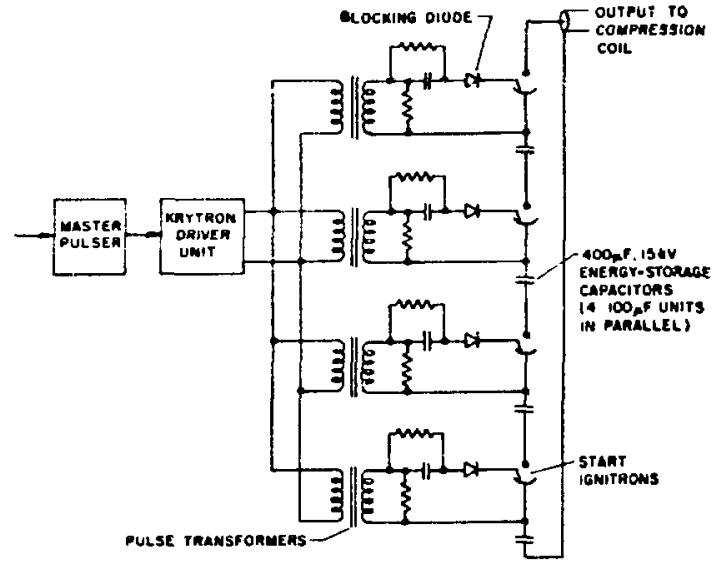

Fig. 5.4-4.

Trigger systen: for capacitor drive of compression coils.

occurs the blocking diode will prevent an energy pulse from feeding back through the pulse transformers to trigger the other three ignitrons. Although this circuit does not provide complete isolation, it is expected to provide a reasonable com. promise between cost and performance. Should the performance of the circuit be unsatisfactory, an optical system with higher performance can be emploved.

\subsubsection{Magnetic Energy Storage}

\subsubsection{General Description}

Fnergy for driving the compression coils for the adiabatic heating of the theta-pinch plasma in the SFTR is to be supplied from a superconducting magnetic energy transfer and storage (METS) system. The system has 128 cylindrical plastic dewars outside the plasma toroid, each dewar containing 10 energy-storage coils. The energy, to be pulsed into the compression .oils in a $0.7-\mathrm{ms}$ transfer period. is charged from a dc, low voltage power supply into a series-connected, modularized, superconducting inductive energy store. Charging is carried out over a $300-s$ period. The charge-discharge cycle is to be repeated every $\$ 70 \mathrm{~s}$ to provide for refrigeration recovery at the $4.5 \mathrm{~K}$ level. This 900 -s cycle includes the $3(1)$-s charging time and sets the heat load cycle and size of the refrigeration plant for the system.

The refrigerator plant is capable of making liquid helium at a rate of $3045 \mathrm{~kg} / \mathrm{h}$. The refrigeration cycle will use gas turbine expanders in cascade witt intermediate heat exchangers and a reciprocating ex- pander engine in lieu of a I:ule-Thomson valve in the last stage to liquefy the helium. Several principal heat loads deterinine the refrigeration requirements. The $4.5 \mathrm{~K}$ steady-state dewar heat load is $7.6 \mathrm{~kW}, 5.4 \mathrm{~kW}$ of which is from the $25-\mathrm{kA}$ continuous duty electrical leads to each dewar: the 4.5 $\mathrm{K}$ pulsed heat load is $8.6 \mathrm{~kW}, 5.4 \mathrm{~kW}$ of which arises from the cryogenic disconnects; and the transfer lines have a steady-state, $1.1-\mathrm{kW}$ heat load at $4.5 \mathrm{~K}$. Half of the helium, $1517 \mathrm{~kg} / \mathrm{h}$, is returned to the refrigerator as cold gas at a temperature just above $4.5 \mathrm{~K}$ and the remainder as warm gas. Thus ball the heat load is handled efficiently in a refrigeration cy. cle and the remainder is cooled and liquefied from ambient temperature as in a liquefier cycle. The liquefier-refrigerator is about 22 times larger than those in existence at present and will require 16000 hp or $12 \mathrm{MW}$ electrical power.

Components for switching and transfer of the energy from the storage coils to the compression coils are arranged in a sectionalized or modular array around the system between the plasma discharge tube and the storage coils. The sectional arrangement provides access for assembly and servicing and is sized to maintain reasonable modular interconnections between the energy-storage coils and the compression coils. Basic to this module concept are the high voltage and high current limits of $60 \mathrm{kV}$ and $26 \mathrm{kA}$ established for the superconducting energystorage coils with a 2.5-T magnetic field. When these limits are considered in connection with the required 5.5)-T peak plasma adiahatic compression field, the required $250 \cdot \mathrm{ms} \mathrm{L} / \mathrm{R}$ decay time for the compression field, transfer inefficiencies, erergy stored in the compression coil windings, and corrections for the applied multipole field to correct for the plasma behivior, then the storage coil module, its stored energy, and the associated switching and transfer components are defined.

The total stored energy in the 1280 superconducting coils around the torus is 488 MJ.

Switching of the energy from the superconducting energy-storage coils to the compression coils on the plasma is accomplished by disconnecting the seriesconnected storage coils and connecting them in parallel with the compression coils. Each storage coil is connected in parallel with a transfer capacitor. This arrangement creates an L-C.L resonant circuit with nearly 100 ; $;$ transfer efficiency. Once the energy is transferred into the compression coils, each coil is shorted or crowbarred so that the magnetic field is trapped in it for the plasma confinement.

Because charging of the storage coils takes a long time compared with discharge- $300 \mathrm{~s}$ versus 0.7 $\mathrm{ms}-$ it is necessary to connect the storage coils in series within the liquid helium dewar. To bring the 
26-kA current wut of the dwwar to an external seriesconnected switch or interrupter at aach storage coil would creste a prohibilive heat load from the electricial leads entering tha cryogenic sistem and would require a very large refrigerator. A compromise arrangement is prosided with groups of 10 coils in separat. lowars. Thus only les pairs of' $26-k$ A leads are "Ised during the charging cycle. Wit hin the liquid heliom dewar. iow resistance cryogenic disconnects. rain with dissipating peak power of less than 2415 . are used 10 make the series comnections batween coils in the separate dewars. These crygenic disconnects are connected in parallel with $\mathrm{HVJ}$ vacuum interrupters external to the dewar. In the switching covle the disconnects are opened first. bypassing the current through the HVlx interrupters which art then opened, and most of the encrgy dissipated in switehing is outside the liquid helium sviem.

The switching time required for opening the crvogenic discomnect and the $\mathrm{HVI}$ ( interrupter is of the order of $0.1 \mathrm{~s}$. The subsequent $0.7-\mathrm{ms}$ energy transfer poriod is relatively short. The electrical leads carrying the current during transter are small since they carry a pulsed load for only (1. I s. and their thermal conduction heat leaks and resistive electrical heat loads are small.

Siwitches for the HVDC interrupter bank are to be state-of-the-art vacuum breakers. Conventional counterpulse circuitry holds the current in the hreakers at zero with a very low time rate of change of voltage during deionization. Crowbar switches fur the compression coils will also use state-nf-the-art ignitrons like those now ased in the 1.Asl..ClK arugrair.

The transter enpacitor for the L.-C-L resonant cirruit tur the superconducting METS system will use the capacitur bank described in Siec. 5.4.1. It will be connected to store approximately half ihe $488-\mathbf{M}$. energy at bill $\mathrm{kl}$ during the 0.7 -ms energy transter period.

Table 5.4-1 lists the MFTS-SFTR circuit components and describes their functions. The notation of Fig. 5.1-5, which is a MFTS-SFTR circuit diagram. Jocates these elements in the circuit. The following operational sequence of the system describes the energy-storage and transfer process.

In the initial state the cryogenic disconnects $B_{1}$ are closed. and the vacuum interrupters $B$ : and istulating switches $B_{3}$ are open. The electrically operpted line contactors $B_{i}$ are open during charge. The storage inductors are charged in series by ranping the current from the main energy supply to 26 $k A$ in $3(0):$. At the same time, the counterpulse energy is supplied to the transfer capacitor bank $C$, hy charging to several kilovolts. Atter charging. the counterpulse supply is disconnected. The high ioltage vacuum interrupters are closed, the line contactors to yround are closed, and the cryogenic discomnects are opened, in that sequence. This transfers the $26 \mathrm{kA}$ to the breaker circuit external to the liquid helium inductive storage coil dewar. The vacuum in. terrupters are then opened and draw a low voltage arc for several milliseconds until the gap spacing is sufficient to hold oft the high voltage that develops during the transfer cycle. At this time, the counterpulse is trigfered trum the $C_{1}$ capacitor bank. The $26-\mathrm{kA}$ current in the interrupter is commutated through zero at the proper rate as determined by $\mathrm{L}$ : and $C$ : to allow for deionization and complete arc interruption. The current then transfers resonant]y with some small cable or transmission line losses from the storage coil module through the transfer capacitor into the compression coii. At the end of the 0.7 -ms transfer period the fast crowbar $B_{4}$ is closed, trapping the current and energy in the compression coil. Shortly thereafter, the slow crowbar $\mathrm{B}_{5}$ closes. relieving the $B_{4}$ crowbar. The crowbar headers wiil be designed in such a way that with minor cable changes slow crowbar $B_{i}$ can be connected between the compression coils L.2, if desired. This creates a series circuit assuring equal $\mathrm{dB} / \mathrm{dt}$ decay in the full plasma torus.

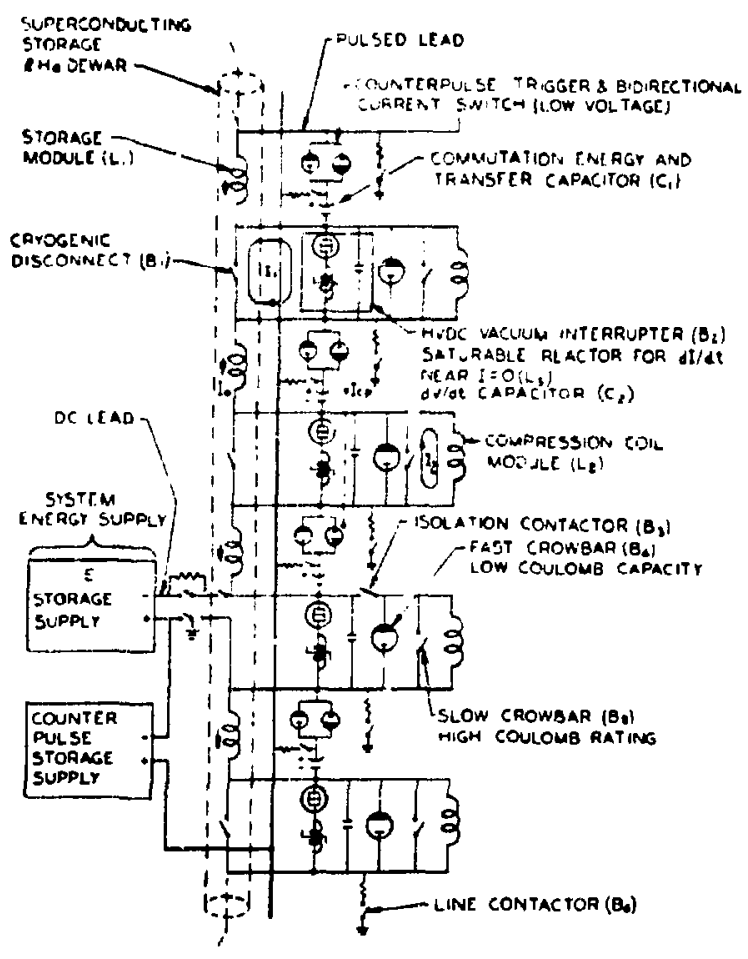

Fig. 5.t-5.

METS-SFTR circuit diagram. 
TABLE 5.4-1

\section{METS-SFTR CIRCLIT COMPONENTS}

\section{Encrgy Supply}

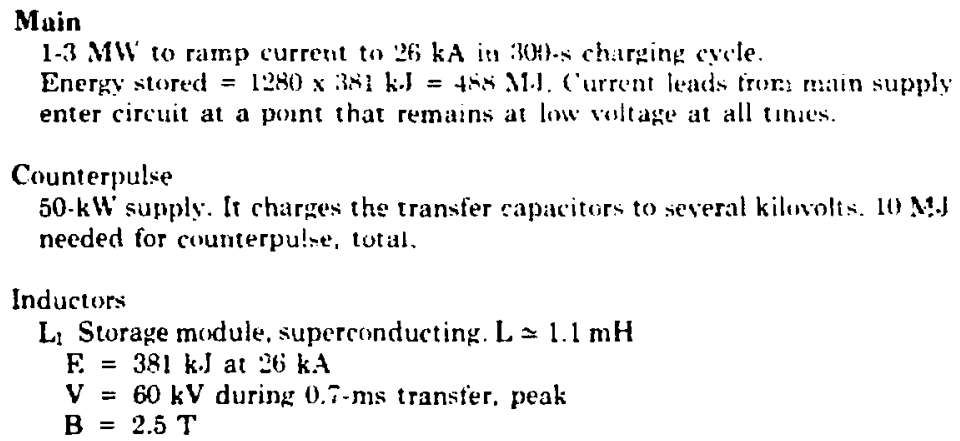

L. Compression module, multiturn copper. $L=1.1 \mathrm{mH}, 14.75 \mathrm{~cm}$ toroidal circumference.

$$
\begin{aligned}
& B=5.5 \mathrm{~T} \text { in } 34 . \mathrm{cm} \mathrm{i.d.} \\
& \mathrm{L} / \mathrm{K} \leq 2 \% \mathrm{~ms} \\
& \mathbf{E}=345 \mathrm{~kJ} \text { when } \mathrm{B}=5.5 \mathrm{~T} \text { inside bure. }
\end{aligned}
$$

Io Saturable reactor to allow preferred $\mathrm{dl} / \mathrm{dt}$ near $\mathrm{l}=0 \mathrm{during}$ commutation.

Parameters to be determined.

\section{Capacitors}

$C_{l}$ Transfer capacitor sets transfer time and peak voltage during tran fer.

It is reverse charged to $\leq 5 \mathrm{kl}$ to supply counterpulice current.

$$
\begin{aligned}
& C_{1}=100 \mu F \\
& V=60 k V
\end{aligned}
$$

C. Transient capacitor to handle the overcurrent during commutation and iimit solsage on HVIC interrupter during deionization perion. several tens of microseconds. The small series damping resistor eliminates ringing.

\section{Breakers, Contactors, Croubars}

$B_{1}$ Cryngenic disconnect within liquid helium cryostat. It cat ries the char:ing cycle current to $26 \mathrm{kA}$. It is to be designed for a power loss $<24$ W at full current. Resistance $\leq 3.5(10)^{-k}$.2.

$B_{2}$ HVLC: sacuum interrupter. or equivalent. Closes just hefore $B_{1}$ disconnects, current transters into the pulsed lead ambient temptrature loop to B.

Bu Isolation contactor. Reinoves energy-storage voltage from compression coil module during charging cycle.

B, Fast crowbar. Crowbars compression coil module at peak current. $(\mathrm{I}=26$ C/ms.)

$B_{\text {S }}$ Slow crowbar. Relieves $B_{4}$ after several milliseconds. Voltage drop ecross each $B_{;}$is insufficient to maintuin $B_{;}$conduction, extinguishing all $\mathrm{B}_{1} ` \mathrm{~s}$.

B. Electrically operated line cuntactor. The series resistor provides the proper $\mathrm{L} / \mathrm{R}$ to discharge the storage coil in a short time it the cryogenic disconnect $B_{1}$ fails co open. 


\subsubsection{Storage Coils}

Superconducting energy-storage coils, each storing $381 \mathrm{~kJ}$, form the basic element of the SFTR energy source. One thousand two hundred and eighty of these are to be located around the plasma torus, 10 each in 128 vertical axis, plastic, cylindrical dewars at a radius of $57.2 \mathrm{~m}$. Separation of the coils into groups of 10 in individual dewars permits system construction and tesring as well as operation by modules or by sections of a number of modules without interference with work being done on other segments of the system. Maintenance can be performed by replacement of preassembled sets of st orage coils with their electrical leads, cryogenic disconnects, hydraulic actuators, etc.. attached to a dewar lid. If necessary, individual dewars can also be easily replaced.

Design of these superconducting energy-storage coils considers:

1. The various components requiring energy to establish the storige requirements:

2. The techrologically atiainable current and voltage levels for operation of the coils:

3. The superconducting cable design and the individual superconductor strand design as they relate (1) the magnetic field wi!hin the coil, enerory losses during charge and discharge, and the liquid helium temperature level:

4. Magnetic forces and stresses;

5. Physical dimensions and variation of the length

as a function of location in the 10-coil stack;

6. Inductance matching to the compression coils:

7. Electrical insulation; and

8. Forces between coils in adjacent dewars.

The $381 . \mathrm{k}$.J eneigy stored in each coil is based upon the four major components listed in Table 5.4 2. The largest item. $325 \mathrm{~kJ}$. is the energy delivered to the compression coil to provide the $5.5-T$. thetapinch plasma adiabatic heating magnetic field and to maintain the lield for a $270 \cdot \mathrm{ms} \mathrm{L} / \mathrm{R}$ decay time for the compression covil. With the attached circuit impedance. this reduces to a $250-\mathrm{ms} \mathrm{l}$, $/ \mathrm{R}$ time constant. 'The compression coil is described in detail in sec. i. 4.3 .6 .
Multipole effects create both helicai and sinusoidal radial modulations of the plasma along the discharge tube torus. Compression coil design to accommodate this perturbation increases the energy-storage requirement by $6^{\prime} ;$.

Transfer losses in resistances of cables, etc., but excluding the transfer interrupter losses, increase the required stored energy by $5 \%$.

Losses in the transfer interrupters (vacuum breakers). cryogenic disconnect, and transient leads increase the energy-storage requirtment by $6.3^{\circ} \mathrm{i}$. Descriptions of these circuit elements are given in subsequent sections.

Pulsed, short transfer period, high current-high voltage superconducting coils have been operated at LASl. This and related experience indicates that reliability of electrical components in the energy: transfer circuit and the overall transfer circuit design poses rapidly increasing problems at voltages much in excess of $60 \mathrm{kV}$.

With this maximum voltage limitation. studies of superconducting METS systems indicate a brciad cost minimum at storage coil fields in the 5-T range. Fields reduced to $2.5 \mathrm{~T}$ increase the cost by less than $2 " ;$. Because of the complex structural and electrical insulation problems at the ends of the coils for multilaver coils, a single-laver, edge-wiound coil is to be used. To obtain even the low end of the 2.5-T and 5 $T$ range for a single laver coil and to achieve an adequate ampere-turns value, the superconductor must have a 2.41:1.0 rectangular aspect ratio and carry 26 $\mathrm{kA}$ to have the proper energy stored wit b reasonable coil dimensions. This cumbination can be developed. given the state of the art for $\mathrm{Nb}$. Ti superconductors. For these conditions, the peak transfer voltage is 60 $\mathrm{kV}$.

The superconductor to carry the $26 \mathrm{kA}$ is built of individual st rands of twisted multifilament $\mathrm{Nb}-\mathrm{Ti}$ in a complex $\mathrm{Cu}$. Cu-30 $\mathrm{Ni}$ matrix.

Energy loss requirements for the superconductor itself during charge and discharge have been set at less than $0.3^{\prime}$, of the stored energy. These losses arise mostly from hysteresis and eddy currents induced in the conductor during the storage coils"

TABLE 5.4-2

\section{ENERGY REQUIREMENT FOR MAGNETIC STORAGE COILS (kJ)}

Compression coil without multipole correction

Multipole correction. $66^{\circ} ;$

Transfer inefficiency, 5 ;

Transfer interrupter, cryogenic disconnect, and transient lead losses. 6.8\%
324.8

19.5

16.2

20.5 
cyclic operation. The $0.3^{\circ}$ ' criterion limits this heat load co about $10^{\circ}$; of the $4.5 \mathrm{~K}$ reirigeration capacity.

In the complex $\mathrm{Cu}, \mathrm{Cu}-\mathrm{Ni}$ matrix, each $\mathrm{Nb}-\mathrm{Ti}$ filament is surrounded by copper and then $\mathrm{Cu} \cdot \mathrm{Ni}$. The copper stabilizes the superconductor, and the relatively high resistance $\mathrm{Cu}-\mathrm{Ni}$ decouples the $\mathrm{Nb}$ Ti filaments. The twist rate of the strands, the size of the strands; the $\mathrm{Cu}, \mathrm{Cu}-\mathrm{Ni}$ to $\mathrm{Nb}-\mathrm{Ti}$ ratios, the size and number of $\mathrm{Nb}$. $\mathrm{Ti}$ filaments, the current density at operating temperature, and the number and transposition of the strands in the superconducting cable characterize the conductor. 'These parameters must be carefully specified to provide for the transport current of $26 \mathrm{kA}$ and to hold the energy loss below the $0.3{ }^{\circ}$; level. The possibility of using a low resistivity impure copper matrix conductor is not without merit. Energy losses during transfer could be low and such a conductor warrants exploration. The gross characteristics of the superconducting cable with a copper matrix are given in Table 5.4-3.

Because the energy-storage coils are arranged in groups of 10 as a solenoid. there is an appreciable field gradient at the ends of the solenoid. This gives an $\mathrm{I} \times \mathrm{B}$ compression force which is high in the end coils and integrates to $6.41(10)^{9} \mathrm{~N}$ over the entral plane of the solenoid. A 3.94-cm-thick inver coil tirm inade of a NEMA G-10 epoxy-fiber glass laminate gives an acceptable stress level of $5(10)^{8}$ $\mathrm{Pa}$. The maximum hoop force in the conductor occurs at the center of the solenoid and a structural shell support must be added over the conductor to hold it in place. A NEMA G-10 shell of $1 . \mathrm{cm}$ thickness will hold the conductor with a stress level (i) +110$)^{x} \mathrm{~Pa}$.

Physical dimensions of the energy-storage coils are determined by the energy to be stored, the field level at which it is to be stored, and, in turn, the current

TABLE 5.4-3

\section{SUPERCONDUCTING CABLE CHARACTERISTICS}

level of operation. For optimum transfer of energy. the storage coil inductance must match that of the compression coil it drives. The coil volume is fixed by matching this parameter. after the field and current have been fixed, as discussed above. Because of the finite solenoid length for 10 coils, the mutual inductance of the outer coils is low. These coils are iengthened to match the inductance to the compression coils. The electrical and physical parameters of the coils are given in Table 5.4-4. Number " 1 " coils are the innermost pair, and the number " 5 " coils are the outermost of the stack of 10 .

High voltage electrical insulation of the storage coils is provided in several ways. The conductor will have $0.76 \mathrm{~mm}$ of insulation between turns. The coil form material will be epoxy-fiber glass laminate. generally NEMA G-10, which has good insulating properties. The dielectric strength of liquid helium in which the coils are immersed is good. The electrical leads exit the coil at opposite ends. Alternate coils are reverse-wound. This permits the high voltage terminals of the anjacent coils to be close together.

Fringe fields at the plasma discharge tube for the 128 assemblies of storage coils located at a $57.2-\mathrm{m}$ radius are of the order of $1 \mathrm{G}$ if all coils are oriented with the same polarity and more than an order of magnitude lower if the polarity is alternated in adja. cent dewars around the circle. Either arrangement is acceptable.

Forces between adjacent dewars are largest during startup and testing when energy may be stored in only two sets of solenoids. For the reversed polarity situation, the attractive force will be of the order of $3500 \mathrm{~N}$, which is not excessive. Some extra structural support will be designed into the coil mounting.

\section{TABLE 5.4-4}

\section{ENERGY-STORAGE COII. PAIAMETERS}

\author{
Transport current \\ Operating current density \\ Short sample critical current \\ Cable cross-sectional area \\ Cable thickness \\ Insulation thickness, each face \\ St rand filling factor
}

$26 \mathrm{kA}$

$1.41(10)^{5} \mathrm{~A} / \mathrm{cm}^{2}$

$2.22(10)^{5} \mathrm{~A} / \mathrm{cm}^{2}$

$2.83 \mathrm{~cm}^{2}$

$1.08 \mathrm{~cm}$

$0.076 \mathrm{~mm}$

0.33

4.0

$\begin{array}{ll}\text { Energy } & 381 \mathrm{~kJ} \\ \text { Transport current } & 26 \mathrm{kA} \\ \text { Maximum operating voltage } & 60 \mathrm{kV} \\ \text { Maximum magnetic field } & 2.5 \mathrm{~T} \\ \text { Inductance } & 1.13 \mathrm{mH} \\ \text { Radius (mean) } & 33.64 \mathrm{~cm} \\ \text { Lengths - coils 1, 2, and } 3 & 57.9 \mathrm{~cm} \\ \text { coil } 4 & 58.9 \mathrm{~cm} \\ \text { coil } 5 & 67.4 \mathrm{~cm}\end{array}$




\subsubsection{Cryogenic Disconnect}

The crygenic disconnect is a very low resistance contactor which switches the 26-kA storage coil rurrent from its charging path through the disconnect to a low impedance path through the vacuum interrupter. Atter opening, the disconnect stands off the $60-\mathrm{kV}$ do pulse generated across its contacts.

The disconnect operates in liquid helium and will be located inmediately outside the bore of the storage coils it serves. I! wil! be operated by an arm connected to an actuator mounted on the lid of the storage coil dewar.

The disconnect will open fully in as short a time as practicable to minimize steady-state heating of the pulse leads. An opening time of 0.1 s or less has been selected for a design criterion. System cycling time will ailow the disconnect to be open for $600 \mathrm{~s}$ betore it begins to close. Closing will be slow. on the order of a few seconds. In the normal svstem cucle, the disconnect will remain closed during the storage coil charging period of $300 \mathrm{~s}$. The disconnect should be capable of being cycled 5000 times without signifjcant deterioration of performance.

From the literature ${ }^{l}$ it appears reasonable to expect that with contact pressures on the order of 2.7 $\mathrm{x} 10^{\bar{T}} \mathrm{~Pa}$ between carefully prepared, normal conductor surfaces of the proper materials, contact interface resistances as low as $10^{-9} \Omega$ can be obt ained.

Published information on the dielectric properties of liquid helium and cold gaseous helium indicates that a $20-\mathrm{mm}$ gap between the contacts should stand off $60 \mathrm{kV}$ with a reasonable margin of safety.

Accordingly, a disconnect is being designed that will have a contact area approximately $8 \times 10^{-3} \mathrm{~m}^{2}$, a contact spacing greater than $20 \mathrm{~mm}$ when open. and will be capable of applying contact interface pressures as high as $3.8 \times 10^{7} \mathrm{~Pa}\left(5.5 \times 10^{33} \mathrm{psi}\right)$. It is a toggle mechanism driving two moving contacts ugainst two fixed contacts and is illustrated in Fig. $5.4-6$.

The mechanism is designed for rolling contact between its highly loaded components during final contact closure. It will provide force amplification of approximately 10 to 1 . thereby keeping the external actuator to a size compatible with rapid opening and keeping the heat leak ciswn the actuator arm to a reasonable minimum. The mechanisms are designed to maximize actual surface areas in contact. Contact materials will be selected partly on the basis of previous work ${ }^{1}$ and partly from results of tests now in progress here at I.ASL.
Initial plans are to split each contact into two regions: cre of very low resistance which will open first; a secorid which will be connected to the first region by a very low impedance path and which will open last. Any arcing will occur only across the second region so that the contact surfaces of the first region will remain unscarred and uncontaminated. If necessary, a third contact region can be incorporated to reduce arcing. Magnetic arc quench also can be incorporated if experiments indicate the need.

Wiih maximum contact loading, the closing force required for the disconnect is $3 \times 10^{4} \mathrm{~N}\left(6.8 \times 10^{3} \mathrm{lb}\right)$. In the SFTR, 10 storage coils with their 10 disconnects are stacked vertically in each dewar. The 10 disconnects will be ganged. All 10 will be operated by a common arm which, in turn, is tied to a single actuator mounted on the dewar lid. With 10 aisconnects, an actuator must develop a closing force of up to $3 \times 10^{5} \mathrm{~N}\left(6.8 \times 10^{4} \mathrm{lb}\right)$. This is within a reasonable range for hydraulic actuators.

The moving parts of 10 disconnects, an actuating arm, a hydraulic piston, and a pneumatic piston are estimated to weigh $100 \mathrm{~kg}$. Calculations using a cosine displacement curve show that the maximum forse required to open the disconnect in $0.1 \mathrm{~s}$ is $3.2 \mathrm{x}$ $10^{3} \mathrm{~N}(14000 \mathrm{lb})$, a force well within the range of a reasonably sized pneumatic actuator.

A hydraulic-pneumatic system for actuating 10 disconnects is shown in Fig. 5.4-7. A hydraulic actuator is used to close the disconnects, and a hydraulic buffer cushions the opening shock.

\subsubsection{HVDC Interrupters}

The HVDC current interrupter modules in the METS-SFTR svstem serve to disconnect the inductive energy-storage series loop, thus initiating the energy transfer pulse into the plasma compression coil. There is one interrupter module for each st orage coil module, and a total of 1280 in the complete system. Ten interrupter modules are grouped into a submodule for compatibility with the system in cable runs and access for maintenance.

The interrupter module parallels the cruogenic discornect electrically through a pair of pulsed current leads into the liquid helium dewar. During a typical energy transfer cycle, the interrupter is closed mechanically to create en electrical short circuit around the cryogenic disconnect just before the disconnect is opened. During actuation of the disconnect, the $26-\mathrm{kA}$ storage current is diverted through the pulsed current leads into the external lonp containing the vacuum interrupter.

When the cryogenic disconnect has separated to a gap capable of withst anding $60 \mathrm{kV}$. the count arpulse 


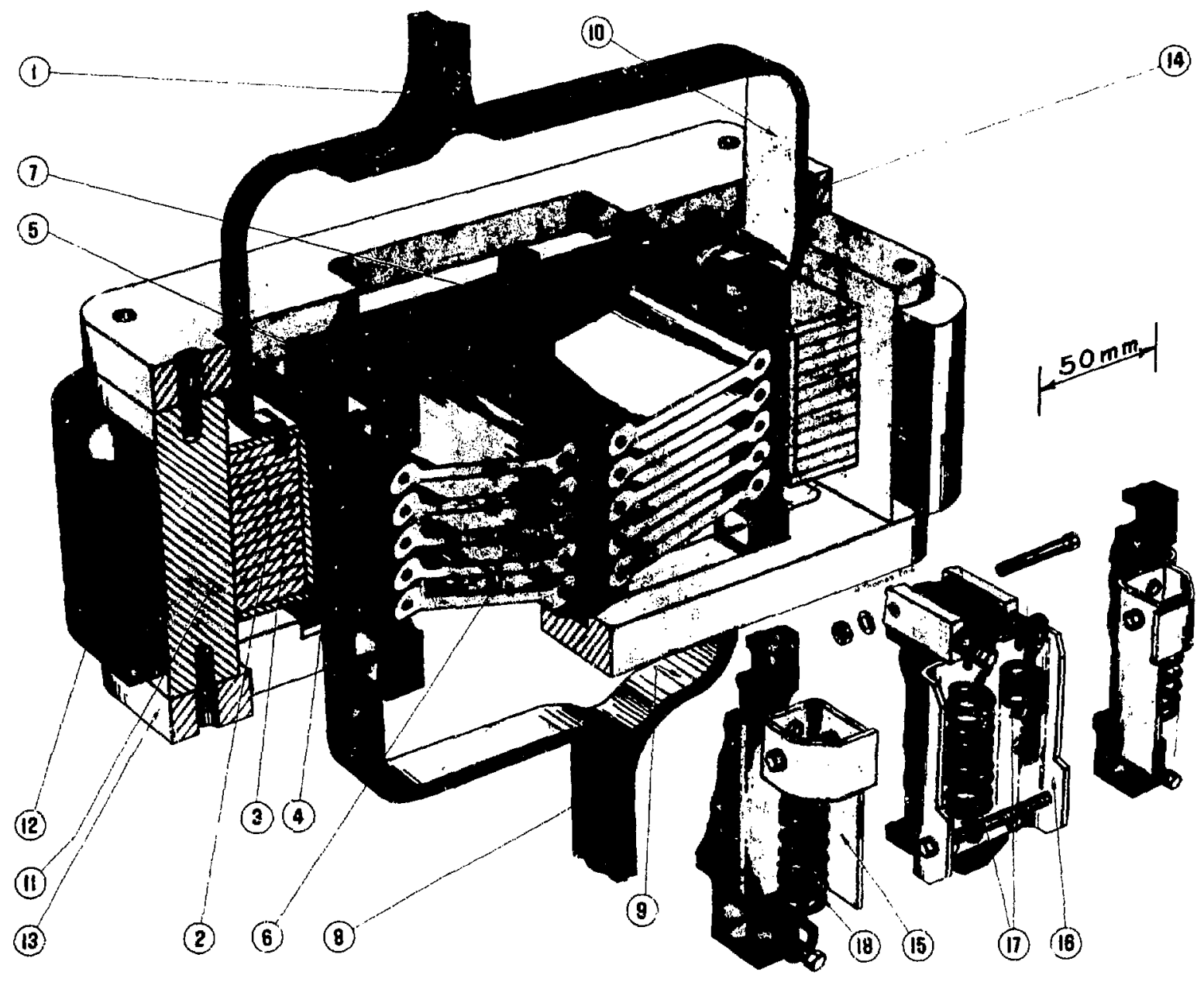

Fig. 5.4-6.

Crvogenic disconnecting switch: (1) and (10) stationary superconductors; (2) lnad distribution pad; (3) fixed contacts; (4) movable contacts; (5) contact slides/guides; (6) toggle bars; (7) operating arm and plate; (8) and (9) flexible superconductors; (11)-(14) structural members; (15)-(18) spring return mechanism details.

circuitry initiates fast current interruption of the vacuum breakers, presenting an open circuit to the storage coil current. The $0.7-\mathrm{ms}$ energy transfer pulse begins at this point, and the interrupter completes its function.

The major components within the interrupter module are shown in Fig. 5.4-8. A vacuum interrupter of a design used in the ac power distribution industry is the interruption element. The vacuum bottle actuators are capacitor discharge solenoid drivers triggered by the submaster control. All other components are passive, with the exception of the triggerable fuse.
Each of the four vacuum interrupters conducts 13 . $\mathrm{kA}$ current and holds off $30 \mathrm{kV}$ voltage after interruption. The saturable reactor, $\mathrm{dV} / \mathrm{dt}$ capacitor, and damping resistor operate when the counterpulse current is applied to off-commutate the arc current (see Sec. 5.4.3.5). The voltage grading network assures equal voltages across all interrupters during the energy transfer pulse. A vacuum breaker has already been successfully tested to a $17.0-\mathrm{kA}, 44-\mathrm{kV}$ level.

The 100- $\mu \mathrm{H}$ bucking transformer serves two important purposes in successful interrupter operation. 


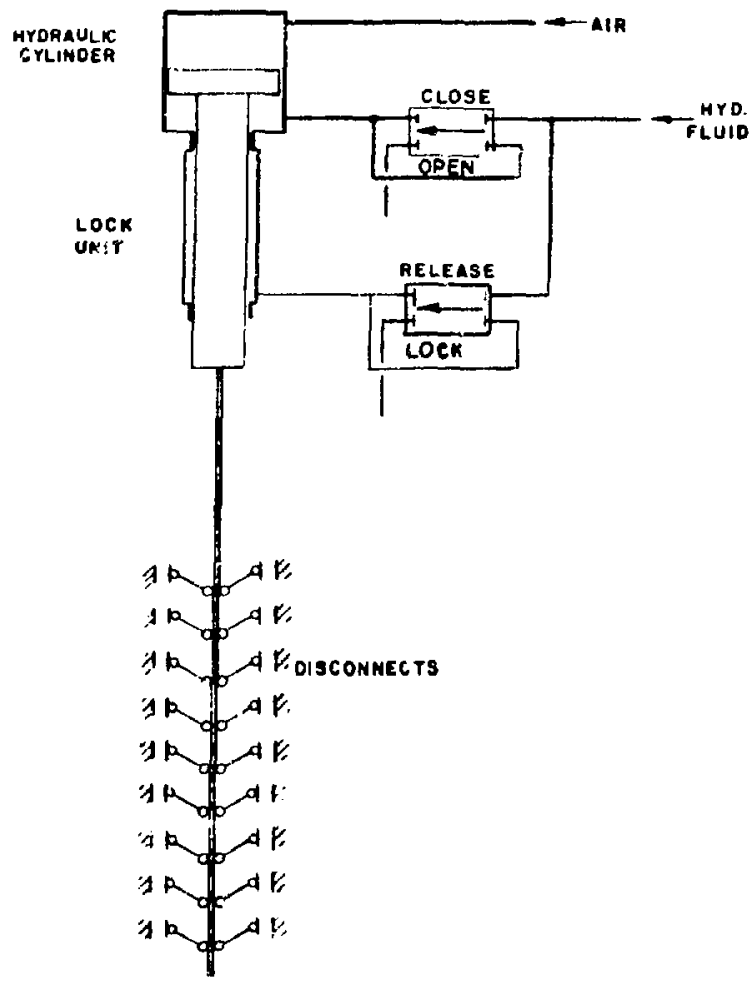

Fig. 5.4-7

Mechanism for actuating the disconnect.

First. it assures equal currents in both interrupter legs during current transfer from the cryogenic disconnect circuit. where current sharing is determined mainly by leg inductances. Joule heating starts evenly in each interrupter during the $0.1 \mathrm{~s}$ before the cryogenic discounect separates, During this time. resistive stabilization through contact heating assures current sharing. All interrupters enter the contact break and arc phase with uniform contact thermal conditioning. A fraction of a millisecund iitter is to be expected in the mechanical actuation of contact breaking between interrupters. The second purpose of the transformer is to impede inductively a current outrush from one leg due to a premat ure aic voltage strike, and to hold the current balance within $\pm 1 \mathrm{kA}$.

The triggerable fuse is a one-shot replaceable safety device to assure successful current interruption. Since the SFTR plasma rynamics require a fairly uniform rise in the conpression magnetic field from all modules, an unsuccessful current interruption in one module leading to complete loss of field rise in

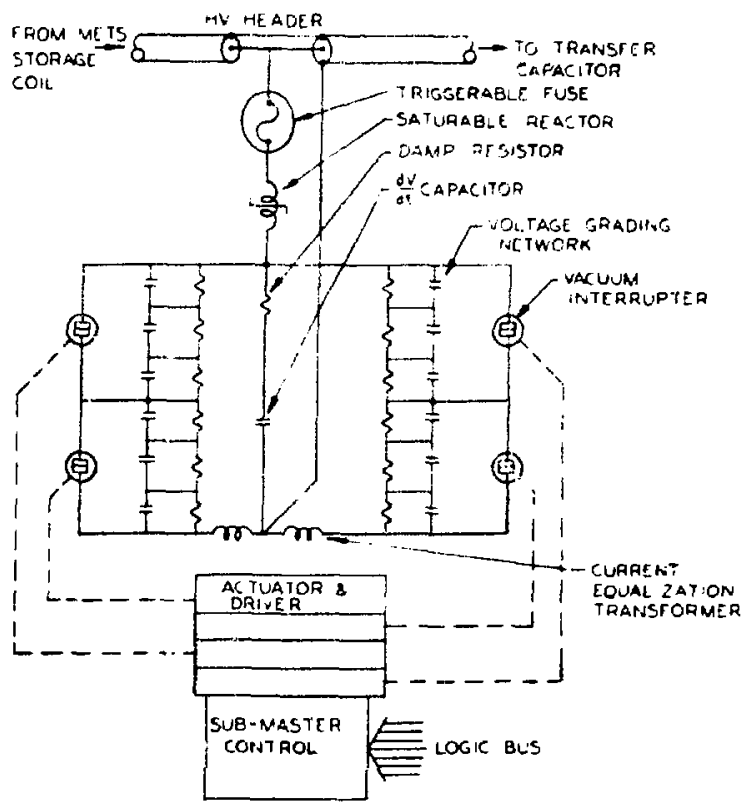

Fig. 5.4.8.

IivDC interrupter module.

it: corresponding compression coil would dest roy the plasma containment. The submaster control references each module's energy transfer voltage waveform to the st andard waveform. Voltage lag indicates unsuccesstul current interruption, and a drop or loss of voltage indicates an arc restrike. In both cases. the fuse would be opened fast enough to assure plasma containment.

An arc restrike is potent, $: v$ the most troublesome failure mode of the system iswever, the arc current rise is impeded during the first several microseconds by the saturable reactor, time enough to detect such a failure and trigzer the fuse. A current shunt or Rogowski loop in each module may provide a much fastes. more reliable failure indicator.

The mechanical layout of a 10 -unit interrupter submodule is shown in Figs. 5.4-9 and 5.4-10. The high current bus runs are so located as to minimize inductance and to isolate high voltages from grounded structures. The submaster control and triggerable fuses are easily accessible. The high voltage header is located nearest the overhead cable runs. A complete module can be removed from the main enclosure and exchanged to minimize downtime during the occasional maintenance expected on interrupter bottles and actuators. 


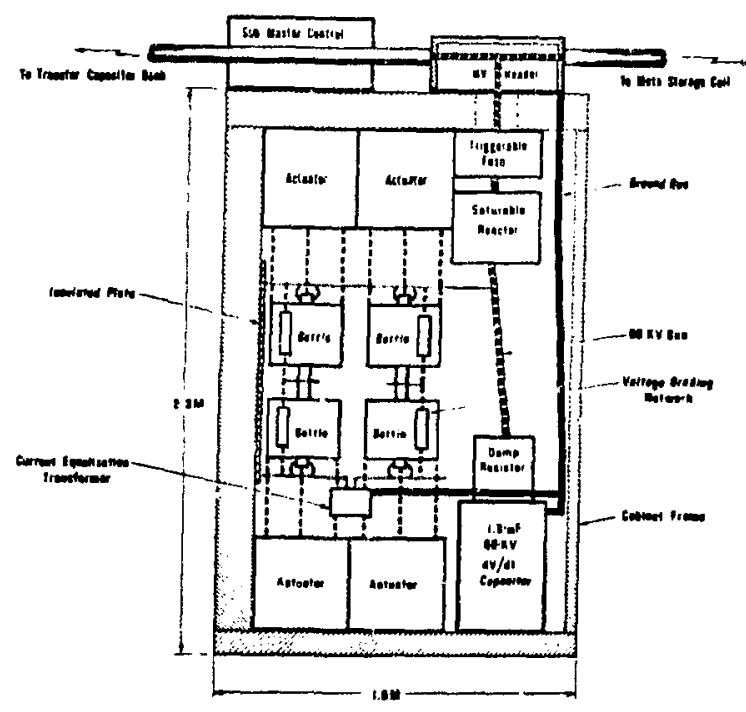

Fig. 5.4-9.

Physical lavout of an interrupter module.

\subsubsection{Interrupter Counterpulse System}

A vacuum interrupter conducting a high current arc requires that the current be forced through zero to regain its high voltage vacuum dielectric st rength. In the de current circuit of METS, as opposed to an ac circuit, the current zero within the interrupter must be provided artificially by switching in a countercurrent from an external source.

The arc extinction parameters of the countercurrent required to maintain $I=0$ in the interupter during a time dt to allow arc plasma dejonization and dispersal set a coulomb value $Q=I \mathrm{dt}$ to be met by the current source. A caparitor discharge circuit used as the current source will require nunimum energy $Q^{2} / 2 C$ with maximum capacitance.

The addition of ignitron switches to each bank of transfer capacitors results in a minimum cost counterpulse circuit. No udditional capacitance is needed heyond that required for the energy transfer circuit and one $d V / d t$ capacitor per module. With

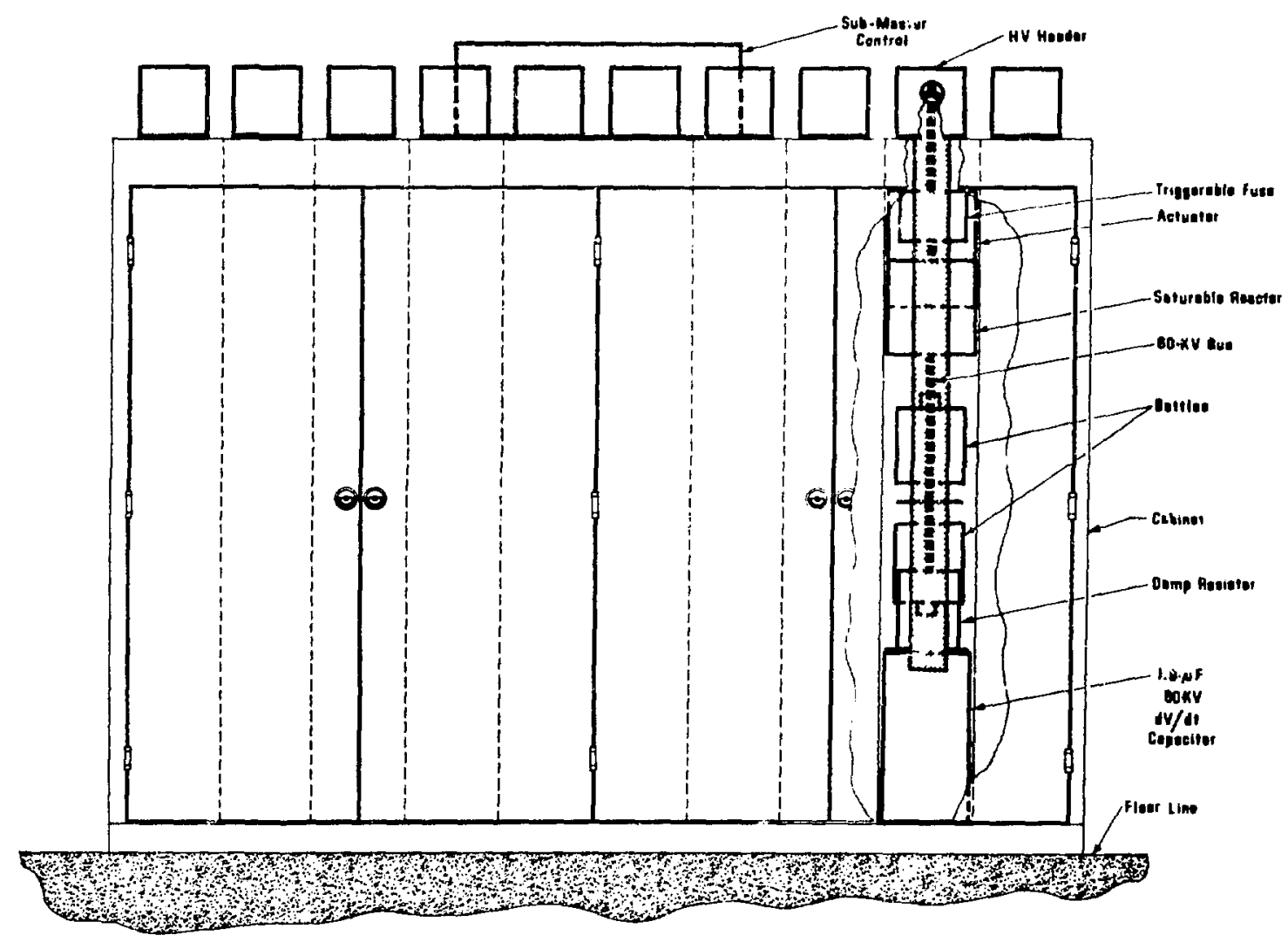

Fig. 5.4-10.

Interrupter module. 
this large capacitance, a minimal counterpulse energy is required.

The transfer capacitors (Fig. 5.4-5) are reversecharged to several kilovolts befoce the energy transter cycle. The capacitor discharge ignitron switch is closed when the $26-\mathrm{kA}$ storage current is tlowing in the storage coil and interrupte: branches if the circuit and when the interrupter has been mechanically opened and is arcing. Because the inductive impedance of the interrupter leg is negligible compared to the storage coil and compression coil branches, virtually all of the capacitor discharge current flows into the interrupter, bucking the 26-kA de current. The i.tterrupter current drops rapidly to the $1-\mathrm{kA}$ level when the saturable reactor becomes unsaturated, forcing a lower $\mathrm{dI} / \mathrm{dt}$ as the current approcaches $\mathrm{I}=0$.

As the current goes through $\mathbf{l}=0$ the interrupter stops conducting. The arc begins to deionize, and $-i 0) \mu$ s are required to reestablish vacuum dielect ric strength. During this time, large voltage transients would cause arc restrikes. A small $\mathrm{dV} / \mathrm{dt}$ capacitor is placed across the interrupter to absorb the counterpulse circuit over-current following the $I=0$ transition. thus limit ing the reverse voltage transient. The damping resistor quenches the high frequency parasitic voltage oscillations impressed on the interrupter by the saturable reactor and $\mathrm{dV} / \mathrm{dt}$ capacitor.

A saturable reactor is used instead of a standard inductor to furt her minimize the required counterpulse energy and. hence, the reverse volt age applied 16 the transfer capacitors. High values of $\mathrm{d} I / \mathrm{dt}$ can be tolerated by the interrupter until close to the $I=0$ interruption and dejonization time. The detailed physic's of the arc plasma interruption process sets limits on the $\mathrm{dl} / \mathrm{dt}$ near $\mathrm{I}=0$, the quiescent recovery time, and the $\mathrm{dV} / \mathrm{dt}$ following $\mathrm{I}=0$. "The st ray inductance of the count erpulse loop will limit $\mathrm{dL} / \mathrm{dt}$ to less than $1 \mathrm{kA} / \mu \mathrm{s}$ until near $\mathrm{I}=0$ when the saturable reactor comes ou of saturation. The large inductance of the saturable reactor at low current will limit the $\mathrm{dl} / \mathrm{dt}$ to less than $100 \mathrm{~A} / \mu$ s as the current goes $t$ hrough zero, within the ideal range for vacuum interrupter reliability. Since the counterpulse circuit has laros inductance only near $I=0$, the energy required from the transfer capacitor is minimal.

Typical rates of recovery voltage for vacuum breakers are in excess of $1 \mathrm{kV} / \mu \mathrm{s}$. The maximum rate of recovery voltage following interruption within the METS energy transfer circuit is $95 \mathrm{~V} / \mu \mathrm{s}$ on each vacuum breaker. It is extremely unlikely that an arc restrike would occur with the METS voltage waveform.

The $\mathrm{dV} / \mathrm{dt}$ capacitor is a standard Scyllac $60-\mathrm{kV}$ capacitor of $1.8-\mu \mathrm{F}$ value. This size represents less than $2 \%$ of the transfer capacitor size, and the capacitors are readily available. The capacitor will hoid the voltage on the breaker during deionization below several kilovolts even with a $1-k A$ excess counterpulse current beyond $\mathrm{I}=0$.

Research on vacuum interrupters at the $12.5-\mathrm{kA}$, $30-\mathrm{kV}$ level indicates that the dam. ping resistor is not necessary. The final determination will be made by reliability testing. So far, the characteristic parasitic oscillation of $500-\mathrm{V}$ amplitude has not caused a single loss of interruption.

\subsubsection{Compression Coil}

The compression cuil which provides the magnetic field for adiabatic heating of the theta-pinch plasma is in the form of many small pancake coils made from copper st rap insulated with layers of epoxy and fiber glass. Each pancake coil is energized by a separate module of the METS system to provide the magnetic field for $20 \mathrm{~cm}$ along the toroidal discharge tube. Each pancake coil is actually a so-called "split-pancake" coil. i.e.. it is made of two coils, une wound clockwise and the other counterclockwise with an electrical connection at the inside turns. Thus, all other electrical connections can be con. veniently brought to the suriace at the outer turns of the two coils of the split pancake. Each pancake coii has 18 clockwise and 18 counterclockwise turns.

Insulation of the coils will be conservative. A thickness of $1.5 \mathrm{~cm}$ of epoxy-fiber glass will insulate the clockwise from the counterclockwise turns within each pancake. and $1.0 \mathrm{~mm}$ of epoxy will be vacuum impregnated into fiber-glass cloth between turns. The insulation thickness along the outside radius and aiong the side of the pancake cortaining the high voltage terminal will be $1.5 \mathrm{~cm}$. whereas in sulation thickness on the inside radius and low voltage side need be only $0.75 \mathrm{~cm}$ to stand off half the peak voltage.

Radiation damage effects to the insulation after several years of operation under pessimistic dose rates are still well within the levels found tolerable for use in high-energy particle accelerarors. The average hoop stress on the coil at $5.5 \mathrm{~T}$. including dynamic stress, is only $4.82(10)^{\top} \mathrm{Pa}(7 \mathrm{kpsi})$, which puts half-hard OFHC copper below the fatigue endurance limit by at least a factor of 2. Additional support will be provided from the epoxy-fiber glass insulation.

Average dimensions of a completed pancake coil with epoxy-fiber glass insulation are $34 \mathrm{~cm}$ i.d.. 55.7 $\mathrm{cm}$ o.d., and $14.8 \mathrm{~cm}$ length. The copper strap used in the conductor is $3.8 \mathrm{~mm}$ thick and $5.5 \mathrm{~cm}$ wide. Energy stored in each compression coil amounts to $345 \mathrm{k} . J$. including the field energy stored outside the bore in the windings and gaps between coils. 
The compression coil must be shaperi to follow the dimensional variations of the shock heating coils which themselves are shaped to provide the mulripole tields for plasma equilibrium. These multipole fields will alter the plasma surface while still maintaining its cross section as a circle. The center of this -ircle describes a helix tiong the turoidal discharge tube. with a wavelength of $3.2 \mathrm{~m}$. Relationships betceen the flux surfaces at the plasma edge and the inner surface of the compression coils have been derived $: 1: 3$

For the present choice of plasma parameters. the amplitude of the radial moduation on of the tux surface at the compression coil is $0.7 \mathrm{~cm}$. while the radius of the helieal modulation $\delta_{1}$ is $3.1 \mathrm{~cm}$. lncorporation of these spatial modulations into the compression coil complicates its construction only minimally. Several mandrels of different sizes will be needed for winding the pancake coils. and each stuccessive pancake will need to be oftset radially from its neighbor by about $7.0 \mathrm{~mm}$.

Table $5.4-5$ is a summary of the compression coil design parameters.

TABLE 5.4-5

\section{SUMMARY DESCRIPTION OF COMPRESSION COIL DESIGN}

\section{Parameters of Pancake Coils}

Type of coil

Mean outside diameter

Mean inside diameter

Length

Number of turns

Magnetic field

Peak voltage

Peak current

Total inductarice

L/R decay time

Amplitude of $\delta_{0}$ modulation

Amplitude of $\delta_{1}$ modulation

Copper conductor width

Conductor thickness

Stored energy

\section{Materials Required}

Total number of pancake coils

Copper for each coil

Epoxy for each coil

Total mass of copper

Total volume of epoxy
$60 \mathrm{kV}$

$24.3 \mathrm{kA}$

$1.10 \mathrm{mH}$

$0.27 \mathrm{~s}^{*}$

$0.7 \mathrm{~cm}$

$3.1 \mathrm{~cm}$

$5.5 \mathrm{~cm}$

$3.8 \mathrm{~mm}$

$345 \mathrm{~kJ}$

$$
\begin{gathered}
1280 \\
92.6 \mathrm{~kg} \\
9.84 / \\
1.18 \times 10^{5} \mathrm{~kg} \\
1.25(10)^{4} /
\end{gathered}
$$

\subsubsection{Fast and Slow Compression Coil Crow-}

har Switches

The crowbar switches used in the sirtem transform the L,-( - L METS transfer circuit to an L. $\mathrm{R}$ circuit with an $\mathrm{I} / \mathrm{R} \geq 2 \mathrm{20}) \mathrm{m}$. This is acconiplished by shorting f(cowbarring) the compression coil at time of peak current in the compression coil with a dual ignitron, metai-to-metal switch design The ignitrons are arranged to carry the initial corrent tfatst compression soil crowbar switchl and a metal-tometal type switch to carry the final total current tslow compression coil switchl. An attempt is being inade at LASL, to increase the coulomb rating of the comnercial-size D ignitron so that the ignitrons will be able to withstand the maximum coulomb load posible during the $L / R$ decay cycle.

The critical operating parameters of the crowbar ignitron are to maintain $26 \mathrm{kA}$ for a 10 -rns $\mathrm{L} / \mathrm{R}$ (ime, which is equivalent 10250 (C. and to withstand a pulse voltage of $60 \mathrm{kT}$. Single ignitrons have been tested as 400-(c. 15-k\%, 2-ms? switches: hence. a series arrangement of ignit rons will suffice o provide the necessary crowbar swit'h. Fach compression covil module will have four ignitrons arranged in series and installed to fire automatically ihrough solid state rectifiers in series with each of the ignitron igniters. The header will be designed so that the crowbar can be applied directly acrosis the compression coil for single coil operation: and. with minor reconnection. the crowbar can connect the compression coils in a series or loop mode if this proves to be the desired circuit. The metal-to-metal crowbar will be a commercially available switch capable of closing within $10 \mathrm{~ms}$, withstanding the SFTR $6(0 . \mathrm{kJ}$ pulse voltage. and carrying the $25-k A$ current with a contact resistance of $100 \mu \mathrm{\Omega}$.

\subsubsection{Charging Power Supply for Storage Coils}

*0.25 ms when connected to crowbar circuit.

The METS storage coils will be charged to $26 \mathrm{kA}$ in $300 \mathrm{~s}$ by using a $125-\mathrm{V}, 26-\mathrm{kA}$, dc power supply. The power supply probably will consist of a 13.2. $\mathrm{kV} / 480-\mathrm{V}$ distribution transformer. $480-\mathrm{V}$ cont actor. 6- $\phi Y$ Y $Y$ transformer and three modules, $125 \vee$ and 9 kA SCR each. The duty cycle will consist of $300 \mathrm{~s}$ charge and $600 \mathrm{~s}$ off in the most optimistic mode. The control system shall permit remote operation from a central cont rol area and shall provide current and voltage regulation in a flexible manner so that maximum current out put and rate of charging each storage coil can be monitored and adjusted. The power supply is to be connected to the storage coil through a normally open charging switch. The charging switch is to be connected in parallel with an 
Hectrolvic dump resincor capable of abserbing the stured enerery in the event af a power failure or a desire to cump the stored energy. The normally eneen switch would be clused whale the system is being charged. and would open only under emergency situations.

As is characteristic of charging superconducting storage coils, the circuit resistance is a low value comsinting primarily of do bu resisiance and some loid resistance. The load inductance is quite high with L storage $=1.4 \mathrm{H}$, and the maximum voltage rating is determined by the rate of change of load curre'ne and loat inductance; therefore $I=26 \mathrm{kA} / 300)$ $s=86.7 \mathrm{~A} / \mathrm{s}$ and $\mathrm{V}=1 . \mathrm{t}=1.4 \times 86.7=1.2 \mathrm{~V}$. A power supply rated at $125 \mathrm{~V}$ and $26 \mathrm{kA}$ will supply the requirements with some spare capacity and the total $121 \mathrm{~V} \times 26 \mathrm{kA}=3.1 \mathrm{MW}$ peak will not exceed the power distribution sistem capabilities. In addition. Wpical charging characteristics of superconducting systems call for a higher $i$ initially. with a decreass in $\dot{I}$ at the end of the charging period. This will permit a substantial decrease in the peak rurrent power, particularly if the power supily is designed (o) have a volt age rating as high as 200$)$ for the early charge period.

The operating sequence of the power supply consists of closing the contaclor and energizing the rectifier with an input volt ange determined by the preset charge rate and maximum hadiett ings. While the load coil current increases. the controller will automatically decrease cutput voltage until the preset current level is achieved. At that time the power supply output voltage will be only a tew vilts... enumgh (1) offeet the IR drop of bus and lead resistances. The charge complete signal will be transmitted upom achieving the preset current level and withir 10 s the firing sequence will be initiated. Aiter a normal shot the contactors will open and the powersupply and controller wili be reset for the next shot. In the event of a delaved test. the current can continue to circulate in the charging loop for many hours. limited to the $I / R$ decay time $(15 \mathrm{~h})$ of the charge loop. As mentioned previously, the wistem 'ncrgy can be dumped more rapidly by opening the charging switch. thereby inserting the dump resistor in series with the storage coils.

\subsubsection{Counte:pulse Storage Power Supply}

The count erpulse storage power supply consists of one or two capacitor charging supplies carried over from the Phase I system as described in Sec. 5.t.1.

Approximately $10 \mathrm{M}$.l total energy must be supplied to the transfer capacitor banks for the counterpulse energy: The energy is delivered in $300 \mathrm{~s}$ during the same period that the superconducting eniergy-storage sistem is charged. Whenever a fault "cours during the enersy-strrage ramp and a fiat (tump) is required. the counterpulse circuit is set with the proper reverse woltage for current incerruption.

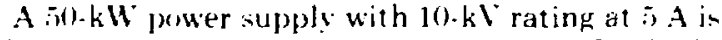
adequate to charge all tranafer capacitor banks in parallel. Remole programming will be required in make the charging voltage follow the entrgy-storage current.

\subsubsection{1) Capacitor Transfer Bank}

The METS capacitor transter bank is used primarily to provide for efficient energy transfer from the METS storage coils to the load coils. The bank serves the secondary purporse of providing an chergy source for the counterpulse circuit isee Sec. i.4.3.51. A description of the bank is given in Sec. i.4.1. The main difference between Phase I, where the transfer bank is the primary energy source. and Phase II, where the METS storage croil is the primary energy source, is the connection scheme of the capacitors. Ltilized as the transfer bank with wital $\mathrm{C}=0.13 \mathrm{~F}$. energy $=230 \mathrm{MJ}$, each of the 1280 capacitor modules consists of 16 each. $15-\mathrm{k} l, 100-\mu \mathrm{F}$ capacitors with a total $\mathrm{C}=100 \mu \mathrm{F}$ per module. The (apaciturs are arranged in a 4 series by 4-parallel array and the module is connected to the transfer circuit through the counterpulse trigger and bidirectisnal current switch. The capacitor module is charged by the storage coil during the $0.7-\mathrm{ms}$ energy transfer pulse from storage coil to load coil. The module is charged to a maximum of $60 \mathrm{~kJ}$ during that pulse and the peak energy stored is $180 \mathrm{~kJ}$. Energy-storage capacitor banks have been used successfully for many years in LASL's CTR program, and experience with relatively large banks has shown that the total energy required per module is well within state-of-the-art techniques.

\subsubsection{Electrical Leads}

Two basic types of electrical leads will be required in the METS system: 128 pairs of $26-\mathrm{kA}$ continuous duty leads and 1280 coaxial-type $26-\mathrm{kA}$ pulse leads. The $26-\mathrm{kA}$ continuous duty leads will connect the bus of the storage coil charging power supply (see Sec. 5.4.3.8) to the storage coils and provide interconnection between storage coils and their adjacent dewars. To carry the rated $26 \mathrm{kA}$ in a continuous mode. the leads will necessarily be rather large. The 26-kA coaxial pulse leads are much smaller: however, the relatively large number of pulse leads required for the complete system introduces a significant source of heat load. The pulse leads connect each storage coil module with coaxial cabies to 
the corresponding HVDC interrupter. An additional lead complication for the system is the fact that the pulse leads will be required to withstand a $60-\mathrm{kV}$, 0.7 -rus pulse during the discharge sequence. This high voltage pulse introduces dielectric probiems that are considered in the lead design.

A. 26-kA Continuous Duty Lead: These leads will be similar to commercially available leads to 10 . kA current ratings. Each consists of a cylindrical conductor with a number of small-diameter cooling channels running the length of the conductor. The bottoms of the cooling channels are located above the liquid helium level and the helium gas is directed upward through the channels and out the top of the lead where it is collected and returned to the refrigerator system. The heat lood of these leads is $43 \mathrm{~W}$ per pair at the liquid helium level. A computer program has been used in the design of these leads to consider the steady-state heat transfer and fluid flow for evaluating the temperature and pressure profiles.

B. 26-kA, 60-kV Pulse Leads: These leads will be coaxial to limit the number of dewar penetrations and to linit the amount of mechanical stress present with conventional parallel leads. Figure $5.4-11$ is a representation of the pulse lead. Note that the center portion of the lead carries the $60-\mathrm{kV}$ pulse and that it is cooled by belium gas flowing through the center of the lead. It is surrounded by a dielectric medium that prevents voltage arcing 0 the outside ret:irn lead. Consideration must also be given to possible voltage tracking across the dielectric both at the lower end of the lead in the telium gas and at the upper end: therefore. sperial attention is given to providing a rather lengthy tracking path hetween the two leads. Any frost problem will be eliminated by adequate insulation in the upper region. The out er lead is cooled by passing helium gas around its outside through the channel between the outer lead and outer membrane. The upper portion of the lead is designed to comnect to an adjacent coaxial header for direct coaxial cable routing to the do interrupter.

The refrigeration heat load from each of these leads is calculated to be $0.5 \mathrm{~W}$ at the liquid helium level. Most of the heat load results from the fact that the lead is connected to the storage coil permanentIv, and the heat conduction along the lead from roum temperature to the liquid helium is far greater than any joule heating experienced during pulsed operation.

A computer program has also been used in the design of the pulse leads to consider the transient heat transter and fluid flow to determine the transient temperature profiles.

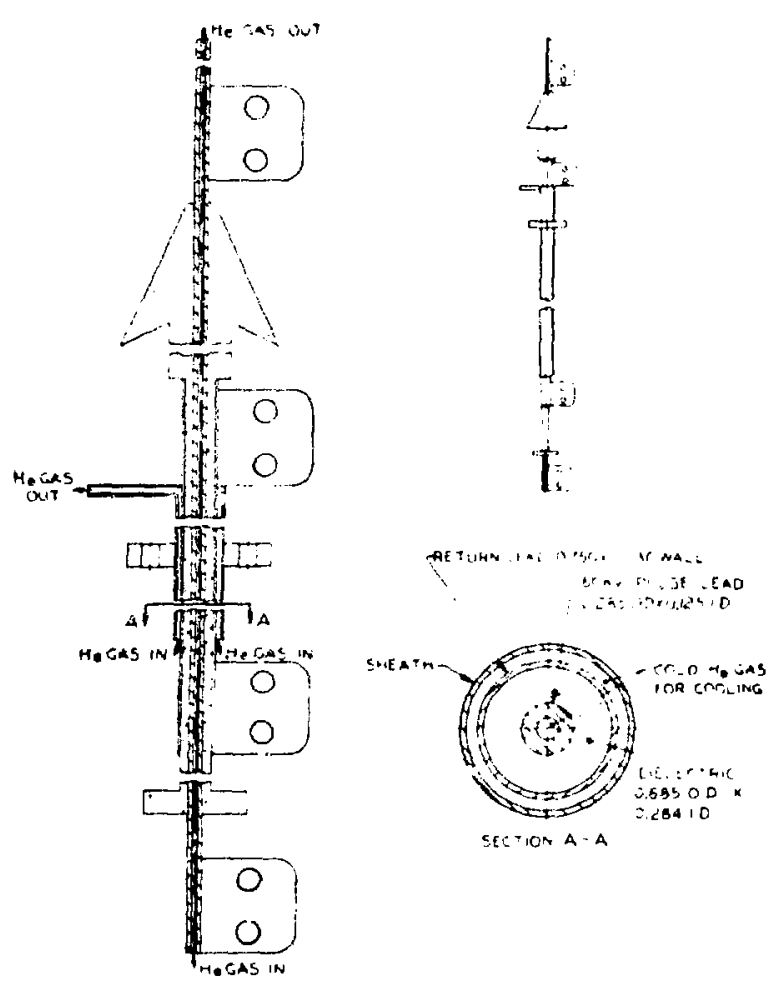

Fig. 5.f-il.

A $6(1)-k V, 26-k A$ coaxial pulse lead.

\subsubsection{Component Conrections and Cabling}

Most of the interconnections to be marte in the MFT'S sy'stem from the storage coil through the compression coil will be accomplished by the use of high volt age. pulsed-type coaxial cable. The types of eoaxial cables proposed were developed for use in the I.ASL. CTR program and consist of RO; 17/14 and RC; 19 cable. Bus-type interconnections will be used only. on the de charging system connecting the MFis power supply with the storage coil modules and the final connections of slow crowbar to compresision coil.

The entire METS circuit can be separated into five primary interconnection modes: (1) storage coil to HVDC interrupter. (2) HVDC interrupter to transfer capacitor bank. (3) transfer capacitor bank (o fast crowhar switches. (1) tast crowbar swit ches to slow crowbar switches, and (5) slow crowbar switch to compression coil. The schematic of Fig. $5.4-\overline{3}$ shows that each of the five interconnections has a different purpose and duty cycle.

1. Storage coil to HVDC interrupter: The cabling required for this connection must necessarily be of 
lise recistance and inductance because the eneryy dissipated in the arc that is initiated during the "lpening of the crygenic disconnect is a function of the resiviance and inductance in the storage coil to interrupter loup. These cables are also in series with the pulsed leads and must ordinarily be able to carry a $26-k \mathrm{~A}, 10 \mathrm{k}-\mathrm{ms}$ pulse without appreciable temperature rise. The most practical, lowest indurtance $6(0 . \mathrm{kV}$ pulsed cable is $\mathrm{RG} 17 / 14$. A total of 17 parallel RG: $17 / 14$ cables per storage coil is required in prevent a temperature rise per charge cycle of more than $15^{\circ} \mathrm{C}^{\circ}$.

2 . HVICC interrupter to transter capacitor hank: This contection requires a minimum amount of cabling in terms of current-carrying capacity. l'nder normal operating conditions. current will be carried for the relatively short transter time of $0 . \overrightarrow{7}$ ins. These cables must also carry the counterpulse current af $26 \mathrm{kA}$ for $100 \mu \mathrm{s}$. Thus the $26 \mathrm{kA}$ for $0.7 \mathrm{~ms}$ is the cont rolling requirement and can be handled by four Ro; $17 / 14$ cables. The use of $\mathrm{RG} 17 / 14$ cables is proposed becaldse of their unique tusing characteristics. Inder fault conditions they can be used as a fuse to protect the ircuit.

3. Transfer capacitor bank to fast crawbar switches: The conductor function is cimilar to that described in the previous section except that it does not carry any counterpulse current.

4. Fast crowbar switches to slow crowbar switches: The cabling for this section must have a large $L / R$ ratio to provide for the longest possible decay in the crowbarred compression coil loop. In additim, the cable must be capable of providing satisiactory current terminating characteristics for the large coulomb capacity of the crowbarred section. The RG 19 cable has these capabilitie: The RG; 19 cable with an $\mathrm{L} / \mathrm{R}$ ratio $=8.87 \times 10^{-5}$ is approximately two and one-half times better than RG $17 / 14$ with an $L / R$ ratio $=3.64 \times 10^{-5}$. Only eight RG 19 cables are required for the crowbar section: consequently, fewer cell wall penetrations are needeil.

5. Slow crowbar switches to compression coil: This connection will be acconılished with a commercial aluminum bus, $0.5 \mathrm{~cm} \times 25 \mathrm{~cm} \times 4.6 \mathrm{~m}$ long, :o provide for a negligible $\mathrm{L} / \mathrm{R}$ decay factor when compared to the $L / R$ of the compression coil itself. For each of the 1280 runs. $\mathrm{L}=45 \mathrm{nH}$ and $\mathrm{R}=$ $0.2 \mathrm{~m} !$ !.

Table 5.4-6 summarizes cable requirements for each section.

TABLE 5.4-6

\section{INTERCONNECTION MODE PER COMPRESSION COIL MODULE}

\begin{tabular}{|c|c|c|c|c|}
\hline Item & $\begin{array}{c}\text { Storage Coil } \\
\text { to HVDC } \\
\text { lnterrupter } \\
\end{array}$ & $\begin{array}{l}\text { HVDC Interr. } \\
\text { to Transfer Ca- } \\
\text { pacitor Bank }\end{array}$ & $\begin{array}{c}\text { Transfer Ca- } \\
\text { pacitor Bank to } \\
\text { Slow Crowbar } \\
\end{array}$ & 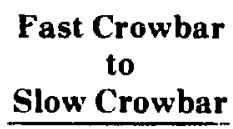 \\
\hline Tipe of atble & $\mathrm{RG} 17 / 14$ & $\mathrm{RG} 17 / 14$ & $\mathrm{RG} 17 / 14$ & RG 19 \\
\hline L chad requirement & $26 \mathrm{kA}-100 \mathrm{~ms}$ & $26 \mathrm{kA}-1 \mathrm{~ms}$ & $26 \mathrm{kA}-1 \mathrm{~ms}$ & $26 \mathrm{kA} \cdot 10 \mathrm{~ms}$ \\
\hline $\begin{array}{l}\text { Number of } \\
\text { pilrallel calbles }\end{array}$ & 17 & 4 & 4 & 8 \\
\hline $\begin{array}{l}\text { Temperature rise } \\
\text { ner pulse ( } 1 \text { ) }\end{array}$ & 15 & 2.7 & 2.7 & 6.8 \\
\hline $\begin{array}{l}\text { Fusing time for } \\
2(i k A(s)\end{array}$ & 3.6 & 0.2 & 0.2 & 3.9 \\
\hline $\begin{array}{l}\text { Total length of } \\
\text { ablo per module (m) }\end{array}$ & 18.3 & 4.9 & 3.7 & 3.7 \\
\hline Remist ance and induc- & $3.9 \mathrm{~m} \Omega$ & $4.4 \mathrm{~m} \Omega$ & $3.3 \mathrm{~m} \Omega$ & $1.46 \mathrm{~m} \Omega$ \\
\hline $\begin{array}{l}\text { tance equivalent } \\
\text { of run }\end{array}$ & $141 \mathrm{nH}$ & $160 \mathrm{nH}$ & $160 \mathrm{nH}$ & $129 \mathrm{nH}$ \\
\hline 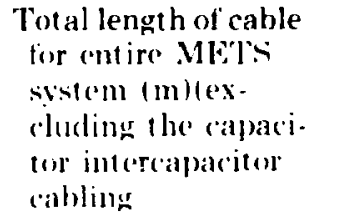 & 438000 & 27500 & 20600 & 41200 \\
\hline
\end{tabular}




\subsubsection{Dewars}

A series of 128 crlindrical, epoxy-fiber glass dewar: will provide the $4.5 \mathrm{~K}$ environment for the superconducting magnetic energy-storage coils and the associated cryogenic disconnects. The dewars will be lowated at a major radius of $57.2 \mathrm{~m}$ and will acconmrodate 1280 coil-cryogenic disconnect pairs 110 pairs per dewar). Fach dewar will have a pressure rating of $i$ atm.

The details of a storage coil dewar are shown in Fig. 5.4-12. The dewar walls and lid are constructed of fiber glass-reinforced epoxy. The selection of a specific resin sistem and a specific reinforcement configuration depends to some extent upon the process used to manufacture the structure, which in this case is filament winding. There are other strus:tural material requirements such as the dewar lids and vacuum pumpout ports, the construction of which is not amenable to the filanent winding

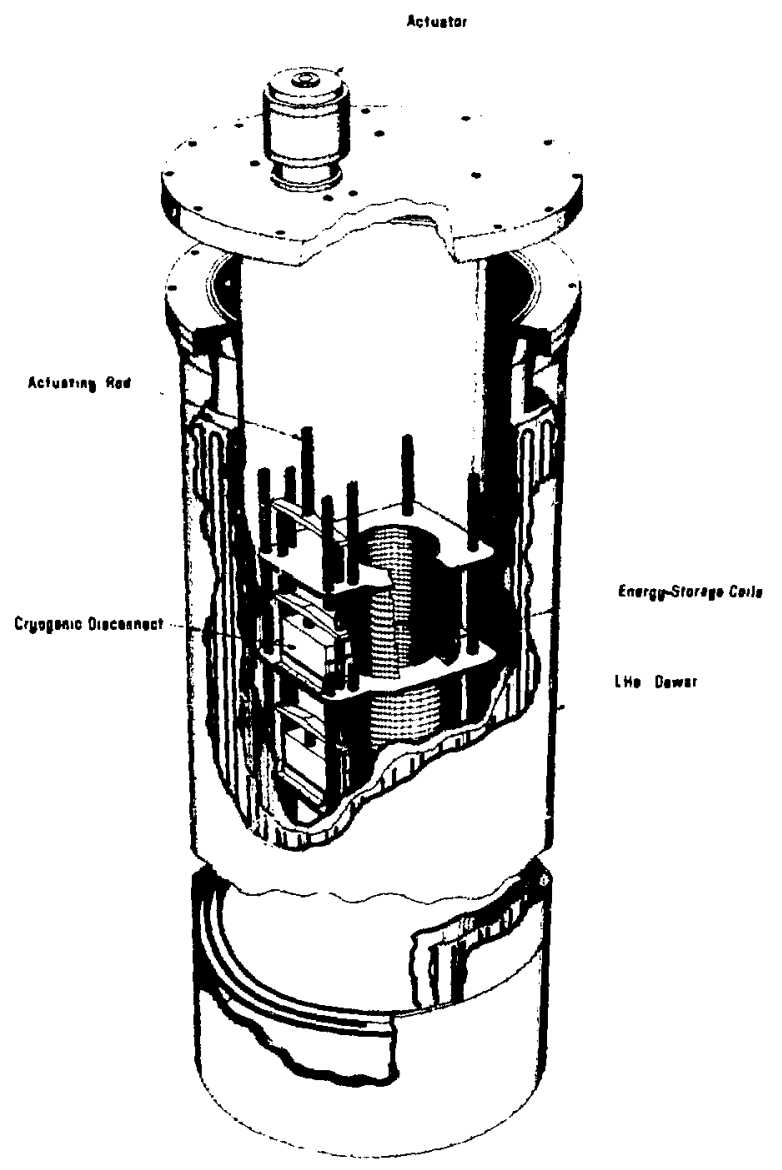

Fig. 5.4-12.

A 10-coil dewar assembly. technique. These parts will be mathined from that laminates (NEMA (i-10) made of epoxy-liber glass cloth. The resin sistem fiee Table $5.4-71$ chosen for filament winding of the cylindrical dewar vessels was developed by soffer and Malho and modified by Westinghouse."

The two types of glass fiber: that may be conl. sidered for use in this application are $E$-glass and $S$. glass. S-glass has about $30 \%$, higher strength and 201 higher modulus than E-glass. S-glass is used for critical aerospace applications, is made under highly cont rolled conditions, and is, therefore. about is to t times as expensive as F-glass. A commercial version of $S$-glass known as $\$ .2$ glass is also available but is not manufactured under the exacting quality control conditions of S-glass: nor does it have the high performance finish of the S-glass.

The glass chosen for this application is S-904. It has a high pertormance finish which acts as a wet ting agent for the epoxy and which is neither temperat $d r e$ nor humidity sensitive. It is particularly useful for slow winding operations.

The basic dimensions of each dewar are $1.17-m$ i.d. by $6.96-\mathrm{m}$ depth. The depth of liquid helium above the top coil is regulated to $0.1 \pm 0.0125 \mathrm{~m}$ and the tillage space is $0.61 \mathrm{~m}$ in depth. Severn phenolic shields are placed in the ullage volume to disrupt natural convection currents between the liquid helium surface and the warm lid and to act as thermal radiation barriers. The 10 coils in each dewar will be placed over an epoxy cylinder which will be an integral part of the inner shell. This cylinder will reduce the helium inventory

An $80 \mathrm{~K}$ shield is located between the inner and outer vacuum walls. It is tied thermally to the inner shell at a point $0.8 \mathrm{~m}$ from the top and consists of several layers of etched conductor on a plastic substrate. The layers are separated to prevent field coupling and eddy current losses during coil pulsiı.g. Three layers of overlapping but not continuous superinsulation (0.066:35-mm-thick Mylar aluminized on one side) are placed on the rutside of the inner vessel to improve the emissivity and to minimize the radiation heat load from 80 to $4.5 \mathrm{~K}$.

Two levels of heat interception are available: one at $80 \mathrm{~K}$ by gaseous helium and the other at $4.5 \mathrm{~K}$ by liquid helium. The $80 \mathrm{~K}$ steady-state heat loacis in the dewars are summarized in Table 5.4-8.

The $4.5 \mathrm{~K}$ steady-state heat loads to the liquid helium in the dewars are summarized in Table 5.4-9.

In addition to the steady-stace heat loads, load factors result from the pulsed operation of the system at the $4.5 \mathrm{~K}$ refrigeration level. To determine these loads, it was assumed that one charge and discharge cycle occurred every $900 \mathrm{~s}$ and that each cryogenic disconnect had a power loss of $24 \mathrm{~W}$ when carrying $26 \mathrm{kA}$. Table 5.4-10 summarizes the pulsed $4.5 \mathrm{~K}$ refrigeration requirements. The heat loads in the table have been averaged over the 900 -s cycle 
TABLE 5.4-7

DEWAR RESIN SYSTEM

\begin{tabular}{|c|c|c|c|}
\hline Constituent & Supplier & $\begin{array}{c}\begin{array}{c}\text { Composition of } \\
\text { Constituent Parts } \\
\text { by Weight }\end{array} \\
\end{array}$ & Function \\
\hline IDER:32:2 & Dow Chemical Co. & 100.00 & $\begin{array}{l}\text { Base epoxy resin: } \\
\text { diglycidyl ether of } \\
\text { bisphenol-A }\end{array}$ \\
\hline $\begin{array}{l}\text { Dodecanyl Succinic } \\
\text { Anhydride }\end{array}$ & $\begin{array}{l}\text { Allied Chemical } \\
\text { Corp. }\end{array}$ & 115.9 & $\begin{array}{l}\text { High molecular } \\
\text { weight curing } \\
\text { agent }\end{array}$ \\
\hline Empol 1041 & $\begin{array}{l}\text { Energy lndus- } \\
\text { tries, Inc. }\end{array}$ & 20.0 & $\begin{array}{l}\text { For flexibility: } \\
\text { trimer acid }\end{array}$ \\
\hline Benzyldimethylamine & $\begin{array}{l}\text { Miller-Stephenson } \\
\text { Co. }\end{array}$ & 1.0 & Catalyst \\
\hline
\end{tabular}

TABISE $5.4-8$

MU K STFADY-STATE DEWAR REFRIGERATION REQUIREMENTS

\begin{tabular}{|c|c|c|}
\hline & $\begin{array}{c}\begin{array}{c}\text { Single Dewar } \\
(w)\end{array} \\
\end{array}$ & $\begin{array}{c}128 \text { Dewars } \\
\text { (W) }\end{array}$ \\
\hline $\begin{array}{l}\text { Radiation from the } \\
\text { warm walls and lid }\end{array}$ & 273 & 34944 \\
\hline Transfer lines & 0 & 318 \\
\hline Toral & 273 & 35262 \\
\hline
\end{tabular}

TABLE 5.4-9

\subsection{K STEADY-STATE DEWAR REARIGERATION REQUIREMENTS}

Single Dewar

(W)

Wall conduction 80 e $4.5 \mathrm{~K}$

Radiation from the walls

(⿻) $11,4,5 \mathrm{~K}$

Radiation from the lid

Continuous leads conduction

Inst rument at ion

Support st ruct ures

Fill, blowdown. and vent lines

Pulsed leads:

$$
\text { Total }
$$

\begin{tabular}{rr}
1.8 & 226 \\
1.0 & 128 \\
6.0 & 768 \\
42.5 & 5440 \\
0.6 & 77 \\
0.3 & 38 \\
2.6 & 8338 \\
4.8 & 614 \\
\hline 59.6 & \\
\hline
\end{tabular}


TABLE 5.4-10

\subsection{PULSED REFRIGERATION REQUIREMFN'S}

\begin{tabular}{|c|c|c|}
\hline & $\begin{array}{l}\text { Single Dewar } \\
\qquad(W)\end{array}$ & $\begin{array}{l}\text { 12x Dewars } \\
\text { (W) }\end{array}$ \\
\hline $\begin{array}{l}\text { Pulsed leads above } \\
\text { liquid surfare }\end{array}$ & 9.8 & 1254 \\
\hline Pulsed leads below & 1.8 & $2: 30$ \\
\hline $\begin{array}{l}\text { liquid surface } \\
\text { Continuous leads } \\
\text { joule heat ing }\end{array}$ & 0.08 & 10 \\
\hline $\begin{array}{l}\text { Cryogenic disconnect } \\
\text { joule heating }\end{array}$ & 26.2 & $3: 354$ \\
\hline $\begin{array}{l}\text { Cryogenic disconnect } \\
\text { apening losses }\end{array}$ & 16.7 & 21388 \\
\hline $\begin{array}{l}0.3^{\prime} \text {; ciectromagnetic } \\
\text { losises }\end{array}$ & 12.7 & $16 \geq 7$ \\
\hline Total & 67.3 & 86113 \\
\hline
\end{tabular}

time and have assumed 10 coil-cryogenic disconnect pairs per dewar. 'The pulsed leads are coaxial, with one per coil.

The liquid helium evaporated at the $4.5 \mathrm{~K}$ level is a valuable contributor to refrigeration because of the high value of $C_{p} J T$ het ween $4.5 \mathrm{~K}$ and $300 \mathrm{~K}$, i.e.. $1485 \mathrm{~J} / \mathrm{g}$. as compared to its heat of vaporization of $20.4 \mathrm{~J} / \mathrm{g}$. It is, therefore, used to cool the continuous and pulsed leads. The total heat deposited in a pulsed lead during a pulse is $60500 \mathrm{~J}$ maximum. The average power ovc: the 900 -s cycle period is $68 \mathrm{~W}$. The system is designed so that $90 \%$; of this power is abs roed by warming helium gas as it passes through the leads, and $10^{\circ} \circ$ of this power manifests itself as an additional $4.5 \mathrm{~K}$ refrigeration requirement.

All items placed within the dewar are to be inounted from the lid to facilitate assembly. 'The major components are the coils and cryogenic disconnects. NEiviA G-10 epoxy-fiber glass is to be used for support structure for these items. The pulsed electrical leads wili be coaxial and superconducting below the liquid helium level. Each will terminate opposite the coil to which it attaches.

Each dewar will be provided with a $0.33-\mathrm{m}$ vent sized to handle the helium gas evolved from the failure mode in which all 10 of the storage coils go normal and derosit their full $3.9 \times 10^{6}$ f of stored energy in the liquid helium. Other penetrations throuigh the lid include the pulsed and continuous leads, liquirl helium fill line, instrumentation feedthroughs, and the actuator drive mechanism for the cryogenic disconnects.

Each dewar is equipped with appropriate safety relief valves and/or rupture discs on both the liquid and vacuum spaces.

\subsubsection{Liquid and Gas Distribution Systems}

Liquid helium must be proviced to each of the 128 roil dewars. In addition, a helium recovery and purification system is required. Liquid helium siorage dewars will provide a tutal capacity of 500 000 --equal to the total capacity of the coil dewars - and will be used to maintain the helium inventory as described in Sec. 5.4.3.17.

The liquid helium will flow through vacuumjacketed, 38.1-mm-i.d. transfer dines through individual dewar control valves and into the dewars. The liquid level in the individual dewars will be controlled by carbon resistor level sensors whose output will control the position of the feed valves. The $4.5 \mathrm{~K}$ transfer line refirigeration requirements are summarized in Table 5.4-11.

Evacuation and backfill purges will be used to reduce the size of the required gas purification system. With this procedure the purification system will have to accommodate only the small leakage of

TABLE 5.4-11

\subsection{K TRANSFER LINE REFRIGERATION REQUIREMENTS}

\begin{tabular}{l} 
Item \\
\hline Line radiation \\
Joirsts, val ves \\
Refrigarator in-line \\
liquid helium storage
\end{tabular}

Total

Refrigarator in-line
liquid helium storage
Heat Load (W)

314

717

33 
impurities into the helium system during normal operation.

A summary of the transfer line parameters is given in Table 5.4-12.

\subsubsection{Application of Refrigeration}

To apply the refrigeration from the helium refrigerators to the storage coil dewars under steadystate conditions is routine and typical of standard practice in the cryogenic indusiry. However, the transient heat loads arising from heat generation in the pulsed leads, from operation of the cryogenic disconnects, and from the $0.3 \%$ electromagnetic losses complicate the system.

Varying the rate of refrigeration in the refrigerators would be undesirable and difficult to execute. The pulses should be accommodated with as little disturbance as possible to either the refrigerator $\omega^{\prime \prime}$ the dewar refrigeration balance.

A large reservoir for absurbing the pulsed heat load exists in the liquic helium inventory within the dewars. The liquid iuventory per dewar at operating conditions is $3900 \%$. If, during a pulse, the liquid is permitted to warm to a temperature of $5.16 \mathrm{~K}$ (2.2 atm) from $4.22 \mathrm{~K}(1 \mathrm{~atm}), 6 \mathrm{MJ}$ would be absorbed without vaporization. This compares to $0.06 \mathrm{M}$ J generated in each pulse and $3.8 \mathrm{MJ}$ that would be released should all 10 coils in a dewar go normal and release all of their stored energy to the liquid helium. The probability of the coils releasing all of their stored energy to the liquid helium is very small because a safety circuit. will pulse the stored enırgy to an external load if any coil begins to go normal.

In this analysis it is assumed that most of the heat generated in the pulses results in the immediate vaporization of liquid helium because bubbles of gaseous helium will rise through the liquid with little condensation. Approximately $20 \%$ of this heat is assumed to remain in the coil and surrounding liquid and will be withdrawn as vaporized helium before the next pulse. Because most of the gas is generated rapidly, it will not absorb much heat as it passes out of the dewar, and thus it will be returned cold to the appropriate heat exchanger in the refrigerator through the vacuuni-jacketed transfer lines.

TABLE 5.4-12

TRANSFER LINE PARAMETERS

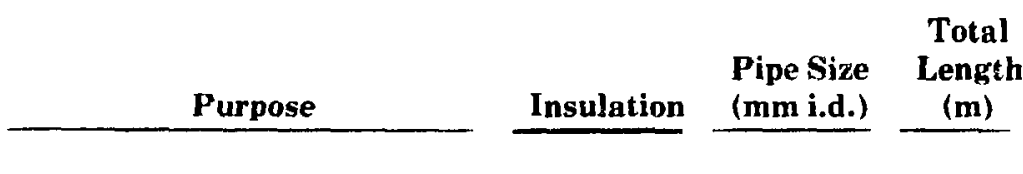

1. Liquid helium from the refrigerator to the coil dewars:

Main distribution

Feeders $t$ " each dewar

2. $5 \mathrm{~K}$ gaseous helium return line (1) refrigerator:

Feeders from dewars

Collection header

Header to surge tank

Surge tank to efrigerator

3. $300 \mathrm{~K}$ gaseous helium

return line:

Feeders from dewars

Collection header

Header to refrigerator
L. N.traced, vacuum i.N.2 1 raced. vacuum 38.1 371

$12.7 \quad 117$

LN. traced. vacuum 12.. 117

LN. traced. vacuum

6i3.5

L. N. traced. vacuum

63.5 5.3 L.N. Iraced. vacuum

38.1 8.11

none

none

12.7

117

none

101.6

$37 \mathrm{i}$

$127.0 \quad 119.6$ 


\subsubsection{Refrigerator Design}

The helium liquelier refrigerator will provide 3045 $\mathrm{kg} / \mathrm{h}$ of liquid helium. All of the helium vaporized as a result of the steady-state heat load will be returned as warm gas to the compressors, and in this respect the unit will operate as a liquefier. The gas evolved as a resuit of the current pulse will be returned to an intermediate heat exchanger at a temperature of 25 $\mathrm{K}$ or less.

The refrigerator for thir application is much larger than any equipment in existence. At present, the largest systems are liquefiers with a capacity of 1000 $i$ of liquid helium per hour.

When designing a liquefier-refrigerator of this size, it is important to use commercially available components, the three most important of which are the compressors, expanders, and heat exchangers.

The cortpressors chosen are three-stage, nonlubricated, reciprocating machines with balanced horizontally opposed cylinders. Each cylinder is equipped with a double rod seal to reduce the leakage of helium gas from the machine and to return this gas through a small purification system to the main process stream. The total required cumpressor input power is $12 \mathrm{MW}$. One backup com. pressor will be installed to permit maintenance on the other compressors while maintaining full refrigerator capacity.

The expansion engines used in the refrigerators will be centripetal, gas bearing turbines. In these machines half of the enthalpy drop of the gas occurs in the nozzles and the other half in the wheel. The machines are designed such that the velocity of the gas at the exit of the nozzle does not exceed the speed of sound. For steady-state operation with pulsing once every $900 \mathrm{~s}$, expanders 1 and 2 (Fig. 5.4-13) will be operating at a pressure ratio of approximately 2.8 . well below the theoretical maximum value of 5.6 calculated from ideal gas considerations. Fxpander 3 will be operating near the maximum pressure ratio value and its operation will not he optimum. At reduced output the machines will operate at decreased efficiency. Under certain conditions it may also be possible to bypass one of the expanders.

The heat exchangers are not large by commercial standards and will be of the plate-fin type shown in Fig. 5.4-14. They are considerably cheaper than any other type of heat exchanger that might be used.

The basic refrigeration process is shown in Fig. 5.4-13. The process points with sigrificant fluid properties are listed in Table 5.4-13. The following prints should be noted.

1. Liquid nitrugen is used to provide refirigeration at the $80 \mathrm{~K}$ level.

2. Refrigeration generated by expanders 1 and 2 ran be varied by changing the pressure ratio across the expanders. This is done by varying the speed of each machine.

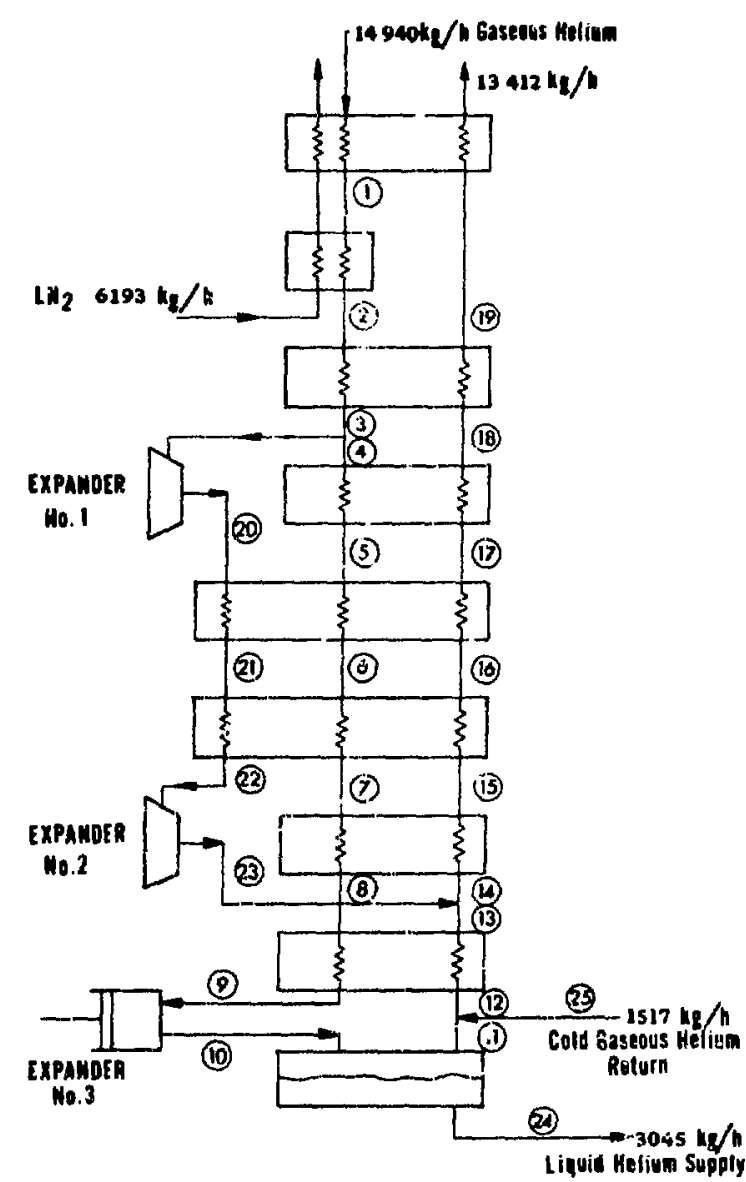

Fig. 5.4-13.

Schematic of refrigerator.

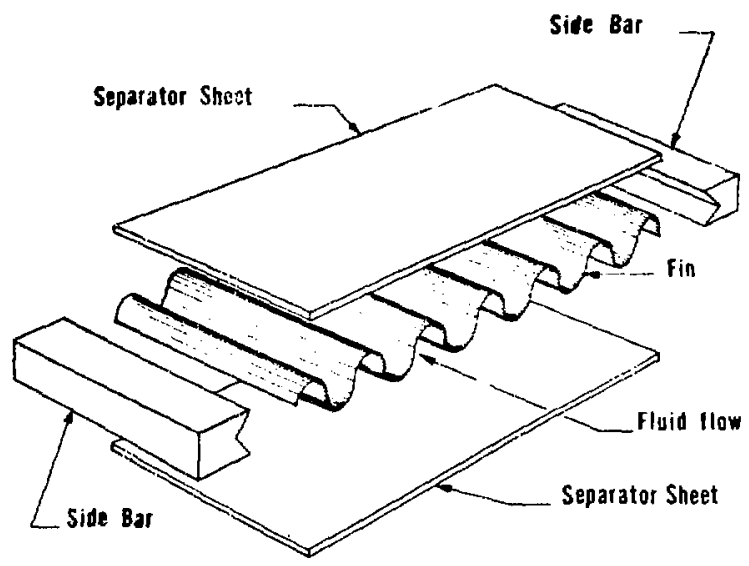

Fig. 5.4-14.

Plate-fin type heat exchanger. 
TABLE 5.4-13

TABLE 5.4-14

\section{REFRIGERATION PROCESS POINTS}

\begin{tabular}{|c|c|c|c|c|}
\hline & $\begin{array}{c}\text { Flowrate } \\
(\mathrm{kg} / \mathrm{h})\end{array}$ & $\underset{(\mathbf{a t m})}{\mathbf{P}}$ & $\begin{array}{c}T \\
(\mathbf{K}) \\
\end{array}$ & $\begin{array}{c}\text { Enthalpy } \\
(\mathrm{J} / \mathrm{g})\end{array}$ \\
\hline 1 & 149.40 & 12.4 & 91.0 & 491.1 \\
\hline 2 & 14940 & 1.2 .4 & 81.4 & 413.7 \\
\hline :3 & 14940 & $i 2.3$ & 36.9 & $2 j 4.7$ \\
\hline 4 & $7018:$ & 12.3 & 36.9 & 204.7 \\
\hline 5 & 7082 & 12.2 & $31 . \overline{1}$ & 177.4 \\
\hline 6 & 7082 & 12.1 & 23.6 & 32.6 \\
\hline 7 & 7082 & 12.1 & 14.2 & 77.0 \\
\hline 8 & 7082 & 12.0 & 12.3 & 64.8 \\
\hline 9 & 7082 & 12.0 & 7.9 & 31.4 \\
\hline 10 & 7082 & $\ldots$ & ... & $\ldots$ \\
\hline 11 & 4037 & 1.40 & 4.6 & 29.6 \\
\hline 12 & 5554 & 1.40 & 4.6 & 29.6 \\
\hline 13 & 5554 & 1.38 & 11.5 & 72.1 \\
\hline 14 & 13412 & 1.38 & $11 . \tilde{3}$ & 72.1 \\
\hline 15 & $1: 3412$ & 1.34 & 12.6 & 78.6 \\
\hline 16 & 13412 & 1.32 & 23.6 & 136.6 \\
\hline 17 & 13412 & 1.31 & 31.5 & 177.9 \\
\hline 18 & 13412 & 1.28 & 34.2 & 192.3 \\
\hline 19 & 13412 & 1.25 & 79.1 & 425.2 \\
\hline 20 & 7858 & 4.05 & 31.5 & 177.6 \\
\hline 21 & 7858 & 4.03 & 24.3 & 140.4 \\
\hline 22 & 7858 & 4.01 & 15.5 & 91.5 \\
\hline 23 & 7858 & 1.38 & 11.5 & 72.1 \\
\hline 24 & 3045 & 1.40 & 4.6 & 11.9 \\
\hline 25 & 1517 & 1.40 & 4.6 & 29.6 \\
\hline
\end{tabular}

\section{ELECTRICAL POWER REQUIREMENTS FOR FULL REFRIGERATION}

\begin{tabular}{|c|c|c|}
\hline & (hp) & $(\mathrm{kW})$ \\
\hline $\begin{array}{l}\text { Helium refrigerator } \\
\text { compressis: }\end{array}$ & 15600 & 11633 \\
\hline Cooling water system & 350 & 261 \\
\hline Purification system & 30 & 22.2 \\
\hline Miscellaneous & 65 & 48.5 \\
\hline Tirtal & 16045 & 11964.7 \\
\hline
\end{tabular}

by the helium compressors and other auxiliary equipment. A cooling watur system was sized by using two prepackaged mechanical draft cooling towers as shown in Fig. 5.4-15. These towers are constructed of galvanized steel and induce flow trom the bot tom to the top of the tower, counter to the water flow. These modular units meet normal fireproof ratings and are shipped in modular form. which minimizes field fabrication time. Each modular cell is equipped with a $37.3-\mathrm{kW}$. 50-hp fan and has an integral pump.

The units required for this installation were sized to accommodate a $11.1^{\circ} \mathrm{C}\left(20^{\circ} \mathrm{F}\right)$ temperature rise for $44.4^{\circ} \mathrm{C}\left(80^{\circ} \mathrm{F}\right)$ water with a wet bulb temperature of $36.7^{\circ} \mathrm{C}\left(66^{\circ} \mathrm{F}\right)$, which corresponds to summertime conditions in L,os Alamos. Two 18 925- $1 / \mathrm{min}$ (5000gpm) pumps would be required to attain the i:iaximum capacity of the tiswer modules. Thus the power requirements for this system would include $149.6 \mathrm{~kW}(200 \mathrm{hp})$ for the fans and another $112.0 \mathrm{~kW}$

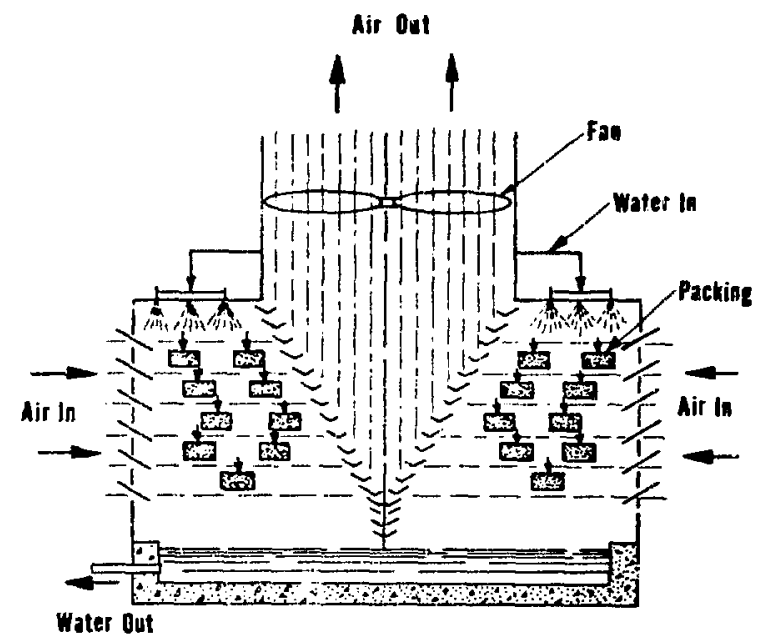

Fig. 5.4-15.

Cross flow cooling tower. 
$(150) \mathrm{hp})$ for the circulat ing pump, for a total of $26 !$ ! 6 kIt (3i) (l hpl.

Hakeup water will be required to replace that lost by evaporation. from drift, and by blowdown. The periodic draining (blowdown) of water from a cooling tower is necessary to prevent buildup of insoluble minerals and chemicals that eventually coat the tube walls and cause corrosion in heat exchangers of the tower basin. A: the minimum heat load. the tot al water makeup will be approximately $\$ 11.01 / \mathrm{min}$ (1:35 gpm).

Nitrogen System. Large quantities of liquid nitrogein will be required for the helium refrigerator. and incidental quantities for the purifiers. helium storage tanks, and primary dewar. The highest consumption will occur during plant cooldown and coil pulsing.

The maximum usage rate. $77333 / \mathrm{h}(2043 \mathrm{gph})$. can be met by either on-site production or direct purchase of nitrogen from large industrial gas producers.

Liquid nitrogen is produced concurrently with oxygen in air separation plants, few of which produce more than 120 tons/day. Most have committed at least $80^{\prime}$; of their capacities and usually can only neet short-duration demands by careful advance scheduling.

Liquid nitrogen is transported by railcars of :39:364-r (10 400-gal) canacity or by over-the-road tankers capable of hauling up to $22710 /(6000$ gal). For our purposes, tankers would probably be the prefeered carrier.

To obtain the maximum desired quantity of $773: 3$ $1 / \mathrm{h}$ (2043 gph) the following logistics must be considered. Assuming that a tanker requires at least $: 2 \mathrm{2}$ $\mathrm{h}$ for a round trip, five tankers would have to unload every 24 h to supply 185592 d/day (49 0344 gal/day). and the supplier would have to commit at least 10 tankers to the job. Also, because of the uncertain timing of the tests, it is unreasonal.ir to expect a supplier to stand by for delivery of such large quantities.

The alternative is to purchase the product in more conventional quantities and store enough to make a complete test run. On two weeks' notice, a delivery rate of 60 tons/day would be reasonable to expect. Therefore, two $378500-1$ (100 000-gal) tanks would store enough nitrogen for 4 to 5 days of normal operation. Three types of liquid nitrogen storage tanks are available: shop-fabricated units with capacities up to 189250 / ( $50000 \mathrm{gal})$, field-erected flat-bottomed tanks, and field-erected spherical or pressure tanks. The most economical tank would be a llat-bot tomed field-erected tank provided by concerns such as Chicago Bridge and Iron Co. or Graver Tank.

Instead of buying liquid nitrogen, one can recover and reliquefy warm nitrogen gas by using a system consisting of a $2982.8-\mathrm{kW}$ (4000-hp) compressor, a $223.7-\mathrm{kW}(3(30-\mathrm{hp})$ Freon system, a cold bc $\times 2.438 \mathrm{~m}$

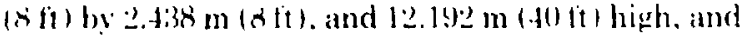
a 151 f(1)-1 (40) (0)(0)-gall storage tank. The instablation rost for such al system, exclusive of the storage tank, would be approximately $\$ 1 \mathrm{M}$. The operational cost would be in the range of $\$(10052 / 6$ (so.0.2/gall) withoul considering the power demand. If the sustem were ruin wily 1 wh a month, the cost would increase to

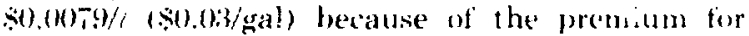
hisher power demand.

In today's market, liçuid nitrogen can be purchatsed for $\$ 0.049 / / 150.19 /$ gal . cielivered; consequently. oll-site production of liquid nit rogen would save approximately \$(0.04.1/. (\$0).17/2al), exclusive of investment costs. In addition. on-site storage capacity

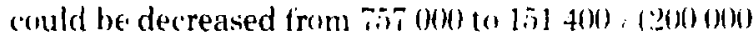
to 40) (mo) gall. so the net additional investment would be $\$ 900000$. In other words, the nitrogen reliquefier would have to be run for 1 bs dass before it became economical: Purchased liquid nitrogen cost $/ \mathrm{day}=\$ 0.19 / \mathrm{gal} x+90: 4 \mathrm{gal} / \mathrm{day}=\$ 9316 / \mathrm{day}$. On-site liquelaction costsiday $=\$ 0.03 / \mathrm{gal} \times 49034$ gal/day $=\$ 1+71 /$ day. So.

$$
\frac{\$ 900000}{\$ 9316 \cdot \$ 1+71}=115 \text { days of operation }
$$

Ender the criterion of 100 to 1060 maximum pulses. there is little justification for on-site production of liquid nitrogen.

Helium Supply. Helium for the initial charge and normal makeup reriuirements will be supplied by liquid-carrying tankors, each capable of transporting $37850,(10000$ gal). Tube trailers are impractical because of the large wuantities of helium required and the distance from the production plants. Hauling charges for a tariker and a gas-carrying trailer are the same, but the larger capacity of the canker decreases the unit transportation cost by a factor of 8. Helium comes from one or more of three commercial liquid helium plants in Kansas. Our requirements of $499200 /(132000$ gal) represent a 33day output from a plant.

Once the system is operating, replacement helium will be required. Losses will occur along the shafts and seals of the compressors, through valve stems and packing glands, and through flanges and other mechanical joints. Losses will also occur during safety valve releases and during equipment maintenance.

The helium inventory required for the proposed system is about three times that required for 1000 MW gas-cooled nuclear reactors now under design. Inventory losses for nuclear reactors range from 0.03 to $0.5 \%$ /day which implies an inventory of 136 to $2271 \mathrm{~d} / \mathrm{day}$ ( 36.0 to $600 \mathrm{gal} / \mathrm{day})$ for the proposed system. However, the short-duration runs and the pulsing in the proposed system will probably result in losses closer to $1 " \%$, or $4996 / /$ day (1320 gal/day). 
The lost helium could be replaced from a $37850-1$ (10) 000-gal) on-site tanker. If losses are $1 c_{i}$, each tanker load would last one week.

The cost for liquid helium in the required quantities would be approximately $\$ 1.06 / /(\$ 4.11 / \mathrm{gal})$, f.o.b. the production plant. Freight charges would increase the cost to approximately $\$ 1.11 / 1$ $(\$ 4.20 /$ gal). Total cost for the helium required to charge the system should be approximately $\$ 554000$.

Liquid helium would be unloaded into on-site storage tanks near the periphery of the experimental area, except during operation. From there, it could be vaporized into the compressor suction lines by the compressor housing as necessary.

Liquid will be transferred from the cold storage dewar to the storage coil dewars by pressurizing the cold storage dewars. By the reverse process one can empty the storage coil dewars. Very little pressure is required for these transfers.

Provision must be made to contain all the helium even when the experimental facility is not operating. One way would be $t o$ operate the refrigeration system continuously. storing the liquid helium in four $151400-1$ (40 000-gal) dewars. However, that may not be economically feasible when the research facility is not operating, and certainly there will be times when the refrigeration plant will need maintenance or repairs. 'This suggests a need for a helium gas storage system. Such a system could be assembled from six surplus L.ASL high pressure gas storage tanks. The tanks would cost nothing, lut there would be installation costs for the tanks as well as the cost of a conpressor to complete the system.

Adequate tankage is available for $17275000 /(610$ $000 \mathrm{scf}$ ) of helium gas at a pressure of $2812400 \mathrm{~kg} / \mathrm{m}^{2}$ $1.8000 \mathrm{psi}$ ). A 1\% $\%$ /day dewar boiloff, if pumped into gas bottles, permits approximately 5 days of refrigeration system downtime, and most repairs can he completed in that time.

To run the refrigeration system with 5-day downcycles saves electrical power, but is practical only when the research facility is to be shut down for an extended period or when the refrigeration system is in need of maintenance or repairs.

As long as dewar boiloff is being pumped into gas storage bottle's. the dewar itself is venting at constant pressure and there is very little temperat ure rise in the hiquid helium stored in the dewar. Additional downtime can be obtained by closing off the dewar vent line when the storage tanks are full, thereby allowing the dewar pressure to build up. By setting the upper pressure limit at the critical pressure of helium, assuming a heat leak equivalent to $1{ }^{\prime} i /$ day boiloff. and estimating a $50 \%$ efficiency from mixing in the dewar (some baffling would be required), the helium can be contained for 5 to 10 additional davs.
However, at the end of that time, a large fraction of the helium has become a saturated vapor. Also, the temperature of the remaining liquid has increased to approximately $5.2 \mathrm{~K}$. Consequently, after the refrigeration system has been restaried. some running time is required to reduce the dewar temperature to $4.5 \mathrm{~K}$. This is a worst-case situation and would arise only when the refrigeration system had been off for more than 5 days.

\subsubsection{Cryogenic Sysiem Operation}

A flow sheet (Fig. 5.4-16) shows the interaction of the various system components and helps in developing instrumentation cont rol philosophy.

All components of the refrigeration system-individual coil storage dewars, helium storage tanks. surge tanks, and all lines-will be purged by evacuation and gas flushing to minimize the purification required.

For start up, one of the liyuid helium storage tanks will be cooled by circulating cold helium from the refrigerator through the tank. As the system cools. liquid helium will collect in the dewar to the desired level.

Indiv:dual coil dewars will be cooled by circulating gaseous helium from the refrigerator through the main storage space of the dewars. Liquid helium will then be transferred from the storage tanks to the coil dewars at a rate limited by the capacity of the refrigerator compressors to recompress the vaporized helium. To cool the $89600 \mathrm{~kg}$ total mass to $4.5 \mathrm{k}$ will require the removal of $7.2 \times 10^{9} \mathrm{~J}$. Cooldown time is calculated to be $72 \mathrm{~h}$.

Once the dewars are cooled, filled with liquid helium, and stabilized, little attention will be required. If the refrigerator is liquefying more helium than is required, the liquid inventory in the storage tanks will increase and the amount of vapor leaving the coil dewars will decrease. The recycle valve on the refrigerator compressors will then open and the helium flowrate of the refrigerator will be lowered.

The amount of expander tlow is determined by the refrigeration requirements and will increase or decrease as necessary to maintain the temperature of the first expander. This is accomplished by opening or closing the adjustable nozzles on the turbines. The nozzles provide a way to decrease the flow through the expanders without decreasing efficiency. The cross-sectional area of the nozzles is changed so that the pressure of the gas at the expander wheel is constant at all flowrates.

Once the pulsing has begun, the increased flow from the dewar will increase the compressor suction pressure and cause the recycle valve to close and 


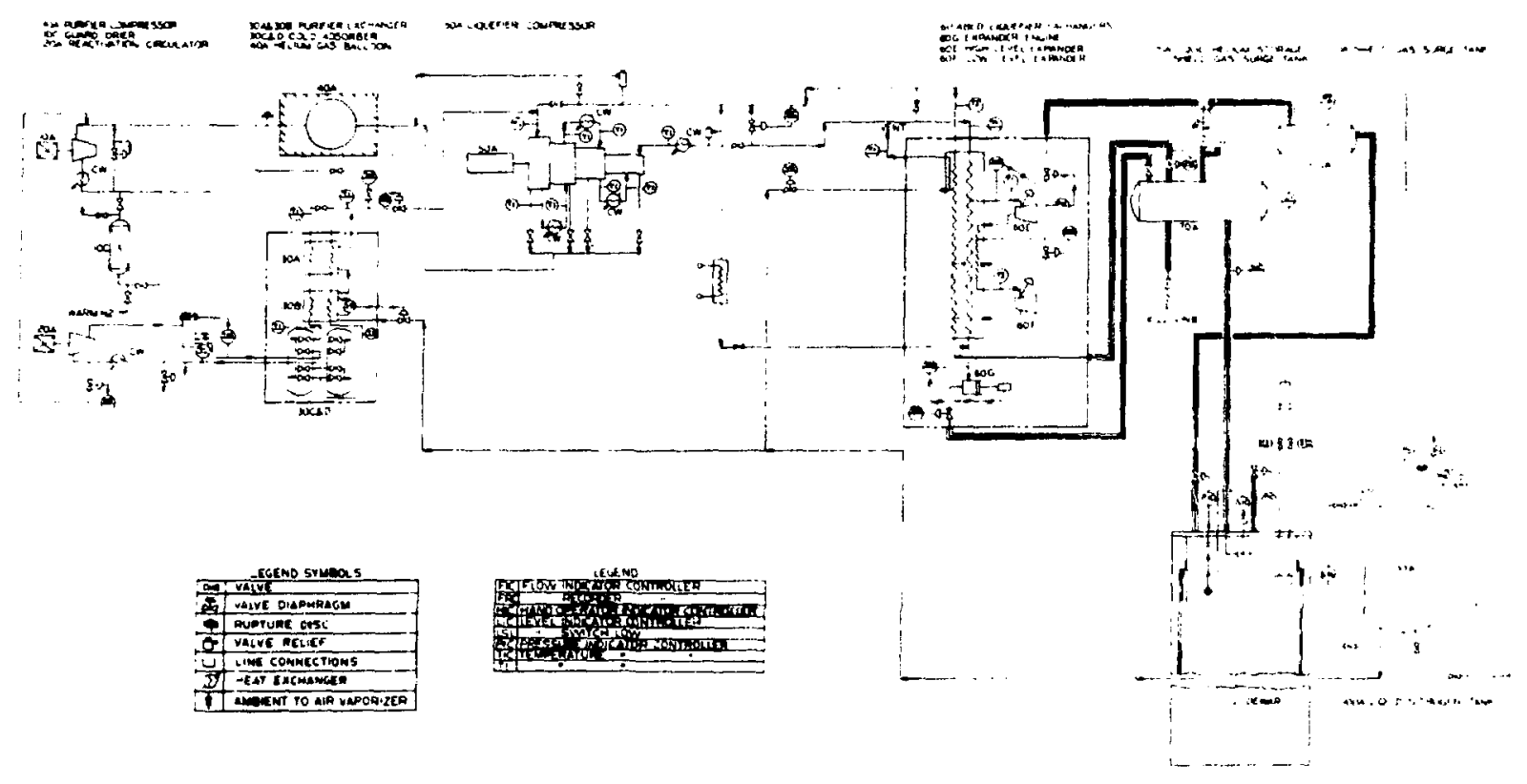

Fig. 5.4-16.

Sichematic of SFTR crogenic system.

deliver more flow to the refrigerator. The compressor discharge pressure will increase and cause the expanders to speed up.

For warmup. the liquid helium will be transferred from the coil dewars to the strrage tanks by pressurizing the dewars. During the transfer the refrigerator must liquefy both the helium saporized during thi transfer and the cold gas displaced from the storage tanks. The coil dewars could be emptied in approximately $40 \mathrm{~h}$. Circulating helium gas would be used to warm up the individual coil dewars. At a flowrate of $i 4900 \mathrm{~kg} / \mathrm{h}$ the warming period would be about 3 days.

\subsubsection{Assembly Area}

Practically all METS components will be preassembled into modules for installation in the SFTR building. Some of these modules will be assembled by vendors under LASL contracts; ot hers will be assembled by LASL personnel. Regardless of where the modules are assembled, some testing and calibration work will be required. This work will be done in a small test facility to be constructed early in the assembly program and to be used for adjusting and testing complete modules. Faults may be detected in some modules during testing. Minor faults will be corrected while the units are in the checkout stand; more complex problems may require rebuilding of the modules.

All of this work can be done in the assembly area-a bay providing $\left.929 \mathrm{~m}^{2}(100 \%) \mathrm{ft}^{2}\right)$ of work sprace. A 9072-kg (10-ton) traveling bridge crane will be available for moving dewar modules and heavy transfer capacitor banks. Test fixtures, stands, and facilities such as vacuum pumping stations will be provided in the bay for the necessary assembly and maintenance work on the MFTS components.

For separation of assemblies, clearance is needed in an amount equivalent to twice the height of a dewar. and a $\mu^{\text {it }}$ area will therefore be included. The pit area will include $t$ wo hudraulic lift platforms for storage coil assembly. These lifts will be used to position coil assemblies so the working area along the assembly is always at floor height, thus eliminating the need for elevated platforms and at tendant safety equipment.

Spare modular coil assemblies will be stored in the assembly area, ready for immediate installation in the main facility if component failures occur. This capability is essential to minimize machine downtime.

Some office space will be provided in the assembly area for a mechanical engineer. an electrical engineer, a cryogenic engineer. draftsman. and building foreman. These individuals, along with 
several technicians, will be responsible for the assembly, checkout, and installation of all METS components in the main facility. They will also have the primary responsibility for the operation and maintenance of the METS refrigeration and gas storage s.'stems located adjacent to the assembly area.

\subsubsection{System Cost Analysis}

In this section, additional costs of the METSSFTlR system are presented, exclusive of the costs of the Phase I, capacitor-driven system described in Sec. 5.4.1. Some Phase I components are to be rearranged or reconnected for the Phase II system: however, since such changes are considered operating expenses, no cost factor is included here. Thus the costs for the following components are not included here:

1. Interrupter counterpulse system (Sec. 5.4.3.5)

2 . Fast and slow compression coil crowbar switches (See. 5.4.3.7)

3. Counterpulse storage power s.spply isec. 5.4 .3 .91

4. Capacitance transfer bank (Sec. 5.4.3.10).

Connections and cabling between components will he the same or will use the same material to some degree in both phases. The cabling costs given here are separated for the Phase I and Phase II systems.

Details of the costs are given in Tables 5.4-15 through $5.4-25$ and are summarized in Table 5.4 .26 .

TABLE 5.4-15

STORAGE COIL COSTS

$\begin{array}{cc}\text { Superconductor } & \$ 5.6(10)^{6} \\ \text { Coil material, man:s } & 16.9 \\ \text { fiacturer. and test } & \\ \text { Total } & \$ 2.5(10)^{6}\end{array}$

Not included are field overhead, contractors' fees, engineering fees, and site preparation.

REFERENCES (Chap. V, Sec. 5.4)

1. Jacob L. Zar, "Electrical Switch Contact Resistance at $4.2 \mathrm{~K}$," Avco Everett Research Laboratory report AMP 234 (July 1967).

2. F. L. Ribe, "Free-Boundary Solutions for HighBeta Stellerators of Large Aspect Ratio," Los Alamos Scientific Laboratory report LA.4098 (May 1969).

3. F. L. Ribe, W. H. Borkenhagen, W. R. Ellis, and K. S. Hanks, "Initial Equilibrium Configuration of the Scyllac 5-Meter Toroidal-Sector Experiment," Los Alamos Scientific Laboratory report LA-4597MS (February 1971).

4. L. M. Soffer and L. Malho, "Cryogenic Resins for Glass-Filament.Wound Composites." NASA-CR. 72114 (1967).

5. E. Mullan. "Design and Fabrication of $300 \mathrm{~kJ}$ Superconducting Energy Storage Coil." Westinghouse Electric Corp., Cheswick, PA, report E.M. 4617 (1974).

TABLE 5.4-16

CRYOGENIC DISCONNECT COSTS

\author{
Disconnects $11: 28011$ \\ Actuators and \\ hydraulic system
}

Total
$5.3 .9(10)^{5}$
0.9

$\$ 4.8(16)^{6}$ 
TABI, :5.4-17

HVDC INTERRUPTER COSTS PER MODULE, I280-MOLUIE SYSTEM, IO MODULES PER CABINET UNIT

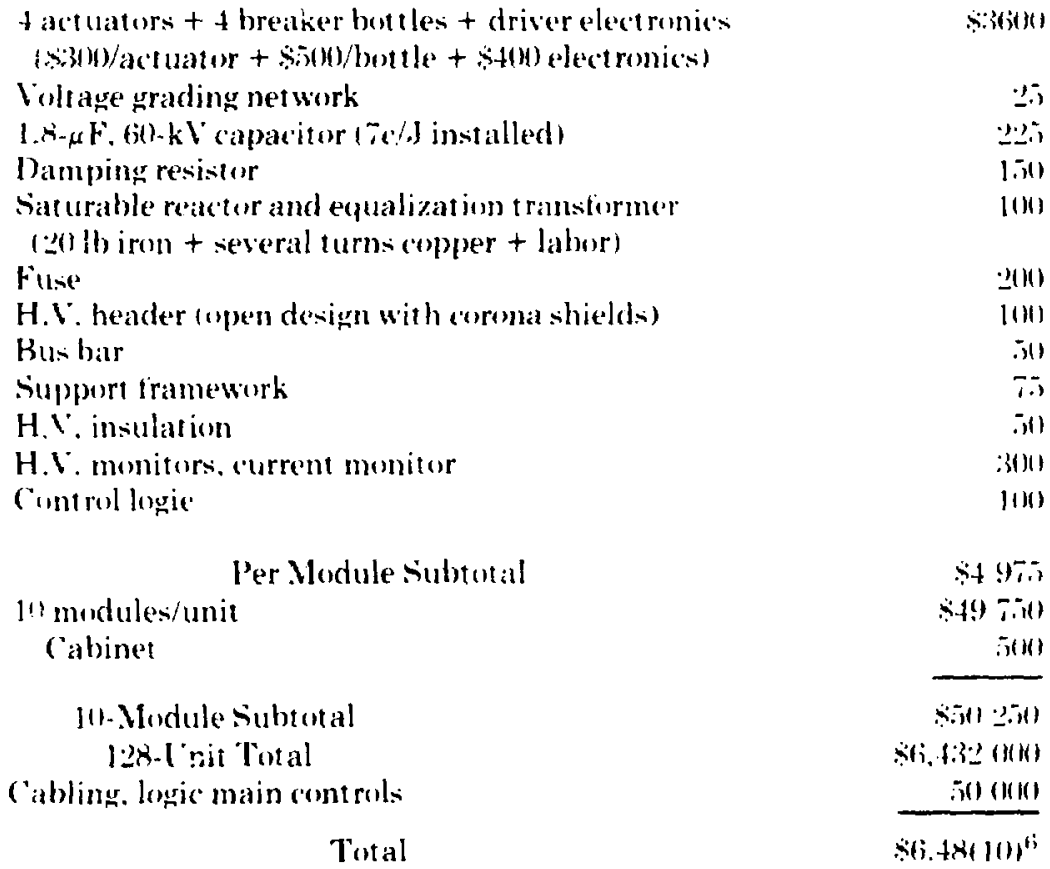

TABLE $\mathbf{5 . 4 - 1 8}$

TABI.E 5.4-1!

\section{COMPRESAION COLI, COSTS}

\begin{abstract}
Material/coil
Labor/coil

Material for 1280 enils

Labor for 1:280 coils
\end{abstract}

Total

411
708
526000
9060000

CHARGING POWER STPPI,Y FOR STORAGE COILS, COSTS

Rectifier power supply. $26 \mathrm{kA}$, I25 $\mathrm{V}$

$51 .+5(H)$

III $(\theta(H)$

17 (6)

$\therefore(m)$

$30(0)$

$\$(\$))(0)$ 


\section{HI.ECTRICAL. I.EAI) ('OSTS}

('momumus. 2fikA. lespairs

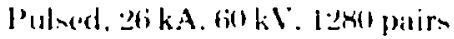
Toral

TABLE 5.1-21

\section{COMPONHNT CONNHCTIONA}

ANI) CAIBIING; COSTS

\begin{tabular}{|c|c|c|}
\hline & Material & labor \\
\hline 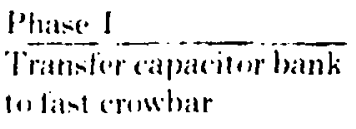 & $\therefore \operatorname{lis}(01101$ & $\therefore$ fit $(1611)$ \\
\hline $\begin{array}{l}\text { Fast cruwbar to slow } \\
\text { crowthar }\end{array}$ & 1:3is 01010 & la \\
\hline $\begin{array}{l}\text { Slow erowbar lo } \\
\text { compression conil }\end{array}$ & 5401010 & 2 T \\
\hline $\begin{array}{l}\text { Subtotals } \\
\text { 'lotal }\end{array}$ & 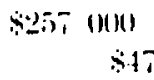 & (W) \\
\hline
\end{tabular}

Phase II

Siurage coil to

interrupter

Int (irrupterto

capaceitor bank Subtorials Total

\begin{tabular}{|c|c|}
\hline (1) & 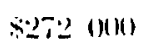 \\
\hline 90200 & bit $(A))$ \\
\hline 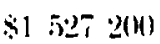 & şiz:3ti (n) \\
\hline
\end{tabular}

TABLE $\mathbf{5 . 4 - 2 2}$

Storige (oil I)owar Costs

$\$ 3.2(10))^{i}$

\section{I.IQ(ID) ANI) GAS DISTRIBL TION COSTS}

Tutal (ryogenic piping
Warm gas piping
Sx(M) (NH)

$2.5(H) ;$

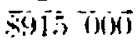

TABLE $3.1-2.1$

\section{RFFRIGERATOR SYSTEM AND CRYOGENIC STORAGE COATS}

Retrigerator cold bex and compressors $\$ 5.2416116$

thesturage. i-361(m)l gal 1.25

A. strrage. 1-1(H)ofolgal (1.2.)

Purificationssistem terix

comtrols, valies. instrumentation $0.3 x$

Vacuum pumps

Toral

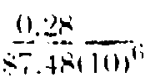

TABI.E: 5.4-25

rouling tower conts linstalled) Sis ant

TABLE. 5.4-26

\section{SLMMARY OF COSTS}

Storage coils

Cryogrenic disconnects

+2.2011010 .

HVDC interrupters

$4.81)$

Compression coils

6.48

Charging power supply 0.18

Flectrical leads

1.02

$\begin{array}{ll}\text { Isolation contaciors } & 0.32 \\ \text { Component comnections and } & 1.86\end{array}$

cabling (Phase II)

Storage coil dewars

3.20

liquid and gas distribution

0.92

Refrigerator and cryourenic

7.48

storage

Cooling towers

Total
$0.0 \%$

Sivo.261 $1011^{6}$ 


\section{ד.5 SHIELDING DESIGN}

\section{i.j.1 Design Objectives}

Seseral radiation protection criteria must be met during operation of the SFTR. These include permissible neutron and yamma-ray dose's to the public and to site workers. control of radioactive etfluents in air and water, dispowal of radioactive wast tes, and prevention of excessive radiation damage to elec. irical insulators. Preliminary design calculations: have been pertormed to specify biological shielding (via the concrete cell wall) which will reduce dose rates to levels below vaues anticipated to be allowable in the $19800^{\circ}$. Activation of magnet coils. capacitors. and other st ructures in the cell was found to be excessively high if no primary shield were placed around the coils. Thus. a primary shield. $0.5 \mathrm{~m}$ thick. was designed to allow access to the cell shortly after a pulse. to reduce alir sctivation in the cell. and to reduce radiation damage in capacitor insulation.

\section{5.s.1.1 Biological Shield Design Criteria}

Present ERDA radiation protection standards ${ }^{1}$ allow an occupational dose equivalent (DF) limit. usually called simply. "dose." of '3 rem/quarter. with a practical yearly limit of 5 rem. Legally. the latter limit can be relaxed $t o$ an accumulated dose of $(x$. 18) is rem, where $X$ is the employee's age. but this limit is not meaningful for shield design purposes. Consultation with health physics personnel revealed that current ERDA "as low as practical" requirements have been translated into a design criterion of $<1 \mathrm{rem} / \mathrm{yr}$ for recently constructed FRDA facilities. Informal inquiries from ERDA have suggested more stringent limits of $0 . \therefore \mathrm{rem} / \mathrm{quarter}$. Considering this trend. we have selected a conservative occupational exposure limit from direct neutron and gamma-ray doses of $0.5 \mathrm{rem} / \mathrm{yr}$ in this design. This does not include doses from activated components. Such additional doses might be received during maintenance operations in the cell or during handling of activated components. Also. the 0.5 $\mathrm{rem} / \mathrm{yr}$ direct dose will be a maximum at any point on the outer surface of the concrete secondary shield: most exposures will be less.

Because the maximum potential dose will be delivered in $\sim 10^{3}$ insiantaneous pulses, rather than in a continuous exposure, more leeway exists in which to notify personnel of anticipated pulses. Thus, further dosage reduction can he achieved during the pulse if personnel are stationed in locations of minimum dosigge consistent with their work assigmments. Dose rate criteria for long-term lowlevel exposire are clearly not applieable for pulsed operations. However, radiation biologists were consulted toverity that high dose rates tas ciuring a very short prulsel would not significanty thange the biulonical eftects to be expected witen the silme toral dose is delivered slowly user a iong period $w$ th a low dose rate. At the extremely low allowathle dose 111. i rem/yr) it is difficult to detect any somatic cffects. and dose rate effects are unobservatile.

Doses to the general public must be kept to less than is mrem/sr. and no problems are expected in this regard. However, quantitative predictions most await detailed facility design and location. fence locations. and other administrative control procedures.

\section{j.5.1.2 Activation and Kadiation Damage Criteria}

Air activation is limited by the allowable release of activity into the airshed, and is ultimately determined by allowable exposure to the public. Sisecific quant itative criteria for allowable airborne radioac. tive materials are gicen in Ret'. 1 as maximum permissible concentrations (MPC"s) for a long list of isotopes. At the low total neutron fluences from the SFTR this will pose no problem. Calculat ions reveal that air activities in the cell are within occupational brea:hing tolerances several seconds after a pulse.

Design criteria for biological doses wo personnel from activated components are much more complex. They must take into account factors such as time of access to the cell after a pulse, dose rate falloff as a function of time. length of time required for maintenance or other operation, location of the exposed person relative to numerous activated $\mathrm{com}$ ponents. Simplifications were therefore necessary to reduce the problem to tractable proportions. The quantitative criteria for radiation protection of personnel are then given by the ERDA standards for occupational dose limits as discussed in the previous section.

Design limits for physical radiation doses to coil and capacitor insulation are difficult to establish due to lack of experimental data. Since the primary shield must be placed outside the magnet coils, shielding can do little to alleviate radiation damage to organic insulation in the coils; radiation-resistant materials must be selected. For example, doses of $9 x$ $10^{5}$ gray $\left(9 \times 10^{7} \mathrm{rad}\right)$ will cause $25 \%$; elongation in polyethylene. ${ }^{2}$ Further complicatirg the problem are gamma-ray doses, which will be of comparable 
masnitude to the neutron doses. The addition of a primars shield wili probably aggravate the prothem sightle by retlecting more neut rons back to the coils and radiating gamma ravs back to the coils. However. the primary shield is designed ti reduce phyicical doses to the capacitors to levels below those which cause ans significant damage in liquid ormanics. Csing as criteria the gas powlution and viscrusity increase due 10 radiation." one tinds that doses of - $10^{6)}$ (iv $110^{*}$ rad) cause gas evolution rates

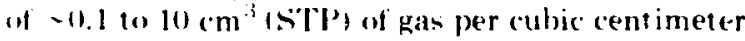
if liquid. which may well be unacceptable for offthe shelf capacitors. Due to a lack of data on radiatiom effecets to the capaciturs. a con.ervative criterion wis itdepted. The maximum allowable physical dose to the capacions is limited $1010^{3}$ (iy/yr. which sheruld cause barely detectable radiation effects in the castor oil insulation.

\subsubsection{Shielding Concept and Layout}

Toidentify approximate shielding requirements to satioly the above-mentioned criteria. preliminary sonping calculations were portormed. The toroidal surce of 1.4-M.'. [).'T neut roms was treated as all rquivalent line surce with strength 1 . is $\times 10^{\text {to }}$ neut romeim per pulse. Fxcluding all shields and uning ompirical removal cross nections for coils. insula-

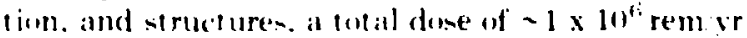
was calculated at 4 me distance from the plasma

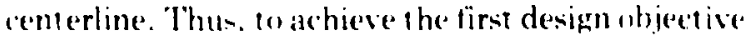
(1) $11.5 \mathrm{rem} / \mathrm{vr}$. shiedding must be introduced which provide an estective duse attentation lactor of is $x$ 10 "This attenuation could be abtained by an ordimars concrete shield of approximately $2-m$ thickness." Howeser. to meet the activation and radtianom damage criteria it is necessary to employ sime shielding as close to the plasma as pussible. Thene requirements lead to the concept of a close-in primary shield in addtition to a hiolugieal shiced larther out from the plasma. The positioning of such a primary shield is dictated primarily by the reyuirements of the plasma containment system li.e.. the coil design). To minimize interference of the primary shield with the magnetic llux paths of in phesion and compression coils. the closest possible locition to the plasma is just outside the compression coil at a radius $R>28 \mathrm{~cm}$. Access requirements: to the coils limit t'a primary shield thickness to about in cm. This. in turn. reduces the thickness of the secondary shield to ahout $1.5 \mathrm{~m}$ of ordinary concrete. To further identify specifications for the primary and secondary shield components. it was necessiry to perform more detailed calculations and also to assess additional design limitations.

\subsubsection{The Primary Shield}

First, the dose rate at $\sim 1 \mathrm{~m}$ from the coils was computed from radionuclides in the corils and $\mathrm{Fe}-\mathrm{Ni}$ alloy only, assuming no primary shield is provided. ( naccept ably high doses $(>1 \mathrm{rem} / \mathrm{h}$ ) at 1 day after a puise immediately led to the requirement for a primary shield around the coils. This shield serves multiple purposes. as discussed below, including the reduction of activation dose rates in the cell to the urder of a feu $\mathrm{mrem} / \mathrm{h}$. The shield keeps gamma radiation from the cois structure (vessel) and reduces capacitor and structural activation.

Minimi:ing activation aad radiation damage of the capaciturs and structure is the principal function of the primary shield. There is no way to shield iite implosion and compression coils from the 14 . Mo neu rons, so radiation-resistan insulators must be chosen for this application. However. reflected. low energy neutrons from the primary shield can be suppresised to some extent by judicicus chosice of shield materials. For example. boronimprenated graphite and polvethylene will sup)press thermal and epithermal neutron fluxes. and these materials were included in the primars shield. suipir sion of low energy lluxes also reduces activation of the coils, and ceil maintenance operations are thereby simplified. Incorporated in the primary shield design is a commercial lead-impreonated molvethylene which reduces dose rates in the cell from the activated coils to $\leq 1 \mathrm{mrem} / \mathrm{h}$. Orls when the primary shield is removed or streaming paths are created through the shield te.g.. through the optical ports do the activated coils cont ribute sionnilicantly to tho dose rate in the cell.

Once the necessity for a primary shield was established. a conceptual design was evolved to satisfy seseral design criteria. Some requirements for the materials of the primary shield are:

1. Low conductivity of the shield material located within the first few centimeters immediately outboard of the compression coil so as not to interfere with the magnetic flux paths.

2. Radiation resistance of the shield material with respect to dimensional stabilits, gas evolution. etc.

3. Reasonable cost and fabricability. i.e., com. mercially avalable materials which can be formed with st andard machining and const ruction methods.

4. Effective gamma-ray a' enuating properties for activation gamma rass from the vessel.

5. High cross section for neutron sloruing down and absorption.

6. Minimum reflection of neutrons back to the coils. and minimun prompt gamma-ray production (radiating to coils). 
7. Low potential for activarion within the shield materials of long-lived gamma-ray emitters and radiologically hazardous isotopes (for waste disposal considerations).

The application of these criteria is reflected in the final primary shield design described in Table $5 . \overline{5}-1$.

Graphite was selected for the first lamination in the shield principally for the following properties:

1. Low conductivity can be built in if desired (. $\sim 5001) \mu ? \cdot \mathrm{cm})$ :

2. Negligible activation and moderate gamma-ray production:

3. High resistance to radiation, especially as opposed to polyethylene:

4. Moderately good neutron attenuating properties: and

5. Excellent structural properlies and machinability.
Boron at $1 \mathrm{wt}$, was added to the graphite to suppress thermal (and epithermal to sime extemt) neutron return to the vessel. which return could result in subsequent activation of the copper. nickel. and iron. Aluminum structure was placed in ewo shells to support the shield and tie the laminations together. yet not activate significantly itself. Because of its high conductivity, the nearest aluminum shell was located about $26 \mathrm{~cm}$ cutboard of the compression coil. Finally, borated and loaded polvet hylene were used in places where neut ron tluxes would be sufficiently attenuated by the graphite and aluminum to preclude excessive radiation damage 10 the polyethylene. The graphite and aluminum also shield the polyetholene somewhat from capture gamna rays in the coils. Locaded polverhylene was selected as the outermost lamination in order $(0)$ shield against prompt famma ravs.

TABLE 5.5-1

PRIMARY SHIELD DESIGN AND COMPOSITION

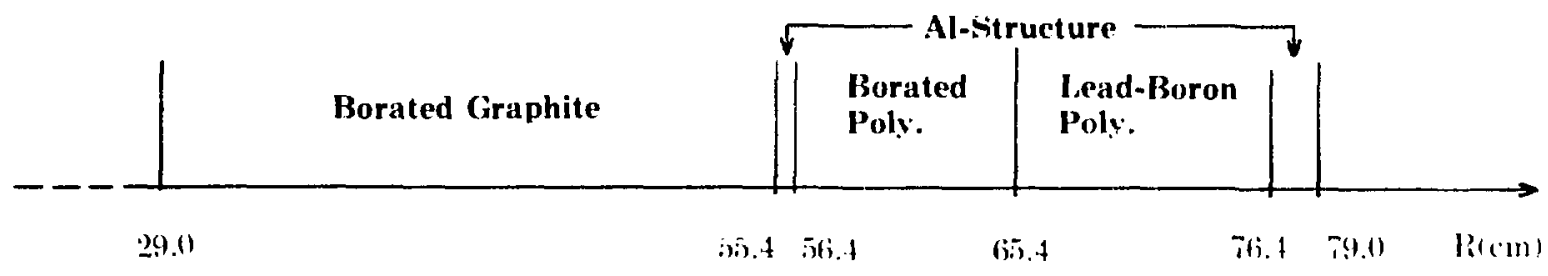

\begin{tabular}{|c|c|c|c|}
\hline $\begin{array}{c}\text { Zone } \\
\text { Description }\end{array}$ & $\begin{array}{l}\text { Density } \\
\left(\mathrm{g} / \mathrm{cm}^{3}\right) \\
\end{array}$ & Composition & $\begin{array}{l}\text { Number-Densit } \\
\text { (atoms/barn - cm }\end{array}$ \\
\hline Borated Graphite & 1.6 & $\begin{array}{c}99 \text { wt'; Carbon } \\
1 \text { wt'; Boron }\end{array}$ & 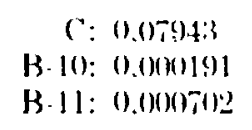 \\
\hline Al-Structure & 2.7 & $100^{r} ;$ Aluminum & $\mathrm{Al}: 0.0601027$ \\
\hline $\begin{array}{l}\text { Borated Poly- } \\
\text { ethylene }\end{array}$ & 0.94 & $\begin{array}{l}95 \text { st } \% \text { Polyethylene } \\
5 \text { wt“; Boron } \\
\text { Impurities }\end{array}$ & 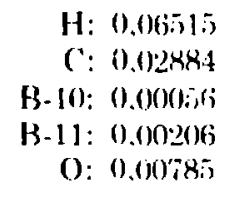 \\
\hline $\begin{array}{l}\text { Lead-Boron- } \\
\text { Polyethylene }\end{array}$ & 4.4 & $\begin{array}{l}80 \mathrm{wt}^{\prime} ; \text { Lead } \\
12.5 \mathrm{wt} \text { ' Polyethulene } \\
1 \mathrm{wt} \% \text { Boron } \\
6.5 \mathrm{wt} \% \text {, Mortar }\end{array}$ & 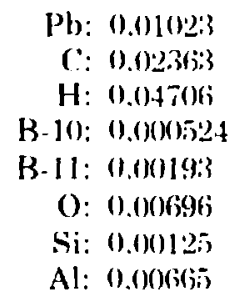 \\
\hline
\end{tabular}


and more important, activat ion gamma ravs during access to the celi after a pulse.

\subsubsection{The Secondary, Biological Shield}

Initial shielding st udies concent rated on sizing the concrete cell walls and ceiling which serve as the biological shield. Ordinary concrote was chosen on the basis of several considereious, including st ructural requirements. cost, and extensive experience with its use in conventiond fission power plants. A schenatic of the cell cross section and its concrete biological shield is shown in Fig. 5.5-1

(alculations were first performed without a primary shield, and with only the coils between the plasma and the concrete walls (i.e., streaming paths which do not intersect capacitors or structure were assumed for the sake of conservatism). Biological doses per pulse from neutrons were calculated assuming that the concrete secondary shield extend- ed from $\mathrm{r}=397 \mathrm{to} 50 / 0 \mathrm{~cm}$. It was found that this amount of concrete would not be an adequate hiological shield because the calculated neutron dose out side the wall was $\sim 0.6 \mathrm{rem} / \mathrm{pulse}$, or $6(10 \mathrm{rem} / \mathrm{yr}$. The gamma-ray dose would be a comparable value. No at tempt was made at this stage to size the secondary shield; however, it could be inferred that a 50 (). cm-thick primary shield would reduce the neutron dose outside the secondary shield to $-6 \mathrm{rem} / \mathrm{yr}$. I'nder current guidelines for occupational doses icf. Sec. 5.5.1.1) anot her $\sim 40 \mathrm{~cm}$ of concrete $($ i.e.. $1433 \mathrm{~cm}$ totall would be required in addition to : $50-\mathrm{cm}$ primary shield if access to the concrete wall is desired during a pulse. The $40-\mathrm{cm}$ estimate assumes that the gamma dose is approximately equal to the neutron dose, a conservative assumption in this case.

A $1.5-\mathrm{m}$-thick secondary shield was thus maintained throughout several iterations on the primary shield design. coil material. thickness chu;ns, etc.

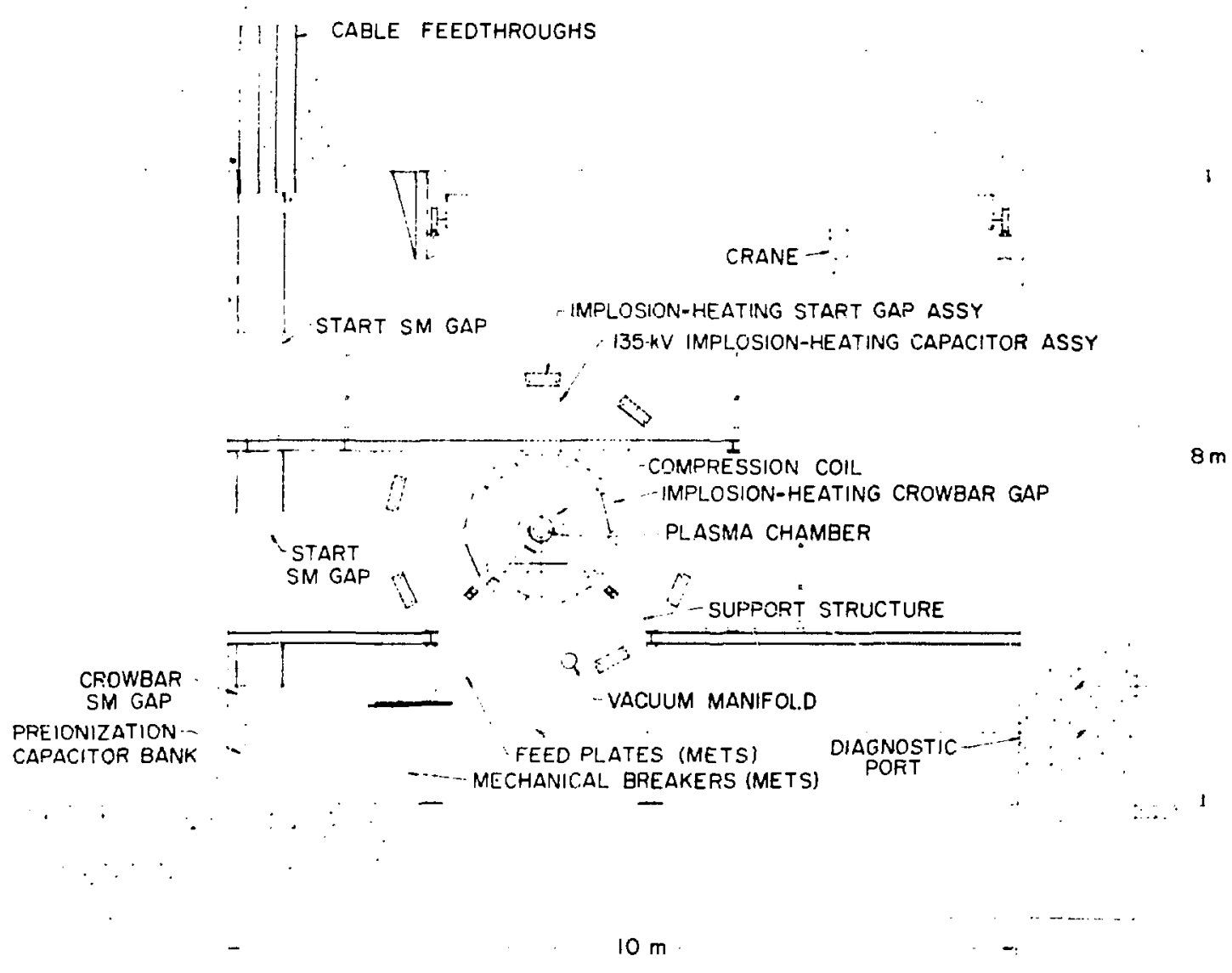

Fig. 5.5-1.

Cross section through SFTR cell. 
After the current reterence vessel module, teedplate. and capacitor designs were selected, a complete and comprehensive shield analssis was pertormed. Since the capacitors outside the primary shield do not torm a closed ring around the torus, their shielding cffect for biological dose calculations ontside the corcrete is very limited. Therefore, to maintain convervarism in the dose calculation through the biulugical shield. only streaming paths which cir"umvent the capacitors were considered.

Although calculational models assume a monolit hic concrete secondary shield, there obviousIy will be many penetrations for optical ports. cables. etc. Some thought has been given, for example. (1) shadow-shielding major penetrations to avoid direct streaming of neutrons from the vessel to the penet ration entrance. Alsu, penetrations are made as nurmal to the streaming line as possible, and ducts are bent or oflset where possible. On the basis of lission reartor experience. these measures should prove adochuate. However, at the detailed design stage. precise calculations of neutron and gamma-ray streaming and scattering will need to be performed.

\subsubsection{Radiation Transport Analysis and Results}

On the basis of the shielding concept and lavout discusised in Sec. 5.5.2. detailed radiation transport calculations were performed to confirm the required performance of both primary and secondary shields. The methods and data applied for this analysis are described below. In presenting the results of the crmputations we will often incorporate results of calculations that do rot include the primary shield tor purposes of easy comparison and to justify the necessity of a primary shield structure.

\subsubsection{Method of Analysis and Nuclear Data}

All radiation transport calculations are performed with codes available in the LASL CTR Nuclear Analvisis System described in detail elsewhere. Transport and activation cross sections, together with other nuclear data. are retrieved from the I.ASI. CTR library, ${ }^{4}$ which is based on most recent E.NDF/B evaluations, and the LASL TD library. The 10()-group neutron cross-section sets in the CTR library are collapsed to 25 groups for the final shield design analysis. All transport calculations are performed in one-dimensional cylindrica! geometry from the plasma centerline outward along radial traverses. Such one-dimensional computational models are considered adequate at this stage of the SFIR design. The application of two- or three- dimensional transport methods is not warranted until a later design stage, because the numerous geometric complexities which can be incorporated into mult idimensional calculat ions will undoubtedly have to undergo se'veral additional design iterat ${ }^{\circ}$ ns before they can be firmly established. The consideration of such complex geometric structures at this conceptual design stage would only comolicate the shield analysis disproportionately and handicap the interpretation of the results.

Figure 5.5-2 shows an R-Z cross secion through the magnet coils, primary shield, and capacitors, ex. tending radially from the plasma centerline to the outer edge of the capacitors and axially over tw 40 $\mathrm{cm}$ modules. It can be seen that many streaming paths exist which allow radiation to circumven? coils, primary shield, and capacitors. Whenever possible, design solutions were sought to block or eliminate such radiation strearing paths. For example, the line-of-sight penetrations required through the primary shield so that the aluminum feedplates can reach the coils inside the shield are blocked by shield sections between adjacent capacitors. An

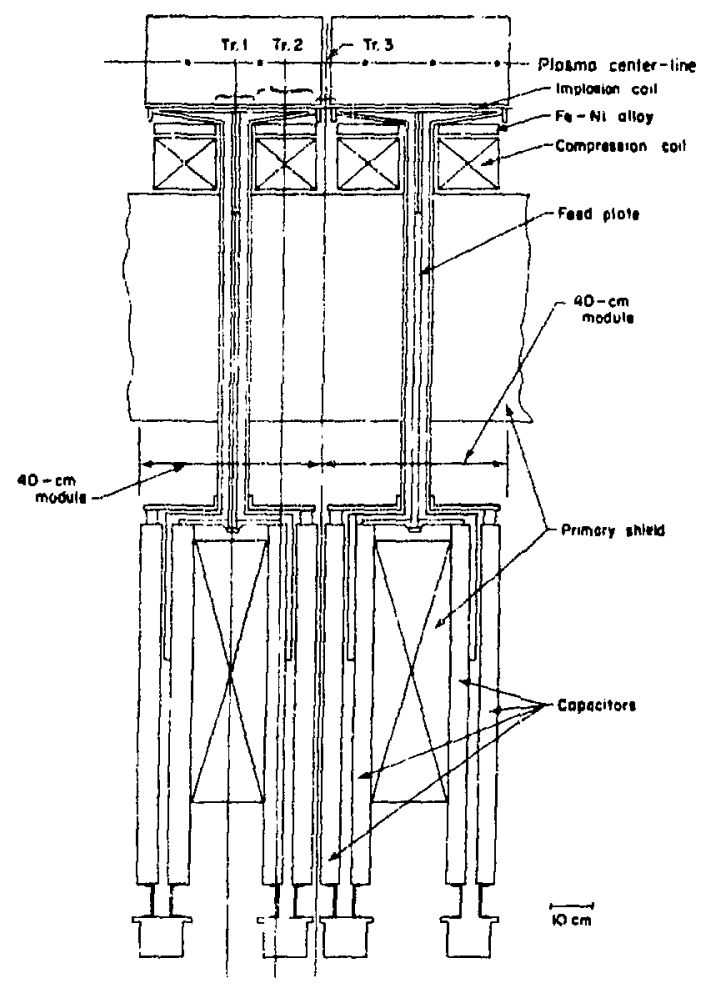

Fig. 5.5.2.

Cross section through two adjacent SFTR modules including coils, primary shield, and capacitors. 
attempt was made to incorporate the effects of the remaining streaming paths into our computational analysis by selecting several radial traverses for the cylindrical model calculations. As indicated in Fig. 5.5-2, for the computation of air activation and radiation effects to the capacitors, we considered three different traverses, Tr. 1, T'r. 2, and Tr. 3, for which separate radiation transport calculations were performed. The calculated neutron flux spectra $\phi_{1}$. ton were then linearly combined to calculate the radiation effects of interest. The coefficients $a_{i}$ for this linear combination are determined from the fraction of first-wall area per module associated with each traverse: $a_{1}=A_{i} /\left(A_{1}+A_{2}+A_{3}\right)$. For the three traverses shown in Fig. 5.5-2 we obtain

$$
\phi=0.56875 \phi_{1}+0.20625 \phi_{2}+0.125 \phi_{3} .
$$

The integral effects of interest are then calculated frum

$$
\mathbf{R}=\left\langle\Sigma_{\mathrm{R}}, \phi\right\rangle,
$$

where $\breve{S}_{H}$ stands for the macroscopic response cros: section and the symbol<.> indicates integrations over all energies and the spatial zones of interest.

\subsubsection{Computational Results}

All transport calculations performed with the D'TF-IV' code were originally normalized to a source strengt h of one 14-Mel neutrun emitted per centimeter of torus. The flux distributions resulting from these compurations were then processed through a pust-processor code. TR3. which performs the linear combinations for streaming corrections ingether with the energy and spatial integrations described above. and renormalizes the source strength as desired. Usually, results are presented per pulse assuming a neutron source strength of 1.78 $x \quad 10^{1 / 4}$ neutrons/cm-pulse. For time-integrated effects a total of 1000 pulses/yr is assumed. In some instances it is convenient to quote equivalent timeaveraged quantities per second which may be obtained by multiplying the results per pulse by $3.17 \mathrm{x}$ $10^{-5}$ pulses/s, or assuming an equivalent timeaveraged neutron source strength of $5.64 \times 10^{9}$ neutrons $/ \mathrm{cm}$ s.

\subsection{Neutron Flux Distribution Inside SFTR ('ell}

The radial distribution of the neutron fluence per pulse is shown in Fig. 5.5-3 for positions inside the

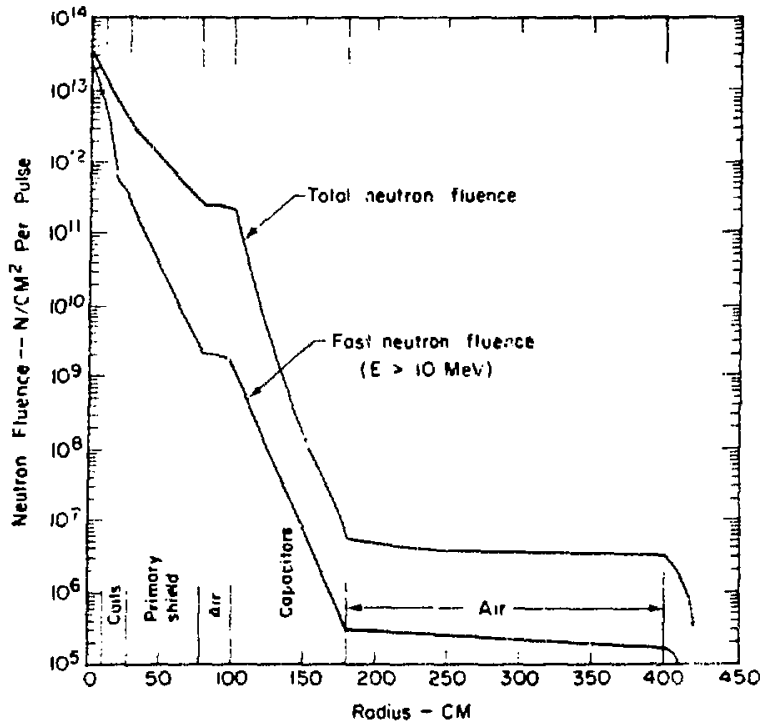

Fig. 5.5-3.

Radial distribution of total and fast neutron fluence per pulse inside SFTR cell.

SFTR cell. In addition to the total neutron fluence (integrated over ail energies), the fast neutron fluence is also plotted for neutron energies above 10 $\mathrm{MeV}$. This high-energy radiation component may be of special interest in estimating threshold activation reactions which may not be specifically quoted in this report.

\subsection{B:ological Dose Distribution}

The radiai distribution of the neutron duse per pulse delivered at any point within $5.5 \mathrm{~m}$ of the plasma is given in Fig. 5.5-4. A conservative assumption has been made for this computation: the shielding effectiveness of the capacitors is totally neglected and the space between the primary shield and the concrete cell wall is assumed to be air. Even with this stringent assumption the calculated neut ron dose at the outer surface of the concrete wall meets the biological shield design criterion of 0.5 $\mathrm{rem} / \mathrm{yr}$ (equivalent to $0.5 \mathrm{mrem} /$ pulse), as seen from Fig. 5.5-4. This calculation also confirms that the I.5-m-thick biological shield, together with the 50 cm-thick primary shield, is sufficient to meet radiation exposure limits for personnel access areas ont. side the SFTR rell. This conclusion holds even for the ext reme case in which personnel are present during all 1000 (assumed) pulses per year, immediately: outside the concrete cell wall and at the maximum dose location. 


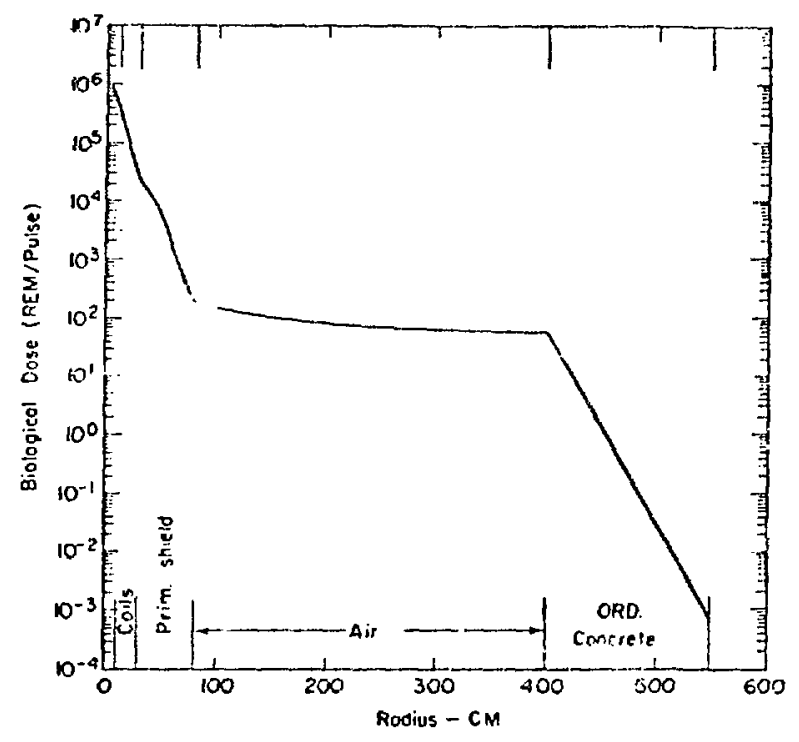

Fig. 5.5.4.

Radial distrioution of biolegical dose per pulse.

\subsection{Air Activation in the SFTR Cell}

Table 5.5-2 present:s a summary of the radioactivity generated in the air inside the SFTR cell per pulse and per year. The air circulating in the cell is assumed to be well filtered so that activation of dust particles need not be corisidered. Unless removed, the activared isotopes in the cell air will eventually be released into the containment at mosphere and into the environment. Therefore, Table 5.5-2 alsc; list: the total annual radiological impact from air activation. assuming 1000 pulses/yr. For the short.lived isotopes (N-13 and N-16) the radioar rive decay will in itself reduce the generated activity to negligible values between pulses. However, the envirunmental impact of the isotopes with longer half-lives must be considered. Therefore. the MP's's for occupational exposure as defined in Rel. 1 are also given in Table 5.5-2. No MPC is given in Ref. 1 for "29 Ar: it was calculated assuming the lens of the eye and the skin to be the most critical organs and therefore represents a conservative limit. Defining a $\mathrm{RBH}$ (relative biological hazard) as

$$
\mathrm{RBH}=\frac{A\left(\mathrm{Ci} / \mathrm{m}^{3}\right)}{\operatorname{MPC}\left(\mathrm{Ci} / \mathrm{m}^{3}\right)}
$$

where $A$ is the calculated specific activity per pulse, it can $w$. seen that for all isotopes the RBH is less than 1.0. This indicates that even if the activated air from the SFTR cel! were released immediately after each pulse, its activity would not create any biological hazard. This conclusion is true, even if no dilution of the cell air is assumed. Due to the long half-lives of ${ }^{39} \mathrm{Ar}$ and ${ }^{14} \mathrm{C}$ isotopes, their activity will be cumulative for multiple pulses. But even after 1000 pulses/yr with the same cell air environment. $\mathrm{RBH} \ll 1$. To express the radiological impact of the long-lived isotopes in air mure quantitatively we define an IHP (integrated hazard potentiai) as

TABLE: 5,5-2

SFTR AIR ACTIVATION, EXCLLIDING; DUST

\begin{tabular}{|c|c|c|c|c|c|c|c|c|c|c|}
\hline Isotope & $\begin{array}{l}\text { Production } \\
\text { Reaction }\end{array}$ & $\begin{array}{c}\text { Half- } \\
\text { Life } \\
\left(\mathbf{T}_{1 / 2}\right)\end{array}$ & $\begin{array}{l}\text { nean- } \\
\text { Rife } \\
(t(s))\end{array}$ & $\begin{array}{c}\text { Principal } \\
\text { Radiation } \\
\text { (Evpe; MeV) }\end{array}$ & $\begin{array}{l}\mathrm{M} \Gamma^{\prime} \mathbf{C}^{3} \\
\left(\mathbf{C i} / \mathbf{m}^{3}\right)\end{array}$ & $\begin{array}{l}\text { Spec. } \\
\text { Act. } \\
\text { per } \\
\text { Pulse } \\
\text { (Ci/mi) }\end{array}$ & $\begin{array}{l}\text { Tot. } \\
\text { Act. } \\
\text { per } \\
\text { Pulse } \\
\text { (Ci) }\end{array}$ & $\begin{array}{l}\text { Tot. } \\
\text { Act. } \\
\text { per } \\
\text { Yatar } \\
\text { (Ci/sr) }\end{array}$ & $\begin{array}{c}\text { RFill } \\
(1,1)\end{array}$ & $\begin{array}{l}\text { IIP } \\
(s)\end{array}$ \\
\hline $13 N$ & ${ }^{14} N(n, 2 n)$ & $9.9 \mathrm{~min}$ & $8.6 \mathrm{E}+2$ & $\beta^{+} 1.2$ & $\cdots$ & $3.0 ; 3 k_{-1-5}$ & 1.08E:-1 & $\ldots$ & $\ldots$ & $\ldots$ \\
\hline${ }^{16} \mathrm{~N}$ & "16in.p) & $7.1 \mathrm{~s}$ & $1.02 \mathrm{E}+1$ & $\left\{\begin{array}{l}\beta-4.3 .10 .4 \\
\gamma 6.1,7.1\end{array}\right.$ & $\cdots$ & $1.62 \mathrm{E}-3$ & $1.68 \mathrm{~F}+\mathrm{I}$ & $\cdots$ & $\ldots$ & $\ldots$ \\
\hline${ }^{14} \mathrm{C}$ & ${ }^{14} \mathrm{~N}(\mathrm{n}, \mathrm{p})$ & $5730 \mathrm{yr}$ & $2.6 \mathrm{E}+11$ & $\beta-0 . i 6$ & $4.0 \mathrm{E}-6$ & $2.9 \mathrm{E}-11$ & $3.11 \mathrm{~N}-5$ & $3.1) E-4$ & $7.3 F_{-1}$ & $1.9 \mathrm{~F}+6$ \\
\hline${ }^{39} \mathrm{Ar}$ & ${ }^{40} \mathrm{Ar}(\mathrm{n} \cdot 2 \mathrm{2n})$ & $269 \mathrm{yr}$ & $1.2 \mathrm{E}+10$ & $\beta-0.5 f$ & $1.0 \mathrm{E}-4$ & $4.3 \mathrm{E}-13$ & 4.5E-9 & $4.5 \mathrm{E}-6$ & $4.3 \mathrm{E}-9$ & $\therefore 2 B+1$ \\
\hline${ }^{41} \mathrm{Ar}$ & ${ }^{40} \operatorname{Ar}(n, y)$ & $1.8 \mathrm{~h}$ & $9.5 \mathrm{E}+3$ & & $2.0 \mathrm{E}-6$ & $1.7 F-6$ & $1.8 \mathrm{~F}-2$ & $\cdots$ & 0.85 & $8.1 \mathrm{~F}+3$ \\
\hline
\end{tabular}

Note: $8.6 \mathrm{E}+2$ means $8.6 \times 10^{+2}$. 


$$
\begin{aligned}
I H T^{2} & =R B I \int_{0}^{\infty} e^{-i t} d t \\
& =\operatorname{RisH} \cdot \tau,
\end{aligned}
$$

where $t$ is the mean-life of the isotope in seconds ( $T$ $=1 / \lambda=T_{1} / / n 21$. These values are also given in Tabie $5.5-2.2$.

A comparison of the activicies given in Table 5.5 .2 with values calculated without a primary shield confijrms the effectiveness of this shield. The unsnielded air activities are approximately a factor of 100 higher than the values in Table $5.5-2$.

\subsection{Activation of Coils, Structures, and Capacitors}

Seventy different nuclear reactions of neutrons with materials surrounding the plasma were considered for our activation analysis. In the process of selecting the most important radioactive isotopes generated in the SFTR cell we dismissed a number of production mechanisms on the basis of (a) very small activation cross section. (b) very low abundance of parent-isotope in natural element. or $(c)$ pure beta emission of product isotope. 'The remaining activation reactions are listed in Table 5.5-3 for materials expected to be present in the magnet coils. the primary shield structure. and the capacitors. Isotopic production rates, $P_{i j}$, as the number of product isotopes, $\mathrm{j}$. generated per parent isctope, $\mathrm{i}$. per pulse. are given at various locations in the cell for every activation reaction considered relevant for the sFI'R. The table is organized according to increasing half-lives of the product isotopes. Potential activation reactions with ${ }^{59} \mathrm{Co}$ are also included because cohalt is expected to be contained in nickel as :m impurity with 0.3 .0 .5 vol', From the production rates given in Table 5.52 it is easy to calculate specific as well as total detivities for certain integral components inside the cell:

$$
\begin{aligned}
& \Lambda_{\text {spec, } j i}(r)=1, \cdot \lambda_{j} \sum_{i} r_{i} P_{i j}(i) \\
& { }_{\text {sot }}=\sum_{j} A_{\text {spec. }, j}^{(r) d V}
\end{aligned}
$$

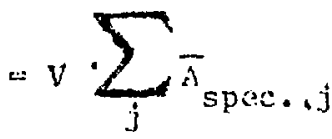

where

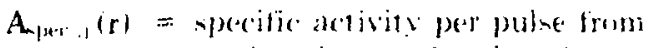

$$
\begin{aligned}
& \text { predue isengere } i \text { al location } r \text {. } \\
& \text { Ci'cm" pular } \\
& A_{1<11}=\text { total activity por pulse. Cispulse } \\
& \mathrm{K} \quad=\text { conversion factor } \\
& 2.7 \times 10^{-11} \quad \text { Ci/kisintegrations/s } \\
& N_{1}=\text { number density of parent isotopes } \mathrm{i} \text {. } \\
& =\text { parent isotones } / \mathrm{cm}^{i} \\
& \lambda_{1}=\text { decay constant for procluct isotope } j \text {. } \\
& \text { disintegrations/sifproduct isotope i } \\
& P_{i}(r)=\text { production rate of isotope } j \text { irom } \\
& \text { imifrupe } i \text { at location } r \text { per pulse. } \\
& \text { product jsotopes/parent } \\
& \text { isotope - pulise } \\
& V=\text { volume of material containing } \\
& \text { parent isotopes at density } \dot{N}_{1}, \mathrm{~cm}^{: t} \text {. }
\end{aligned}
$$

In evaluating the number densities $N_{1}$. the abundance of the particular isotope of interest in the naturally occurring element must he considered, as well as the volume fraction of that element when a chemical compound or phsisical mixture is considered:

$$
N_{i}=\frac{N \rho_{i}}{\Lambda_{j}} \cdot a_{i} f_{i}
$$

where

$$
\begin{aligned}
\mathrm{N}= & \text { Avogadro number } \\
= & 0.60225 \times 10^{24} \text { atoms/mol } \\
\rho_{\mathrm{i}}= & \text { mixture density. } \mathrm{g} / \mathrm{cm}^{3} \\
\mathrm{~A}_{\mathrm{i}}= & \text { atomic weight of element } \mathrm{i}, \mathrm{g} / \mathrm{mol} \\
\mathrm{a}_{\mathrm{i}}= & \text { volume fraction of element } \mathrm{i} \text { in mix } \\
c_{\mathrm{i}}= & \text { fraction of isotopic abundance of specitic } \\
& \text { isotope in element } \mathrm{i} .
\end{aligned}
$$

The above formulas are given intentionally in this much detail ici allow the designer to estimate induced activities in the process of future design iteritions, and to provide the hasis for determination of possible activations of instiliments located throughout the SFTR cell but not shown in the present design drawings.

The so-calculated activities per pulse are the basis for determining (a) safe access of personnel to the rell. (b) potential biological exposures during maintenance and repair inside the cell, and $(c)$ requirements for the handling and disposal of activated components. These criteria are discussed in Chapter VII. 
TABLF 5.5-3

PRODUCTION RATE OF RADIOACTIVE ISOTOPES PLR PUIASE

\begin{tabular}{|c|c|c|c|c|c|c|c|c|c|c|}
\hline \multirow{3}{*}{$\begin{array}{c}\text { Product } \\
\text { Isotope } \\
\text { (j) }\end{array}$} & \multirow{3}{*}{$\begin{array}{l}\text { Production } \\
\text { Mechanism } \\
\text { (i(react.)j) }\end{array}$} & \multirow[b]{3}{*}{$\mathbf{T}_{1 / 2}$} & \multicolumn{8}{|c|}{$\begin{array}{c}\text { Production Kate Pij (product-isotope/parenl-isolope pulse) } \\
\text { at Distance } \mathbf{K}(\mathrm{cm}) \text { from Plasm; }\end{array}$} \\
\hline & & & \multirow{2}{*}{$\begin{array}{l}\text { I.C } \\
10.52\end{array}$} & \multirow{2}{*}{$\begin{array}{c}\text { Fe-Ni } \\
13.77\end{array}$} & \multicolumn{2}{|c|}{ Comp. Coil } & \multirow{2}{*}{$\begin{array}{c}\text { Sh. } \\
5.5 .6 .5\end{array}$} & \multicolumn{3}{|c|}{ Capacitors } \\
\hline & & & & & 17.5\% & 27.15 & & 101.0 & 111.0 & 179.0 \\
\hline $\mathrm{Cu}-66$ & Cut & $5.1 \mathrm{~min}$ & $1.3-12$ & $1.2-12$ & $1.8-12$ & $8.1-1.3$ & $2.1-13$ & $2.5-13$ & $3.7-16$ & $6.2-18$ \\
\hline Cu-62 & $\operatorname{C.uf3}(n, 2 n)$ & $9.8 \mathrm{~min}$ & $2.2-12$ & $9.9-1.3$ & $5.0-13$ & $1.7-1.3$ & $6.9-15$ & $4.5-16$ & $\therefore .2-18$ & $7.7-2(1)$ \\
\hline $\operatorname{Co} 62$ & $\operatorname{Cuf5}(n, \alpha)$ & $1: 3.9 \mathrm{~min}$ & $1.9-1.3$ & $8.7-14$ & $4.4-1.4$ & $1.6-14$ & $8.8-i 9$ & $6.3-17$ & $94-19$ & $1,(i-2)$ \\
\hline $\mathrm{Mg} \cdot 27$ & $A \mid 2 T(n, p)$ & $9.5 \mathrm{~min}$ & $4.3-1.3$ & $2.0-1: 3$ & $1.0-13$ & $? 9-14$ & $2.5-15$ & $1.9-1 d j$ & $2.9-18$ & $5.3 \quad-\ldots .2(1$ \\
\hline Al-28 & $\operatorname{Al} 27(n, \gamma)$ & $23.0 \mathrm{~min}$ & $3.7-14$ & $3 . \overline{3}-14$ & $3.1-1.4$ & $1.4-14$ & $4.3-15$ & $7.9-16$ & $3.7-19$ & $2.7-2 !$ \\
\hline $\mathrm{Ni}-65$ & Cu65(n & $2.6 \mathrm{~h}$ & $1.1-13$ & $5.0-14$ & $2.6-14$ & $9.2-15$ & $5.1-16$ & $3.7-17$ & $5.4-19$ & $9.4-21$ \\
\hline$M n-56$ & $\mathrm{Fes}$ & $2.6 \mathrm{~h}$ & $5.4-1: 3$ & $2.5-1.3$ & $1.3-1: 3$ & $4.5-14$ & $2.4-15$ & $1 . \bar{z}-16$ & $2.5-$ & $4.2-20$ \\
\hline$M n-56$ & $\mathrm{Mn}$ & $2.6 \mathrm{~h}$ & $6.7-12$ & $6.4-12$ & $1.0-11$ & $4.5-12$ & $1.2-12$ & $1 \therefore-12$ & $3 . \therefore-$ & $38-17$ \\
\hline$\Lambda$ & (x) & $2.6 \mathrm{~b}$ & $1.4-1: 3$ & $9.6-14$ & $3.4-14$ & $1.2-14$ & $6.1-16$ & $4.3-17$ & $6.2-19$ & $1.0-20$ \\
\hline Cu-64 & $(n, \gamma)$ & $12.7 \mathrm{~h}$ & $2.9-12$ & $2.7-12$ & $4.0-12$ & $1.8-12$ & $4.8-1.3$ & $5.2-13$ & $1.2-15$ & $1.3-17$ \\
\hline Cul-64 & $\operatorname{Cu} 65(n, 2 n)$ & $12.7 \mathrm{~h}$ & $4.5-12$ & $2.0-12$ & $1.0-12$ & $3 . \overline{5}-13$ & $1.6-14$ & $1.1-15$ & $1.3-17$ & $2.1-19$ \\
\hline $\mathrm{Na}-24$ & $\mathrm{Al} 27$ & $15 \mathrm{~h}$ & $6.3-1.3$ & $2.9-13$ & $1.5-13$ & $5.2-14$ & $2.7-15$ & $1.9-16$ & $2 x-18$ & $+x-2.0$ \\
\hline $\mathrm{Ni}-57$ & $\mathrm{Ni58}(n, 2 n)$ & $36 \mathrm{~h}$ & $1.0-1: 3$ & $4.6-14$ & $2.3-14$ & $8.0-15$ & $30-16$ & $1.9-17$ & $\therefore 0-19$ & $2.9-21$ \\
\hline Cu-5l & $\mathrm{Fe} 5$ & 28 days & $7: 3-13$ & $3.3-1.3$ & $1.7-1.3$ & $6.0-14$ & $2.9-15$ & $2.1-16$ & $2.9-18$ & $4.8-20$ \\
\hline $\mathrm{Cr}-5 ̋]$ & $\operatorname{Cr52(n.2n)}$ & 28 days & $1.2-12$ & $5.2-1: 3$ & $2.6-13$ & $9.1-14$ & $3.4-15$ & $22-16$ & $2.27-18$ & $3.2-2(1)$ \\
\hline Fe-59 & $N i 6^{2}(n, c)$ & 45 days & $9.4-14$ & $4.3-14$ & $2.2-14$ & $7.5-15$ & $3.5-16$ & $2.4-17$ & $3.2-19$ & $\bar{I}: 2-\underline{1}$ \\
\hline $\mathrm{Fe}-59$ & Fe5 & 45 days & $1.3-12$ & $1 . i)-12$ & $1.2-12$ & $5.2-13$ & $1.3-1.3$ & $1 . \overline{1}-13$ & $3.4-16$ & $3 . i-18$ \\
\hline $\mathbf{F}$ & $\mathrm{CoS}$ & 45 days & $3.8-13$ & $1.7-1: 3$ & $8.9-1.4$ & $3.2-14$ & $1.7-15$ & $1.2-16$ & $1.4-18$ & $3.01-201$ \\
\hline $\mathrm{C}$ & $2 \mathrm{n})$ & I1 diais & $2.7-12$ & $1.2-12$ & (i.) -13 & $2.1-13$ & $8.8-15$ & $5.9-16$ & $7.1-1 x$ & $1.1-19$ \\
\hline$C(0-58$ & $\operatorname{Ni58(n,p)}$ & It days & $2.6-12$ & $1.3-1.2$ & $(i .9-1: 3$ & $2.7-1.3$ & $2.1-1.1$ & $1 \therefore-1 \overline{1}$ & $24-17$ & $4.4-19$ \\
\hline Co.57 & $\operatorname{Nis8(n,np)}$ & 271 day & $7.1-1.3$ & $3.2-1: 3$ & $1.6-1.3$ & $5.6-14$ & $2.5-15$ & $1.7-16$ & $22 .-18$ & $\therefore .5-11$ \\
\hline$M n-54$ & $\mathrm{Fe} 54$ & 31:3 days & $2.4-12$ & $1.2-1.2$ & $6.4-13$ & $2.5-1.3$ & $1.9-1$. & $1.4-15$ & $2.2-17$ & $4.1-19$ \\
\hline $\mathrm{Mn}-54$ & $\operatorname{Mn55}(n, 2 n)$ & 31:3 days & $4.6-12$ & $2.0-12$ & $1.0-12$ & $3.6-1: 3$ & $1.6-11$ & $1.1-1 . i$ & $1.3-17$ & $2.11-19$ \\
\hline & $\mathrm{Ni6}$ & $5.2 \mathrm{vr}$ & $7.8-13$ & $3.6-1: 3$ & $1.8-1.3$ & $6.6-14$ & $36-15$ & $26-16$ & $3.8-18$ & $(i . \overline{1}-2)$ \\
\hline & $\operatorname{Cos} 9(n, \gamma)$ & $5.2 \mathrm{yr}$ & $2.6-11$ & $2.5-11$ & $3.6-11$ & $1 . i-11$ & $4.1-12$ & $4.5-1.2$ & $9 . \overline{-}-1 i$ & $1.1-16$ \\
\hline & $\operatorname{Cu63}(n, k)$ & $5.2 y$ & $1.9-1.3$ & $8.6-14$ & $4.4-1.4$ & $1.6-14$ & $R . x-16 i$ & $6.3-17$ & $9.4-19$ & $1.6-2(1$ \\
\hline & Al:2T(n,2n) & $7.3 \times 10^{5} \mathrm{yr}$ & $1.6-13$ & $6.8-14$ & $3.5-14$ & $1.2-1.4$ & $4.1-16$ & $2.6-17$ & $2.5-19$ & $3.3-3-21$ \\
\hline
\end{tabular}

Note: $1.3-12$ means $1.3 \times 10^{-12}$ 


\subsection{Radiation Effects to Coils and} (apaciturs

Radiation effects which may change mechanical or chemical properties of materials are considered in this section. Most of these effects can be quantitarively related to the physical dose absorbed in such material and which can be expressed in units of Gy 11 ciy $\equiv 1$ gray $=100$ rads $)$ or as an energy production density in units of erg/g or $\mathrm{M} \cdot \mathrm{J} / \mathrm{m}^{3}$ (1) Gy. $\left.=10^{-4} \mathrm{erg} / \mathrm{g}\right)$.

linergy production densities from neatron and gamma-ray deposition are everywere less than ! $\mathrm{M} \cdot \mathrm{J} / \mathrm{m}^{\prime \prime}$ per pulse. causing only negligible radiation heating rates so that temperature rises caused by nuclear harating are $<1 \mathrm{k}$ per pulse at any location inside the SFTR cell. likewise, the time-averaged 14-Mel neutron wall loading on the first wall is only $2 \times 10^{-6} \mathrm{MW} / \mathrm{m}^{2}$, so radiation damage to metals is inconseyuential. Due to the small neutron fluence levels. the tran:smutation effects at any location are below $1 \mathrm{ppm} / \mathrm{sr}$ and cause no noticeable changes of mechanical or electrical properties in the copper coils or the Fe-Ni allos: Also. maximum atom disNlacements in the iron. nickel. or copper are -2 $x$ $10^{-x}$ displacements/atom/pulse or $2 \times 10^{-.}$ displacements/atom/yr. which are negligible values. The only remaining radiation effect that may impose a significant burden on the SFTR design is the radiation damage to organic materials being used as insulating and shielding materials.

'The radial distribution of the neutron physical dose absurbed in orranics per pulse is shown in Fig. 5.5-5. Organic insulation may be used in the Fe-Ni allow as well as in the compression eroil and he capacitors. The maximum dose wo an organic insulator in the Fe-Ni alloy at $\mathrm{r}=13.6 \mathrm{~cm}$ is is $\mathrm{x} 10^{-2}$ (ivpulse: to the compresion coil insulatur at $1=17$

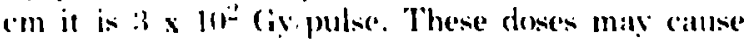
sionificant damane and impair the insulating propurtices of organic materials if used as insulators in these locations. However. no information exist: 10 identily a limiting damage mechanism le.g.. gats generalion. sludge tormation. dimensional growth) and no firm design criterion has been established. The pulyethylene in the primary sheld is subiect to a maximum physical dose of -10 (iy/pulse at its lockation closest to the plasma. However. since there are no other than shiolding requirements established fin these polvethylene regions. an ahsorbed dose of 110 Civ/pulse is of no concern.

The maximum physical dose to the capacitor insulation is $1.5 \mathrm{G} \mathrm{g} /$ pulse at $\mathrm{R}=100 \mathrm{~cm}$ and falls off by abuut 4 decades $108 \times 10^{-i}$ ( iv/pulse at $R=180$ cm. Comparing this maximum dose of $1.5 \mathrm{G}$./pulse with the radiation damage criterion established in

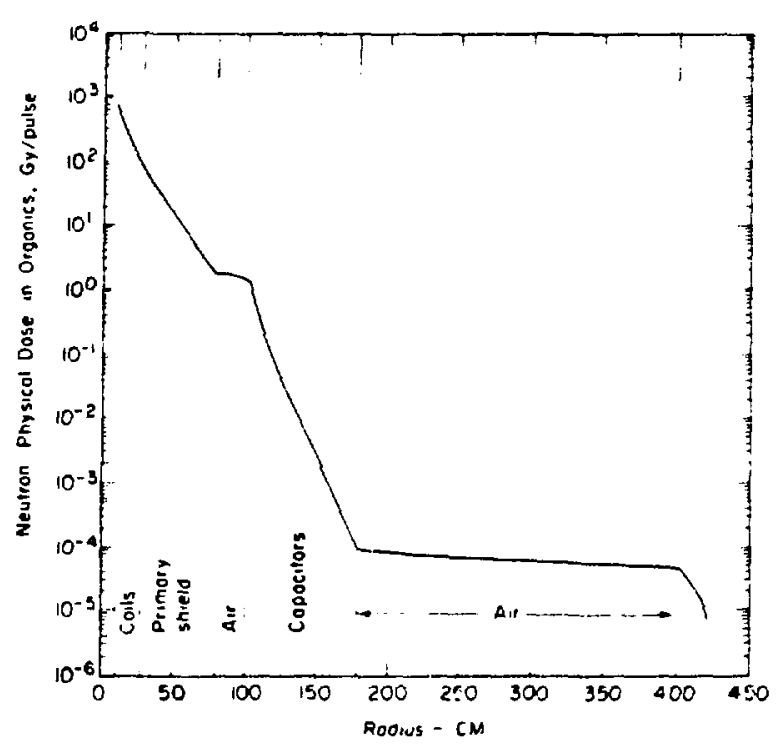

Fig. 5.5-5.

Ratial distribution of neut on physical dose absurbed in organic material $l$ (i) $=10(1)$ radss.

Sec. 5.5.2.1 for the capacitors, $10^{3}$ Giv/r. it is seen that this conservative criterion is almost met. even for the maximum value. $W_{1}$. therefore conclude that radiat ion damage to the capacitors is not expected to be a problem. but the presence of the primary shield is absolutely necessary to meet this objective.

\section{RFFFRENCES (Chap. V. Sec. 5.5)}

1. "Standards for Radiation Protection. Rules and Regulations," 'Title 10. Part 20. I'.S. Alomic Energy Commission 11970$)$.

\section{Harold Etherington (Ed.). Nuclear Engineering Handbook (Mc(iraw.Hill. New York. 1958). pp. 10- $1: 36 \mathrm{ft}$.}

3. Donald .I. Dudziak. "Fusion Reactor Nuclear Analysis Methods and Applications." Proc. Sth Symp. on Fusion Technology. Noordwijkerhout. The Netherlands. 17-21 June 1974 .

4. D. W. Muir et al. "Nuclear Data for the Controlled Fusion Program." in Applied Nuclear Data R\&D Quanerly Progress Report. July 1 through Sept. 30. 1974. Los Alamos Scientific Laboratory report I.A-5804-PR (Der. 1974). 
5. P. X. Stevens and D. K. Trubes. "Methods fir Calculating Neutron and famma-kay Attenuation. Chapter $: 3$ in Weapons Radiation Shielding Hand!hok. DNA.180\%-3. Rev. 1 (197\%).

\subsection{TRITIEM HANDLING SYSTEM}

\subsubsection{Overview of Tritium Handling System Function, Design Requirements, and Layout}

The major functions of the tritium handling system are: (i) (1) inject precisely measured (D).T\%. mixtures into the SFTR discharge tube in preparation for a D-T discharge: (ii) to recover and/or to account for all unburned tritium after a D-T discharge: (iii) to provide safe sturage of gram yuantities of tritium: (iv) to monitor and to control the chronic release of tritium and. (v) to present safeguards and cleanup capabilities in the event of a sjgnificant accident al release of tritium. The major subsistems that make up the tritium handling ststem are:

- Passive Storage Facility or Vault (PSF)

- Tritium Iniection system (TISi

- Tritium Recovery sistem ITRSi

- Vacuum sistem

- Tritium Waste Treatment (TWT) Facility

- Cell Cleanup Facility (CCF).

Figure 5.6.1 depicts schematically the interrelationship of components of the tritium handling ssistem. In addition to the more obvious func. tions of sturage, injection. and recovery. the SFIR vacuum system. (D.T), impurity control. and posidischarge gas analyses are considered important portions of the tritium handling system.

The interactions between these subsustems may be multifarious andior diffuse: for instance, the vacuum system per se p!ays important roles in both tritium injection and recovery and to some extent impinges upon the design of the TWT and CCF.

Tritium injection will be made by the use of stan. dard volumes and vacuum transfer techniques. A uranium bed will supply clean tritium to the injection sistem. Recovery of used (D.T) 2 gas will occur at the cryogenic vacuum pump station, and the (D.T)., so collected will be stored on a second uranium bed. In addition to metal hydride storage of tritium, a vault-like structure will be used for a more permanent, secure storage of gasenus tritium. Chronic tritium releases ar expected to Gccur at the vacuum pump stations (four stations are located within (he SFTR cell) and at the locations of major tritium handling components (cleanup units, uranium beds. etc.) Such components will be located in ventilation hoods, and tritium released

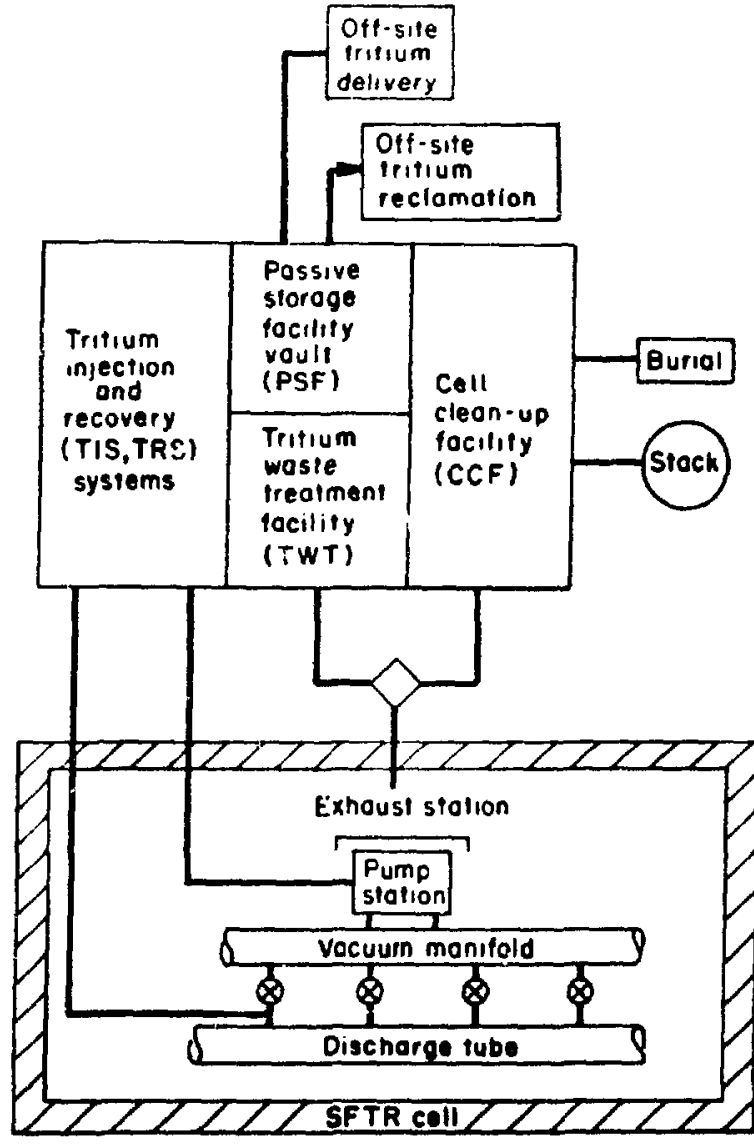

Fig. 5.6.1.

Schomatic of tritium handling sustem.

during routine maintenance of these componemts

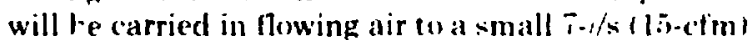

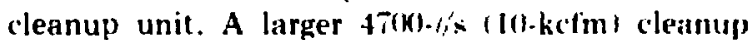
unit will be provided to remove tritiun from the recirculating air in the SFTR cell in the event the discharge tube fails during a D.'T' discharge: the larger cleanup facility will be available for nonscheduled action associated with the failure of other components of the tritium handling ssstem.

The system described above is desiuned to provide for $1000 \mathrm{D}$-T discharges/yr, each discharge requiring on the average $15 \mathrm{mg}$ of $\mathrm{T}_{2}$. On the hasis of $20 \mathrm{D}$ - $\mathrm{T}$ discharges/week, a total triium supply of $3 \mathrm{~g}$ is anticipated; $0.3 \mathrm{~g}$ (one week's supply) will be stored as uranium tritide and $2.7 \mathrm{~g}$ (two months' supply) will be in gaseous form.

In many respects the design for the tritium handling system presented here is very conservative in view of the low inventory of tritium on-site $(3 \mathrm{~g}$, or a 
maximum af T ge induding that tritium nut vet sell oft-sile tor receserst and the relatively small number

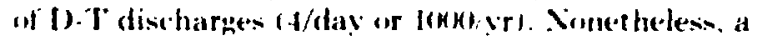

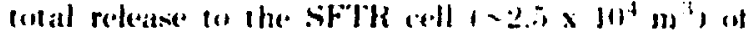
tritium associaled with a 1 ).'T discharge 1 - lis mgl

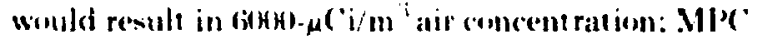

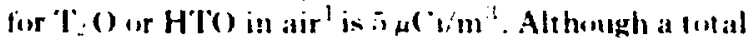
release of the I ritium wo the cell serems hiyhly unlike. iv for any discuptions short of a lotal domolitions of the sfoll diseharge lute dice.. the vacuum system should colleet the major portion of the tritium before the $\left.-x^{+}\right)^{*}$ discharge lube reaches atmospheric

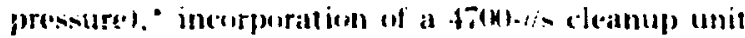
in the cr provides a eapability ut a - $1.5 \cdot h$ tur. never time fur the redl atmesphere in the evemt al at whal release of the lit mis of e ritium. The similaty conservative design of the vatum sysem dictastes a 1-s time comblant in assure lil rapid recovery of Iritium within the discharge tube, tiil a discharge. erele time of lis min. and liiil a base presure law

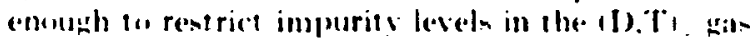
to below 11.1 al. $\therefore$ for a relat ively high outgassing ratc and los leetwed isclation at the discharge nube and the erecurregece of the plasma discharece Assump). tims of a similarls comservalive nature have been made in wher substatem desiens as well as in prupuserl monituring. control, and malntenatmes procedures. The freedinn to select ann-rvative parameters is in part afforded by the preliminary.

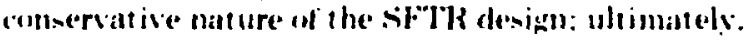
buverer. the rost is benelit relationship must be examined in more aetail.

\subsubsection{Physical, Chemical, and Radiological Nature of Tritium, ${ }^{1,2,3}$}

Tritium is a radioactive hydrogen iscolope of mass three. It decays by emission of a $\sigma^{-}$particle forming three-helium. The emilted $f^{-}$particle has a maximum energy of $18.6 \mathrm{ke}+\mathrm{l}^{\circ}$ and an average energy: of $5.6 \mathrm{kel}$. The half-life of tritium is $-12.3 \mathrm{yr}$. ('he-micalls, t ritium undergnes the same reactions as normal hidrogen."

The riddological hazard: associated with tritium are related to the fact that tritium gas will oxidize to

*To insure a reliable cacuum cleanup of tritium in 'cent of a local rupture of the discharge tube, the gettering of $(D . T)$. along with any air which ingresse's must be done by the Lo. $V_{2}$-cooled roughing pump. Therefore, the roughing pump must be main. tained at $L N_{-}$temperatures during the $D \cdot T$ exporiment. and the cost of this readiness may be hish. form tritilled wate. " Tritium will also underen an isotupice exchange reaction with water:"

$$
\mathrm{I}_{2}+\mathrm{H}_{2} \mathrm{O}+\mathrm{HI}+\mathrm{I} \mathrm{TO}
$$

This water mixes rapidly with body water and is then rapidly eliminated from the buds. The hiolugiral nalf-life of trilium in uxide form is 6-1.t dars."

The maximum permissible cuncentration ${ }^{\text {t: }}$ of

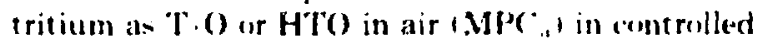
areas is $s u\left({ }^{\circ} \mathrm{i} / \mathrm{m}\right.$ '. In practice this number is used in

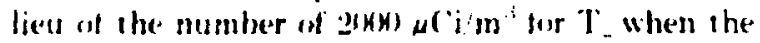
chemical form of the tritium is not known with cer. tainty. The cocupational maximum permissble bedy burden fur individuals is $1 \mathrm{ml}$ "i."

In uncont rolled areas the $\mathbf{M l l}{ }^{\circ}$, for tritium as the cxide is $0.2 \mu$ ( $\mathrm{i}$ im? which is the maximum $2 \mathrm{t} \cdot \mathrm{h}$ average concentratioll that will exist in the sFJ]? stack. For individuals in comtrolled or uncontrolled areas. the maximum dose equivalent resulting from uperation of the SFll tacility will not esceed the then-current gevernment limits.

Fidence foxist- that tritium entering the bods as the oxide slowly exchanges with the hidrogen on the organic molecules. Tritium trom tritiated water is kmown in be assimilated in the organic constifuents of other forms of plant and animal lite. ${ }^{1:}$ Should tritiated proteins enter the human body in vegetalble, dairs: or meat products. the in-bods residence time for tritum in human tissues might be increased considerably. As a resule the decay of tritium so incorporated in biologically active sub. stances could conceisably result in increased somatic and genetic te.g. tritium in thymidine:" damage to body tissue. Clearly. further experimen. tal work is needed on the effects of low levels at tritium on biological sistems.

Keleases of iritium io the ensironment must be sutficiently low that tritium concentrations in man or his foud chains do not increase fo critical levels. The maximum permissible water and air concelltrations listed in lable $\mathbf{5 . 6} \mathbf{6} 1$ retlect this concem. since tritium appears in the envirunment predominantly as tritiated water (HTO). the related radiation drise to man is strongly dependent on the local hudrologic cycle. An atmospheric source of tritium may contaminate a local landscape either by direct deposition of moisture or by exchange of tritiated water molecules of the at mosphere with ordinary water of soils. plant. animals. and surface waters. Once rontaminated. soils. plants. and animals can carry tritium to man through the fond 
TABLE: $5.6-1$

SUMMARY OF IMPORTANT RADIOLOCICAL. BIOLOGICAL, ANI) (HEMIC'AI. CHARACTERISTIC'S OF THITILM

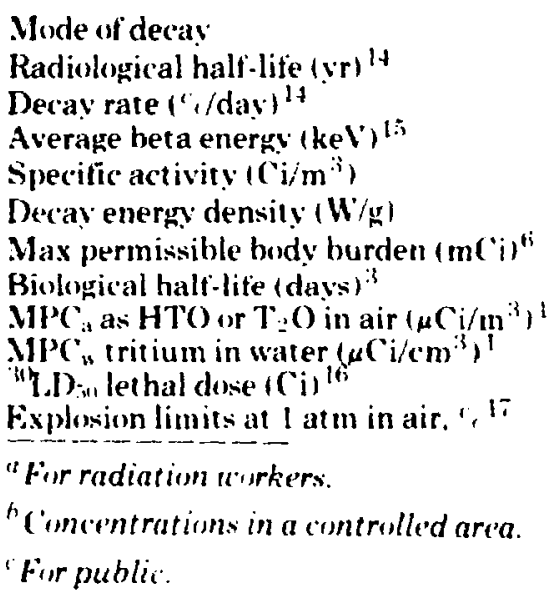

\author{
'H ${ }^{2} \mathrm{He}+\mathrm{y}^{-}$ \\ 12.26 \\ 0.01548 \\ i. in \\ 2.tix $10^{6 i}(36,7)(\mathrm{i} / \mathrm{g})$ \\ (1),3:2()ti

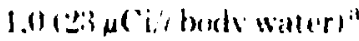 \\ (i. 1.4 \\ $-3.0^{\mathrm{h}} \cdot 11.2 \mathrm{.g}$ \\ $0.1^{b} \cdot 11.010 .3$ \\ 4.5 \\ 4.11. 74.2
}

chain. An example of such a pathway is the soil. plant-cow-milk-man food chain. The iotal dose to man from such a pathway depends on the turnover and replacement rate of tritium in each link of the pathway. In general. the overall turnover rate in a given ecopathway depends on the rate of the slowest transport mechanism. Tritiated soil water has turnover times ranging from 24 day's in the trupics to 1.7 vr in the desert. In temperate zones, turnover time for soil water in the rooting zone of plants may vary from 30 to 120 day's, depending on location. soil type. and season of the year. Intrinsic lumover rates in plants, animals. and man are much faster.

Table 5.6-1 summarizes those characteristics of tritium which will have a direct impact on the SFTR design. An ever-present problem associated with routine tritium handling is the tendency toward isotopic exchange with normal hydrogen in any hydrogenous material. Subsequent to isotopic exchange, the hydrogenous materials will undergo selfirradiation and thereby sufter radiation damage." Also the daughter 'He resulting from decay will tend (1) collect in the material. The low solubility of helium in most materials generally causes extreme property changes (swelling, emhrittlement, etc.). Oils and plast ics are particularly susceptible to these problems. It is therefore highly advisable to design tritium facilities so that the use of organic materials is minimized. ${ }^{1 k}$ This means using metal gaskets on nlanges and connectors, using all-metal valves and fittings whenever possible, and emploving oil-free vacuum systems. In essence, all component" used in a tritium facility must be carefully scrutinized with respect to possible tritium pickup either by isotopic exchange or by tritium adsorption on surfaces. ${ }^{\text {in }}$
Hydrogen embrittlement is an additimal concern in systems handling hydrogen gas and, in particular. tritium gas. 'To avoid this proble'm the low $3(1)$-series st ainless st eels ur copper are recommended for use in tritium systems. There is evidence that the presence of tritium can lead to enhatrced hydrogen embrittement, "1 bu: systems can be easily designed to avoid this prohlem. The primary mechanism for enhanced hydrogen embrittemem by tritium is the reduction of oxide films on the metal. Such films retard the diffusion of hydrogen by acting as a protective laver in which the diffusion of hydrogen is molecular and hence slow. A second factur results from the helium formed by decay of the tritium dissolved in the metal. (The helium is insoluble and has a low permeability constant in metals. "21, Also. gaseous hydrogen and. in particular. tritium. will tend to leach carbon from stainless steels thus forming tritiated methane. This reaction is more probable with ss-3014 than with si-316. The use of s. -316 throughout the tritium handling tacility is therefore recommended.

The intent of this summary is to present the major const raints associated with the use of gram quantities of tritium. Detailed descriptions of significant. specific experiences associated with handling gram quantities of I ritium can be found elsewhere. ${ }^{*}$.The problems associated with tritium containment are widespread, particularly for high-temperature applications. But the SFTR is a rom temperature device and many of the containment problems can be alleviated by proper design and anticipated maintenance procedures. so tritium containment le.g., against low chronic releakest should not be difficult in the SFTR. 


\section{i.ti.A Description of Subsystems}

The primary functions of the tritium handling sistem are the storage injection and recovery of tritium gas. (leamup and waste treat ment function. are ideneified as eit her routine or nunscheduled. The following sections describe the operation lstorage. injection, recoveryl as well as the cleanup function of tritium handling.

Figure ioti-g illustrates the fueling/recovery aspe'ts of the tritium handling system. (iaseous tritium from a passive storage vault is transferred woekly to a uranicum bed in - $(1), 3-\mathrm{g}$ quantilies. The yuant ity of tritium gas required for a daily series of 1) T discharges ltypically 4 discharges/day at -1 is mo/dischargel is transterred from the uranium bed (1) a preinjection space. where measurements and sampling areur. An amount of gas desired for a given discharge is metered and mixed with $\mathrm{I}$ ), and transferred to four holding volumes located at each of four vacuum pumping stations within the SFTR cell. "The vacuum sistem is isolated from the SFTR discharge tube, the (D.T) gas at each of the four staging areas is injected into the discharge tube, and the discharge is triggered. The spent (D.T): gas $1-0.01-(1)\left(0: 3^{\prime}\right.$, burnup, (H.D, T) $\mathrm{O}$ contaminated $\mid$ is collected at the crovgenic pumping stations, which have been open to the discharge tube subsequent to

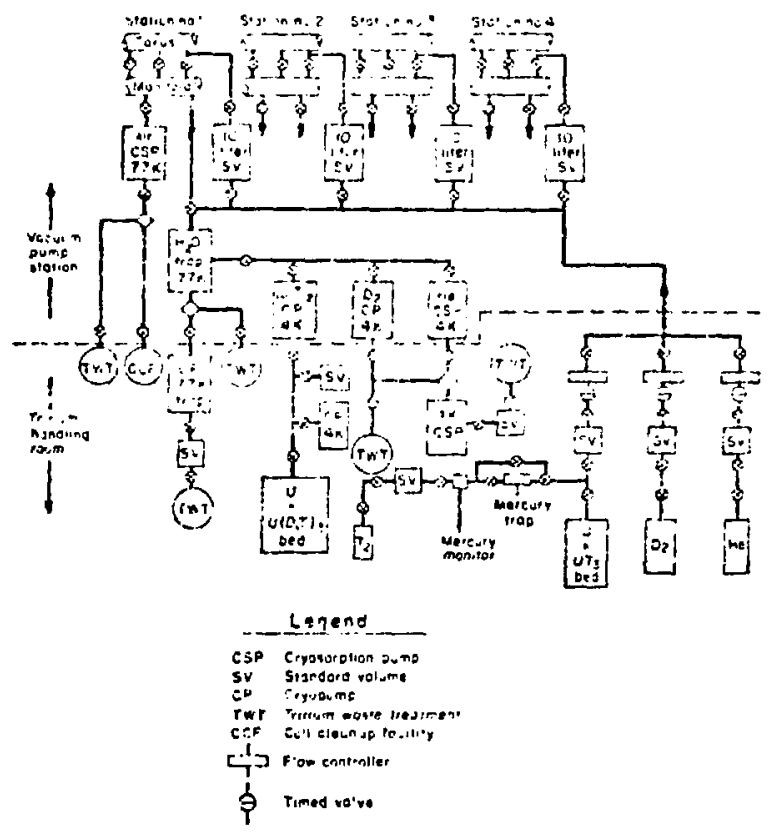

Fig. 5.6-2.

Schematic diagram of tritium fueling/recovery system. the discharge. The rollected (D), $\mathrm{T}$ ), gas undergoes a vacuum transfer via a cryogenic pump to a second uranium bed and is stored thereon for one week: samples of spent (D.T) are easily retrieved for posidischarge diagnostics (Sec. 5.6.4.3). After a week of operation the $\sim 10.3 \mathrm{~g}$ of tritium that was initially absorbed in the uranium bed used for tritium supply now is absorbed in the recovery bed. Each week the tritium in the recovery bed is transferred and stored in the passive storage facility vault and a ciean. 0.3-g tritium supply is transferred to the tritium supply bed. Approximately every 2 months the tritium in the vault $(-.3 \mathrm{~g})$ is exchanged for a new tritium supply obtained from an off-site source.

The tritium clean $1 \mathrm{p}$ scheme operates at two levels: a low-flow cleanup (i / / $/$ ) Tritium Waste Treatment (TWT) system for routine and chronic Iritium release and a high llow cleanup $47(10-1 / \mathrm{s}$ Cell (leanup) Facility ( $C(F)$ for accidental releases. In both sistems the tritium is first oxidized in the flowing air stream and then dried. All tritium handling equipment is located in hooded chambers which ventilate to the TWT. The air within the SFTR cell is continually replaced and monitored for - itium and other activities. The tritium concentration within the exhaust air is continually monitored to determine whether cleanup in the CCF is required before stacking.

\subsubsection{Tritium Storage Facility}

The tritium storage facility comprises two major components: a passive storage vault for secure storage of gaseous tritium. and an active tritium supply which enables tritium to be stored on a uranium-uranium tritide bed. Tritium gas will be desorbed from the uranium bed on demand to provide gas for the SFTR inijector system, whereas reserve or spent tritiuin will be kept in the vault.

Tritium gas will be received from the ERDA supplier as gas in DOT-approved tanks and will be stored in the vault. A portion of this gas $(\sim 390 \mathrm{mg})$ will be absorbed in the uranium bed each week, and the tank with its contained gaseous tritium will be returned to the vault. The vault is secured with fireproof and blastproof walls and security door. This vault will pernit close control over access to the bulk of the $7 \mathrm{~g}$ maximum of tritium expected to be on-site at any given time. When the bulk of the tritium is stored in this area. it will not be subject to release in any credible accident.

The vault (Fig. 5.6-3) will have a ventilation system $(\sim 7 / / \mathrm{s})$ so the air in the vault can be processed by the TWT or CCF if necessary. For instance, if a tritium tank should develop a leak, the tritium can 


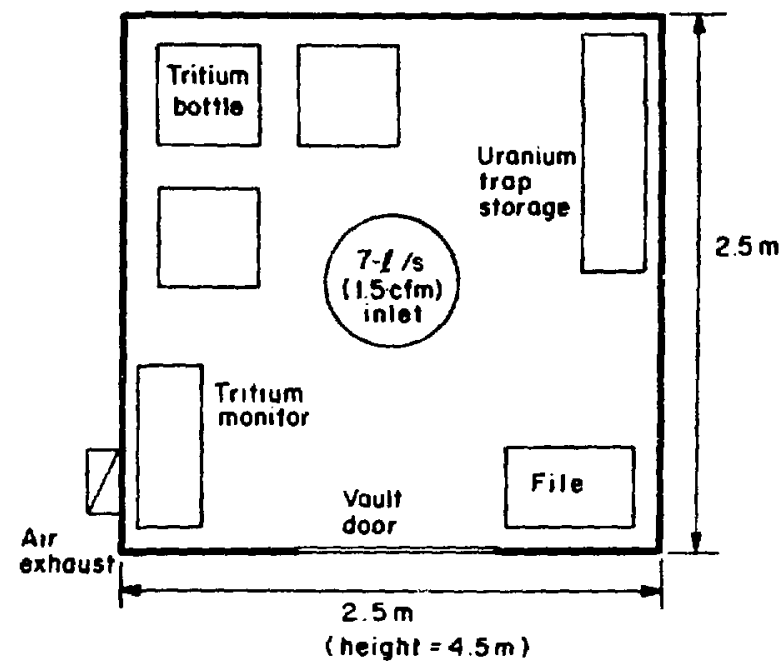

Fig. 5.6-3.

Lavout of tritium vault.

be isolated in the vault by appropriate valve closures, and the air in the room processed at a slow rate to remove all tritium before release to the at mosphere. The vault air will be continuously monitored for tritium.

The active storage facility consists of an allwelded. SS-316 uranium bed on which a 1-wk supply $(\sim 0.3 \mathrm{~g})$ of tritium can be stored as the tritide. The details of the uranium bed are shown in Fig. 5.6-4. The uranium bed will contain $\sim 50 \mathrm{~g}$ of uranium. which can absorb about $2 \mathrm{~g}$ of tritium, although the bed will not be charged with more than the 1-wk supply $(\sim 0.3 \mathrm{~g})$.

Tritium will be reacted with the uranium bed as follows. A tank containing gaseous tritium will be removed from the vault, connected to the uranium bed, and the gas lines evacuated. Tritium gas will then be admitted to the calibrated volume on the in. let line until the pressure in that volume corresponds to a tritium charge of $0.3 \mathrm{~g}$. After equilibrium is reached in the calibrated volume, the pressure readings will be recorded and the gas composition determined by a mass spectrometric analysis. The tritium within the standard volume will then be admitted to the uranium bed at $a$ rate such that no significant temperature rise occurs in the uranium bed. This charging process will occur each week, and careful records will be maintained on the quant ity of tritium added to the uranium bed at each charging.

Each day the uranium-uranium tritide bed will be heated $(623-673 \mathrm{~K})$ to decompose the uranium tritide to produce the daily tritium requirement. The

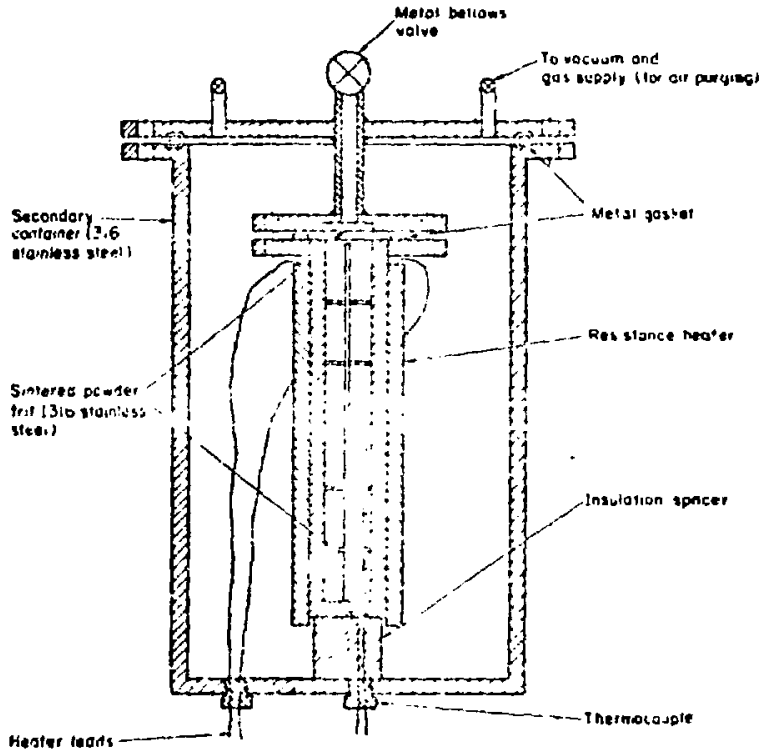

Fig. 5.6.4.

Detail cie't of uranium bed.

dissociation pressure of trit um vs uranium is 101.33 $\mathrm{kPa}$ and $19.98 \mathrm{kPa}$. respectively, at 665 and $395 \mathrm{k}$. 'The equilibrium dissociation pressure of tritium is uranium is given by the equation

$$
\log P_{\text {atm }}-\frac{-3372.6}{T}+4.974
$$

for the temperature range 393 to $623 \mathrm{~K}$ and by

$$
\log P_{\text {atm }}=\frac{-4035.2}{\mathrm{~T}}+6.074
$$

in the temperat ure range $6 \% 310845 k .21$ The I ritium gas desorbed from the uranium bed will be essentially free of 'He formed by tritium decay. Included in the uranium bed heater circuit will be a thermal over-ride switch set at $-70 \% \mathrm{~K}$ so that all elect rical power to the bed heaters will be cut off in event of a thermal excursion.

The uranium bed will be heated once a dax, at which time an amount of tritium adequate for all an ticipated D.T shots for that day is desorbed. This tritium gas will pass directly into the thermustated. calibrated volume in the injector system.

As with wher major components shat handle tritium. the uranium bed will be redundant and ono hed will always be availahle. The uranium beds will he connected to the gas lines by compression fittings 


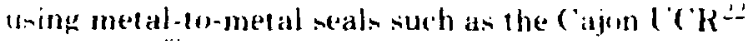

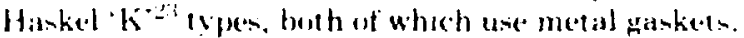
A stindard purge system with valses on both sides ot the connectors will be used. The valves will be of the metal bellows variecy enimilar to the Nupro l' s'riess. - These values will iperate effectively at the temperarures and pressures involved. The gas presinge in the svistem will be measured by an elec. tronie rapacitance manometer such as the Baratron sistem made by Mkilnstriments. Inc.

The entire active storage sistem will be enclused in a houd that will also enclose the main portion of the tritium injectorsistem. The hend can be inclated from the roum air. permitting the processing of hord air through the $7-1 / \mathrm{s}$ TH"l sistem. The hood air can also be sent directly wo the biarger cell cleanup nistem in (ates a llarges tritium -pill dictates rapid cleanup) ot the atr. Howeser. during routine majontenance and couipment replacenent the F-tis sistem is an. ticipalted (a) adequately remove trition from the bueslair.

A sequence of events must be considered which would result in the release to the houd of all of the iritum on the uranium bed. Such a releass would corrempond to a maximum of $6: 3 \mathrm{~g}$. it it were to cocur immediately atter the uranium hed had heen chargad. It the spill bypasied the hoed and entered the irnium handling roum the larger cell cleanup system would be used to remose tritium from the air. However. the spill could be expected to be comfined to the houd area, and the smaller TWT would be used 11 remewe the tritium. This $10.3 \mathrm{~g}$ or $3(k \mathrm{k})$ ( $\mathrm{i}$ would gu to the T-1/s TWT ballast tank where it would he mixed with withe $2 \mathrm{~m}^{\prime \prime}$ of air at $-2 . \mathrm{i}^{\prime}$. relative humidity. The tritium concentration weold then he link! $\mathrm{Ci} / \mathrm{m}^{3}$. At the exhatst trom the "TW"I' the concereration would hase been reduced by a tac-

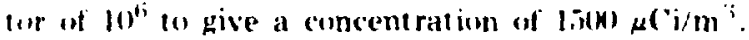
This air from the 7 -lis unit would then be mixed with the stack air to give a momentary concentration of $2.2 \mu\left(\mathrm{i} / \mathrm{m}^{.}\right.$. The integrated stack release alter several minutes weuld amoumt to:3 mCi. and would be lar belew the daily tolerance of $80 \mathrm{mCi}$. Fixeply fir the $: 3$ mo " $i$ the entire $3(k$ k) ( $i$ release would be contained within the TW"I" an the molecular sieve bed.

\subsubsection{Tritium Injection System}

The tritium injection sistem will be supplied with tritium gas from the uranium -uranium tritide bed: deuterium and hetium will he provided directly from gas bottleis. The primary function of the injection sistem is t: leed a predetermined amount of the ap)propriate gas mixture into the discharge tube. Sichematic diawings of the flow pattern and spers- toon of the injector sistem are given in Figs. $5.65-2$ and $5,(i-i)$.

The discharge tube, on the average. will be filled (1) a pressiure of $10^{-2}$ torr for each shot. The worus vilume is $7.89 \mathrm{~m}^{3}$. so each shot wil require $1114 \mathrm{~cm}$

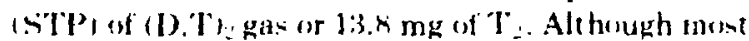

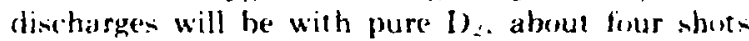
each day will he 1!).T.. Occasionally the injection gas may be a deuterium helium mixture. The tritium gas will be supplied from the uranian bed. Fach day a quantity of tritium gas sufficient to fuel all planned D T discharges for that day will be ther. mally desorbed from the uranium bed and collected in the thermostated. calibrated volume shown in Fig. 5.6-5. Typically, each discharge will require lis $\mathrm{mg}$ of $\mathrm{T}$. including auxiliary systems: hence. four

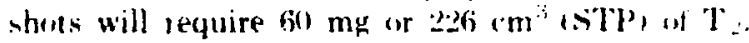
These tigures do non include the gas which must be supplied to the standard colume 1 provide a sut ficient pressure yradient thr the gas lo huw irum the standard volume intu the injector system. Alno. some gas must be left in the injectur sistem aller in ieclion. but this quantity is a small froction of the

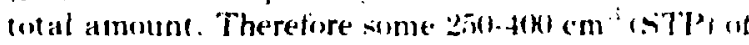
$T$ : will have to be collected in the standard wolume at the start of each day. After the gas has been collected in the standard wolume. time will be allew. ed for the gas to reach thermal equilibrium. and the temperature and presivure will be recorded. An indicated on Fig. 5.6.5. an electrinic capacitance manometer will be used 10 monitor pressure.

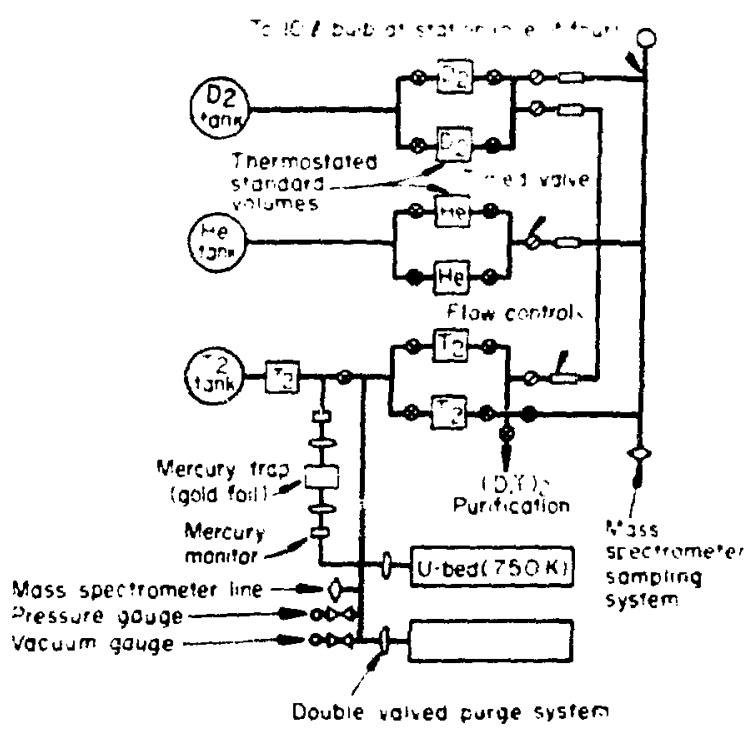

Fig. 5.6-5.5

Tritium injection sivstem. 
Deuterium gas from a gas tank will be collected similarly in a calibrated volume on the deuterium inlet line and the temperature and pressure record. ed. At a predetermined time before a discharge the electronically controlled timing valve on the deuterium line will open, and deuterium gas will flow through the timed valve to the flow controller. The flow controller will be similar to the Series 8240 Brooks Thermal Mass Flow Controller ${ }^{26}$ and will permit a predetermined flow rate of gas to now through the controller into the lines leading to $10-1$ gas mixing bulbs located at four pumping stations adjacent to the discharge tube. The timed valve will shut automatically when the mass of gas tlowing through the controller corresponds to the amount of deuterium desired for the shot.

Tritium gas from the standard volume also will be admitted to a commion gas line accordin ${ }_{4}$ to the above-mentioned sequence. The deuterium and tritium will then flow simultaneously through the common gas line into the $10 . /$ injection bulhs. In this way the deuterium and tritium will be mixed. and additional mixing will occur in the 10.1 bulh hetore injection into the discharge lube. Pressures and temperatures will be recorded routinely once equilibrium is obtained in the 10.6 bulbs, and the aption to perform mass spectrometric analysis will be arailable at that time. Once the gas mixture is in the 10.- gas bulb. fast-acting valves located on the vacuum downcomers (Fig. 5.6-2) immediately adjarent in each pump station will close. therety isolating the vacuum system manifold from the discharge tube. A tast valve on the bulb will then opt $n$, allowing the gas mixture to tlow into the discharge tube. During the period after closure of the vacutim valves and hefore discharge of the next plasma. the tirst wall will outgas water at an tinknown rate. The goal is to have less than 0.10 at.' ' water in the (1).T) gas mixture at the time of discharge. Therefore. the shot must oceur as sown as possible $(<10$ s) atter the gas mixture is introduced into the discharge tube. After the valse on the injection sistem is closed and as soon as the gas presisure in the discharge tube stabilizes, the machine will be fired. Immediately. after the shut the vacusum valses will open and the vacuum system will begin to evacuate the discharge tube. Al the same time the $10-\mathrm{s}$ bulbs will be opened to the (D.T): cryopump to evacuate the bulbs and associaled gas lines (Fig. 5.6-2). A cryosorption pump will then be useri to remove the traces of the frum the D.T reaction. the $\mathrm{He}$ frum tritiun decay. and ${ }^{3}$ He from D.D reactions. Numerical estimates of rnaximum allowable outgassing rates. injection equilibration times. and vacuum time constants are given in Sec. 5.6.3.3. At the end of the day any tritium gas remaining in the st andard volume will be reabsorbed in the uranium bed and the volume evacuated to remove the traces of helitum.

All transfer lines on the gas injector ststem will he stainless-steel or ETP couper lubing." "The censt ruction of the transfer lines will be such that all permanent joints will he welded. Joints that miglat have lo be upened with any trequency will have metal gasket connectors. The standart purge sistem will be installed at each connector so the line can be opened with a minimum expoware in personnel. "Th* $10-i$ bulbs will be of st ainless-sleel construction with all-welded joints, and the bults will be carefully calibratced so that accurate POT measurements can be performed. Capacitance manometers will be used for measuring pressures in these bulbs.

These 10 - injection bulbs will be located at eact. of lour acuum stations to minimize pressure equilibration times after !he $1 \mathrm{I}), T$, injection. This scheme will require considerable piping to transfer the gas from the fluw cont roller in the injection houd wo the find stations spaced areund the discharge tube see fig. siti-23. In addition. these measurement. wili be recorded on the deulerium and tritiom standard solumess snce the timed salie's have clesed and the sas has flowed into the injector bulbs. This procedure will provide an additional cherk on the amount of gas I ransfered to the discharye tube 'sl each sher.

As with the storage ststem, all major compmenent: of the injectur systern will be redundant and will be litted to the sistem by a standard puroce sistem which uses druble valving arrose all removabls juinis. All manual valves in the sistem will be metal

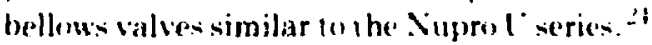

The maximum eredible release of tritium trum the injector sistem lexclusive of the uranium bed, would he the 1-day supply stered in the standard volume. This corresponds lo some 25il.pth cm $^{\text {: }}$ ISTl' or a maximum of $1 \mathrm{kl}$ 'iof tritium gas. The in. jector svistem will he enclosed in the same hend as the uranium bed. The action co be taken in the event of a spill in the hood is described in more detail in

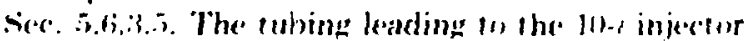
bulbs at the vacuum stations wit! not be in houded areas. but in all cass:s will be in the SFFT cell , in in rowms where the air can be processed through the cell cleanup system and where the air is com inumus. Iy monitured for tritium. The injector bullss will be. located in the hoods at each of four vacuum stations. where the air circulating through the houd is direceled either to the : ritjum waste treat inent or to the cell cleanup system. The transfer tubing from the ll-, bulbs w the dis:harge qube will he inside the 
SFrlK cell sis that any leak in this tubing will bo contained inside the SFTR cell, and the tritium can be wrubbed from this air by the cell cleanup system isec. 5.ti.3.i.).

At this point the insulator material for the first wall has not been detined, although a ceramic/glass conposite is a prime candidate from a fabrication viewprint. Hence, the amusunt of outgassing to be expected from the discharge tube is uncertain. Also. the rate at which the unburned $D$ and $T$ atoms from the plasma will react with the wall to form water is not known. If subsequent experience shows tha! this reaction rate is high, then the outgassing from the lirst wall will be correspondingly high. and the vacuum system or the discharge tube may have to be redesigned to meet the goal of $\leq 0.1$ at. $i$ water in the (I).T1., mixt:are. Also, if this outgassing rate is subst itutial, the water formed unijormly along the discharge tuhe may be sivept along by the gas front whon the fuel is injected ints the discharge tube at the four pumping stations. This phenomenon can result in potentially severe cuncentration gradients of Water within the discharge tube, thereby aftectint: the homoneneity of the final plasma. In any case. the goral will be to initiate the discharge as soun as powibie alter the gas is injected.

The llow rate uf gas from the flow controllers to the 111. injector bulbs may be quite low through the - yint $n$ of small-diameter tubing: this question musi be more extensively investigated. In any event. millicient time must be allowed for the gas mixture in reach thermal equilibrium in the injectur bulh: tiefire the pressure-temperature measurements are nade.

\section{ธ.fi.3.3 SFTR Vacuum System}

Although the fundamental modes of operation for tho tritiom injection and recovery systems have been quilitailicaly discussed in other sections, the vilctum stisten is hasio to tritium injection and ren's:ry. Also. butgassing phenomena. pumping eftiriencies and tine constants. and tritium injection rales are strongly dependent upon the peculiarities of the victum system design. Iltimately, the ancess uf a given plasma discharge will he depen. tiemt on the time required between tritium injection and the plasma dincharge; outgassing rates and suctuten time comstamts play a significant role in this respert.

The following sect ions give an outgassing criterion hy which the gross pumping schemes are considered. and a de:atiled description of a pumping station is presented. For the vactum sistem selected. an estimate of the tritium injection time is made.

\section{General Requirements}

The essential elements of the SFTR vactum system are shown in Fig. 5.6-65. In the sinpiest terms, a tube of radius $R_{;}$and length $L_{1}$ must be evacuated by a pump of speed $S\left(\mathrm{~cm}^{3} / \mathrm{s}\right)$ uncler conditions where the tube is outgassing at rale $G$ $\left(\mathrm{cm}^{3} 3\right.$ rorr $\left./ \mathrm{cm}^{2} \mathrm{~s}\right)$.* The high aspect ratio of the SFTR discharge lube $\left(R_{1}, R_{1} \sim 400\right)$ suggests $\left(v_{i}\right.$ evacuation schemes. shown sc'hematically in Figs. 5.6-6(A) and 5.6-6(B). Figure 5.6-6(A) illustrates, a discrete puinping system, whereby $N$ independer.t pumping stations operate on $n$ downcomers. which are separated by a minimum distance $L_{0} \sim 0.4 \mathrm{~m}$. The alternative system. illustrated in Fig. 5.fi-f(B). envisions a continuous system wherein $n$ downcomers are evenly spaced around the discharge tubte at a distance $L_{\text {s }}$ equal to a multiple of the minimu?n distance 1., The downcomers are attached to a continuous soroidal manifold of minor radius $R:$ onto which are affixed $\mathbf{N}$ pumping stations. For both schemes the distance $L_{1}=2 \pi R_{1} / N$. The minimum distance $I_{4}$, is fixed by the SFTR module length. as indicated schematically in Fig. 5.6-7.

$* 1 \mathrm{~cm}^{3} \mathrm{torr}=3.22 \times 10^{17} \mathrm{H}$, molecules at $300 \mathrm{~K}$.
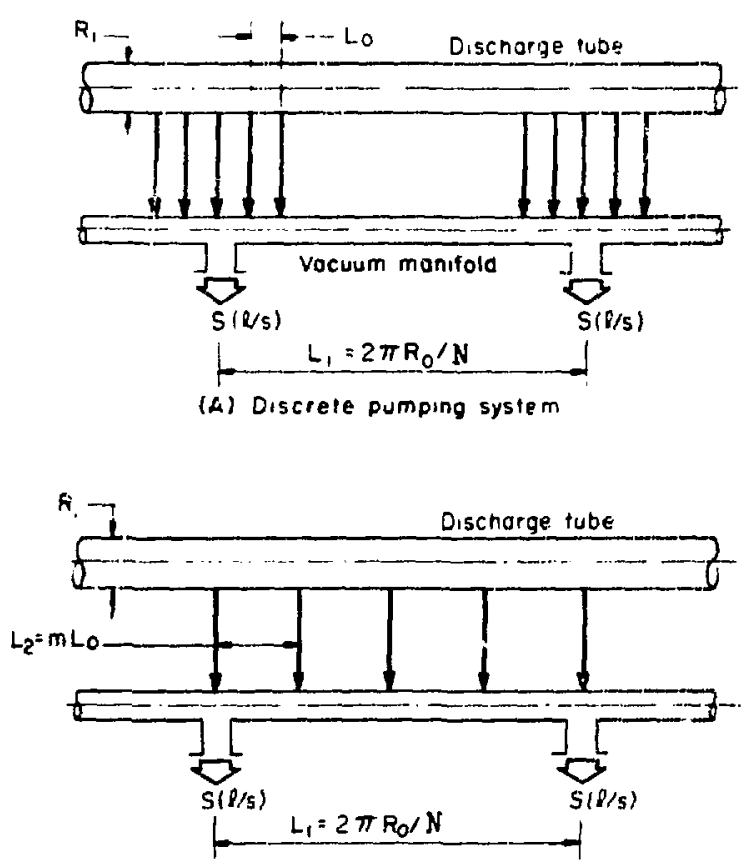

(B) Continuous pumping system

Fig. 5.6-6.

Essintial elements of the SFTR tacuum sistem. 

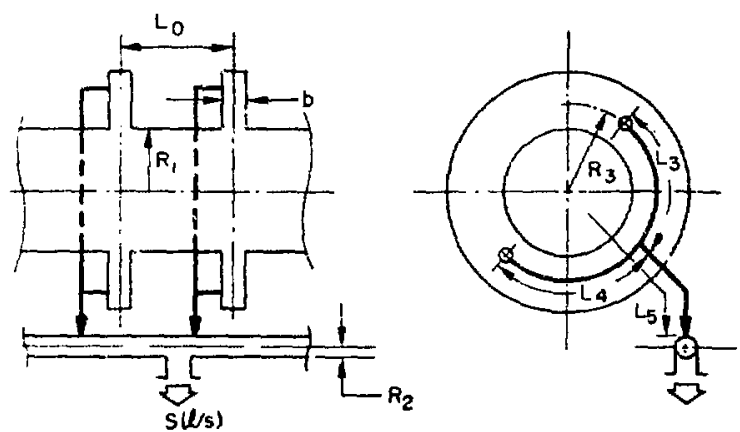

Fig. 5.6-7.

Shematic of a cacuum douncomer and its assiociated dimensions $\left(b=2 \mathrm{~cm} ; R_{1}=R_{2}=10\right.$ c $m: R_{1}=30 \mathrm{~cm} ; L_{11}=40 \mathrm{~cm} ; L_{33}=34 \mathrm{~cm} ; L_{t}=$ $l_{0 ;}=(i)(\mathrm{cm})$.

The following design criteria and system functions must be met by the SFTR vacuum system:

- The SFTR must be capable of a 15-m duty cycle; hence the pumping time constants must be on the order of 1 to 10 s for a practical (i.e., outgassing) vacuum syslem.

- The use of ceramic or glass discharge tubes in conjinction with (H.D.T): portends a serious outgassing problem. The pump speed selected for the system must produce an ultimate vacuum [i.e., (H,D,T) $2 \mathrm{O}$ pressure (commensurate with $0.1{ }^{\prime}$; impurity in the (D. T): was just before a discharge and after the vacuum pumps have been isolated from the discharge tube for $\sim 10$ s. The discrete scheme depicted in Fin. $5.6 .61 \mathrm{Al}$ involves a lower total volume than the continuous scheme, although a higher vacuum conductance is associated with the shorter tube length $l_{1}$ jor the continuous system. A steady-state impurity level will exist along the constantly outgassing discharge tube for the discrete scheme. The pressure gradient associated with large values of $L_{l}$ is considerably reduced by the continuous scheme of Fig. 5.6-6(B), although the added manifold volume may be a disadvantage. When the design criteria are satislied. the choice between the two schemes must be based on relative simpiicity, determined by the system with a minimum number of downcomers, $n$, tir a given number of pumping stations, $N$, as well as the complexity of the plenum or manifold.

Before the system is determined, a qualitative estimate of the overall pumping requirements must be made. If $s\left(\mathrm{~cm}^{3} / \mathrm{s}\right)$ is the total pumping speed recuuired for the system which is outgassing at a rate ( $11 \mathrm{~cm}^{3}$ torr $\left./ \mathrm{cm}^{2} \mathrm{~s}\right)$, the time response of the pressure in the generalized system shown in Fig. 5.6-6(A) can be described by

$$
\left(\pi R_{1}^{2} J_{1}\right) d P / d t=-S P+\left(2 \pi R_{1} L_{1}\right) G .
$$

At equilibrium the pressure is given by $\mathrm{PEQ}_{\mathrm{EQ}}=$ $2 \pi \mathrm{K}_{\mathrm{i}} \mathrm{L}_{1} \mathrm{G} / \mathrm{S}$. If the outgassing rate continues once the vacuum pump is isolated from the discharge tube, the pressure at some time $t$ after the valve closure is given by

$$
P(t)=\left(2 / R_{1}\right) G\left[\tau_{p} \div t\right],
$$

where $\tau_{p}=\left(\pi R_{1}^{2} L_{1}\right) / S$ is defined as a pumping time const ant.

If $P_{1}$, represents the $(D, T)_{2}$ filling pressure and $f=$ $P\left(t_{B}\right) / P_{0}$ represents the fractional impurity level aliowable at the time $t_{B}$ when discharge occurs, $E q$. (i) can be used to estimate $\tau_{p}$, given the outgassing rate $G$. For instance, if the fraction $f_{R}$ of the $(D, T)$ : injected during a previous discharge reacts with the discharge tube to form (D,T) ${ }_{2} \mathrm{O}$, which is subsequentiy adsorbed onto the tube wall, then the mean outgassing rate established at equilibrium for a pumpout time $t_{p}$ between discharges is given by

$$
G=\left(R_{1} / 2\right) f_{R_{0}^{p}} / t_{p} .
$$

Substituting Eq. (4) into Eq. (3) and rearranging yields the following expression for $\tau_{p}$

$$
\tau_{p}=\left(f / t_{R}\right) t_{p}-t_{B} \cdot
$$

Hence, for $t_{B}=10 \mathrm{~s}, t_{p}=600 \mathrm{~s}, f=1.0 \times 10^{-3}$, and $f_{R}=5.5 \times 10^{-2}, \tau_{p}=1 \mathrm{~s}$. Since the volume of the SFTR discharge tube is $\left(\pi R_{1}^{2}\right)\left(2 \pi R_{o}\right)=7.89 \times 10^{6}$ $\mathrm{cm}^{3}$, the total pumping speed should be at least 7.89 $\times 10^{6} \mathrm{~cm}^{3} / \mathrm{s}(7900 / / \mathrm{s})$. For this minimum value of pumping speed, the base pressure in the discharge tube would be $1.83 \times 10^{-6}$ torr and the mean outgassing rate would be $9.17 \times 10^{-7} \mathrm{~cm}^{3}$ torr $/ \mathrm{cm}^{2} \mathrm{~s}$ $\left(2.95 \times 10^{10}\right.$ molecules $\left./ \mathrm{cm}^{2} \mathrm{~s}\right)$ for a filling pressure $P_{\text {。 }}$ of $10^{-2}$ torr.

Because of the simplifying assumptions upon which Eq. (2) and the estimate of $G$ are based, the above numerical results are probably accurate within an order of magnitude. The chemistry of water formation, adsorption, and desorption is complex and not well understood for the conditions anticipated for the SFTR vacuum wall. Furthermore, the distributed vacuum conductance represented by the model given in Fig. 5.6-6(A) will lead to pressure gradients at equilibrium when the wall is outgassing. For the latter rase Eq.(2) must be replaced by

$$
\begin{aligned}
& \left(F L_{1} / \pi R_{1}^{2}\right)\left(d^{2} P / d x^{2}\right)= \\
& \left.-\left(2 \pi R_{1} L_{1} G\right) / \pi R_{l}^{2} L_{1}\right)+(d P / d t),
\end{aligned}
$$


where $F\left(\mathrm{~cm}^{3} / \mathrm{s}\right)$ is the vacuum conductance of the discharge tube, ${ }^{211}$

$$
\begin{aligned}
& \mathrm{F}=3638 \sqrt{\mathrm{T} / \mathrm{M}} \pi \mathrm{R}_{1}^{2} \kappa\left(\mathrm{R}_{1} / \mathrm{L}_{1}\right) \\
& \kappa\left(\mathrm{R}_{1} / \mathrm{L}_{1}\right)=1 /\left(1+3 \mathrm{~L}_{1} / 8 \mathrm{R}_{1}\right)
\end{aligned}
$$

and $\mathrm{x}$ is the distance along the tube. If $\mathrm{P}_{i}$ represents the initially uniform pressire and $P$. represents the llowl pressure at the pump stations the solution to Ey. $(6)$ is $^{2}$ )

$$
\begin{aligned}
& \frac{p(x, t)-p(x, \infty)}{P_{i}-P_{s}}=\sum_{n=1}^{\infty}\left(\frac{4}{\beta_{n}}\right) \\
& \cdot\left[i-\alpha /\left(\pi \beta_{n}\right)^{2}\right] e^{-\beta_{n}^{2} \tau} \text { sin }\left(\beta_{n} \xi\right),
\end{aligned}
$$

where

$$
\begin{aligned}
\alpha & =2 w R_{1} L_{1} G / P_{i} F \\
\dot{P}_{n} & =(3 n+1) w / 2 \\
\xi & =x / L_{1} \\
\tau & =\left(F / \pi R_{i}{ }^{2} L_{1}\right) t \\
P_{(x, \infty)} & =p_{5}+(\alpha / 2) \xi_{2}(l-\xi) .
\end{aligned}
$$

Defining an average pressure within the tube.

$$
\begin{aligned}
& \langle\mathrm{F}\rangle \equiv \int_{0}^{\mathrm{L}_{1}} \mathrm{P}(\mathrm{x}, \mathrm{t}) \mathrm{dx} / \mathrm{L}_{1} \\
& \frac{\langle\mathrm{P}\rangle_{\tau}-\langle\mathrm{P}\rangle_{\infty}}{\mathrm{P}_{\mathrm{i}}-\mathrm{P}_{\mathrm{S}}}= \\
& \sum_{n=1}^{\infty} \frac{\mathrm{s}}{\beta_{\mathrm{n}}^{2}}\left[1-\frac{\alpha}{\left(\pi \beta_{n}\right)^{2}}\right] \mathrm{c}^{-\beta_{n}^{2} \tau} \\
& \langle P\rangle_{\infty}=P_{s}+(\alpha / 12)\left(P_{i}-P_{s}\right) .
\end{aligned}
$$

At equilibrium, $\langle\mathrm{P}\rangle_{\infty}=\pi \mathrm{R}_{1} \mathrm{~L}_{1} \mathrm{G} / 6 \mathrm{~F}$, which equals $S / 12 F \sim 1 /\left|12 \kappa\left(R_{1} / L_{1}\right)\right|$ times $P_{\infty}$ obtained from 1. (2). For $R_{1} / L_{1} \ll 1$, this difference amounts (1) $\sim \mathrm{L}_{1} / ; 2 \mathrm{R}_{1}$. Furthermore, the maximum value of pressure at equilibrium equals $\pi \mathrm{K}_{1} \mathrm{I}_{1} \mathrm{G} / 4 \mathrm{f}$. or Pmax $/\langle P\rangle=3 / 2$. 'These differences tend to underestimate pump sizes and time constants predicted by Eq. (2). The assumed $\sim \bar{r}$, reaction of (I).T\% to form water is undoubtedly high and the overestimation of $G$ will compensate the nonconservative errors introduced by the use of Eq. (2). Furthermore, the major outgassing problem will probably occur during the thermonuclear burn as a result of bremsstrahlung and ion irradiation of the vacuum wall.

\section{Determination of Manifolding Scheme and Pum- ping Requirements}

The calculations and results of the previous section give the gross vacuum requirements based on a relatively conservative outgassing restriction. Whether a 1-s time constant can be achieved with $80001 / \mathrm{s}$ of total pumping must be determined by calculations which pertain to a specific arrangement of pumping stations and downcomers. As indicated in Figs. 5.6-6(A) and 5.6-6(B), the posisibility exists of either discretely or continuously manifolding the downcomers from a module to the pumping station. The system with the minimum number of dowricomers per pumping station, $n$, and the minimum number of pumping stations, $N$, is obriously prefierred.

Since a fast-acting isolation valve is associated with each downcomer, maintenance and reliability requirements dictate as few downcomers as possible. Each pumping station, described in the following section, must be rated at $\sim 8000 / \mathcal{N}(/ / \mathrm{s})$ and will function as a separate, self-contained unit that can be separately removed and replaced. The desirability of minimizing the tritium injection/recoverv function and the cryogenic (liquid helium and liquid nitrogen) requirements associated with each pump station provide a strong motivation for minimizing $N$. On the basis of the latter logistical considerations, $N$ is fixed at $t$, giving $S=2000 / / \mathrm{s}$ for each pump station.

By neglecting the vacuum conductance associated with the length 1.2 of discharge and manifolding between downcomers. an optimistic upper limit can be placed on $\mathrm{n}$. Since the maximum number of downcomers equals $2 \pi R_{1} / 2 L_{0}=314\left(L_{11}=40 \mathrm{~cm} . R_{c}\right.$ $=40 \mathrm{~m}$ ). the fraction of modules with downcomers. fic , equals

$$
f_{D C}=\left(S / F_{D C}\right)\left(L_{o} / \pi R_{0}\right)
$$


where $F_{D C}$ is the downcomer conductance and $S$ is the pumping speed of a pump. Figure 5.6-7 illustrates the downcomer geometry and nominal dimensions. The vacuum conductance of the "brim" of the "top hat" was estimated using a square duct geometry ${ }^{20}$ with dimensions $a=\pi\left(R_{1}+R_{3}\right), b$, and $c=\left(R_{1}-R_{i}\right)$. Standard tabulations ${ }^{20,29}$ were used for the conductances of elbows and tubing. In this way the downcomer conductance $F_{\text {gc }}$ from the discharge tube to the vacuum manifoid was estimated to be $\sim 80 / / \mathrm{s}$ for the dimensions given in Fig. 5.6-7. Introduction of two high conductance valves $(130 / / \mathrm{s})$ into the downcomer reduces $\mathrm{F}_{D C}$ to $-50 / / \mathrm{s}$. By plumbing both sides of a given "top hat," $F_{D C}$ in principle can be increased to $\sim 73 \mathrm{l} / \mathrm{s}$. For $\mathrm{F}_{\mathrm{UC}}=50$ $1 / \mathrm{s}$, the fraction of modules with downcomers given by optimistic predictions of Eq. (12) is $51 \%$; if $\mathrm{F}_{\mathrm{DC}}=$ 73 lis, then foc becomes $36^{\circ}{ }^{\circ}$.

These preliminary estimates neglect the conductance of the discharge tube and vacuum manifolding located between downcomers, and indicate that under the most optimistic conductions -110 downcomers will be required to achieve the $1-\mathrm{s}$ time constant. With $\mathrm{N}=4$ pump stations, $\mathrm{L}_{3}=62.8 \mathrm{~m}$ of torus are evacuated by each pump station, and 28 downcomers/station would be required. The discrete pumping scheme would require $22.4 \mathrm{~m}$ of manifolding for each quadrant of the discharge tube, whereas for the continuous scheme one downcomer would be required for every $\sim 4$ modules $(L)=$, m).

To account for !oroidal vacuum conductance and the vacuum dynamies, the NET-2 computer code was used to solve the equivalent electrical circuit depicted in Fin, 5.6-8 for the continuous and discrete schemes depicted in Fig. 5,6-6. The effective toroidal conductances bet ween downcomers $\left(F_{T}\right.$ or $F_{y 1}$ ) were rblained by using mean pressures, differentiation of F(1. (10) after the higher harmonirs have decaved. and identification of an effective conductance. The effective conductance equals the conductance of a tube of length $L$ and radius $R$ (Eq. (7)/, increased by

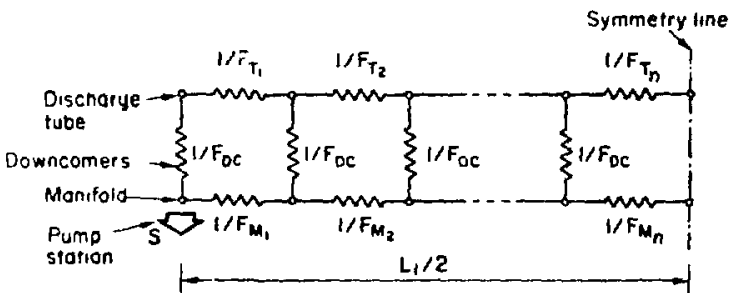

Fig. 5.6-8.

Elertrical analogue used to model the SFTR cacuum sistim with the NET-2 computer code. 3 " the factor $\pi^{2}$. Figure $5.6-9$ gives the dependence of the punping time consiant, $r$, on fix for $N=4$ and equal discharge-tube and manifold radii $R_{R_{1}}=R_{2}=$ $10 \mathrm{~cm}$ ) for the two manifold schemes comsidered and for 1 hree values of $\left.F_{1 K} \cdot(50), 75,100 / / \mathrm{s}\right)$.

As with the sizing of most complex vacuum systems, the numerical results presented here must be considered approximate. Although theoretically sound, these computations often give results which are invalidated when the physical constraints of assembly and the outgassing of real surfaces occur in the actual system. The Scyllac experiment ${ }^{31}$ provides a vacuum experience with which to relate the foregoing computations. Table 5.6-2 compares the vacuum characteristics of Scyllac with those computed for the SFTR.

For all cases considered, the discrete manifolding scheme offers the potential for smaller vacuum time constants when compared to the continuous manifolding scheme for similar values of fix (total numoer of downcomers), $\mathbf{n} \mathbf{F}_{\mathrm{DC}}$ (downcomer vacuum conductance). For $F_{D C}=100 / / \mathrm{s}$ the desired $1-s$ time constant can be achieved with $\mathrm{f}_{\mathrm{D}} \mathrm{C} \sim 60^{\circ} ;$ ( $\mathrm{nN}=188$ downcomers). For each quadrant $\left(L_{1}=2 \pi R_{1} / N=\right.$ $62.75 \mathrm{~m}) 37.6 \mathrm{~m}$ of the torus are occupied by downcomers: the toroidal distance between extreme dowricomers, therefore, is $25.15 \mathrm{~m}$. As noted previously, the maximum pressure at the center of an outgassing tube of lengt $\mathrm{L}$ and radius $\mathrm{R}$ is given by $\pi \mathrm{RLG} / 4 \mathrm{~F}$, where $\mathrm{G}\left(\mathrm{cm}^{3} \mathrm{torr} / \mathrm{cm}^{2} \mathrm{~s}\right)$ is the constant outgassing rate and $\mathrm{F}$ is the tube conductance |Eq. (7)|. Ising the maxim!n allowable value of $G\left(9.17 \times 10^{-7}\right)$ previously determined, and equating $L$ and $R$ to $2515 \mathrm{~cm}$ and $10 \mathrm{~cm}$, respectiveJy, an estimate of the maximum pressure differential can be obtained. The (D, T $)_{2} \mathrm{O}$ pressure between

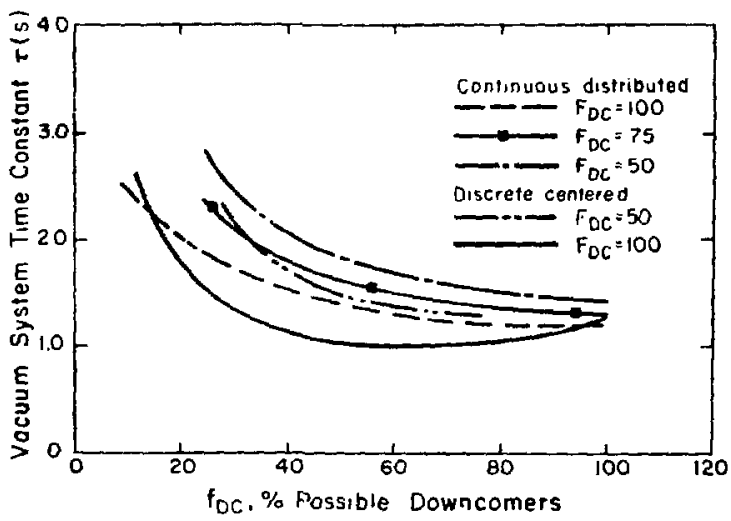

Fig. 5.6-9.

Dependence of the vacuum time constant on $f_{1 x} \cdot$ for 4 pumping stations and $F_{1 x}=50,75$, and $100 \mathrm{l} / \mathrm{s}$. A comparison between continuous and divcret' pumping schemes is shoun. 
TABIE 5.6-2

\section{COMPAKISON OF SFTR VACUUM DESIGN WITH SCYLIAC}

\begin{tabular}{|c|c|c|}
\hline Parameter & Seyllac ${ }^{(31)}$ & SFTR \\
\hline Major radius. $R_{1,}(\mathrm{~m})$ & 4 & \#11 \\
\hline Minur radius (sacusum) $\mathrm{K}_{1}(\mathrm{~cm})$ & 1.4 & 10) \\
\hline Vinlume vil & $1: 13$ & $79(1)$ \\
\hline Discharge tube surface area, $A\left(\mathrm{~m}^{2}\right)$ & 6.9 & 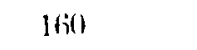 \\
\hline ('ircumference. $(\mathrm{im})$ & 25.1 & 251 \\
\hline Downcomer con ?uctance. Fix $(/ / s)$ & $10.12^{\text {it }}$ & $5(1-7: 3$ \\
\hline Number of pumpingstations. $N$ & 3 & 4 \\
\hline Number of downeomers/station. n & 3.3 & 47 \\
\hline Tinal number of downeomers, nX & 10 & $\operatorname{lin} x$ \\
\hline Pumping speed/station. S(/s) & 260 & $20(10)$ \\
\hline Thutal pumping speed. NS(t/s) & 780 & $x(1)$ \\
\hline $\begin{array}{l}\text { Distance bet ween pumpstat ions. } \\
L_{d}\left(\mathrm{~m} l=2 \pi R_{1} / N\right.\end{array}$ & 8.73 & 6.8 \\
\hline $\begin{array}{l}\text { Distince bet ween disuncomers. } \\
\mathrm{L}_{-1}(\mathrm{~m})=\mathrm{L}_{1} / \mathrm{n}\end{array}$ & $2, \bar{i} 1$ & 1.34 (av) \\
\hline Vacuum time constant, $\tau(s)$ & 1 & 1 \\
\hline
\end{tabular}

pump stations will be approximately twice the latter value. Since previous estimates indicate that a base pressure of $\sim 1.86 \times 10^{-6}$ torr would be adequate for the above value of $\mathrm{G}$ and $\mathrm{a} 0.1^{\circ}$; impurity level in the predischarge (D,T) : gas, the pressure difference of the order of $1.25 \times 10^{-6}$ torr should present no problems.

In summary, the design for a 1-s time constant and $-5 c_{i} /$ shot $(\mathrm{D}, \mathrm{T})_{2}$ reaction with the wall presents a conservative syst em; this design will probably give the desired preshot purity and the facility for rapid (15-min) discharge rate. The major cost of these design restrictions and conservatism is embodied in the large number of isolation valves and downcomers $\left.\left(f_{i x} \cdot=60\right)^{\prime}, \mathrm{nN}=188\right)$ required. By either decreasing the requirements on $\tau$ or increasing $F_{D C}, N$ can be decreased and the cost reduced. In view of the uncertainties associated with vacuum wall outgassing and reac: ..n rates, the conservatism built into the above results is warranted in the absence of definitive experimental results.

\section{Estimation of $(D, T)_{2}$ Injection Time Constant}

As discussed in Sec. 5.6.3.2. the premeasured (D.T). volume will be injected into the SFTR dis. charge tube through 4 downoromer sets located at each pumping station. The last stage of the injector volume, $V_{1}$, and the preinjection pressure. $P_{1}$, within this volume is such that $P_{1} V_{1}$ equals $1 / N=0.25$ of the $(D, T)_{2}$ gas requirement for a given shot (i.e.. $2 P_{1} R_{1}, \pi R_{1}-1, \mathrm{~N}$. Although tlow in the vicinity of a given injector downcomer will be first turbulent and then viscous, the estimate of the injection time given below is based solely on free molecular flow and should, therefore. give conservative results.

The geometry used for the estimation is depicted in Fig. 5.6-10 where the distance between pump stations is $L_{1}=2 \pi R_{1} / \mathrm{N}(31.4 \mathrm{~m})$. After the discharge tube is evacuated to a low pressure $\left(10^{-6}\right.$ to $10^{-7}$ torr), all $\mathrm{nN}$ downcomers are isolated from the vacuum manifold, and four prefilled volumes. $V_{1}$, of (D.T). are opened through a high conductance line and valved to the evacuated discharge tube. The (D.T) : filling gas is discharged through the primary conductances $F_{1}$, and $F_{D C}$. The pressure within the discharge tube at the injection point. $P\left(x= \pm L_{1} / 2\right.$, $t$, is assumed to be described by

$$
P\left(x= \pm L_{1} / 2, E\right)=P_{0}\left(I-e^{-t / \tau} I\right), 11: 1
$$


where $P_{1}$ is the final (D.T), filling pressure and $\tau_{1}$ is the injector time constant, assumed here to equal $V_{1} / F_{1 C}$. Neglecting outgassing during the (I).T).2 injection stage. the pressure within the discharge tube at a nondimensional position $\xi=2 \mathrm{x} / \mathrm{L}_{1}$ and time $\tau=\left(2 F_{1} / \pi R_{1}{ }^{2} L_{1}\right) t$ is given by a solution to

$$
d^{2} P / d \xi^{2}=d P / d \tau \text {. }
$$

Equation (1:3) gives the boundary conditions. and a zero pressure initial condition is assumed. The solution of $\mathrm{Eq}$. (14) is, 28

$$
\begin{aligned}
& P(x, \tau) / P_{0}=1-\frac{\cos \left(\rho^{1 / 2} \xi\right)}{\cos \left(\rho^{1 / 2} / 2\right)} e^{-\rho \tau} \\
& -4 \rho \sum_{n=0}^{\infty} \frac{(-1)^{n} e^{-\beta_{n}^{2} \tau}\left(\cos \beta_{n} \xi\right)}{\beta_{n}\left(\rho-f_{n}^{2}\right)}
\end{aligned}
$$

where

$$
\begin{aligned}
& \rho=\tau_{L} / \tau_{I} \\
& \tau_{L}=\pi R_{1}^{2} L_{1} / 2 F_{L} \\
& \tau_{I}=V_{I} / F_{D C} \\
& \beta_{n}=\pi(2 n+1) .
\end{aligned}
$$

The wotal amount of gas injected into a quadrant of the discharge tube at time 1 equals

$M(L)=\pi R_{1}^{2} \int \begin{aligned} & +l_{1} / 2 \\ & -1_{1} / 2\end{aligned} P(x, t) d x=V\langle p\rangle_{L}$,

where $\langle\mathrm{P}\rangle$, is the spatially averaged pressure at a given time. Integration of $\mathrm{Eq}$. (15) gives

$$
\begin{aligned}
& M(t) / V P_{0}=1-\left(2 / \rho^{1 / 2}\right) \\
& \cdot \operatorname{tin}\left(\rho^{1 / 2} / 2\right) a^{-\infty i} \\
& +8 \sum_{n=1}^{\infty} \frac{e^{-\beta^{2} t}}{\theta_{n}^{2}\left(1-\delta_{n}^{2} / 0\right)} .
\end{aligned}
$$

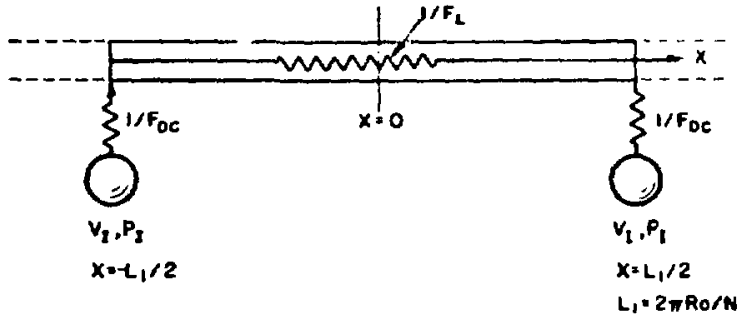

Fig. 5.6-10.

Sichematic of model used to estimate DT injec. tion time

The dependence of $\mathrm{M}(1) / \mathrm{M}(\infty)$ on tine and $p=r_{1} / \mathrm{r}$ is given ${ }^{28}$ in Fig. 5.6-11. Ising $\mathrm{Eq}_{1}$. 17 for $\mathrm{F}_{1}$ 1 $-75 / / s)$, $r_{1}$, is estimated to be $13.2 \mathrm{~s}$. If $f_{1}$ is the coll. ume ratio $\pi \mathrm{R}_{i}^{-2} L_{1} / 2 \mathrm{~V}_{1}$. then $\tau_{1} / r_{1}=$ $\left.\left(F_{1} / F_{1}\right) / f_{1}\right)$. Since $F_{1 K}$ equals a()-7s $1 / \mathrm{s}$. the ratio $r d / \tau$ is approximately expual to $i$. which in turn is 16.5-25. For these system parameters pressure eyuilibrium is expected concour for $2\left(1 / \tau_{1}\right)^{1}-1 . \bar{i}$. or $t / \tau_{1}=0.5 f$ and $1-7.4$. The 7.t-s value is within the $10-s$ period allocaled between the isolation of the discharge lube and the actual plasma discharge.

\section{Description of Pumping Stations}

Four pumping stations will be equally spaced around the torus. After a shot, gas will be pumend from the discharge tube viat dewneomers thate conneet the discharge tube to a pump manifold. The manifold mav circle the discharge lube.

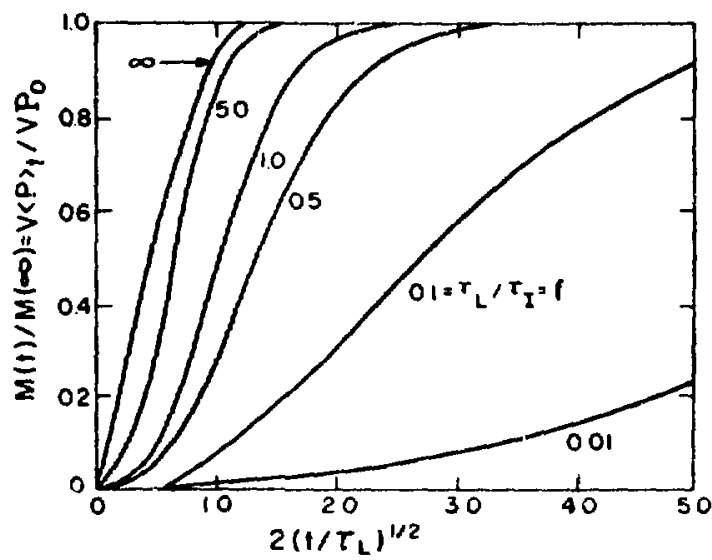

Fig. 5.6-11.

Dopendence of $D . T$ injection time on injector cime constant and lacuum iime constant of the dischargr lube. ${ }^{2 x}$ 


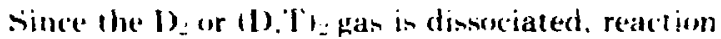
of alumic hydrogen with the discharge tube wall is cortain to take place $1 * 2.63$ (1) a degree that depends on the life of the atomic hedrogen, the nature of the inner wall, and the time of contact. Further, the (1). Ti. () that is formed will be adoorbed on the wall i1. an extent determined by the pressure of the (I). II. (1) the nature of the wall. and the adsorption time. Thus. to minimize the int roduction of (I).T, 0 impurits in a subsequent gas charge. the time between firing and gas evacualion should be minimized. Also. as discussed in See. 5.6.3.2. the time between cessation of pemping and the firing of a charge should be minimized. These considerations: indicate the desirability of using tast-acting valses: ind the evacuation ports.

Acordingly, each downcromer will be equipped with a pneumatically operated fast-acting valve with offective opening and closing times of 1 sor less. Hecallsc walde seat replacement mat be frequent. a s'cond value will be located between the first valve and the discharge tube pump-rust disc oo allow inclation of the discharge tube during such periods. This secend valve serves the same function during valve soitt replatcement in fast acting injoction values. The "Through" leak rate of these values is nest critical. and ('an be as high as $10^{-1}$ and $10^{-2} / / \mathrm{s}$ for the inlet and all exhaust valves. respedtively.

The manifoldist connected to the valved downcomers is joined to a pumpiag station that serves to remexe the gils rapidly from the discharge tube. In addition. the station serves to separate the com. coments of the gas 10 allow botler recovery and allalvsis of the (1).T): analsisis of the amount of wall reaction. and a PVIT analysis of the degree of burm in the 1).T reaction. A drawing of the pumping station is shown in Fig. 5.6-1.2. The station is surrounded by a rentilated hood to isolate accidental tritium leaks in the injection and evacuat iun lines and to provide protection for personnel during maintenance on these lines. Higure 5.6-1:3 gives a schematic layout of the sFlR vacuum sistem.

Redundancy in the pumping stations is provided by four pumping stations l this mumber was selected for ot her reasons, nariely, to increase the speed of evacuat ion to a predetermined value, as discussed in Sec. $5 .(3,3,3)$. Ajso some redundaney is provided in the station itself.

The evacuated gas is assumed to have a pressure of $10^{-3}$ torr at the tralss and pumps to assure effective separations of gas components. Final plans may possibly require a short-term use of a throttling value during a given pump-down to achieve this pressure.

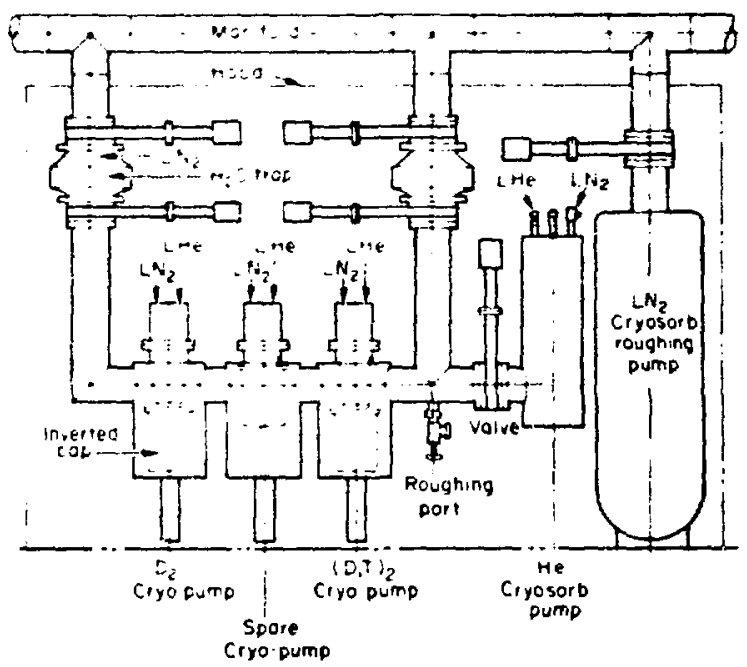

Fins. 5.6-12.

Sormetric drawing of one of four SFTR pum-

pins statimes.

The pumping sistem of the SFTR is completely cryogenic, with cryogenic separation of most of the compunent- of the gats. At each station the (1).Ti. toget her with any $\mathrm{O}_{2}$ or $\mathrm{N}_{2}$ is pumped by a Balser ${ }^{34}$ for equivalent) liquid helium cryopump with a 2000 . is pumping speed. The (D.Ti:O is trapped by a

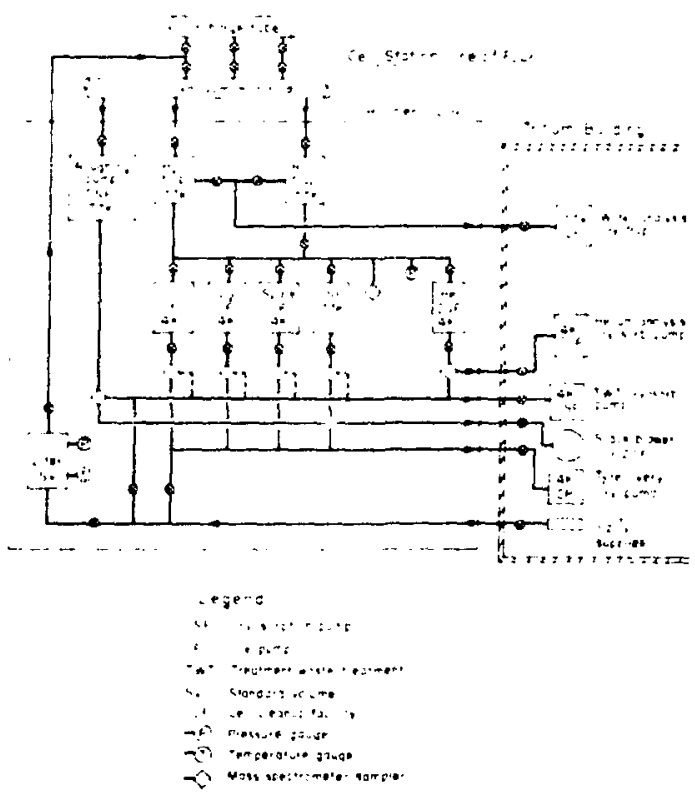

Fig. 5.6.13.

Detailed laveut of SFTR pumping station and associated plumbing. 
liquid nit:ogen Varian cryotrap $^{35}$ with similar capacity. (A spare trap is also provided.) After the pressure is constant, the the is pumped with a 1000 . i/s Excaliber liquid helium cryosorb pump. ${ }^{36}$ (This pump uses a bonded molecular sieve on the cryopanels.) The time needed to pump the $\mathrm{He}$ is about 1 min of the 10 -min evacuation time between runs.

A teature of the Balser cryopumps not apparent in the drawing is a type of construction that gives an economy of space, a minimization of the increased pumping resistance usually associated with pumps, and a dual role in that it also serves as a valve. An inverted cap is pneumatically raised to close off the cryogenic pumping surface (within the pumping svstem) and effect a seal against the flange on the pump. The core of the pump can than be removed for cleaning without affecting the vacuum, or the condensate can be exhausted.

The (D, T) $)_{2} \mathrm{O}$ trap is isolated at the end of the day (or sooner, if desired) with pneumatically operated valves, the trap is warmed, and the water is transferred cryogenically to the tritium handling facility for measurement, decomposition, and possible (D.T) recovery.

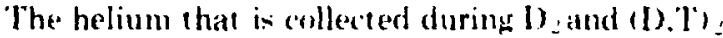
runs will nurmally be evacuated daily from the warmed pump. On ()-T shot.. the heium can be expanded frum the warmed pump. separated from any (1). T1.. and M"l-measised in a thermentated con. tainer tor a determination of the perceme burn.

The [l, also, will normally be evienated trem the warmed pump on a daily basis. It will be cryasorb. pumped wh the 7 -is waste treatment sistem. The mixture of 11).'T) will be eractuated atter sinch rum. and $P " l$ and mass spect rometer moasurements will sive an approvimale accolluting of tritium. The. 11). T), will then be absorbed on a cranium trap. which wiil allow subsequent reowery (by E.Rl)A.

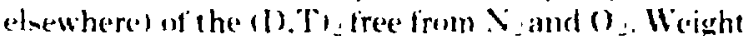
changes of the uranium comitainer. bugether with both the known lassuming a kmown mixt do.'T': content and a persible subseguent amalysis. can give the oxyen content. This together with the meatsured water content gives the extent of the atomic l) and T reaction with the discharge tube. Finally, the tranium ermtainer will be of a standard size that is commonls calurimetered for an inde'endent moia-urement of the iritium contcont.

An independent requirement of the pumping station is the evacuation of the discharge tube from at mospheric pressure. An Excalibur or equivalent crvosorb pump with an appropriate capacity 18 -in.

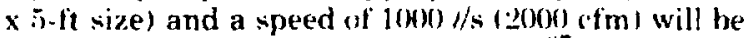
used. A separate cryosorption pump. ${ }^{35}$ attached to the tlange shown in Fig. 5.6-12. will be used for culgassing purposes during nonworking hours. In this (ase. the outgassing of 1 H.D, T) may be sufficienty high to require liquid helium cooling. No recovery or tritium measurements are planned here unless: monitoring equipment during waste processing indicates a high tritium comtent.

Gauges to measure pressuren from 10 $10^{-i}$ torr to 1 atm will be required at various ponts on the vacuum system. Some temperature measurements will also be required.

Routine operation of the vacuum system will be antomatic, with automatic recording of temperat ure and pressures, together with mass spectrometer or residual gas analyer analyses. Special operations. usua!ly once a day with D-T runs, will be manual. All operations will be remote-cont rolled except for maintenance operations during nomoperating periods of the fusion experiments.

Selection of relatively large volume enclosures for the cryopumps is planned to allow the daily collec. tion of deuterium with oxygen ffrom the decomposition of and outgassing from the discharge tube inner wall be particle bombardment? without danger resulting from a shock-indused explesion of the solid mixture. The volume of the container should he aboul a factor of 7 larger than the volume (STP') of. fir example, a two to one hydroges. to axyigen mixture. Air leakage would cause the geeatest hazard: if a massive leak of air is assumed. the combined volume of the enclosure should be about a factor of 16 larger than the 2 , of deuterium of provide reasciable safety. In the (ase of the (D).T) mixtures. the 0.08 , will be processed after each run and can in no case present a problem.

\subsubsection{Tritium Hecovery System}

A strong effort is being made to recoser the purest posisible (D.'T): from the plasina ash. The use of crvogenic punping and the reaction of the spent plasma gas with a uranium bed allows the recovery of (D.T): with protium as the only likely impurity. This separation method should operate with relative ease and high efficiency. A further henefit is the separation and recovery of the helium 10 provide an additional and simple means of measuring the degree of hurn of the D-T plasma.

Part of the discharge tuhe exhaust gas will he (I) T), $\mathrm{O}$ from the reaction of atomic $\mathrm{D}$ and $\mathrm{T}$ with the inner wall. Althoukil this will constitute a major portion of the tritium waste handled by the $7-1 / s$ tritium waste treatment unit. the value of the tritium will amount to only a few dollars per day. Tritium from mass spectrometer samples will be a 
second major source of waste tritium. Ayain the quantity is small and also will be discharged into the waste treatment system. In this case the daily discharge is marginally accountable. The (D.T).2 from the mass spectrometer and $t: 1$ (D, T),2 $O$ analysis gas will be crvosorption-pumped to the tritium waste treat ment system. If desired, the (D,T), $\mathrm{O}$ from these sources could be recovered by the addition of a hot bed of copper turnings / for removing oxygen from the (I).TH. Ol and minor piping revisions.

The final major source of waste tritium may be (D.T): in collected helium. Should this be ap. preciable, it could be piped directly to the tritium recovery uranium bed.

Primary tritium recovery will handle the (D.T): that has been collected in the cryopump. together with traces of nit rogen from outgassing and perhaps a fow tent hs of a percent of oxygen from the first -wall decomposition. The cropoump will be allowed to warm up, and the pressure and temperature of the was in the previousily calibrated volume will be measured. The quantity of gas will be determined with an accuracy of a few percent. A mass spectrumeter analysis will determine the purity of the gas. 'The gas will then be reacted with a uranium bed. Fig. i.6-14. The uranium trap will be of a standard size for precise calorimetric determination of the tritium content. Not only the mass spect rometer analyses but also weight changes in the trap could be useful for crude oxyen determination if wall deconnposition is large. Alternatively, a cryotsap (with an additiona! valve). conled with heliuin at $20 \mathrm{~K}$ and located between the liquid nitrogen trap and the croopumps. could be used to collect the oxygen for subsaquent PVT measurements. Sivllac measurements could be devised to measure oxide decompusition, at least for one type of inner wall.

The uranium beds normally would be shipped elsewhere. Alternatively. they could be heated to regenerate the (I).T): gas for collection in standard tritium gas shipping containers or for direct reuse in the SFTR. Nitrogen and oxygen would not be released during this heating. so the uranium bed acts to purify the (D.T): gas. The operation of the uranium beds in the recovery operation is like that used for fuel injection. except that wice as much gas would be absorbed (but to give the same tritium content). as discussed in Sec. 5.3.

\subsubsection{SFTR Cleanup Systems}

The design of a facility to clean up tritium waste incurred during routine and abnormal operations is made difficult by the disparity het ween the required tlow rates anticipated for the iwo : ases. The cleanup

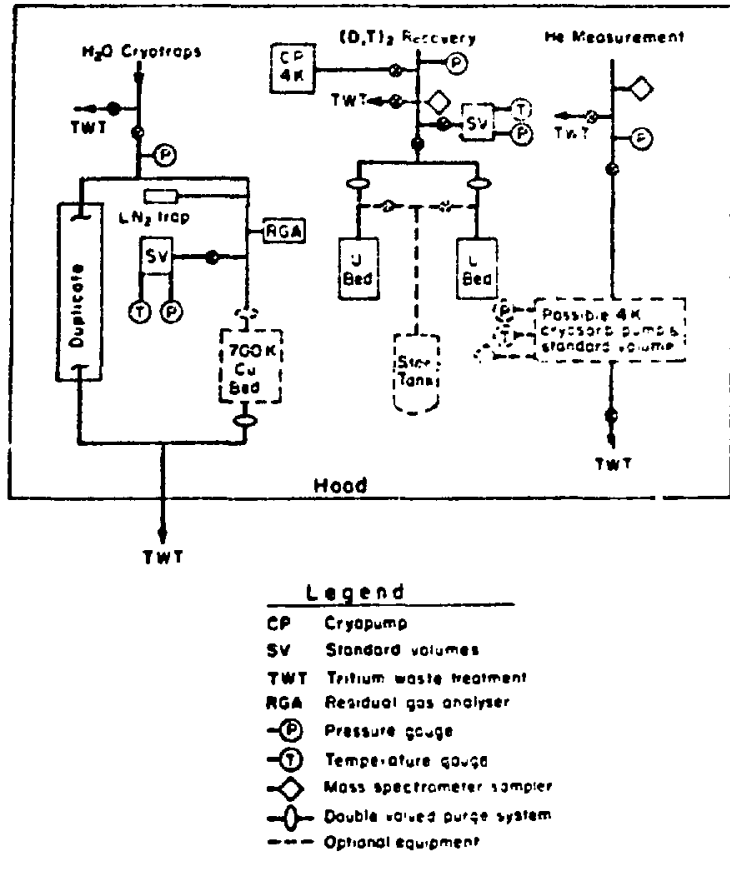

Fig. 5.6-14.

Analysis and $D . T$ recolery sistem.

of routine waste associated with equipment maintenance, intentional leaks (sece. i.ti.:3.t1, (I).T) diffusion through walls of process equipnent. liss from contaminated surfaces, and accidental leaks can be made by means of a relatively small unit $i^{\prime}$ 1/s) used in conjunction with a ballast or storage tank and proper scheduling. This approach is useless. of course in event of a rapid. large release to the SFTR cell or I ritium handiing area. Cleanup of the latter release must be reasonably rapid and large volumes: of air must be treated. Two cleanup sistems. therefore. are anticipated: routine waste will be treated by a small capacity TWT system, with a $T-d / \mathrm{s}$ capacity. whereas large spills will be subjected to cleanup br a large $47001 / \mathrm{s}$ unit. For cases such as an accidental spill in the vault, the TWT system could still be employed ower a period of dass. If. however. the SFTh cell atmosphere suddenly becomes contaminated with tritium. the $47(K)-i / s$ cleanup unit must be used to effect a rapid cleanup of a large volume of air. This section describes both the $7-. . s$ TW"I and the $4700-1 / \mathrm{s}$ C CF. Operation of these $t$ wo sistems in conjunction with a monitoring sistem under accident-type conditions is described below. The Fingelhard Corp. manufactures similar but simpier sistems. is 


\section{Cleanup System for Scheduled Tritium Releases ('TW')}

The TllT sytem is an automatically actuated tritium remowal sistem based on a precilons metal analstic recombiner which comverts all hydrogen inotopes in air to water. and organic materials tw water and carhon dioxide. The water is then adorrbed on molecular sieve drying cowers. This -salem will handle ot herwise moneollectable residues. and effluents from the vachum syitems. injector ststem. purge sistem. uranium heds, malss speectrometer ssistem. and the relatively low air thow: through the houds associared with these systems during maintenance periods. Air circulating through these houds will then inter the ventilation duets and 'an be atutomaticalls diserted inew the TH!l, in tromt of minor tritium spill in these areas. Figure i.titis is a thew diagram of the Tll"l and its remationship 10 the larger cell cleanup tacilits

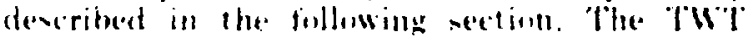
dencribed here is -imilar 10 a sintems which i: nperational at l.Asl. is

(iats trem the various alowe mentioned soures will tirst flow into a large storage and ballatst ank. The exygen, hydrogen isotope. and watter comeen. trations in the tank air will be continumusly monitured and maintained at a lesel sufticient id provide enough oxygen to comsert catalstically all on the hydrogen isolofes 1 water: the wxygen and hydrogen are cont rollec. Io leverts belem the explusise limits: and the water is kept $1,2.51$, relative humidi19. minimum. (o effect dilutions of the (I).T1.0 without unnesessary loading of the dryer with wat er.

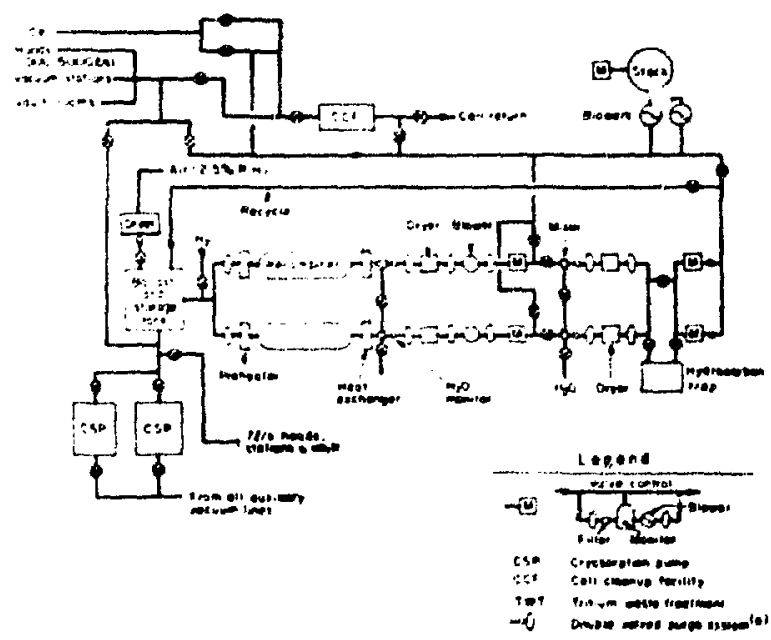

Fig. 5.6.1.5.

Fine diastam of the tritium waste treatment sisto'm.
Tritialled water is removed with greater efliciency with an increase in the mumber of drsers and a derrease in the ammunt of water injectionst The pressure in the ballast tank will be maintained between 27 fi and rix.s $k t_{a}$ be electrical valses. Whes the poessure in the tank reaches at presen cuppers limit 1 - intil'al the walves will open and the circulas. tion bluwer on the system will come on and pull gits wit of the tank through the Tll"l. The gas mow through the TWT will com inue umt the pressure in the ballast tank falls to the lewer pressure limit 1 - 28 kt'al an which time the attematric valse on the tank fluese and the T.en blewer is lurned ofl.

The recombiner preheater arran;erment and thes

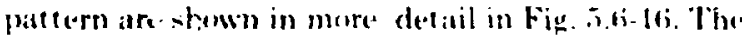
caltaletic reconbiner combans a precions metal tplatimum-hased cillalys dintributed on :he subt.

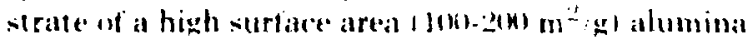
support material." The hed eperate at at temperature of - tiol k. The gas leaven the recomhimer and palswes threngh a watcrecolded he:al exrhanger to reduce the lemperature 11 - 2egt $k$ hetore the gas enters the muleevlar sene drying tuwer. At this point all but = 1 ppb of the herirogen isoncopes will have heen converted to willer. The gate exiting from the drsing wewer will comtain - 1 prm watter. This gas must the'n pass throuph ion chamber tritium monitors an determine the extent of tritium contamination in this exit gas st rean. At thi- prist the "ll"l" lugic sistem will route the gas to one of two possible paths depending on the tritium level still present in the exit gas. If the tritiom lesel is accep.

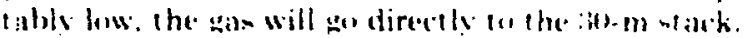
If the tritium level is high, the gats will go to a second drver. If the tritium level is mon lew enoush. the was will be stacked. If the tritium is tom high and the

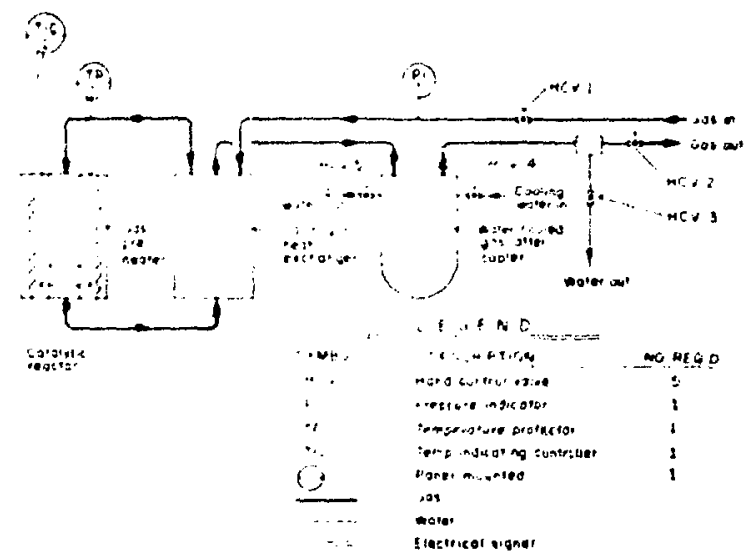

Fig. 5.6.16.

\section{Find dianram for the tritium recom. biner/prehioler.}


water level low, the gas will then be recycled, going back to the ballast tank, or it necessary, iransferred to the second line. Alsu, if the tritium level is high and the water concentration is correspondingly high. the gas will flow through the second set of recombiners, drying lowers, and ion chambers. At this point the gas will go one of two routes. If the tritium level is sufficienty low. the gas goes up the stack. If tritium still renaains in the gas stream, the flow is directed hack to the ballast tank. At this point more efficiene tritiated-hodrocarbon removal by a $1: 3 \mathrm{X}$ drveris, or longer recirculation may be required. The system will be designed with an autonated alarm system such that operating personnel will be immediatoly alerted it the system goes into a recycle mode. "The activation of the alarm will be indicative of a sistem problem requiring immediate attention.

The drying towers will have redundant beds containing regenerable molecular sieve similar or equal to the Linde +-A product. "These molecular sieves: are vers effective for water adsorption. The redun. dancy in drying towers will permit regeneration ail a saturated tower while the TW"I sistem eontinues to operate with a second processing line. The molesular sieve bed can be regenemated by heating the bed to firl $k$ and thwing a dry purge gas fargonl wer the siece bed. "This regeneration function sweeps out the adsorbed water. which can then be condensed from he purge gas and collected in a cont ainer approved for if-site disposal. The container may be shipped (1) an approved recovery laboratory or it may be shipped to an approved burial site.

Buth the reombiner. and the drying towers will have heaters. The recombiner operates at elevated fomperature $(-4 i) \mathrm{k})$ and the drying towers must be heated to -450 if for regeneration. These units will be designed so that the heaters are external and san be replaced without opening the active beds to the outside air. This design eliminates the need for personnel exposure to tritium during heater change. out. which could conceivably occur with some tre. quency. These units will be equipped with ower. temperature thermestats that will shut off all elec. tricul power to the unit in the esent of a thermal excursion. These units will also be built such that the active material, either cataly'st or molecular sieve, can be changed in situ. Fach unit will have removal purts at the bottom of the columns and fill ports at the (in). A second option is the complete replacement of the unit since the tritiated water in the unit would be well sontained and in a solid form.

The blowers to be used in the system will be oilless compressors. The main compressor providing the $T$ i/s gas fhow will be an oilless. sealed compressor. $A$ unit of this type provides adequate gas flow, suffers relatively little radiation damage. and does not introduce any organic materials into the system. A pais of these comprcssors will be used in the TW" system; one compressor will be in use and the other will be a standby unit that can quickly be placed anline. All components in the TWT system will have to be specially designed or moditied to insure that they are gastight lleak rate $\leq 10^{-4} \mathrm{~cm}^{3}$ torr/s). The equipment will be in an isolated romm, but any tirtium leakage to the open rom will be a potential hazard.

All equipment will be connected to the TWT by means of a standard purge system deseribed in Sec. 5.t.t.4. The piping within the TWT will be either (upper or stainless-steel tubing. Wherever fossible. the joints will be permane?t. e.y.. soldered or welded. Flanges which require gaskets will use "(irafoil" gaskets. Where possible the nonpermanent joints will be made with Cajon-type metal gasket connectors. The use of elastomers as gasket material will be avoided. The manual valves on the low-pressure side of the compressor will be quarter-turn stainless-steel ball valves with a Tellon seat. On the high-pressure side of the compressor. metal-bellows valves such as the Nupro or Hoke packless belluws valves will be used.

\section{Cleanup System for Accidental Tritium Releases}

In the event of a signilicant release of tritiun to the SFTR cell or to one of the tritium handling rooms. a rapid cleanup of a large volume of air will be required. $A+7(0)-1 /$ s cleanup system will be used for this purpose: the most likely function of this unit will be to remove rapidly tritium which has been released to the SFTH cell as a result of a large-scale failure of the SFTK discharge tube during a D.T plasma pulse. The unit proposed tor this purpose is similar to a system available commercially from the Fingelhard Corp.. ${ }^{37}$ and is probably conservatively. sale. even for a massive discharge tube failure during a D-T shot.

This facility will be activated in any of the following cases: an accidental tritium spill in the cell surrounding the discharge cube. as a backup for the handling of a large spill in the hoods. or tritium handling rooms. or possibly for the emergency handling of a major spill in the vault. Figure 5.6-17 gives a how diagram for the cell cleanup facility.

This tacility has a low probability of use because:

(1) The discharge tube, vacuun system. and most other components of the injection. recovery. and postdischarge gas diagnosis systems are normally under a partial vacuum. so that all leaks would be into the system. 


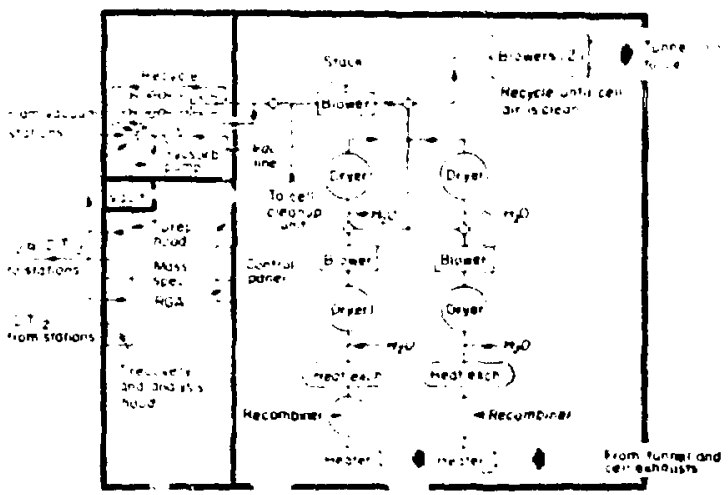

Fig. 5.6.17.

Tritium facility flow plan and ce'll ileanup facilits.

(2) Only mimor quantities of tritium are inwived in the SFTR (no breeding).

(3) Should a large break occur in the discharge tube, the enclosed gas could be evacualed im. mediately. into the four cryosorb pumps tser. 5.6.3.31 which have a capacity of several thousand liters ISTP' and a speed of l(MK) is. Continual operation of these pumps might not be ecoummical.

(4) Long gas lines which carry tritium will be of copper of reduce the permeation of Iritium: Sis.316; is another opcion. ${ }^{19}$

(i) In the speration of the hoxds, which are normally closed. a low llow of gas exhaust s inu the $7.4 / \mathrm{s}$ unit described in the previous section. Thus, ac. cidental leaks would be woll contined.

(ii) A large spill in the vault could be handled by the T-is unit.

The accidental release that would he the must diffiecult to handle would be a massive rupture of an ap. preciable fraction of the discharge fube (o relesse. about lin (i) of tritium. Only 16 , of the runs. huwever. will involve tritium. This release would probahi: be into an undamaged cell, the solume of which is 20 $900 \mathrm{~m}^{3}$. The essulting average tritium concentration would be is $\mathrm{m}\left(\mathrm{i} / \mathrm{m}^{-3}\right.$. or a solume traction of $3 \times 10^{-5}$. The volume fraction of $\mathrm{T} .0$ in air suitable for public release is: $0.2 \mu \mathrm{Ci} / \mathrm{m}^{i}$ ur a fraction of $11^{-3}$. It follows that a difution of aboust :30 (KM) would be required belore the gals conidi be stacked.

The safest methed of eleaning such an enelewure is (n) a "once-t hrough" basis with an inlet of tresh atir. it precessing of the air wo remese I ritiun. and a liwal external exhatsi of the eleaned air. In this rase.

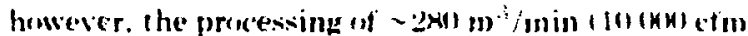

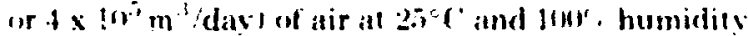
would prodene ! I this of tritiated water pure day with allendant waler handlung problems. trexpent drses

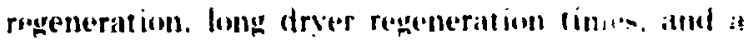
requirement for massive drvers. Acourdmols, it is planned le recirculate the irealled gats. The Blow patern of the timbse unit is shows in Fin. i.ti-1:

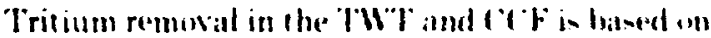

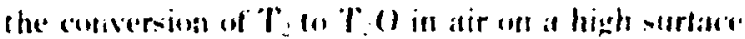
atrea material impregnated with precions metal

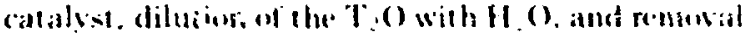
of the tritiated water with une or more drsers, wheh

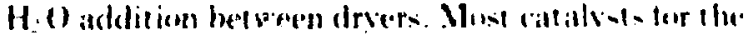

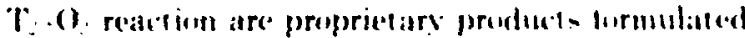

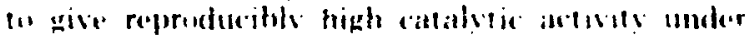

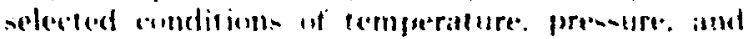
nin.

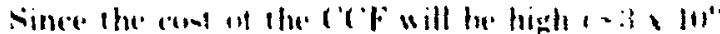
didlarsi. alernative aperatimal moder mipht well be considered for the tinal te-ign. A- a gudele ter the

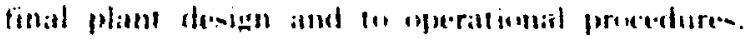

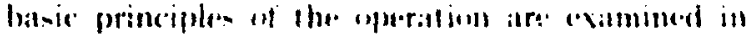
dintiil:

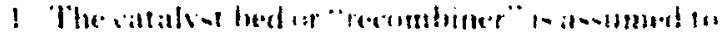

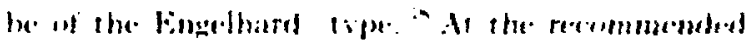

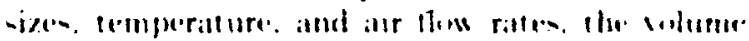

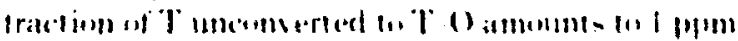

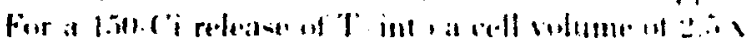

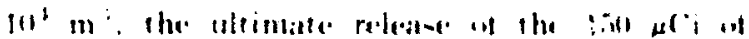

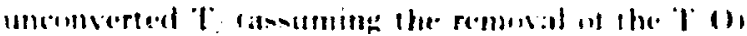

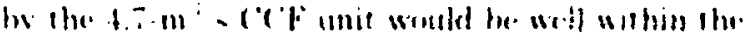

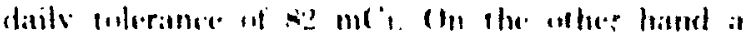

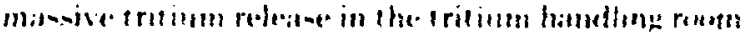

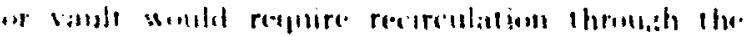

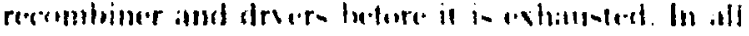

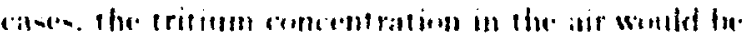

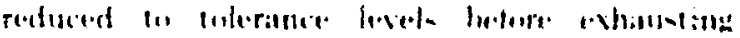

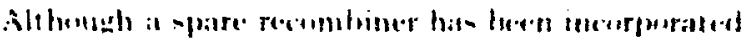

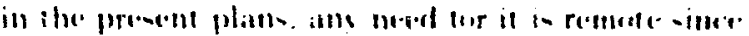

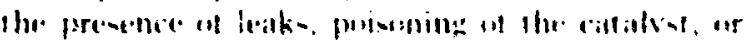

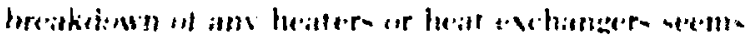
inlikels.

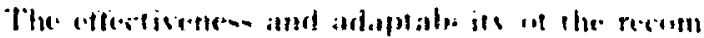

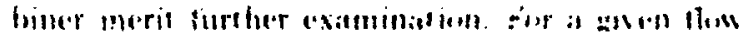
fermerature. and hed velume. the tanetional conver.

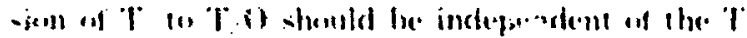

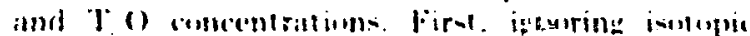

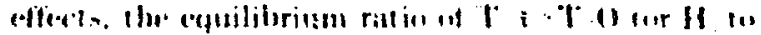

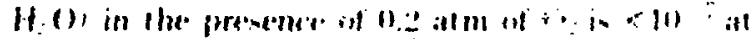

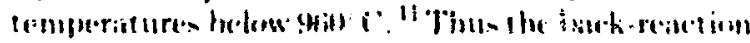

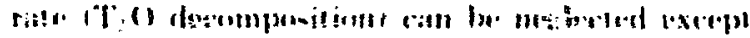

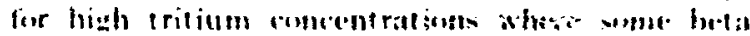

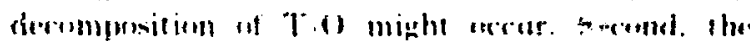

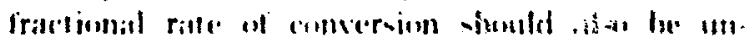

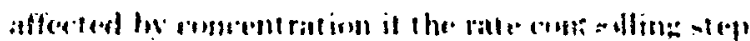

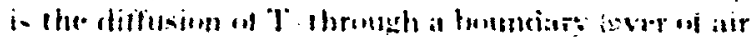

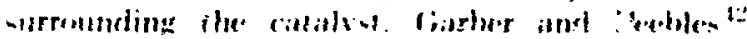


found thin to be the (asie for $H_{2}$ at wimewhat clavated

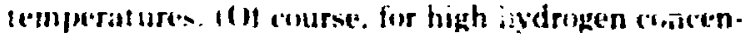
tratichs. sutnc increase in rate would be caused by the rhatuge in aumpustion wi the boundary layer,

2. Sinne Enge-llatrol recombiners can be aperated aicer at rampe of lemperaluses is but reaction rales are men poven ter thedi catalysts. Since the (CF unil is uned ond for sandly, continasus heating of the matsone recombuser and preherater would be

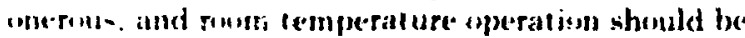
comsingreded.

It the reactions rate is controbled be diffunion

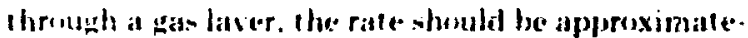
iv propurtional to the spuare ot the aboctule lemperatlore." fixperiments bs fiarber and

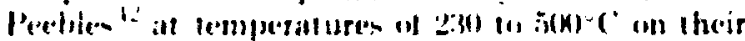

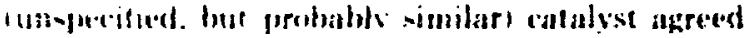
with thi-lunctumal dequendence. Acoordingly a 20 .

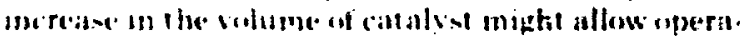

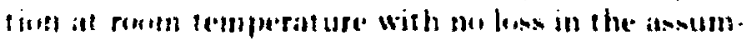

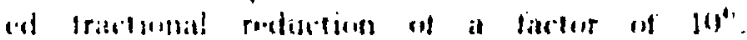

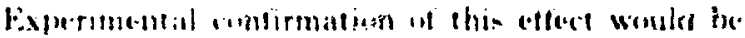
chentritila.

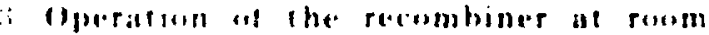
temperature and the omission at a lix molecular

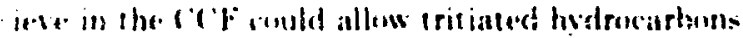

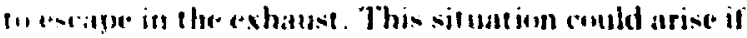

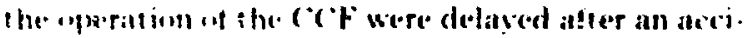
do.ne This problem probably shmuld lue reasoessed as the stelte platro beenme linal.

1. l'rusiojul has heren made for the addigion at hudrugent to the romtaminated air that conters the recumbinger. This might he useful an tal inerease the rafe nt reatcien due la the temperature rise that

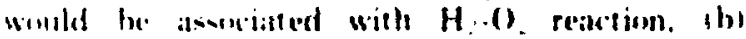
mereane this ratte markedly if the caltallist is stet.

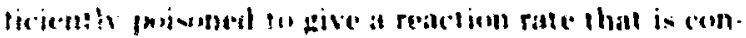
antrat is ntt alceidentalls comblentel water. and all reactivalte

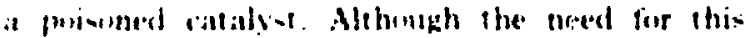

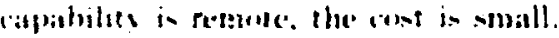

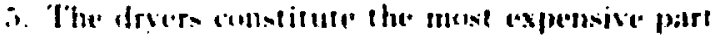
if the (1\% and varinus schemes for their aperation

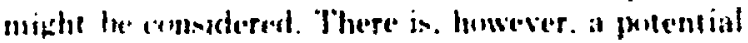

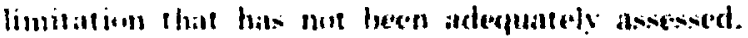
bul which could prohebly be studied at I.ASI. in cime ta be usetul for the titiol (CF plans. The prohisest relates to the rate at which water from the hed it the drver exchanges with the water in the pronesesed anir. Fur examples a dryer that is highly colltaminited with tritiated water might well add tritiun to a gas having a low lesed of tritium contaminatum. This exchange should be studied using tre-h. ersugenically dried air in fouls combining varicuss wialer fratediums.
If this exchange rate is high. one must be very careful to limit the tritium contamination of the final dryer to low levels. On the oither hand. it the rale is cxceedingly low, one might eledt to use only (wo or threc dryers. This saving. combined with it:e use of anly inte recombiner could reduce equipmont. building. and maintenance cesis by perhaps a million clollars. The option to recirculate the air, using "batch" processing. is als" attractive but soind increaste the processing lime.

It has been assumed that the air entering each dryer ia iuld have a water content of $l(x)$ ppm. cor-

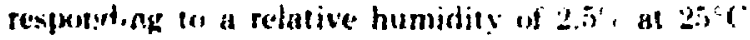
and (BW) (arr. and that the exil wuld have a comtent of 1 ppen. The walce content entering each dryes sould bo adjusted fo this value by mixing with a continuous water arr steatm spras. Thus each drser weuld reduce the tritium content by a factos at 10 : This cleaning rectunique can be exionded ro give air with a tritium level equal to the natural hackiground level of aboul three parts of $\mathrm{T}$. $\mathrm{O}$ in $10^{\text {th }}$ of $\mathrm{H.}, \mathrm{O}^{-}$ lar salurated with $T$, wo wold require six such drsefs in series. It the inlet air lo each dryer were

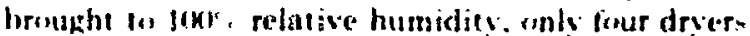
wowld he required. Thas: the chuice af lext pent H. () in the inles is arbitrasy. An increase in the inlet humidicy would reduce the number of strots. the number of recirculations. the sparce requirements. and tho presiure drup, but wiuld increase the colume af tritiated water coliested atod trequeney of drver regeneration. An a furthes examplo: the ust ul buth $-2=$ relative humidity ius the indet water con-

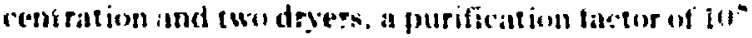
wotuld be netaiced. Alternatively. using 2.51 . rolative humidits and thres cirvers. a purifientum factor of $10^{t}$ would he obtained with mily 151 as much waste water heing collected. and there wisuld be a corresponding reciumion in the need for the regeneration of the drvers.

The proposed ofjerating procedure is as follows:

1. The cell ait, ideally of bew humidits. is process.

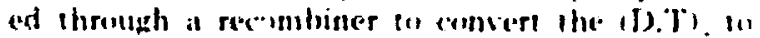
(1), It, (1). The relative humidits is comtrolied at more than 2.51 .

$\therefore$ The air is passed through a molecular sieve dever.

3. Nater is sprayed iato the exhaust to give a relative humidity of about $2.5 \%$.

1. The gas is pasised through a socond dryer and then into the coll. Appropriate sensing and control devices are implied.)

5. Occasionally the druers are renzuerated. the water is collected in cans on abs ahsorbeut. and the product huried in doubly sealed comainers. For the asto issumed, the tritium content wuld he list ( $i$ per majur acrident. 
Althugh present plans all fir a tilk -. - process.

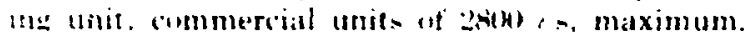

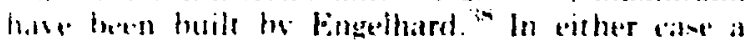
lluw of $14.5 \mathrm{~m}$ : 1 lok to mint in a 1 - $\mathrm{m}$ - ligh bed of linde mulecular sieve tA "in each of lwo dryers in ereres would be used. The pressure drup alderose the

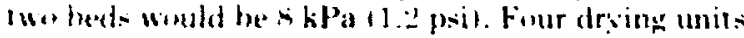
wonld be repuired to proside redundancy. Weight

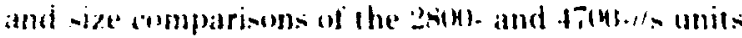
are shenn in Table i.ti-3.

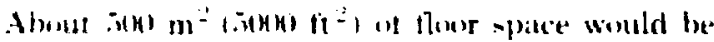
recatlared for handing and installation of the four targe droins units. for the cwo recombiners, the biewer- and stakekts. and for the 7 . - tritium waste treartmesst unit.

\section{i.6. Instrumentation, Control, and Operational Procedures}

The instrumentation. control, and operational atpect. of each subststem defining the tritium hatmiting motem hase been described in previous sections. This section treats specistically tour aspeets if the tritium hatndling sistem which interface with mute than whe if the tritium handling sub. -ritems: tritiun monitoring. nanscheduled action. pomedischarge diagnosis of gases, and maintenance pronedures.

\section{j.fi.1.1 Tritium Monitoring}

Permanent thw-through ionization-chamber-type tritium air momulors for personnel protection will be lucated in the SFIR cell and in the tritium handling room. These will be rate instruments, giving concenuations directl: Figure $5.6-18$ gives the thow patterns and monitur stations. A cell monitor will be kwated at each of the two cell entrances, and readouts will be available at both entrances and at the SFTR control room. These monitors will sample

T.ABL.F. . . . (i-3

\section{(OMPARISON OF 2XI01- A.NU IJIH-//S DRYERS}

\begin{tabular}{|c|c|c|}
\hline & $\begin{array}{l}2 x(111 / / s \\
\text { Dryer }\end{array}$ & $\begin{array}{l}\text { I7106//s } \\
\text { Dryer }\end{array}$ \\
\hline rea) of bed. in & $616010^{2}-1$ & $10130619 t^{2}-1$ \\
\hline Wiameter of bed. no & $\therefore=18(t)$ & $3 .+111 \mathrm{hi}$ \\
\hline Wuight of bed. MIg & $11+$ tonst & 717 (otins) \\
\hline
\end{tabular}

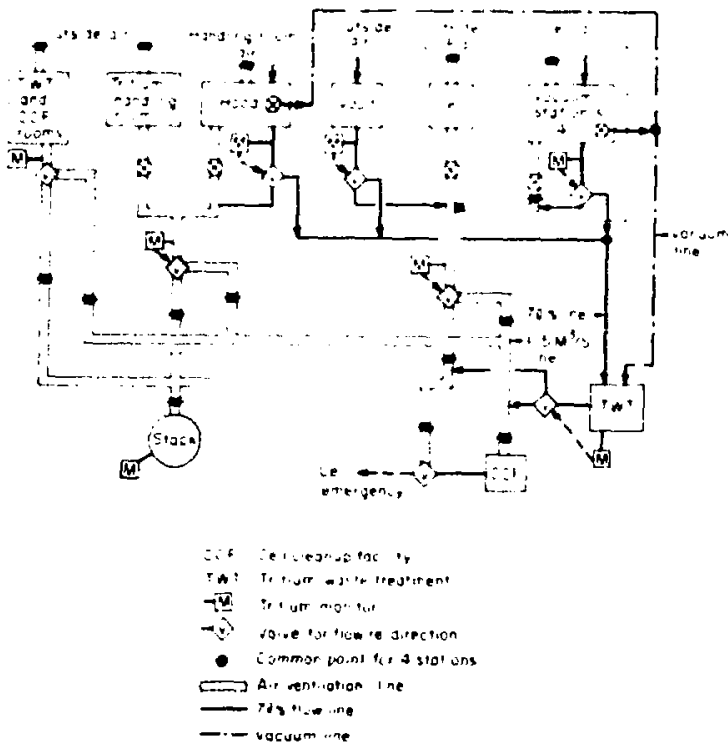

Fin. . . th-lo

Trusum flue and momituring sistem for .SFTR.

the air at selected points in the cell secpuentially and automatically through sampling hosen. The monitur in the tritium l.andling room will also have readouts located locally and in the control roum. 'The tive houds will alsi, he monitored with rate ins? ruments with local readouts in addition $t 0$ : hose in the cont rol roum. These air monitors will have sampling hoses 10 enable the person working with the equipment inside the hood. sometimes through glove ports. to monitor any part of the equipment inside.

The vault and rooms where the two tritium recovery systems (TWT and $C(F)$ are located will be monitored only as needed with portable or semiportable air monitors. However. the exhausts of these rooms will be continuously monitored with ionization-chamber-type integrating air monitors which will route the exhausts to one of the recovery systems if the tritium being exhausted past a particular monitor exceeds $20 \mathrm{mCi}$ /day. This and similar action by the other integrating monitors fexcept the stack monitorl will assure that the concentration in the stack is kept below the maxi" sum permissible concentration of tritium in air $10.2 \mu \mathrm{Ci} / \mathrm{m}^{3}$, averaged over $24 \mathrm{~h}$. This stack concentration monitored for a day corresponds to a release of 82 $\mathrm{mCi}$.

Integrating monitors will also be located in the exhaust ducts of the cell and the five honds. Similar monitors will also be used in the recovery systems to check their performance and route the air to redundant, backup components as necessary to keep the 
concentration at the exhaust of the recovery systems within design levels.

The mormal flow through the five hoods and the vault will loe kept at less than $7 / \mathrm{s}$ si) that spills in these places can be handled by the smalier $i-1 / \mathrm{s}$ TH\%. Tukard this end. thexible ducts will be used in the howds to increase ventilation near any highly ronlamimated parts being handled when glove ports

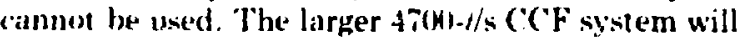
back up the ' $\mathrm{WW}$ and handle any released tritium that camnot be handled by the 'TWT. Figure 5.6-18 shows the ventilation system for the SFTR cell and the tritium rooms and indicates the loseation of the Various air monjtors.

Monituring of lisw-level tritium in the presence of activaled air procilued by neutrons during a D-T pulse or in the presence of a high natural radon hackground can present some problems. For a dis:usion of these and of 1 ritium monitoring in general at the sfF'll tacilits. see Sec. 7.3.1.1.

\subsubsection{Nonscheduled Action}

Sicclion 5.6 .4 .1 outlines the purposes of the tritium monitoring equipment and refers to the ventilation and tritium (eleasup) sistems to which the equipment is comnected. Accidentai releases stritum will nor. mally be trapped and on's insignificant quantities will bo released to the en $;$ ironment $(<82 \mathrm{mC} /$ day).

should a release occü in a room $v$ here persunnel are uresent, the roun will be evacuated and reentry permitted only after the concentration has dropped II an acceptable level. Supplied air suits ${ }^{4: 3}$ will be worn if teentry is required sooner than swould otherwise be allowed. Tritium is stored not only in the vault and the tritium recovery systems. but in houds at each pumping station. A release in any of these will mot require evacuation but only assurance that the hood doros: are closed. Action will be taken whenever possible to minimize the amount of tritium released io the ventilation system.

In case of tailure of the discharge tube during a $\mathrm{D}$. T pulse. - 10-1ii ny of iritium at most would be released in the unciceupied cell and automatically exhausted in the $47(0)-1 / \mathrm{s}$ CCF. A potent release in the even: of a minor failure of the discharge tube could be handled by having the downcomer and cryosorb roughing pump valves open immediately, directing most of the tritium into the crvosorb pumps (refer to fontnote in Sec. 5.6.1); only a small fraction of the tritium inventory within the discharge tube would be expected to escape into the cell in this case.

linles: a tire or explosion is directly involved. the tritium in most of the signiticant releases would he in the form of the gas. which is orders of magnitude lest toxic than the wicte. Surmal conversion of the gas 10 the oxide by oxidation or exchange is very slow and this releane can be neglected. However. all sateiv guides will assume that tritium measured by the monitors is in the oxide form.

\section{1i.4.3 Postshot Diagnosis}

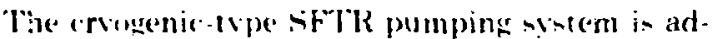
mirably suited for ajding in postshor analysis of the fuel ash. First. the sistem itself introduces no impurities into the exhaust gas. except possibly tor trace amounts of outgased material. st that neither is protium int roduced into the yas nor are (I). T) subsituted methanes (or other organics) introduced that might cause ambiguity in tho water (or oxygen content in mass specometric analsses. Furthor, a -rvogenic pump can be used nol only tor evacuating. but also tor iransferring gas at at mospheric pressure. Finally. by suitable design. cryogenic pumping can be ased to soparate gas components. The degree of separation depends on pumping rates, gas presisures. relative concent rations of components, temperatare comt rol. and gas solubilities in the condensed sulds. Fur the svisem considered here. evidence fur elfec. tive separation is strong. except possibly in the case of heliuns.

sume of the postshot diagnostic's that would be unctul follow:

1. A determination of the amount of axyon formed in a run wisuld show the extent of ciecomposition of the wall maters? or the magnitude of leaks. Such ixygen might be formed by a combination of alpha. deuteron. and ir son bunbardment. neutron sputering, and brensistrahlung and other high intensity radiation. "Thr-extent of this formation is expeckex to be of minor siuniticance. Outgassing of oxygf $n$ is also of minor concern. given the planned injecrion and evacuation schedule. Of major imort ance is the early detection land repairl of leaks.

2. The determination of the amount of water in the ash is critical. The amount of water in the ash could be quite large (perhaps a tew percent) since the inter wall will almost rertainly he an oxide. and since existing thermodynamic data. ${ }^{22.3} 33^{2}$ indicate that all oxides are unstable in the presence of the anticipmted presstares of atomic hydrogen. The reaction is kijelically controlled. and the vield might well he governed hy the relative rates of reactions such as: $D+D i+$ wall $\ldots D .$. and $D+D+$ axide .. . D.. + metal. suboxide. oxyhydroxide. etc. The relative rates of these wo reactions will certainly depend on the ceramic used and on additives that might pur. posely bo used in the ceramic. A selection for the inner wall material would be based on research on 
ceramies tailored to mmiraize water formation and still give the required electrical conductivity, thermal stability. etc.

The rate of corrosion of the inner wall by atomic hydregen attack should be extremely low (less than 31) ann/grt unlessi nonuniform attack or pitting of the coat by preferential reaction should occur.

The critical problem with water formation is that the water formed will most likely be adsorbed on the oxide wall and will then slowly outgas to contaminate the subsequent plasma gas charge. Since heary-ion contamination of the plasma significantly lowers it temperature by bremsst rahlung radiation. it is imperative that the oxvgen impurity content of the plasma be kept low. A steady state will be obtained if shot intervals, injection times, and evacuation times are constant, to give an amount of out. gatsied water during evacuation that is equal to the amount formed during a shot. Thus, the measure. ment of the (D.T) $2 \mathrm{O}$ content of the plasma ash can be used to calculate the amount of water contamination in the plasma. If a constant outgassing rate is assumed. the calculated contamination would tend to be too large. If the differential outgassing rate during the evacuation period is measured, then a more accurate knowledge of the actual contamination would be obtained. Since the wall temperature would be expected to drop during evacuation. a knowledge of the change in outgassing rate during this period is clearly advisable.

3. The helium content of the ash gives the D-T burnup) of the fuel. A helium measurement thus allows a direct determinat ion of the degree of burn of the $\mathrm{I}$. $\mathrm{T}$ reaction in a particular run as a check awainst other diagnost ic methods. It is assumed that such measurements will be made only occasionally.

4. The measurement of other impurities by mass spect rometer and/or quadrupoie mass spect rometer residual gas analyzer will be made to

(:. Determine protium contents to estimate not only the protium content of the plasma but also to give information on the purity of the plasma charges and (I).T', recovered gas.

(b) Measure the tritium and deuterium contents of the injected gas $(t o$ check the volumetric meisurements) and of recovered tritium.

(c) Estimate the amount of contaminants, such as ( (). CO... $\mathrm{N}_{\mathrm{g}}$, in the exhaust (from outgassing). Sucin measurement will be of particular use after the discharge tube has been exposed to air and the first lesw runs havr been made. Of major importance is the detection of leaks. in conjunction with oxygen determination discussed in item 1.

(d) Assist in the determination of helium in the exhaust gas. Such determinations are not unamhiguous since $H D$ interferes with any ${ }^{3} \mathrm{He}$ determination but, of far more importance, the presence of $D_{2}$ in large quantities negates the usofulness of a ${ }^{4} \mathrm{He}$ determination. However the (D.T). can be oxidized to (D.T) $\mathrm{O}$ and easily separated from the helium.

Figure 5.6-14 shows a schematic of the post shot diagnosis apparatus. Mass spectrometer or RGA samples will be taken where shown and also at the pumping stations (Fig. 5.6-13). Details of the mass spectrometer analytical techniques have not been selected. A Nier-type and an RGA-type spectrometer are to be located in the tritium handling room.

Cryot rapped $(\mathrm{D}, \mathrm{T})_{2} \mathrm{O}$ will be transferred, at least every day, to a liquid nitrogen trap. ${ }^{29}$ After warming. the gas will be expanded into a calibrated thermostated standard volume for PVT measurements. The water will then be transferred via a liquid helium cryosorption pump to the TWT, as shown in Fig. 5.6-15. As knowledge is gained on normal water contents of the ash. water determinations will be made by expanding water directly into a calibrated volume at ambient temperature for PVT measurements. (It should be noted that such expansions must be handled carefully to avoid the extremely slow sublimation that occurs from ice.) If water contents are low or well known, the water can be expanded directly to the TWT. If the (D.T) 20 centent is sufticiently high, it might be desirable to pass the vapor over hot copper for recovery of the (D.T):. Indeed, such a technique might be used as the analytica! method for obtaining average water contents. This could be obtained uy measuring weight changes in the copper (or hopcalite tube. This option is also shown in Fig. 5.6-14.

Initially, it is planned to make helium PVT measurements in a calibrated volume at ambient temperature as shown in Fig. 5.6.14. Assuming a need for an accurate measurement of the helium. a standard thermostated volume and an additional cryesorb pump would be added, as shown in Fig. 5.6-14. In both cases, mass spectrometer analyses would be required. If (D.T). contamination is significant, the (D, T).2 would be converted to (D.T) $\mathrm{O}$ and removed as indicated above. Helium from $D_{2}$ runs could be measured the same way. but since the quantity of gas is so small, PVT measurements will be made only occasionally in a calibrated volume at ambient temperature. More extensive measurements would require an additional liquid helium cryosorption pump at the pumping stations.

Finally. $\sim 1$-in.-diam tubing for gas transfers will be located in the cell. Having four pumping stations allows the use of only one station for a given analysis. with a rotation of sampling points to speed operating 
times. Alterratively, specific products from the four stations can be combined for analysis.

\subsubsection{Maintenance of Tritium Handling Equipment}

The entire tritium haidling facility is designed with the goal of minimizing routine maintenance. For this reason organic materials will not be used in those sections of the system with which tritium might come in contact. The cryogenic pumping svstem will eliminate vacuum pump oils from the system and will provide a clean, fast, and efficient mode of evacuation.

Wherever possible the tritium lines will be of welded construction to eliminate as many joints as possible. Where it is necessary to have joints, the connectors or flanges used in the joints will have metal gaskets. Where flanges and connectors must be used in TWT and CCF areas and where a hard vacuum is not required, the use of Grafoil gasketing material $^{44}$ will be specified. This all-graphite material will not absorb tritium by isotopic exchange: furthermore, it will suffer relatively little radiation damage. Grafoil is currently in use at the I.ASL tritium facility. ${ }^{39}$ Throughout the tritium svistem considerable redundancy has been incorporated into those major components that might preseut frequent maintenance probleins. This procedure was adopted so that a standby unit would alwavs be available in event of a critical component failure. Also, this procedure allows routine maintenance to be performed on a component without shutting down the entire system.

The components of the tritium storage, injector. and vacuum systems will be enclosed in hoods. These hoods are designed such that during routire maintenance and component change-out, the air flowing through the hoods can go to either the $7-1 / \mathrm{s}$ TWT or the $4700.1 / \mathrm{s} \mathrm{CCF}$ for removal of any released tritium before stacking. If the tritium release during these operations can be kept low enough the air will, of course, go directly to the stack rather than be processed. This routing of the air will be automatically determined by the ventilation logic system once the air has been monitored for tritium (Fig. 5.6i-18).

'The tritium handling system has been designed so that all major components can be isolated by a set of valves. This procedure leads to the use of many valves but means greater personnel protection against tritium-contaminated air and surfaces during maintenance. Fach colnponent in the system will be joined to the main tritium system by a double-valved. purge sustem as illustrated in Fig. $5.6-19$. When a component has to be changed, valves
To ouxiliary vacuum system or clean argon supply

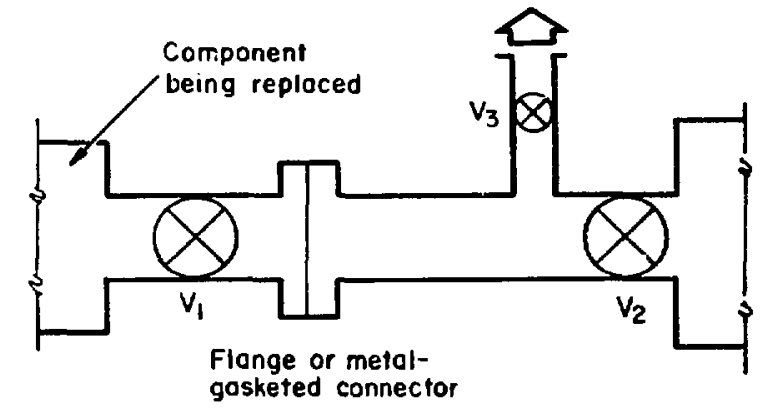

Fig. 5.6-19.

Typical double-valued purge system to be used throughout SFTR tritium handling system.

$V_{1}$ and $V_{2}$ will be closed and valve $V_{3}$ opened to the auxiliary vacuum system. When the volume between $V_{1}$ and $V_{2}$ has been evacuated, this volume will be back-filled with clean argon. The same sequence of events will occur at all other joints leading into or out of the component. The joints can then be opened with a rninimum hazard to personnel, and the maintenance and/or change-out can proceed. This procedure has the added advantage of keeping the remainder of the system isolated so that working vacuum or pressure can be maintained. After the maintenance is finished, the component can be easily rejoined to the system. The auxiliary vacuum svstem will then be used to evacuate the room air from the volume defined by valves $V_{1}$ and $V_{2}$, valve $V_{3}$ will be closed, and valves $V_{1}$ and $V_{2}$ can be opened to bring the system back to operating condition. This purge system is being used in the LASL tritium facility ${ }^{39}$ with great success (reduced personnel exposure, tritium releases to the atmosphere, and maintenance times).

The four vacuum stations located around the discharge tube will be situated in semimobile hoods. These hoods are designed in such a way that the vacuum station will be assembled in the hood at a remute assembly station in the tritium handling area. The hood will then be transported to the working location in the SFTR cell. Here the hood will be connected to the ventilation system ductwork and the vacuum system connected to the vacuum manifold leading to the discharge tube. Conversely, when major overhaul of a vacuum station is necessary, the vacuum svstem and hood can be isolated and the hood moved out of the cell to a more favorable working area in the tritium facility for performance of the necessary maintenance. Here again the hood will be connected to the main ventilation 
system so an adequate supply of tresh air will be pulled through the hood. In this scheme the air can be processed by a cleanup sustem in the event of a tritium release during the maintenance operation. During this maintenance the remaining three vacuum stations will be adequate to keep the SFIR operating. It will be desirable however to have a spare hood and vacuum assembly readied for installation. Once the required maintenance is conplete, this svitem will be available as the standby unit. It must be emphasized that replacement of an entire vacuum station would occur only in the event of major maintenance requirements. Most maintenance and the replacement of cryogenic heads, valve seats, and molecular sieve should be done in situ. During maintenance the air in the area will be carefully monitored to assure personnei safety. When maintenance is being performed in situ on any part of the tritium handling facility the maintenance personnel will work in supplied-air bubble suits. These suits and the associated air supply will be similar to those described by Mound Laboratory. ${ }^{1: 3}$

Components of other portions of the tritium handling srstem, (e.g., the injector system, TWT system. etc.) will be interconnected with the standard purge system illustrated in Fig. 5.6-2. The tritium storage, injection, recovery, and diagnostic svstems will be enclosed in a hood that will have air fins from tront to back to pull the air away from working personnel. The hoods will be relatively shallow so that a person working at the face of the hood can easily reach all components. Provision will be made at each hood for attachment of a small flexible duct through which air can be pulled on its way to the TWT for tritium removal. This will allow one to hold the duct next to the working area, thereby giving a more positive air replacement in the critical area. A plastic covering containing gloves will be used over the door opening in some cases to reduce the air flow sufficiently for the TWT to be used.

The fast-acting pneumatic valves on the injection line and downcomers will require periodic maintenance. Because these valves are not located in the vacuum station hoods, provision must be made for the safe handling of tritium released during the maintenance. Once again, flexible ducting from the vacuum station hood will be run to the valve area and positioned next to the valve during maintenance. The backup valves at the injection station will permit closure and isolation of tritiumcontaminated surfaces, thereby exposing a minimum of contaminated area.

\section{REFERENCES (Chap. V, Sec. 5.6)}

1. Federal Register. Title 10, Part 20 (10('FR20). "Standards for Protection Against Radiation" (1968).

2. D. G. Jacobs, "Sources of 'Tritium and Its Behavior Upon Release to the Enviromment," USAEC report TID-24635 (September 1971).

3. E. A. Evans, Tritium and Its Compounds (van Nostrand Co., Inc., Princeton, NJ. 1966).

4. G. J. Casaletto, L. H. Gevantman. and J. B. Nash, "The Self-Radiation Oxidation of Tritium in Oxygen and Air." U.S. Naval Radiological Defense Laboratory report USNRDL-TR-565. San Francisco. CA (May 1972).

5. J. Y. Yang and L. H. Gavantman, "Tritium $\beta$ Radiation-Induced Isotopic Exchange in the $\mathrm{T}_{2,2}-\mathrm{H}_{2} \mathrm{O}$ System." U.S. Naval Radiological Defense Laboratory report USNRDL-TR-471, Sar Francisco, CA (September 1960).

6. Maximum Permissible Body Burdens and Maximum Permissible Concentrations of Radionuclides in Air and Water for Occupational Exposure. NBS Handbook 69, K. Z. M.rgan. Chairman (Superintendent of U.S. Documents, L.S. Gov. Printing Office. Washington. DC, IJunc 1959).

7. A. G. Evans, "New Dose Estimates from Chronic Tritium Exposures," Health Phys. 16. 57 (1969).

8. W. Minder, "Interne Kontamination mit Tritium," Strahlentherapie 137, 700 (1969).

9. W. S. Snyder, B. R. Fish. S. R. Bernard. M. R. Ford, and .J. R. Muir, "Urinary Excretion of Tritium Following Fxposure of Man to HTO-A Tivo Exponential Model," Phys. Med. Biol. 13, 547 (1968).

10. B. E. Lambert, H. B. A. Sharpe, and K. B. Dawson, "An Accidental Intake of Tritium Water," J. Am. Ind. Hyg. Assoc. 32, 682 (1971)

11. S. M. Sanders and W. C. Reinig. "Diagnosis and Treatment of Deposited Radionuclides," Excerpta Medica Foundation (1968), p. 534.

12. A. A. Moghissi, M. W. Carter, and R. Lieberman, "Long-Term Evaluation of Biological HalfLife of Tritium," Health Phys. 21, 57 (1971). 
13. J. J. Koranda, "Residual Tritium at Sedan Crater," USAEC report UCRL-70292 (April 1967).

14. W. M. Jones, "Half-Life of Tritium," Phys. Rev. 100, 124 (1955).

15. G. H. Jenks, F. H. Sweeton, and J. A. Gormley, "A Precise Determination of the Half-Life and Average Energy of Tritium Decay," Phys. Rev. 80, 990 (1950).

16. Subcommittee on Permissible Exposure to Radiation in an Emergency Condition, G. V. LeRoy, Chairman, " Exposure to Radiation in an Emergency," Report No. 29, National Committee on Radiation Protection and Measurements, Univ. of Chicago, Chicagu, IL (January 1962).

17. R. C. Weast, S. M. Selby, and C. D. Hodgman, Eds. Handbook of Chemistry and Physics, 46th Ed. (The Chemical Rubber Co., Cleveland, OH, 1965-1966).

18. T. B. Rhinehammer, P. H. Lamberger. Eds., "Tritium Control Technology," USAEC report WASH-1269, Mound Laboratory. Miamisburg. $\mathrm{OH}$ (December 1973).

19. R. M. Alire, Los Alamos Scien iic Laboratory, personal communication, October 1974.

2!. S. Dushman, J. M. Lafferty, Scientific Foundations of Vacuum Technique (J. Wiley and Sons. Inc.. New York. I96i2).

21. R. S. Carlson, Mound Laboratory. Miamisburg. OH. personal communication. October 1974 .

22. Cajon Vacuum Products. Cat. No. CA-1171. Cajon Co.. Cleveland, OH (1972).

23. Haskel Engineering and Supply Co., Bulletin S6. Burbank. CA, 1966.

24. Nupro Manually Operated Bellows Valves. Bulletin N-473, Nupro Co., Cleveland. OH (1973).

25. MKS Instrume'tts. Inc.. Applications Note No. 1 MKS Instruments. Inc. Burlington MA (1973).

26. Matheson Gas Products Enpineering Report G1:11. Matheson Gas Products, East Rutherford. NM (1973).
27. "Properties of Copper and Copper Alloys." Metals Handbook, Vol. I, Am. Suc. Metals, Metals Park, OH (1961), pp. 1007-1010.

28. J. Crank, The Mathematies of Diffusion $(0 x-$ ford at the Clarendon Press, 1967).

29. D. H. Davis, "Monte Carlo Calculation of Molecular Flow Rates through a Cylindrical Elbow and Pipes of Other Shapes," J. Appl. Phys. 31. 1169 (1960).

30. Net ? A. F. Malmberg "I'sers Manual Vet -2. Network Analysis Program." Reicase No. 9. HDL050.1. Harry Diamond Laboratr.1es. Washington. DC. September 1973.

31. W. E. Quinn, Los Alamos Scientific Laboratory. personal comrunication. September 19it.

32. J. W. Tester. R. C. Feber, and C. ('. Herrick. "Heat Transfer and Chemical Stability Calculation: for Controlled Thermonuclear Reacturn (CTR)." Los Alamos Scientific I,aboratory report LA-5i328-MS (.July 19731 .

:33. J. P. Coughlin. Contributions to the Data on Theoretical Metallurgy, XII. Heats and Free Energies of Formation of Inorganic Oxides. Bureau of Mine: Bulletin 54: 1L.S. Gov. Printing Office. Washington. DC. 19it)

34. Balsers Bulle:ial 1'31-14-iloge, Balsers High Vacuun Corp. Santa Ana. CA 1 = 197ïi.

35. Viarian Bulletin VAC-1005, “.NRC 316 series. Circular (hevron (rototraps. Varian Vacuum Division. NRC Operation. Lexington. MA $1 \cdot 1973)$.

36. The Excalibur Corp. bulletins 3-750-(01 and "Cryo-lacuum Punps Excalibur series CVR-1100." The Excalibur Corp.. Los Alios. CA Avrember $197: 3)$.

37. The Excalibur Corp.. private communication. 1974. Pump description: linde Cryosorption pump 14000 / capacity): Linde bulietin F2891 "Linde S.X. 150 Crysosorption Vacuum Roughing Pump." I'nion Carbide Corp. Linde Division. New York $(-197 ; 3)$.

38. H. M. Rogers and R. Michalek, "Tritium Removal Systems." bullet in EM 1(k)54. Fingelhard Industries Division. Engelhard Minerals and Chemical Corp.. East Newark. X.J ( 1972). 
39. .1. L. Anderson, personal communication. Los Alamos Sicientific Laboratory. September 1974.

111. Linde C'. Bulletin Flon:6B. The Linde ('o.. New Yirh 119621.

H. 1). K. situll and H. inophet. Project Directors, JANAF Thermochemical Tables, 2nd Ed.. NSRISS-NBS 3T tsuperintendent of Documents. I.S. Government Printing Office. Washington. DC. 19711.

42. I. H. Garber and F. .. Peebles, "The Cumbination of Hydrogen and Oxygen in Platinum Catalyzed Fluw Reactors." Oak Ridge National Laboratory report ORN1. 1796 iOctober l9it).

43. C. I. Phillabaum and P. C. Adams, "The Development of the Mound Laboratory SuppliedAir Bubble Suit." Mound Laboratory Report MIL.M. 2(M) (February 19-3).

4. Carbon Products Div. Bullet in 524-203ha. Inion Carbide Corp.. . N.Y. .Y. 197 I.

\section{T DIAGNOSTICS}

\section{\$.7.1 Introduction}

\subsubsection{Experience at Los Alamos}

Progress in cont rolled thermonuclear research has been inextricably tied to the development of plasma diagnostics. Researchers at Los Alamos have an expertise in diagnost ies accumulated from many years of experimental work and are uniquely qualitied tor the task of inst rumenting and diagnosing the SFTR.

The original Scylla experiments pioneered the use of streak cameras and neutron detectors. 1,2 The results were excellent, and very nearly the same techniques are in use as standard methods today. Careful studies of $x$-ray emissions from Scylla established the electron temperature, impurity levels, and other plasma features. $3-6$ The double-foil or twoabsorber technique $\bar{i}$ for electron temperature measurements grew out of the work and was the standard method until the advent of Thomson scattering. During this same early period. work on the Perhapsatron S-4 involved elaborate visible and ult raviolei spectroscopv for determination of impurity le vels and energy losses due to radiation.

Significant improvement in diagnostics was made pussible with the use of lasers. Their brightness and nonperturbing interaction with a plasma led to a hest of applications. One of the first and mest impressive applications at L.A.it, was the use of a rubs. laser as an illumination source in a Mach-Zehnder interferometer on sivila $\mathbb{I N}^{:}$"The resulting interferongrams showed the density profile in a striking manner-both qualitatively and quantitatively. The method was later combined with a $\mathrm{Ho} \cdot$. Te laser measurement of Faraday rotation to obtain the magnetic field int ernal to the plasma. "

One of the next laser technigues developed was the coupled-cavity intertersmeter tusing the $3.399-\mu$ wave $^{\text {nn }}$ ath of a He-Ne laser) which is fairly easy to align and ofters signiticantly greater response 10 plasma than does visible radiation. "Examples of results using this method are found in the Sivila 111 experiment ${ }^{12.13}$ and sillac. ${ }^{14}$

Many other interferometric methords have been used. such as a Mach-Zchnder on a linear Z-pinch. ${ }^{\text {i. }}$ a coupled-cavity with a CO.2 laser ${ }^{16}$ a Michelson interlerometer with a $\mathrm{CO}$. laser. ${ }^{i}$ and a MachZehnder interferometer using a pulsed $\mathrm{CO}$. laser. ${ }^{\text {th }}$

At the same time that lasers were beingr used, refinements were sought in other measurements. Plasma luminosity was made more quantitative by the use of fiber optics and phot omultiplier:s. Comhined measurements of luminosity and magnetic excluded flux determined the plasma beta in theta pinches. ${ }^{12.14}$ External field measurements have also been applied to the Columba Z,-pinch to determine internal current characteristics. ${ }^{19}$

Interferometry with a Mach-Zehnder, while extremely useful, is a difficult technique because of practical requirements of alignment and mechanical stability. Due to the pioneering effort at los Alamos. ${ }^{20,21}$ essentially the same data are now obtained routinely and much more easily by the use of double-exposure holographic interferometry. The technique has been used on Scylla IV-3, a 3-m thet a pinch, ${ }^{22}$ using in some cases as many as three lasers for three time intervals: $:^{23.24}$ on the $5-\mathrm{m}$ linear

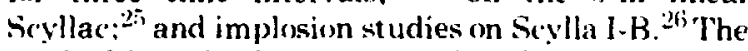
method has also been extended to $C_{0} O_{2}$ pulsed laser light, ${ }^{2 i}$ and recently to HF laser radiation ot $3.39 \mu$. which permits side-on density profile measurements in Scyllac. ${ }^{28}$

In recent years Thomson scattering has become one of the favored plasma diagnostic schemes because of its highly localized and fundamental nature. On Scylla III the cooperative scattering phenomena were studied:29,30 $90^{\circ}$ Thomson scattering was used on the linear 5-m Scyllac: ${ }^{25}$ the electron temperature was measured with a unique technique on the Scyllac toroidal experiment; ${ }^{14.31}$ and on $\mathrm{ZT}-1$ the electron temperature was measured. ${ }^{32}$ 


\subsubsection{Importance of Diagnostics}

In the thinking and planning for this experiment it was clear that elaborate diagnostics would be needed. This need comes from the level of uncertainty in predicting the results, and a realization that only by careful instrumentation and observation can one effectively identify the inevitable problems.

The proper role of diagnosticians is to test hypotheses advanced to predict plasma behavior. Experience shows that first interpretations can change completely when sufficient data are examined. Since much time and expense are involved in modifying a device the size of SFTR, careful diagnostics greatly facilitate the process of defining problems and seeking their solutions. In so doing, the effort and expense of thorough instrumentation are more than repaid.

\subsubsection{Increased Role of Automatic Data Processing and Its Significance}

In recent vears. many of the improvements in diagnostic methods consist of better dat a acquisition and analysis by the use of computers. Previously, the st andard approach for taking data had been to use oscilloscopes with cameras and Polaroid film. As soon as fast (submicrosecond intervals) analog-to. digital converiers became commercially available, it was possible to acquire data and transfer them to a small dedicated computer immediately (a second or less) after an experimental discharge. The im. plications of this technique are many and farreaching. The computer can be programmed to display results in an intelligible manner. Data analyses can range from simple tasks such as scaling to more substantial feats such as curve fitting.

Consider, for example, the data obtained from a coupled-cavity interferoneter. On an oscilloscope, one sees essentially a sine wave of voltage representing the optical path length vs time. To express the signal as plasma density vs time, one compares the phase timing with and without plasma. The difference is proportional to plasma derisity and is virtually impossible to see by simply looking at an oscilloscope trace, but it does show up when digitally recorded data are put through a straightforward computer operation. After every discharge, one immediately sees the plasma density at the preionization tirne and after main bank time. Any change from the expected values is immediately noted and can be investigated. In the older mode of taking data, it might happen, for example, that abrupt changes in preionization conditions would go unnoticed since analysis was time-consuming and attention was directed elsewhere. Clearly, it is possible to waste effort, lose valuable operating time, and temporarily misinterpret results while certain dat: await analysis. Such problems are largely avoided by automatic data acquisition and on-line computing.

Table 5.7-1 lists some comparisons between conventional and computer-oriented data acquisition. Overall, the automated data acquisition provides a more organized and fail-safe system with sophisticated feedback to the experimenter, permitting him to direct the experiment more intelligently.

Under development at the present time is automated digitization of cptical information such as streak-camera results, Thomson scattering, interferograms, etc. The tremendous quantity of information in s'dch two-dimensional displays of intensity have heretofore been largely unused because of practical constraints. By the use of dedicated minicomputers and modern television tube technology, this situation should change dramatically.

The present revolution in technique is fortunate. even essential, for a large device such as the SFTR. Conventional methods would be difficult if not impossible to extrapolate to such a large experiment with so many channels of information. Consequently all planning for diagnostics assumes the use of digital acquisition and computer control.

\subsubsection{Basic Measurements and Equipment Re- quired}

5.7.2.1 Plasma Luminosity for Profile and Location of Density

A hot deuterium or D-T plasma (Te $z 100 \mathrm{eV}$ ) emits radiation in the visible portion of the spectrum, which for the most part is free of lines and varies as $1 / \lambda^{2}$. The intensity can be estimated as that due to bremsst rahlung although small amounts of impurities inevitably increase the level. The estimate for a deuterium plasma integrated from 4000 to $7000 \AA$ is ${ }^{33}$

$$
P_{\text {Brem }}=6.4 \times 10^{-34} \frac{n_{e}^{2}}{T_{e}^{1 / 2}}\left(\mathrm{~W} / \mathrm{cm}^{3}\right)
$$

$\left(\mathrm{n}_{\mathrm{e}}, \mathrm{cm}^{-3} ; \mathrm{T}_{\mathrm{e}}, \mathrm{keV}\right)$. The relatively stronger dependence of emitted power upon density than upon temperature is the basis for using visible light to determine density profiles.

In the SFTR experiment. we expect substantially lower density and higher temperatures than in 
TABLE : 3.7-1

\section{COMPARISON OF DIAGNOSTIC PROCEDERES LSING CONVENTIONAI. MLANA (WITHOUT COMPUTER) AND MODERN MEANS (WITH (OMPI"TEK)}

\author{
Step in Diagnostics \\ 1. Prepare instruments \\ for dat a collection
}

2. Calibrate response

3. Record data with plasma

4. Make preliminary check of proper recording after each discharge

5. Recheck calibrations

6. Analvze clata

7. Re-analyze or further analyze in light of questions raised by study of the results

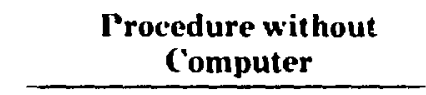

(ienerally easier because interfacing with an oncilloscope is easy.

Done on occasion depending upon attention of experimenter.

I lse oscillowcope, Accuracy is fair.

Look at photographs of oscilloscope traces.

Done according to need as determined by experience.

Vormally done one of more days after the event.

Often requires returning to original data, Process is usually tedious.
Procedure with Computer

Generally hadder but improvenents are constantly being made in $A / J)$ equipment. see boloss.

Typically dome on every discharge by computer control. May require complicat ions in the instrument ation.

L'se analow-to-digital device. Accuracy can vary' 10.suit the experiment and be better than ascillesicopes: if needed.

Program computel 10 portorm tests and display information uguide the experimenter.

Done either on every shot or intermittently with the aid of the computer.

Done within seconds or minutes and displaved.

Original dat a is archived and analssis can be performed by programming. previous experiments, but the level of intensity is still readily detectable. Section 5.7.4 gives some typical numbers based on the above formula. Image intensifiers are required in front of conventional streak cameras to record the expected level of luminosity. Furthermore, the time required to obtain enough photoelectrons to form an image of the plasma will limi: the timing resolution to about $1 \mu \mathrm{s}$ unless the plasma is much brighter because of im. purities.

At present, an image-converter streak camera with intensifier and digital readout is under develop- ment. Such a device satisfies all the requirements of luminosity measurements in the SFTR. It offers the same type of quantitative information as the present luminosity apparatus (using fiber optics, photomultipliers, and analog-to-digital converters on each photomultiplier output). The new approach is less bulky, less expensive, and potentially more accurate because of greater spatial resolution.

We therefore propose to use such cameras at a variety of locations around the torus to observe the time history of the density profile. Any variations in the equilibrium around the torus will be detectable. 
Instabilities of any (ype alferling the densily profile and its evolution in time will also be detectable. Just as conventional st reak cameras have been so useful heretofore, plasma luminosity is expected to be a basic diagnost ic in the SFIR.

special purpose instruments also can be readily designed. Position detectors for feedback and a framing canera to observe many slits simultaneousIf for mode structure have been used in the past. Because of the large time seale involved, we plan to develop a special television camera with instant replay features for qualitative observations. A single line of 'T' intormation can be seanned in ill $\mu s$. If that line is parallel to the image of plasma through a slit, the resulting video signal stored with a recorder contains the same informations as a conventional st reak camera would, except that the time resolution is limited to the $5(1) \mu$ s framing time. NearIf standard video recorders can be used in this manner as inexpensive monitors of luminosity time history.

\subsubsection{Neutron Detectors for Ion Temperature}

'The neutron flux will, of course. depend upon whether or not the filling gas includes tritium. In ejther case. the expected temperature and density: are such that the neutron flux will be substantially higher than in previous experiments.

In many experiments the temperature derived from neutrons has been suspect because the time scale of the experiment did not appreciably exceed the "self-collision time" as given by Spitzer. ${ }^{34}$ Some typical values are given in Table 5.7-2.

In the SFTR, the time scale will be sufficiently long that the ion velocity distribution should be truly Maxwellian; thus, the neutron emission rate should provide a reasonable measure of temperarure. The reaction rates and cross sections are given by Glasstone and Lovberg ${ }^{35}$

$$
\begin{aligned}
& R=1 / 4 \mathrm{n}^{2}(\overline{\sigma v}) \mathrm{DD} \\
& \text { (neutrons emitted } / \mathrm{cn}^{3}-s \text { frola } \mathrm{D}-\mathrm{D} \text { ), }
\end{aligned}
$$

or

$$
\begin{aligned}
& \mathrm{R}=\mathrm{n}_{\mathrm{D}} \mathrm{n}_{\mathrm{T}}(\overline{\mathrm{OV}})_{\mathrm{DT}} \\
& \text { (neutrons emitted } / \mathrm{cm}^{3}-\mathrm{s} \text { from } \mathrm{D}-\mathrm{T} \text { ), }
\end{aligned}
$$

where
TABBSE $5.7-2$

\section{ION-ION SELF-COLLISION TIME FOR DEITERONS}

$$
\begin{aligned}
& \text { Temperature } \\
& \begin{array}{ccc}
\begin{array}{c}
\text { (keV) } \\
1
\end{array} & \frac{10^{13}\left(\mathrm{~m}^{-3}\right.}{10^{16}\left(\mathrm{~m}^{-3}\right.} \\
+ & \delta(1) \mu \mathrm{s} & 5 \mu \mathrm{s} \\
f(1) \mu \mathrm{s} & 4(1) \mathrm{s}
\end{array} \\
& (\overline{\sigma v})_{D D}=\frac{2.33 \times 10^{-14}}{T^{2 / 3}} \\
& . \exp -\frac{18.76}{T^{1 / 3}} \mathrm{cr}^{3} / \mathrm{s}, \\
& \overline{(\sigma v}_{\mathrm{DT}}=\frac{3.68 \times 3.0^{-12}}{\mathrm{~T}^{2 / 3}} \\
& \cdot \exp -\frac{19.94}{T^{i / 3}} \mathrm{~cm}^{3} / \mathrm{s} \text {, }
\end{aligned}
$$

with $\mathrm{T}$ in $\mathrm{keV}$.

The temperature dependence is very sirong: at 4 $\mathrm{keV}$ the neutron flux is 400 times greater than at 1 keV. Furthermore, the D.T cross section is approximately 75 times larger than the D-D cross section. Thus, we expect SFTR neutron fluxes to be $10^{2}$ to $10^{4}$ times larger than in previous experiments with time integrated yields $10^{8}$ times larger because of the longer time scale.

The experimental apparatus for this diagnostic consists of scintillators and photomultipliers to determine the time history of the neutron emission and silver foils with counters to determine activation and thus total yield.

Spatial resolution, although clearly limited, is not as irad as one might think. Since the solid angle of neut ron collection falls as $1 / r^{2}$, the effective length of plasma column providing the signal is only a few times greater than the distance from the detector to the plasma axis. For example, a detector $1 \mathrm{~m}$ from the axis receives $99.9 \%$; of its signal from $3 \mathrm{~m}$ of length along the column.

\subsubsection{Interferometry for Plasma Density}

The basic interferometry method for plasma density measurement consists of measuring the change 
in optical path length caused by the presence of plasma electrons. Taking $p$ as the number of fringe shifts due to plasma (i.e.. phase shift of light divided by $2 \pi), n$. as the electron density along a ray of light $\left(\mathrm{c} \mathrm{m}^{-3}\right)$, we have 36

$$
p=4.5 \times 10^{-1.8} \lambda \int n_{e} d \ell \text { (friinges) }
$$

where $\lambda$ is in microns and length is in centimeters. For example.

$$
\begin{aligned}
\int n_{e} d l & =\left(2 \times 10^{15} \mathrm{~cm}^{-3}\right) \times(6 \mathrm{~cm}) \\
& =1.2 \times 10^{16} \mathrm{~cm}^{-2}, \\
\lambda & =3.4 \mathrm{\mu}, \\
p & =0.18 \text { fringes. }
\end{aligned}
$$

This may be too small for a holographic interferometer using HF laser light, but it is an arequate signal level for a coupled-cavity interferometer or similar device. It is reasonable to seek $1 / 100$ fringe resolution using a $\mathrm{He}-\mathrm{Ne}$ coupled-cavity interierometer operating at $3.39 \mu$. Normally one measures $\int n_{e} d t$ as a function of time along a single line, e.g.. a chord through the center of the discharge tube. However. techniques are being developed to permit more than one chord to be scanned simultaneously, thus permitting density profiles to be measured.

\subsubsection{Light Scattering for Temperature and Density Profile}

Present techniques of Thomson scattering used on Scyllac should be directly applicable to SFTR. The scattered intensity for Thomson scattering is well known: at $90^{\circ}$, using a ruby laser, we have ${ }^{37}$

$$
\begin{gathered}
P_{s} d \lambda d \Omega d V=4.48 \times 10^{-26} P_{i} n_{e} \\
. \frac{e^{-\left(\Delta \lambda / \lambda_{0}\right)^{2}}}{\lambda_{0}} d \lambda d \Omega d V \text { (w) }
\end{gathered}
$$

where

$$
\begin{aligned}
& P_{s}=\text { scattered power }\left(W / \AA-s t e r-\mathrm{cm}^{3}\right) \\
& P_{i}=\text { incident power }\left(W / \mathrm{cm}^{2}\right) \\
& n_{e}=\text { electron density }\left(\mathrm{cm}^{-3}\right) \\
& \lambda_{1}=19.4 \sqrt{T_{e}}(\AA) \text { with } T_{e} \text { in electron volts. } \\
& \lambda \lambda=\text { wavelength shift from } 6943 \AA .
\end{aligned}
$$

In Sec. 5.7.5 we show that the intensity of the scattered light can be measured using reasimable parameters. By resolving the scattered light in space as well as wavelength, we obtain from a single laser pulse the density profile from the intensity of scattered light as well as the electron temperaiure profile from the spect ral distribution of the scat!ered light. This is indeed a powerful diagnostic.

In principle, the density measurement can be calibrated absolutely by using Rayleigh scattering. but for the calibration one needs a nuch smaller level of stray light than is necessary to measure the spectrally shifted Thomson scat tered light. By combining the interferometry data for absolute density with Thomson scattering for relative density. Rayleigh scattering calibrations are unnecessary.

For the time scale of SFTR, the electron and ion temperatures will be nearly equal if energy losses and energy exchange between the two species are classical. Ion temperature measurements using neutrons, excluded flux measurements, and electron temperature measurements will permit a study of the actual energy confinement.

\subsubsection{Excluded Flux Loops}

The significant feature of excluded flux measurements in SFTR is the very large signal available compared with standard theta pinches. Because of the large plasma radius, the excluded flux will be on the order of 10 '; of the flux through a loop around the plasma, rather than $1 \%$; as in present-day experiments. For this reason, the measurements should be significantly more accurate.

By combining excluded flux with an independent estimate of the relative pressure profile, the aboslute level of plasma pressure, or beta, can be determined. The relative pressure profile can be determined from luminosity as a function of time and checked by Thomson scattering at one instant of time. Plasma beta is an important parameter in all theories of equilibrium and stability.

\subsubsection{Diagnostic Apparatus and Cost}

Two levels of resolution are required in SFTR. Important phenomena surely occur within the basic helical wavelength, and these require a concentration of diagnostics within a single wavelength. Other variations can be expected over many wavelengths and thus require diagnostics periodically placed around the torus. The diagnostic apparatus described in Table $5.7-3$ assumes that two distinct regions will be instrumented in as detailed a way as possible. 
TABL.F 5.7-:3

\section{I.IST OF DIACNOSTIC'S AND REQLIRED EQLIPMENT}

\begin{tabular}{|c|c|}
\hline Diagnosties & Required \\
\hline $\begin{array}{l}\text { 1)ierital streak } \\
\text { ammera }\end{array}$ & $\begin{array}{l}\text { Image-inte } \\
\text { convention } \\
\text { camera. dig } \\
\text { systems. m }\end{array}$ \\
\hline $\begin{array}{l}\text { Plisma luminemity } \\
\text { l'lmonitor }\end{array}$ & $\begin{array}{l}\text { I.L.TY cam } \\
\text { recerder. di } \\
\text { system. }\end{array}$ \\
\hline $\begin{array}{l}\text { Neut ron scintil- } \\
\text { lattors }\end{array}$ & $\begin{array}{l}\text { Sointillator } \\
\text { multiplier. } \\
\text { comverter. }\end{array}$ \\
\hline Neut ron counters & $\begin{array}{l}\text { Silver foil. } \\
\text { rube, count } \\
\text { digital out }\end{array}$ \\
\hline $\begin{array}{l}\text { Coupled-cavity } \\
\text { interferometer }\end{array}$ & $\begin{array}{l}\text { He-Ne lased } \\
\text { detedtor, fa } \\
\text { converter. }\end{array}$ \\
\hline Thumson scattering & $\begin{array}{l}\text { Ruby laser. } \\
\text { 3-prating pr } \\
\text { meter. imag } \\
\text { sifier, digit: } \\
\text { systen. mit }\end{array}$ \\
\hline Fxoluded flux & $\begin{array}{l}\text { Loops, proh } \\
\text { converter. }\end{array}$ \\
\hline \multicolumn{2}{|c|}{$\begin{array}{l}\text { emainder of the torus will be monitored at } \\
\text { rvals using relatively less expensive } \\
\text { on to equipment costs, there is a substan- } \\
\text { pment cost for a large and sophisticated } \\
\text { proximately } 20 \text { man-years would be need- } \\
\text { ing and calibration of prototypes. Tire } \\
\text { or instrumentation of } \$ F T R \text { is, therefore, } \\
\text { to be } \$ 3.21 \mathrm{M} \text {. In addition, an estimated } \\
\text { ld be used for advanced development (see } \\
\text { he total cost for diagnostics is estimated } \\
M \text {. }\end{array}$} \\
\hline
\end{tabular}

\begin{tabular}{|c|c|}
\hline $\begin{array}{l}\text { Systems } \\
\text { Seeded }\end{array}$ & $\begin{array}{l}\text { Fistimated } \\
\text { Cost }\end{array}$ \\
\hline 4 & $\therefore 4 x$ T11K \\
\hline
\end{tabular}

8II

and $\mathrm{xij}$

$8(1$

(i) $\times 1.0 \%$

$\underline{\mathbf{u}} \mathbf{1}$

-11 $\times 11 . i k$

4

$4 \times 2016$

4

$4 \times 11116$

Total $\quad \frac{811 \times 2 \mathrm{~K}}{\$ 2210 \mathrm{~K}}$

while the remainder of the torus will be monitored at equal intervals using relatively less expensive diagnostics.

In addition to equipment costs, there is a substantial development cost for a large and sophisticated system. Approximately 20 man-years would be needed for testing and calibration of prototypes. The total cost for instrumentation of SFTR is, therefore, estimated to be $\$ 3.21 \mathrm{M}$. In addition, an estimated $\$ 1.0 \mathrm{M}$ would be used for advanced development (see below) so the total cost for diagnostics is estimated to be $\$ 4.2 \mathrm{M}$.

\subsubsection{Advanced Techniques for Development}

\subsubsection{Light Scattering at 10.2 Microns}

Light scattering measurements in the cooperative regime (Salpeter parameter $\alpha \equiv\left(\Delta \mathrm{k} \lambda_{D}\right)^{-1} \approx 1$ ) can be used to measure ion temperature and/or plasma turbulence. For the SFTR parameters, a CO.2laser at $10.6 \mu$ can be used at a scattering angle of about $10^{\circ}$ for such a measurement. The technique has been applied elsewhere and should be applicable to SFTR if windows for the $\mathrm{CO}_{2}$ wavelength are carefully incorporated into the vacuum system. 
The measurement would establish the level of tur. bulence at wavelengths close to the Debye length and might be important to transport processes. In the absence of turbulence. the meastrement world provide a localized determination of ion temperature.

\subsubsection{Determination of Wall Interaction}

A difficult but important question concerns the plasma/gas conditions outside the main column and near the wall. Appropriate diagnostics are unknown hecause little can be predicted about the conditions (1) be found. Spectroscopy: specialized Thomson scattering. wall pressure and temperature sensors, and magnetic field probe measurements come to mind. such measurements will be carefully planned and retined as experience is obtained.

\subsubsection{Neutron Measurements}

The increased thux of neut rons will doubt less allow. a number of refinements in neutron measurements. For example, significant improvements in spatial resolution mas be possible.

An interesting technique for temperature measurements using neutron activation of materials has been discussed at I.ASI by Group T.2. "it The energy spread of emitted neut rons is significantly in. creased by the thermal motion of the interacting ions. I sing the "Be(n,p) ${ }^{4} \mathrm{Li}$ reaction, which has a threshold of $14.26 \mathrm{MeV}$, it is possible to determine the ion temperature at $5 \mathrm{kel}$ with a stat istical uncertaint $y$ of ' 2 '. This new approach should be tried and may prove very valuable.

\subsubsection{Predicted Bremsstrahlung Level of Luminous Rudiation in SFTR}

Ling Eq. (1) in Sec. 5.7.2.1 we can est imate the effective surface brightness by using expected plasma parameters. Present-day theta pinches radiate 10 to 50 times this estimated level because of small levels of impurities, but we will not include this factor because the higher temperature and longer time scale of SFTR may give different results. Assuming.

$$
\begin{aligned}
\mathrm{n}_{\mathbf{e}} & =2 \times 10^{15} \mathrm{~cm}^{-3}, \\
\mathrm{~T}_{\mathbf{e}} & =4.0 \mathrm{keV} \\
\mathrm{R}_{\text {flasina }} & =4 \mathrm{~cm},
\end{aligned}
$$

we estimate a source bright ness of

$$
\begin{aligned}
& B=\left(P_{\text {Brem }} / 4 \pi\right) \times R_{\text {plasin }}= \\
& 0.4 \mathrm{mth} / \mathrm{cm}^{2}-\text { ster }
\end{aligned}
$$

For example. if one uses an image of the $20-1 \mathrm{~m}$ discharge tube un a $2-c \mathrm{~m}$ photocathorle imannification $=0.1)$. and the lens aperture is $t / 1.6$ if/lti in the plasma), the light tlux $F$ on the cathode is

$$
\begin{aligned}
& \text { Soljd angle }=\frac{\pi}{4 E^{2}}=\frac{\pi}{4(1.6)^{2}}=0.307 \\
& F=B \times \text { solid angle }=0.123 \mathrm{mi} / \mathrm{cm}^{2} .
\end{aligned}
$$

Typical cathodes give $50 \mathrm{~mA} / \mathrm{W}$ so the elec:rom thux from the cathode is $6.1 \mu \mathrm{A} / \mathrm{cm}^{2}$. If we seek spatial resolution of $0.01 \mathrm{~cm}$ on the cathode $10.1 \mathrm{~cm}$ in the plasina). we must detect $0.61 \mathrm{nA}$ per picture element.

This current correspounds to one photoelectron every $0.25 \mathrm{~ns}$. A good image requires about l(H)1 photoelectrons per picture element so the time resolution is limited to about $0.25 \mu$ s. This is not expected to be an important limitation. but working at such a low level of intensity does require more gain than is offered by conventional streak cameras. Various types of image intensifiers are available $(1)$ permit operation at the limit of quantum statistic's so we see no fundamental dificulty in the measurement.

\subsubsection{Predicted Intensity for Thomson Scattering}

Assuming that a (wo-dimensional detector is used to obtain spatial resolution as well as wavelength resolution, we estimate the signal level at the first photocathode of the detector. The intensity of light per unit area may be increased by demagnilication of the image until either the necessary lens aperture becomes impractical or the spatial resolution becomes unacceptable. For this numerical example. we assume collection at $\mathrm{f} / \mathrm{R}$, magnification of $1 / 5$. spatial resolution of $2.5 \mathrm{~mm}$ in the plasma (0).5 $\mathrm{mm}$ on the cathodel, and wavelength resolution of $100 \AA \AA$ (200 $\mathrm{A} / \mathrm{mm}$ linear dispersion on the cathode). We write $\phi$, the flux oil light per unit area on the cathode. as

$$
\phi=g m^{-2} P_{s} d \Omega\left(\frac{d \lambda}{d y}\right)\left(\frac{d V}{d x}\right)
$$


where

$$
\begin{aligned}
& \text { ¿ } \quad=\text { efticiency of optical system. } \\
& \text { m = marnilication from jalasma torathocie. } \\
& \text { ads = linear wablemith dimpersion an } \\
& \text { rathude I Acm) } \\
& \text { dI } d x=A \text {. The area ut the incident laser heam } \\
& \text { 1.m: I. }
\end{aligned}
$$

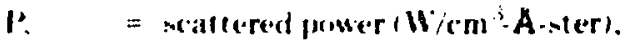

$$
\begin{aligned}
& \text { d!! = solid angie of cullection islert. }
\end{aligned}
$$

Taking

$$
\begin{aligned}
& \text { ISA }=\text { ) } \\
& \text { It. }=11)^{1:}(\mathrm{m}) \\
& 0^{2} \cdot 3 \cdot 0^{2}=1 \% 3 \\
& \text { I. } \quad=4 \text { HKI }+1 \\
& \text { ․ }=12(x) \mathrm{A} \\
& \text { d!! }=0.111: 3: 3 \\
& \text { mi } \quad=0.2 \\
& \text { div/dy }=2(x) A /(\cdot m \\
& \text { H. }=0 .(1)
\end{aligned}
$$

and using the equation in sea. 5.7.3.4. We obsain

$$
\because=11.69 \mathrm{mH} / \mathrm{cm}^{2}
$$

'This assumes that an image of the incident laser heam is as small as the entrance slit of a grating polvehrometer. Assuming we use an si.z) pherocathode with $20 . \mathrm{mA} / \mathrm{K}$ sensitivity. We would expect a 2()$-n s$ laser pulse to give $N$ photofect rons in each resiolution element $(1 / 2 \mathrm{~mm} \times 1 / 2 \mathrm{~mm})$

$$
\begin{aligned}
N & =\left(\frac{0.0 . \mathrm{A} / \mathrm{W}}{1.6 \times 10^{-19} \mathrm{c} / \mathrm{e}}\right) \mathrm{CAt} \\
& =5600 \mathrm{~F} . \mathrm{e} .
\end{aligned}
$$

This signal level is adequate with regard to statistical fluctuations $\left(1^{\prime}\right.$ ') and detectability using image intensitiers.

\section{REFFRENCES (chap. V', Sec, 5.7)}

i. K. Boyer. H. C. Elmore. E. M. Little, W. F. (Quinn. and .1. I. Tuck. Phys. Rev. 189. 831 (1960).

2. E. M. Iittle, W. F. Quinn. and F. I. Ribe. Phys. Fluids 4. 711 (1961).

3. K. Boyer. F. M. Litle, W. E. Quinn, G. A. Sawyer, and T. F. Stratton. Phys. Rev, Lett . 2, 279 (1959).
4. F. ('. Iahoda, E. M. I.ittle. W. F. Quinn, (;. A. Sawyer. and T. F. Siratton. Phys. Rev. 119. 44.3 1) 146ill.

․ A. . Hearden. F. L. Kihe (;. A. Siawyer, and T. F itrat.un. Phys. hes. Itett. 6, 25:- (19til)

fi. (i. A. Sawver, A. J. Bearderi. I. Henins, F. (' Iahoda, and F. I. Ribe. Mhws. Rev 131. 1891 1196i31.

7. F. M. Linle W. F. Quinn. F. L. Kibe and (; A siaver. Nuclear Fusion: 1962 supplement. Part 2. p. $44 \%$

x. H. J. Karr. F. A. Knapp. and J. F. Osher. Phys. Fluid 4. 424 119611.

9. F. C. Jahoda. F. M. Little. W. F. Quinn. F. L. Rihe. and (i. A siawier. I. Appl. Phes. 35. 2:3i] I!s6ith.

III. R. F. Giribble. F. M. Little. R. I. Morse and $\mathbb{W}$. F. Quim, Phes. Fluids 11. 1221 119tix)

11. I). A. Baker. J. F. Hammel, and F. C. Jahoda. Res. Sici. Instrum. 36. 39i 1196i).

12. (i. A Sawyer I. A. Finlayson. F. C. Jahoda. and $k$. s. Thomas, Phys. Fluids 10. 1ifit 11967 .

1:3. k. S. Thomas, Phys. Fluids 11. 1125 1968).

1.1. H. R. Fllis. F. C. Jahoda. R. Kristal. W. E. Quinn. F. L. Ribe (i. A. Sawyer, and R. E. Siemon. Nucl. Fusion 14. 841 11974).

15. P. K. Forman. A. Haberstich. H. J. Karr. J. A. Phillips. and A. E. Schotield. Phys. Fluids 14. 684 11971).

16. H. Herold and F. C. Jahoda. Rev. Sci. Instrum. (4). $14 i)(1969)$.

17. J. F. Hammel. R. W. Peterson, and A. R. Sherwomd. Status Report of the ILASL Controlled Thermonuclear Research Program for a 12-Month Period Finding October 1970. Los Alamos Scient ific Liaboratory repert L.A-4iBSi-MS.

18. P. R. Forman. F. C. Jahoda. and R. W. Peterson. Appl. Opt. 11, 47\% (1972).

19. A. Haberstich and P. R. Forman. Bull. Amer. Phys. Soc. 13. 1489 (1968). 
20. F. ('. Jahoda, K. A. Ifeftries, and (; A. Siawser. Appl. Opties 6. $1+11$ \% $196 \%$ ).

21. T. D. Butler, I. Henins, F. $e^{\circ}$. Inluda, I. Marshall. and K. L. Murse. Phys. Fluids 12. 15k., (1969).

22. R. F. Gribble. H. E. Quinn, and R. E. Siemon, Phys. Fluids 14. 2042 (1971).

23. (' R. Hardér, F. L. Rihe. R. E. Siemon. and I:. S. Thomas, Phys. Rev. Lett. 27. 386 \&1971).

24. K. S. Thomas, C. R. Harder, H. E. Quinn, and R. F. Siemon. Phỳs. Fluids 1\%, 1658 (1972).

25. K. S. Thomas, H. W. Harris, F. C. Jahoda, G. A. Sawyer, and R. E. Siemon. Phys. Fluids 17. 1:314 $1197+1$.

26. K. F. Mckenna, R. Kristal, and K. S. Thomas, Prys. Rev. Lett. 32, 409 (1974).

27. P. R. Forman, S. Humphries. Jr., and R. W. Peterson. Appl. Phys. Lett. 22. 537 (1973).

2R. R. Kristal. Appl. Opt. 14. 628 (1975).

23. M. Daehler and F. L. Ribe, Phys. Rev. 161, 117 $(1967)$.

30. M. Daehler. G. A. Sawyer, and $k$. S. Thomas. Fris's. Fluids 12. 225 (1969).

31. R. E. Siemon. Appl. Opt. 13. 697 (1974).

32. .J. X. DiMarco. I. C. Burkhardı, P. K. Forman, R. B. Howell, and H. .J. Karr. Jr., Bull. Amer. Phys. soc. 18, 13:27 (1973).

33. Glasstone and Lovberg. Controlled Thermonuclear Reactions. D. van Nost rand. Princeton (1960), p. 32.

3-4. L. Spitzer, Physics of Fully Ionized Gasses. (Interscience, NY, 1962) p. 133.

$\therefore$. Glasstone and Lovberg, op. cit., pp. 10-20.

36. F. C. Jahoda and G. A. Sawyer in Methods of Experimental Physics. R. Lovherg. Ed. (Academic Press, New York, 1971), Vol. 9B, p. 1.
37. A. W. Desilva and (i. C. Cioldenbaum. in Methods of Experimental Physics, H. K. Ciriem and R. H. Lwoberg. Eds. Arademic Press, New Fork. 1970) Vol. 9. Part A. Chap. 3.

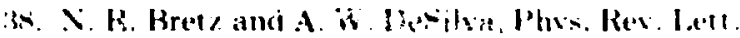
32. $138(195+1$

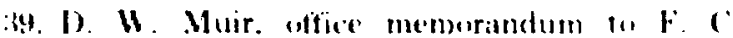
labodas. Mar. 4. 1974.

\section{5. $\times$ CONTKÖ.}

\section{K.I Introduction}

The purpose of the control s-sotem is to provide tor the proper operational sequencing of the various. systems within the SFIR. as well as 11 monitor for improper operation of the sistem and to implement a safe shutdown procedure when necessalry. The control system could be a completely analing sistem emploving relay operation. meter realdouts. signal lamps, and audible alamis with provisions for operator interactions al each siep similar it early Scyllac operation. Comversels, it could be completely computer-controlled, with dat a being recorded and stored in menory, and requiring noopesator action except to initiate the operation. Finalls, the control system could consist of some combination of these Iwo ext remes, similar wo presens iscrllac nperation. The advantage of the analog system is its apparent simplicity and reduced cost. However. such a system is intlexible, slow, and requires much effort on the part of the oporater. thus easily leading to operator error. In addition, such a sistem in an experimental machine sush as the SFIR will undoubtedly require many changes and modifications to accommodate an evolving experimental physies program and thus what was intended to be a simple inexpensive system quickly becomes a complicated costly swstem requiring much effort to document and maintain.

The advantage of the computer-based system is the complete automation of all actions with the greatly reduced probability of error and the high degree of flexibility inherent in a computer-based system. The disadvantage is the effort required in precisely defining all control functions. in the programming of the computer. the documentation. and maintenance effort required. It is sometimes argued that the lack of operator interaction in an experimental procedure results in a loss of insight in 
the process: however. similar arguments can be made for the increases of insight gained through empliving computer control. A combination analog and digital crint rol system could also be designed to have stome of the advantages of hoth types, but such a syst :-n wuld also have some of the disadvaniages. The present sicyllac cont rol system follows the latter approach.

A machine as large as the SFTK will require the monitoring and accumulation of data from thousands of sensors. The assimilation of these data by an cperator on the lime scale required to take appropriate action in an emergency procedure is beyond the capability of one or even a battery of "perators. Computer operation wiil therefore be a necessity for many of the functions, and the advan. tage's of a complotoly computerized syscem appear to out weigh the disadvantage.s. Thus, we opt for a completely computerized system with the capability of strong operator interaction through the computer during nuemal uperation of the experiment.

The control systeril will be designed as a disirihuted multicomputer network. A multicomputer design will be the most flexible and can easily accommodate future design changes and moditicatuons. The svitem will provide for a rapid data reduction with graphic display to enable the "1)erator (1) study the data between each discharge. Because of the high speed and precise timing requirements. the spark gap trigger system will be an analug system as discussed in Sec. 5.3.5.1. with the initiation signal being provided by the computer. The safety interlock system will also be essentially analog te.g., interlock switches, electrical lockouts), but will be monitored by the computer so that the machine operatur can quickly Jocate the cause of any operational shutdown.

The control system will be designed to allow the systematic testing of the various subcomponents of the machine to aid in the location of malfunctioning components. This will decrease the time required to locate and repair a malfunction and thus increase the overall availability of the machine.

\subsubsection{System Design}

The hasic design of the control system consists of a main control computer, which is interconnected via a com munication bus to several system computers. The sustem computers provide the detailed control funct ions with the master computer handling system timing functions. monitoring for and interrupting on fault conditions, and determining the status of the system. The main control computer will be located in the cont rol room, with the subsystem computers typically located is shielded areas near the system being cont rolled.

The major systems in the machine to be controlled or motititured are:

- Vacuum and Tritium Handling System

- METS Refrigeration System (Phase I!)

- METS Energy Transfer System (Phase II)

- Compression Bank Energy Transfer System (Phase I)

- Implosion-Heating System

- Preionization System

- Precision Timing System

- Diagnostics System

- Safety Interlock System

- Display Cumputer

Various subsystems can be controlled independently. e.g., the compression bank charge subsystem or the pulse charge subsystem of the implosion-heating system: however. these stand-alone subsisterns will be categorized with the major systems with which they are associated. The degree of control or monitoring that a system requires will vary significantly throughout the machine and therefore the size and complexity of the associated computers will also vary. For example, the preionization system computer will only be required to charge the PI banks and monitor for malfunctions in 6.10 capacitor and start gaps. whereas the compression bank computer must control the charging of 19200 capacitors from 8 power supplies and monitor the aperation of 4800 start switches and 1280 crowbar switches. The PI computer will therefore be much smaller than the compression bank computer and may. in fact, be combined with one of the other system computers.

Figure 5.8-1 shows the various interconnections in the control system. An important consideration in this type of design is to define carefully the interface standards botween the various subcomponents. $T^{\prime} H_{1}$ is includes the interface standard between the main control computer and various system control computers, as well as the interface between the computers and the machine. The interfacing standard probably will be the CAMAC standard now in existence. Use of the CAMAC standard will allow the use of equipment that has already been developed under this standard and will also allow devices developed for this system to be used in other systems. Also, component and software development can proceed independently of the actual computer system to be used.

The CAMAC interface wili be serial for those sections of the machine with many points to monitor and no critical timing requirements. It will be parallel for those areas of control where timing is a problem. In addition, a microprocessor can be embedded in the control system to insure very rapid 


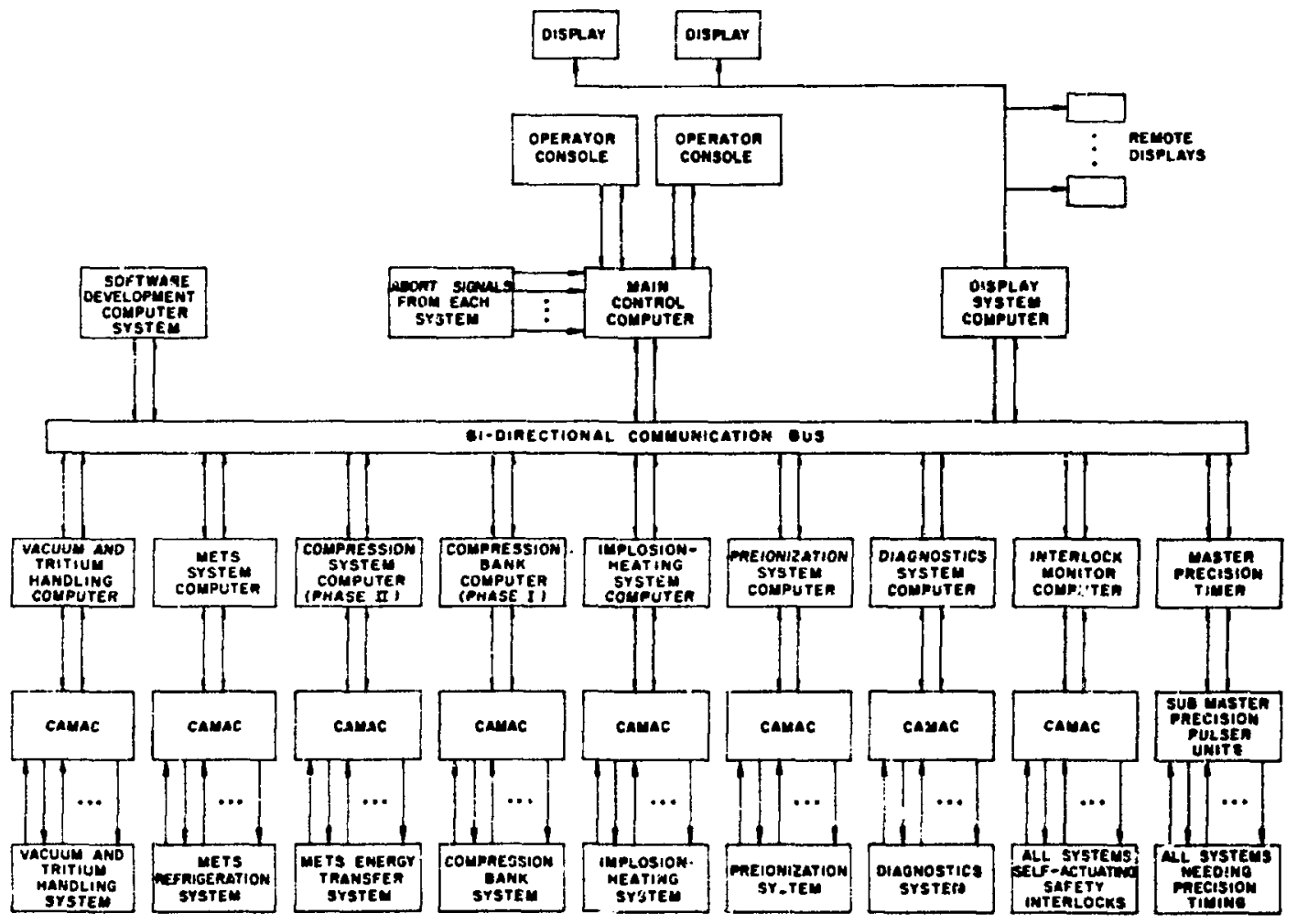

Fig. .5.8.1.

Block diagram of the SFTR control system.

response when needed. For applications such as the trigger systems, where th microprocessor is not fast enough, analog circuitry will be used as indicated by the precision timing system. Analog circuitry will also be used in personnel ariù machine safety applications. This analog circuitry will then be monitored by computer so that the operator will be informed of the cause of any malfunction and can take appropriate action.

It should also be noted that the CAMAC standard is being used on the Scylla IV.P experiment, which will provide a technology base for its use ot. the SFTR.

The requirements and control of the systems within the machine will be discussed in the following sections.

\subsubsection{Tritium Handling and Vacuum System}

The tritium handling and vacuum system control is an integral part of the system itself isee Sec. i.6.41. Thus, the function of the control computer is that of system monitoring, timing, and determination of operating conditions. The normal mode of operation of the system is to evacuate the plasma chamber, introduce the proper mixture and volume of gas. and diagnose the data acquired during the event. These actions will be initiated by the control computer. The progress of the actions will be followed by the control computer and displaved at the control console for the machine operator.

In the event of a failure of a part of the system or an accidental release of tritium, the tritium handling syst em wiil immediately revert to a failure mode of operation to clean up the tritium spill and will signal the main control somputer via a high priority interrupt to go into a failure mode of operation and bring the machine to a nonoperational status. Such a procedure will include ceasing all charging, d:ımping all stored energy, shorting all capacitor banks. and opening the METS energy-storage coils. After the failure mode actions have been taken. the status of the machine will be displayed at the control console.

\subsubsection{METS Refrigeration System}

As with the tritium handling and vacuum system. the METS refrigeration system is an integral part of 
the system it:elf. The primary function of the control computer is system monitoring. The overall purpose of the refrigeration system is to provide and store cruguens for the METS supereonducting coils 1.tec. 5.4.3). The comt rol computer will determine whether or not the system: is operating properly and will display the stat us on the operator console. Fault conditions detected by the control computer will result in failure mode of operation that disarms the machine until the trouble is corrected or the MFTs sistem is locked out.

\section{j.8.2.3 METS Energy Transfer System}

The Mlits energy transier system is completely controlled and monitored by the control computer. The computer will insure that the storage coils are superconducting. cont rol the charging of the storage ails. and initiate the switching of the current from the storage coils into the compression coils. All components in the system will be monitored simult aneously to insure proper operation. The shot will be aborted via a high priority interrupt in the erent of a compoment failure. The normal operating sepuence of the system is described in siec. 5.4.3.1.

\section{X.2.4 Compression Bank System}

The compression bank sistem supplies the energy fir the compression field during !hase 1 of system "peration. as discussed in Sec. 5.4. The cont roi computer performs alt control function as well as system nonitoring. The computer will cont rol the charging of the compression bank. and the initiation of the switching operation. Monitoring for proper operation of the components will be carried out and the shot ahorted in the event of component failure. The "perat ion consists of charging the conıpression bank. which is carried out in 30 s. and which triggers the master ignitron $530 \% \mu$ s before the time required for beat compression tield. The crowbar switch is closed when the field reaches its peak. The compression bunk consists of 20 480 caparitors, 5120) start ignitrons. and $5 ! 20$ (rowbar ignitrons. All of these components will be monitored for proper operation and the machine operator alerted to any malfunctioning component.

\subsubsection{Implosion-Heating System}

The implosion-heating system consists of the implosion-heating capacilor bank as welt as the pulse charge system and the trigger systems for the start gaps and crowbar gaps. The control computer controls the slow charging of the pulse charge system. but the actual triggering of the system must be accomplished with more precision than can be accomplished by the computer. The triggering is carried out by the precision pulser system. The computer will monitor the system for proper operation and will alert the machine operator to any malfunctioning components.

\subsubsection{Preionization System}

The preionization sistem is small and simple. The computer will control the charge cycie and provide component monitoring. Triggering will be accomplished by the precision pulser system.

\subsubsection{Diagnosties System}

The diagnosties system consists of a series of instruments: streak cameras, neutron counters, etc. (see Sec. 5.7.2). The diagnostics system computer will be used to calibrate the diagnostic equipment in addition to its use in acquiring and reducing the data. It will also provide data storage for a few tens of events to aid the experimenter in evaluating the data. The precision timing pulses required by the diagnostic equipment. such as in the Thomson scattering measurement, will be provided by the precision pulser system.

\section{X.2.8 Presision Pulser System}

This system provide; pulses with nanosecond resolution for triggering the implosion-heating system and precise timing of the diagnostics system. The pulser system is cont rolled directly by the main control computer. The main control computer checks that all machine systems are ready and then triggers the precision pulser. which initiates the plasma burn cycle. Intil the precision pulser is triggered. it is possible to abort the shot. but once the pulser is triggered, the shot inust be completed.

\subsubsection{Safety Interlock System}

The satety interlock system provides for personnel and machine safety and can be considered to consist of two parts. The machine is protected by both fast analog protection circuit ry and computer programming. The computer provides the main protection: however. the machine operator can also terminate a shot via the analog circuitry in the event of a dangerous condition.

The personnel safety interlocks must be handled differently-people can walk into dangerous situations at any time even when the machine is turned off: therefore, a fail-safe personnel safety interlock svitem will be installed and monitored. but 
not cont rolled, by the computer. A warning system that consists of horn, bells, and flashing lights to alert personnel of dangerous situations will be part of the safety interlock svstem.

\subsubsection{Basic Computer Design}

\subsubsection{Computer Protection}

The environment in which the computers must function is very harsh and several specifications have been made to give the computer as much protection as possible. First. "clean power" is specified tor the computer and diagnostics, and "dirty power" for the rest of the machine. The clean power is to be split from the dirty power at the 1:3.8. $k V$ transformers and kept separate thereafter. Also, each rack of computer equipment is to be fed from an isolation transformer. Within each rack the power is to be distributed on one side of the rack and logic signals on the other. A double-electrically-isolated shield room is specified to house the computer and data acquisition equipment. In addition. 1:2()-dB power line filters are specified tor each leg of the incoming power. Strict attention will be paid to the grounding system to keep ground-loop problems to a minimum.

The CAMAC equipment will be mounted in racks which will appear as extensions of the outside wall of the shield room, and clean power will be fed through copper pipes from inside the shield room.

In addition. the control signals to the power supplies and other systems will be coupled. using techniques that have proved successful in other LASL, fusion experiments.

\subsubsection{Standard Nomenclature}

Standard systems of nomenclature have proved valuable in the design and maintenance of large systems: therefore, a uniform system of notation to be used throughout the entire design has been defined. This uniform nomenclature is valuable because it enables personnel of diverse backgrounds to communicate. It also aids in teaching the operation of machine to new personnel and the technicians who help run and maintain the system.

The basic idea is to have a uniform nomenclat ure that can communicate the information desired. but that is not so cumbersome as to fall into disuse. Also. it should be versatile enough to be usable. Therefore. the following eight-character standard format was chosen:

$$
\begin{array}{rrrrrrrr}
1 & 2 & 3 & 4 & 5 & 6 & 7 & K \\
V & V & W & W & X & Y & Y & Z
\end{array}
$$

where $V V$ is a two-character abbreviation for the major system. WW is the device name. $X$ is the device type, $Y Y$ are digits to distinguish between multiple units, and $Z$ is the signal type. The abbreviations used to date are shown in Table $5.8-1$. The standard 8-character nomenclature can be preceded by two more characters, making a 10 . character, or larger, name capable of handling multiple identical systems.

A typical power supply cycle will now be described. A preionization primary bank supply will serve as an example. A typical cycle would be:

1. Open the shorting balls.

2. Open the dump Jennings switch.

3. Close the load Jennings switch.

4. Close the ac contactors.

5. Monitor the vollage on the bank.

6. Wait for full charge.

7 . Open the ac contactor.

8. Open the load Jennings.

9. Fire the machine.

10. Close the jump dennings.

11. Drop the shorting balls.

This power suppls. named PlKlistll, is shown in Fig. 5.x-2. The typical operating cycle can now be described using the signal numes that cause the varisus actions to excur. The first signal sent by the computer would be PIBli Aoll which would actuate the air cylinder and open the shorting balls. The computer now rhecks PIBkAOIS to conlirm the desired effect. If the shorting balls fail to open, the computer would now flag the operator and take the proper error exit. If the shorting balls opened. the computer would now send PIBK,J(12D, which would open the dump Jennings switch: the proper sense signal PIBK.J02S would now be tested to confirm that the proper action occurred. As each function is performed. the computer tests the proper input signal to confirm proper action. The remainder of the write-up will ignore the test signals.

PlBk.Joll is now transmitted. connecting the power supply to the load. The load must be con. nected before the ac power energizes the power supply since the supply will overvolt if not connected to a load. Signal PIBKo1P is now sent and the supply begins to charge the Pl bank. The volt age atross the hank is monitored by the computer using signal PIBKM01V, which is an input to an analog-todigital converter. Again, if this signal fails to show the proper behavior the computer can abort the 
TABLE: $5 . X-1$

ABBREVIATIONS USED IN THE SYSTEM OF SITANDARD NOMEN(IATT HE:

A. Column 1 and 2 describe system

\begin{tabular}{|c|c|c|c|}
\hline l'R & primary & Als & air \\
\hline$(A$ & crowbar-A & 10 & vateutum \\
\hline $\mathrm{CH}$ & (ruwhar-H & 11. & interlinek \\
\hline |'| & preiunization & SF & sulfur hexatluuride. \\
\hline III & implosion heating & 10 & (a)mprespiun \\
\hline (id & gas: & |) & diatumenties \\
\hline
\end{tabular}

B. Column 3 and $\$$ for device name

\begin{tabular}{|c|c|c|c|}
\hline BK & bank & $\%$ & 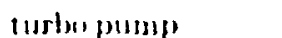 \\
\hline $\mathrm{HF}$ & bulfer & היא & premente semsor \\
\hline (30) & honester & If ; & imn қанце \\
\hline ("I & cable tost & $\mathrm{T}^{\circ}$ & thermocentple. \\
\hline $\mathbf{B I}$ & hials & $M$ & mamual vilve \\
\hline$f^{\circ}$ & pulse charge & HR & horn \\
\hline Tlk & irigger & BI. & betll \\
\hline$(0)$ & crumpressor & $B E$ & beticontil \\
\hline il. & value & $\mathrm{WW}$ & water temperat ure \\
\hline$F V^{\prime}$ & firepump & $\mathrm{WF}$ & water fluw \\
\hline MK & manual regulator & $P()$ & puwerol \\
\hline
\end{tabular}

C. Column i for device type

\begin{tabular}{|c|c|c|c|}
\hline$\therefore$ & supply. & I. & light \\
\hline 11 & divider & .1 & iemings switch \\
\hline (; & gatuge & r & eoritiletor \\
\hline l & protection & B & breaker \\
\hline i & $\begin{array}{l}\text { actuator } \\
\text { momitor }\end{array}$ & WN & warnims \\
\hline
\end{tabular}

D. Columns 6 and 7, 2-digit number that distinguishes between multiple units

F. Column 8 signal type

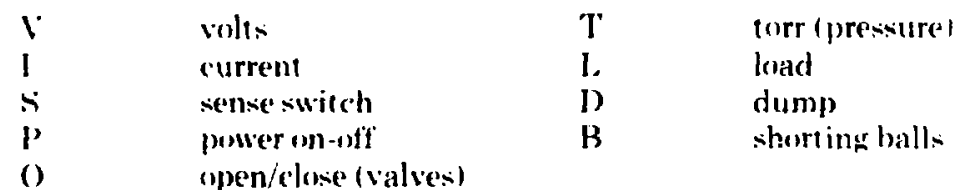

charging of the power supply. When the supply reaches the proper bank voltage. the computer drops signal PIBKCOlP disconnecting the ac power. Next. the computer drops PIBK.J01L disconnecting the load. This is done to protect the power supply from transients when the capacitor bank is dumped into the load coil.

The PI ready signal is now sent to the main control computer, which can then initialize the Fire Pulse if the machine is in an operational condition. If the machine were undergoing testing. the PI bank Fire Pulse could originate from the PI subsystem. If a failure has been detected, the computer can send PIBK.J02D and the energy in the bank will be dissipated in the water resistors. If it is a successful shot. the dump Jennings are closed to drain any remaining charge in the capacitors. In either case. PIBKJI:2D is the next signal to be sent by the computer, then PIBKA01B is dropped, closing the shorting balls, and the cycle is now complete. 


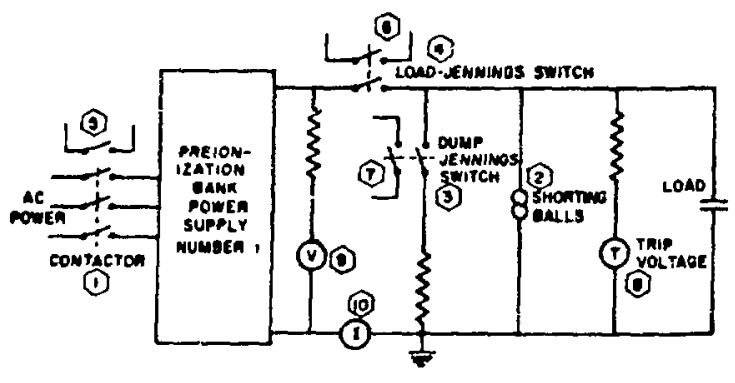

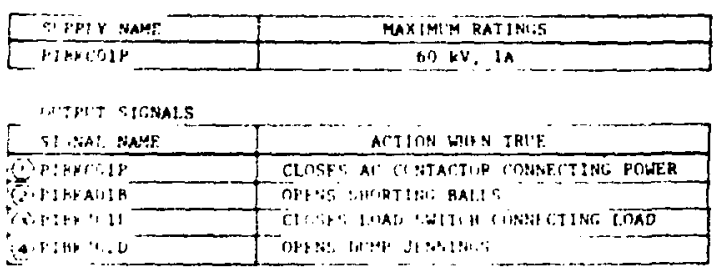

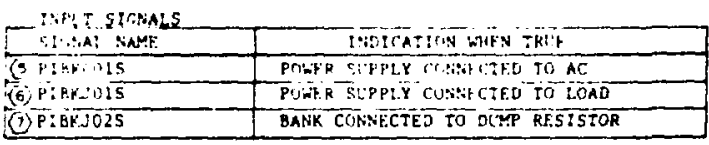

\begin{tabular}{|c|c|c|}
\hline SIGNAL NAKE & SHLNT VALLE & INOICATION \\
\hline (A) PIBKMO14 & 10 vorts & MONITORS BAHK \\
\hline (9) PIBKM $02 \mathrm{~V}$ & 10 VOLIS & MONITORS SUPPLY \\
\hline (id PIBNAO3I & 10 VOLTS & MONITORS SUPPLY CURRENT \\
\hline
\end{tabular}

Fig. 5..4.2.

Typical potcer supply schematic.

\subsubsection{Main Control Computer and Operator Consoles}

The operator consoles will interface to the main control computer through the defined standard interface. This will allow the control console to be treated as just another input/out put device and will allow the machine to begin operation with a very simple control console, which can then be easily replaced in the future by more elegant ones. It also allows the machine to be easily reconfigured by the operating program through redefinition of the input and output signals.

The main control console will communicate with the subsystem computer on two different paths. It will transmst and receive information on the standard communication path in those situations where there is sufficient time. In addition, a fast interrupt svstem will be used to transmit abort signals between the control computers and the various subsystem machines. The control computer should have a vectored interrupt structure to enable it to respond quickly to the abort signals from the subsystem control computers.

It should be stressed that the subsystems will protect themselves, and only after each subsvstem has given an all-is-well signal will the main control svstem start the precision pulser firing system. This will insure that the entire system is vulnerable to a subsystem malfunction for only a minimal time.

\subsubsection{Cathode Ray Tube Display System Computer}

Graphic displays are a powerful means of man. machine communication. Graphics devices. however. cause a heavy load on a cont rol computer. Therefore, to insure the control computer's rapid response to varying situations, the graphics devices will be driven by a separate computer system.

Two types of displays will be used. Live displays, which have selective erase. will be used to :ommunicate with the main control console. This will allow the maximum man-machine communication where it is needed.

Storage tube displays will be scattered throughout the machine and will allow users to interrogate the control computer and view results from many stations throughout the machine with a much smaller amount of overhead than that required by the live displays.

\subsubsection{Precision Pulser (Timing) System}

The timing measurements of the various subsystems vary from tens of minutes to nanoseconds. To accommodate such a wide range of timing requirements, the timing is split into coarse and fine or precision timing systems.

The coarse timing is provided by the main control computer. The main control computer can communicate with the various subsystem control computers and vary the timing to accommodate the various systems.

Once all the subsystems are in a "go" condition. the main computer sends a pulse that triggers the precision pulser system.

The precision pulser system contains a master pulser which is capable of driving several submasters. Both the master and submaster pulsers have variable delays that can be used to synchronize the start pulses. The submaster channels can then be delayed in increments of $10 \mathrm{~ns}$. The output of the submaster is boosted to a $8-\mathrm{kV}$ pulse, which is then used to fire the various systems.

The system submaster pulsers will be cabled radially from the master pulser, centrally located in 
the master control room. to insure simultaneity of the submaster st art pulses. The variable delays built into the submaster units can then be used to control the timing to within $10 \mathrm{~ns}$.

\subsubsection{Software Development Machine}

Since the cost of the software (programming) usually far exceeds the cost of the hardware for a Iypical system, a soft ware development machine will sion pay for itself many times over by reducing the proyram development time for the system. This machine should have all the core storage necessary (1) supjort developmental software. such as editors. high-level languages, and debug programs that aid program riseckout such as trace and patch routines.

This machine should be capable of running in the time-sharing mode with multiple input/output devices such as Tektronix Model 4010 CRT units. Time-sharing will further reduce program development time by allowing many programmers "simultaneous" access to the machine.

The development machine will, in turn, reduce the amount of core storage needed on the worker computers. It will also reduce the need for sophisticated input/output devices on these machines. Figure $5.8-3$ is a schematic of this subsystem.

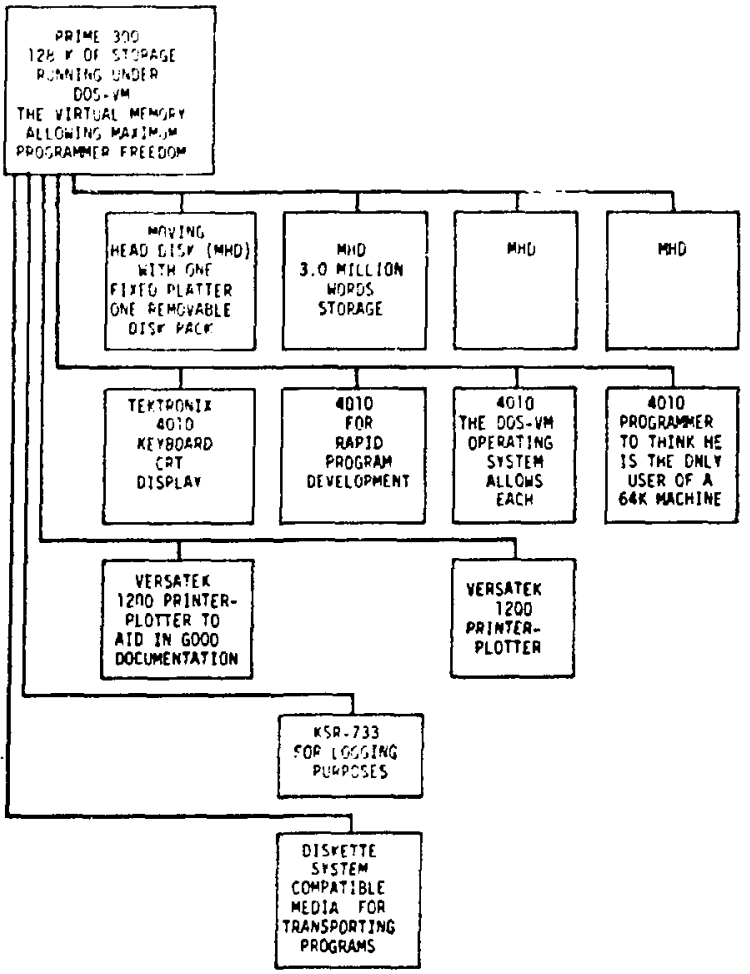

Fig. 5.8-3.

Softu'ure development system. 


\section{FACILITIES}

\subsection{GENERAL DESCRIPTION}

The facilities for the SFTR include not only a large amount or covered area and associated utilities to house the experimental equipment, such as the plasma chamber, trigger systems, and compression system, but also include the construction required for the handling of tritium and activated materials. The major elements making up the facilities are: Concrete Shielding Structure and Remote Hardling Equipment, Tritium Handling Installatior., METS Refrigeration Installation, METS Maintenance Area, Compression System Area, Trigger System Area, Diagnostics and Control Room, Shops Area, Warebouse and Receiving, and Administrative Area.

Several approaches to SFTR structure design were considered. The initial approach placed the entire installation inside a large square structure approximately $120 \mathrm{~m}$ on a side and four stories high; however, it was found that such a structure was larger than needed, and did not lend itself to economical construction techniques so that the cost of the facilities was unduly high. Removing the corner of the structure came close to satisfying the space requirements, but placing al' areas inside the torus presented egress problems for the personnel involvec in the operation and, again, was not well suited for economical construction techniques. In the layout that was finally selected, a toroidalshaped structure is placed over the main experimental equipment, with the support facilities located in inexpensive buildings around the outside of the torus.

The overall facilities layout is illustrated in Fig. 6 1 . with plan views of the various levels shown in Figs. 6-2 through 6-4. The basic approach is to place a main toroidal building around the plasma chamber and shielding structure, the compression system area, and diagnostics area. Room is also provided for the trigger system, with floor space availaple for limited maintenance operations. The diagnostics area is located on the ground floor adjacent to the inside of the shielding structure, and a single-story control room is located at ground level in the center of the building to allow for uniform cable runs between the control room and the torus. A hot cell is located on the basement level inside the torus to handle radioactive components from the plasma chamber. The hot cell is also located adjacent to a main 3-m-wide basement-level passage which runs beneath the shielding structure from the receiving area to two elevators inside the torus so that equipment and material can be moved in and out of the inner area of the torus. Little maintenance will be carriad out inside the torus so that only a limited number of personnel will normally be working in this inner area. Egress from the torus is provided by the $3-\mathrm{m}$-wide main passage plus three additional $1.8-\mathrm{m}$ wide personnel passages running under the shielding structure to the periphery of the main building. The METS refrigeration equiprnent, METS maintenance area, tritium facility, shops and maintenance area, receiving and storage areas, and administrative areas are placed in appropriately sized structures around the outside of the main toroidal building. Such an approach allows a more conventional building design to be followed, thus resulting in lower structural costs.

Table 6-1 lists the square footage associated with each of the major areas within the facility. Experience gained from the present Scyllac facility has been used in determining the proper size of the support areas. In general, a support area for SFTR which is three to four times that of the present Scyllac experiment is felt to be sufficient. Shop support can also be provided as required from the CTR Office Building, located close to the SFTR. The office building will also provide the office space requirements for SFTR personnel.

\subsection{MAIN STRUCTURE}

The main toroidal structure houses not only the plasma chamber and shielding structure, but also the compression system consisting of the 128 METS dewars, the vacuum interrupters and transfer capacitor banks, the trigger system for the implosion-heating system, and the diagnostics area. The building is a 12-sided structure in a toroidal shape. The enclosure is $30 \mathrm{~m}$ wide. $12 \mathrm{~m}$ high, and the periphery of the building is $50.3 \mathrm{~m}$ from the center of the torus. A cross section of the structure is shown in Fig. 6-5. The plasma chamber and shockheating system are located inside a concrete structure which provides a biological shield from neutron radiation and also a secondary containment vessel in 


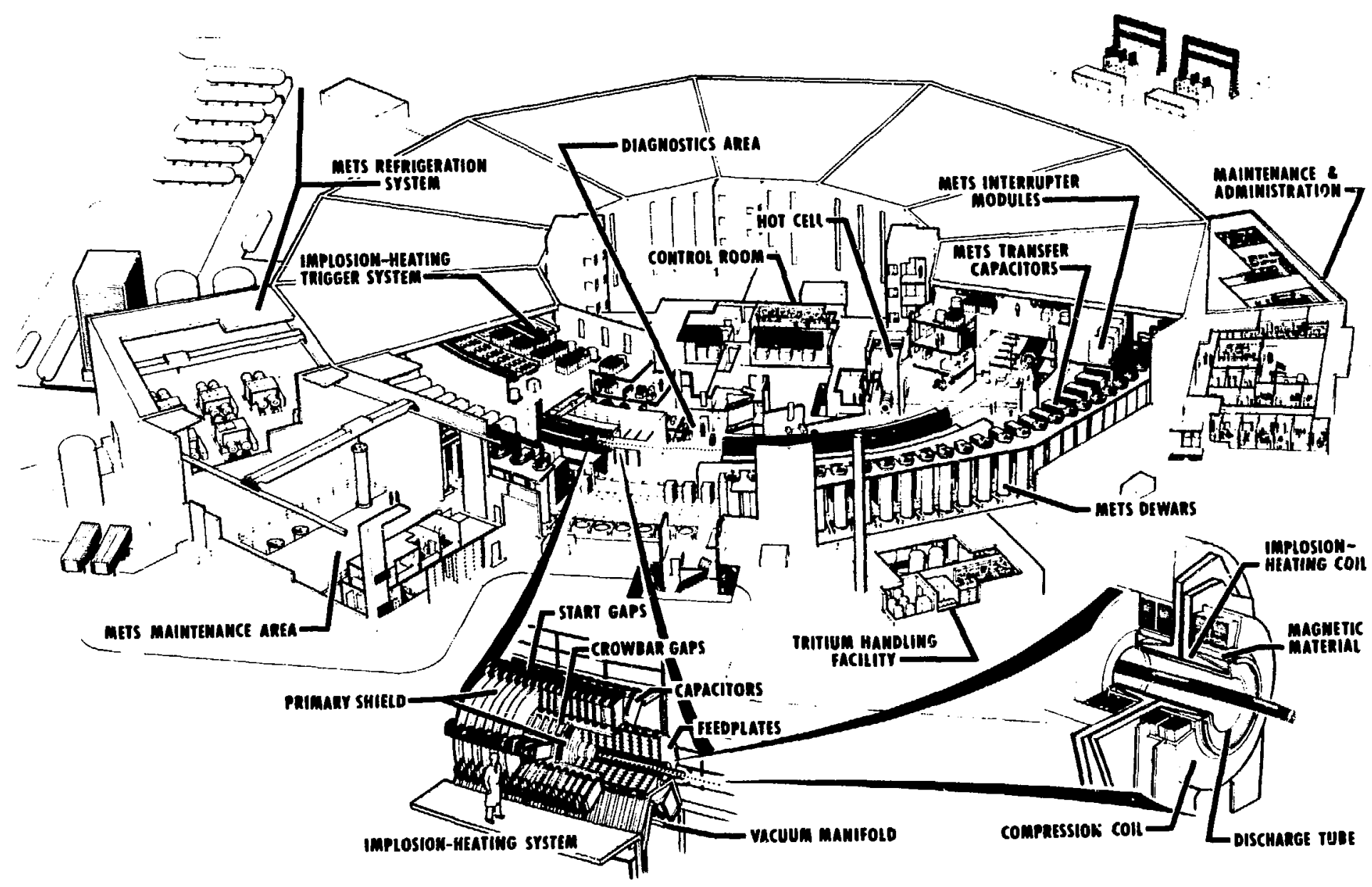

Fig. 6-1. 


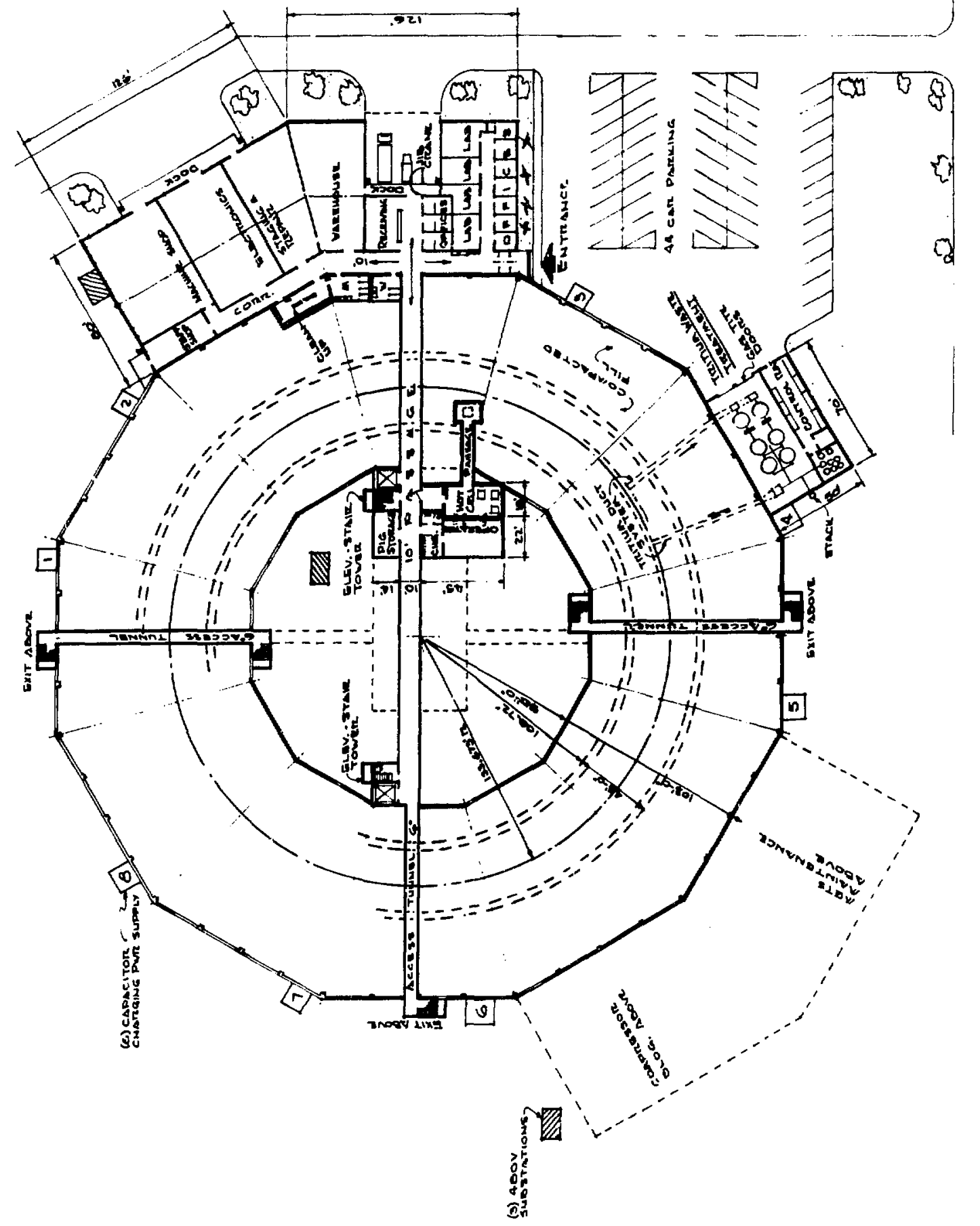

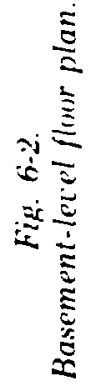



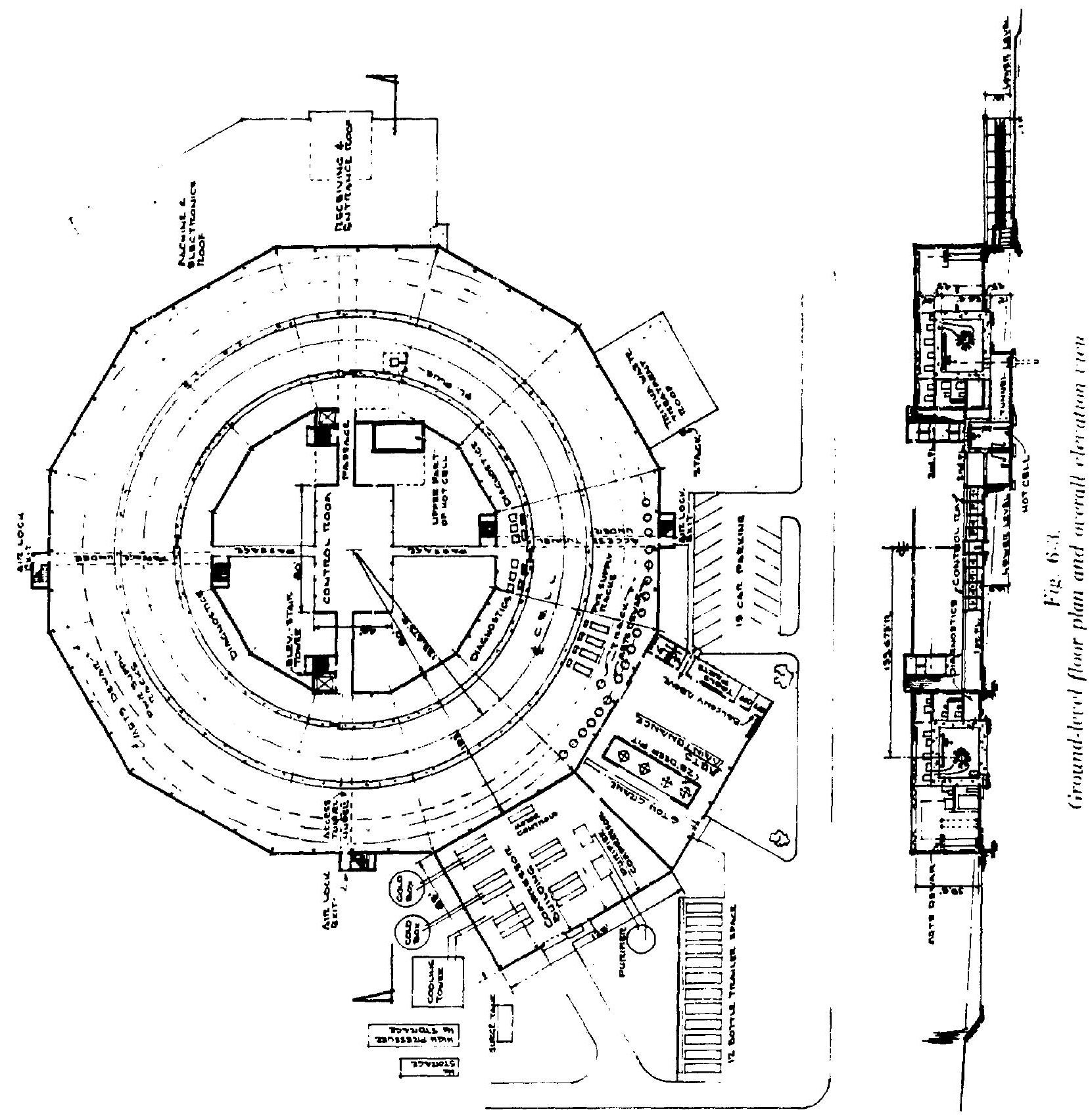


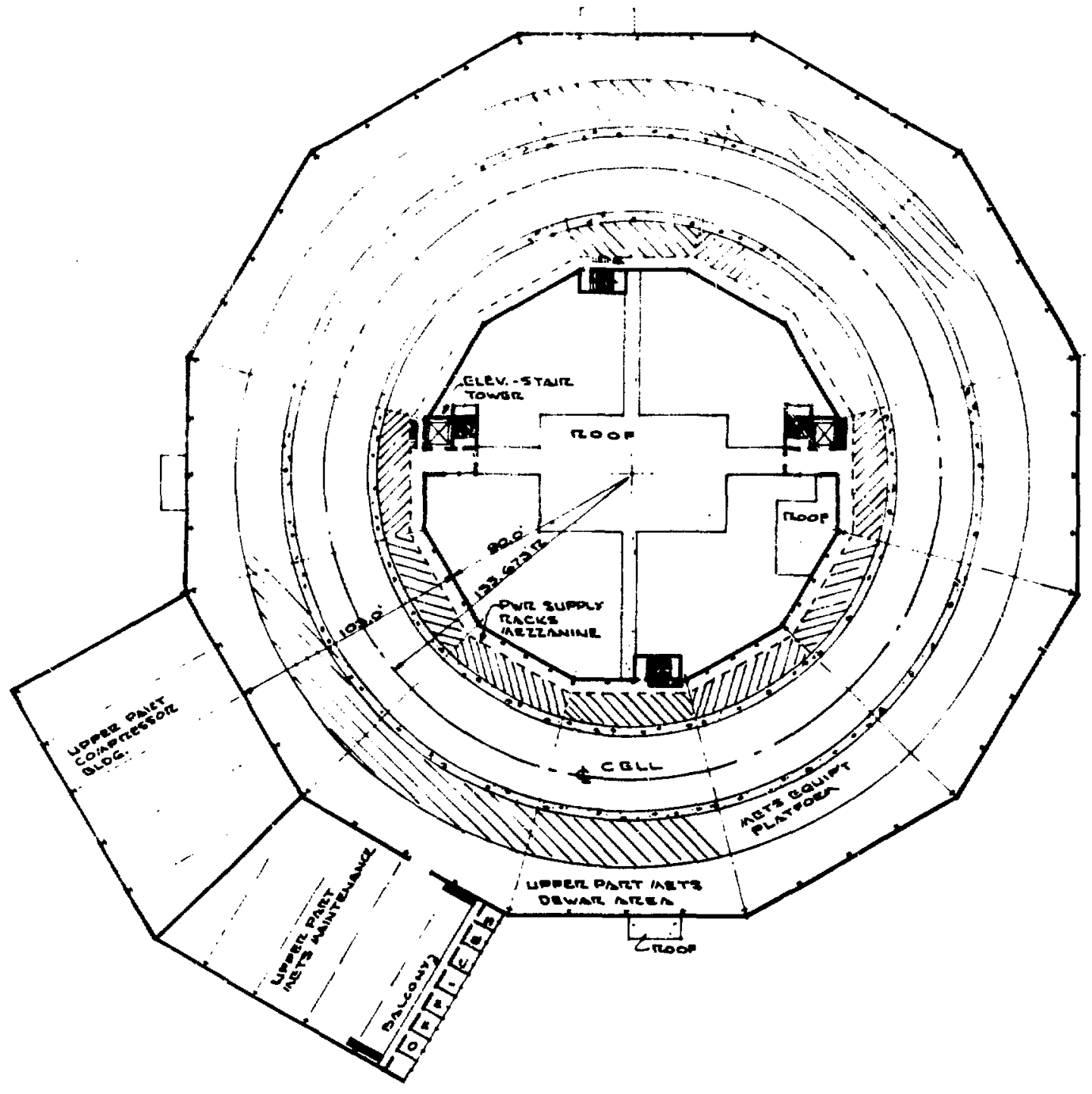

Fig. 6-4.

l'pper-level floor plan.

the event of a tritium spill from the plasma chamber. The wall thickness of the concrete shield is approximately $1.5 \mathrm{~m}$, the inside height is $8 \mathrm{~m}$, and inside width is $10 \mathrm{~m}$. The plasma chamber will be placed on a steel support structure to be erected after the structure has been completed. The submaster gaps for the trigger system will also be located inside the structure. Four overhead cranes, which can be controlled remotely, will provide for initial installation, as well as maintenance during operation. After operation with DT, the plasma chamber will be radioactive, and maintenance functions must be handled remotely. As long as the major shieid is in place, personnel will have access to the inside of the concrete shield structure so that capacitor and spark-gap maintenance can be done without the need for remote handling equipment. In the event that a portion of the plasma chamber must be replaced, the capacitors and gaps will be removed from the module and the cabling and vacuum system disconnected. A remote handling fixture on the crane will permit removal of the main shielding 
TABI.Et t I

\section{SQLARE FOOTAGE OF MAJOR ELEMENTS IN THE SFI'H FACIIITY}

\begin{tabular}{|c|c|}
\hline Main toroida! building & $916(x) \mathrm{ft}^{2}$ \\
\hline Pritium handling & $3 i(H)$ \\
\hline MlÖls refrigerat iun sistem & $9 \delta(H)$ \\
\hline HEOY mainlenance area & $9: 5(n)$ \\
\hline Comprol rumm & 3 रінl \\
\hline Mathine and st aff shey? & $36(k)$ \\
\hline lelectronics maintenance arid repoin & $f \leqq(k)$ \\
\hline Recoiving and watrehsuse & $2 x(k)$ \\
\hline Simall laburatury space & $1 \pi(k)$ \\
\hline Office'spaces & 1 (6) $(1)$ \\
\hline 1. & 1823314 fit \\
\hline
\end{tabular}

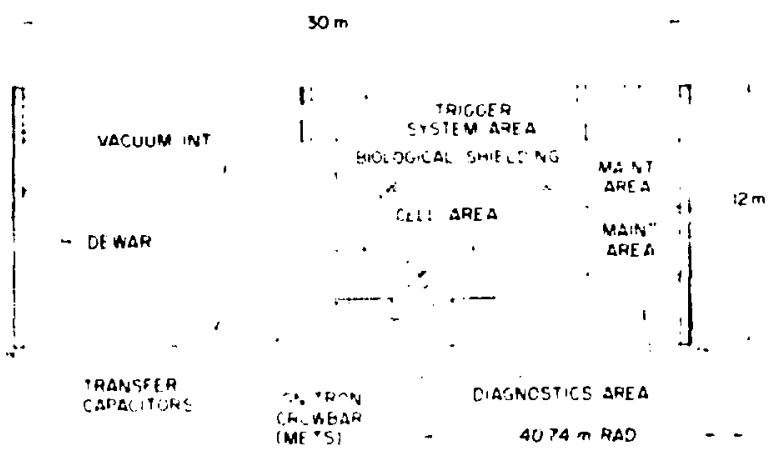

Fig. 6-5.

(rosit section of main toroidal structure.

and the monule section will be unwelded. As many as four $40 . \mathrm{cm}$ modules can be handled as a single unit by the remote handling facilities. Four modules are estimated to weigh 4 tons so that a 5 -ton crane will be adequate. When the module section has been unwelded. the crane will move the module to the hot-cell entrance located in the floor of the concrete shielding structure !see Fig. 6-2). The module will then be placed on a dollv in the hot cell for repair. A repaired module section will be introduced into the shielding structure and welded into the plasma chamber in the same manner.

A large number of openings will be required in the concrete shielding structure. Cable feedthroughs, as shown in Fig. 6-5. will be required for the implosior. heating trigger system cables, the pulse-charge system cables, and the compression system cables. Diagnostic ports through the inside wall will be necessary. Personnel and equipment access will be through four doors, one in each quadsan an the inside wall of the structure. The cables penet rating tis structure will require a $15-\mathrm{cm}^{2}$ conduit every $38 \mathrm{~cm}$ arourd the torus, both in the ceiling and in the bot tom. The cable seal system will be sufficiently tight (1) satisfy the tritium release requirements (see App. II). A $45-\mathrm{cm}$ diagnostic port will be provided every $3.2 \mathrm{~m}$ or ever: wavelength for a total of 80 ports.

Two maintenance levels are shown in Fig. 6-5 above the diagnostics area. Only a verv low level of maintenance activity, requiring a minimum of personnel, will be carried out in these areas. The areas will also be used for $\mathrm{st}$ ging during const ruction and rar provide space for additional experimental equipment.

The trigger sistem must be located as close as possible to the implosion-heating capacitors and gaps in order to reduce cable lengths: thus, the trigger system is placed on top of the shielding st ructurc with cable feedthroughs, as discussed earlier.

The energy-slorage sustem will be adjacent to the outside wali of the shielding structure. with the crowbar ignitrons placed in clusters next to the wall it the cable penetraisins. The capacitor banks wiil be just outside the ignit ron clusters. and the vacuum interrupters will be on a steel rack over the transfer capacitors. The dewars containing the superconducting energy-storage coils will he located adjacent to the outer wall of the building. The piping required for the cryogenic coolants will be on the outside wall. over the dewars. The cabling between the various components of the compression syst em will be run in floor ducts wherever possible. Cranes will not be provided in the compression system area. Instead. hydraulic lifts and dollies will be used for the vacuum interrupters and capacitors, while the ignitrons can be handled directly by personnel. The dewars will be mounted on individual dollies and moved to maintenance facilities on permanent tracks installed in the floor around the torus.

\subsection{METS REFRIGERATION AND MAIN- TENANCE AREA}

METS refrigeration system components. such as the compressors and control panels, requiring protection from the elements are to be placed in a structure approximately $900 \mathrm{~m}^{2}$ and $12 \mathrm{~m}$ high. The METS maintenance area will be in an adjoining structure of similar size. The two structures will be located next to the main toroidal structure. as shown in Fig. 6-3. Those refrigeration system components. such as the gas-storage tanks and cooling tnwer, are 
10 be plated ontside, next to the refrigeration building.

An access way between the MFIS mainia:. once area and the main struture allows a dewar to be mosed on the irack system ints the maintenance irta. The maintenance building will not be tall

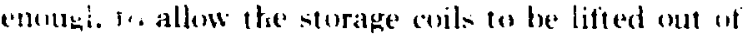
the dewar dire tis. wo the dewar is to be placed in an $\therefore$-m-deep pit to provicte sutticient overhead clearance. The pit will accommote four dewars simultaneousiy. A 6 -tun crane provided in the METS Inain!enance area will handle the dewar:s and storage coils. A small office space and toul rom: lecated in the MFIS maintenance building will have storage space on a baleony ahove the otifice and tul rowin.

\subsection{HOT-CELL TRITIUM FACILITY AND CONTROL. ROOM}

The hot cell is to be located inside the torus at the basement level adjacent to the raain $3-\mathrm{m}$ access corridor. A corridor also leads from the hot cell to a donr in the floer of the conire!e shielding structure, as shown in Fig. 6-2. through which modules of the plasma chamber can pass. The hot cell facilities can handle as many as four $40 \cdot \mathrm{cm}$ modules as a single unit.

The main purpesse of the hot-cell facilities is to handle the defective radioactive components of the plasma chamber which are activated after several operations with DT. The parts to be handled by the hot ceil are the first wall, Marshall coil and feedplates, maynetic materia!, and compression coii. Maintenance will be required if the first wall cracks or if an electrical failure occurs in the Marshall coil or compression coil. The hot cell will have two maintenance positions and one checkout position. Positions for storing up to 10 spare units of 4 modules each are also provided in the hot cell. The hot cell has a total area of approximately $144 \mathrm{~m}^{2}$, not including the ronnecting corridors.

Defective modules will be lowered through the doorway in the concrete shielding structure onto a remote-controlied dolly in the corridor leading to the hot cell. The dolly will then be moved to one of the iwo maintenance positions, where work will be carried out by manipulators. It is envisioned that the maintenance operation will consist of unwelding the components of the module as reauired and replacing the defective components with new components which have been brought in through the main access corridor and introduced into the hot cell. The refurbished group of four modules will then be moved to the rheckont position where the coils will be griven a high voltage test and the vacuum seals tested. Alter successfulty passing the tests. the modules will be placed in the storage racks as spares for hater use. The detective components which were remowed from the modules will be placed in "grigr." which will then be moved by truck to the radiontctive citmo fir dispusal.

The strueture required to house the tritium handling facilits will be adjacent to the outside of the main coroidal structure on the hasement level as shewn in Fig. 6-2. The structure will have dours large enough to permit the processing equipment to be aneved in and out of the building. The dowrs will be gistiphis to allow the structure to serse as a secondary confinithent ressel in the event of a tritium spill. The tritiun, waste ! reat ment facility is to be in an area of approximairly $14+\mathrm{m}^{2}$. A duct system will

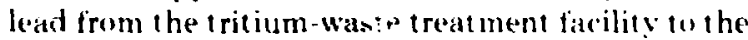
shielding st ructure so that an. spilled tritium can be circulated from the plasma chimber through the treatment facility. A 25 -m st ack wit? vent the output of the facility when the tritium leve! bas reached a saristactorily low level. A $7 \bar{n}-\mathrm{m}^{2}$ contrul som for the tritium facility will be adjacent on the wase treatment facility. A $21-\mathrm{m}^{2}$ tritium storage vatblt for sturing the inventory of gaseous tritium will aiso be in the facility.

The control room is so he a single-story structure with a flour space of approximately $355 \mathrm{~m}^{2}$ on the ground floor at the center of the lorus. as shown in Fig. 6-83. Four covered passiggeways lead from the cont rol room directly into the diagnostics area of the main building. These passageways will provide personnel access from the control room to the diagnostics area and also provide sheltered runs for cable ducts between the control room and the main buildings. The control room will contain the main computer equipment in a sireen room and the main cont rol panel with appropriate display equipment.

\subsection{GENERAL MAINTENANCE AND ADMINI- STRÁTIVE AREAS}

The maintenance activities will require a substantial machine shop and electronics shop, such as shown in Fig. 6-2. A 265. $\mathrm{m}^{2}$ machine shop for production machining johs and a $50-\mathrm{m}^{2}$ staff shop where the CTR staff has access to machine tools are expected to support the normal activities of the facilities. Overload machine work can be handled by the shops in the CTR office building located near the SFTR site or can be done in the main laboratory shops. The electronics shop is housed in a $250-\mathrm{m}^{2}$ 
area where electronic items will be developed and buils as required. Xornal repair of existing elecironics components will be handled in a separats $190 \mathrm{~m}^{2}$ area containing sturage shelves for spare (omponents and parts. In addition to the above shop areas, four small laboratories, with a total floor space of approximately $125 \mathrm{~m}^{2}$. are provided primarily for diagnostuc purposes. The total area dewted to maintenance functions, including the haboratory space. is approximately $880 \mathrm{~m}^{2}$.

The receiving area stown in Fig. 6.2. is located at the end of the main $3-\mathrm{m}$. wide passage running under the cincrete shielding st ructure to t wo elevators. one on each side of the iorus. Equipment can thus be offloaded trom trucks and taken directly to the appropriate level in the main toroidal building. A 42 $\mathrm{m}^{2}$ reseiving area and $1901-\mathrm{m}$ " warehouse are provided. but these storage areas are for incoming inspections and temporary storage only. Most of the equipomesnt probably wili be moved from a laboratory staping arca directly to it sermanent location in the facility. During operation of the experiment. the warehouse will be used for storage of spare parts.

It is expected that the major administrative sup. port area for the SFTK will be in the CTK oftice huilding. Ten olfices are incladed for those few st aff members spending $\mathrm{I}(\mathrm{K})^{\prime}$, of their time at the facility.

\subsection{STRUCTURES AND UTILITIES}

The main toroidal building and attached auxiliary structures are designed to take all possible advan. tage of prefabricated building techniques in order 10 at'hieve the lowest possible structural cost. The basic construction will be a structural steel frame with appropriate steel siding and insulation. The roof will consist of insulated built -up roofing supported by open-web steel joints. Modular design will be employed to achieve flexibility as well as economy. The architectural and structural requirements, the heating and ventilating systems, the piped disiributinn systems. and the electrical service systems will be basically the same for each module. The design will be in accordance with the spprupriate ERDA standards and codes. Earthquake loadings will be determined in accordance with the uniform building code for seismic risk zone 2; wind loan's will be in accordance with the A.N.S.I. code A58.1, with a 1000 . yr mean recurrence leve!, a basic wind speed of 100 mph with exposure $C$.

The concrete shielcting structure will be constructed from reinforced concrete and will be designed to support the trigger system as well as to provide basic structural integrity. Care has been taken to eliminate as mech mansive foundation construction as possible. The shielding structure will rest directly on the ground and be sufficiently massive (o provide its own foundation. The flooring of the main building and auxiliary buildings is simple reinforced-concrete slat).

Two basic ventilation system: are provided. A special system provides the necessary ventilation for the tritium handling facilits. stich as for tritium hoods and tritium cleânup in the shielding structure. This svstem will have a minimum of two supply and two return/exhaust fans. and will also be designed to: have reserve cepacity for emergency conditions. The design will allow the shutdown of ans one fan fin maintenance and repair without reducing the required ventilation rate of the system.

The normal ventilation system will furnish air in the quantity and at the temperature and humidity meressary lo sat isfy the requirements for comfort and: the processes and operation in the structure liefrigerated air conditioning is not expected to be required except in the control rom where the cornputer equipment is located.

Litile chilled water will be required for the facility except for the METS refrigeration system. An appropriately sized cooling cower will be prowided as. discussed in Sec. 5.4.33.37.

litities for the SFTR will provide for domesti: water, fire water, natural gas, electric power, storn' drainage, and sanitation. Due io the size and impor. tance of the elec!rical power requirements, the electrical power utilities are discussed separately in Sec. 6.7. Domestic water and fire water will be connected (1) a water system to be installed at the proposed Two-Mile Mesa site prior to the construction of this farility. Back-flow prevention devires will he installed as required. The fire-water line will be ioped $t "$ insure a reliable flow and will include all necessary hydrants and P.I.V. valves.

The natural-gas line will be connerted to a gits main that presently exists on Two-Mile Mesa.

Sanitary waste will be connected to a system to be constructed on Two-Mile Mesa prior to the construction of the SFTR. The system will collect all sanitury waste on Two-Mile Mesa and convey it to the existing Snnitary Waste Treatment Farility at TA-3. which presently has the capacity to handle the additional load.

Storm drainage will be accomplished through open ditches and site grading, except at walks and roads, where drains will be provided.

Chemical waste will be collected locally and transported to the Laboratory's industrial waste treatment group tor disposal. A holding tank will be 
installed for collecting waste tritiated water. Depen. ding on its tritium concentration, the water will either be exhausted through the stack at the facility or transported to the Laboratory's industrial waste treatment group for solidification and burial.

\subsection{ELECTRICAL POWER}

The operation of SFTR will put a significant and continuously changing demand on the local power supply system. Since the facility is expected to operate only over a period of a few years, puwer lines, transformers, and other long-lived distribution equipment must be carefully chosen to avoid expensive devices that cannot be used in future projects. The power supply system will be designed to operate at voltages compatible with existing LASL distribution equipment. That is, $115-\mathrm{kV}$, three -phase power line, 13.2-kV primary distribution system to several points within the facility, $13.2-\mathrm{kV}$ to $480-\mathrm{V}$ stepdown and secondary distribution substations, $480-\mathrm{V}$ power to large motors, dc supplies, etc., and 208/120$\mathrm{V}$ supply for lights and other small loads as required. Installed capacity will be 20 MVA to handle the estimated load, including the METS system.

\subsubsection{Power Requirements}

The planned operating phases will require significantly different amounts of power. Phase I operation requires 2-M continuous plus $16-\mathrm{MW}$ pulsed loads, whereas Phase II requires 9-MW continuous plus $9 \cdot \mathrm{MW}$ pulsed loads. Table 6.2 lists the various loads to be supplied and the expected operating condition. Pulse loads are, in general, linearly rising ramps : nproximately $30 \mathrm{~s}$ long with a cirop to zero load at the end. The power shown in Table $6-2$ is the peak load at the end of the ramp. Reprtition rate will not exceed one pulse every 15 min. A continuous load of $2 \mathrm{MW}$ is expected to supply building services during any phase $s_{t}^{\prime}$ operation. An additional continuous load of 6-8 MW will be needed for the METS refrigeration system.

\subsubsection{Impact on Electric Power System}

The Los Alamos area is currently connected into the Public Service Co. of New Mexico (PSCNM' distribution network by two $115-\mathrm{kV}$ lines; one from Santa Fe rated at $20 \mathrm{MW}$, and one from Albuquerque rated at 65 MW. Present planniriog includes two new lines from a PSCNM substation called Nor- ton, approximately 12 miles east of Los Alamos. These new $115-\mathrm{kV}$ lines will increase the total available power from PSCNM to $115 \mathrm{MW}$ by 1981 and to approximately $200 \mathrm{MW}$ by 1985 . The Norton substation will be part of the PSCNM 345-kV network connecting Albuquerque. Farmington, and Ojo Caliente. Figure h-6 shows the arrangement forecast for 1980 . The $345 / 115 \mathrm{kV}$ transformer at Norton has been ordered.

Power available for the SFTR in FY- 81 will be 42 MW if the firsi $115-\mathrm{kV}$ line to Norton is completed and LASL Inad grows, as forecast, from the current $50 \mathrm{MW}$ to $88 \mathrm{MW}$. The total power available includes the $20-\mathrm{MW}$ generating capacity at the Zia power plant in Los Alamos on a short time load basis (15 MW continuous). Since the expected SFTR load is $20 \mathrm{MW}$ or less, the currently planned power source wiil be adequate.

In the event that the Warm METS option is adopted, a separate, dedicated $115-\mathrm{kV}$ line will be required. This is discussed in App. I.

\subsubsection{Power Distribution System}

Figure 6-7 shows the proposed electrical distrihution system at the SFTR site. The incoming 115-kV power line from ETA substation (see Fig. 6-6) will supply all power to the facility through a $115-\mathrm{kV}$ to 13.2-kV, 20-MVA transformer; $13.2-\mathrm{kV}$ switchgear will be used to control power to 11 locations within the area. Locatiors numbered 1 to 8 on Fig. 6.7 are for 2-MW dc power supplies used to charge the transfer capacitor banks during $P$ hase I operations. The three shaded blocks in Fig. 6.7 represent 13.2$\mathrm{kV}$ to $480-\mathrm{V}$ substations. Each substation will contain one 2000-l:VA, 13.2-kV/480-V step-down transformer and $480-\mathrm{V}$ circuit breakers to feed the various loads in one third of the building. The substation near the control room will supply the instrument and control power. 'The two substations outside the torus will supply mast of the motors. lights, and building services. Power for motor control ceitters and other large loads will be supplied at the 480-V level. Power for lights, receptacles, and other small loads will be supplied by 480/120/208-V transformers located throughout the building. Clean power panels will be provided for critical inst ument loads. These panels will be fed by $480 / 120,208-\mathrm{V}$ isolating transformers. The substation near the METS refrigeration area will also contain transformers and motor starters for the large motors driving compressors in this area. and will have a total capacity of $10 \mathrm{MVA}$. 
TABLE 6-2

\section{ESTIMATED POWER REQIIREMENTS FOR SFTK}

\begin{tabular}{|c|c|c|}
\hline Load & $\begin{array}{c}\text { Power Required } \\
\text { (MW) }\end{array}$ & Notes \\
\hline $\begin{array}{l}\text { Shock heating } \\
\text { Charge system }\end{array}$ & 0.7 & 60-s pulse \\
\hline $\begin{array}{l}\text { Compression sysiem } \\
\text { Capacitor charging } \\
\text { Phase I } \\
\text { Phase II }\end{array}$ & $\begin{array}{r}16 \\
5\end{array}$ & $\begin{array}{l}\text { 30-s pulse } \\
30-\text { s pulse }\end{array}$ \\
\hline $\begin{array}{l}\text { Cold METS storage } \\
\text { Coil charping }\end{array}$ & 3 & $30-5$ pulse \\
\hline $\begin{array}{l}\text { Cold METS } \\
\text { Refrigeration }\end{array}$ & $6-8$ & Continuorus \\
\hline $\begin{array}{l}\text { Tritium handling } \\
\text { Vaculm pumps } \\
\text { Cleanup pumps }\end{array}$ & 0.1 & $\begin{array}{l}\text { Continuous } \\
\text { during clean-up } \\
\text { operation only }\end{array}$ \\
\hline $\begin{array}{l}\text { Building heating \& } \\
\text { Air conditioning }\end{array}$ & 0.5 & Continuotes \\
\hline Inst rument power & 1 & Continuous \\
\hline $\begin{array}{l}\text { Building lights. } \\
\text { Rerept acles. and misc. }\end{array}$ & 0.7 & On as needed \\
\hline
\end{tabular}

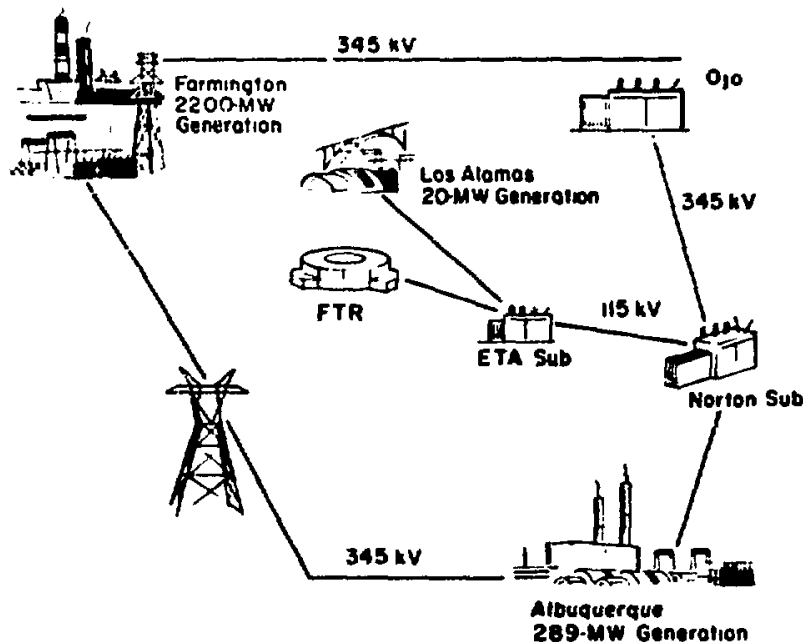

Fig. 6-6.

Northern Neu: Mexico high voltage power distribution network as planned for 1980.
All substations and the eight capacitor charging dc power supplies will be outdoor equipment. Weather protection will be provided by sheet meial factory-built housing similar to the "protected aisle" switchgear used in high voltage switchyard.. Switching equipment, housing for the power supplies, and mantenance personnel weather protection will be built in modules that bolt together and that can be readily disassembled for removal or replacement. Concrete pads or piers will be decigned to suit the equipment selected. All 13.2-kV interconnecting cables will be under ground.

Three existing 13.2-kV power lines near the SFTR site on Two Mile Mesa can be tapped and brought into the outdoor substation for back-up power if the $115-k V$ primary line should fail. Sufficient power for essential building services can thus be provided although these sources are not sufficient for normal operations. 


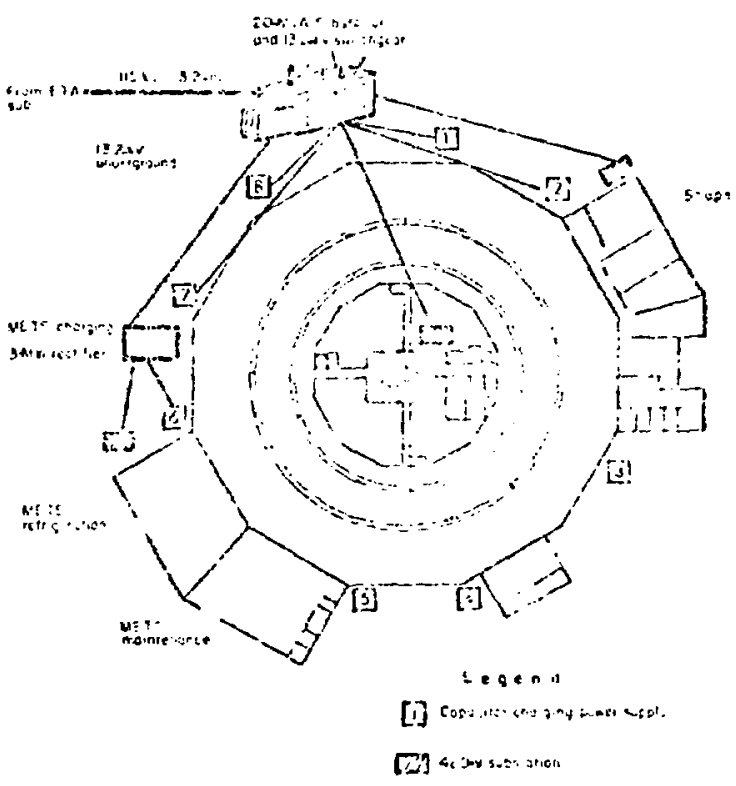

Fig. 6-7.

Pouer distribution at the SFTR site.

Certain loads. such as those for the tritium handling facility, cannot be turned off for the time re. quired to correct a power failure or to switch over to emergency power manually. These loads will be conaected to a $480-\mathrm{V}$ power source with automatic switching to an engine-driven alternator that will start in a few seconds and carry the load until normal power can be restored.

\subsection{SITE SELECTION}

\section{f.8.1 Genera]}

A detailed site selection study for the SFTR was made by the LASL Engineering Department, and the results presented in an internal report. Criteria for the site selection were developed and presented in a separate report. In accordance with these criteria, consideration was given to population distribution, proximity to other facilities and operations, utilities and services, and to the meteorology, hydrology, geology, and seismicity of the candidate locations. Special attention was given to consequences of hypothetical tritium release.

The area of site selection search was limited tc the ERDA-controlled land of Los Alamos County (Fig. 6-8). This area represents approximately $104 \mathrm{~km}^{2}(40$ miles $\left.{ }^{2}\right)$ of the county's $285-\mathrm{km}^{2}\left(110-\right.$ mile $\left.^{2}\right)$ area. and many lORDA research tacilities are lecated there. 'The city of Los Alamos, with a residential populat ion of approximately 11 (K), lies to the north. The residential areas of White Rock. La Vista, and Pajarito Acres, with a combined population of about fo(k), lie to the south and east.

\subsubsection{Selection of Sites for Initial Study}

Eight sites, all within the Technical Area of the ERDA-controiled land in Los Alamos ('ounts. were selected for initial study. Detailed maps, atrial phortographs, and utility drawings of the Terehnical Area sreatly shortened the time required for this phase of the work. In addition. information was used front the Fluor Corporation report and the Dames and Moore studies for both the New Pluonium Facility and the New Pulsed Iiltra-High Flux Facility.

The estimated area required for the new site was bei ween 10 and is acres, depending on topography. On early review, it was apparent that a number of lavorable locations could be found within the Technical Area. As a result, selection requirements for the sites to be included in the study were made somewhat restrictive. Lucations were selected with consideration given to the following.

Population. Favorable distance to population center of Los Alamos, White Rock, and the trailer court on East Jemez Road was required. (It is recognized that safety features to be built into the new facility will make it feasible to use locations ad. jacent to population centers.)

Hazards. Sufficient distance is needed from areas of potential hazards, such as the Los Alamos airport operation or explosion research being conducted within the Technical Area, so as not to warrant special design considerations in the facility.

Topography. Favorable topography and plot shape were considered so as to minimize the cost of grading and costs associated with general facility layout or operation.

Accessibility. Favorable location is needed with respect to the center of Technical Area operations. including access over all-weat her roads.

Ground Rules and Criteria

AECM 6301 (General Design Criteria).

AECM 6203 (Site Development Planning).

AECM 2406 (Physical Protection AEC Property).

AECM 0552 (Industrial Fire Protection).

Relatively "flat" area is desirable.

Proximity to the Intense Neutron Facility is desirable.

Proximity io piesent opcrations is desirahle. 


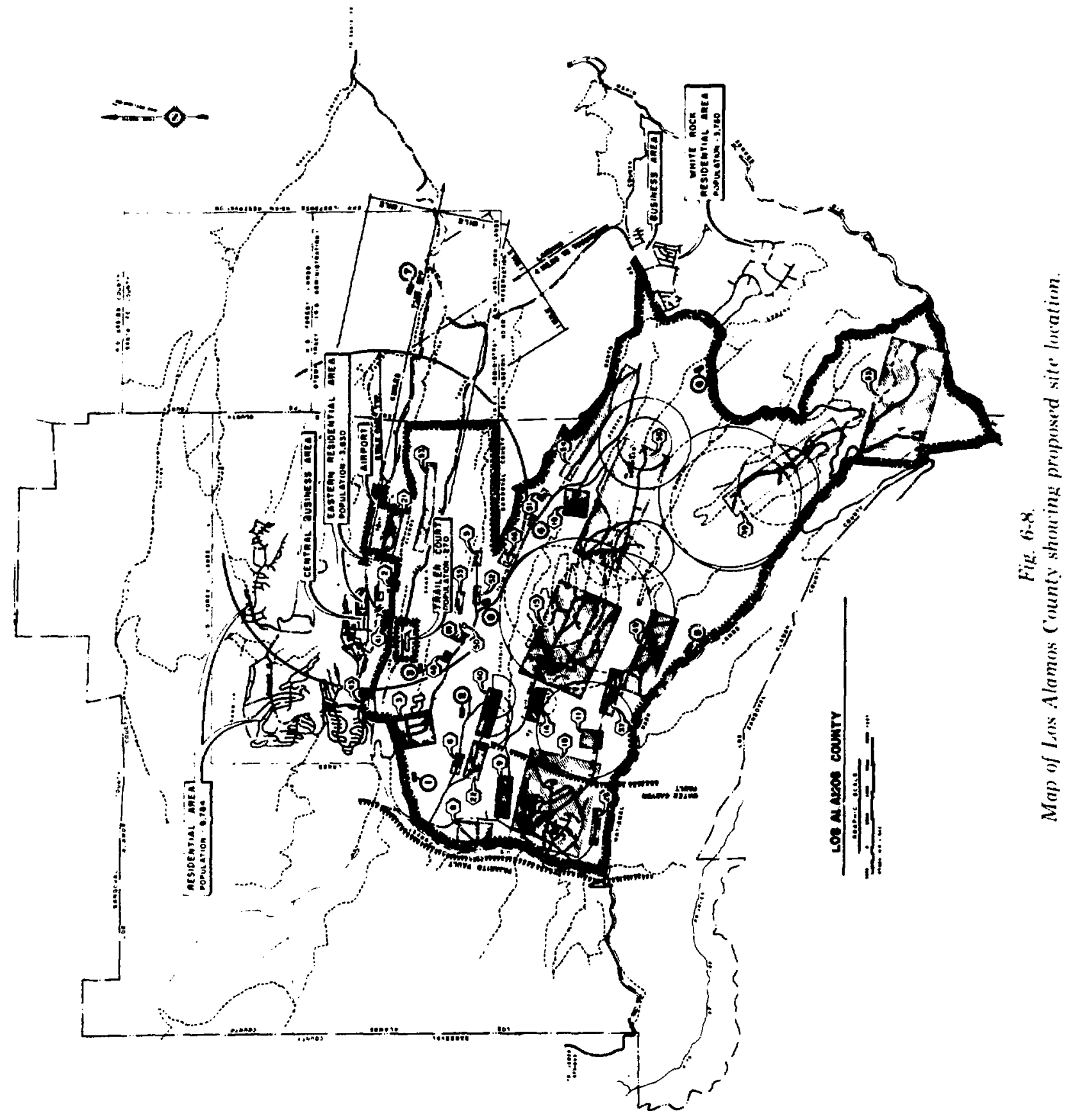


Good vehicular access is required.

Must be outside a security area so that Soviet Bloc visitors can have access.

Future expansion (at least double) must be available.

\subsubsection{Description of Study Sites}

The eight sites selected for study are shown in Fig. 6-8. The following description of each site is based on available maps, utility drawings, and visual inspection.

Site 1 is located on the west edge of the present T.A-3 area. adjacent to and on the south side of State Road Loop 4 . The area is heavily wooded with ponderosa pine trees and slopes upward to the west at $10^{\prime} ;$ or less. Excavation of the welded tuff rock in the area is assumed to be difficult. The site is located within a chain-link fenced area but is not in a securitV. area. Fire Station No. 1 is no more than a 2 -min run distant. The nearest fault is Pajarito fault, about $1.067 \mathrm{~km}(0.663 \mathrm{mile})$ to the west. No access to the arca exists at the present time. necessitating the construstion of a new paved roadway across TwoMile Canyon. Itilities in the area include a $13.2-\mathrm{kV}$ power line (S-17) of limited capacity approximately $450 \mathrm{~m}(1500 \mathrm{ft})$ to the southwest. A telephone service manhole is located across Two-Mile Canyon from this site. and extension to this site would be under ground by way of the fill anticipated for the road across the canyon. A $0.305-\mathrm{m}$ (12-in.), 100-psi natural-gas line crosses the site parallel to the water line.

Site 2 is located within a security area and is on the north side of Two-Mile Mesa Road approximately $1.372 \mathrm{~km}(0.85$ mile) east of the road turnoff to TA-22. The area is relatively flat for 0.091 to 0.122 $\mathrm{km}(0.06$ to 0.08 mile) adjacent to the road and then slopes off approximately $10^{\prime} ;$ to the south. A $13.2-\mathrm{kV}$ pole line crosses the area. No gas or water lines were noted. A shot impact area limits the southern boundary of this site. The Pajarito fault is located ap. proximately $2.743 \mathrm{~km}(1.7$ miles) to the west.

Site 3 is located on the mesa top to the east of TA. 3 and the Sigma Building (SM-66), about midway between the trailer court and TA-48. The only access to the area is a dirt trail which skirts the emergency radio installation. The area is generaliy clear exce $a t$ for a scattering of juniper and pinon trees, and is relatively flat, sloping gently to the east. The area is essentially open to public access. Fire Station No. 1 is within a 5-min run. Excavation in the tuff rock at this site would be routine. The nearest fault is the Los Alamos fault, located $1.93 \mathrm{~km}$ (1.2 miles) to the northeast. The only utility in the area is a dedicated $13.2-\mathrm{k} V$ pole line $(S-20)$ which traverses the site in an east-west direction.

Site 4 is located on the north side of Pajarito Road, approximately $0.7 \mathrm{~km}$ (0.4 mile) west of Rex Drive. The area is relatively flat with a heavy covering of pinon, pine, and juniper trees. A $115-\mathrm{kV}$ pole line runs along the north edge of the site. Another $115-\mathrm{kV}$ pole line traverses the southwest corner. The Pajarito fault lies approximately $7.010 \mathrm{~km} \quad 4.36$ miles) to the west. A $0.203-\mathrm{m}(8$-in.) water line and 0.076-m (3-in.) gas line run parallel to and north of Pajarito Road. A $0.400-\mathrm{m}$ (16-in. 1 water line russ parallel to and south of Pajarito Road. TA-51, a radiation exposure experimental facility, is located to the east. The site contains four Indian ruins. The boundary of ERDA-controlled land is approximately $0.304 \mathrm{~km}(0.19 \mathrm{mile})$ to the north. The adjacent land is designated Indian Sacred Area. Excavation in the tuff rock would be routine.

Site 5 is located approximately $1.61 \mathrm{~km}$ ( 1 mile) southeast of the entrance road to TA-49, on the north side of State Road 4. The area is cut by arroyos and is of moderate slope, generally to the east. It is covered with a moderatc stand of pinon and juniper trees. Excavation in the tuff rock at this site would be routine. The nearest fault is the Water Canyon fault located about $3.54 \mathrm{~km}$ ( 2.2 miles) to the west. The utilities in the area are limited to a $13.2-\mathrm{kV}$ aerial electrical line (S-17), an aerial telephone line running along State Route 4 at this location. A 0.152-m (6-in.) cast-iron water main and a $0.076-\mathrm{m}$ (3-in.) 100-psi natural-gas line parallel State Road 4 on the north side in the vicinity of the site. Neither of these lines is aderjuate to serve the proposed facility.

Site 6 is located on the north side of State Road 4 opposite Pajarito Acres in the White Rock area. It has a moderate slope to the east and a moderate cover of pinon and juniper trees. Excavation in the tuff rock and hasalt 2 at this site would be difficult. The fire station at White Rock is within a 5-min run. The nearest fault is the Water Canyon fault, located approximately $10 \mathrm{~km}(6.2 \mathrm{miles})$ to the west. There are no utilities in this area except those serving the Pajarito Acres residential area.

Site 7 is located in the Otowi tract of the Bandelier National Monument which is under General Services Administration control and presently reserved for LASL development. The area under consideration is near the "Y" where State Road 4 branches to Los Alamos or White Rock, and is on the north side of the Los Alamos branch. The area is subject to the $100-\mathrm{yr}$ flood problem. A moderate cover of pinon and juniper exists at this site which also has moderate to steep slopes. The tuff 
rock and conglomerate are assumed to be difficult to excavate. The nearest fault is the Guaje Mountain fault located about $7.24 \mathrm{~km}$ (4.5 miles) to the northwest. There is no access to the area and there are no utilities there.

Site 8 is located on the northeast corner of the intersection of Pajarito Road and Puye Drive. It is 2.6 $\mathrm{km}$ (1.6 miles) south of the Los Alamos airport runway. The site has sparse vegetation and is relatively flat, with a gentle slope to the east. Two $115-\mathrm{kV}$ power lines, a $13.2-\mathrm{kV}$ pole line, and a 24.8 $\mathrm{MPa}(3600$-psi) hydrogen line traverse the area. A $0.20: 3-\mathrm{m}(8$-in. $)$ water line and a $0.076-\mathrm{m} 13$-in. $)$ gas line run yarallel to and north of Pajarito Road. A $0.400-\mathrm{m}$ (16-in.) water line runs to a test reactor. presently shut down, and a classified material disposal plant. A Zia branch office is located to the north. across Puye Drive. The Pajarito fault is approximately $4.876 \mathrm{~km}$ (3.03 miles) to the west. Fxcavation in the tuff rock at this site would be routine.

\subsubsection{Site Selection}

During the early phase of the Site Selection Study, Sites 3, 4, 5, 6, 7, and 8 e eliminated.

Site 3 was eliminated becaust the buildable area is tow small for both the SFTR and a planned CTR office building. Future expansion obviously would be precluded. Access to the site is difficult and a new road would have to be built in an ares inconvenien! for traffic control. Not only is this site too remote from the present CTR tecinical operation area and the Intense Neutron Source Facility, but it is too close to the residential trailer court.
Sites 4 and 8 were eliminated because they also are already too small for both the SFTR and the office building and are too far from present technical CTR operations and the proposed site for the Intense Neutron Source Facility.

Sites 5, 6, and 7 were eliminated because they are extremely remote from CTR technical operations and the Intense Neutron Source Facility and their use would involve long travel times. Excavation in much of these three areas is difficult, although in parts it would be routine. Site 6 is subject to noise and pressure pulses from explosive testing at TA-36, and Site 7 is subject to the possibility of a $100-\mathrm{yr}$ flood. Basically, these sites are poor construction sites because of the moderate to steep slopes.

A population distribution study of the sites under consideration was made and appears in Sec. 2 of the Site Selection Study report. The results of this study show that radiation doses at the sites considered, as a consequence of various postulated facility accidents, are far below established exposure guidelines. Thus, the population distribution had no bearing on either of the two remaining sites.

Cost estimates for the development of Sites 1 and 2 included the cost of

1. Electitic power.

2. Water supplies from two separate sources,

3. Gas supply from a single source.

4. Site grading and access roads.

The estimate showed that the difference in development costs for the two sites is approximately $\$ 1 \mathrm{M}$, a sum not of major significance when compared to the total cost of the facility. Location of the facility is therefore proposed for either Site 1 or 2 on Two-Mile Mesa. 


\section{SAFETY AND ENVIRONMENTAL CONSIDERATIONS}

\subsection{OVERVIEW}

Because of the preliminary nature of the SFTR design. a comprehensive environmental impact statement cannot be formulated at this time. However. in this section we consider those safety and environmental implications of the SFTR experiment that can be estimated from existing design detail. Although our conclusions may change somewhat with subsequent analysis, major aspects of the SFTR safety and environmental impact described below are not expected to alter significantly.

The SFTR site will be loceted within $2 \mathrm{~km}$ of the Los Alamos (population 1.5000 ) town site and ap. proximately $40 \mathrm{~km}$ from Santa Fe (population 55 000). Approximately $3 \mathrm{~g}\left(3 \times 10^{4} \mathrm{Ci}\right)$ of tritium will be stored at the SFTR site. If suddenly released, the tritium could result in a maximum integrated population dose of 200 man-rem. The operational radioactive hazard will arise from (14-MeV) fusion neutrons, $x$-radiation, and neutron-induced gamma radiation. all of which are reduced to or below background radiation levels by $\sim 2 \mathrm{~m}$ of concrete, biological shielding. This radiation spectrum will present no public hazard. Radioactive structural waste will be generated at a rate of $20 \mathrm{Ci} / \mathrm{yr}$ and approximately $15 \mathrm{~g} \mathrm{~T}_{2} / \mathrm{yr}$ will be transported to and from the SFTR site. The chemical and sanitary waste will be similar to that generated by the ongoing Scyllac experiment at LASi and, therefore, is considered negligible. The major occupational hazard will be associated with the high voltages needed to operate the SFTR experiment, and will also be similar to those encountered by the present Scyllac experiment. The potential for a thermonuclear "runaway" is zero, although mechanical failure of magnets and high-current leads may occur; protection of personnel from electrical hazards and missiles generated by mechanical failures will be provided according to LASL procedure and practices. The potential for normal and off-normal safety and environmental hazard is quantified in the following sections.

\subsection{DESCRIPTION OF SFTR SITE}

The SFTR site is on the western edge of the Pajarito Plateau adjacent to flanks of the Sierra de los
Valles; a map of the area is shown in Fig. $7-1$. The area is at an elevation of about $76(K) \mathrm{ft}$ and cont ains a heavy cover of pines and 0.3 to $1 \mathrm{~m}$ of soil. The slope is 10 degrees or less eastward. In places the tuff bedrock is exposed. The surface is cut by a few small channels that drain into Two-Mile Canyon.

\subsubsection{Demographic Description}

Approximately 400000 people inhabit a curcle ot $\sim 100-\mathrm{km}$ radius centered on Los Alamıs. Nearly two-thirds of this population is concentrated in Albuquerque, about $100 \mathrm{~km}$ to the soutt. Another one-ninth is located in Santa Fe, about $41 \mathrm{~km} \mathrm{to}$ the southeast. Except for the 15000 residents of Los Alamos, the remaining population is distributed among small towns, ranging in size from a few hundred to a few thousand people, and Indian Puebles of several hundred people each. The nearest community is Espanola, a town of about 2000 people. located about $20 \mathrm{~km}$ to the northeast. About 11000 of the los Alamos population live in the residential area of Los Alamos proper and the remaining 4000 reside in the developments of the White Rock conmunity.

At any particular time these people are scattered throughout the Los Alamos area. It is impossible to predict their exact locations, but it is meaningful to distinguish between a daytime population distribution and a nighttime distribution. The distributions for angular sections $45^{\circ}$ wide and $1 \mathrm{~km}$ deep with respect to the LASL meteorological tower are shown in Table $7-1$. Movements of people to work and to school and the influx of workers into the area have been considered.

The economy of the Santa Fe-Los Alamos area is based largely on government (federal and state) operations, a large tourist trade, arts and crafts, agriculture, and some light and service industry (mostly associated with the tourist trade). The Los Alamos Scientific Laboratory accounts for much of the federal employment, while the New Mexico state governmental offices in Santa Fe provide the state jobs.

Agriculture is practiced only to a limited extent in the immediate vicinity (within $20-40 \mathrm{~km}$ of Los Alamos). In this area many people raise vegetables in home gardens, but rarely depend on this activity for more than half of their subsistence. Outside this area, agriculture is practiced by much of the rural 


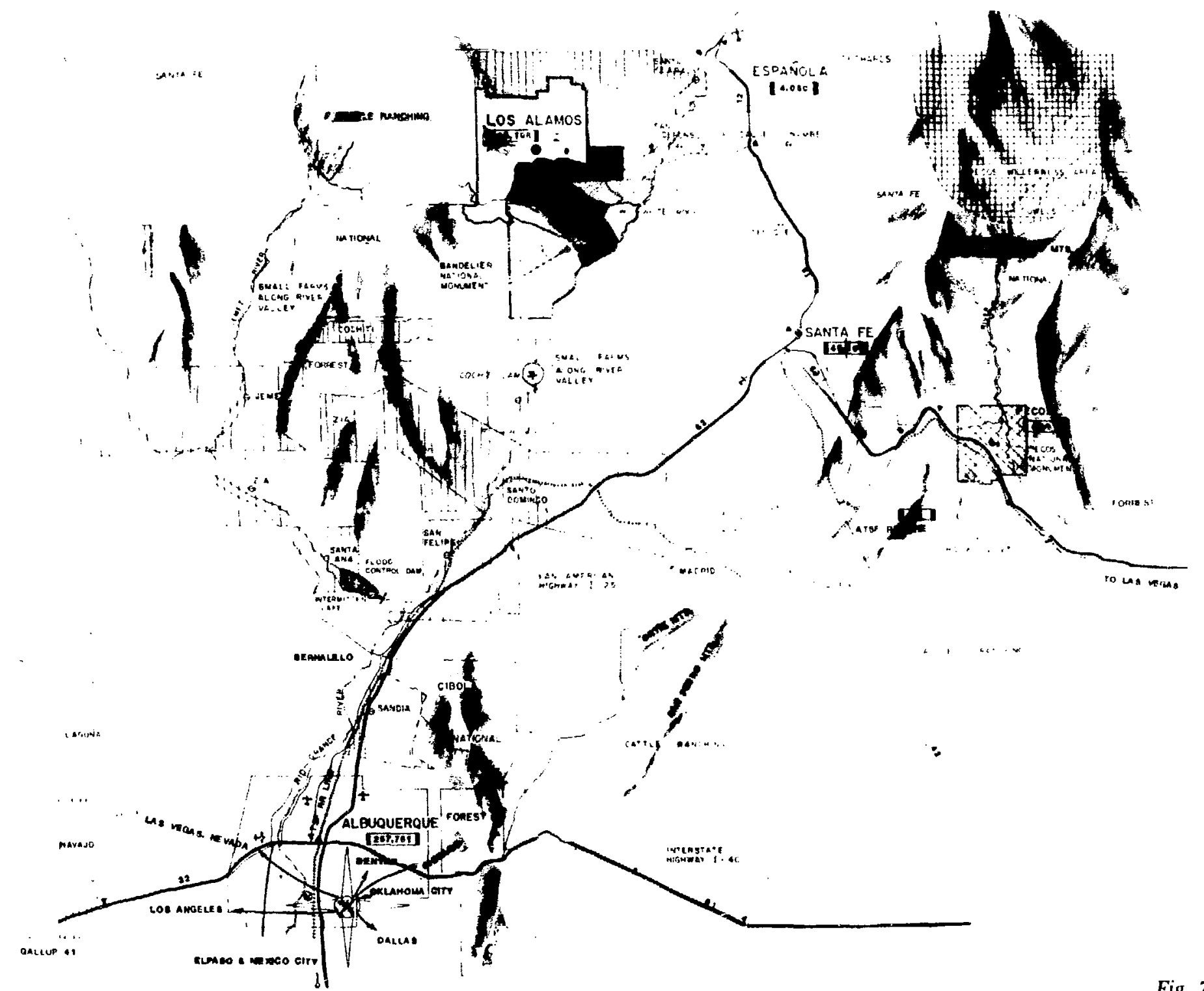


TARI.E 7-I

POPUI.ATION DISTRIBUTION IN I,OS AL.AMOS, NM, I.OCAI.F:

\begin{tabular}{|c|c|c|c|c|c|c|c|c|}
\hline \multirow{2}{*}{$\begin{array}{c}\text { Distance } \\
\text { Interval } \\
\text { (miles) } \\
\end{array}$} & \multicolumn{8}{|c|}{ Total Population in Segment,Daytime/Nighttime } \\
\hline & $0^{\circ}-45^{\circ}$ & $15^{\circ}-91^{\circ}$ & $90^{\circ}-135^{\circ}$ & $135^{\circ}-180^{c}$ & $180^{\circ}-225^{\circ}$ & $225^{\circ}-270^{\circ}$ & $270^{\circ}-315=$ & $315^{\circ}-3360^{\circ}$ \\
\hline 0.1 & $694 / 448$ & $46 ; 0$ & 32010 & $26 j /(1$ & $: 310 / 0$ & $107 / 0$ & $i \cdot \pi / 41 / 1$ & $1957 / 8(1)$ \\
\hline $1+2$ & $3464 / 27: 35$ & $926 / 1467$ & $244 / 1$ & $42 / 0$ & $8 i / 11$ & $62 / 1)$ & $(1 / 1)$ & $12: 1.1 / 2(1) 19$ \\
\hline $2-3$ & $1747 / 1874$ & $1042 / 1965$ & $175 / 0$ & $15 / 0$ & $9 \div 2 / 11$ & $129 / 10$ & $(1 / 1)$ & $(1 / 1)$ \\
\hline $3-4$ & $0 / 0$ & $496 / 0$ & $39: 3 / 6$ & $9 / 0$ & $(\mathbf{0} / \mathbf{0}$ & $(1 / 1)$ & $(1 / 1)$ & $0 / 0$ \\
\hline $4-5$ & $0 / 0$ & $0 / 0$ & $2 / 0$ & $(1 / 1)$ & $0 / 11$ & $(1 / 0$ & $0 / 1)$ & $01 / 11$ \\
\hline $5-6$ & $0 / 0$ & $0 /(1$ & $(1 / 0$ & $(1 / 0)$ & $(1 / 1)$ & $01 / 11$ & $0 / 0$ & $01 / 11$ \\
\hline $6-7$ & $0 / 0$ & $0 / 0$ & $(1 / 0)$ & $: 39 / 0$ & $(1 / 1)$ & $(1 / 1)$ & $01 / 1)$ & 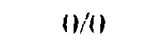 \\
\hline $7 \cdot 8$ & $0 / 0$ & $0 /(1$ & $2624 / 33861$ & $46 / 27$ & $(1 / 0)$ & $(1 / 0)$ & $01 / 11$ & $(1) / 61$ \\
\hline $8-9$ & $0 / 0$ & $0 / 0$ & $0 / 0$ & $9 / 11$ & $(0 / 0)$ & $0 /(1)$ & $01 / 1)$ & $(1) / 6$ \\
\hline 9.10 & $(3 / 0$ & $0 /(1$ & $0 / 0$ & $(1 / 0$ & $(1 / 1)$ & $(1 / 0)$ & $01 / 11$ & $11 / 11$ \\
\hline $10-11$ & $0 / 0$ & $0 / 0$ & $0 / 0$ & $(1 / 1)$ & $(0 / 11$ & $(1 / 1)$ & $01 / 0$ & $(1) / 11$ \\
\hline $11-12$ & $0 / \mathrm{c}$ & $0 / 0$ & $0 / 0$ & $(1) / 0$ & $(1 / 1)$ & $0 /(1)$ & $(1 / 0)$ & $01 / 11$ \\
\hline $12-13$ & $0 / 0$ & $215 / 215$ & $(1 / 0)$ & $(1 /)$ & $(1 / 1)$ & $0 / 0$ & $0 / 0$ & $(1 / 1)$ \\
\hline $13-14$ & $0 / 0$ & $0 / 0$ & $(1 / 0)$ & $(1 / 1)$ & $(0 / 0$ & $0 /(0$ & $(1 / 1)$ & $0 / 11$ \\
\hline $14-15$ & $0 / 0$ & $0 / 0$ & $0 / 0$ & $(1 / 41$ & $0 / 0$ & $(1 / 0)$ & $(1 /()$ & $01 / 11$ \\
\hline $15-16$ & $0 / 0$ & $5478 / 5478$ & $0 / 0$ & $(1 /(1$ & $(1 / 0$ & $(1 / 0$ & $(1 / 0)$ & $(1 / 1)$ \\
\hline $16 \cdot 17$ & $4(10 / 400$ & $1000 / 10(K)$ & $(1 / 0$ & $(1 / 1)$ & $(1) /(1)$ & $(1 / 1)$ & $(1 / 0)$ & $(1 / 1)$ \\
\hline $17-18$ & $0 / 0$ & $450 / 450$ & $0 / 0$ & $15(1 / 150$ & $(1 /()$ & $(1 / 0)$ & $(1 / 1)$ & $01 / 61$ \\
\hline $18-19$ & $0 / 0$ & $6(10 / 600)$ & $(1 / 1)$ & $(\mathrm{l} / \mathrm{(})$ & (1)/1) & $0 / 1 !$ & $(b / 0)$ & $0 / 1$ \\
\hline $19-20$ & $0 / 0$ & $0 /(1$ & $(1 / 0)$ & $(1 / 0)$ & $(1) / 11$ & $0 / 11$ & $(1 /)$ & $01 / 11$ \\
\hline $20-21$ & $0 / 0$ & $0 /(1$ & $0 / 0$ & $(1 / 0)$ & $(1 / 1)$ & $(1 / 11$ & $(1 / 0)$ & $0 / 1)$ \\
\hline $21-22$ & $150 / 1: 0$ & $\left(6 \overline{50} / 65_{5}\right)$ & $0 / 0$ & $(1) /(1)$ & $3: 3(1) / 3 \sin (1)$ & $(1 / 0)$ & $(1 / 0)$ & $!(K) / l(m)$ \\
\hline $22-23$ & $0 / 0$ & $(1) / 0$ & $545 / 545$ & $(1 / 0)$ & (1)/0 & $0 / 10$ & $(1 / 11$ & 2 :311:-in \\
\hline $2: 3-24$ & $0 / 0$ & $7(0) / 7(0)$ & $(1 / 0$ & $0 /(0)$ & $(i / 1)$ & $481 / 481$ & $(1 / 1)$ & $(1 / 0)$ \\
\hline $24-25$ & $0 / 0$ & $0 / 9$ & F.,3:39/5:53:39 & $0,(0)$ & i(1)/:00 & $0 / 11$ & $(1 / 0)$ & 政湴 \\
\hline
\end{tabular}


population. some for subsistence and income augmen'ation and some on a strictly commercial basis. Limited truck farming has been made possible in the river valleys by irrigation. Tree fruits, chili peppers, beans, corn, and alfalfa are the principal crops. Viry little of this produce enters interstate commerce. Most of the unforested land, although sparsely vegetated, is used for low-density grazing of beef cattle. Some milk is produced in the Santa Fe area. but none in the immediate vicinity of los Alamos. A small dairy in Nambe. about $25 \mathrm{~km}$ east of Los Alamos. and another in Sasta Fe sell milk produced in the area. The only ot her agricult urally oriented activity in the area is logging in some of the highland forests.

\subsubsection{Meteorological Description}

The important meteorological elements in environmental assessment are winds, from the viewpoint of pollutant transport and physical damage. precipitation (as it affects the hydrology), and light ning: temperature. humidity. and pressure are of secondary importance.

Major spaital variation of surface winds in Los Alamos is caused by the unusual terrain. Inder moderate and strong almospheric pressure gradients. flow is channeled by the terrain features of' the area, while under weak gradient flow. a distinct diurnal slope wind cycle exists. The interaction of these two domains gives rise to a westerly flow predominance on the western part of the Laboratory sitc and a southerly component at the east end of the mesas. At most sites near-calm conditions exist 10 . $15)^{\prime} ;$ of the time. 80$)^{\prime}$, of the wind speeds are less than $3 \mathrm{~m} / \mathrm{s}$, and less than $1 \%$ of the time 10 -min-averaged winds are greater than $16 \mathrm{~m} / \mathrm{s}$. Figure 7.2 is an example of wind roses for one location at TA-3 for conditions stratified by gross thermal stability. Table 7 2 gives the model inputs for estimates of $x / Q$ vs distance curves, and the resulting curves are given in Figs. 7-3 and 7-4. The point source strengt $n$ is $Q$, and $x$ is the concentration of a given released substance. The recorded peak gusts are $42 \mathrm{~m} / \mathrm{s}$, and gusts exceeding $20 \mathrm{~m} / \mathrm{s}$ occur on an average of 47 days during the year.

The precipitation averages $0.46 \mathrm{~m} / \mathrm{yr}$. with nearly $40{ }^{\prime} r$ occurring as thundershowers in July and August. Thunder is reported 60 days/yr. The temperature and humidity are typical of the altitude and geographic region. Monthly averaged temperatures range from $-2^{\circ} \mathrm{C}$ to $20^{\circ} \mathrm{C}$, and diurnal changes of $13^{\circ} \mathrm{C}$ are typical. The average relative
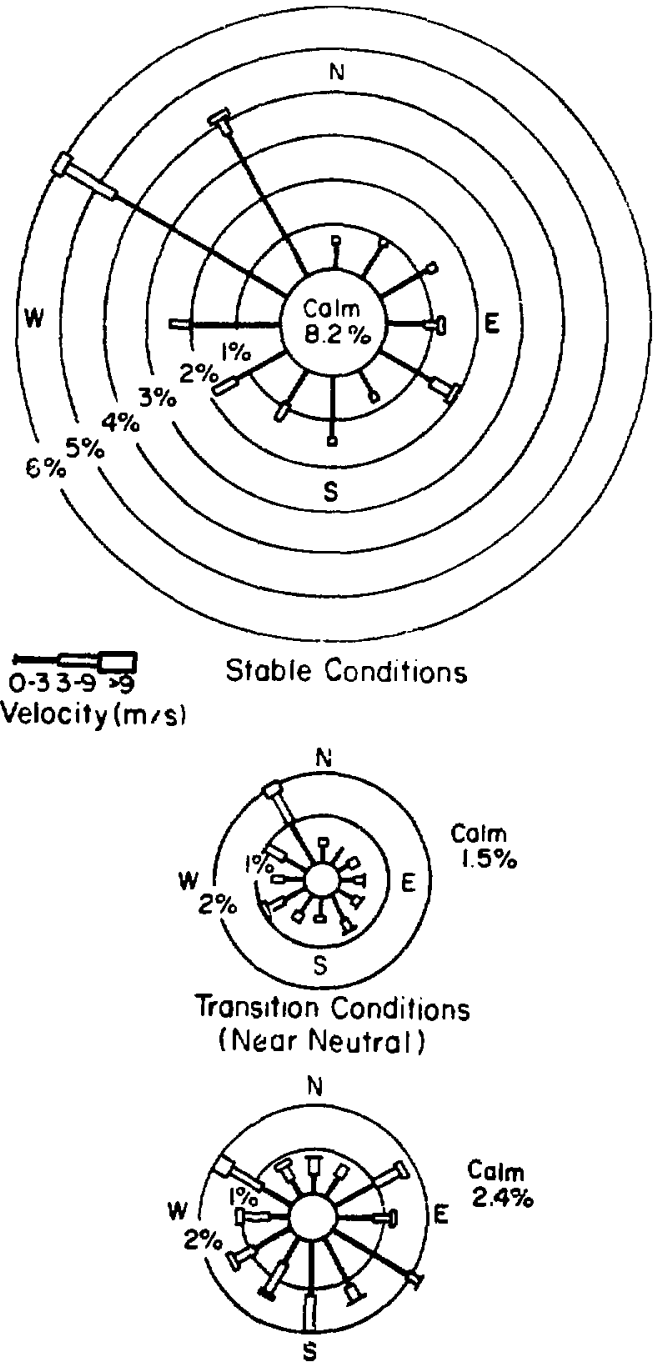

Unstuble Conditions

Fig. $7-2$.

Wind roses for TA-3 location of SF'T'R (see Fig. $7-1)$.

humidity is $40{ }^{c}$. Figures $7-5$ and $7-6$ present relevant temperature, humidity, and precipitation parameters on a monthly basis.

There are three atmospheric transport routes by which a moderately large population could be exposed to radioactive releases from the proposed SFTR site. One route is toward the NNE and the western residential area. Air flow from the SFTR site to the Western Area must cross the head of Pajarito $\mathrm{Ca}$ nyon and Los Alamos Canyon (see Fig. 7-7, 030 
TABIE $7-2$

\section{MODEL INPUTS FOR V I/Q ESTIMATES}

Tramsport direction

Primary receptor

Sisurce bejght

Mtein wind

Dilution rate

Mixing depth

Approximate frequency

ot inceurrence
NNE

IVtistem Area

$30 \mathrm{~m}$. $\mathrm{i} 10 \mathrm{~m}$

$1 \mathrm{~m} / \mathrm{s}$

Pasejuill $\mathrm{B}$

$100 \mathrm{~m}$

$12 \cdot$
F.

TA:R Trater (1)

$30 \mathrm{~m}$. iii $\mathrm{m}$

$1 \mathrm{~m} / \mathrm{s}$

Pit:ifuill $\mathrm{F}$ :

l(II) m

t.it.
Eist:

White Rink

inl 111

$0.5 \mathrm{~m} / \mathrm{s}$

Piafuill $\mathrm{F}$

in! II!

$21 \%$.

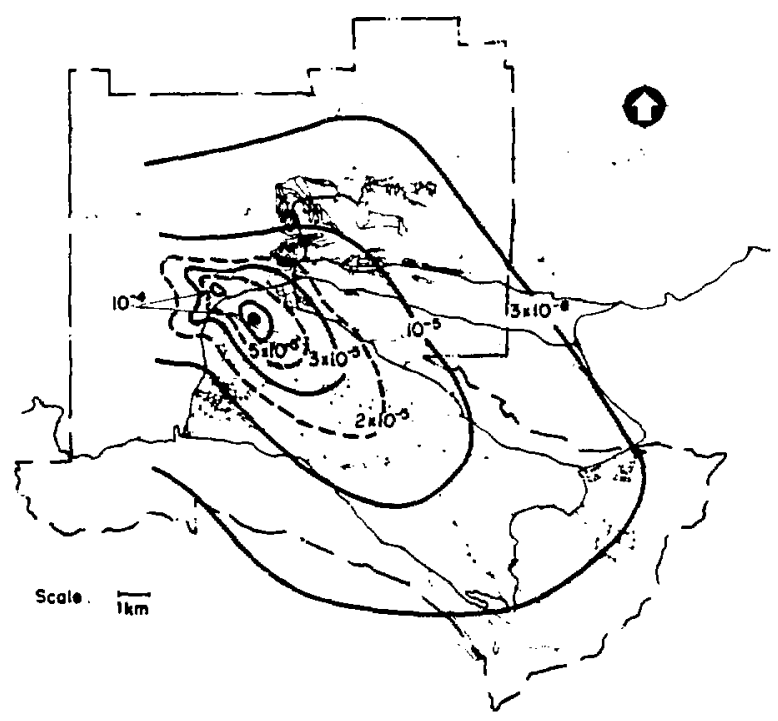

Fig. 7.3.

Annual ac erage $\times / Q\left(\mathrm{~s} / \mathrm{m}^{3}\right)$ for $S F T R$ source at 30.m stack height.

azimuth). The circulations induced by flow over such terrain account for significant mixing and dilution of containment clouds. The second path is across TA-3 and the Trailer Court in an easterly direction. The potential release cloud would encounter the heads of Pajarito and Sandia canyons as shown in Fig. $7.7\left(085^{\circ}\right.$ azimuth) and present smaller perturbations to the flow than would Los Alamos Canyon. In both cases a decoupled tlow within the canyons is likely. An injection of contaminant (e.g., $\mathrm{T}_{2} \mathrm{O}$ or HTO) into such a flow would reduce the quantity of material transported to the residential sites, but would also carry it down (or up) the canyon under different and probably more

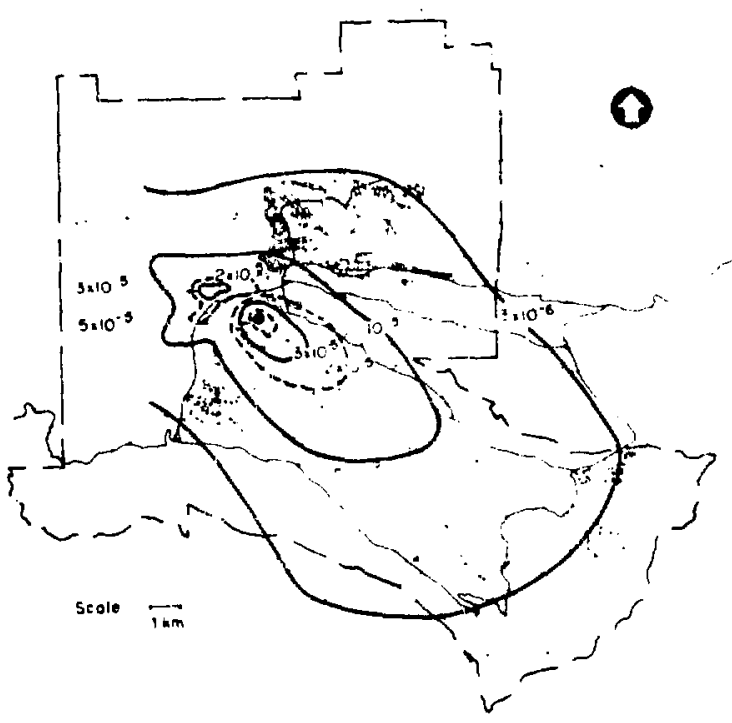

Fig. 7.4.

Annual acerage $\times / Q\left(\mathrm{~s} / \mathrm{m}^{3}\right)$ for SFTR source at jol-m stack height.

restrictive mixing conditions. This situation represents the third condition: a down-canyon drainage wind. The canvon now occurs frequently and is characterized by light winds and reduced turbulence. In addit ion, the lateral dispersion is bounded by canyon walls, giving potentially high concentrations downstream. The most likely canyon for at mospheric drainage from the SFTR site is Pajarito Canyon. which opens in the White Rock community about $10 \mathrm{~km}$ down-canyon; similar effects will hold for any canyon traversed by the cloud.

To establish confidence in concentration estimates, certain source factors must be determined, such as source size and duration, associated heat 


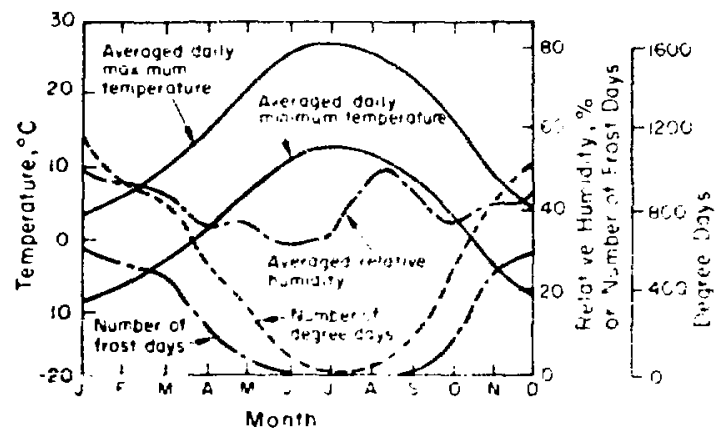

Fig. 7.5 .

Acerage annual cariation of thermal and humidia conditions at Los Alamos. N.M.

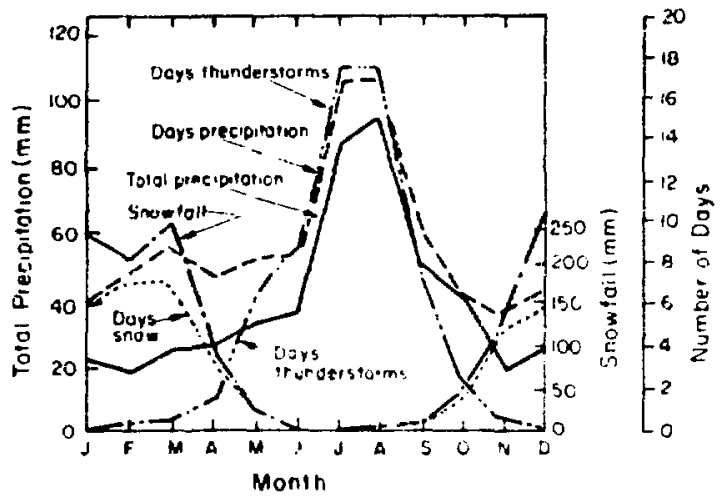

Fig. 7.6

Alrrager annual lariation of precipitation at Law Alumes. N.M.

release. parucle sizes, and some elements of the chemist ry of the released material for scavenging estimates. Also, many of the meteorological data on the influence of terrain, as shown in Fig. 7-7. are speculative and need to be upgraded b: fieic studies and computational models. Despite these potential shortcomings, tatimates of downwind concentration can be made. In the sample calculations presented here, a Gaussian plume model is used for a continuous, elevated-point source, as described in Ref. 1. Inituai buoyancy, particle settling, deposition, and transformation during travel are ignored. Sections 7.3.1 anci 7.4 .1 give calculational results for chronic and accidental releases, respectively.
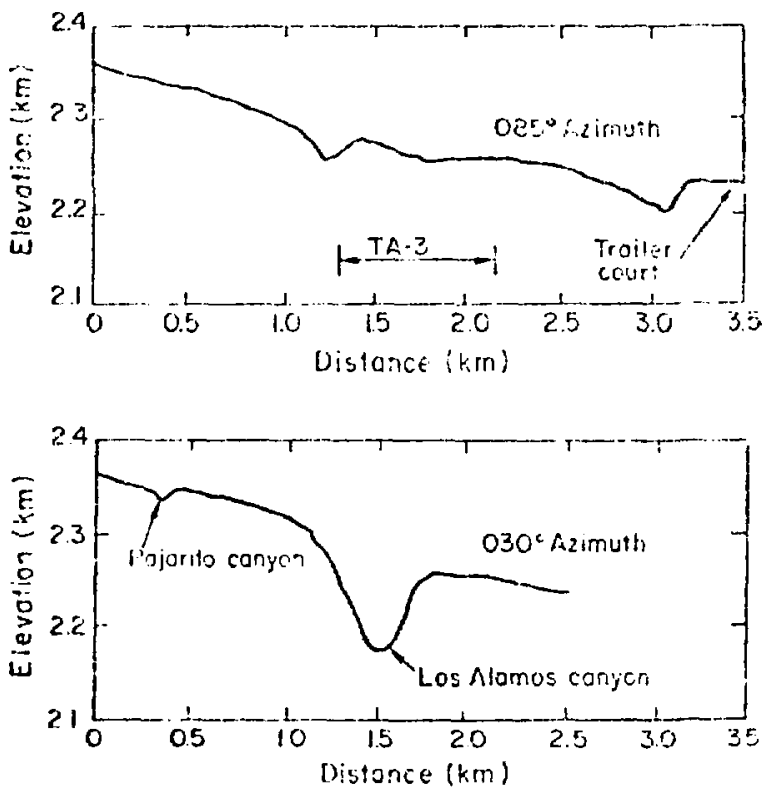

Fig. 7.7.

Terrain cariation along the $030^{\circ}$ and $085^{\circ}$ azimuths from SFTR site.

\subsubsection{Geologic and Hydrologic Descrijition}

Volcanic-flow rock and sediments underlie the site at the western edge of the plateat. A generalized section showing rock units and their thicknesses is given in Table 7.3 and is based on a test hole located ahout I mile northeast of the SFTR site.

The Bandelier Tuff consists of three inembers: Tshirege, Otowi, and Guaje youngest to oldest I. The Tshirege member is composed of a series of nonwelded to welded ashflows of rhyolite tuffs. The flow underlying the site is a weided tuff. The Otowi member is a nonwelded pumiceous ashflow of rhyolite tuff, and the Guaje member is made of an ashfall rhyolite pumice.

The Tschicoma Formation is composed of latite and quartz latite flows and pyroclastic rocks. The Puye Formation is composed of gravels and boulders of latite, quartz latite, rhyolite, and pumice in a matrix of silt and sand.

The individual ashflows of the Bandelier Tuff dip gently eastward off the flank of the mountains formed by the Tschicoma Formation. Because the tuffs are volcanic in origin, they have become highly 
TABLE 7-3

GENERALIZED SECTION OF SITE UNDERLAY
Handelier Tunf

Thirege member

()towi member

(iunje member

?orhicuma Furmation

Paice Formation

Tischicoma Formation

Puve Formation

Techicoma Formation

\begin{tabular}{|c|c|}
\hline $\begin{array}{l}\text { Thickness } \\
\text { (m) }\end{array}$ & $\begin{array}{c}\text { Depth } \\
\text { (m) }\end{array}$ \\
\hline 189 & IST \\
\hline fifi & והיטי \\
\hline is & 283 \\
\hline 115 & 3901 \\
\hline 119 & 148 \\
\hline Xַ & נהו \\
\hline 3 & $58: 3$ \\
\hline 1.60 & $7: 1$ \\
\hline
\end{tabular}

jointed during cooling. The Pajarito Fault is a northsouth trending fault about $1070 \mathrm{~m}$ west of the site. It is a normal fault and is down-thrown to the east. The estimated displacement is $122 \mathrm{~m}$. The Water Ca. nyon Fault also has a north-south trend and terminates about 1 mile southeast of the site. This fault is also a normal fault with maximum displacement of $24 \mathrm{~m}$. The movement on both faults has been post Bandelier.

Drainage from the site goes into Two-Mile Canyon, into Pajarito Canyon, and eventually into the Rio Grande. The stream in Pajarito Canyon is intermittent. The alluvium is thin but may contain small a mounts of wate: seasonally. The water is perched on the underlying tuff, but no water is perched in the volcanic rocks and sediments above the main aquifer. The main aquifer of the Los Alamos area is the only aquifer that can supply municipal and industrial water. The top of the aquifer at the site lies at a depth of about $110 \mathrm{~m}$ in the lower part of the T'schicoma Formation or upper part of the Puye Formation. The aunifer slopes gently eastward from the recharge area in the Jemez Mountains to the discharge area along the Rio Grande.

Los Alamos falls within seisnic zone 2 of the Cniform Building Code. ${ }^{2}$ On the basis of geologic evidence, historical data, and seismic records since 1962, an earthquake of 5.5 magnitude will occur every $100 \mathrm{yr}$, with an epicenter located somewhere in the Rio Grande depression between Questa and Albuquerque (an area extending $80 \mathrm{~km}$ north and 80 $\mathrm{km}$ south of Los Alamos and which is 32 to $64 \mathrm{~km}$ wide).

The Los Alamos area lies in the Rio Grande depression. which was formed by a complex series of faults. The seismicity of this part of the depression is (1) less than tire Albuquerque to Socorrusegment fot recorded earthquakes in New Mexico, $95^{\prime}$; oceurred in this latter segment and (2) substantially less than similar areas in Southern California.

Ground subsidence caused by the withdrawal of water, by landslides, and by renewed volcanism at the SFTR site has been considered. Ground subsidence is unlikely trecause of the already low ground water level and phvisical structure of the waterhearing levels. Landslides, texcluding rock falls at the canyom lip. do nor oceur because of the geological itructure and dry condition of the mesa rock. Indications are that volcanic as ivity is decreasing: in the past 20 yr fumaroles have not been active and thermal springs have become cooler.

\subsubsection{Access and Security Aspects}

The site is located on the west edge of the TA-13 area (Fig. $7-1$ ) and adjacent to the east sicte of siate Road 4. It is $1.22 \mathrm{~km}$ from the LASL Administ ration Building and $1.53 \mathrm{~km}$ from the nearest fire station. A paved ruad to TA 6 i provides direct access of the site, In addition, a paced road is to be const ructed ac ross Two-Mile Canyon to shorten the access to TA-3. Because the site lies within a serurity area, a fence will be required to the south to separate it from classifird technical areas, and a second fence to the north will be required fo restrict public access.

\subsection{SAFETY AND ENVIRONMENTAI. CONSIDERATIONS DURING NOHMAI. OPERATION}

Operation of the SFTR is not expected to create any significant personnel safely problems not already encountered an $I$ successfully dealt with at existing reactor or accelerator facititips Tho dose equivnlent (DF) from direct radiation will be under $500 \mathrm{mrem} / \mathrm{yr}$. less than the design goal of $1 \mathrm{rem} / \mathrm{yr}$ for new facilities." Exposures from other sources of radiation (tritium-induced activity) wili be minimized by shielding, remote handling. glovebox. es. personnel cont rol, etc.

Environmentally, the DE's to the public from routine operation of the SFTR will be the sum of the effects of small releases of activated air and tritium and of the direct neutron radiation during D-T power pulses. These DE's are analyred in detail in the sections that follow and are summarized helow. 


\section{ANNCAL, WHOLE-BODY DOSE RQUIVALENT \\ (mrem/yr)}

\begin{tabular}{|c|c|c|c|c|}
\hline & $\begin{array}{l}\text { Tritium } \\
\text { (max) }\end{array}$ & $\begin{array}{l}\text { Activated } \\
\text { Air }\end{array}$ & $\begin{array}{l}\text { Direct Neutrons } \\
\text { (estimated) }\end{array}$ & Total \\
\hline 110 & 0.2 & 11.1165 & $<4$ & $<4$ \\
\hline Fodgo al liwnsife & $0.0: 3$ & 0.61 & $<1$ & $<1$ \\
\hline
\end{tabular}

These DF:s are very small compared with normal background radiation in los Alamos county 11301xi) $\mathrm{mrem} / \mathrm{yr}$ ) and with the radiation protection standards for the public given in ERDA Manual Appendix $(52+15(*) \mathrm{mrem} / \mathrm{yr}$ (maximum/ or 170 mrem/sr laverage)l:" and do not constitute a signifirant risk to pablic health. In addition. it should be emphasized that [3.T operation of the sFTl is expected to last snly about 1 yr, or long emough to produce the radiation doses given in the abue lable.

\subsubsection{Tritium Inventory and Release?}

The quantities of tritium con-site in the SFT'R will amount to tens of kilocuries Every effort has been made to keep the tritium inventory to a minimum. to keep most of the tritium in accident-proof configurations. to allow an absolute minimum in the firm of gas. and to exercise all necessary care in the release of tritium to the enviromment. Some of the measuras incorporated in the design and operational procedures in the SFTR facuity are given below.

The normal plant inventory of (ritium ( $T$, ) is expected to be $3 \mathrm{~g}$. with a projected absolute maximum of $7 \mathrm{~g}$ or $70(90) \mathrm{Ci}$. About $90^{\prime} ;$ will be secured in a fue - and blast-prowf vault having a combination lock: the vault may be alarmed. Fizure $7-8$ is a schematic of the distribution of the tritium inventory throughout the facility.

The $15 \mathrm{mg}$. or $150 \mathrm{Ci}$. of tritium that will be used in a burn pulse will be transferred to the cell. plasma chamber, and associated piping and pumps lor 10 min. Four such D.T shots are planned per day. After each shot the (D,T): mixt ure will be t ransferred from the SFTR cell for processing and reclamation. The remainder will be in the form of tritiated water on molecular sieve beds or as uranium hydride. About 10 ', of this remaining tritium will be in a tritide form. Except for tritium in the SFTR discharge tulse, all tritium will be in metal containers or metal piping.

Tritium handling. shipping. and release to the environment will be carefully controlled to meet ap-

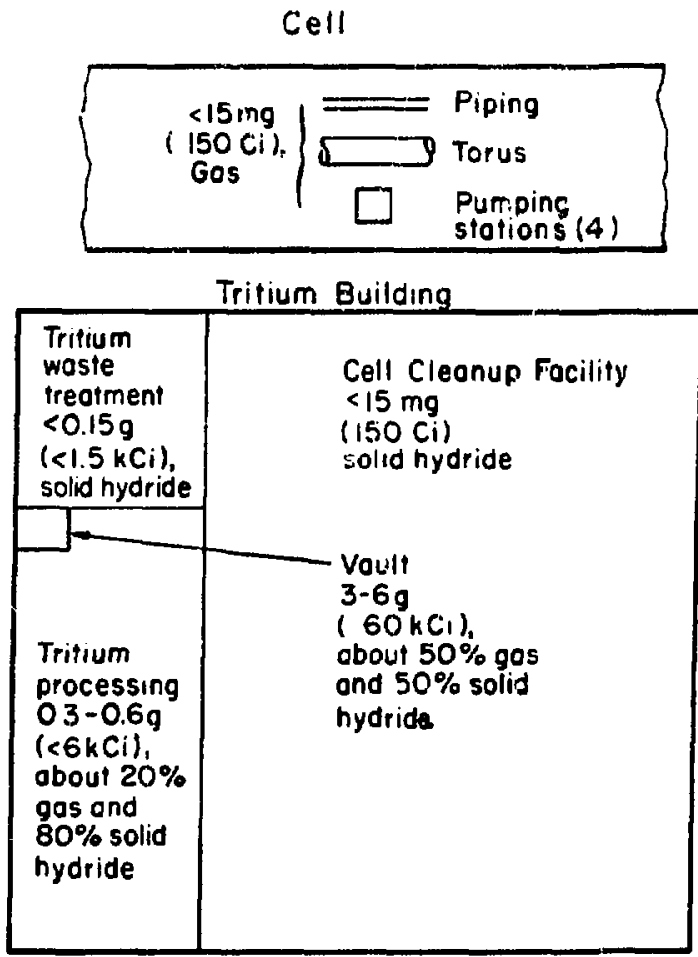

Fig. 7-8.

Schematic diagram of tritium inventory in the SFTR facility.

propriate federal regulations. All contaminated gas exhausted from the SFTR facility will have an average tritium concent ration less than $0.2 \mu \mathrm{Ci}, \mathrm{m}^{3}$. Since the stack exhaust rate will be $4.7 \mathrm{~m}^{3} / \mathrm{s}(10000$ (fin), the daily tritium release will be less than 82 mCi. Stack gas will be continuously monitored, and the discharges will be recorded by the LASL Health Division on a monthly basis.

A second form of tritium waste will be tritiated water from the tritium waste treatment dryers and cell cleanup dryers. The waste treatment dryers should operate for several months to a year without 
rexeneration. The tritium content in these units will depend almost exclusively on the degree of the (I).T).. (hemical reaction with the plasma chamber wall. If it is assumed that $l^{c}$, of the (D. T) ., mixture reatets with the wall to form (D.T), O, 6 ( $\mathrm{ci} / \mathrm{day}$ will be collected, or $1500 \mathrm{Ci} / \mathrm{yr}$. The water collected in the units would consist mostly of $\mathrm{D}_{2} \mathrm{O}$ collected from the deuterium pulses. and would amount to 8 /day . vapor, or $\_\mathrm{kg} / \mathrm{mi}$. Thus, if no other releases of t ritium anurred. regeneration of the drvers would give 1:000) C $i$ of tritium in a few pounds of water. This water might be processed elsewhere. but probably would be sealed in double containers for burial.

Maintenance operations in the hoods might release tor much tritium to be stacked. An effort will be made to process such gas in the tritium waste treatment (TWT) unit which handles $T / 1 / \mathrm{s}(1.5 \mathrm{~cm})$ at a pressure of 600 torr. If such an operation processed air of $30 \%$, relative humidity at $25^{\circ} \mathrm{C}$ for 15 min. about $40 \mathrm{~g}$ of water would be collected. The result would be more frequent regeneration of the drver with a corresponding reduction in the tritium cuncentration in the collected water.

Accident al tritium releases in the tritium preparation hood ' $\mathrm{r}$ in the vault might release up to $3 \mathrm{~g}$ of $\mathrm{T}$. which woild be handled by the 7 -i/s TWT unit. Tritium releases in hoods would be handled similar$l y$. but would result in a smaller inventory in the $\bar{r} / / / \mathrm{s}$ drver. In these cases. regeneration of the dryers would depend on the humidity of the air and the processing time: for a release in the vault, regeneration yrobably would be initiated following collection of the tritium.

Aside trom stack gas and tritiated water collected by the drvers, the only tritium waste routinely released from the site would be found on contaminated hardware and equipment which would be buried in sealed containers according to standard LASL procedures. Valve seats might require routine replac ement. Cryosorb panels in the cryosorption pumps might also have a limited life: the tritium contamination here would probubly be less than a curie.

Central to the successful control of the relatively small quantities of tritium used by the SFTR experiment are (1) the monitoring of small quantities of tritium and (2) the institution of unambiguous and accurate control and inventory procedures. Contro! and inventory procedures are discussed in more detail in the following subsections. Radiation doses from "routine" tritium releases are also discussed.

\subsubsection{Tritium Control and Inventory Procedures}

Tritium Assignments. Tritium inventories will be assigned to individuals. and transfers between individuais will be handed by signed receipt, using forms similar to those presently in use by LASI. Group CMB-3. Monthly summaries of these inventories will be compared with a phrsical inventory taken at the same time. Permanent tritium inventory records will be kept. No deuterium inventory will be kept: deuterium will be considered expended upon receipt.

Tritium Security. All tritium that is easily portable will be stored in a vault with a combination lock. It will be treated as classified material, and the vault may be subject to rout ine security checks dur. ing nonworking hours. Fach person is respomsible for the security of tritiom assigned to him.

Physical Inventory. A physical inventory of the iritium in the SFTR will be made each month. Several of the quantities will be estimated. The inventory will normally consist of the following:

1. One or two 12-i containers of $T:$ stored in the vault. No more than one container will be full.

2. Tritium recovered as uranium deutero-tritide and stored in steel containers in the vault. Normally. any tritium to be sent out for recovery will be picked up at the time of delivery of a shipment (a 12-/ container of $T, 2$ would normally be received once every two or three months).

3. Tritium recovered possibly as (D.T). stored in one or two 12-/ cont ainers either for reuse or for shipment. U(D,T $)_{3}$ storage would be correspondingly reduced.

4. Tritium located in the preparation hood as solid on one or two uranium beds within metal containers, and tritium (during working hours) in one or two metal storage bulbs.

5. Tritium as solid on one or two uranium beds (traps) within metal containers in the recovery hood. These traps would be transferred to the vault each week.

6. Tritium gas within the discharge tube and in transfer lines and metal containers. A few times a day $100-200 \mathrm{Ci}$ of tritium would be at pumping stations, in lines in the cell, or in the discharge tube for short intervals during working hours

7. Tritiated water in the TWT unit.

8. Tritiated water, possibly in the Cell Cleanup Facility (CCF). 


\section{Tritium Measurements.}

1. Tritium receipts: Tritium will be received in standard $12-1$ containers at 2 atm pressure. The tritium content as assigned by the supplier will constitute the primary measure of the tritium in each container. This content will be checked by a PV'? measurement. Mass spectrometer analysis of the gas will be made periodically as the gas is used. Since the gas will be measured as it is used, a cumulative accounting of this tritium will provide an independent determination of the tritium in a given containcr. When significant ifew percent) differencess appear between assigned and measured values. aq)propriate measures will be laken to determine the cause.

2. Trizium stored as [T(D.T) 3 and [(H.D.T): The stainless steel containers of uranium tritiated hydride will be measured calorimetrically. Mass spectrometer analyses will give the composition of the hydrogen isotopic concentrations. For low tritium concentrations, PITT and mass sipectrometer analyses unight be used without calorimetry. Tritium on $\mathrm{C}(\mathrm{D}, \mathrm{T})_{33}$ traps in the preparation and recovery hoods will be culculated from PVI and mass spectrometer analyses and careful accounting of gas $t$ ransfers onto and off the traps.

3. (ias storage containers in vault: If (D, T). is collected as the gas. either for reuse or for shipment off-site for recovery. $\mathrm{P} \backslash \mathrm{T}$ and mass spectrometer analyses will determine the quant ities. The receiver. however, will normally assign a more accurate value in the contents, and this value will be accepted. In case of unreasomable variances, the $(\mathrm{DD}, \mathrm{T})_{3}$ container could be measured calorimetrically betore decomposition. collection, and shipment.

4. Storage coutainers in hoods (standard volume containers): Again. PVT and mass spectrometer antalysis and careful accounting will be used to determine tritium stored in these containrs.

5. Tritium Waste Treatment Facility (TW'T): Any water collected from regeneration cycles will he sampled and the tritium content determined by scintillation counting or other standard techmiques by Group H.1. Tritiated water going into the TWT wili be measured by PVT methods and analyzed occasionally. lnaccou ted-for tritium will probably be assigned to this uatit except in case of knuwn losses or unreasonably large quantities.

The careful accounting of the tritium contained in the large number of mass spectrometer samples-each of low tritium content-sicarcely seems warranted. Also the error is large in mass spectrometer analyses of samples containing ap- preciable quantities of protium such as might be expected in overnight outgassing, etc.

6. Cell Cleanup Facility (CCF): Excellent estimates of the tritium content in this facility can be expected, since transfers to this unit will usually involve the transfer of accidentally released. but measured, quantities of tritium. This unit probably will be little used.

7. Labeling of piping and ducting: All piping and ducting will be painted a characteristic color and carefully laheled for immediate identification.

8. Alarms: The alarm system has not been designed in detail. In general, tritium air concentrations above predetermined values will activate visual and audio alarms in areas where overconcentrations occur and at control points. The following section describes the tritium monitoring system envisioned for the SFTR facility.

9. Group H-1 responaibilities: Group $\mathrm{H}-1$ will have control of tritium safety measures. routine maintenance of counting equipment, monitoring of personnel, and preservation of records.

\subsubsection{Tritium Monitoring Procedures}

Monitoring for tritium will be done by state-ofthe-art. flow-through ionization chambers modified as noted beiow when monitoring for tritium in activated air. Such instruments are available with electrometer sensitivities of $10^{-15}$ to $10^{-17}$ A. A 20-/ chamber can measure a tritium concentration of 0.2 $\mu \mathrm{Ci} / \mathrm{m}^{33}$ resuiting in chamber current of $4 \times 10^{-15} \mathrm{~A}$. Such instruments can be used for stack monitoring with sensitivities down to the maximum permissib!e concentration $\left(\mathrm{MPC}_{\mathrm{a}}\right)$ for the public $\left.0.2 \mu \mathrm{Ci} / \mathrm{m}^{3}\right)$.

Tritium monitoring in the cell and cell exhaust air presents a special problem. During a D-T pulse of 4.5 $\times 10^{18} \mathrm{n}$. approximately $20 \mathrm{Ci}$ of $16 \mathrm{~N} .100 \mathrm{mCi}$ of ${ }^{12} \mathrm{~N}$. and $20 \mathrm{mCi}$ of ${ }^{41} \mathrm{Ar}$ would be produced in the cell air. Most of the ${ }^{10} \mathrm{~N}$ produced is partially trapped in the air spaces of the inner confines of the primary shield and capacitors. Because of its short half-life. only the amount formed outside the shield needs to be considered during monitoring for tritium in the cell in the presence of the activated air. Thus, the maximum total concentration of high-energy beta radiation out side the shield is about $6 \mu \mathrm{Ci} / \mathrm{m}^{\text {? }}$.

Tritium in such an atmosphere can be monitored easily by a technique recently developed at LASL. ${ }^{4}$ The method employs conceit ric ion chambers with a thin common wall separating the inner flow-through signal chamber from the outer compensating chamber. The currents produced by the high-energy radiation from the two chambers can be completely 
subtracted, leaving only the curront from the tritium in the inner chamber. In the above atmosphere, a tritium concentration of less than $0.1 \mu \mathrm{Ci} / \mathrm{m}^{: 3}$ can be measured with a $2(0)-i$ (inner) chamber using a 30 -s time contant.

Monitors with dual chambers will be used for the cell and possibly for the stack. The cell hood monitors, because of their lower tritium sensitivity requirements, will not be equipped with dual chambers. Single-chamber monitors will also be used in the tritium-handling hoods and in rooms where air activation does not occur.

The signal to the valves in the exhaust air ducts that route the tritium to the recovery sistems must sometimes be given rapidly when, within a $24-h$ period, about $20 \mathrm{mCi}$ of tritium have pasised a sampling point. Although this action could be accomplished with rate instruments, system response will be faster if the current coming directly from the ion chamber is integrated. Such integrating circuits ar common and can be wired to give a signal at the desired instant. These integrating monitors are planned for all five tritium hood exhaust ducts, the $2810-1 / \mathrm{s}$ duct, and for monitoring the performance of both tritium, recovery systems. Where air activation is a problem, dual concentric chambers will be employed. Figure 7-9 summarizes the location. type. and function of each tritium momnitor at the SFTR facility.

When ion chambers are used to ineasure tritium concentrations of less than $1 \mu \mathrm{Ci} / \mathrm{m}^{3}$, radon in the air can be a serious prob!em. Therefore, the concrete walls in the cell will be coated with a sealant to reduce the cell concentration from this source to less than $10^{-13} \mathrm{Ci} / !$. Radon in the inlet air will be at the loutdoor) ambient concentration $\left(\sim 10^{-14} \mathrm{Ci} / /\right)$, and will be no problem.

Alternative methods tn ionization chambers for monitoring tritium are available. Proportional counters and plastic scintillators have been developed for continuous monitoring of airborne tritium. The counters have the advantage of pulsehejght discrimination and, therefore, may be useful in removing much of the radon alpha and highenergy beta radiation background. Unfortunately, these counters also have disadvantages which may include strong memory effect, a requirement for a continuous gas supply, a long waiting time or long time constant, and lack of sensitivity.

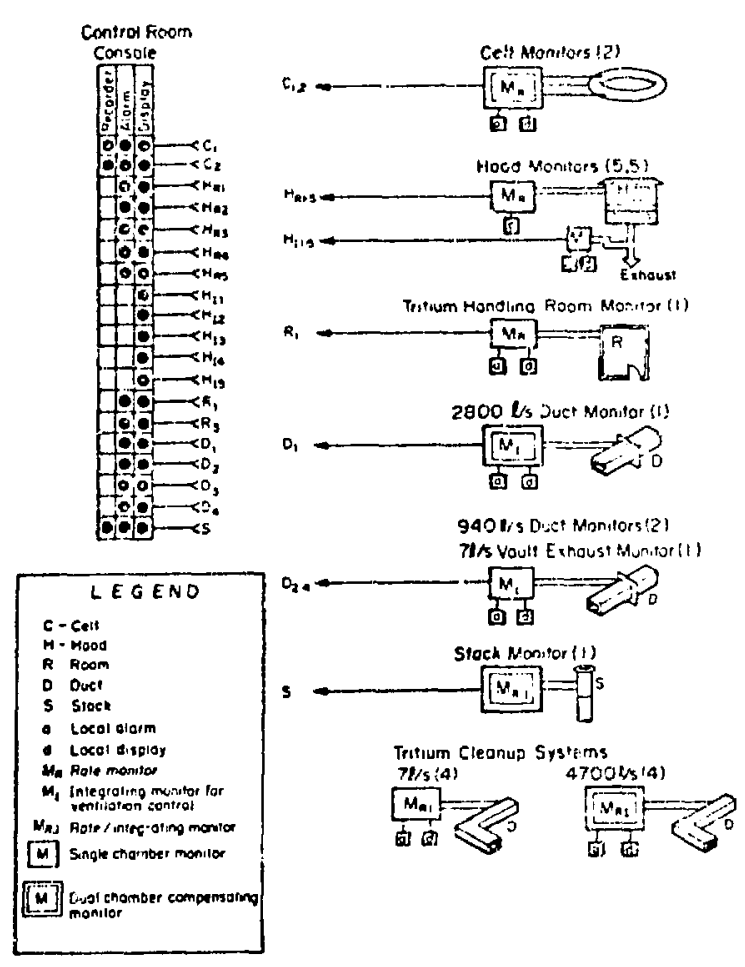

Fig. 7-9.

Schematic of tritiumi monitoring system and control functions.

\subsubsection{Radiation Dose Rate Kesulting from Routine Tritium Releases}

No simple method is available to estimate the amount of tritium that will routinely leak of diffuse from the various containers, pumps, or plumbing. Whatever this leakage rate, the tritium recovery systems will trap all but an insignificant amount before the tritium containment air is exhausted into the atmosphere through the stack. The design of the claanup/recovery systems is such that the average daily concentration in the stack will not exceed the maximum permissible concentration for the public of $0.2 \mu \mathrm{Ci} / \mathrm{m}^{3}$ for tritium as $\mathrm{H}^{\prime} \mathrm{TO}$ or $\mathrm{T}_{2} \mathrm{O}$ in air. With a stack tlow of $4.7 \mathrm{~m}^{3} / \mathrm{s}$ the maximum discharge rate will be about $82 \mathrm{mCi} /$ day. It is expected that the long-term average concentration in the stack will be substantially less than the $\mathrm{MPC}_{\mathrm{a}}$. However, the 
'TW' and C CF will be operated to keep the stack exhaust tritium under the daily limit of $82 \mathrm{mCi}$. This maximum discharge rate is expected to be maintained in the event of accidental releases. Such releases may involve, for instance, part of the $150 \mathrm{Ci}$ of trititim in the reaction cliamber resulting from mechanical failure of the chamber wall during a D-T pulse. Sitch a failure may occur $2-3$ times per month during the 1 yr of D.T operations. Other significant releases are extremely improbable. Small releases involvin less than $1 \mathrm{Ci}$ are expected when contaminated plumbing is disassembled.

Annual average iscoleths $(x / Q)$ in $\left.s / \mathrm{m}^{3}\right)$ were calculated for the SFTR site based on wind and wher at mospheric data compiled at the LASL Ad. ministration Building Weather Station llocated about $i .5 \mathrm{~km}$ from the proposed site). These data are plotted in Figs. $7-3$ and $7-4$ for two stack heights $(30$ and $50 \mathrm{~m}$. No allowance was made for terrain features which would interrupt the normal flow toward the population centers.

From the isopleths for the $30-\mathrm{m}$ stack, the annual doses to persons persistently exposed to tritium being chronically released at the maximum discharge rate from the SFTR stack were calculated and transferred to the same isopleth curves. The tritium was assumed to be completely oxidized, and absorption : hrough the skin was included. Figure 7.10 gives the chronic tritium whnle-body dose curves. On the basis of these calculat,ons no member of the public would receive over $1 \mathrm{mrem} / \mathrm{yr}$ even if the maximum average release rate were to be increased manyfold.

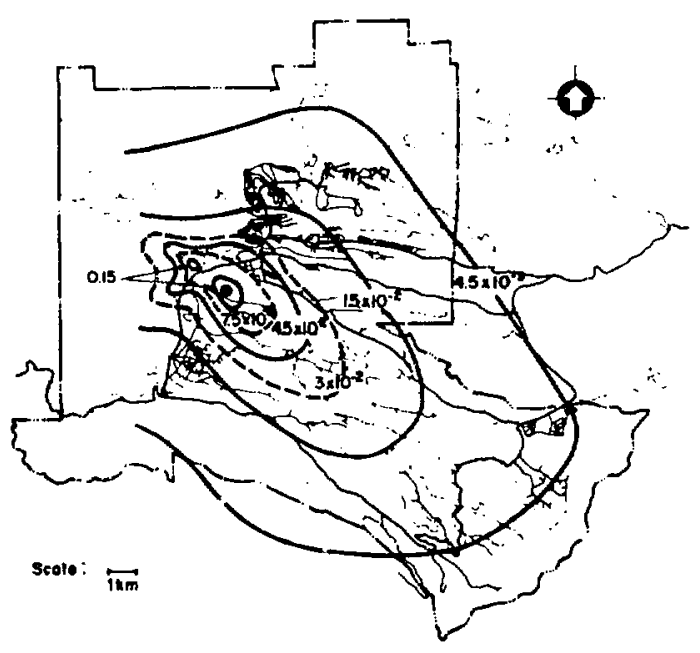

Fig. 7-10.

Chronic tritium whole-body isodose curves ( $\mathrm{mrem} / \mathrm{yr}$ ) for SFTR tritium released from a 30-m stack.

\subsubsection{Direct Neutron Irradiation Exterior to the SFTR Cell}

The neutron source is conservatively esimated to he $4.5 \times 10^{14} \mathrm{n}$ per D.T burn pulse and $10^{3}$ pulses/vr. These neutrons will be generated in a toroidal plasma chamber (minor raditis $0.1 \mathrm{~m}$. major radius $40 \mathrm{~m}$ ) surrounded by magnet coils, electrical insulation, and a primary shield.

The main function of the primary shield (Sec. 5.3.2. laminated aluminum, graphite, and borated!eaded polvethylene) is to limit the aciivation of capacitors and reduce damage 10 organic insulators. Secondary but important iunctions of the shield are the reduction of air and wail activation in the cell and the shielding of personnel from the high gamma exposure rates of the activated toroidal structure. This problem is discussed in some detail in Sec. 7.3.3.

The neutron dose per $4.5 \times 10^{18} \mathrm{D}$-T burn pulses in the cell (Fig. $7-11$ ) is given in Fig. $7-12$. The effectiveness of the primary shield in reducing the neutron dose can be seen. Combined with the primary shield, the $1.5-\mathrm{m}$-thick cell wall of ordinary concrete pruvides sufficient shielding to reduce the dose equivalent (DE) immediately outside the tunnel to less than the design value of $0.5 \mathrm{rem} / \mathrm{yr}$. Since the SFT'R experiment is expected to be completed with feurer than $4.5 \times 10^{21} \mathrm{D}-\mathrm{T}$ neutrons produced, the 0.5 rem can be assumed as the maximum DE for the experiment, since the D-D neutrons are not expected to add much to the background radiation by virtue of their lower numbers and energy. The corresponding dose values in the parking area and at the closest site boundary, without additional shieiding, are approximately 100 $\mathrm{mrem} / \mathrm{yr}$ and $8 \mathrm{mrem} / \mathrm{yr}$, respectively. The coriservativeness in the shielding calculations and in the assumptions of no additional intervening walls or equipment strongly suggests a further reduction of these neutron doses by at least a factor of 2 or 3 .

\subsubsection{Neutron Activation of Equipment, Cell At- mosphere, and Shielding}

The neutron activation in the cell and resulting DE rates to personnel following D-T burn pulses were calculated using the same neutron transport code used for Fig. 7-12. The calculations assume a speciully designed, $0.5-\mathrm{m}$-thick laminated primary shield (Sec. 5.3.2) which immediately and almost completely surrounds the toroidal structure. Because of electrical and vacuum lines, complete shielding is unattainable. 


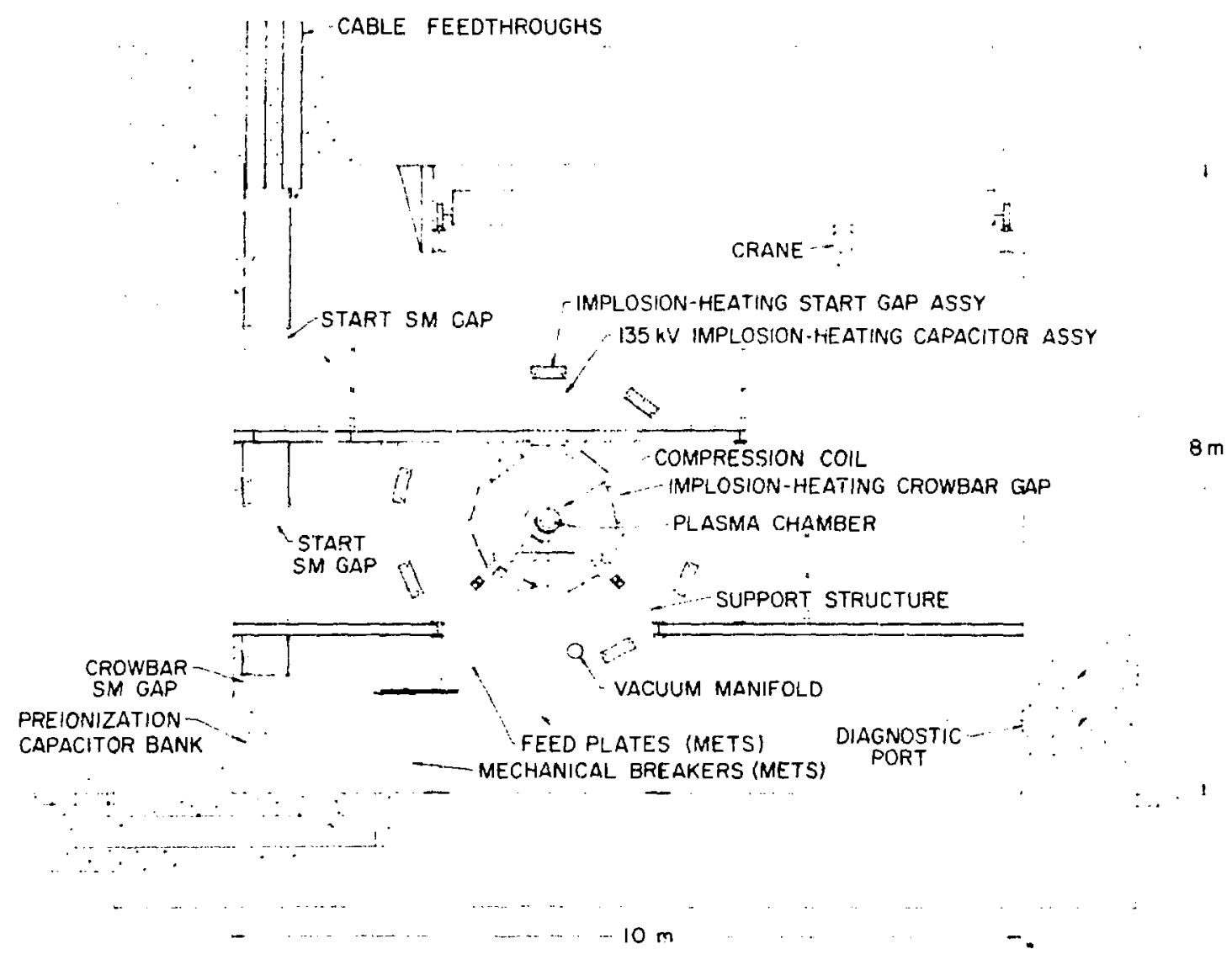

Fig. 7.11.

Cross section of SFTR cell, radiation shielding, and structure that is susceptible to activation by neutrons.

Calculations show that activation products outside of the primary shield present ro significant exposure problems to personnel in the cell. Similarly, the quantities of radioactive gases discharged to the environment will be minimal. The only appreciable radiation levels occur when the primary shield is removed for maintenance or replacement of sections of the activated toroidal structure.

\subsubsection{Activation of Cell Ajr}

Table 7-4 summarizes the results of calculations made to determine the quantitias of radioactive gases formed in the cell during a D-T burn pulse yielding $4.5 \times 10^{18} 14-\mathrm{MeV}$ neutrons. This is considered a maximum yield. The planned spacing between pulses will not permit concentrations of the radioactive gases to build up within the cell.
Reentry into the cell following a pulse will not be restricted because of the gases, since even for the case of ${ }^{13} \mathrm{~N}$ the maximum $\mathrm{DE}$ rate is less than 3 $\mathrm{mrem} / \mathrm{h}$. Of greater concern to persunnel is the structural activation following a pulse (Sec. 7.3.3.2).

Of the nuclides listed in Table 7.4 only ${ }^{1.3} \mathrm{~N}$ and ${ }^{41} \mathrm{Ar}$ are of local environmental concern; ${ }^{16} \mathrm{~N}$ (halflife, $7.1 \mathrm{~s}$ ) will decay to insignificant levels before reaching anyone outside the SFTR ceil. Even the activity of $13 \mathrm{~N}$ (half-life of $10 \mathrm{~min}$ ) will decrease appreciably as it is slowly removed from the cell and carried downwind from the SFTR site. Nitrogen-13 is a pure positron emitter and. like ${ }^{41} \mathrm{Ar}$, which is both a beta and gamma emitter, will be responsible for both skin and : $:$ hole body doses received by ar. exposed person downwind. The 110 -min half-life of ${ }^{41} \mathrm{~A}$ will be reduced by one-half by the time it reaches the environment. 


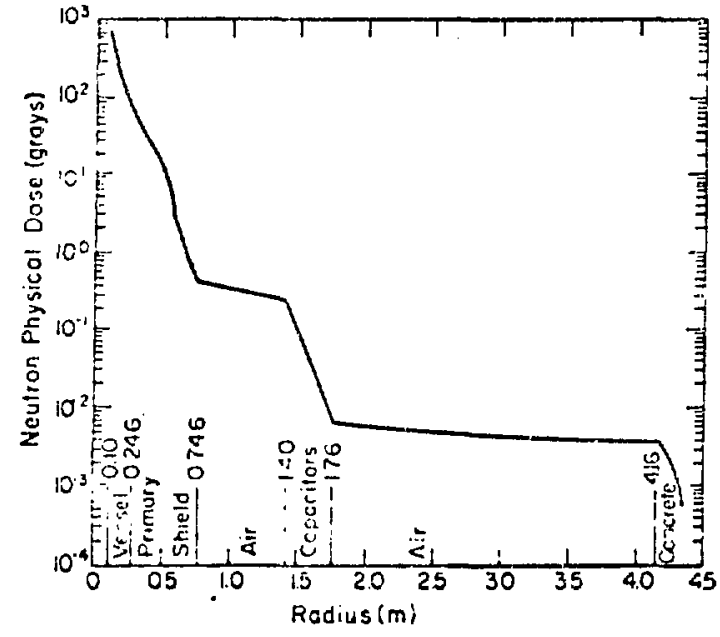

Fig. 7-12.

Veutron phvsical dose per $4.5 \times 10^{18}$ D.T neutrons deposited in the SFTR cell.

Using an average wind speed of $2 \mathrm{~m} / \mathrm{s}$ to calculate the decay of the $13 \mathrm{~N}$ and ${ }^{41} \mathrm{~A}$ as they are carried downwind, and the isopleths in Fig. 7-3 for a $30-\mathrm{m}$ stack. the annual beta skin and gamma whole body
DE's were calculated for an annual production of 4.5 $\times 10^{21} \mathrm{D}$-T neutrons and plotted in Figs. 7-13 and 714. From these it is concluded that the activated tunnal atmosphere does not. constitute a significant public hazard.

The activity induced in the cell wall (biological shield) is not expected to contribsto to the exposure rates resulting from the total neutron-induced radioactivity in the tunnel. As in the case of the capacitors, the neutron fluence per shot outside of the primary shield is reduced to the point where the resultant activities in the tunnel wall are insignificant.

\subsubsection{Structural Activation}

Activation of structural equipment and material within the SFTR cell is considerably reduced by the laminated primary shield that surrounos the plasma chamber. For instance, the activity of each of the capacitors closest to the shield following a maximum yield D-T pulse $\left(4.5 \times 10^{18} \mathrm{n} /\right.$ pulse $)$ is only about 0.2 inCi. Thus. the initial exposure rate from all capacitors at floor level is less than $1 \mathrm{mR} / \mathrm{h} .^{*}$ and quickly decays.

At floor level and with the shield in place, the ex. posure rates after $10^{3}$ pulses over $1 \mathrm{yr}$ from the copper compression coils, plasma chamber, and primary shield structure are somewhat higher: about $250 \mathrm{mR} / \mathrm{h}$ initially, $40 \mathrm{mR} / \mathrm{h}$ in $1 \mathrm{~h} .5$

${ }^{*} I-m R / h$ exposure rate is equivalent to a $I-m r e m / h$ DE rate.

TABLE $7-4$

RADIOACIVE GASES FORMED IN THE CELL FOLLOWING A MAYIMUM-YIELD D-T BURN PULSE

\begin{tabular}{|c|c|c|c|c|c|c|}
\hline \multirow[b]{2}{*}{ Nuclide } & \multirow{2}{*}{$\begin{array}{c}\text { Initial } \\
\text { Activity } \\
\text { (Ci) } \\
\end{array}$} & \multirow{2}{*}{$\begin{array}{c}\text { Physical } \\
\text { Half-Life } \\
\text { (min) } \\
\end{array}$} & \multirow{2}{*}{$\begin{array}{l}\text { Effective } \\
\text { Half-Life } \\
\text { (min) } \\
\end{array}$} & \multicolumn{2}{|c|}{$\begin{array}{c}\text { Initial DE } \\
\text { Rate } \\
\text { (mrem } / \mathbf{h})\end{array}$} & \multirow{2}{*}{$\begin{array}{c}\text { Total Activity } \\
\text { Released at Stack } \\
\text { Exhaust } / 10^{3} \text { D-T } \\
\text { Pulses }{ }^{b} \\
\text { (Ci) }\end{array}$} \\
\hline & & & & Gamma & Beta & \\
\hline $\operatorname{lin}$ & 0.11 & 10 & 8.6 & 9.2 & $: 3.3$ & 15 \\
\hline$" N$ & 17 & 0.12 & 0.12 & $5 \times 10^{4}$ & $1.7 \times 11^{3}$ & $0.0444^{\circ}$ \\
\hline${ }^{H} \mathrm{Ar}$ & $1.8 \times 10^{-2}$ & 110 & 40 & 2 & 0.57 & 12 \\
\hline $\mathrm{Ar}$ & $4.5 \times 10^{-9}$ & $1.4 \times 10^{8}$ & 62 & $\sim 0$ & $\sim 0$ & $4.5 \times 10^{-65}$ \\
\hline $14 \mathrm{C}$ & $3.0 \times 10^{-7}$ & $3.0 \times 10^{9}$ & 62 & 0 & -11 & $3.0 \times 10^{-1}$ \\
\hline
\end{tabular}




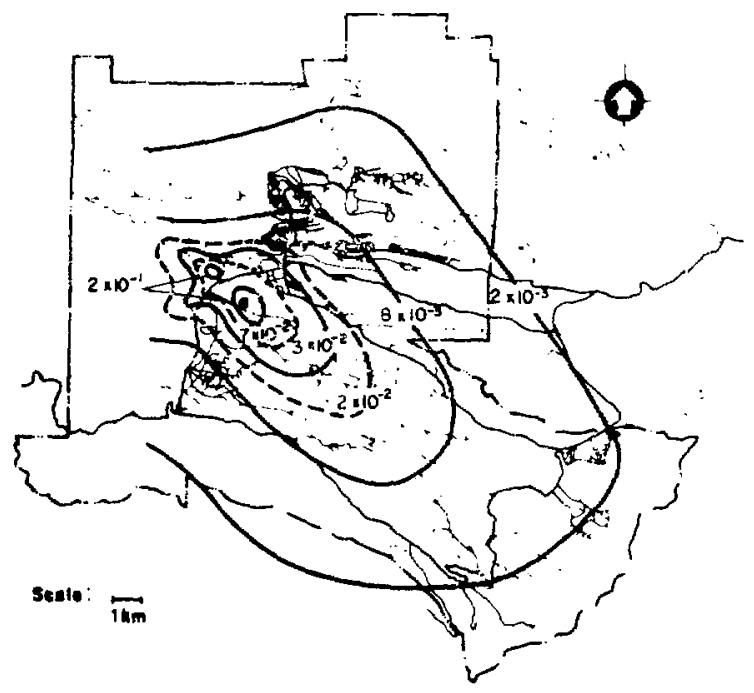

Fig. $7-13.3$

Beta radiation skin isodose curces (mrem/yr) for SFTR acticated air released from a $30 \cdot \mathrm{m}$ stack.

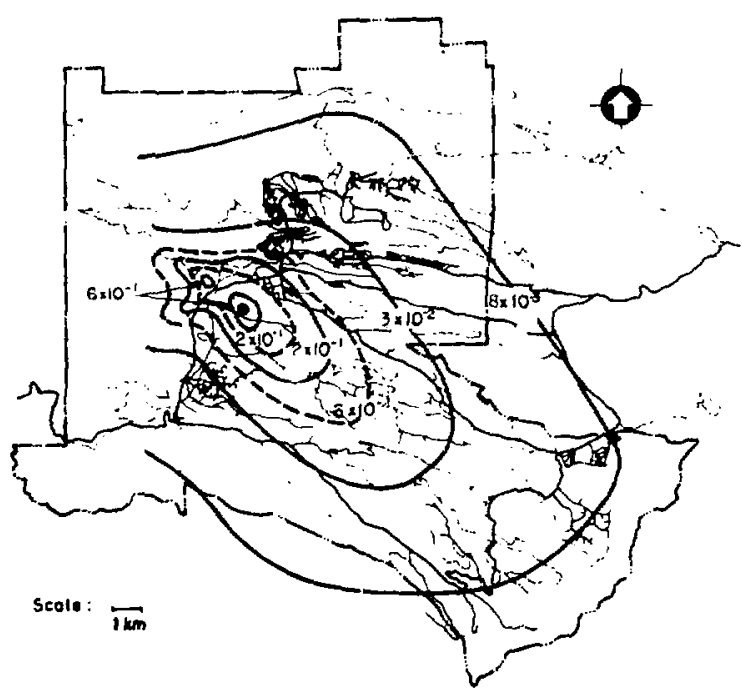

fig. 7-14.

Whole-body gamma-ray isodose curves (mrem/yr) for SFTR activated air released from a $30-m$ stack.

$\mathrm{mR} / \mathrm{h}$ the following day, and about $2 \mathrm{mR} / \mathrm{h}$ in a week. The shielding effect of the capacitors is not included but is expected to be significant. They wouid be helpful in supplementing any local shielding provided for those working in the cell room after shutdown.
Within the primary she od the activities are as expected. quite high. When the shield is removed lor maintenance o: replacement of a section of the plasma chamber. remote handling will be reyuirerl. The exposture rate from a $10.4-m$ coil section wil be high initially $(-15 \mathrm{R} / \mathrm{h}$ at $1 \mathrm{~m}$ ). bat will ciecas 10 $-100 \mathrm{mR} / \mathrm{h} 24 \mathrm{~h}$ later. On the other hand, a $9.4-\mathrm{m}$ discharge tube would have corresponding rates of 1 and $\dot{g}(1) \mathrm{mK} / \mathrm{h}$. Handling ot these tuhe and coil sections with the primary shield remored wonld be dome remotely.

The above estimates are the result of calculations based on $10^{: 3}$ D-T maxinum yield pulses per year and are thus high compared to those expected during the early part of the D-T phase of the experiment. This is particularly true for the activities and corresponding exposure rates a day or mure after shutdown when the long half-life isotopess begin to dominate.

\subsubsection{Power Hequirements and Thermal Effects}

Power requirements of SFTR are described in

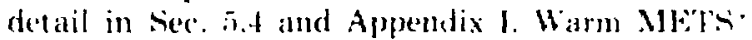
requires a dedicated $115-\mathrm{kV}$ transmission line from the Vorton switch line (near Buckman Mesal tu carry power to the ETA switchyard and ultimately to a switchyard at the SFTR site. The peak power requirement will be $1+1$ MVA. with an average power of 7 MWe. Most of this power will be dissipated at the SFTR site and will have insignificant environmental effects. For instance, the energy dissipated on each charging operation equals 7.4 iimes the magnetic field energy stored in the Warm METS energy-storage inductor. The field energy, in turn, is dissipated eventually in the inductor itself, the com pression coil, and the crowbar. Therefore, a total energy of 4.2 G.J is dissipated during each pulse, accumulating to $8.4 \mathrm{TJ}(2.3 \mathrm{MWh})$ over the 2000 shot life-expectancy for the experiment. This accumulated energy is small when compared, for example, with the lighting load of of an industrial building complex (lighting load of is $\mathrm{W} / \mathrm{m}=$ is assumed 1. "This implies that a thorr space of $80 \mathrm{~m}^{2}$ will represent the same integrated load over a $2-y r$ period, if the year consists of $2112 \mathrm{~h}$ of lighting. On the other hand, the duty factor for 2000 shots ower a 2 -yr period is 0.12 : hence the inst antaneous thermal output of the environment is the equivalent of the light load of a $670-\mathrm{m}^{2}$ noor space. The power requirements and associated thermal effects. therefore, will not be serious.

* If the carious options for energizing the compression coils, the "Warm METS" scheme requires the most power, so we assess its impact. 


\subsubsection{Radioactive Waste}

Routine operations of the SFTR will result in the generation of sigrificant quantities of both tritium. contaminated and neutron-activated waste materials, possibly requiring some special handling and disposal techniques. ${ }^{6.7}$ At present the Liboratory generates small volumes of both waste types which are routinely disposed of by controlled bural in pits and shafts at the Laboratory's Radioactive Solid Waste Disposal Area. The technique required depends upon both the amount and type of contamination present. The projected contaminated and/or acrivated waste materials originating from SFTR operations will be handled and disposed of in a like manner. The quantity of radionactivity disposed of in the waste burial area will not le increased significantly as a consequence of the normal SFTR operation.

Once operations with tritium are initiated, it is anticipated that several tens of milliliters of tritiated water. containing up to several curies of tritium, wil! be generated daily. This material will be absorbed for dispowal as a solidified matrix. Disposal will be accomplished by packaging in special asphalted containers and deposited into special asphalt -lined deep shafts drilled in the LASL Radioactive Waste Burial Ground. Asphalt has been shown in LASI tests to provide excellent long-term containment of tritium. Alternative means of solidification and stabilization of the tritium for disposal will continue to be investigated.

Activated hardware requiring replacement and disposal will probably include sections of the reaction chamber, compression coils, capacitors, and (ables. Total waste volume for these materials may range hetween as little as 56 and as much as 280 $\mathrm{m}^{1 / 4} \mathrm{sr}$, depending largely upon the ability to reduce volume in the more bulky pieces, e.g., crushing or cutting of reaction chamber sections requiring dis. posal. A capability to store these activated hardware pieces at the SFTR site for short times will be provided, as necessary. to effect a reduction in radiation shielding required for handling and transport of these pieces.

Calculations based upon projected neutron vields and the known materiais of construction of the chamber and associated components show initial induced activities ranging from about 3 and $60 \mathrm{Ci}$ for chamber sections and compression coils, respectively. down to less than $100 \mathrm{mCi}$ for cables and capacitors. Activities. however, will be short-lived, decaying to levels ranging from about $400 \mathrm{mCi}$ to a few microcuries in 1 wk or less. Consequently. routine disposal by pit burial at the LASL Radioactive Waste Burial Area can be accomplished with a minimum of handling difficulty, shielding requirements, or risk of personnel exposure problems.

Calculations also show that there will be an in. significant buildup of long-lived activities in these pieces over the lifetime of the SFTR operations. Final disposal of the hardware also vill not pose major problems other than as a result iff the sheer bulk of material involved.

\subsubsection{Chemical, Sanitary, and Other Wastes}

Chemical Waste. Chemical waste will be collected at the place of use and transported to the laboratory's industrial waste treatment group for disposal.

Sanitary Waste. Sanitary waste will be handled by a system to be constructed on Two-Mile Mesa before the construction of SFTR. The system will collect all sanitary waste on Two-Mile Mesa and convey it to the existing TA-3 Sanitary Waste Treat ment Facility which at present has the capacity to handle the additional load.

Radioactive Contaminated Liquid Waste. A holding tank will be installed to collect waste water from those rooms where tritiated water may be collected. Depending on the tritium concentration. the water will either be stacked at the facility or transported to the Laboratory's industrial waste treatment group for solidification and burial. No radioactive contaminants other than tritium are expected in the waste water.

\subsubsection{Transport of Radioactive Materials}

The main radioactive materials to be transported will be the tritium received at the SFTR site and the radioactive solid waste materials requiring disposal. No problems are anticipated in the routine handling and transport of these materials. DOT transport requirements will be met in all such operations. Tritium will be received from the suppliers as molecular tritium gas contained in DOT-approved shipping containers. All tritium transported and stored at the LASL site will be packaged in the approved containers.

Both tritium-contaminated and activated waste materials will require transport to the LASL waste disposal site. Wastes of these types are handled routinely at the Laboratory. Calculations indicate that after decay times of 1 wk or less, activated hardware requiring disposal will have exposure rates of $<200 \mathrm{mR} / \mathrm{h}$ at $1 \mathrm{~m}$. As a result, only a minimum of shielding, if any, will be required for safe transport to the waste disposal area. No transport problems 
are anticipated. Tritium waste will be packaged at the SFTR site in approved transport and disposal containers.

\subsubsection{Land Usage and Despoilment}

About 4 acres will be required for the building and parking lot, and trees and underbrush will have to be removed. The pine trees at the site are not old enough to be used for lumber. Consideration will be given to the maintenance of green belts wherever possible to enhance the esthetics of the area and to promote recovery of vegetation.

There will be excess top soil and tuff after the site is leveled. Usable top soil will be stockpiled for rehabilitation of the area around the buildings. The tuff will be used in construction of the access road across Two-Mile Canyen.

The urbanization resulting from construction of the site will increase the surface runoff into TwoMile Canyon. Surface drainage will be maintained during and after completion of construction to minimize erosion as well as to transport sediments into the canyon.

\subsection{SAFETY AND ENVIRONMENTAL CONSIDERATIONS DURING OFF-NORMAL OR ACCIDENT CONDITIONS}

On the basis of past experience and coujecture with the Scyllac experiment, the major accident hazards associated with the SFTR will be: (1) mechanical failure of magnetic coils and vacuum enclosure. (2) high voltages, and (3) fire. With careful design and operational procedure, the probability of an accident from any of these three possibilities has been reduced to near zero for Sryllac. Although the same low probabilities will exist for the SFTR experiment, the following subsections address specific operational hazards recognized for the SFTR, and, when possible, the consequences of related, hypothetical accidents are described.

\subsubsection{Consequences of a Sudden and Total Release of Tritium}

Once D-T operations are under way, tritium will be found in storage containers in the vault, on uranium beds in the tritium handling hoods, cryogenically trapped within pumping stations, and on molecular sieve beds ir the tritium cleanup systems. The entire inventory $(\sim 3 \mathrm{~g}) \mathrm{might}$ be released from these various containers by an act of sabotage or a major accident, such as the crash of a large aircyati into the building. Howerer, the relesine of the SFTK tritium inventory in lote would not be catast rephic.

Exposure to personnel in the building is almost iripossible to predict because of the possible variations and complexities of any accident scenario. Furthermore, property damage and probable tire. with accompanying injury and death, would probably overshadow any danger from expositre to tritium. Gaseous tritium is many orders of magnitude le:s toxic than tritiated water vapor and should present no significant exposure problem. A ly tritium oxidized in a fire would rapidly escape from the building with other combustion products. Such a fire would have to be controlled from an uywind position, or self-contained breathing apparatus would be needed.

In case of sabotage, the tritium cleanup sytems would be called into service automatically unless thay were out of commission. In that event, the tritium, as gas or oxide, would find its way to the environment either slowly at ground level or up the staek, depending on the coldition of the ventilation system.

Calculations were made to est imate the personnel exposure outside the building. assuming that all tritium is in the form of HTO. Three standard meteorological conditions, release heights of 0 and 30 $\mathrm{m}$, and a wind speed of $1 \mathrm{~m} / \mathrm{s}$ were used. Biologrical parameters included a biological half-life for $\mathrm{HTO}$ of 10 days, a tritium quality-factor of 1 , and a breathing rate of $3.47 \times 10^{-3} \mathrm{~m}^{3} / \mathrm{s}$. Absiorption through the skin was assumed only for the case of the extended release. The result for the rapid stack release is plotted in Fig. 7-15. In the case of an extended release via stack, the average time-integrated concentration at any point downwind is lower by a factor of 1.5 to 2 . Consequently, the intake through the lungs is decreased proportionately. Intake through the skin, however, is increased from zero for a very short release to an amount nearly equal to that taken in through the lungs for an extended release. Hence, Fig. $i-15$ may also be used for the case of the extended release to arrive at expusure estimates correct within a factor of 2 .

A release at ground level can occur only very slow. ly and this situation is treated in Fig. 7-16. The dose equivalent (DE) is based on a release lasting up to 8 $h$, but should the release take longer, the DE is decreased further because of increased lateral dispersion.

The largest DE would occur at the site boundary (approximately $300 \mathrm{~m}$ from the SFTR building). The $\mathrm{DE}$ to an individual at this location for releases up the stack or at ground level as a function of the fraction of the total inventory $(3 \mathrm{~g})$ released is plotted in 


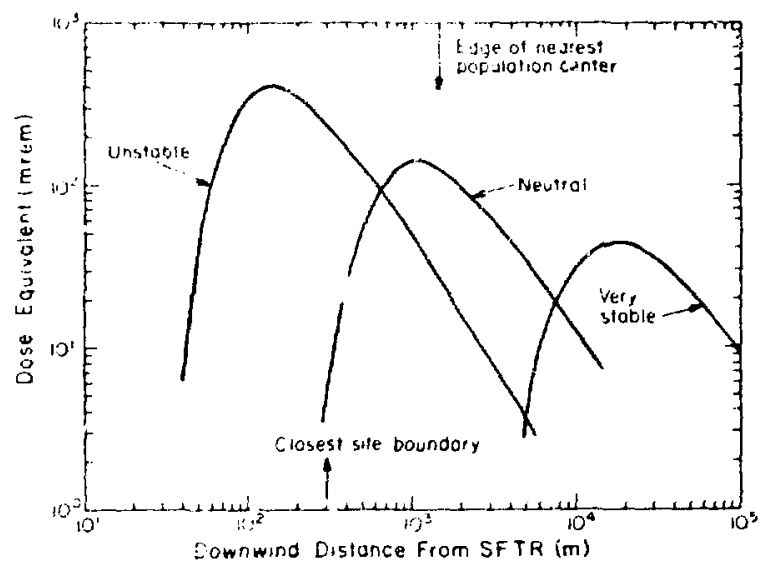

Fig. 7-7.5.

Dese' e'quilalent dounuind from an instan(ane')us release' of $3 \mathrm{~g}$ or $T$ ' as $H T$ () as a function "f distance for three meteor losical conditions, a $\mathrm{l} \cdot \mathrm{m} / \mathrm{s}$ wind speed, and a $30 \cdot \mathrm{m}$ stack height.

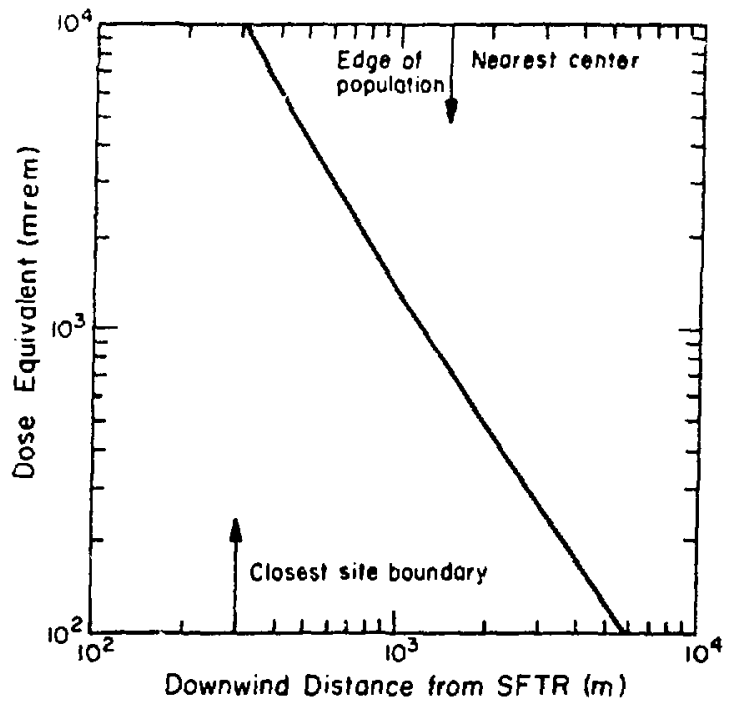

Fig. 7-16.

Dose equivalent downuind from a ground release of $3 \mathrm{~g}$ of $\mathrm{T}$ as HTO extended over a period of $8 \mathrm{~h}$ for a meteorological condition and a $\mathrm{l} \cdot \mathrm{m} / \mathrm{s}$ uind speed.

Fig. 7-17. The worst atmospheric condition for this distance was used in estimating the doses: approximately neutral for a release up the stack: stable (Pasquill F) for the ground release. A wind specd of 1 $\mathrm{m} / \mathrm{s}$ and a stack height of $30 \mathrm{~m}$ were used. A gradual

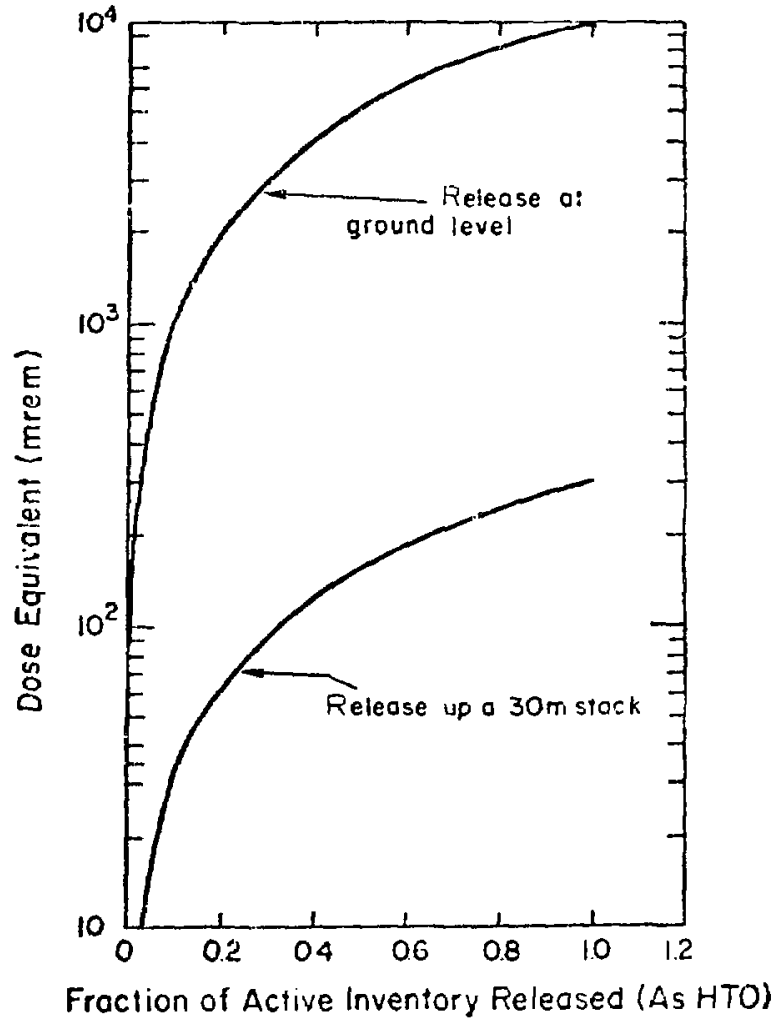

Fig. 7.17.

Infinite-time $D E$ at the closest site boundam $(.3(j i) \mathrm{m})$ as a function of the fractional release of the tritum inc'entiony (i g) released as HTO.

release lasting no longer than $8 \mathrm{~h}$ was again assumed. If an extended release were to take place. ample time would be available to evacuate people from the downwind area.

In the event of such releases, a large segment of the local population could be exposed. albeit the in. dividual doses would be quite small. The population dose was calculated for two cases: a release in the direction of the Los Alamos townsite and one in the direction of Santa Fe $(40 \mathrm{~km}$ to the suutheast). In the first case, a neutral meteorological condition was assumed; in the second case. a moderately stable condition was used. These two conditions represent the worst situations for the two population centers. assuming that the release occurs instantaneonsly up a $30-\mathrm{m}$ stack. The maximum DE at Los Alamos is about $110 \mathrm{mrem}$ and the population dose is estimated to be about 200 man-rem. In the Santa Fe case. the corresponding values are 4 mrem (average) and 160 man-rem. No allowance was made for 
terain features that would dilute the concentration or for protection afforded by shelters.

Given that the local radiation background DE is approximately $150 \mathrm{mrem} / \mathrm{yr}$, the tritium exposure resulting from the (highly improbable) release of a substantial fraction of the active tritium inventory does not constitute an unreasonable risk to the public.

\subsubsection{Catastrophic Failure of Discharge Tube During a D-T Experiment}

Loss of vacuum containment from crowbar failures is expected to occur relatively frequently during the early check-out phase of the SFTR operation. Reliability of the SFTR firing sequence and vaculim wall will be established before D-T experiments proceed. As discussed in Sec. 5.2, local vacuum-wall failures during a D-T discharge would release only a small fraction of the $-15-\mathrm{mg} T$ : inventory. The bulk of the tritium would be collected on the cryogenic roughing pumps. In the unlikely event of a massive !ailure of the discharge tube and subsequent release to the SFTR cell of all tritium used in that shot, the tritium concentration within the cell would exceed 6000 times MPC $_{\text {a }}$. The cell cleanup facility $(\mathrm{CCF})$ would then be activated and the tritium removed from the cell air in a matter of hours. Details of this procedure are discussed in Sec. 5.2. Vo serious consequences to operat ing personnel or the general public are envisioned for a (highly improbable) massive failure of the SFTR vacuum.

\subsubsection{Fire Hazards within the Cell}

During assembly, calibration, and initial operatirn of the toroidai structure, the fuel will be crmbustibles usually associated with construction. Although these combustibles will be kept to a minimum. they will constitute a significant fire hazard. A temporary or portable automatic sprinkler system is a feasible method of protection during this period. In addition, hose lines and extinguishers (water type, $\mathrm{CO}_{2}$, and $\mathrm{ABC}$ type powder units) will be provided. There will be no release of radioactive material as a result of a fire during this time, and ventilation and air exhaust systems will be adequate to cope with any smoke and heat.

Once the facility is in operation, an automatic fire detection and suppression system will be installed in the cell. The likeliest cause of fire is "fire fuel" (rags, thin plastic sheet, or shavings, paper packing materials, etc.) that become oil-soaked and are ignited electrically. A less likely source of fire is spark. gap failure during which a low energy fireball of hydrogen is released. Although such a fire could ig. nite tissue paper, the cables or capacitor oil probably would not ignite.

The cell will contain approximately $4000 \mathrm{gal}$ if castor oil-a relatively stable hidrocarbon that re. quires preheating before ignition. Its flash point is about $204^{\circ} \mathrm{C}$ and its ignition lomperature about $445^{\circ} \mathrm{C}$. In case of fire, castor oil is no more hazardorits to health than ordinary combustibles, normally remains stable, and does not reac't with water. It has a slight tendency toward auto-ignition when left on saturated cellulose waste in poorly ventilated closures. In a cell fire. we assunte that no more than $\mathbf{5 0} \mathrm{gal}$ of castor oil would be available for burning. such an oil fire could be extinguished by standard foam, high expansion foam, dry chemical. or vaporizing liquid type agent. First consideration will be given to Halon 1301, and the somewhat cheaper. but more toxic. Halon 1211 also will be considered.

The celt will contain a significant amount of polyvinyl chloride insulated cable which will soften at about $811^{\circ} \mathrm{C}$ and melt at $104^{\circ} \mathrm{C}^{\circ}$ but which is difticult to ignite. When it does ignite. it produces acrid, highly corrosive fumes which are mainly sydrogen chloride. The extinguishing arents mentuned above will also cont rol the buining P(. but they must be applied quickly because of the corrrosive gas.

Other combustible and corrusive materials. such as polyethylene cable insulat ion or neoprene insulation, are likely to be present in the cell, but will not be of consequence.

The cell ventilation system will be designed to exhaust the bulk of the hot gases and smokt under most fire conditions. Some exhaust filtration? will be necessary, as will drains and retention tanks to handle any water used in fire-fighting.

\subsubsection{Fire Hazards External to the SFTR Cell}

\subsubsection{Fire within Tritium Storage and Treat-} ment Facilities of SFTR

In the presenco of tritium, deuterium, and cryogenic fluids, mocification of normal fire-cont rol measures will be necessary in the tritium storage and treatment sections of the SFTR. These modifications involve the control of personnel entry and fire hose usage. Otherwise, safety measures will be typical of labotatory and equipment rooms, and should include an antomatic sprinkler or $\mathrm{CO}$. system. Activation of any suppression system should automatically interrupt the ventilation until the ventilation is restarted b $;$ a manual override. High expansion foam, $\mathrm{CO}$, , and Halon 1301 systems could 
he used in areas of specialized hazard and limited size. In areas where capacitors are stored or used, and where there are large numbers of cables that would be vulnerable to water, a Halon 1301 suppression system like that for the present Scyllac experjment will be the choice.

The tritium inventory in the storage vault poses the largest radiological hazard. Part of the tritium will be a gas under 2 atm pressure in a rugged metal container. The remainder $(<3 \mathrm{~g})$ will be (11), T); a pyrophoric hydride, also in a metal container stored within a closed metal cahinet. The likelihord of fire in the vault is very small. The main fire risk is from combustible materials brought in during shut-down and renovation periods.

In case of fire. personnel entry may be limited to mandatory usage of supplied-air suits because of the high potential hut localized danger.

The quantity of tritium in the Iritium processing roum (a tew thousand curies) will be an order of magnitude lower than that in the vault. All but a small fraction will be uranium tritide or deuterotritide in metal containers within a vented enrlosure. Supplied air and controlled use of spraytype fire nozzles (particularly on the hooded enclosure) constitute the special fire-cont rol feat ures in this room, although high expansion foam systems will also be considered.

In the TWT and C.C.F rooms, tritium levels should be sufficiently low (one or two orders of magnitude lower than that in the tritium processing room) and the confinement of the tritium in the dryers should be sufficiently secure to eliminate the need for special fire.cont rol measures.

The deuterium supply will be in a single cylinder (280 / STP), and normally only one tank (several months' supply) will be on hand. Deuterium. likt' hydrocen and helium, has a segative Joule-Thomson coefficient at room temperature, so that rapid expansion of deuterium or hycirogen in air at romm temperature can lead to self-ignition. Such fires might be left to burn (so) long as the valve on the cylinder is kept cooll to avoid the creation of an explosive deuterium-air mixture. The tank ot deuterium is also a hazard if it explodes or if deuterium is somehow released to aggravate a fire or (1) give an explosive deuterium-air mixture. Deuterium Jeaks can be hazardous, but should be rare.

Ten to twenty liters of liquid helium and liquid nitrogen will be contained in dewars and equipment in the tritium processing and TWT rooms. Because these liquids could vaporize rapidly during a fire and burst their containers. emergency procedures must be developed to deal with this hazard. If the containers burst, about $1 \mathrm{Ci}$ of tritium would be released (in the hood), and occasionally a maxumum of $150 \mathrm{Ci}$ could be released during operation.

Special dry-chemical and standard carbon dioxide extinguishers can te placed thrcughout the facility with no restriction on use, except possibly on a deuterium fire. Noncombustible and fire-proofed equipment should be used where practicable. consistent with the degree of tritium hazard (highest in the storage vault). A limited number of emergency showers will be :nstalled.

In sum mary, the major fire hazard for the SFTR is expected to be the PFN and transfer capacitor banks (high voltage, nil) which are far removed from the major tritium hazard (storage vault). A great deal of experience has been accumulated at LASL in the sate handling of both large capacitor banks and tritium. With only a few extrapolations from present 1.ASL practice, the fire and tritium control expert ise required for the SFTR facility is already available.

\subsubsection{Fires Associated with the Transfer Capacitor System}

The transfer capacitor systen is composed of low voltage capacitors connected in a series-parallel arrangement to provide a $100-\mu \mathrm{F}$ capacitance at 60 $\mathrm{kV}$. The capacitor is cor.uposed of materials present Iy used in the standard Scyllac-type energy-storage capacitor. The capacitor plates are aluminum foils which are separated by several layers of $\mathrm{Kraft}$ paper impregnated with castor oil. The switches used on the capacitor bank are standard. commercially available ignitrons.

There is little fire hazard associated with the capacitor bank. The major hazard arises if. for some reason, a capacitor should rupture and eifust the castor oil onto an area where there might be a possibility of ignition. A capacitor case can be ruptured if the capacitor shorts and energy from parallel connected units is dissipated in the ruptured capacitor. Experience shows that up to $30 \mathrm{hJ}$ of energy can be dissipated in one of these capacitors with little danger of rupture. Each unit contains approximately $11 \mathrm{~kJ}$ of energy. which is not sufficient to cause rupture. and the energy from parallel units will be limited by a cable connection which will act as a fuse under fault conditions.

The probability of a unit rupturing is therefore very small: consequently. the fire hazard associated with the transfer capacior bank is very small.

\subsubsection{Hydrogen Explosions}

The quantities of deuterium and tritium that will be handled in the SFTR are minor by normal stan. dards. Nevertheless, explosions involving these 
materials. give concern because of the potential release of tritium in quantities that may be radiologically hazardous. The conditions that might lead to such explosions are discussed below.

Sume limits of tlamrnability (fl.) and detonability (de.) tor hydrogen and deuterium in air anci oxygen are as follows ${ }^{8}$ (voli): fl. $H_{2}$ in air, 4.00-74.2); $11 . \mathrm{H}$ in $0.2 .65-93.9 ;$ fl. D. in $\mathrm{O}_{2}, 5.00-95.0 ;$ de. $\mathrm{H}_{2}$ in air. 1.59. For hydrogen in air, other properties are": ignition temperature, $858 \mathrm{~K}$; ignition energy (:30 vol\%; H.2), $0.02 \mathrm{~mJ}$; heat of combustion, 285 $\mathrm{k} . / \mathrm{mol}$; and flame temperature, $2318 \mathrm{~K}$. Because the llammability limits of hydrogen-air mixtures are relatively insensitive to pressure. ${ }^{9}$ the limits for a lu-ternperature solid mixture are not expected 10 be markedly different.

The largest hydrogen source is the $0.28-\mathrm{m}^{3} \mathrm{STP}$ deuterium supply cylinder discussed in Sec. 7.4.4.1. Because the attendant tritium hazard is only in. cidental, no special precautions are required, particularly since the tritium handling room is well ventilated and the tank is normal!y gaged so that an appreciable loss could be noticed. The small quantity of ( $(D . T)_{1}$ (several liters of $\mathrm{T}_{2}$ equivalent) in metal (antainers and under hoods is also of little concern. especially since careful PVT and analytica! measurements will be made several times a day in the preparation ane? use of this material.

Tritium will be received in gaseous form in a metal (ontainer with $3 \mathrm{~g}(11$ / STP) of tritium at a pressure of 2 atm. Only one filled container will be on hand in the vault at any one time. A leak in this container w.suld be immediately detected by a tritium monitor, and an alarm would be souried. Alter mose than half the tritium has been used, an air leak into the container could create an explosive mixture containing less than 5 / of tritium. Since some of this giti is used and analyzed weekly. an air leak would be detectea fairly soon. Ignition of this gas is unlikely. and use of a smail-diameter tube during transfer would prevent flame propagation into the storage container. ${ }^{9}$ The remaining tritium stored in the vault as $U(D . T)_{3}$ should present to hazard except in calie of fire.

A fraction of a liter of deuterium and tritium will be stored in metal containers in the tritium handling room hood. Analytical and PVT measurements would quickly datect any air contamination in these containers. About 0.1 / of this gas is transferred at low pressure to four $10-j$ injection containers. Hecause of the low pressures involved, no hazard exists either in the injection system or the discharge tube.

Perhaps the highest potential for a hydrogen explosion exists in the cryogenic fuel-ash exhaust system. Here, outgassed oxygen, oxygen from the decomposed wall of the discharge tube, or air trom equipment and piping leaks could mix with the 1) or (D.T) exhaust gas to form explosive mixt ures. In the D-T runs the gas is cryogenically pumped into cryopumps at $4 \mathrm{~K}$ at each of four stat ions. About (1).? I of (D, T), wili be condersed as solid in these pumps. Since the pumps have a tot al volume of $201040 \%$, the volume of (D,T).2 is too small to create physical damage were an explosion tu occur. Also, the gas is subsequently expanded and analyzed before it is cryogenically pumped for more accurate measurements in a standard volume, if desired) or reacted with uranium. Thus, the chance for a damaging tritium explosion in the tritium exhaust and recoverv operarions is extremely remote.

Because of the rapid sequencing of the denterium runs (one every $15 \mathrm{~m}$ ), the deuterium-ash will be collected daily on four cryopumps (5- to 10 -( volume) located in the hooded pumping stations. The volume of deuterium gas collected daily would be 3 /, based on a duty cycle of $15-\mathrm{m}, 8-\mathrm{h}$ operation and a deuterium volume per shot averaging about 0.1 \% Thus, the pump to deuterium gas volume ratio would be less than 13. At one stage a cap will be placed over the pump to give a volume ratio of only 6. This compares with a $\mathrm{D}, \mathrm{O}$ (at $273 \mathrm{~K}$ ) to $\mathrm{D}_{2}$ (at 273 K) ratio of 8.5 , a volume ratio marginal in its ability to avoid explosion pressures significantly above 1 atm. Instantaneous pressures might be expected to be markedly higher. ${ }^{10}$ Further, since the deuterium will be collected as a solid, an intimate mixture of oxygen and other gases with deuterium could be condensed as a solid, in which case the solid could be shock-sensitive. Since less than 1 / of deuterium would be in a pump, the energy of the explosion would be less than about $10 \mathrm{ke}$. Because the pump will be enclosed in heavy piping, an explosion resuliting in the release of liquid nitrogen and helium into the pump enclosure could cause a rapid discharge of the gas (and liquid) through filling ports in the pump and into the hooded enclosure. Although less than a curie of tritium would be involved in such an accident, it might be sater to transfer deuterium to the TWT more frequently than planned. This problem will be investigated thoroughly before $D . T$ experiments are begur.

The deuterium will be traneferred uncer low pressiare to the large TWT cryosorb pump, an operation that presents no obvious explosion problems. Every day the cryosorb pump will be warmed, the deuterium and other gases will expand, and heliurn will be flushed into the $2000-1$ TWT hallast tank. Although potentially explosive mixtures are possible in this operation, the energy generated will be relatively small, the equipment strong, and the likelihood of initiation of the reaction in the closed 
system remote. Sufficient air will be present or added during this operation to keep the final average deuterium concentration well below the lower exolo. sion limit of $\sim 5$ vol $\%$. A similar condition may arise if hydrogen is injected to aid in the $\mathrm{D}_{2}-\mathrm{O}_{2}$ reaction as discussed in Sec. 5.6.3.5. Here, again, the injected quantities would be kept smail.

In summary, some possibility exists for a deuterium explosion, but the energy would be small in mast cases $(40 \mathrm{~kJ}$ ). Furthermore, the explosion would be confined in most ases by strong equipment and hooded enclosures. The probability of a hazard from direct tritium release is very low. Redunciancy of equipment, supply lines, and pumping stations should eliminate interruption of operational schedules. Added safety measures, such as more frequent deuterium transfer from the slations, oxidation of the devierium by hot copper oxide before mixing with air, or provision of barriers, would not be difficult but seem unwarranted. Grounding of equipment and selection of fireresistant equipment are worth consideration.

\subsubsection{Magnet Malfunctions}

Most of the mechanical energy that could be released as kinetic energy is stored within the compression field magnets. Table 5.4-5 gives the energy storage, size, and number of magnets in the compression system. A $14.8 \mathrm{~cm}$ coil stores $345 \mathrm{~kJ}$, and this hoop strain energy amounts to $2.33 \mathrm{MJ} / \mathrm{m}$ or $25.6 \mathrm{MJ} / \mathrm{m}^{3}(3.8 \mathrm{~J} / \mathrm{g})$ of compression coil. This compares to $370 \mathrm{~J} / \mathrm{g}$ for $\mathrm{H}_{2}$. Hence, the potential exists for slight and localized damage to surrounding equipment if a structural failure should occur within the coil and this stored energy were released as kinetic energy. The resulting destruction would be limited to a highly localized area of the SFTR cell a a time when personnel would not be present. The primary radiation shielding surrounding a module probably would be sufficient to contain the energy release, and local damage to the implosion-heating coil and/or vacuum wall might occur. Similar kinetic energy releases assoriated with leads and cables are possible, but simple design of support and restraining structures should eliminate this problem. Past experience in this area with Scyllac and Scylla experiments at LASL can be applied directly to the SFTR.

The SFTR magnets may fail from overheating and localized electrical breakdown, as happens in current CTR experiments at LASL, but such failures are considered experimental nuisances rather than hazards solong as proper precautions are instituted.

\subsubsection{Failure of Cryogenic Energy-Storage and Transfer System}

The temperature of liquid nitrogen and liquid helium is low enough to damage human tissue. If surfaces cooled by these fluids or their evolved gases are touched, the tissue temperature may be lowered below $273 \mathrm{~K}$, where damage occurs. Such cooling does not occur immediately because the blood supply acts as a heat source to the tissue and the contact of a cryogen with warm tissue creates a gas film which is not a good heat conductor (if cold metal is touched, the tissue is damaged much more rapidly!. This delay occasionally permits successful counteractive measures to be taken when an exposed part of the body is splashed by cryogenic fluid. Two nieasures contribute significantly to an individual's safety: (1) shutting off the flow of cryogen or escaping the area, or both, (2) immediate flooding of the tissue or clothing with water. Water's enormous heat capacity, its inertness and availability make it vital in safe cryogenic operations.

Safety glasses will be worn whein personnel are working with laboratory and industrial cryogenic and pressurized, or potentially pressurized, systems. Where splashing is a definite possibility, face shields will be worn. Individuals will be trained not to Jook directly into open cryogenic lines without adequate eye protertion, since even small mechanical or thermal disturbances can cause ejection of cryogenic fluid. Persoririel may also be required to wear special clothing. gloves, or fontwear. Gloves should be clean and dry. Loose-fitting gloves are preferred because they can be removed quickly.

Spills and leaks are by far the most common failures in cryogenic systems. Ruptures, although fairly rare, create the greatest safety hazards. A serious condition would exist if the coil dewar were not properly vented and if the 10 coils in one of the 128 METS dewars should go normal, thereby causing the liquid helium in the dewar to vaporizt. Releasing this much helium gas into the area of the main building would reduce the oxyger concentration to $14^{\circ} c$ if mixing were uniform. But complete mixing would be difficult to achieve, and consequently local oxygen concentrations would be much lower than $14 \%$. For this reason the $0.33-\mathrm{m}$ (13-in.) dewar vent lines will be piped to the outside of the building to eliminate the danger of asphyxiation. In addition, although the storage coil dewar area will be safe for normal activities, it is to be an exclusion area when coils are charged or otherwise tested. All outrioor cryogenic. storage areas will conform to stan. dard industrial safety practice for cryogenic storage and will be fenced exclusion areas. 
Liquefied helium and nitrogen are capable of condensing (and solidifying in the case of helium) air that may come in contact with them. The liquid, and especially the solid, air can block vent paths and jeopardize vessels because of pressure buildup. In the METS system the liquefied helium will be transferred in clcsed piping loops pressurized to greater than atmospheric pressure to prevent the backflow of air. Relief devices will be designed to prevent any back leakage of air.

Air coming in contact with a surface cooled below $82 \mathrm{~K}$ will partially condense, with the condensate containing approxirnately $52 \%$ oxygen, and will continue to condense as long as air with $21 \%$ oxygen is available to the surface. Such an oxygen-enriched mixture will significantly enhance burning rates of combustibles and even permit some materials to become combustible that might not qualify as such in normal air. The system will be designed to preclude condensate formation and to ensure that the runoff, if any, does not create a hazard. This can be accomplished by making sure that the runoff does not come in contact with combustibles or components which become embrittled or cease to function at low temperatures.

It may not be desirable to have vacuum jackets around all components which carry cryogenic fluids, but at least the lines must be covered with blankets or possibly foams. Since most foams are cellular structures, oxygen-enriched air may accumulate in the cells, particularly if the foam is of the open cell type. This effect can be minimized by providing a barrier against the infusion of air. Foams for this application will be selected on the basis of their fire resistance. If glass or niineral wool blankets are used, care will be taken to assure that they contain no oil to react with the oxygen-rich condensate.

Cryogenic systems and components probably will not cause or contribute to any fires which might occur in the experimental area. On the other hand. these units can be damaged by adjacent fires. In fact, the plastic dewars could fail and discharge their contents if subjected to high temperatures for extended periods. In most cases. the protective overpressure blowout discs would release the pressure buildup within the dewar before the dewar itself ruptured. The released gas would be either nitrogen or helium and there would be no increase in the fire intensity. Such a release of cold inert gas might actually help to control a fire.

\subsubsection{Fuel Injection Accidents}

If the amount of $(D, T)_{2}$ fuel supplied to the SFTR is too small or too large, the discharge would result in an off-optimum thermonuclear burn. For a given set of operating perameters (shock heating, compression field, etc.), the (D,T) filling pressure is selected to maximize the fusion yield and any deviation from this optimum filling pressure will result in a poor yield. In essence, the plasra represents an important element in a highly tuned electrical circuit, and either under-or over-fueling will de-tune that circuit and result in an off-optimum thermonuclear yield. Hence, the possibility of nuclear or thermal "runaway" for the SFTR is zero

\subsubsection{Consequences of Utility Fuilures}

The SFTR power supply is protected against overvoitages and short circuits between phases or to ground in conventionai fashion. If bulk power ceases to be supplied during charging of the energy-storage inductor, then charging simply discont inues and the current maintains the existing magnetic field in the inductor by circulating through the free-wheeling diodes. This current decreases exponentially with the time constant $L / R=7.4 \mathrm{~s}$, and the magnetis field energy is dissipated in the inductor winding and busses. This dissipation amounts to an additional less of no more than $14^{\circ}$; over that occurring normally on charging when the marnetic field energy is eventually dissipated eisewhere in the system. Apperidix I describes in detail the consequences of a utility failure at the SFTR site. Genecally, no significant environmental erfect or safety hazard can be identified as a result of a massive and sudden utility (electrical power, water, etc.l interruption.

\subsubsection{Unusual Oecurrences}

Although detailed accident analyses have not been made. the above-mentioned descriptions, calculations, and conjectures are indicative of the worst consequences possible in the event of a cataclysm (earthquake, tornado, airplane crash, volcanic eruption, act of war, dedicated vandalism). The damage. injury, and death caused by such unusual occurrences will undoubtedly be caused direct ly by the occurrence itself rather than by the SFTR facility. The radioactive inventory (active structure, tritium) will be secured in limited access areas (SFTR cell, tritium storage vault, etc.) which will be surrounded by thick concrete walls and substantial siructure. "The small quantity of tritium is probably the only item of interest to international bandits, and the few grams of tritium stored on the site will be safely secured and inventoried (Ser. 5.2) 


\subsection{SUMMARY AND CONCLUSIONS}

From the viewpoint of safety and environment, operational and aceident aspects of the SFT'R do not differ significantly from the current Scyllac experiment. Although the use of tritium and the production of moderate amounts of structural activation present one major difference, these hazards can be controlled with existing and well-tried techniques. Even with the unlikely simultaneous failure of control and release of the total tritium inventory, the consequences to the public and the environment would be minimal: Radioactive hazards outside the SFIR cell during a D-T shot are nonexistent, and the more serious dangers of fire hazard and high voltage can be controlled by existing procedures. Any perturbation created by the SFTR on the demographic, meteorological, hydrologic, and geologic character of the site will be insignificant. Terrain, vegetaticr, and wildlife will not be adversely affected if appropriate conservation and consi ruction methods are used in SFTR construction.

\section{REFERENCES (Chap. VII)}

1. D. H. Slade, Ed., "Meteorology and Atomic Eriergy," USAEC report TID-24190 (July 1968).

2. "Uniform Building Code," Intern. Conf. Building Officials (1973).
3. "Standards tor Radiation Protection," CSERDA Manual Chapter U524 (April 1975).

4. R. A. Jalbert, "Monitoring Tritium in Air Containing High-Energy Beta Emitters Using. lon Chambers." Proc. Am. Nucl. Soc. (to be published).

5. D. G. Fink and J. M. Carroll, Standard Handbook for Electrical Engineers, iOth ed. IMcGraw Hill, New York, 1968i.

6. "Radioactive Solid Waste Management Policy." Los Alamos Sciantific Laboratory internal document (Sept. 27, 1973).

7. L. J. Johnsen, "Information on Snlid Radioactive Waste Management at i.ASL." Los Alamos Scientific Laboratory internal document (May 13, 1974).

8. C. D. Hodgman, R. C. Weast, S. M. Selby, Eds.. Handbook Chemistry and Physics, 39th ed. (Chemical Rubber Pub. Co.. Cleveland, OH, 1956).

9. D. B. Chelton, "Safety in the Use of Liquid Hydrogen," in Technology and Uses of Liquid Hydrogen (MacMillan, New York, 1965).

10. F. J. Webb, "The Use of Liquid Hydrogen for the Production of Cold Neutrons Inside a Nuclear Reactor," ibid. 


\section{COS'I ESTIMATE}

\subsection{INTRODUCTION}

The :iesign of SFTR is sufficiently detailed for a cost est:mate to be made. Syst $\epsilon$.ns, such as the vacuum system, that use commercially available components or systems, such as the METS refrigeration system, that have components similar to exising equipment can, of course, be costed with more confidence than those requiring further developmeni, such as the implosion-heating system or the first wall and Marshall coil. Cost estimates for commercially available systems were made from catalog prices when possible and from estimates based on experience with projects such as Scyllac. For components or systems requiring developmental work, cost estimates were made by extrapolating from costs for existing equipment where possible. Estimated custs of the facilities, manpower, and raw material required for the components are more susceptible to error than are commercially available items: consequently, conservatively high estimates have been made.

\subsection{SÜBSYSTEM COSTS}

Cust estimates are divided into the following areas: First Wall and Marshall Coil Assembly; Implosion-Heating System; Preionization System; Primary Shitiu; Compression Banks (Phase I): METS (Phase Il): Tritium Handling and Vacuum System: Diagnostics System; Control System; Facilities. The cost estimate for each system will be discussed in the following sections.

\subsubsection{First Wall and Marshall Coil Assembly}

The most difficult and uncertain cost estimate in the project is that of the first wall and Marshall coil assembly. Ceramics will probably be used to enable the first wall and Marshall coil to withstand the high neutron flux and still maintain adequate electrical insulation strength: however, the processing, manufacture, and assembly of these ceramic components have not yet been done for theta pinches, and such stevelopment may require several manyears of effort. Initial efforts are under way and simple ceramic first walls will be used in 1976 in LASL Z-pinch and staging experiments.

The cost estimate is based on present concepts of materials and construction. Development of the coils will require an effort of ronghly $50 \mathrm{~m}$ an-years, and since it must be done befure the machine can be funded, development costs are not included. The cost of the ceramic wili be negligible compared to the cost of the fabrication facilities and time required for fabrication. The major expense will be that for furnaces and grinding marhines and the labor cost for operating the equipment. The cost estinate is shown in Table 8.1.

The estimate includes the cost of labor, capital equipment, and material. It is assumed that the assemblies will be constructed over a period of $2 \mathrm{yr}$ and that only half of the total assemblies will be usable. A $40{ }^{\prime} i$ contingency factor is included to cover the high uncertainty in the overali process and design.

\subsubsection{Implosion-Heating System}

The implosion-heating system consists of the current feedplates between the implosion-heating capacitors and the Marshall coil, capacitors and start gaps, rowbar gaps, pulse-charge system. trigger syster:, and structural material in the plasma chamber and for the trigger and pulse-charge system. The ferrous material between the implosion-

TABLE $8-1$

\section{COST ESTIMATE OF FIRST WAi. AND MARSHALL COII}

Material

('opper plating equpment

Casting equipment

Ceramic furnaces:

Metalizing furnaces

Bronzing furnaces

Grinding equipment

Miscellaneous equipment

Facilities

Iabor (2 vr)

Equipment assembly and testing $20^{\prime}$ 。

Contingency $40 \%$,
$\$ 0.60 \mathrm{M}$

$0.1: 3$

0.10

0.78

0.78

0.78

0.39

0.25

(0.)

3.065

1.47

3.54

Total $\quad \frac{12.38 \mathrm{M}}{\$ 12.38}$ 
heat ing system and the compression coils is also included in this cost estimate.

Many components, such as the trigger system, are commercially available and require little or no development. while whers. such as the $1: 3 i 5-\mathrm{kV}$ (apacitors and gaps, require moderate development efforts which are not included in the estimate. The low engineering estimate is due to the repetitive nat ure of the system. The estimate is given in Table 8.2.

\subsubsection{Preionizatio: System}

The preionization system is small, and components are already developed, and only a moderate amount of engineering will be required. Costs are given in Table 8-3.

\subsubsection{Primary Shield}

Although the primary shield cannot be purchased from a commercial vendor, the required material is available commercially and the fabrication could possibly be carried out on contract. The estimate in Table 8-4 was made by costing the required volume of materials and estimating the fabrication and installation costs. Little development work will be required, but a large contingency is used because of lack of experience with the manufacture of such systems.

TABLE 8-2

\section{COST ESTIMATE FOR IMPLOSION-HEATING SYSTEM}

Current feed plates

Capacilor connectors

li3)-kl ('apacitors

Sitirt gelps

Crowlar yaps

Siart-gap) trigger system

('rowbar l rigger system

Pulse-charge system

Ferrous material

Siructural material

Fquipryent assembly and testing $20{ }^{\prime}$,

Fingineering $10 \%$,

Contingency $1 \pi^{\circ}$
$\$ 1.211 \mathrm{M}$

0.86

1.15

7.7

3.8

3.65

1.50

$0.9: 3$

0.17

$\frac{1}{22.1}$

4.41

$\frac{2.21}{28.68}$

4.30
TABLE 8 -4

\section{COST RSTIMATE FOR PREIONIZATION SYSTEM}

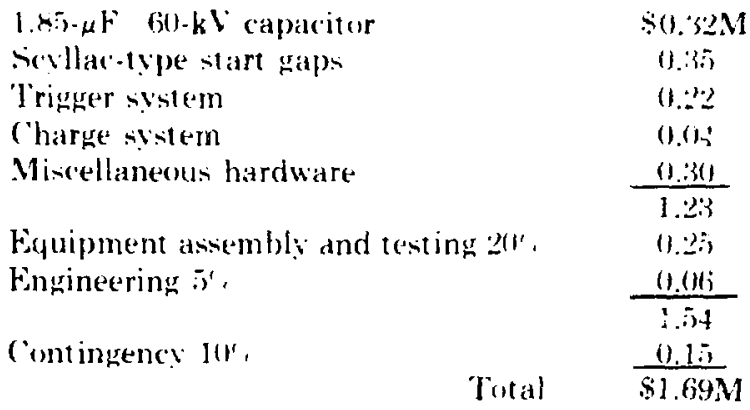

\section{COST ESTIMATE FOR PRIMARY SHIEID}

Burated graphite

Aluminum structure

$\$ 3.6411$

$B$, rated polvethylene

0.64

l.ad-horated polvethylene

Engineering

$1 .(0)$

Fabrication

2.56

16.51

Assembly 5',

Contingency $30 \%$

$\frac{0.8 .3}{17.34}$

Total $\frac{5.20}{522.54 M}$

\subsubsection{Compression Bank (Phase I)}

The compression bank is made up entirely of commercially available components for which reliable cost information is available. Efforts are under way at Los Alamos to develop a lower priced capacitor: however, in Table 8-5 the present price of capacitors is assumed. The only innovation required in this system is the optical fiber isolated trigger system which will require a small amount of development work, and the first metal-to-metal crowbar switch which will require a larger development effort. As in the implosion-heating system, a small engineering effort is assumed because of the highly repetitive nature of the system. 


\section{COST ESTIMATE FUK COMPRESSION BANK (PHASE 1)}

\begin{tabular}{|c|c|}
\hline Eneres storatse capacitors & $\therefore 7.17 \mathrm{M}$ \\
\hline Stant ienitions & 0.48 \\
\hline Truwbar ignitroms & 2.69 \\
\hline Metal-to-metal crowbar switch & 3.2 \\
\hline Cumpression coils & 1.43 \\
\hline Tripuer systems & 1.26 \\
\hline Cabling & 0.80 \\
\hline Charge silstem & 3.15 \\
\hline Charge racks & (1.6.t. \\
\hline Miscellaneosus hardware & 1.92 \\
\hline & 21.74 \\
\hline Equipment ansembly and testing 20 , & $4.3 \overline{5}$ \\
\hline Fingineering $10 \%$ & 2.17 \\
\hline & 8.26 \\
\hline C'omtingency $10 \%$ & $\stackrel{2}{2} .83$ \\
\hline
\end{tabular}

\subsubsection{METS (Phase II)}

The METS system consists of components requiring little work, such as the refrigeration system, as well as components requiring a substantial amount of development, such as the HVDC interrupter and the superconducting coils. The development effort has not been included in the cost estimate for each component. The estimated cost of system installation also includes the small amount of labor required to modify the cabling on the compression capacitor banks. The low engineering cost reflects the fact that the equipment is purchased commercially and the engineering is that required for the installation and system interconnections. Table 8-6 lists the METS costs.

\subsubsection{Tritium Handling and Vacuum System}

The major equipment cost in the tritium and vacuum system is that of the cell cleanup facility. Unfortunately, the cost estimate for this equipment has the highest error because the unit is significantly larger than any that is presently available. Little development effort is required, and estimates can he made by extrapriating from smaller existing systems.

Most of the remaining equipment is available commercially and can be costed with greater confidence. The main uncertainty is in the exact number of control functions, such as readout, alar-

\section{COST ESTIMATE FOR METS (PHASE II)}

Sitorage conits

Cryugenic disconnects

HVid interrupters

('harging puwer supply

Electricil leads

Isolation connectors

(atbliner

Storage coil dewars

Liquid and gas distribution

Refirigeration and crygenic storage

Equipment assembly and testing 20$)^{\prime}$,

Fingrineerings $10 \%$

Contimgency ise,

ming, and sampling frequency. which will achieve optimal efficiency and safety in system operation. The estimate includes the cost of the storage and transfer system, tritium purification equipment, gas metering and mixing, and the analytical equipment. Sufticient uranium beds are included to allow for some $T_{?}$ in storage as well as in off-site tritium recovery facilities.

A high maintenance item is the fast operating downcomer valve which must endure approximately 800 closures a month. As a result, a second valve is included on the downsomer to allow replacement of the Viton gasket in the fast operating valves.

Table 8-7 is a summary of the estimates for the system. Cleanup system costs constitute by far the major portion of the cost, and a partial breakdown of that system is listed, although more detailed costs were generated.

\subsubsection{Diagnostics System}

The diagnostics system will be made up of presently available instruments integrated into a large sophisticated system which will require a development effort of approximately 12 man-years. The estimate is given in Table 8-8.

\subsubsection{Control System}

The control system consists of a main computer and a number of satellite computers with the 


\section{COS'I' ESTIMATE FOR TRITIUM HANDLING AND VACUUM SYSTEM}

\begin{tabular}{|c|c|}
\hline liranium beds & $\$ 0.04 \mathrm{M}$ \\
\hline (ryosernic pumping systems & $0.1: 3$ \\
\hline Dewars & $0,0)$ \\
\hline Piping and valves & 0.16 \\
\hline Diagnostic equipment & 0.02 \\
\hline Gas cont rol equipment & 0.04 \\
\hline Hond enciositires: & 0.116 \\
\hline System cont rol equipment & 0.0 .3 \\
\hline Tritium waste treatment system & 0.13 \\
\hline \multicolumn{2}{|l|}{ ('rll cleanup facility } \\
\hline 2 l'reheaters & 0.52 \\
\hline 2 Catalytie reactors & 0.44 \\
\hline 2 Heat exchangers & 0.18 \\
\hline 2 Blowers & 0.17 \\
\hline \multirow[t]{2}{*}{+ Dryer towers } & 0.44 \\
\hline & 2.38 \\
\hline Fingrineering $2 u^{4}$ & 0.47 \\
\hline Equipment assembly testing 25 & 0.59 \\
\hline \multirow[t]{2}{*}{ ('ontingeney lis', } & $\begin{array}{l}3.44 \\
0.52\end{array}$ \\
\hline & $\$ 3.96 \mathrm{M}$ \\
\hline
\end{tabular}

TABI.E 8-8

\section{COST ESTIMATE FOR DIAGNOSTIC EQLIPMENT}

lligit al st reak cameras (4)

Plasma luminusity TV monitors (80)

$\$ 0.28 \mathrm{M}$

Neut ron scintillaturs (8(1)

1.20

0.08

Vout ron comenters (20)

(coupled-cavity interferometers (4)

0.01

0.08

(1. 40

Thomsinn scattering 14

Fixcluded flux 1801)

Fipuipment assembly and testing 2()$^{x}$,

$\frac{0.16}{2.21}$

0.44

3.25

(ontingene' 15$)^{\prime}$

Total
COST ESTIMATE FOR CONTROL SYSTEM

Main cont rol computer system

Soft ware development system

$\$ 0.127 \mathrm{M}$

CRT cont rol

0.216

Pl cont rol

Implosion-heating cont rol

Tritium and vacuum cont rol

0.171

0.100

0.378

$0.1(k)$

METS crint rol

0.111

Compression bank cont rol

1.654

Diagnostics cont rol

0.505

Precision pulser system

0.861

Cabling

Fquipment assembly and testing $2 r^{r}$,

$\frac{0.048}{4.28}$

4.22

Contigenn $10 \%$;

$\frac{0.84}{5.06}$

Tinal

$\frac{0.51}{\$ 5.57 \mathrm{M}}$

\subsubsection{Facilities}

The facilities cost estimate includes not only items relating to the structure itself, such as the cell. remote handling facilities, control room. experimental enclosure, and maintenance bays, but also site preparation, utility connections, and access roads. The construction of the facilities is straightforward. with the exception of the concrete cell which has 1 1/2-m-thick walls. Care has been taken to keop the size of the building to a minimum and to take a cost effective approach in the design and type of construction.

Table 8.10 gives a summary of the facilities estimate. The building estimates are based on cost per square foot for similar types of structures. whereas the concrete estimates are based on volume costs. The utilities estimate has been determined by using a structural percentage figure of the structural costs plus cost for length of runs to the nearest existing utility that can handle the facility adequately. Because of the large elect rical power requirement. a separate estimate has been made for the electrical utilities, including an appropriately sized substation and distribution sustem.

\subsection{SFTR SYSTEM COST}

The cost estimate for the entire SFTR experiment is given in Table 8.11. The cost for Phase I operation is $\$ 133.16 \mathrm{M}$. and an additiona: $\$ 72.80 \mathrm{M}$ is required

associated interface equipment and cabling. All control sistem components are available commercially from computer manufacturers and the costs are bas. ed on catalog prices. System development and engineering are included in most of the subsystem costs. The engineering required for system interfacing is assumed to be $15 \%$ \% Table $8-9$ lists the costs. 
TABLE 8-10

\section{FACILITIES COST ESTIMATE}

\section{Cell}

Remoce handling facilities

Torus st ructure

MFTS system buildings

Receiving. assembly, and maintenance areas

Tritium waste building

control room

Electrical power

I'tilities

Site development

Engineering $20 \%$

contigency 10 .

TABLE 8-11

\section{COST ESTIMATF FOR SFTR SYSTEM}

First wall and Marshall coil

Implesion-heating system

Preionization system

Primary shield

Cumpression bank (Phase I)

Tritium handling and vacuu $m$ svistem

Diagnostics system

Cont rol system

Facilities

METS șistem (Phase II)

Total

for Phase II. The cost of the experiment is therefore $\$ 205.96 \mathrm{M}$. Some of the cost associated with the Phase I compression bank ( $\$ 31.09 \mathrm{M}$ ) could be saved by going directly to a METS system.

\subsection{CONSTRUCTION SCHEDULE}

Figure 8-1 shows a simplified logic schedule for the SFlR project. The timing is based on the assumption that funding for early facility design and site preparation will be available by Octoher $197 \pi$, and major procurement and construction funds approved by October 1978. The logic diagram shows the milestones for the various subsystems in the project. as well as the more critical design review points and information restrictions.

Certain equipment items will require the longest possible development al time, and will determine the schedule's critical paths:

- Plasma tube first wall (ceramic)

- Marshall coil assembly

- Compression field coil

- Compression field metal-to-metal crowbar switch

- $150-\mathrm{kV}$ shock heating field capacitors

- Superconductive energy-storage coil

- Cryogenic disconnect switch.

The schedule assumes that much of the materials research and fabrication development for these items will proceed well in advance of any specific construction funding for SFTR. Actually, much work is already in progress.

Facility construction (Title III) is estimated to require $3 \mathrm{yr}$. So that ecuipment installation and checkout can begin as early as possible, it is assumed that beneficial occupancy is obtained 9 months before facility cornpletion. This will allow sufficient time for Phase I operations (capacitor-driven compression bank) to begin by April 1984. Certain systems closely associated with the facility. e.g., cell cleanup system, remote handling equipment, and the cryogenic system refrigeration machinery, may be installed even before the beneficial occupancy date.

After an estimated 1 yr of Phase I experimental operations, the facility will be converted for Phase II (superconductive-coil-driven compression bank) operation. This will involve retrofitting the compression capacitor bank for operation as a transfer bank. and a period of integrated systems checkout with the METS system. Phase II operations should begin by January 1986. 


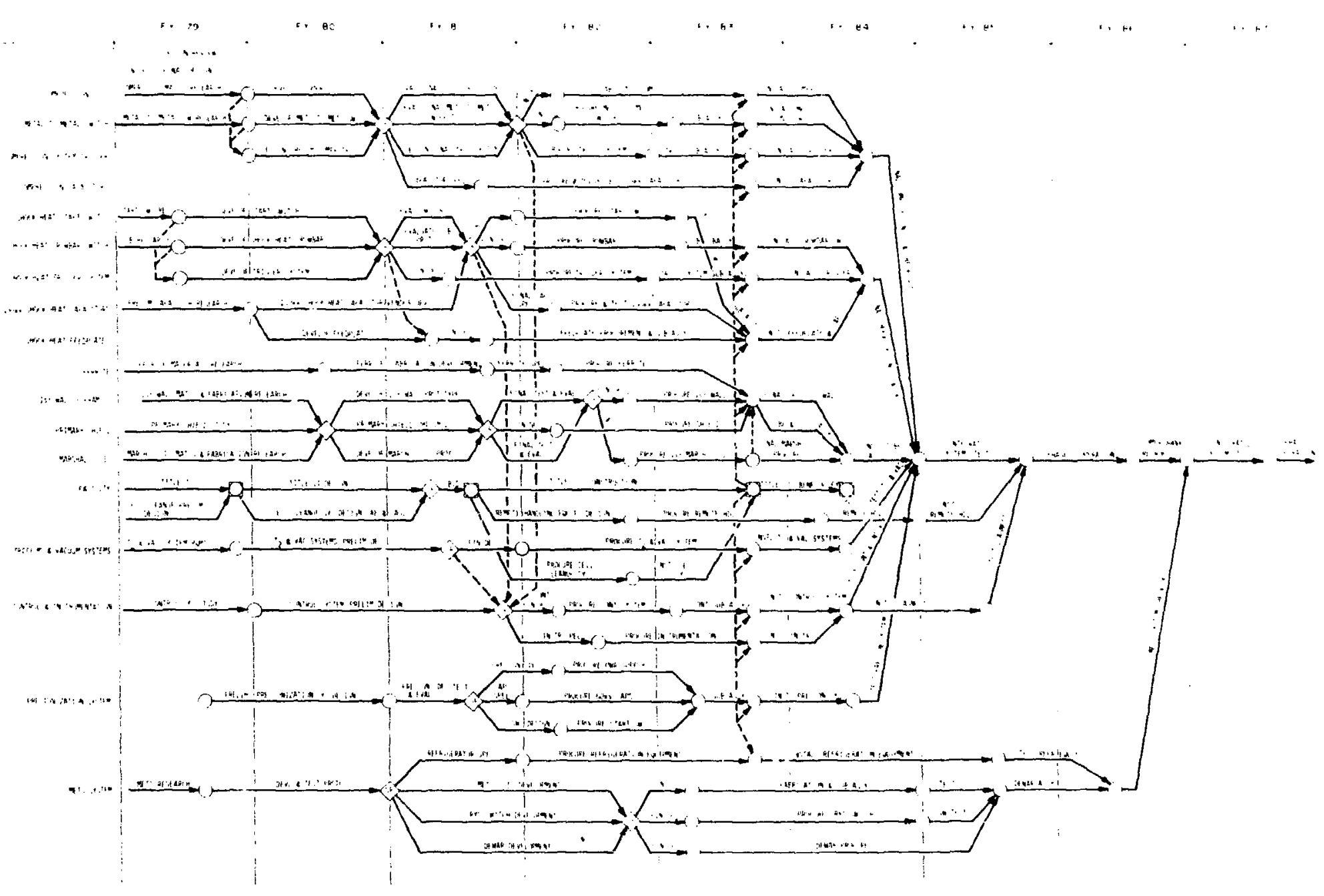

Fig. 8- $I$.

SFTR construction schedule. 


\section{APPENDIX 1}

\section{ROOM T'EMPERATURE MAGNETIC ENERGY STORAGE}

\section{A.1 SUMMARY}

The Warm METS system proposed here is a viable magnetic energy transfer and storage alternative for SFTR. It will provide the compression energy by conventional technology, using a room temperature 500-MJ $r$ nergy-storage inductor. The inductor is to be charged on-line from the Public Service Co. of New Mexico's 115-kV transmission system. This system is feasible and when built with stationary equipment it is the most economical system for magnetic energy storage. Rotating machinery is therefore not discussed. The compression coil, energy transfer capacitors, and transfer initiating current interrupters are the same as for the superconducting METS.

\section{A.2 CIRCUIT DESCRIPTION}

The circuit is shown in Fig. A-1. 'T he energystorage inductor is described in Sec. A.3. its dc resistance and inductance are $0.18 \Omega$ and $1.344 \mathrm{H}$. During energy transfer, the resist ance rises to $140 \Omega$ because of eddy currents.

The inductor is charged by the rectifier transformer circuit shown in Fig. A-1. The transformer is enc rgized from the $115-\mathrm{kV}$ bulk power system. The load seen by the $115-\mathrm{kV}$ system is plotted in Fig. A-2, where the time constant $T=7.4$ $s$ and the time interval over which charging takes place is $34 \mathrm{~s}$. The maximum load occurs at the end of charging just before the load is dropped. Turn-on and load dropping are both effected by the $115-\mathrm{kV}$ circuit breaker $\mathrm{Cbl}$. The switching sequence is given in Table A-1. The load dropped at the end of charg. ing is $141 \mathrm{MVA}, 136 \mathrm{MW}$ as seen in Fig. A-2.

The 12-phase rectifier transformer uses freewheeling diodes and is rated at two-thirds the peak load or 90 MVA with $8^{\prime \prime}$ impedance. The out put rectifier uses solid state diodes.

A load study ${ }^{1}$ shows that light flicker and the possible cumulative effect of the 2000 mechanical pulses on the inrbine generators at Los Alamos when SFTR fires must be considered in the design. Both effects can be tolerated if:

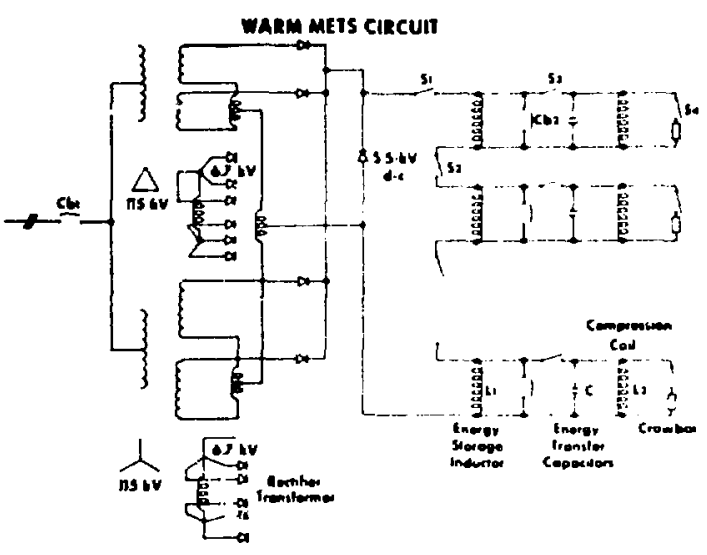

Fig. A-1.

('ircuit for direct line charging of un inductice' energy'storage ring?.

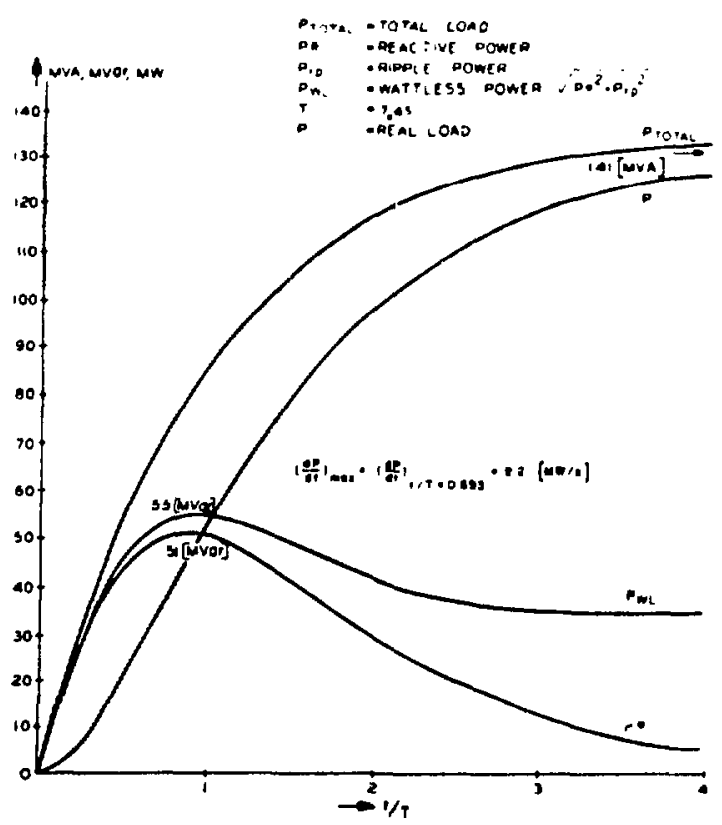

Fig. A-2.

Power load to the 115-kV line. 
TABIE A-1

\section{SWITCHING SEQUENCE:}

\begin{tabular}{|c|c|}
\hline \multicolumn{2}{|r|}{ Operation } \\
\hline No. & Description \\
\hline 1 & Prepare circuit cireuit tor eharging \\
\hline$\underline{2}$ & Start charging \\
\hline 3 & Find charging \\
\hline+ & Reduce I'R loss at idling \\
\hline$i$ & Prepare fur energy transfer \\
\hline fi & Initiate transfer \\
\hline$\tau$ & ('rowbar \\
\hline
\end{tabular}

1. The $345 . \mathrm{kl}$ transmission line from the Four Cirners plant to Alhuquerque with a $34.3 / 115-k$ l transformer is in operation in 1980: and

2 . One $115-\mathrm{kl}$ transmission line from Xorton to las Alamos is dedicated at least during the hours that the experiment is run or an additional $11 \mathrm{r}-\mathrm{k} \mathrm{V}^{\circ}$ transmission line is inst alled from . Norton at a cost of

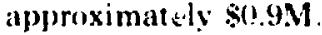

The New Mexico Public Service Company bulk power system as planned for !980 is shown in Fig. A3. 'This ststem includes the $345 / 115-\mathrm{kl}$ ' substation extension at Norton. The load study indicates the voltage levels listed in Table A-2. The table shows that the voltage drop on the $115 \cdot \mathrm{kV}$ side of the experiment will be $9.6^{\circ}$, and the flicker when the load is dropped will be 2.:3', at Santa Fe 1Zial. This njocker is close to the limil of acceptability.

The circuil breaker $\mathrm{Ch} /$ is a $115-\mathrm{k} \mathrm{l}^{\circ}$ breaker with vacuum cells and with a maximum interrupting rating of $4 \mathrm{kA}$ and momentary rating of $40 \mathrm{kA}$. The circuit breakers (b2 are 300 . to 400 -k.J units as developed tor the MFTS program.

\section{A.3 ENERGY-STORAGE INDUCTOR}

\section{A.3.1 General Jeseription}

Only the most important design equations and features of the energy-storage inductor are given here. All derivations and many design details are

$\frac{\text { Breakers }^{\mathrm{a}}}{\text { CBi CB2 }} \quad$ S1 S2 S3 S1

$\begin{array}{llllll}0 & 11 & \text { j } & 1 & 11 & 0 \\ 1 & 01 & 1 & 1 & 11 & 11 \\ 11 & 11 & 1 & 1 & 11 & 11 \\ 0 & 1 & 1 & 1 & 11 & 11 \\ 11 & 1 & 0 & 11 & 1 & 11 \\ 0 & 11 & 11 & 11 & 1 & 11 \\ 0 & 11 & 11 & 11 & 1 & 1\end{array}$

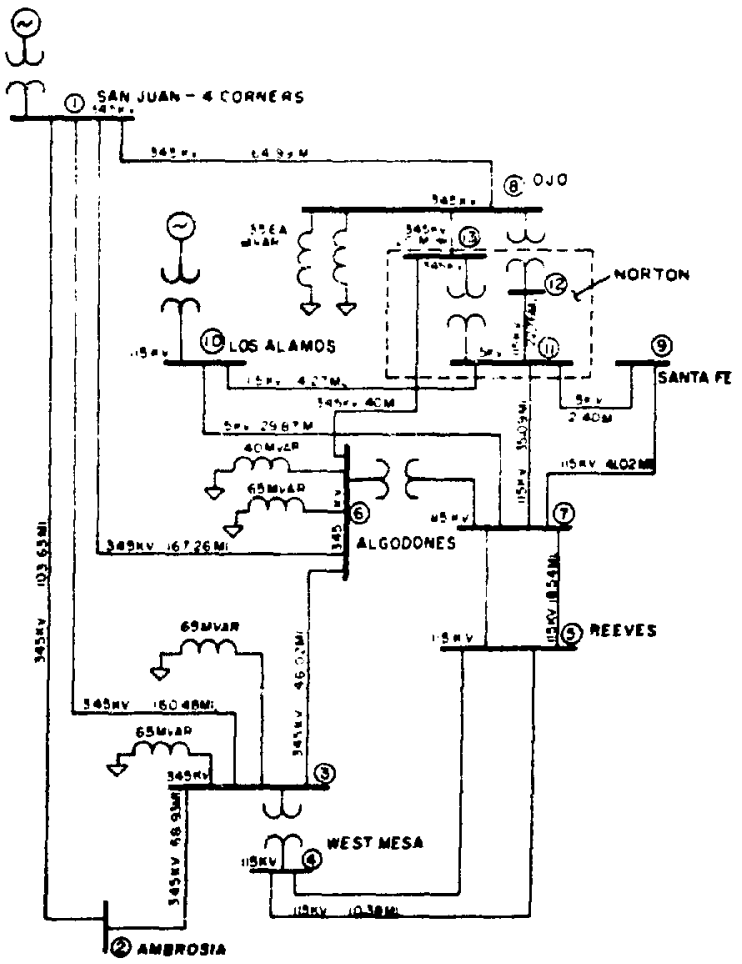

Fig. A.3.

The Vite Mexico Public Sercice Company's poucer sustem for 1980 . 
TABLE A-2

\section{UNIT VOLTAGE AT SEVERAL POINTS IN THE BULK POWER SYSTEM WITH DEDICATED 115-kV LINE NORTON TO LOS ALAMOS}

\begin{tabular}{|c|c|c|c|c|c|}
\hline \multicolumn{2}{|c|}{ Load } & \multicolumn{4}{|c|}{ Voltages at Locations ${ }^{a}$} \\
\hline MW & (reactive) & ETA $^{\mathbf{b}}$ & Zia & Nurton & Experiment \\
\hline 0 & 0 & 1.025 & 1.019 & 1.031 & 1.0332 \\
\hline 55 & 55 & .996 & .991 & .997 & $.9: 36$ \\
\hline $1: 36$ & 10 & 1.002 & .999 & 1.005 & .985 \\
\hline
\end{tabular}

aThese unit voltages have been scaled linearly with load from a similar table given in Ref. 2 for different ioad numbers: for $10 \mathrm{MW}, 40 \mathrm{MV}$ ar and for $100 \mathrm{MW}, 7 \mathrm{MVar}$.

'Eastern Tech Area.

reported in Ref. 2. The inductor consists of 12280 round coils with two turns each, all coils assembled in toroidal fashion. An alternative design. in which each coil is surrounded by a two-turn secondary winding designed for energy transfer at improved transfer efficiency, was rejected because of extra energy requirements for the counterpulse circuit. Economy requires the use of copper conductors for room temperature operation, although a cryogenic aluminum design is feasible.

\section{A.3.2 Charging Círcuit}

The inductor is charged from a virtually constant voltage source. The current is thus given by

$$
i=\frac{V}{R}\left(1-e^{-\delta \tau}\right)
$$

where

$$
\delta \equiv \frac{1}{\mathrm{~T}}=\mathrm{R} / \mathrm{L}
$$

and

$$
\frac{V}{R} \equiv I_{0}
$$

and where $r$ denotes time. The loss over a charging cycle is given by

$$
\begin{aligned}
& R \int_{0}^{t} i^{2} d \tau \\
& =I_{0}^{2} L\left(x+2 e^{-x}-\frac{1}{2} e^{-2 x}-\frac{3}{2}\right)
\end{aligned}
$$

where $\mathrm{x} \equiv \delta \mathrm{t}$.

We have for each of the 1280 coils, $I_{0}=27.1 \mathrm{kA} . \mathrm{t}$ $=34 \mathrm{~s}, \mathrm{~T}=7.4 \mathrm{~s}, \mathrm{~L}=1.05 \mathrm{mH}$. and $\mathrm{R}=0.14 \mathrm{~m} \Omega$.

The total energy fed into the R-L sustem $\left(W_{t o t}\right)$ when expressed in units of the total stored magnetic energy $\left(W_{m}\right)$ has the following limit when $x \ll j$;

$$
\lim _{x<1}\left(\frac{w_{t o t}}{w_{m}}\right) \approx 1+\frac{2}{3} x
$$

For large values of $x$.

$$
\lim _{x \rightarrow 1}\left(\frac{W_{t o t}}{W_{m}}\right) \approx 2(x-1)
$$

The corresponding expressions tor the maximum power demand occurring at the end of the charging cycle are ${ }^{2}$

$$
\lim _{x<1}\left(\frac{P}{W_{m}}\right) \approx \frac{2}{t}+\frac{1}{T}
$$




$$
\lim _{x \rightarrow 1}\left(\frac{P}{W_{m}}\right) \approx \frac{2}{T}
$$

Equations (5) and (6) show that charging is done with minimum power loss, if $x \ll 1$, for which energy input equals energy stored. Though Fq. (T) is a good approximation for the charging cycle of a supercondurting coil, Eq. ( $(8)$ is used for Warm MFIS where $\mathrm{T}=7.4 \mathrm{~s}, \mathrm{x}=4.62$. and hence $W_{1,1}, \mathrm{~W}_{\mathrm{n}}=7.42$ : $\mathrm{P}_{\text {mix }} / \mathrm{W}_{m}=0.275 \mathrm{~s}^{-1}$.

'The inductance of a thick-walled toroid is:'

$$
\mathcal{L}=\lambda, N^{2} \frac{\mathrm{A}}{l}
$$

where

$$
\begin{aligned}
& \mu=0.4 \pi 10^{-8} \mathrm{H} / \mathrm{cm} \\
& \lambda=\text { toroidal form factor } \\
& \therefore=\text { number of turns } \\
& \mathrm{A}=\text { effective flux linkage area } \\
& /=\text { coil length }(\text { if the solenoid consints of } \mathrm{M} \\
&\text { identical coils, we have } \left./=2 \pi \mathrm{R}_{\text {mat }} / \mathrm{M}\right)
\end{aligned}
$$

'The toroidal form factor for the minor aperture radius $R_{4}$ and the major toroidal radius $R_{m a 1}$ is given by

$$
\lambda \approx 1+\frac{r}{4}\left(\frac{R_{i}}{k_{\mathrm{inaj}}}\right)^{2}
$$

where

$$
k=1+\frac{2}{3} \frac{b}{R_{i}}+\frac{1}{6}\left(\frac{b}{R_{i}}\right)^{2}
$$

and where the coil height

$$
\mathbf{b}=\frac{N q}{p l}
$$

with $q$ denoting the conductor cross-sectional area, and $p$ the coil packing factor.

The tlux linkage area is then

$$
A=R_{i}^{2} \pi k \text {. }
$$

We choose $b=41.2 \mathrm{~cm}, R_{i}=166.0 \mathrm{~cm}, R_{\text {maj }}=1001$ cm, $p=0.336, \lambda=1.007, k=1.1755, N=2$, and use a total of 1280 coils.

The maximum flux density is $B_{\max }=1.68 \mathrm{~T}$, with a corresponding minimum in the aperture $B_{\min }=$
1.20 $\mathrm{T}$. The center value within the minor circle is $\mathrm{B}$. $=1.40 \mathrm{~T}$.

The dc resistance of each of th. 1280 coils is given by

$$
R_{d c}=\rho N \frac{2 \pi\left(R_{i}+\frac{b}{2}\right)}{q}
$$

where

$$
p=p_{0}(1+\alpha \theta)
$$

and

$$
\begin{aligned}
& p_{11}=10^{-4} / 61 ! ? \cdot \mathrm{m} \\
& a=1 / 234.5)^{\circ}(-1 \\
& \theta=\text { average copper temperature }
\end{aligned}
$$

The pulse resistance can be approximated by an ac resistance consistent with the frequency spectrum described in Sec. A. 3.3 . The ac resistance is given by

$R_{a c}=R_{d c}\left[\zeta(\zeta)+\psi(\zeta) \frac{n}{m} \sum_{v=1}^{m} v(v-1)\right]$

where

$$
\zeta=\frac{h}{\delta}
$$

with

$$
\begin{aligned}
\mathrm{h} & =\text { strand thickness } \\
\delta & =\text { skin depth } \\
& =\sqrt{\left(2 /(\omega) /\left(\frac{\mathrm{a}}{\mathrm{a}}\right)\right.} \approx\left\{\begin{array}{l}
28 \mathrm{~cm} \text { for charging } \\
0.25 \mathrm{~cm} \text { for discharging }
\end{array}\right.
\end{aligned}
$$

$a=\Sigma$ copper widths over /

$\mathrm{m}=$ (number of conductors)(number oi strands stacked radially)

$\eta=$ transposition weight $\mathrm{f}=1$ for the perfect . ly transposed winding: $=\mathrm{m}$ for the nontransposed winding)

and

$$
\phi(\zeta)=\zeta \frac{\sinh 2 \zeta+\sin 2 \zeta}{\cosh 2 \zeta-\cos 2 \zeta}
$$




$$
\dot{\psi}(\zeta)=2 \zeta \frac{\sinh \zeta-\sin \zeta}{\cosh \zeta+\cos \zeta} .
$$

Of course, $\phi(0)=1 ; \psi(0)=0$. Equations (18) and (19) show that with our time constant $T=7.38 \mathrm{~s}$ there is basically no need for stranding, because we get $R_{a c} / R_{d c}=1.001$. However, because of the requirement that the flux be extracted within 0.7 ms. the winding must be stranded, as shown in the following sections.

\section{A.3.3 Flux Extraction}

The energy is transferred from the storage inductor to the compression coil by way of the transfer capacitors C shown in Fig. A-1. The current in the compression coil for this oscillatory transfer is easily derived, and may be written

$$
\begin{aligned}
\frac{i_{2}}{I_{10}} & =\left(\frac{\omega_{2}}{\Omega_{0}}\right)^{2} \\
& \cdot\left[1-\left(\frac{\Delta}{\Omega} \sin \Omega t+\cos \Omega t\right) e^{-\Delta t}\right]
\end{aligned}
$$

where $I_{10}$ is the initial current in the energy-storage coil, and

$$
\begin{array}{ll}
\omega_{1}^{2}=1 /\left(L_{1} C\right) & \Omega_{0}^{2}=\omega_{1}^{2}+\omega_{2}^{2} \\
\omega_{2}^{2}=1 /\left(L_{2} C\right) & \Delta=\delta_{1}+\delta_{2} \\
\delta_{1}=R_{1} / L_{1} & \Omega^{2}=\Omega_{0}^{2}-\Delta^{2} . \\
\delta_{2}=R_{2} / L_{2} &
\end{array}
$$

The following numbers apply for the present design:

$$
\mathrm{L}_{1}=1.05 \mathrm{mH} ; \quad \mathrm{L}_{2}=0.98 \mathrm{mH} ; \quad \mathrm{C}=0.101 \mathrm{mF}
$$

for which the required stored energy $1 / 2 L_{1} I_{10}^{2}$ may be approximated by the empirical formula with $R_{2}=$ $50 \mathrm{~m} ! 2$ being the fixed parameter:

$$
\begin{aligned}
& W_{1} / W_{c} \approx \\
& \left.\left(1+0.033 R_{2} / R_{2}\right)\right|_{R_{2}}=0.05 \Omega,
\end{aligned}
$$

which is ensily verified with Eq. (20) for a limited range in $W_{1}$. Here, $W_{o}=459.8 \mathrm{MJ}$. Equation (21) shows the importance of low ac resistance. With $R_{1} / K_{2}=2$, for example, $6.6^{\circ}$ of the stored energy is dissipated during the transfer.

It is important to consider the eddy current problem because the 7.4-s time constant requires substantial radial thickness of the winding (i.e.. 41.2 $\mathrm{cm}$ ), and because the $0.7-\mathrm{ms}$ transfer time implies a frequency of $0.7 \mathrm{kHz}$. Figure A-4 shows ihe frequency spectrum for the compressior coil current pulse, and the $0.7-\mathrm{ms}$ rising pulse. The current derays with a 250-ms time constant following the crowbar of the coil. The low frequency components are generated by the duration of the pulse, and the high frequency components by the fast rise. The $0.7-\mathrm{kHz}$ frequency used for ac resistance calculations corresponds to the one indicated by an arrow in the frequency spectrum. It can be seen that the area under the frequency spectrum is divided approximately in half by the frequency chosen for the resistance calculations.

We propose 100 conductor strands and obtain from Eqs. (16)-(19) $\zeta=0.928, \phi=1.064, \psi=0.240$ for $\omega=4490 \mathrm{~s}^{-1}$, which yields a ratio of ac resistance to dc resistance of $R_{a c} / R_{d c}=801$, and thus $R_{a r}=$ 0.111 S2. For this set of numbers we obtain from Eq.
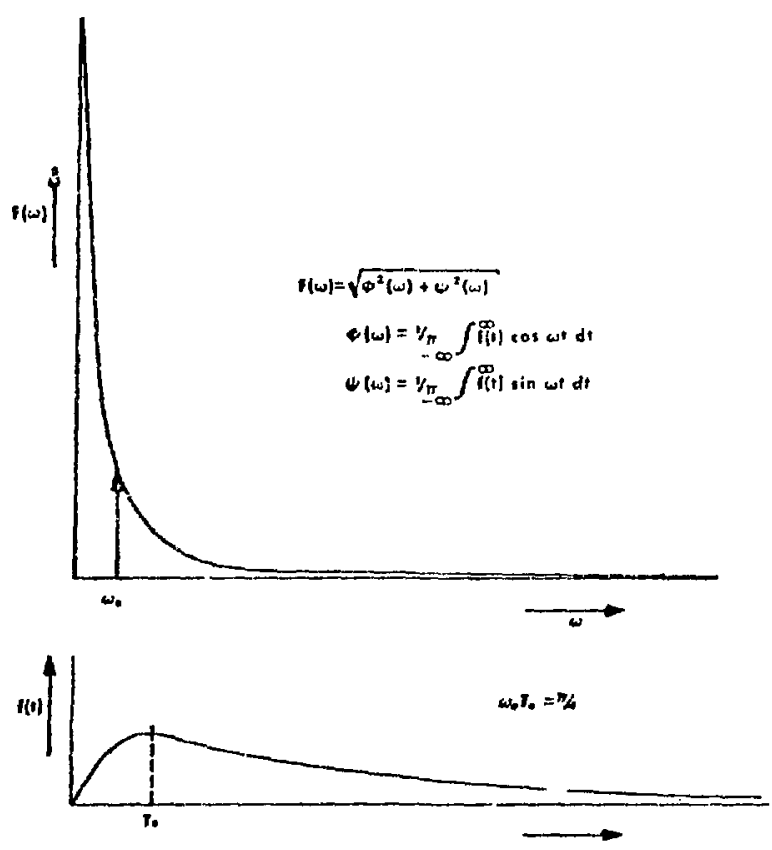

Fig. A-4.

Frequency spectrum (above) of the compression coil current waveform (below). 
(21) the energy loss in the storage coil $\left(W_{1} / W_{0}-1\right)$ $=7.4 \%$, and the total transfer loss in both energystorage coil and compression coil $\left(W_{1} / W_{2}-1\right)=$ 11.6. With the compression coil design current $I_{2}=$ $26.6 \mathrm{kA}$, corresponding to a compression field energy $W_{2}=44: 3 \mathrm{M} \cdot \mathrm{J}$, and a central field of $5.1 \mathrm{~T}$, the stored energy is $W_{1}=494 \mathrm{MJ}$.

The coil winding is shown in Figs. A-5, 6, and 7, with Fig. A-5 indicating the dimensions of the toroidal winding, and Figs. A-6 and 7 showing the dimensions of an individual coil (all dimensions in millimeters).

\section{A.3.4 Temperature Rise and Cooling}

The resistivity of the coils is given by $\mathrm{Eq}$. (14) so one can determine the instantaneous temperature rise $\$ 0$ resulting when all

$$
\int_{0}^{T} I^{2} R d t
$$

energy is stored in the thermal mass of the conductor.

$$
\Delta \theta=\frac{1}{\alpha}\left(\mathrm{e}^{\alpha \mathrm{F}(\tau)}-1\right)
$$

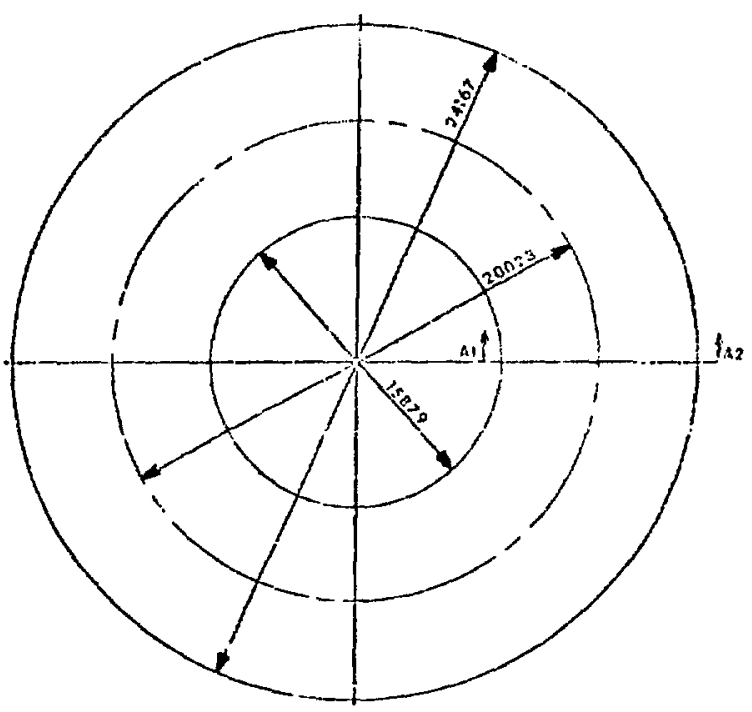

Fig. A-5.

A 2.1 -m-diam toroidal inductive storage ring consisting of 1280 coils. One of the coils making up this torus is shown in Fig. A-6.

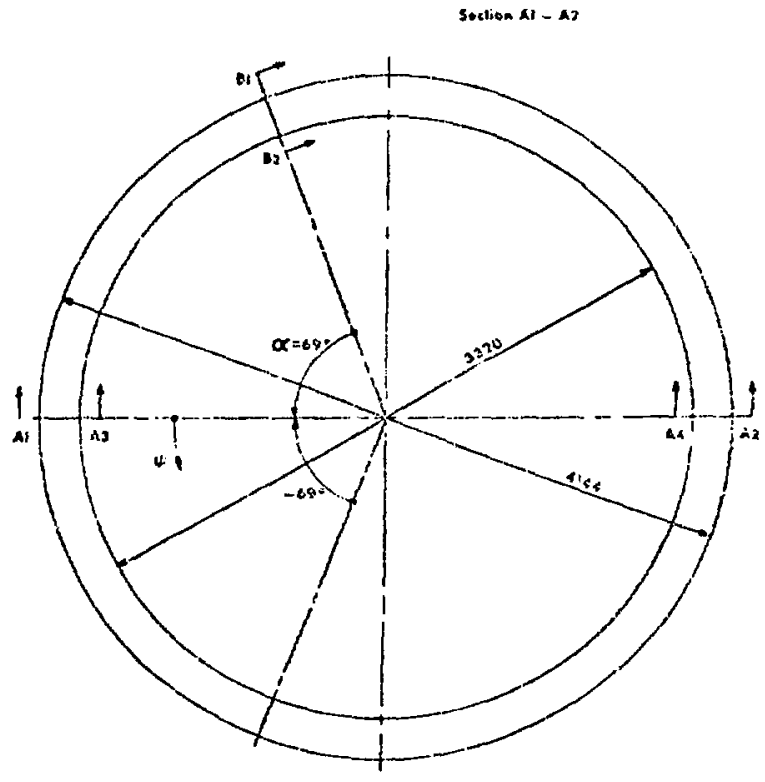

Fig. A-6.

Dimensions of each of the 1280 individual coils of the storage ring.

where

$$
\begin{aligned}
F(\tau)= & \frac{\rho_{o} T}{\gamma C_{m}}\left(\frac{I_{o}}{q}\right)^{2} \\
& \left(x+2 e^{-x}-\frac{1}{2} e^{-2 x}-\frac{3}{2}\right)
\end{aligned}
$$

with

$$
\begin{aligned}
& \left.\begin{array}{l}
\mathrm{C}_{\mathrm{n}}=0.389 \mathrm{~J} / \mathrm{g}^{\circ} \mathrm{C} \\
\gamma=8.9 \mathrm{~g} / \mathrm{cm}^{3}
\end{array}\right\} \quad \text { for copper } \\
& \mathrm{T}=\mathrm{L} / \mathrm{R} \\
& \mathrm{x}=\pi / \mathrm{T} \\
& q=\text { conductor cross-sectional area } \mathrm{cm}^{2} \\
& p_{t}=\text { resistivity at start of pulse. }
\end{aligned}
$$

The temperature rise per pulse is $\lambda \theta=7.6^{\circ} \mathrm{C}$, and the temperature rise for any number of pulses is less than $20^{\circ} \mathrm{C}$ if the winding is water-cooled at a flow rate of $20 / / \mathrm{s}$. The cooling water requirement is given by this flow rate at an average load of $6 \mathrm{MW}$. The consequences of coolant flow interruption are discussed later. 

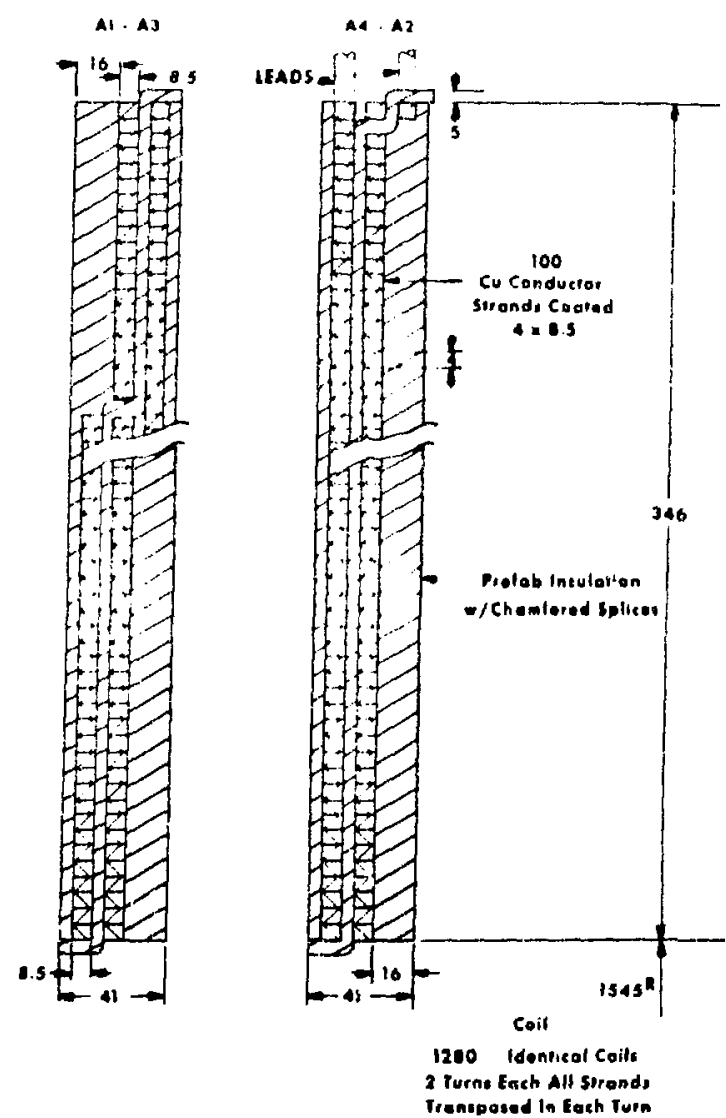

Fig. A.7.

Cross sections of the individual coils.

\section{A.3.5 Structural Considerations}

The basic structural design requirements have been developed in conceptual form from the preliminary' design data of this proposal. These considerations include:

1. Support of the torus against magnetic pulse forces tending to move each coil inward towards the major axis.

2. Support of each coil against hoop stresses developed by magnetic pulse forces.

3. Support of the torus weight as a whole.

The analysis in this report covers concepts related to the above three structural considerations, including the selection of materials and sizing studies.

\section{A.3.5.1 Torus Inner Support Structure}

A concrete ring structure is envisioned for supporting the torus against the net magnetic force in the midplane. This magnetic force tends to move each turn towards the major axis. Figure A-8 illust rates the concept. The niagnetic force per turn is

$$
F=2 \pi B_{0} I k_{m a j}\left(\frac{1}{\sqrt{1-y^{2}}}-1\right)
$$

with $\mathrm{B}_{1}=1.4 \mathrm{~T}, \mathrm{I}=27.1 \mathrm{kA}$, and where $\mathrm{y}=0.195$ is the ratio of minoe $\mathrm{t}$ major diameter. We find $\mathrm{F}=$ $46.6 \mathrm{kN} / \mathrm{turn}(10.5 \mathrm{kips} / \mathrm{turn})$. Since there are two turns per coil, $F_{r}=2 F=9.3 .2 \mathrm{kN} / \mathrm{coil}$ ( $21 \mathrm{kips} / \mathrm{coil}$ ).

There are 1280 pie-shaped coils around the major axis of the torus. The average width of each slice is given by

$$
\bar{W}_{c}=\frac{2 \pi R_{\text {naj }}}{1.280} \text {. }
$$

$\mathrm{I}^{\circ} \mathrm{g}(25)$ gives $\bar{w}_{\mathrm{c}}=0.0491 \mathrm{~m}$. The innermost radius of the toriss is $b=7.94 \mathrm{~m}$, and the width of each coil at that point is $w_{c^{\prime i}}=0.0390 \mathrm{~m}$.

Assuming that we select a concrete cylinder of outer radius b. (conservatively i) to support a $90^{\circ}$ arc of the inner wall of the torus as shown in Fin. A-8, and that a uniform pressure $p$ is imparted to the contact surface of the concrete cylinder, we have

$$
F_{c}=\int_{\frac{\pi}{4}}^{\frac{3 \pi}{4}} p \sin \theta \cdot r d \theta \cdot w_{c i}
$$

which gives for each coil

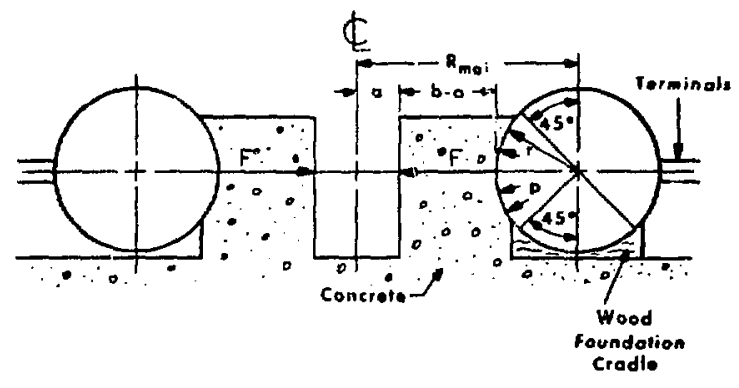

kig. $A-8$.

Concrete retaining structure to support the radial magnetic forces $\boldsymbol{F}$. 


$$
\begin{aligned}
& F_{c}=\sqrt{2} \operatorname{pr} \mathrm{ci} \\
& r=2.001 \mathrm{~m} .
\end{aligned}
$$

Solving (27) for the pressure $p$, we have

$$
\mathrm{p}=\frac{\mathrm{F}_{\mathrm{c}}}{\sqrt{2} \mathrm{rw}_{\mathrm{ci}}}
$$

Substituting the dai a into Eq. (28) gives $p=128$ psi.

The maximum compressive hoop stress ! in the concretel for a thick-walled cylinder under uniform external pressure is ${ }^{3}$

$$
S_{2 \max }=-p \frac{2 b^{2}}{b^{2}-a^{2}} .
$$

The maximun radial stress is

$$
\mathrm{S}_{3 \max }=\frac{1}{2} \mathrm{~S}_{2 \max }
$$

Selecting 3000-psi concrete material, and a concrete inner radius $a=7.100 \mathrm{~m}$. we obtain

$$
\begin{aligned}
& S_{\text {max }}=-1100 \mathrm{psi} \\
& S_{\text {intax }}=-550 \text { psi. }
\end{aligned}
$$

The minimum factor of safety (F.S.) is

$$
\text { F.S. }=3000 / 1100=2.7
$$

This factor of safety appears to be adequately conservative.

\section{A.3.5.2 Hoop Stress in Coil}

Because of the concrete support structure shown in Fig. A-8, and internal pressure in each coil tending to cause uniform dilation of the coils, there will be discontinuity stresses at $\theta=45^{\circ}$ and $\theta=135^{\circ}$. It is desirable to know the magnitude of the stress discontinuities, and whether the windings will be subject to collapse from net compressive stress. To avoid buckling at these points, the magnetic pressure on the coil must exceed the pressure $p|\mathrm{Eq} .(28)|$. Then lle coil is always in tension.

Since the peak field at the inside of the torus is $1.68 \mathrm{~T}$, the magnetic pressure is

$\mathrm{P}_{\mathrm{m}}=\frac{\mathrm{B}^{2}}{2 \mu_{\mathrm{O}}}=1.12 \times 10^{5} \mathrm{~N} / \mathrm{m}^{2}=163 \mathrm{psi}$.
This exceeds the pressure $p=128 \mathrm{psi}$, hence no buckling occurs. Note that the hoop stress is $\sim 5.6$ (radius thickness) times $p_{n}$ where the coil is unsupported, and 5.6 times $p_{m}-p$ at the concrete support. The discontinuity is therefore 714 psi. which is low. The yield st rength of annealed copper is : ? (b) psi.

\section{A.3.5.3 Cradle Support Design for Foundation of Toroid}

The wood foundation cradile in Fig. A-8 musi support the coil weight, which is $W_{1}=280 \mathrm{~kg}(1721 \mathrm{lb})$. The bearing area of material supporting each coil is $A_{b}=0.1355 \mathrm{~m}^{2}\left(210\right.$ in. $\left.{ }^{2}\right)$. Thus, the bearing unit st ress will be $S_{\mathrm{h}}=W_{\mathrm{t}} / \mathrm{A}_{\mathrm{b}}=7.1 \mathrm{psi}$. A toroidal cradle foundation of yellow pine fyield strength is $440 \mathrm{psi}$ foi compression across grain) about $6 \mathrm{in}$. thich at the thinnest point should be sufficient to support the coils. The grain orientation is immaterial hecause of the low unit stress. Care should be taken to avoid contact with soi!. and the wood should be prepared against recay. The foundation could be built in pieshaped sections for each individual coil. Two circumferential bands of mild steel can be used to ensure against slippage of the pie-shaped sections. There should be negligible meridional stress. so these bands should be pretensioned only enough to provide a compact foundation.

\section{A.3.6 Optimization}

The cost vs minor diameter is given in Fig. A. 9 for the approximate design parameters, where the maximum flux density in the aperture is the variable: $L$. $R$, and field energy heing consiant. The cost. number of turns, major diameter, and conductor cross section are dependent variables. The cost varies over a range $5 \div 1$ as one varies the diameter over a feasible range of values. The coil with the least number of turns is the least expensive. and to all intents and purposes the minimum number of turns per section is 2. A torus with only single turn sections would have a minor radius approximately equal to the major toroidal radius and no room would be left for the winding.

For an aluminum conductor, the minimum number of turns per section is 4 ( 3 not being considered because of poor coil space utilization at 3.5 . 7 turns in double pancake coils) costing approximately $\$ 2.8 \mathrm{M}$ on the scales of Fig. A-9. Though this number amounts to a savings of approximately $40{ }^{\prime} ;$ of the 4-turn copper coil, the 4-turn aluminum coil costs still more than the 2-turn copper coil. 


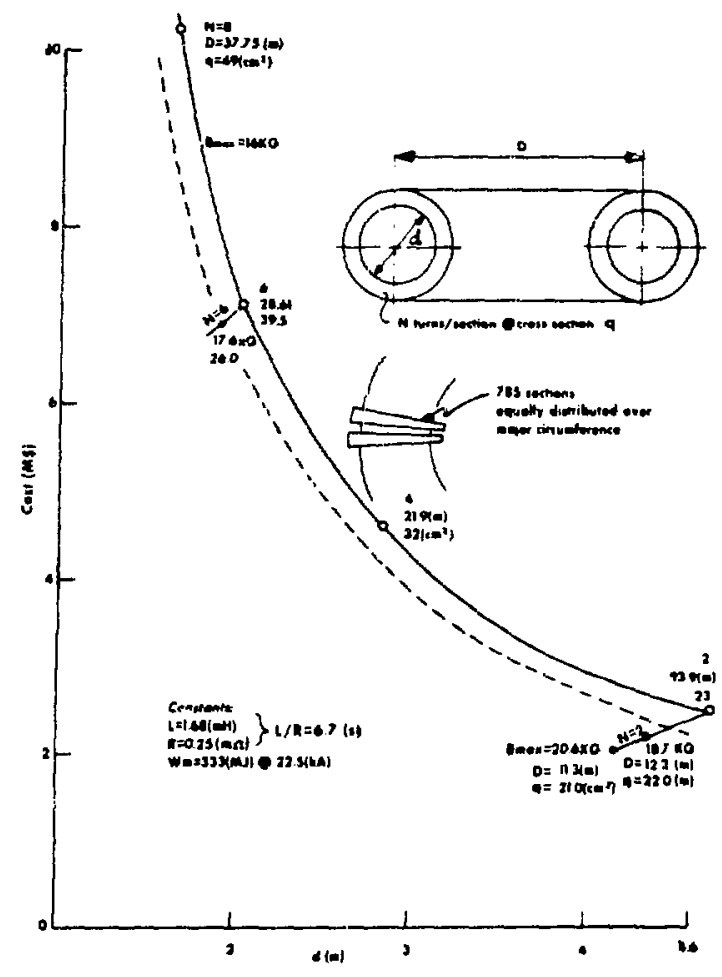

Fig. A-9.

Cost us minor diameter for different magnetic fields and turn ratios of energy-storage coils.
The cost curves have been determined by calculating the copper weight, and rating it at 4.8 $\$ / \mathrm{kg}$. If we base our estimate on $6 \$ / \mathrm{kg}$ of coil mass, then the cost of the energy-storage induclor with the design parameters discussed is $\$ 6.1 \mathrm{M}$. For coun. parison, the outer coils for the 1014 main ring magnets at the Fermilah cost 4.81$) \mathrm{s} / \mathrm{kg}$ for material plus manufacturing in 1970; however the 1975 replacement cost is thought to be $8.80 \mathrm{~s} / \mathrm{kg}$.

\section{A.4 SUMMARY OF COST}

The cost of the Warm METS option is given in Cable A-3. The total cost is equivalent to $7.1 \mathrm{c} / \mathrm{J}$ of mergy transferred into the compression field. The purpose of the switchyard extension at Norton and of the 115.kV transmission line from Nurton is explained in the next section.

\section{A.5 OPERATIONAL CONSMDERATIONS}

Section A.2 mentions the voltage drops and voltage tlicker levels leading to the proposial of the dedicated $115-\mathrm{k} V$ transmission line inentioned there. This line is dedicated to SFTR during its operating hours. The line could in principle be one of the two lines to Los Alamos shown in Fig. A.3. i.e.. the one from Norton or the one from Algodones.

TABLE A-3

\section{WARM METS COS'T SUMMARY}

Switchyard extension at Norton

Overhead 115-kV transmission line Norton to SFTR site $\$ 50 \mathrm{k} / \mathrm{mile}$

Switchyard. outdoor. $115 \mathrm{kV}$, incl. (b)

Rectifier transformer

Rertifier

Auxiliary equipment and cont rols

Engineering

Disconnect switches

Energy -storage induct or

Circuit breaker connecting busses

1280 current interrupters at $\$ 6.5 \mathrm{k}$ each

Cooling

Energy transfer capacitor hank, 2:35 M.J

$0 . . \mathrm{M}$
0.9
11.2
11.6
0.9
0.6
0.2
1.3
6.1
0.7
8.3
0.2
9.4
$5.29 .6 \mathrm{M}$

I.)

0.9

.6

1.3

8.8

.
Total $\$ \frac{9.4}{59.6 \mathrm{M}}$ 
Huwever, during hours of SFTR operation only one transmission line remains for Los Alamos, and that would be insufficient in the event of an outage. For this reason. an additional $115-\mathrm{kV}$ transmission line and the necessary switchyard additions at Norton are listed as line items in the cost summary. The switchyard additions at Norton are to include a 115 $\mathrm{kV}$ line circuit breaker. This transmission line would probably use the same right of way as the existing line to the Eastern Tech Area (ETA) and proceed from there to the SFTR site.

Auxiliary power necessary for corling the water pump. as well as power for the operational controls, would come from the distribution site. but the line circuit breaker $\mathrm{Cbl}$ would receive power from the dedicated $115 \cdot \mathrm{kV}$ svistem. The circuit protection is designed to trip (bl when the site distribution sistem fails. This is necessary to p:event continuation of pulsed operation when auxiliary power is down.
The station battery system for the SFTR switchyard is to be operated from tne site distribution system and not from the dedicated line system. Switch $\mathrm{Cbl}$ is operated from the station battery system as is customary.

\section{REFERENCES (Appendix I)}

1. W. N. Hannah, "Service to Proposed Pulsed Load at Los Alamos," private communication dated March 20, 1975.

2. Herbert F. Vogel, Robert J. Bartholomew, and M. Kristiansen. "500 Megajoule Inductive Energy Storage and Transfer at Rrom is Liquid Nitrogen Temperature." Los Alamos Scientific Laboratory report in preparation.

3. Raymond J. Roark, Formulas for Stress and Strain (McGraw-Hill. 1965). 


\section{APPENDIX II}

\section{CABle PENETKations}

(1) major concern for tritium leakage is the penetration of the biological shield by large numbers of electrical cables. Fach $411-\mathrm{cm}$ module of the reaction ressel will require approximately 36 cables for its operation. These include high soltitge pulse. do charging. control. and diagnostic's types of varying sizes. The largest cables will be pulse types with a diameter of $2.86 \mathrm{~cm}$. For purposes of space allowation it will be assumed that all cables are of this larger size.

The reaction vessel torus will consist of (bt) modules requiring berween $2: 3000$ and $24(N)$ ('abless. most of which must penetrate the biolonical shield while maintaining the integrity of the core st ructure with respect to neutron radiation, tire, and pussible tritium spills.

Conduits for the cables will be located in the ront of the shield and in the lloor (Fig. A-10). The roof conduits will handle the cables reguired for the charging and firing of the shock heating sistem. while those in the thor will connect to the MF.l's banks to drive the compression sistem.

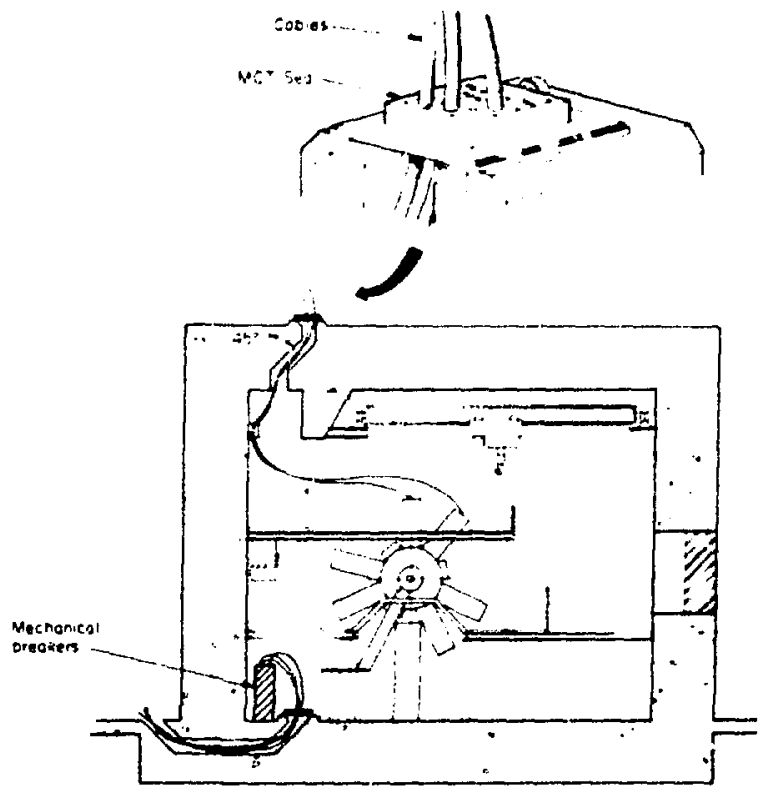

Fig. A-10.

C'ell cross section showing cable penetrations.
'The necessary number of cables will recuire a lis

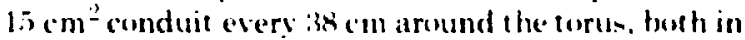
the root and thenr. This will allow rable packing of 40', or less, even assuming the latger cable si\%es. conduits will be steel or ylass-epoxy. Heratloce this number of conduits will remose about fll, of the comcrete along one purtion of the rowit st ructure. ad ditional steel reinforcing will be required betwent the conduits.

The conduits will be direced at an angle of tio from the vertical to prevent direct neut ron st reaming irum the reaction tube. In addition, a comereva beam will be cast as part wi the rowf structure to neutronshadow the condule orifiees and te make up the mass lasis in the shicld caused by the conduit penet rations. The cables themselves will ald in neut rom shielding as their bulk is largely. Pl' and polyethylene.

The cables will he saled by Muldi cable Transit 1.M10"T assemblies. manufactured by Nelscul Filece. erice. The assembly consists of a healy steel frame with modular rubber blocks that are compresed around each eable as shown in Figr. A-I0. The seals accummodatc cable sizes from $5 / 3.210 .3 .3 / 4 \mathrm{in}$. in diameter, and cables can be added or remoned with relative ease. The MC"I units provide an air seal up 11. 2 i psi. and are resistant against $17(x)^{\circ} \mathrm{F}$ fïre fur $1 \mathrm{~h}$ or Ionger.

While the Mo "l assemblies are designed 10 be air. tight. the integrity of the cable penetrations anainst a tritium spill is not entirely dependent on the mechanical perfection of these air seals. (Of equal importance is the maintemance of a negative air presisure of approximately 1 in. of water within the shield st ructure. This effectively prevents diffusion of tritum upstream through a leak in a cable.

Asisume a leak equivalent (n) a $1 / k$-in.-diam round hole:

$$
\begin{aligned}
& d=0.01 \mathrm{ft}(1 / \mathrm{s}-\mathrm{in} . \text { diameter }) \\
& 1=0.2 \mathrm{ft} \text { (l,ength of seal) } \\
& \left.p=5.2 \mathrm{lb} / \mathrm{ft}^{2}-1 \mathrm{in} . \mathrm{H}, \mathrm{O}\right) \\
& p=0.075 \mathrm{lb} / \mathrm{ft}^{*} \text { (Density of air) }
\end{aligned}
$$

Then the equivalent head of air is

$$
h=\frac{p}{\rho}=\frac{5.2}{0.075} \frac{1 \mathrm{~b} / \mathrm{ft}^{2}}{1 \mathrm{~b} / \mathrm{ft}^{3}}=69.3 \mathrm{ft} .
$$


I sing the Darey elpuation fir thew through a duct

$$
\begin{aligned}
& l_{\mathrm{i}}=\mathrm{f} \frac{\ell}{\mathrm{d}} \frac{\mathrm{v}^{2}}{2 \mathrm{~g}} \\
& 69.3=\mathrm{f} \frac{0.2}{0.0 \mathrm{i}}-\frac{\mathrm{v}^{2}}{2(32.2)} \\
& \mathrm{f \textrm {V } ^ { 2 }}=223.55,
\end{aligned}
$$

where

$$
\begin{aligned}
& r=\text { Flenw relecity } \\
& H=32.2 \mathrm{ft} / \mathrm{s}^{2} \\
& i=1 \% \text { triction lactur }
\end{aligned}
$$

and the Revonids number is

$$
\operatorname{Re}=\frac{v d}{v}
$$

where.

$$
\begin{aligned}
& x=\text { Kinemattic viscosity ot air }=1.7 \times 11^{\prime} \mathrm{tt}^{\prime}= \\
& R e=\frac{v(0.01)}{1.7 \times} \frac{10^{-i}}{R e}=58.8 \mathrm{~V} .
\end{aligned}
$$

If we assume laminar fow through the I s.in. diam holle:

$$
f=\frac{6 !}{\operatorname{Re}} \quad \text { and } \quad h=\frac{64}{R e} \quad \frac{\ell v^{2}}{d 2 g} .
$$

Substituting for Re

$$
h=\frac{32}{g d^{2}}
$$

The velucity is

$$
v=\frac{h g d^{2}}{32} \frac{d^{2}}{v \ell}=205 \mathrm{its}
$$

With this velocity the Reymolds number is

$$
\operatorname{Re}=\frac{V d}{v}=\frac{205(0.01)}{1.7 \times 10^{-t}}=12059
$$

which indicates lurbulent flow contrary in the laminar assumption. Wo must therefore use the Dar-

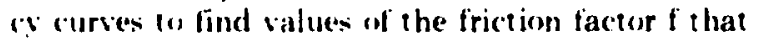
will sat isfy the two equations:

$v=\left[\frac{223.55}{f}\right]^{\mathrm{i} / 2}$ and $\mathrm{Re}=58.82 \mathrm{~V}$.

Relative roughness is assumed to be in the order of 11.14.

A relocits of is nis pives

$$
\begin{aligned}
& R_{0}=32.55 \text { and } i=0.072 \\
& v=\left[\frac{223.55}{0.072}\right]^{\prime \prime}=55.7 \mathrm{ft} / \mathrm{s} .
\end{aligned}
$$

Thus, the flow in a leate of this sige is in the region between laminar and turbulent flow. Actual leaks raused by a misfit in the rubber clamping blocks would be in the form of thin gaps in which the flows. would lie in the laminar range.

Asstuming a laminar flow through the leak and a Hiven tritiun concentrat iun inside the shield struc. cure. the diffusion equation

$$
\frac{\partial c}{\partial c}+v \frac{\partial s}{\partial x}=D \frac{\hat{a}^{2} c}{\partial x^{2}}
$$

caln be used to chbtain the concentration of tritium to anpear at the esurside of the leak.

For steady-state conditions

$$
\frac{\partial c}{\partial t}=0
$$

$$
v \frac{d c}{d x}=D \frac{d^{2} c}{d x^{2}}
$$

The solut ion of this equation ${ }^{1}$ is

$$
\frac{C}{C_{0}}=\exp \left(-\frac{V_{x}}{D}\right)
$$

where

$$
\begin{aligned}
C^{\circ}= & T_{.} \text {concent ration within the containment } \\
C^{\circ}= & T_{3} \text { concentration just outside the } \\
& \text { containment }
\end{aligned}
$$


$Y=$ Gas flow velocity through the leak

$\mathrm{x}=$ Distance along leak

$"$ = Diftusion constant for tritium.

The diffusion constant for hydrogen in air' is 0.611 $\left(\mathrm{cm}^{2} / \mathrm{s}\right.$. Comverting units and adjusting for the higher atomic weight of tritium. we obtain

$$
\mathrm{v}_{\mathrm{i}_{3}} \text { in } \mathrm{H}_{2}=5.37 \times 10^{-4} \mathrm{ft}^{2} i \mathrm{~s} \text {. }
$$

( sing this value of $\mathrm{D}$ and the flow velocity found above. we can get the relative tritium concent ration ratio outside and inside the containtitent:

$$
\frac{c}{C_{0}}=\exp -\left[\begin{array}{rrr}
5.5 & 0.2 \\
5.37 \times & 10-2
\end{array}\right]=e^{-20434}
$$

The value of $\mathrm{C} / \mathrm{C}_{\text {. }}$ is so small as to indieate that the concent ration-driven diffusion of tritium against the pressure-driven bow through the duct is essentially zeru.

If we now postulate a narrow slot-like leak 0.010 in. wide and $1.0 \mathrm{in}$. long and perform the same exercise we get

$$
\begin{aligned}
& \text { hydraulic radius of slot } \\
& \quad d_{r}=\frac{\text { Area of slot }}{\text { Perimeter of slot }} \\
& d_{r}=\frac{(1.0)(0.010)}{2.02} \\
& \cong 0.005 \mathrm{in} .=0.0004 \mathrm{ft} .
\end{aligned}
$$

lsing four times $d_{r}$ for narrow orifices, the velocity is

$$
v=\frac{h \varepsilon(. \mathrm{id})^{2}}{32 \mathrm{LE}}=5.25 \mathrm{ft} / \mathrm{s}
$$

and

$$
\begin{aligned}
\text { Re } & =\frac{\left.v(4)_{r}\right)}{v}=\frac{5.25(0.0018)}{1.7 \times 10^{-4}} \\
& =49.4 \text { (laminar flovi } .
\end{aligned}
$$

Then

$$
\begin{aligned}
& c / c_{0}=\exp \left(-\frac{v_{x}}{D}\right) \\
& =\exp \left(-\frac{(5.25)(0.2)}{5.37 \times 10^{4}}\right)=e^{-1955} .
\end{aligned}
$$

This concentration ratio is still negligihle even with the much lower velocity of flow. Finally. if we assume an extremely narrow leak 0.001 in. wide by 1 in. long we obtain

$$
\begin{aligned}
& d_{y}=\frac{1.0(0.001)}{2.002} \approx 0.0006 \mathrm{in} .=4 \times 10^{-5} \mathrm{ft} \\
& v=\frac{(69.3)(32.2)\left(16 \times 10^{-5}\right)^{2}}{32\left(1.7 \times 10^{-0}\right)(0.2)}=0.053 \mathrm{ft} / \mathrm{s}
\end{aligned}
$$

and the concentration ratio is

$c / c_{0}=\exp \left(-\frac{(0.053)(0.2)}{5.37 \times 10^{-4}}\right)=e^{-39.7}$

$c / c_{o}=2.7 \times 10^{-9}$.

Fven with extremely tine leaks the concentration ratio is lower by more than 8 orders of magnitude. The conclusion to be drawn is that the cable seal syitem will be effective as long as there is negative air pressure within the containment.

Installed cable seals will cost between $\$ 5$ and $\$ 6$ per cable, piving a total cost of $\$ 115000$ to $\$ 144000$ for the complete torus.

\section{REFERENCES (Appendix II)}

1. J. W. Dailey and D. R. F. Harleman, Fluid Dynamics (Addison Wesley, 1966).

2. D. F. Gray, Ed., American Institute of Physics Handbook (McGraw-Hill, 1972). 